\title{
Englischsprachige Literatur im World Wide Web
}

\author{
Dissertation \\ zur Erlangung des philosophischen Doktorgrades \\ der Philosophischen Fakultät \\ der Georg-August-Universität Göttingen
}

\author{
vorgelegt von \\ Angela Ella Schröder \\ aus Kassel
}

Göttingen 2004 


\section{Inhaltsverzeichnis}

I. Einleitung 1

1. Der Literaturbetrieb und die neuen Medien - Mesalliance oder Synergie? .... 1

2. Die Anfangsjahre - Zeit der Prognosen..................................................... 7

3. Der Stand zu Beginn des 21. Jahrhunderts ..................................................... 15

4. Zielsetzung und Methodik .................................................................... 21

II. Arbeitstechnische, rechtliche und dokumentarische Probleme 26

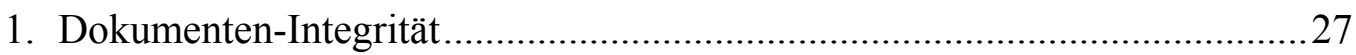

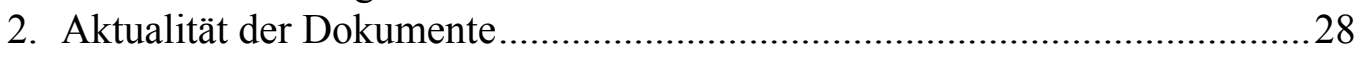

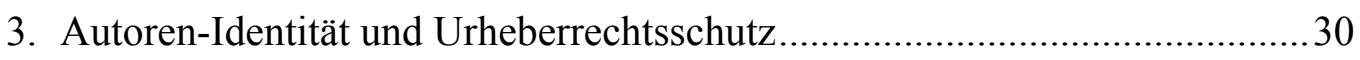

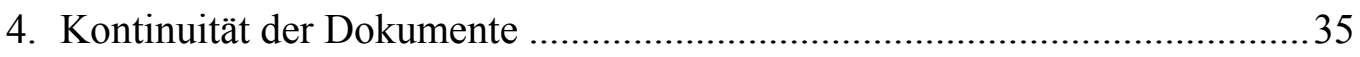

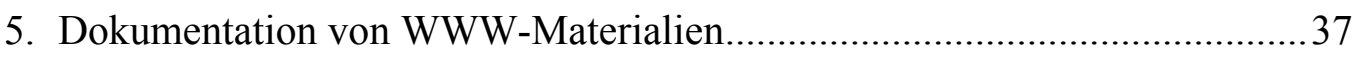

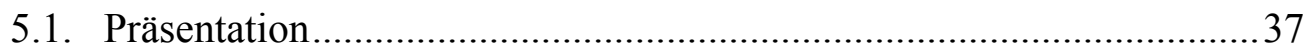

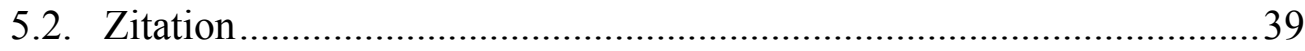

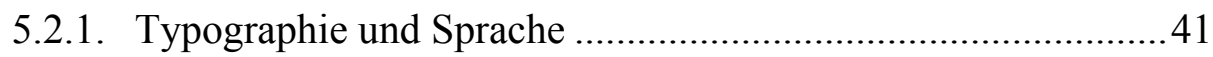

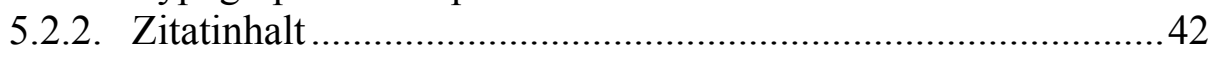

5.2.3. Neue Zitierrichtlinien für literaturwissenschaftliche Arbeiten.....45

III. Die Begriffsproblematik -

Übersicht, Diskussion und Festlegung $\quad 50$

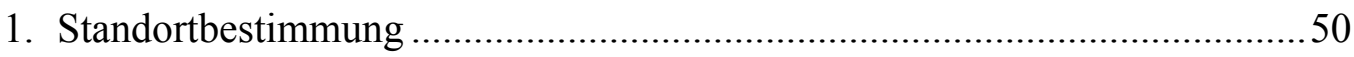

1.1. Online-Literatur zwischen Tradition und Moderne ............................53

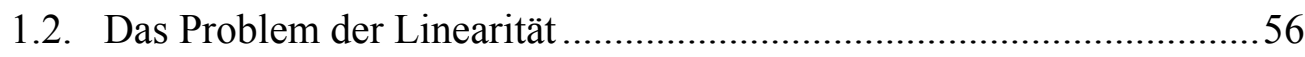

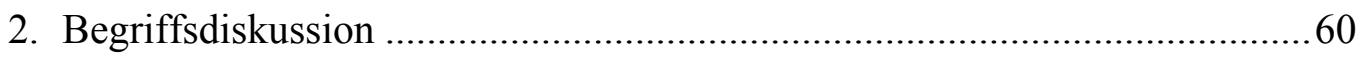

2.1. "Textwucherungen, Rhizome, Konglomerate" .................................61

2.2. Grundsätzliche Tauglichkeit des Begriffes Literatur...........................66

3. Abstimmung auf die Besonderheiten des WWW ........................................69

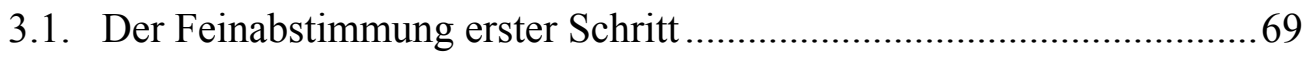

3.2. Der Feinabstimmung zweiter Schritt ............................................. 73

IV. Formen webunabhängiger digitaler Literatur im WWW 75

1. Konventionelle, linear-konzipierte Literatur im Web (Literatur im Web) ....78

1.1. Digitalisierte Druckwerke in Datenbanken und Archiven................... 78

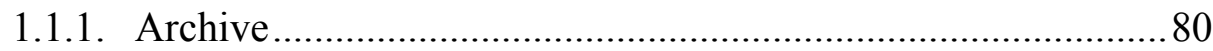

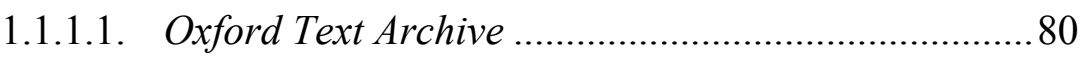

1.1.1.2. Alex Catalog of Electronic Texts ............................. 81

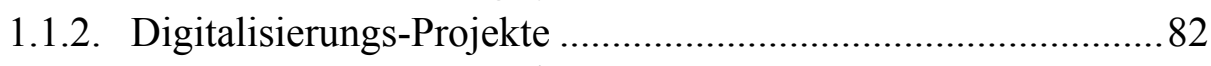

1.1.2.1. Project Gutenberg ................................................ 82

1.1.2.2. Bartleby.com: Great Books Online ...........................8 84

1.1.2.3. Victorian Women Writers Project ............................. 85

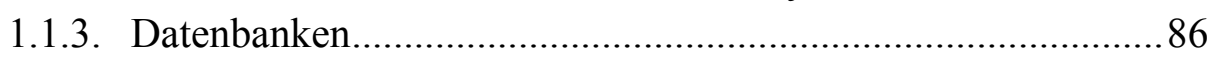

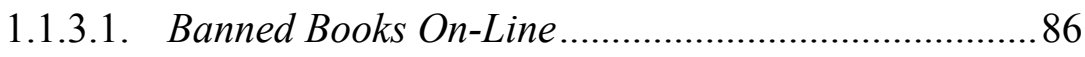

1.1.3.2. Prize-Winning Books On-Line .................................. 87 
1.2. Analoge Publikationen von Monographien, Periodika und Einzelwerken .87

1.3. Linear-konzipierte, in digitaler Form erzeugte zeitgenössische Werke ... 90

1.4. Drucktexte im Web - Cui bono? .92

2. Computergestützte, webunabhängige Literatur im Web (Computergestützte Literatur im Web).... .93

2.1. Programmgesteuerte Literatur ohne Interaktionsmöglichkeiten Dargestellt am Beispiel von "easter offering"

2.2. Programmgesteuerte Literatur mit Interaktionsmöglichkeiten Dargestellt am Beispiel des Heretical Rhyme Generator. 96

2.3. Geschlossene Hypertextsysteme Dargestellt am Beispiel von The Benefactor 97

2.4. Computergestützte Literatur - Spielerei oder Bereicherung? ..... 100

V. Das Phänomen Hypertext - ein Exkurs

1. Strukturierung von Hypertexten 104

2. Inhaltliche Gestaltung - Auf dem schmalen Grat zwischen Leselust und Lesefrust. 107

2.1. Anforderungen an die literarische Arbeit ......................................... 107

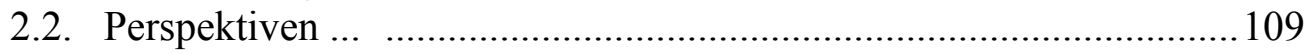

2.3. ... und Grenzen des Hypertextes ........................................................ 110

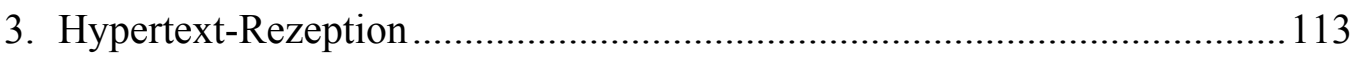

3.1. Formen der konzeptionsentsprechenden Rezeption ......................... 113

3.2. Entfaltungsmöglichkeiten des Nutzers .............................................. 116

3.2.1. Interaktivität .................................................................. 116

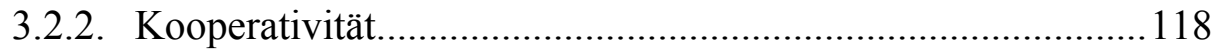

3.3. Text - Kontext - Hypertext ................................................ 120

VI. Webbasierte Literatur Analyse und Bewertung anhand konkreter Fallbeispiele 123

1. Webbasierte Prosa................................................................................... 127

1.1. Ausgewählte Beispiele webbasierter Prosa ....................................... 129

1.1.1. Mahoney the Cat ................................................................. 129

1.1.1.1. Präsentation ...........................................................130

1.1.1.2. Analyse und Auswertung ......................................... 139

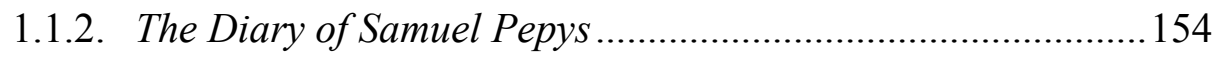

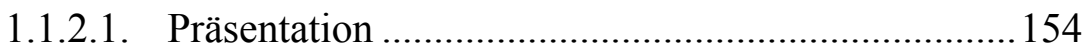

1.1.2.2. Handhabung des Hypertextes................................ 164

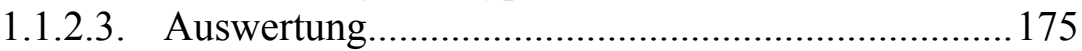

1.1.2.4. Verbesserungsansätze.......................................... 181

1.2. Prosa \& WWW - Zettels Albtraum

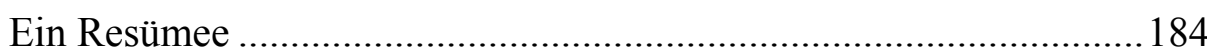

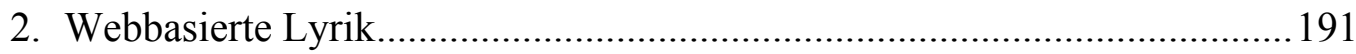

2.1. Vorstellung dreier Foren zur webbasierten Lyrik.............................. 192

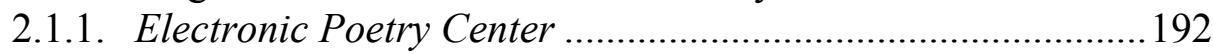

2.1.2. Prosebush-Collaborative Fiction Community....................... 196

2.1.3. Interactive Poetry Pages ...................................................... 198 
2.2. Ausgewählte Beispiele webbasierter Lyrik ...................................202

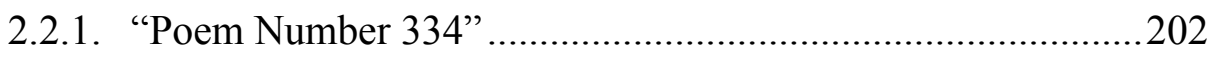

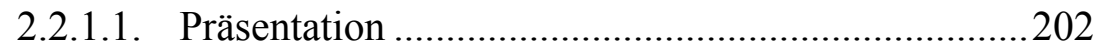

2.2.1.2. Analyse und Auswertung ....................................204

2.2.1.3. Verbesserungsansätze.........................................209

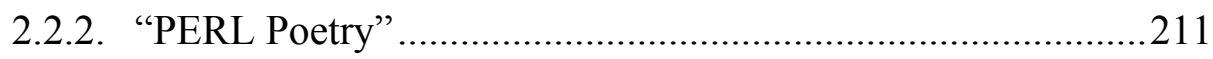

2.2.2.1. Präsentation ........................................................ 211

2.2.2.2. Analyse und Auswertung ......................................2. 217

2.2.2.3. Verbesserungsansätze ...........................................222

2.2.3. The Ultimate Writer ........................................................... 223

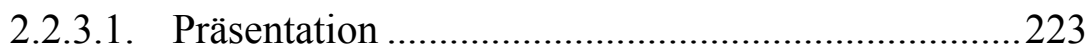

2.2.3.2. Analyse und Auswertung ....................................2239

2.2.3.3. Verbesserungsansätze ..........................................243

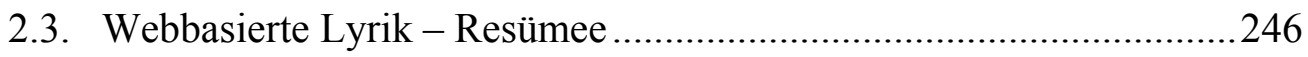

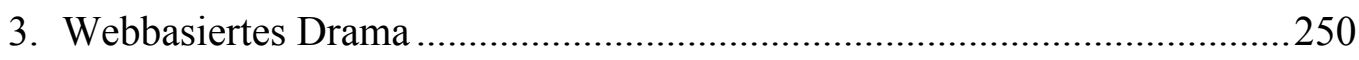

3.1. Ausgewählte Beispiele des webbasierten Dramas............................ 252

3.1.1. The Company Therapist ...................................................252

3.1.1.1. "Production \& Direction".......................................253

3.1.1.2. “Graphics \& Design"............................................264

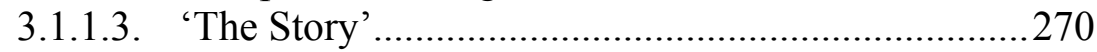

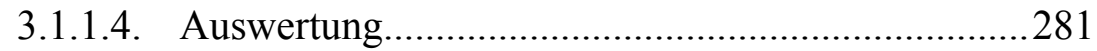

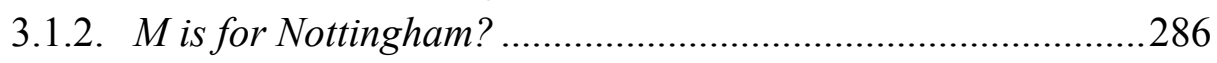

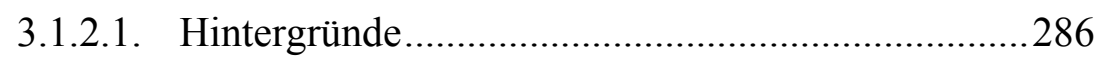

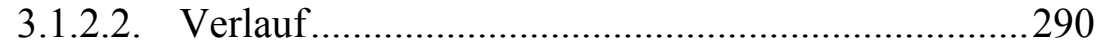

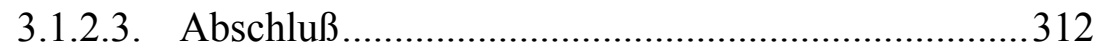

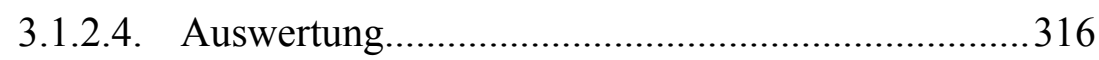

3.2. Webbasiertes Drama - Resümee …................................................ 319

VII. Die Lage der englischsprachigen Literatur im World Wide Web 322

1. Vom allgemeinen Nutzen der neuen Technik für die englischsprachige

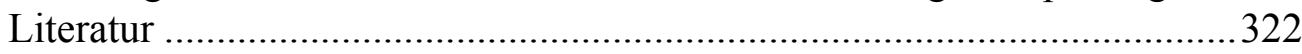

2. Die gegenwärtige englischsprachige Literatur im WWW kritisch gesehen...324

3. Die besondere Situation der webbasierten englischsprachigen Literatur, deren Ursache und Überwindung

3.1. Von der unterschiedlichen Grundeinstellung zu Hypertext und Buch.. 331

3.3. Ein leidenschaftlicher Spieler und mehr -

Oder: Über die Minimalqualifikationen eines erfolgreichen Autors .. 336 


\section{Einleitung}

\section{Der Literaturbetrieb und die neuen Medien - Mesalliance oder Synergie?}

It is highly improbable that fictional joint ventures on the Net will ever be anything other than amusing projects of average quality. Some of the hyperstories written by individual authors [...] already explore the possibilities of hypertext fiction in a convincing way. But the new medium's congenial artist, I think, is yet to be born. ${ }^{1}$

Es war Rudolf Freiburg, Professor für Anglistische Literaturwissenschaft, der sich im Jahre 1996 anläßlich einer Konferenz zum Thema Anglistik im Internet mit den obigen Worten zur Werthaltigkeit literarischer Hypertexte äußerte und eine eher verhaltene Prognose hinsichtlich der Entwicklung schriftstellerischer Gemeinschaftsproduktionen abgab. Tatsächlich ist nach nunmehr einigen Jahren Erfahrung mit Hypertexten in elektronischen Medien festzustellen, daß ihn offensichtlich noch kein Autor zu erschaffen vermochte, den sogenannten "Online-'Ulysses'”, jenes "hypermediale Großwerk, das seinen staunenden Lesern, Betrachtern und Hörern 24 Stunden Erlebniszeit anbietet". ${ }^{2}$ Im Jahre 1997 bereits brachte Hermann Rotermund den aktuellen Sachverhalt mit dieser Äußerung in treffender Weise auf den Punkt und ergänzte seine Erkenntnis anschließend noch durch jene folgerichtige Forderung: "Gesucht wird ein originäres Kunstwerk, das Wellen schlägt wie seinerzeit 'Das [sic!] Leiden des jungen Werthers', 'Madame Bovary' oder eben 'Ulysses'”.3 Geraume Zeit später setzte sich auch Auer mit der Frage nach dem Ergebnis der Verbindung von Literatur und neuen Medien auseinander: "Nach nur drei Jahren also Katzenjammer?

1 Rudolf Freiburg, "Postmodernism on the Internet", in: Doris Feldmann, Fritz-Wilhelm Neumann und Thomas Rommel, Hg., Anglistik im Internet. Proceedings of the 1996 Erfurt Conference on Computing in the Humanities (Heidelberg, 1997), 183-200, hier: 197.

2 Hermann Rotermund, "Warten auf den Online-Ulysses: Über das Problem des Internet, eine eigene Kunstform zu finden”, Die Zeit 41 (1997), 78. - Im Zusammenhang mit dem World Wide Web kommt dem Wortbestandteil Hyper- entscheidende Relevanz zu. Im Jahre 1965 wurde der Terminus Hypertext von Ted Nelson geprägt, der die Entwicklung eines Datenverwaltungs- und Präsentationssystems vermutlich am deutlichsten vorangetrieben hat. Siehe Rainer Kuhlen, Hypertext: Ein nicht-lineares Medium zwischen Buch und Wissensbank (Berlin; Heidelberg, 1991), 69. Hypertext-Systeme dienen zur Verwaltung von Informationseinheiten, die durch sogenannte Links miteinander verbunden sind. Im Hinblick auf die Online-Literatur erweist sich die zentrale Bedeutung dieser Systeme für den Nutzer in Möglichkeiten wie globalem Zugriff, Interaktivität und Kooperativität. Detailliertere Ausführungen zum Thema Hypertext folgen in Kapitel V. dieser Arbeit.

3 Rotermund, "Warten auf den Online-Ulysses", 78. 
Literatur und Internet eine Mesalliance?"4 Sicher nicht. Auch wenn die englischsprachige Literatur bislang noch nicht durch digitale Werke bereichert werden konnte, die auf ihre Art annähernd mit den oben erwähnten gedruckten Publikationen vergleichbar wären, so hat die Integration des Computers in den Literaturbetrieb doch zu ebenso fortschrittlichen wie nachhaltigen Veränderungen in der Literaturpräsentation und -rezeption geführt. Innerhalb der letzten Jahre hat sich das Internet zu einem vielversprechenden, ja zukunftsweisenden Medium entwickelt. Es werden ihm Eigenschaften zugeschrieben, die ebenso einschneidende und bahnbrechende Auswirkungen haben sollen wie seinerzeit der Buchdruck mit beweglichen Lettern nach seiner Erfindung durch Johannes Gutenberg. ${ }^{5}$

Das Internet als das umfangreichste mehrerer weltweiter Computer-Netzwerke faßt Millionen von Computern in lokalen, nationalen und internationalen SubNetzwerken zusammen, die jeweils nach verschiedenartigen, teilweise eigenständigen Techniken funktionieren und durch spezielle Computersysteme zur Datenübertragung zwischen unterschiedlichen Netzwerken, sogenannte Gateways, miteinander verbunden sind. Daß unabhängig von den jeweiligen Hardware-Systemen dennoch ein Datenaustausch stattfinden kann, geht auf gemeinsame Protokolle zurück, die festlegen, in welcher Form die Nachrichten übermittelt werden sollen und welche Funktionen bei Fehlern in Kraft treten. ${ }^{6}$ Ein wichtiger Aspekt des Internet ist zudem, daß es dezentral aufgebaut ist. Sollte es zum Ausfall eines Netz-Teilbereiches kommen, bleibt die Funktion aller weiteren Bereiche gleichwohl erhalten.

Genau in diesem Gedanken hat das Internet seinen Ursprung: Der Wunsch nach einer stabilen Kommunikationsarchitektur, die nicht mit einem Schlag zerstört werden konnte, veranlaßte das amerikanische Forschungsministerium Ende der 50er

4 Johannes Auer, "Der Leser als DJ oder was Internetliteratur mit HipHop verbindet", in: Beat Suter und Michael Böhler, Hg., Hyperfiction. Hyperliterarisches Lesebuch: Internet und Literatur (Basel; Frankfurt a.M., 1999), 173-181, hier: 173. Auer allerdings beantwortet seine provokative Frage selbst umgehend mit einem definitiven Nein, räumt aber auch zugleich ein, die Internet-Literatur sei noch im Begriff, sich zu entwickeln und zu etablieren, und lasse daher nur vorläufige Aussagen zu. Ebd., 173.

5 Siehe Bill Gates (mit Nathan Myhrvold und Peter Rinearson), The Road Ahead (Rockland, MA, 1995), 13. Siehe auch Sven Birkerts, Die Gutenberg-Elegien: Lesen im elektronischen Zeitalter, übers. von Kurt Neff, (Frankfurt a.M., 1997 [The Gutenberg Elegies. The Fate of Reading in an Electronic Age (New York, 1994)]), 259.

6 Als Protokolle werden jene Vereinbarungen bezeichnet, nach denen die Datenübertragungen innerhalb des Internet ablaufen. Hauptsächlich handelt es sich dabei um zwei Protokolle, die allgemein unter der Abkürzung TCP/IP zusammengefaßt werden. TCP bedeutet Transmission Control Protocol, das heißt dieses Protokoll regelt den Datentransport im Internet; IP steht für Internet Protocol und bedeutet, daß dieses Protokoll die Zustellung der Daten steuert. Bernhard Koring, Lernen und Wissenschaft im Internet: Anleitungen und Reflexionen zu neuen Lern-, Forschungs- und Beratungsstrukturen, (Bad Heilbrunn, 1997), 45. 
Jahre zur Gründung der Behörde Advanced Research Projects Agency, kurz ARPA. Im Rahmen der Förderung innovativer Projekte wurde ab 1964 eine Reihe von Forschungsvorhaben auf dem Gebiet der Computer-Netzwerke durchgeführt, woraus im Jahre 1967 die Gründung des sogenannte Arpanet resultierte, das Ende 1969 mit vier Rechnern an unterschiedlichen amerikanischen Universitäten in Betrieb ging. Die Zahl der Knoten nahm in der Folge rasch zu, und neben dem Arpanet entstanden weitere eigenständige Netzwerke, deren Verknüpfung trotz der technischen und organisatorischen Verschiedenartigkeit im Laufe der Zeit realisiert werden konnte. Im Jahre 1986 ging das heute bekannte Internet offiziell aus dem Arpanet hervor und findet seither global eine kontinuierlich zunehmende Verbreitung. ${ }^{7}$

Die entscheidende Veränderung der Präsentationsform des Internet erfolgte ungefähr Mitte der neunziger Jahre durch Installation der wohl bekanntesten und anwenderfreundlichsten Benutzeroberfläche, des Informationssystems World Wide Web (WWW bzw. Web), das Farbe und Animation ermöglichte und damit den Bedürfnissen und Träumen des Menschen in der digitalen Welt näher kam. In seiner Eigenschaft als Hypertext-Dienst präsentiert und verwaltet es assoziativ verknüpfte Informationen textueller, graphischer und audiovisueller Art, was durch die Programmiersprache Hypertext Markup Language (HTML) ermöglicht wird, in der alle Dokumente des World Wide Web verfaßt sind. Über den sogenannten Uniform Resource Locator, kurz $U R L^{8}$, einen Adreß-Schlüssel, der unter anderem Angaben über die

7 Andreas Grandel, "Internet - Grundlagen und Anwendungen”, in: Gudrun Gross, Uwe Langer und Rudolf Seising, Hg., Studieren und Forschen im Internet: Perspektiven für Wissenschaft, Wirtschaft, Kultur und Gesellschaft (Frankfurt a.M., 1997), 51-67, hier: 52f. Martin Rost und Michael Schack, Hg., Der Internet-Praktiker: Referenz und Programme (Hannover, 1995), 50f. Klaus Lipinski, Hg., Lexikon der Datenkommunikation: 2.000 Begriffe von ATM bis Z-Modem3.500 Abkürzungen, 4., aktual. und erw. Aufl. (Bonn, 1996 ['1994]), 271f. Niels Klußmann, Lexikon der Kommunikations- und Informationstechnik: Telekommunikation, Internet, Mobilfunk, Multimedia, Computer, E-Business, 3., neu bearb. und stark erw. Aufl. (Heidelberg, 2001 [1997]), 494f.

8 Die Abkürzung $U R L$ hat sich mittlerweile auch im deutschen Sprachgebrauch etabliert. Als einzige Übersetzungen werden in einschlägigen Online-Wörterbüchern URL-Adresse oder InternetAdresse aufgeführt, wobei URL-Adresse nur eine Aneinanderreihung bedeutungsnaher Begriffe darstellt. Die Bezeichnung Internet-Adresse umschreibt die Eigenschaft eines URL verständlich und prägnant, soll aber in vorliegender Arbeit aufgrund der thematischen Beschränkung auf das World Wide Web keinen Gebrauch finden. Statt dessen wird die Abkürzung URL verwendet werden, allerdings nicht, wie zum Beispiel bei PONSline üblich, mit femininem, sondern in Übereinstimmung mit DicData mit maskulinem Genus. Ernst Klett Sprachen GmbH, Hg., PONSline Das Online-Wörterbuch, <http://www.pons.de/>, cd-rom-produktion, Hg., DicData Wörterbuch Deutsch-Englisch, $<$ http://www.dicdata.de/>. 
Speicheradresse und das Übertragungsverfahren enthält, ist jedes Dokument eindeutig identifizierbar. ${ }^{9}$

Nachdem der Siegeszug des WWW und die verbreitete Nutzung der elektronischen Post $^{10}$ in der ersten Phase der Etablierung des Internet vor allem von den naturwissenschaftlichen Disziplinen getragen worden waren ${ }^{11}$, fanden nach einiger Zeit schließlich die Literatur und sukzessive auch die Literaturwissenschaft ihren Weg in das weltumspannende Datennetz. Das Online-Medium wurde alternativ zum Buchdruck zunehmend zur Verbreitung literarischer Publikationen genutzt. Der in diesem Zusammenhang verwendete Begriff des Elektronischen Publizierens oder electronic publishing entstand vermutlich im Frühjahr 1977 anläßlich einer Konferenz des amerikanischen Institute of Graphic Communication. ${ }^{12}$ Zum heutigen Zeitpunkt scheint man in Literatur- und Literaturwissenschaftsbetrieb die Notwendigkeit der Integration von Literatur in die elektronischen Medien erkannt zu haben, wobei allerdings ein größerer Zuspruch hinsichtlich der Nutzung, vor allem aber der Bereitstellung digitaler Forschungsmaterialien nach wie vor durchaus wünschenswert wäre. ${ }^{13}$ Das World Wide Web stellt nämlich mit hoher Wahrscheinlichkeit das

9 Ein $U R L$ ist folgendermaßen aufgebaut: $<$ Übertragungsverfahren $>: / /<$ Rechneradresse $>/<$ Verzeichnis $>/<$ Dateiname $>$. $<$ Dateityp $>$. Das Beispiel der Abteilung für Neuere Englische Literatur des Seminars für Englische Philologie der Georg-August-Universität zu Göttingen verdeutlicht den Charakter eines URL: <http://www.gwdg.de/ goeengl/nel.htm>. Die WWW-Seiten der Seminar-Abteilung werden mit Hilfe eines standardisierten Verfahrens zur Übertragung von HTML-Dokumenten, dem Hypertext Transfer Protocol (HTTP) gesendet; sie sind auf dem Rechner der Gesellschaft für wissenschaftliche Datenverarbeitung Göttingen gespeichert; über das Verzeichnis des Seminars für Englische Philologie gelangt man an jenes der Literatur-Abteilung; der Dateiname lautet 'nel', wobei es sich um eine html-Datei handelt.

10 Nachfolgend wird diese Form der Nachrichtenübermittlung auch synonym als E-Mail (Abkürzung für Electronic Mail) bezeichnet werden.

11 Vgl. Doris Feldmann, Fritz-Wilhelm Neumann und Thomas Rommel, "Introduction: Hanging in/on to the Net", in: Dies., Hg., Anglistik im Internet, i-iii, hier: i.

12 Joost Kist, Elektronisches Publizieren: Übersicht, Grundlagen, Konzepte, bearb. u. hrsg. Manfred Krüger (Stuttgart, 1988), 7. Kist macht unter anderem den Vorschlag, elektronisches Publizieren als "die Herausgabe einer geschriebenen Arbeit mit Hilfe elektronischer Mittel (insbesondere Computer), sei es direkt oder über ein Kommunikationsnetzwerk", zu definieren. Ebd., 8. Diese Beschreibung erscheint einfach, aber ausreichend und soll unter Beschränkung auf das WWW als Grundlage für alle nachfolgenden Ausführungen dienen.

13 Diese Tatsache wirft nach Auffassung der Autorin vorliegender Arbeit die Frage auf, warum gerade Geisteswissenschaftler den Computer in so vergleichsweise geringem Maße nutzen. Die Antwort mag unter anderem in dem unterschiedlichen Aktualitätsdruck der einzelnen Wissenschaftszweige begründet liegen, innerhalb derer die Naturwissenschaften in höherem Maße auf aktuelle Untersuchungsergebnisse angewiesen sind. So vermag in der Medizin die rasche Kenntnisnahme neuester Forschungsresultate die Entwicklung lebensrettender Medikamente zu beschleunigen. Winter scheint ähnliche Gedankengänge vollzogen zu haben, bezeichnet er die Naturwissenschaften in seinen Ausführungen zu Inhalt und Zielen der Literaturwissenschaft doch als lebenswichtige Wissenschaften. Siehe Helmut Winter, Literaturtheorie und Literaturkritik (Düsseldorf, 1975), 11. 
Publikations- und Kommunikationsmedium der Zukunft dar. ${ }^{14}$ Auch wenn es in seiner gegenwärtigen Konstellation wissenschaftlichen Maßstäben noch nicht gerecht werden kann ..., "in der derzeitigen Phase der deutschen Anglistik sollte man sich auch für Impulse dankbar zeigen, die noch nicht in letzter Konsequenz systematisiert und festgeschrieben worden sind". ${ }^{15}$ Indes sind wir noch weit davon entfernt, jeden Literaturbeflissenen automatisch als "aktiven, navigierenden, surfenden, mit allen chattenden Angehörigen der Cyberkultur"16 anzusehen. ${ }^{17}$ Bereits im Jahre 1996 hatten Botz und Sprengnagel eindringlich davor gewarnt, sich den digitalen Technologien zu verschließen:

Wer sich jedoch heute abkoppelt, muß damit rechnen, daß morgen die globalen Informationsflüsse an einer vorelektronischen 'Insel der Seligen' vorbeilaufen oder erst verspätet erreichen. Dies würde die Peripherien vollends zum kulturellen Hinterland jener Zentren degradieren, an denen ohnehin der Großteil der elektronischen Produkte erzeugt werden und wo auch die kulturelle Definitionsmacht liegt. ${ }^{18}$

Nur durch Anschluß an die technologische Entwicklung kann die Aussage des einstigen US-Vizepräsidenten Al Gore Realität werden, das globale digitale

14 Bill Gates äußerte bereits im Jahre 1995, daß die Datenautobahn bald unverzichtbar sein werde: "The highway will be indispensible because it will offer a combination of information, education services, entertainment, shopping and person-to-person communication." Gates, The Road Ahead, 103. Der Begriff Datenautobahn beziehungsweise Information Highway oder gar Information Superhighway entstand in Anlehnung an das Projekt der Vernetzung der USA durch Bundesautobahnen und wurde schließlich zur feststehenden Redewendung durch Al Gore, dessen Vater den Federal Aid Highway Act im Jahre 1956 unterstützt hatte. Fälschlicherweise führt die Metapher der Autobahn zu Assoziationen wie Weite und Distanz, welche ja mittels der neuen Technologie gerade aufgehoben werden sollen. Ebd., 7.

15 Neumann, "Geisteswissenschaften im Internet", 55.

16 Gudrun Gersmann, "Schöne Welt der bunten Bilder: Kritische Anmerkungen zur Geschichtsdarstellung in den Neuen Medien”, in: Claudia Gemmeke, Hartmut John und Harald Krämer, Hg., Euphorie digital? Aspekte der Wissensvermittlung in Kunst, Kultur und Technologie (Bielefeld, 2001), 105-119, hier: 109.

17 Ein positives Signal ist das Ergebnis einer Studie der Stiftung Lesen, wonach die bundesdeutsche Bevölkerung mit Bildungsabschluß Abitur oder Studium den Computer zu 76 Prozent nutzt und damit zur zentralen Nutzergruppe zählt. Hans-Jürgen Hippler, "Tummelplatz Internet oder: Ist Lesen eine veraltete 'Technologie'?”, in: Stiftung Lesen, Hg., Leseverhalten in Deutschland im neuen Jahrtausend: eine Studie der Stiftung Lesen (Hamburg, 2001), 165-174, hier: 167.

18 Gerhard Botz und Gerald Sprengnagel, "Zeitgeschichte als Multimedia-Geschichte und Hi-TechHistory - Allgemeine Überlegungen zur Implementierung an österreichischen Universitäten”, Zeitschrift für Hochschuldidaktik 1-2 (1996), <http://www.oeghd.or.at/zeitschrift/1996h1-2/16 art.html>, 24.06.2002. - Die Zitierform der bibliographischen Angaben wird in Kapitel II.5.2.3. ausführlich erläutert werden. An dieser Stelle soll nur angemerkt werden, daß sie aus der Überarbeitung der existierenden Zitationsrichtlinien und deren Anpassung an die vorliegender Arbeit zugrundeliegenden Richtlinien für die Anfassung wissenschaftlicher Arbeiten entstanden ist. 
Netzwerk erlaube es, auch von dem "entlegensten Dorf aus in den modernsten Bibliotheken zu stöbern"19.

Angesichts seiner Multifunktionalität verwundert es nicht, daß das World Wide Web nicht allein als technisches Hilfsmittel dient, sondern auch für kreative Zwecke verwendet wird, die vorerst mit dem Begriff Internet-Literatur belegt werden sollen. Mit einem Rekurs auf Suter, der mit der Bezeichnung "Event-ualität" Vielzahl von künstlerischen Phänomenen des WWW erfaßt, soll diese andere Seite des elektronischen Mediums eingeführt werden.

Suter bedient sich des Wortspiels 'Event-ualität', um sprachschöpferisch darzulegen, was Online-Literatur eigentlich ausmacht: “'Event-ualität' heisst Kunst mit Ereignischarakter, Geschwindigkeit, Rastlosigkeit, Punktualität, dauerndes Weitergehen, Aufmerksamkeit, Bühne, Performanz, Spiel."21 Und 'Event-ualität' heißt eben auch Online-Literatur, denn genau diese Bestimmungen sollte ein für das World Wide Web konzipiertes Werk erfüllen: Es sollte einzigartig, unmittelbar, dauerpräsent, interaktiv und kooperativ sein. Es ist nicht das Ergebnis einer Anpassung existierender Dokumente an eine neue Entwicklung, vielmehr verkörpert es eine weiterentwickelte Form der Literatur durch Autoren, die sich ganz bewußt für das World Wide Web als Vermittlungsweg für ihre Werke entschieden haben.

Mit dem Terminus 'Event-ualität' versucht Suter auszudrücken, was sich aufgrund seiner Vielseitigkeit und Mehrschichtigkeit per definitionem einer knappen Beschreibung eigentlich entzieht. So ließe sich seine Liste an Eigenschaften vermutlich um viele weitere Punkte ergänzen, doch ergeben auch die genannten Merkmale bereits ein recht anschauliches Charakterbild: Online-Literatur zeigt Suter zufolge

19 Miriam Meckel, "Schleusenwärter in Digitalien", Zeit-Punkte 5: Der Mensch im Netz: Kultur, Kommerz und Chaos in der digitalen Welt (1996), 64. Diese Vision wird auch von Gates favorisiert: "The highway is going to give us all access to seemingly unlimited information, anytime and anyplace we care to use it." Gates, The Road Ahead, 278. Im Zusammenhang mit dieser Vorstellung eines weltumspannenden Datennetzes fällt immer wieder der Begriff des sogenannten globalen Dorfes. Daß der Zugriff auf die Informationen dieser Welt dank des Datennetzes vereinfacht wird, hält Zimmer durchaus für realistisch, allerdings in der modifizierten Form, daß nicht die Welt zu einem einzigen großen Dorf wird, sondern die Informationsgesellschaft sich vielmehr in zahlreiche einzelne, über die Welt verteilte kleine Dörfer segmentiert. "Statt des einen globalen Dorfs viele global verteilte Dörfer.” Zimmers Ansicht nach wird keine Informationsgleichheit herrschen, sondern die modernen Medien werden vielmehr ausschließlich Privilegierten zur Verfügung stehen: "Die Information teilt die Welt noch einmal in Habende und Habenichtse und verbreitert so die Kluft, die zwischen ihnen sowieso besteht." Dieter E. Zimmer, Die Bibliothek der Zukunft: Text und Schrift in den Zeiten des Internet, 3. Aufl. (Hamburg, 2000 ['2000]), 40f.

20 Beat Suter, "Ein neues Literaturmilieu (zwischen Transfugalität und 'Event-ualität')", in: Johannes Auer, Christiane Heibach und Beat Suter, Hg., netzliteratur.net: Netzliteratur - Internetliteratur Netzkunst ([30.11.2000]), <http://www.netzliteratur.net/suter/kassel.htm>, 07.07.2003.

21 Ebd., 07.07.2003. 
unter anderem "Experimentalität, [...] künstlerischen Gestaltungswillen, [...] Hybridität, [...] narrative Eigenräumlichkeit, [...] Nichtendgültigkeit, [...] Streben nach Interaktivität." ${ }^{22}$ Es läßt sich also feststellen, daß Online-Literatur ein intensives, weitgehend individuelles Lese- und Gestaltungserlebnis bescheren und sich somit durchaus als Event präsentieren kann. ${ }^{23}$

$\mathrm{Zu}$ Beginn des Kapitels war Auer mit der provozierenden Frage zitiert worden, ob das Zusammengehen von Literatur und Internet eine Mesalliance bedeute. Folgt man Suter und seiner Kennzeichnung von Online-Literatur als 'Event-ualität', läßt sich Auers Frage unschwer beantworten: Bei kreativer Ausschöpfung aller Möglichkeiten des Web vermögen Literatur und neue Medien beträchtliche Synergieeffekte zu entwickeln; von einer Mesalliance kann in diesem Falle keine Rede sein.

\section{Die Anfangsjahre - Zeit der Prognosen}

Der Prozeß der graduellen Anerkennung und zunehmenden Nutzung elektronischer Medien und vor allem des World Wide Web, der in Deutschland ungefähr im Jahre 1996 verstärkt einsetzte, ${ }^{24}$ nahm anfänglich einen sehr ambivalenten Verlauf. Die überwiegende Akzeptanz, die man den neuen Medien zum heutigen Zeitpunkt

22 Ebd., 07.07.2003.

23 Grundsätzlich ist unter einem Event ein besonderes kulturelles Ereignis zu verstehen. Ein Event präsentiert sich als eine aus dem Alltäglichen speziell herausgehobene Veranstaltung, da er sich gewissermaßen als eine Steigerung des Besonderen von einer konventionellen kulturellen Darbietung unterscheidet. Eine Filmvorführung beispielsweise kann als eine aus dem Alltag herausragende Vorstellung angesehen werden, ist aber nicht zwangsläufig auch ein Event. Um als solcher zu gelten, müßte sich die Aufführung durch zusätzliche, außergewöhnliche Elemente der Darbietung oder Publikumsintegration hervorheben. Entsprechend dieser Definition bringt Suter die Bezeichnung Event in die allgemeine Diskussion über Internet-Literatur ein, nämlich um ein künstlerisches Phänomen der ganz besonderen Art zu beschreiben. Allgemein wird dieses Wort allerdings gegenwärtig gerne und (zu oft) regelmäßig dann gebraucht, wenn ein Gastgeber den Anspruch erhebt, seinen Gästen eine ausgefallene, zumeist künstlerische, unkonventionelle, dem Zeitgeist entsprechende, gleichzeitig aber auch stilvolle Veranstaltung zu bieten.

24 Die Datierung auf das Jahr 1996 rechtfertigt sich damit, daß die Debatte über die Integration der Computertechnik in den Wissenschaftsbereich zu dieser Zeit verstärkt einsetzte, wenngleich den neuen Medien in der englischsprachigen Literaturwissenschaft zunächst nur vergleichsweise geringe Beachtung zukam. Obwohl in Deutschland die technischen Voraussetzungen für ihre Nutzung gegeben waren, verspürten Glotz und Thomas im Jahre 1994 eine deutliche, generelle Zurückhaltung seitens der Wissenschaften, die sie nach einem Vergleich mit der Aufgeschlossenheit in den USA zu der Erkenntnis führte, die Deutschen bemerkten zwar ebenso wie die Amerikaner, daß die Menschen des 21. Jahrhunderts anders sein würden als die Menschen des 20. Jahrhunderts, sähen aber in dieser Entwicklung weniger Chancen als vielmehr Risiken: "Sie fürchten sich." Peter Glotz und Uwe Thomas, Das dritte Wirtschaftswunder. Aufbruch in eine neue Gründerzeit (Düsseldorf; New York, 1994), 230. Auch noch zwei Jahre später kritisierte Mengel unter besonderem Hinweis auf die Literaturwissenschaft die Zurückhaltung seitens der Forschung, das neue Medium als Arbeitsmittel anzunehmen. Ewald Mengel, "Drama- und Theaterressourcen im Internet", in: Feldmann, Neumann und Rommel, Hg., Anglistik im Internet, 157-182, hier: 179. 
zweifellos zusprechen darf, resultiert aus einer langwierigen, kontroversen Diskussion über Vor- und Nachteile der Computernutzung und deren mögliche Auswirkungen auf Literatur und Wissenschaft. So löste diese Thematik anfänglich eine starke Polarisierung von Befürwortern und Kritikern aus, die mit der Anwendung elektronischer Medien einerseits ungerechtfertigt hohe Erwartungen, andererseits übertriebene Befürchtungen assoziierten. ${ }^{25}$

Auf seiten der Befürworter herrschte teilweise eine regelrechte Euphorie, und der Computer entwickelte sich zu einer Art Mythos, dessen Allmachtspotential die illusorische Hoffnung nährte, der PC sei der ultimative Schlüssel zur Erfüllung aller denkbaren - sowie noch nicht denkbaren - Möglichkeiten ${ }^{26}$. Dabei wurden die Visionen, die sich an den Computer-Einsatz knüpften, nur in seltenen Fällen von einer konkreten Vorstellung über ihre Umsetzung getragen. Vielmehr hielt sich ungebrochen der Glaube an den Beginn eines neuen Zeitalters, und alle damit assoziierten Wünsche gingen auf den einen Gedanken zurück, mit Hilfe des Computers sei grundsätzlich alles möglich. ${ }^{27}$ Verhältnismäßig ahnungslos, welche Ideen technisch überhaupt $\mathrm{zu}$ realisieren waren, ging man oftmals in unkritischer und geradezu naiver Weise davon aus, der Computer sowie alle damit verbundenen Einsatzmöglichkeiten, wie beispielsweise der Zugang zum WWW, führten - bildlich gesprochen - gleichsam automatisch in das Schlaraffenland der Literatur, in dem es lediglich gelte, alle nur denkbaren literarischen Schätze in doppeltem Wortsinne von den Bildschirmseiten zu 'lesen'.

Charakteristisch für derartige Visionen ohne konkrete Konturen waren die Begleitumstände und Resultate der Internet-Literaturwettbewerbe der Wochenzeitung Die Zeit. Erstmals ausgerichtet im Jahre 1996, fand diese alljährliche Initiative bereits nach dem dritten Durchgang ihr Ende, ohne daß es innerhalb dieses Zeitraumes gelungen wäre, das Ziel der Veranstaltung, respektive den Begriff der Internet-Literatur,

25 Kaiser beschreibt diese Meinungsdivergenz mit folgenden Worten: "Den Tanz um das neue goldene Kalb führen aber beide Gruppen gemeinsam auf. Und beide Gruppen bestärken einander darin, dem, was da eventuell ist, eine imaginäre Übergröße und Übermacht anzudichten die einen, um es hochzujubeln, die anderen, um es zu verdammen." Reinhard Kaiser, Literarische Spaziergänge im Internet: Bücher und Bibliotheken online, 2. Aufl. (Frankfurt a.M., 1997 [1996]), 12.

26 Bezüglich jener noch nicht denkbaren Möglichkeiten findet sich bei Gates folgende treffende Äußerung: "The information highway will open undreamed-of artistic and scientific opportunities to a new generation of geniuses." Gates, The Road Ahead, 201.

27 Vgl. auch Claudia Gemmeke, Hartmut John und Harald Krämer, "Vorwort", in: Dies., Hg., Euphorie digital? Aspekte der Wissensvermittlung in Kunst, Kultur und Technologie (Bielefeld, 2001), 9-10, hier: 9. 
präzise und unmißverständlich $\mathrm{zu}$ formulieren. ${ }^{28}$ Ursächlich für diesen Mißerfolg scheint eben jene oben beschriebene, unreflektierte Faszination seitens der Initiatoren gewesen zu sein, die offensichtlich weder ausreichende Kenntnisse der gestalterischen Bedingtheiten des Mediums Internet besaßen noch genau wußten, was sie in diesem damals noch unbekannten Datennetz eigentlich zu finden gedachten. ${ }^{29}$ So mögen der Reiz, der von dieser neuen Technik ausging, und die Tatsache, daß das Thema der neuen elektronischen Medien - gerade auch im Zusammenhang mit Literatur - zu jener Zeit allgegenwärtig und vieldiskutiert war, die Auslöser für diese Veranstaltungen gewesen sein und die Ausschreibung von Internet-Literatur-Wettbewerben geradezu aufgedrängt haben. ${ }^{30}$

Der Gruppe der Computer-'Euphoriker’ gegenüber stand der Kreis jener Skeptiker und Kritiker, die den Computer-Einsatz als unheilvolle Bedrohung aller althergebrachten, elementaren geistigen und materiellen Werte empfanden. So war die Diskussion über die Konkurrenzfähigkeit der gedruckten Medien und den künftigen Stellenwert des Buches fester Bestandteil des Forschungskanons. “Ängste und Phantasien von der Vollstreckung des Todesurteils an der geschriebenen Literatur auf dem elektronischen Stuhl neuer Medien" ${ }^{\prime 1}$ mündeten in der Sorge, der Vorgang des Lesens werde sich dereinst nur noch auf bloße Zeichenerkennung am Bildschirm reduzieren. ${ }^{32}$ Die

28 Bezeichnenderweise unterlag der Titel der Zeit-Wettbewerbe nach dem zweiten Durchgang einem grundsätzlichen Wandel. Um "keine interessante, neue Tendenz auszuschließen", klammerten die Veranstalter den Begriff Literatur gänzlich aus und annoncierten die dritte Ausscheidung unter der Bezeichnung "Pegasus98”. Dirk Schröder, "Was kann die Literatur für das Internet tun?", Universitas: Zeitschrift für interdisziplinäre Wissenschaft 54 (1999), 282-292, hier: 290.

29 Ebd., 283. Die Arbeiten sollten unter Einbeziehung der Mittel des Internet erstellt, in sich geschlossen ohne Hyperlinks nach außen sein und die Größe von vierzig Kilobyte nicht überschreiten Ebd., 284. Nicht nur, daß unerläutert blieb, um welche Mittel des Internet es sich konkret handeln sollte, in Anbetracht der hohen Speicherkapazität, die eine Arbeit erfordert, sobald sie mittels multimedialer und interaktiver Elemente über das auf Papier reproduzierbare Maß hinausgeht, erscheinen vierzig Kilobyte als viel zu geringe Größe ohne Spielraum für Experimente. Zudem stellt sich die Forderung nach Abgeschlossenheit der Werke angesichts der gewünschten Einbeziehung des globalen Netzwerks Internet als ausgesprochen widersinnig und unreflektiert dar.

30 Der Befund "Irgendwie ist alles 'hyper' oder 'cyber'" ist bezeichnend für die damalige Situation in Deutschland. Er basiert auf der Aussage des Cyberpunk-Theoretikers Brooks Landon, der diese Entwicklung in den USA bereits im Jahre 1993 registriert hatte: "You know the list; top-heavy with 'de-' and 'post-' prefixes, it has recently grown fond of 'hyper-' and 'cyber-'anything." Siehe Ruth Mayer und Ernst-Peter Schneck, "Hyperkultur - die ganze Welt ist ein Text. Eine Einleitung", in: Martin Klepper, Ruth Mayer und Ernst-Peter Schneck, Hg., Hyperkultur: Zur Fiktion des Computerzeitalters (Berlin; New York, 1996), 1-13, hier: 4. Siehe auch Brooks Landon, “Hypertext and Science Fiction”, Science Fiction Studies 20 (1993), 449-456, hier: 449.

31 Klaus Modick, “Textnetzwerke. Prolegomena zu einer Literatur des Bildschirmtextes”, in: Ders., Hg., Das Stellen der Schrift. Essays (Siegen, 1988), 91-104, hier: 97.

32 Walter Müller, "Literatur im technisch-elektronischen Zeitalter", Merkur 402 (1981), 1270-1277, hier: 1277. - Die Frage nach dem aktuellen sowie nach einem möglichen künftigen Stellenwert des Buches wird an späterer Stelle noch eingehender behandelt werden. 
sogenannte Technikfolgenabschätzung ${ }^{33}$, das heißt die Analyse der Auswirkungen neuer Technologien, die üblicherweise spätestens zu dem Zeitpunkt vollzogen wird, an dem neue Techniken oder Medien und die dadurch entstehenden technischen und vor allem aber gesellschaftlichen Konsequenzen unübersehbar geworden sind, löste zahlreiche Mutmaßungen über die absorbierenden Eigenschaften des Computers aus. $^{34}$

Birkerts beispielsweise sah von der "vollelektronischen Zukunft"35 gleich mehrere entscheidende Bedrohungen ausgehen: ${ }^{36}$

a) "Spracherosion" 37

Birkerts' Ansicht nach geht die zunehmende Nutzung der elektronischen Kommunikation mit einem Niveauverlust der gesprochenen und geschriebenen Sprache einher. Die durch den Buchdruck geprägte vielschichtige Schriftkultur weiche einer telegrammstilartigen Ausdrucksform, die nurmehr als eine Art "Primitiv-Sprech"38 zu bezeichnen sei. Dabei gehe es nur noch um reinen Informationsaustausch bar jeglicher sprachlicher oder stilistischer Nuancen, der in einem Aussterben der Sprachgewandtheit münde: Ein eloquenter Mensch werde künftig gezwungen sein, sich sprachlich dem allgemeinen Primitivniveau anzupassen. Sprachstil werde somit langfristig nur noch in literarischen Klassikern weiterexistieren. Da diese dann jedoch aufgrund ihres vergleichsweise hohen Niveaus nicht mehr rezipiert werden könnten, werde "die zivilisatorische Energie einer Prosa [...] zwischen geschlossenen Buchdeckeln verpuffen"39.

33 Manfred Faßler und Wulf Halbach, "Vorwort", in: Dies., Hg., Geschichte der Medien (München, 1998), 7-15, hier: 8.

34 Daß Innovationen im Verlauf ihrer Integration in das Alltagsleben durchaus mit Skepsis betrachtet werden, ist nicht erst seit der Verbreitung der neuen Medien zu beobachten. So pflegen die von Pross als sogenannte "Kulturpessimisten" bezeichneten Kritiker gerne auf Platons Phaidros zurückzugreifen. Harry Pross, Der Mensch im Mediennetz: Orientierung in der Vielfalt (Düsseldorf; Zürich, 1996), 165. Auch Boesken erinnert mit ihrem Verweis auf diesen philosophischen Dialog daran, daß sich bereits Platon gegen die Schriftlichkeit ausgesprochen habe, da das menschliche Gedächtnis nicht mehr in ausreichender Weise gefordert und somit das Vergessen gefördert werde. Auch wenn es also schon im 4. Jh. v. Chr. eine Technikfolgenabschätzung gab, so ist der Vergleich doch wenig überzeugend, denn der mit der Einführung der Computermedien verbundene revolutionäre Effekt hat keineswegs das Ausmaß des Übergangs von Oralität zu Verschriftlichung. Gesine Boesken, "Lesen am Bildschirm: Wer ist 'drin', und sind Bücher jetzt 'out'?”, in: Stiftung Lesen, Hg., Leseverhalten in Deutschland im neuen Jahrtausend: eine Studie der Stiftung Lesen (Hamburg, 2001), 127-149, hier: 127.

35 Birkerts, Die Gutenberg-Elegien, 174.

36 Ebd., 174-178; 219f.

37 Ebd., 174.

38 Ebd.

39 Ebd., 175. 
b) "Verflachen der historischen Perspektiven"40

Eine ebenso negative Prognose erteilt Birkerts der allgemeinen Geschichtsauffassung. Die richtige Einschätzung historischer Tragweiten sei bei den Menschen nur in Form augenfälliger und greifbarer Massen von Büchern zu erzielen. Der Umstand, daß durch das weltumspannende Datennetz alle Informationen jederzeit - quasi mit Hilfe eines Knopfdrucks - verfügbar gemacht werden könnten, führe jedoch $\mathrm{zu}$ einem Verlust der räumlichen Vorstellungskraft und lasse damit das Gefühl für die historischen Dimensionen schwinden.

c) "Das Absterben des privaten Ich" 41

Ein weiterer Kritikpunkt betrifft die allmähliche Auflösung der Privatsphäre jedes Einzelnen. Nach Birkerts wird die technikbedingte Distanzverringerung zwischen den Menschen mit einer zunehmenden Veröffentlichung des persönlichen Bereichs einhergehen. Die jederzeitige Erreichbarkeit über ein immer enger werdendes Datennetz führe schließlich dazu, daß die Intimsphäre des Individuums nahezu ausgelöscht werde. ${ }^{42}$

d) "Grobschlächtigkeit"43

Der Mensch büße durch die zunehmende Verbreitung und Nutzung des Computernetzwerks nicht nur seine Sprachfähigkeiten, sein historisches Einschätzungsvermögen und sein Privatleben ein, vielmehr werde sein ästhetisches Empfinden zusätzlich durch unschöne Formatierungen gestört; nicht zuletzt müsse er sich im Umgang mit dem Computer auf den Entwicklungsstand eines Kleinkindes zurückbegeben: Unabhängig davon, wie bedeutungsvoll der damit durchgeführte Vorgang tatsächlich sei, habe der Umgang mit der Computer-Maus in jedem Fall hohe Ähnlichkeit mit einem Reflex-Test in einer Spielhalle.

Ähnlich Birkerts hatte auch Müller zu Beginn der achtziger Jahre die zunehmende Einbindung des Computers in das Arbeits- und Alltagsleben als eine sehr kritische Entwicklung angesehen, die zwar einerseits eine fortschrittliche und bemer-

40 Ebd.

41 Ebd., 177.

42 Als Leitbegriff hat sich in bezug auf die neuen (interaktiven) Medien die Bezeichnung Immersion etabliert. Immersive Medien integrieren den Nutzer als aktiven Teilnehmer in das Geschehen. Er taucht vollständig in den interaktiven Bereich ein und betritt damit quasi eine Bühne, "vor der es keine abgeschatteten Zuschauer mehr gibt”. Pierre Lévy, "Cyberkultur: Universalität ohne Totalität”, in: Stefan Bollmann und Christiane Heibach, Hg., Kursbuch Internet: Anschlüsse an Wirtschaft und Politik, Wissenschaft und Kultur (Reinbek bei Hamburg, 1998), 60-87, hier: 72.

43 Birkerts, Die Gutenberg-Elegien, 219. 
kenswerte Arbeitserleichterung für den Menschen darstelle, die gleichzeitig aber auch aufgrund fragwürdiger Begleitumstände - wie beispielsweise Unpersönlichkeit, Oberflächlichkeit und Vergänglichkeit - zu einer beunruhigenden Entmenschlichung zu führen drohe. ${ }^{44}$ Im Jahre 1988 vertrat Modick zwar eine den neuen Medien deutlich stärker zugewandte Position, gleichwohl hatte auch er Sorge, die neuen Medien könnten eine Bedrohung nicht nur für alle anderen existierenden Medien, sondern vor allem für unsere zwischenmenschlichen Beziehungen darstellen.

Im Jahre 1996 brachte Barth den neuen Medien eine nach wie vor kritische, aber doch wesentlich aufgeschlossenere Haltung entgegen. Er vertrat die Überzeugung, der Computer könne dem Nutzer neue, hilfreiche Möglichkeiten eröffnen, die allerdings - so fügte er einschränkend und gleichsam mahnend hinzu - weder Bücher noch Bibliotheken ersetzen könnten. ${ }^{45}$ Für Gauron stellte sich allerdings zur selben Zeit bereits nicht mehr die Frage, ob und in welchem Maße elektronische Medien eingesetzt werden sollten. In der festen Überzeugung, eine Zukunft ohne elektronische Medien sei weder denkbar noch möglich, befaßte sich der Wirtschaftswissenschaftler vielmehr mit den konkreten technischen, finanziellen und soziologischen Problemen, die diese hervorrufen würden. ${ }^{46}$ Ebenso wie Gauron verstand auch Bürger die neuen Medien als fruchtbare Ergänzung und Erweiterung der nicht-elektronischen Medien. ${ }^{47}$ Klostermann hingegen sah die Nützlichkeit der neuen Medien auch im Jahre 1997 noch ausschließlich in der wissenschaftlichen Kommunikation und war der festen Überzeugung, Publikationen sollten weiterhin auf Papier und nur nach gewissenhafter Prüfung durch Lektoren veröffentlicht werden. ${ }^{48}$ Für seine Position konnte Klostermann jedoch zu diesem Zeitpunkt schon keine überwiegende Unterstützung mehr erwarten.

44 Müller, "Literatur im technisch-elektronischen Zeitalter", 1270-1277.

45 Robert Barth, "Die Bibliothek der Zukunft", in: Peter Rusterholz und Rupert Moser, Hg., Die Bedeutung des Buches - gestern-heute-morgen, Referate einer Vorlesungsreihe des Collegium generale der Universität Bern (Bern; Stuttgart, 1996), 53-75, hier 74f.

46 André Gauron, "Das digitale Zeitalter", in: Stefan Bollmann, Hg., Kursbuch neue Medien. Trends in Wirtschaft und Politik, Wissenschaft und Kultur, 2. Aufl. (Mannheim, 1996 [ $\left.\left.{ }^{1} 1995\right]\right), 24-40$, hier: 25.

47 Thomas Bürger, "Modelle zum Umgang mit originalen und digitalen Drucken. Zur Bereitstellung alter Bücher", in: Sabine Wefers, Hg., Von Gutenberg zum Internet 7. Deutscher Bibliothekskongreß, 87. Deutscher Bibliothekartag in Dortmund 1997, Zeitschrift für Bibliothekswesen und Bibliographie: Sonderheft 68 (Frankfurt a.M., 1997), 51-60, hier: 51.

48 Vittorio E. Klostermann, “'Von Gutenberg zum Internet' - Wie bitte?!”, BuB-Journal 49 (1997), Sonderheft Bibliothekskongreß, 25. 
Neben den allgemeinen Reflexionen über grundsätzliche gesellschaftliche Entwicklungstendenzen sowie ein möglicherweise nahe bevorstehendes Ende des Buches war außerdem eine Ausrichtung auf die Frage nach der Position des Autors in der modernen, multimedialen Welt zu verzeichnen. Durch die neuen Formen der Literaturpräsentation, vor allem aber durch die neue Art der Literaturrezeption, schien die bisherige Autorität des Autors jäh in Frage gestellt zu sein. ${ }^{49}$ Man wurde plötzlich gewahr, daß sich die bis dato übliche klare Trennung zwischen Autor einerseits und Leser andererseits durch den Computer und die dadurch realisierbare Interaktivität, das heißt, "die tendenziell weltweite Möglichkeit, daß jeder zugleich Empfänger und Sender von Informationen wird"50, allmählich aufzulösen begann, und sah darin den Auftakt zum "Abgang des Autors". 51 Allerdings ließen sich in dieser Hinsicht durchaus unterschiedliche Standpunkte beobachten. Die Auflösung der traditionellen Rollenverteilung zwischen Autor und Leser wurde und wird nach wie vor auch als Fortschritt und als seit langem angestrebtes Ziel angesehen, insofern sie dem Leser endlich die Befreiung von der einengenden Autorität des Autors eröffne. ${ }^{52}$ Mayer und Schneck beispielsweise charakterisierten diese Entwicklung als "das euphorische Diktum von der Verschmelzung von Leser- und Autorfunktion in der interaktiven Hyperfiction". ${ }^{53} \mathrm{Da}$ der Leser nunmehr zum Koautor werden konnte und aufgrund der Interaktionsmöglichkeiten der Hypertext-Dokumente die Gelegenheit besaß, zu agieren und zu reagieren, wurde

49 Siehe Ruth Nestvold, "Das Ende des Buches: Hypertext und seine Auswirkungen auf die Literatur”, in: Martin Klepper, Ruth Mayer und Ernst-Peter Schneck, Hg., Hyperkultur: Zur Fiktion des Computerzeitalters (Berlin; New York, 1996), 14-30, hier: 15. Siehe auch Martin Warnke, "Informationstechnologie - das digitale Monopol”, in: Gemmeke, John und Krämer, Hg., Euphorie digital?, 21-33, hier: 28.

50 Landtag von Baden-Württemberg, Bericht und Empfehlungen der Enquête-Kommission 'Entwicklung, Chancen und Auswirkungen neuer Informations- und Kommunikationstechnologien in BadenWürttemberg' (Multimedia-Enquête), Drucksache 11/6400 (1995), 17. - Interaktivität wird jedoch in unterschiedlicher Weise interpretiert. Diese Tatsache wird in Zusammenhang mit der Frage, inwiefern interaktive Angebote diesen Definitionen tatsächlich entsprechen können, im weiteren Verlauf der Arbeit noch ausführlichere Erwähnung finden.

51 Benjamin Woolley, Die Wirklichkeit der virtuellen Welten, übers. von Gabriele Herbst, (Basel; Boston; Berlin, 1994 [Virtual Worlds (Oxford, 1992)]), 178. Woolley kommt zu dem Schluß: "Im Cyberspace sind alle Autoren, was bedeutet, daß keiner ein Autor ist: Der Unterschied, auf dem der Begriff 'Autor' beruht, der Autor als unterschieden vom Leser, verschwindet. Abgang des Autors... ." Ebd. Inwieweit ein Hypertext-Autor tatsächlich die Vollmacht an seinem Werk verliert und bis zu welchem Grad der Leser seine Aufgaben übernimmt, wird im weiteren Verlauf der Arbeit noch intensiver erläutert werden.

52 Rolf Todesco, "Hyperkommunikation: Schrift-Um-Steller statt Schriftsteller", in: Suter und Böhler, Hg., Hyperfiction, 113-124, hier: 122.

53 Mayer und Schneck, "Hyperkultur", 7. 
auf der anderen Seite jedoch nicht nur als Bereicherung, sondern auch als Zwang empfunden. ${ }^{54}$

Wie Schanze bereits im Jahre 1995 vorausgesagt hatte, erwies entgegen allen kritischen Stimmen, daß der "erste Grundsatz der Mediengeschichte" nach wie vor Gültigkeit besitzt, demzufolge die Einführung neuer Medien keinesfalls eine Bedrohung für den Fortbestand der bereits vorhandenen, bewährten Informationsträger und der damit verbundenen tradierten Wertvorstellungen darstellen muß: "Noch nie hat ein Neues [sic!] Medium ein altes [sic!] ersetzt." ${ }^{, 55}$ Vielmehr sind die technischen Innovationen - so der Grundsatz - in der Regel dazu prädestiniert, die schon existierenden Hilfsmittel zu ergänzen und zu vervollständigen, und können somit einer rascheren Kenntnisverbreitung sowie der Förderung des wissenschaftlichen Dialogs dienen; ${ }^{56}$ die Nutzung verlagert sich also nicht von einer gängigen auf eine neue technische Komponente, sondern es kommt vielmehr zu einer Neugestaltung des Nutzungsverhaltens, das heißt dem Anwender steht künftig eine erweiterte Bandbreite an Gebrauchsobjekten zur Verfügung. ${ }^{57}$

Tatsächlich entbehrten die anfänglichen Prophezeiungen, die Print-Medien würden höchstwahrscheinlich eines Tages durch die elektronischen Medien ersetzt werden, mehr und mehr ihrer Brisanz, da die negativen Zukunftsprognosen der Vergangenheit allmählich durch die tatsächlichen Entwicklungen der Gegenwart widerlegt wurden. Ebenso wie um diese Bedenken wurde es auch um die Prognosen wegen eines möglichen Autoritätsverlustes der Autoren allmählich stiller, und nachdem jene Stimmen, die eine Verdrängung des Buches und den Abtritt des

54 Warnke, "Informationstechnologie - das digitale Monopol”, 28.

55 Helmut Schanze, "Was kann ein Geisteswissenschaftler mit einem Informationsnetz anfangen? Vorüberlegungen zu einer Nutzungstheorie eines 'Neuen Mediums',, in: Helmut Jüngling, Hg., Internet und Bibliotheken: Entwicklung - Praxis - Herausforderungen (Köln, 1995), 102-111, hier: 103. Schanze bezieht sich mit seinen Äußerungen ausdrücklich auf die neuen virtuellen Medien: "Noch nie hat ein Neues Medium ein altes ersetzt. Das gilt auch für das virtuelle Forum der Netze." Ebd. Snyder vertritt ebenfalls die These, daß neue und alte Medien ergänzend ineinandergreifen: "The introduction of a new technology of writing does not automatically render older ones obsolete. [...] A more likely scenario is that a number of technologies will continue to co-exist, interact, even complement each other." Ilana Snyder, "Page to Screen", in: Dies., Hg., Page to Screen: Taking literacy into the electronic era (London; New York, 1998), xx-xxxvi, hier: xxf. Auch Hautzinger sagt die Koexistenz beider Medienformen voraus, wobei ihrer Ansicht nach die narrative Literatur zunächst der Buchform vorbehalten bleiben werde, wohingegen sich die wissenschaftliche Literatur vorzugsweise für das neue Medium Hypertext eigne. Nina Hautzinger, Vom Buch zum Internet? Eine Analyse der Auswirkungen hypertextueller Strukturen auf Text und Literatur (St. Ingbert, 1999), 122f.

56 Bürger, "Modelle zum Umgang mit originalen und digitalen Drucken", 51.

57 Hautzinger, Vom Buch zum Internet?, 122. - Die weiteren Ausführungen werden zeigen, daß der Computer mit eigenem Zeitkontingent in Ergänzung zu den etablierten Medien genutzt wird. 
Autors heraufbeschworen hatten, beinahe gänzlich verstummt waren, schienen in jüngerer Vergangenheit nur noch einzelne Autoren oder Verleger provokative Thesen dieser Art aufwerfen zu wollen. ${ }^{58}$ Doch auch in diesen einzelnen Fällen ließ sich beobachten, daß allenfalls die Titel der Veröffentlichungen noch herausfordernd klangen, während die jeweiligen Verfasser den elektronischen Medien inhaltlich bereits ihre vollständige Zustimmung entgegenbrachten. ${ }^{59}$

\section{Der Stand zu Beginn des 21. Jahrhunderts}

Im Anschluß an die aufgezeigten Entwicklungen ist um die Jahrtausendwende die Frage nach den realen Auswirkungen der neuen elektronischen Medien auf den Menschen sowie auf dessen Verhaltensweisen verstärkt in den Mittelpunkt der Betrachtungen gerückt. Thema ist nun nicht mehr die Frage, ob die Print-Medien eines Tages grundsätzlich durch elektronische Medien ersetzt werden könnten, vielmehr geht die Tendenz dahin zu analysieren, inwieweit sich dieser Vorgang im Leben der Menschen praktisch bereits vollzogen hat, das heißt in welchem Maße Print-Medien im Vergleich zu Computer-Medien noch genutzt werden. Die Ergebnisse solcher Untersuchungen weichen zum Teil signifikant von gängigen Annahmen - oder vielmehr Vorbehalten - ab und könnten dazu beitragen, daß nunmehr fundierte Erkenntnisse hinsichtlich des Ist-Zustandes zielgerichtet zur Herbeiführung eines wie auch immer zu bestimmenden Soll-Zustandes verwendet werden.

Eine der Untersuchungen, auf die hier eingegangen werden soll, ist die in Kapitel I.1. bereits erwähnte Studie der Stiftung Lesen aus dem Jahre $2000^{60}$, die sich auch mit dem Verhältnis von PC-Nutzung und Häufigkeit des Bücherlesens bei Jugendlichen bis zum Alter von 29 Jahren befaßte. Das Ergebnis der Studie war, daß

58 Gleichwohl soll in Kapitel V.2.3. dieser Arbeit untersucht werden, ob und in welchem Maße die neuen Formen der elektronischen Literatur einen Autoritätsverlust der Autoren herbeiführen.

59 Uwe Jochum und Gerhard Wagner, Hg., Am Ende - das Buch: semiotische und soziale Aspekte des Internet (Konstanz, 1998). Vgl. Hautzinger, Vom Buch zum Internet?. Siehe auch Spiegel Spezial 10: Vom Buch zum Internet: Die Zukunft des Lesens (1999). Siehe ebenso Spiegel special 3: Info-Sucht - Der Mensch im Netz der Medien (1999). Siehe außerdem Jane Yellowlees Douglas, The End of Books? - Or Books without End?: Reading Interactive Narratives (Ann Arbor, 2000). Vgl. ferner Bernd Guggenberger, Das digitale Nirwana (Hamburg, 1997). Siehe ferner Gemmeke, John und Krämer, "Vorwort", 9, zu den Ergebnissen einer Veranstaltung des Fortbildungszentrums Abtei Brauweiler/Rheinisches Archiv- und Museumsamt und des Heinz Nixdorf MuseumsForums, Paderborn, die am 28./29. September 1998 für mehr als 120 Fachleute und Experten aus Museen, Kunst und Kultur, Wirtschaft und Wissenschaft in Paderborn zum Zwecke eines disziplinübergreifenden Diskurses durchgeführt wurde. Zu einer Bilanz siehe außerdem Warnke, "Informationstechnologie - das digitale Monopol”, 21.

60 Hippler, "Tummelplatz Internet", 167. 
der Computer keineswegs eine Barriere für das Lesen darstellt, sondern daß im Gegenteil jene Jugendlichen, die täglich beziehungsweise mehrmals wöchentlich Bücher lesen, mehrheitlich auch zur Gruppe der PC-Nutzer zählen. ${ }^{61}$ Die Ergebnisse einer anderen Bestandsaufnahme besagen gleichfalls, daß die Nutzung des World Wide Web das allgemeine Leseverhalten nur unwesentlich beeinflußt. Den Befragungen von Langen zufolge wendete im Jahre 1999 lediglich eine relativ geringe Zahl von Web-Nutzern aufgrund verstärkter Online-Anwendungen weniger Zeit für das Lesen auf. ${ }^{62}$ Der Anteil der Bundesbürger, die eine hohe oder sehr hohe Leseneigung zeigten, lag im Jahre 1999 im Vergleich zu einer drei Jahre zuvor durchgeführten Umfrage sogar um drei Prozent höher. ${ }^{63}$ Dieselbe Umfrage ergab zudem, daß der Anteil an Buchlesern im Verlauf der letzten 20 Jahre mehr oder weniger konstant geblieben ist. ${ }^{64}$ Alle diese Befunde werden durch die Rechercheergebnisse von Tullius bekräftigt, wonach "Vielleser" im allgemeinen gleichzeitig auch "Vielmediennutzer" sind, die den PC vorwiegend in Ergänzung zu Büchern einsetzen, wohingegen "Kaumleser" nicht nur weniger Bücher lesen, sondern sich auch seltener der übrigen Medien bedienen. ${ }^{65}$

Andere Untersuchungen liefern jedoch abweichende Ergebnisse. So weisen Bischof und Heidtmann auf Befunde der neueren medienwissenschaftlichen Forschung hin, wonach bei Kindern und Jugendlichen in zunehmendem Alter eine

61 Ebd., $168 f$.

62 Claudia Langen, "Lesen in einer veränderten Medienlandschaft", in: Claudia Langen und Ulrike Bentlage, Hg., Das Lesebarometer - Lesen und Mediennutzung in Deutschland (Gütersloh, 2000), 25-40, hier: 32. 80 Prozent der Befragten gaben an, nach wie vor gleich viel Zeit zum Lesen zu verwenden, ein Anteil von fünf Prozent widmete dem Lesen mehr Zeit und bei 14 Prozent ging die Nutzung des Internet zu Lasten des Lesepensums.

63 Claudia Langen, “Das Lesebarometer 1999 - Tendenz steigend?”, in: Langen Bentlage, Hg., Das Lesebarometer, 11-17, hier: 11. Es handelt sich hier um einen Anstieg von 22 auf 25 Prozent.

64 Ebd., 12. Der Leseranteil innerhalb der bundesdeutschen Bevölkerung ergibt sich nach Auswertung der Antworten auf die Frage: "Haben Sie in den letzten zwölf Monaten ein Buch gelesen?" Ebd.

65 Christiane Tullius, “Typologien der Leser und Mediennutzer”, in: Stiftung Lesen, Hg., Leseverhalten in Deutschland im neuen Jahrtausend: eine Studie der Stiftung Lesen (Hamburg, 2001), 61-83, hier: 65; 68. Vielleser zählen darüber hinaus zu allen drei Motivationsgruppen, das heißt sowohl zu den Unterhaltungs- als auch zu den Informations- und den Weiterbildungsorientierten, während Wenigleser scheinbar vorwiegend aus Informationsmotivation sowie mehr oder weniger gezwungenermaßen aus Fortbildungsgründen lesen. Ebd., 69. - Da die Lektüre von Büchern vielfach die Basis für Medien-Sachverständigkeit bildet, muß befürchtet werden, daß den Kaumlesern der Zugang zur Informationsgesellschaft verwehrt bleiben könnte. Siehe Norbert Rojan und Joachim Schroth, "Leser als Buchkäufer: Wie kommt der Mensch zum Buch?”, in: Stiftung Lesen, Hg., Leseverhalten in Deutschland im neuen Jahrtausend, 85-109, hier: 108. Siehe auch Boesken, "Lesen am Bildschirm", 133. 
Interessen-Verlagerung von der erzählenden Literatur zu anderen Medien zu verzeichnen ist. ${ }^{66}$

Wiederum andere Erhebungen über das Leseverhalten junger Menschen konstatieren eine grundlegende Veränderung des Nutzungsverhaltens, das offenbar einen erweiterten Lesebegriff erforderlich macht. Danach wenden sich jugendliche Leser verstärkt einem neuen Lesemedium zu. So konnten CD-ROMs binnen relativ kurzer Zeit $\mathrm{zu}$ den beliebtesten, am häufigsten nachgefragten sowie umsatzstärksten Medienträgern avancieren ${ }^{67}$. Dieser Erfolg gründete auf der Interaktivität des Mediums, die den Nutzer mit einer bis dato unerreicht hohen Mit- und Eigenverantwortlichkeit betraute und so zu der Einschätzung beitrug, der Computer fordere aufgrund seines Multimedia-Angebots die Aktivität und Kreativität seiner Nutzer und begünstige die "Individualität von Rezeptions- und Lernprozessen". ${ }^{68}$ Tatsächlich scheint man sich mit dem Gedanken vertraut machen zu müssen, daß der Computer zwar offensichtlich keine grundsätzlich verdrängende Wirkung hat, daß er aber gleichwohl von Fall zu Fall als Ersatz für die bislang genutzten Print-Medien fungiert. Dies bedeutet, daß beispielsweise die Online-Ausgaben von Tageszeitungen anstelle der gedruckten Pendants gewählt oder wissenschaftliche Themen im World Wide Web nachgelesen werden.

Allerdings gibt es auch Untersuchungen mit teilweise diametral gegensätzlichen Ergebnissen, weshalb nur eingeschränkte Rückschlüsse auf die momentane Situation in der Frage der gegenseitigen Beeinflussung von Computer- und PrintMedien möglich sind. So scheinen die Erkenntnisse von Gedissmann ${ }^{69}$ Heidtmanns Erfahrungen zu widerlegen: Die verstärkte Nutzung des Computers provoziert danach eine zunehmende Verkümmerung der manuellen und intellektuellen Fähig-

66 Ulrike Bischof und Horst Heidtmann, "Warum sind Film- und Fernsehbücher so erfolgreich? Das IfaK forscht zur Kinder- und Jugendliteratur im Medienverbund", Buch und Bibliothek: Medien, Kommunikation, Kultur 52 (2000), 414-417, hier: 414. Leider liefern Bischof und Heidtmann keinen Beleg für ihre Aussage. - Wenn in diesem Zusammenhang der Begriff 'erzählende Literatur' verwendet wird, so liegt angesichts einer umfangreichen Bandbreite an Lektüreformen die Frage nahe, um welche Art der Buchliteratur es sich bei den genannten Erhebungen eigentlich handelt. Laut Lesebarometer dominiert hier als Resultat eines veränderten Nutzungsinteresses eindeutig das Bedürfnis nach Information (ausschlaggebend für 72 Prozent der Befragten). Das Interesse an Entspannung (63 Prozent) und Unterhaltung (61 Prozent) rangieren demzufolge nur noch auf Platz zwei und drei, gefolgt von dem Wunsch nach Rat und Hilfe (maßgebend für 55 Prozent). Claudia Langen, "Der Griff zum Buch warum wird gelesen?", in: Langen und Bentlage, Hg., Das Lesebarometer, 19-24, hier: 23.

67 Horst Heidtmann, "'Im Buch geht alles ohne dich, aber im Computer nichts...'. Multimedia in Kinder- und Jugendbibliotheken: Ergebnisse einer Umfrage", Buch und Bibliothek: Medien, Kommunikation, Kultur 53 (2001), 406-410, hier: 410. Ebd.

69 "Tumbe Computerkids", Der Spiegel 21 (2002), 187. 
keiten und verursacht dadurch weitreichende, zum Teil irreparable Schäden insbesondere bei Kindern und Jugendlichen. Gedissmann konnte anhand seiner Untersuchungen einen "eindeutigen Zusammenhang zwischen Mediennutzung und der Entwicklung des kindlichen Gehirns" belegen und nachweisen, daß eine intensive Verweildauer vor dem Computermonitor in frühkindlicher Phase eine andere Verknüpfungsform der Nervenzellen und somit eine Verkümmerung von Phantasie, Kreativität und Wortschatz verursacht. ${ }^{70}$ Im Zusammenhang mit dieser Problematik hatten Glotz und Thomas bereits im Jahre 1994 auf einige Beurteilungen verwiesen, denen zufolge die Nutzung des Computers das "Abwürgen der Körpermotorik" verursache und sich, was den Aufbau sowie den Erhalt intellektueller Kompetenzen betreffe, zumindest in der Form auswirke, "daß Computerkinder später keine Zeitungen mehr läsen". ${ }^{71}$ Auch Veröffentlichungen aus dem Jahre 2000 belegen, daß die Problematik der Verkümmerung entscheidender geistiger Fähigkeiten durch Computer-Nutzung tatsächlich besteht. So registrierte man bei Jugendlichen eine deutliche Verschlechterung der Fähigkeit, mehrschichtige Texte zu verstehen. ${ }^{72}$ Es ist in diesem Falle nicht auszuschließen, daß eine Wechselwirkung vorliegt, wonach einerseits der Computer aufgrund seines unterhaltsamen und interaktiven Angebotsspektrums den Befindlichkeiten und Neigungen jener jungen Menschen entgegenkommt, die das Bücherlesen als zu komplex und ermüdend empfinden, wonach aber andererseits diese Jugendlichen ihre intellektuellen Fähigkeiten aufgrund der verstärkten Hinwendung zu Computer-Medien und der gleichzeitigen Vernachlässigung der Print-Literatur nicht mehr ausreichend schulen. So beobachtete man, daß der vermehrte Zugriff auf das World Wide Web offensichtlich speziell bei Kindern und Jugendlichen eine signifikante Verschlechterung der generellen Befähigung zur Wissensbeschaffung bewirkt. Gerster äußerte diesbezüglich den Vorwurf, das WWW leiste dem Erwerb sogenannter "Wissensinselchen"73

70 Ebd.

71 Glotz und Thomas, Das dritte Wirtschaftswunder, 232f.

72 Bischof und Heidtmann, "Warum sind Film- und Fernsehbücher so erfolgreich?", 414.

73 Angela Gatterburg und Karen Andresen, “'Man muß den Charakter bilden.' Die 'heute'-Moderatorin Petra Gerster und ihr Mann, der Journalist Christian Nürnberger, über ihr Buch zum 'Erziehungsnotstand' in Deutschland, die Unsicherheit von Eltern und Lehrern und die Folgen der Globalisierung für die Familie", Der Spiegel 35 (2001), 58-61, hier: 60. Gerster kritisiert, der Computer habe unser Denken sukzessive in der Weise beeinflußt und verändert, daß mittlerweile kein Buch mehr von vorne bis hinten gelesen werde. Es entfalle somit die Möglichkeit, anhand dieses Mediums, in dem eines auf dem anderen aufbaue, analoges und logisches Denken zu üben. "Stattdessen tastet man sich von Link zu Link. Da entstehen im Kopf lauter Wissensinselchen, die gar nicht miteinander verbunden sind." Ebd. 
Vorschub. Es verleite den Nutzer dazu, auf seiner Suche nach Informationen ruhelos von einer elektronischen Ressource zur nächsten überzugehen und somit - getrieben von der unausgesetzten Sorge, entscheidende Informationen zu verfehlen - zusammengefügtes und oberflächliches, wenn nicht gar unverbürgtes Wissen anzusammeln. ${ }^{74}$

Dennoch sollte nicht der Eindruck entstehen, der einschneidende Wandel der Medienlandschaft, der in der Zeit von 1995 bis zum Jahr 2000 vonstatten gegangen ist $^{75}$, habe überwiegend negative Auswirkungen gehabt. Ein vorläufiges Resümee internationaler Forschungen zum Computereinsatz in den Geisteswissenschaften lautet vielmehr, daß das Internet tendenziell mit einem zusätzlichen, das heißt gewissermaßen mit einem 'eigenen' Zeitbudget ${ }^{76}$ in bereichernder Ergänzung $\mathrm{zu}$ den bestehenden Medien genutzt wird. Gerade im wissenschaftlichen Bereich sind im Zuge einer deutlich aufgeschlosseneren Haltung gegenüber den elektronischen Medien verstärkt sehr konkrete Überlegungen zu den spezifischen Nutzungsmöglichkeiten des Computers in den Vordergrund getreten. ${ }^{77}$ Es scheint, als zeichne sich im Wissenschaftsbereich eine vorwiegend positive Nutzungstendenz ab. Vielleicht hat Schön ja wirklich Recht, wenn er sagt, daß negative Prophezeiungen selten einzutreffen pflegen, da sie sich in zu hohem Maße auf die rein “theoretisch-technischen Möglichkeiten der Medien” stützen und die eigentliche Realität sowie das menschliche Verhalten nicht in ausreichender Form berücksichtigen, beziehungsweise sich diese Faktoren als unabwägbar erweisen und somit

74 Ebd.

75 Bertelsmann Stiftung, Hg., Lesen fördern in der Welt von morgen: Modelle für die Partnerschaft von Bibliothek und Schule (Gütersloh, 2000), 170.

76 Langen, "Lesen in einer veränderten Medienlandschaft", 33.

77 Hautzinger, Vom Buch zum Internet?. Siehe diesbezüglich ebenfalls Thomas Sand und Kay Wahlen, Mediennutzungskonzepte im Hochschulbereich: Planung, Organisation, Strategien (Hannover, 2000). Siehe auch Douglas, The End of Books? - Or Books without End?. Siehe außerdem Michael Joyce, Othermindedness: the Emergence of Network Culture (Ann Arbor, 2000). Siehe zudem Bertelsmann Stiftung und Heinz Nixdorf Stiftung, Hg., Studium Online: Hochschulentwicklung durch neue Medien (Gütersloh, 2000). Siehe des weiteren Winfried Sommer, "E-learning: Schöne neue Welt des Lernens", MEORUM: Menschen, Erfolge, Organisationen, Resultate \& Märkte 3 (2001), 12-13. Siehe ferner Hartmut Weber und Gerald Maier, Hg., Digitale Archive und Bibliotheken: Neue Zugangsmöglichkeiten und Nutzungsqualitäten (Stuttgart; Berlin; Köln, 2000). 
dem gedanklichen Kalkül mehr oder weniger entziehen. ${ }^{78}$ Anders formuliert: Es müssen nicht alle Entwicklungen, die denkbar sind, auch tatsächlich eintreten. ${ }^{79}$

Generell ist festzustellen, daß die emotionserfüllte Aufbruchphase der letzten Dekade des 20. Jahrhunderts mittlerweile einem neuen Stadium des nüchternen Abwägens und verhaltenen Abwartens gewichen ist. Gelegentlich noch spürbare Anzeichen von zurückhaltender Skepsis und unterschwelliger Resignation liegen mutmaßlich in den teilweise eher ernüchternden Erfahrungen der vergangenen Jahre begründet, innerhalb derer sich das WWW von einem Medium intensiver Produktions- und Instandhaltungstätigkeit zumindest partiell $\mathrm{zu}$ einem Lagerplatz völlig veralteter Webseiten gewandelt hat. Die anfänglich nahezu überbordende Schöpfungstätigkeit im Web scheint deutlich nachgelassen zu haben; gleichzeitig ist eine Vernach-

78 Vgl. Erich Schön, "Kein Ende von Buch und Lesen: Entwicklungstendenzen des Leseverhaltens in Deutschland - Eine Langzeitbetrachtung", in: Stiftung Lesen, Hg., Lesen im Umbruch - Forschungsperspektiven im Zeitalter von Multimedia (Baden-Baden, 1998), 39-77, hier: 39.

79 Die Autorin vorliegender Arbeit erkennt eine Vielzahl von Betätigungsfeldern, auf denen der Computer einen Zusatznutzen verspricht. Als Beispiele seien genannt: Die Forschung kann sich des Webs als Recherche- und Kooperationsmedium bedienen und es zur Präsentation aktueller Projekte, zu Aufbau und Pflege wissenschaftlicher Kontakte, zur Bitte um Unterstützung bei Nachforschungen oder zur Ausschreibung wissenschaftlicher Stellen einsetzen. In virtuellen Kommunikationsforen können sich Experten wie interessierte Laien zu wissenschaftlichen Themen austauschen und diskutieren. Zur gezielten Information bieten sich vor allem themenspezifisch gegliederte sogenannte Newsgroups an. Sie werden oftmals von Universitäten ins Leben gerufen und sind in der Regel nur für berechtigte Nutzer zugänglich. Damit besteht zugleich die Möglichkeit, Einfluß auf die Zusammensetzung der Diskussionsgruppen zu nehmen und wissenschaftliches Niveau zu garantieren. Virtuelle Kommunikationsforen finden sich aber auch in elektronischen Zeitschriften, wo die wissenschaftliche Diskussion durch Rückmeldungen zu den veröffentlichten Zeitschriftenartikeln eine völlig neue, bereichernde und provokative Dynamik erhält. Auch die Lehre vermag das Web für wissenschaftliche Zwecke zu nutzen: Universitäten und Hochschulen können den Studierenden virtuelle Seminare anbieten, indem sie Lehrveranstaltungen digital aufzeichnen, die an Computerterminals unmittelbar mitverfolgt werden können und anschließend zusammen mit vorlesungsbegleitendem Material online zur Verfügung stehen. Der Umgang mit einem vernetzten, virtuellen Lernraum kann nicht nur zur Entwicklung neuer Lernwege inspirieren, sondern auch dem Problem des übermäßigen Andrangs in einigen Seminaren entgegenwirken und sich für Studierende wie Lehranstalten kostensenkend auszahlen. Über diese ganz allgemeinen Nutzungsvorteile hinaus macht sich der technische Fortschritt speziell auch im Fachbereich Englischsprachige Literaturwissenschaft überaus positiv bemerkbar, indem er beispielweise die weltweite unmittelbare Kontaktaufnahme zu Kollegen ermöglicht, eine Debatte literaturwissenschaftlicher Themen auf internationaler Ebene gestattet oder die Organisation und Auswertung von Kongressen und Literatur-Workshops erleichtert. Durch seine außergewöhnlichen Eigenschaften gewährleistet das Web den Anschluß an andere Wissenschaftsbereiche und begünstigt damit interdisziplinäres Arbeiten und internationale Kooperation. Aufgrund seiner Globalität und Aktualität erweist es sich vor anderen Medien als idealer Datenträger für Nachschlagewerke. Es gestattet dem Nutzer den Zugriff auf eine Vielzahl von Hilfsmitteln wie zum Beispiel Lexika, Wörterbücher oder Bibliographien und unterstützt seine Recherchen mit vielfältigen Suchfunktionen. Durch den globalen Zugriff auf Institutionen zur Bereitstellung wissenschaftlicher Materialien wie beispielsweise Bibliotheken trägt das Web gleichzeitig zur Behebung des nationalen und internationalen Ungleichgewichts in Sachausstattung und Personalumfang bei und erlaubt auf diese Weise einen allgemeinen, gleichberechtigten Anschluß an die weltweite Forschung. Globalität und Aktualität machen das Web darüber hinaus zu einem idealen Publikationsmedium für literaturwissenschaftliche Abhandlungen. Veröffentlichungen finden umgehende internationale Verbreitung und können dadurch zu einer Beschleunigung des Wissenstransfers und einer Synchronisierung von Forschungsprojekten beitragen. 
lässigung des bereits existierenden Bestandes unübersehbar. Dabei ist es nebensächlich, ob die unzureichende regelmäßige Pflege der Webseiten durch Mangel an Personal oder finanziellen Mitteln verursacht wird oder ob sie auf Unwissenheit oder gar Desinteresse zurückzuführen ist. Unerfreuliche Tatsache bleibt, daß der Nutzer bei seiner Recherche im Web nur allzu oft auf sogenannte "Datengräber" Phantomdokumente trifft, das heißt auf Seiten, die entweder seit unverhältnismäßig langer Zeit nicht mehr überarbeitet wurden oder aber unauffindbar sind, weil dem Nutzer die Änderung der Web-Adresse entgangen ist, beziehungsweise die gewünschten Seiten gar nicht mehr existieren. ${ }^{81}$ Diese Tatsache sowie mangelnde Kenntnisse auf dem Gebiet der Web-Recherchen führen dazu, daß einer Studie zufolge offensichtlich ungefähr ein Drittel aller Suchen im WWW nach durchschnittlich 15 Minuten erfolglosen Nachforschens "ohne Ergebnis im Nichts" enden ${ }^{82}$ und somit notwendigerweise zu einer gewissen Resignation seitens der Nutzer führen.

\section{Zielsetzung und Methodik}

Von der Literaturwissenschaft wurden die auf der Basis der Hypertext-Systematik entstandenen Literaturformen zunächst vielfach ignoriert. Allenthalben wurde gemutmaßt, es handele sich bei den neuen Literaturformen lediglich um "alten Wein in neuen Schläuchen". ${ }^{83}$ Da der Literatur im Verlaufe der nachfolgenden Jahre zudem weder ein Erfolgswerk noch irgendeine entscheidende revolutionäre Neuerung, zum Beispiel eine bislang noch nicht dagewesene Präsentationsform, beschieden war, bleiben Zurückhaltung und Skepsis nach wie vor eine nicht zu unterschätzende Barriere für die Akzeptanz von Internet-Literatur.

Die Verfasserin der vorliegenden Arbeit sieht gleichwohl Hinweise dafür, $\mathrm{da}$ die neuen Literaturformen die konventionelle Literatur $\mathrm{zu}$ erweitern und $\mathrm{zu}$ bereichern vermögen. Sie hält es deshalb für notwendig, Hemmungen und Zweifel aufzubrechen und das Interesse verstärkt auf die neuen Literaturformen hinzulenken. Allerdings erfordern diese eine ganz spezifische neue Form des Denkens, Handelns und Schaffens, die an den Computer und das WWW gebunden ist, und setzen somit die Bejahung der elektronischen Medien voraus. Durch das bisherige Angebot an

80 Gersmann, "Schöne Welt der bunten Bilder", 110.

81 Die Unbeständigkeit des WWW wird ein zentrales Thema des Kapitels II. bilden.

82 Basil Wegener, "Chaos im Netz macht der Internet-Ökonomie zu schaffen", heise online, 26.07.2000, <http://www.heise.de/newsticker/data/jk-26.07.00-000 > 09.07.2002.

83 Todesco, "Hyperkommunikation", 115. 
Sekundärliteratur konnte der dringende Informationsbedarf noch nicht gedeckt werden. Ziel der vorliegenden Arbeit ist der Versuch, diese Lücke zumindest teilweise zu schließen.

Die Arbeit soll dazu beitragen, das Literaturangebot im WWW in seinen verschiedenartigen Konzeptions- und Präsentationsformen sowie die daraus resultierenden Möglichkeiten genauer zu erschließen und Rezipienten und potentiellen Autoren unter Erörterung sowohl inhaltlicher als auch formaler Gestaltungs- und Darbietungsalternativen näher zu bringen. Möglicherweise kann es auf diese Weise auch gelingen, die neuen Literaturformen verstärkt in das Blickfeld literaturwissenschaftlicher Betrachtung zu rücken.

Bei der Analyse des Literaturangebots im World Wide Web soll folgendes Problem im Mittelpunkt vorliegender Arbeit stehen: Welche Bereicherungen und Chancen, aber auch welche Konsequenzen und gegebenenfalls Risiken birgt die neue Dimension des Informationsumschlages im Bereich englischsprachiger Literatur?

Als Ausgangspunkte auf dem Wege zur Klärung dieser Sachlage werden die nachstehenden Fragen dienen:

- Wie lassen sich im World Wide Web publizierte Werke angesichts der bestehenden rechtlichen und technischen Schwierigkeiten wie Kontinuität, Zitierform, Urheberrecht oder Archivierung in die literaturwissenschaftliche Arbeit integrieren?

- Ist die Bandbreite der herkömmlichen literaturwissenschaftlichen Begriffe für die Interpretation der neuen Literaturformen ausreichend oder ist gegebenenfalls eine Überarbeitung beziehungsweise eine Anpassung der herkömmlichen Terminologie erforderlich?

- Wie präsentiert sich englischsprachige Literatur im World Wide Web?

- Wie ist dieses Literatur-Angebot zu bewerten? Welche Maßstäbe sind dabei anzuwenden?

- Hat das World Wide Web neue Literaturformen hervorgebracht und wie stellen sich diese dar?

Neben der erwähnten Unübersichtlichkeit des World Wide Web sind vor allem sein kontinuierlicher und sehr schneller Wandel die entscheidenden Gründe dafür, daß es nicht ohne weiteres möglich ist, eine verbindliche Aussage über die derzeitigen Verhältnisse auf dem Gebiet digitaler Medien zu treffen. Tatsächlich ist 
nur sehr schwer zu beurteilen, an welchem Punkt wir uns derzeit befinden und welcher Grad des geistig sowie technisch Machbaren bereits erreicht ist, da die Entwicklung des Datennetzes so schnell und ruhelos verläuft, daß viele Feststellungen nur flüchtigen Wert besitzen können. ${ }^{84}$ Wir befinden uns nach wie vor in einer permanenten Phase des Erprobens und Entwickelns. "Dennoch sind erste Wegmarken gesetzt, Thesen formulierbar", wie Auer bereits erkannt hat. ${ }^{85}$

Möglicherweise ist eben diese Sachlage ursächlich dafür, daß bis zum gegenwärtigen Zeitpunkt keine Untersuchungen vorliegen, die den obigen Fragenkatalog in ausreichender Weise beantworten. Obgleich bereits zahlreiche Abhandlungen das Thema der Literatur in Verbindung mit dem World Wide Web zum Gegenstand hatten, konnten sie den oben erwähnten Vorgaben in der Substanz nicht gerecht werden. ${ }^{86}$ Vielmehr ist in vielen Fällen recht deutlich erkennbar, daß sich die Autoren vor der Erstellung ihrer kritischen Ausführungen nur oberflächlich mit dem WWW und dem dort angebotenen Material auseinandergesetzt haben. ${ }^{87}$ In vielen Fällen

84 Gates stellt hierzu fest: "[...] the Internet of today is not the Internet of even a short time ago. The pace of its evolution is so rapid that a description of the Internet as it existed a year or even six months ago might be seriously out-of-date. This adds to the confusion. It is very hard to stay upto-date with something so dynamic." Gates, The Road Ahead, 140.

85 Vgl. Auer, "Der Leser als DJ", 173.

86 Die Abfassung vorliegender Arbeit ist unter anderem auch durch jene Erfahrungen motiviert, von denen Kaiser berichtet. Dieser war dessen überdrüssig, auf der Suche nach einem adäquaten Internet-Literaturführer fortwährend nur mit einer großen Auswahl an Handbüchern konfrontiert zu werden, die neben einer technischen Beschreibung der elektronischen Medien lediglich eine Anleitung zu ihrer Bedienung anbiete, und fährt fort: "Dieses Buch wollte ich eigentlich nur kaufen - nicht schreiben. Doch im Buchhandel war es nicht zu bekommen. Bücher über das Internet füllten dort zwar schon etliche Regale, aber einen literarischen Wegweiser fand ich unter ihnen nicht." Kaiser, "Vorbemerkung", in: Ders., Literarische Spaziergänge im Internet. Obgleich er seinem Anspruch, ein solches Handbuch zur Literatur im Internet zu liefern, in gewisser Weise gerecht wird, bietet auch er nur eine Auswahl an kommentierten Adressen zu Internet-Ressourcen. Informationen über Art, Form und Inhalt der Literatur geben Kaisers Ausführungen nicht. Weitaus interessantere Aufschlüsse erlauben die Beiträge des Hyperliterarischen Lesebuches von Suter und Böhler. Die Herausgeber haben sich zum Ziel gesetzt, theoretische Abhandlungen in Verbindung mit einem Überblick über das Angebot hyperfiktionaler literarischer Texte zu präsentieren. Die Beiträge liefern in der Tat zahlreiche Denkanstöße, gleichzeitig lassen die Autoren jedoch viele Fragen unbeantwortet. Beat Suter und Michael Böhler, Hyperfiction - Hyperliterarisches Lesebuch: Internet und Literatur (Frankfurt a.M., 1999). Einen ähnlichen Eindruck erhält der Leser der Ausgabe Digitale Literatur der Zeitschrift Text + Kritik. Die Autoren versuchen ausdrücklich, "[d]en Fragestellungen und Herausforderungen, die das neue Medium mit sich bringt", nachzugehen. Auch in diesem Falle aber bleibt oben aufgestellter Fragenkatalog unbeantwortet. Digitale Literatur [Text + Kritik: Zeitschrift für Literatur 152 (2001)]. In Beat Suter, Hyperfiktion und interaktive Narration im frühen Entwicklungsstadium zu einem literarischen Genre (Zürich, 2000) versucht der Autor eine Analyse und Klassifizierung des Resultats der Vereinigung von Literatur und Computermedien, legt den Schwerpunkt allerdings zu sehr auf technische Aspekte der Hypertext-Systematik.

87 Das Gegenteil beweist Wingert, der das digitale Werk afternoon, a story sehr ausführlich untersucht hat, schließlich aber zu der Erkenntnis gelangt ist, daß "afternoon nicht funktioniert”. Bernd Wingert, "Kann man Hypertexte lesen?", in: Matejovski und Kittler, Hg., Literatur im Informationszeitalter (Frankfurt a.M., 1996), 185-218, hier: 212. 
haben wir es offenbar mit dem Phänomen der Self-fulfilling prophecy zu tun, wonach eine Voraussage aufgrund der entsprechenden inneren Einstellung und durch unbewußtes persönliches Zutun genau in jener Konstellation eintritt, die man vorausgesagt oder instinktiv erwartet hat. ${ }^{88}$

In anderen Fällen wird die Beschäftigung mit Literatur im WWW negativ beeinflußt durch falsche Erwartungshaltungen hinsichtlich des angebotenen Materials. Dies bedeutet, daß zahlreiche Nutzer lediglich "Ausschau halten nach dem, was sie kennen und in dem neuen Medium und im Gewand der neuen Technik wiedererkennen können" ${ }^{89}$ Somit sehen sie nur das, was sie instinktiv sehen w o 11 e n.

Im Hinblick auf belletristische Texte beispielsweise erwarten viele Leser elektronische Ressourcen, die sowohl hinsichtlich ihres optischen Aufbaus als auch bezüglich ihrer inhaltlichen Konzeption der bekannten und vertrauten Druckform gleichkommen. Im Zweifelsfalle übersieht der Leser mit einem solcherweise eingeschränkten Blickwinkel einen Großteil der angebotenen Literatur, da das präsentierte Material nicht die traditionellen Charakteristika aufweist. Weil somit die verbleibenden Ressourcen den Erwartungen des Nutzers im allgemeinen nicht entsprechen können, muß dessen Urteil notwendigerweise nachteilig für die neuen Literaturformen ausfallen. Demzufolge mag mancher Todescos Ansicht teilen, Literatur in Hypertext-Form zu lesen sei ein ebensolcher Genuß, wie Konzerte am Telefon $\mathrm{zu}$ hören ${ }^{90}$. Entsprechend lesen sich die Bewertungen wie folgt: "Kleine Texttrümmer"91, "selbsterzeugte Medienbricolagen",92, "Klickeratur"93, "Affären jenseits

88 Bezüglich dieses Umstandes umreißen Suter und Böhler pointiert das Bild eines geplagten Nutzers, der sich rasch wieder von den neuen Formen der Literaturpräsentation abwendet, "begleitet von einer hastigen Erleichterung im Befund des literarästhetisch bisher wenig Überzeugenden an den neuen Produkten". Siehe Suter und Böhler, "Hyperfiction", 7f. Auch Douglas ist sich der Tatsache bewußt, daß die ursprüngliche, traditionelle Vorstellung von Texten die Wahrnehmung von Hypertexten nachhaltig belastet: "Even when addressing the experience of reading actual interactive narratives, critics seem to focus less on the texts than on their own treasured memories of reading, fond recollections of live-action game-playing, or vague notions of what a marriage of digital fluidity and narrative fiction ought, ideally, to achieve." Douglas, The End of Books - Or Books without End?, 3.

89 Suter und Böhler, "Hyperfiction", 8. Auch Hautzinger empfindet es als eine Barriere für die Anerkennung der Netzliteratur, daß diese 'als 'Literatur', und damit als 'Ersatz' für gedruckte Bücher angesehen wird". Hautzinger, Vom Buch zum Internet?, 116.

90 Todesco, "Hyperkommunikation", 115.

91 Markus Krajewski, "Spür-Sinn. Was heißt einen Hypertext lesen?", in: Lorenz Gräf und Markus Krajewski, Hg., Soziologie des Internet: Handeln im elektronischen Web-Werk (Frankfurt a.M.; New York, 1997), 60-78, hier: 67.

92 Zimmer, Die Bibliothek der Zukunft, 56.

93 Dirk Schröder, "Der Link als Herme und Seitensprung - Überlegungen zur Komposition von Webfiction", in: Suter und Böhler, Hg., Hyperfiction, 43-60, hier: 50. 
des Schönen"94, Texte, die "der Schnelligkeit des Mediums entsprechend unsorgfältig und geschwätzig" sind - kurzum: "Alles nur zweite Wahl"96, um lediglich einige wenige Beispiele zu nennen.

So gerät der Nutzer in einen circulus vitiosus: Aufgrund der mangelhaften Qualität der Suchergebnisse kommt ein Sondieren weiterer Literaturressourcen meistens nicht mehr in Frage, so daß es letztlich bei einer oberflächlichen Betrachtung verbleibt.

Daher scheint es geboten, erstmals eine über das bisherige Maß hinausgehende, intensive und genaue Bestandsaufnahme des literarischen Angebots im World Wide Web durchzuführen, um detailliert Aufschluß über Form und Inhalt der für Anglistik und Amerikanistik möglicherweise relevanten elektronischen Dokumente zu erhalten. Zunächst erscheint es allerdings unerläßlich, die Frage nach einer neuen Terminologie sowie das Problem der Gattungseinordnung von Online-Literatur zu klären und damit eine eindeutige Diskussionsgrundlage zu schaffen.

Weiterhin sollen einzelne elektronische Werke exemplarisch herausgegriffen und detailliert analysiert werden. Dies soll nach einer Methode erfolgen, die der Vorgehensweise bei gedruckter Literatur in weitest möglichem Maße entspricht.

Auf diesem Wege sollte es gelingen, unter Berücksichtigung der derzeitigen Probleme hinsichtlich Urheberrecht und Haltbarkeit der Speichermedien sowie Kontinuität und Zitierung der elektronischen Ressourcen eine Prognose für die nahe Zukunft der neuen elektronischen Formen englischsprachiger Literatur zu erstellen. Die vorliegende Studie soll somit einen Beitrag zur Beantwortung der von Luserke gestellten Frage leisten: "Wo ist der Ort der Literatur in den Medien und wo ist der Ort der Medien in unserer Kultur?"97

94 Christa Karpenstein-Eßbach, "Medien als Gegenstand der Literaturwissenschaft. Affären jenseits des Schönen”, in: Griem, Hg., Bildschirmfiktionen, 13-32, hier: 13.

95 Zoë Jenny, "Ein Geschenk für den Leser? - Tatjana Blobel im Interview mit acht Autoren", Spiegel Spezial 10: Die Zukunft des Lesens: Vom Buch zum Internet (1999), 20-24, hier: 23.

96 Suter und Böhler, "Hyperfiction", 8.

97 Matthias Luserke, "Kultur, Literatur, Medien. Aspekte einer verwickelten Beziehung”, in: Renate Glaser und Matthias Luserke, Hg., Literaturwissenschaft - Kulturwissenschaft: Positionen, Themen, Perspektiven (Opladen, 1996), 169-191, hier: 185. 


\section{Arbeitstechnische, rechtliche und dokumentarische Probleme}

Die zunehmende Etablierung des World Wide Web im Wissenschaftsbereich verlangt nicht nur eine verstärkte Anpassungsbereitschaft auf seiten der Nutzer, das neue Online-Medium erfordert gleichzeitig auch seitens der Herausgeber eine Anpassung an die anerkannten wissenschaftlichen Maßstäbe, Richtlinien und Bewertungskriterien. Das Fehlen einer einheitlichen, wissenschaftlich akzeptierten Zitierweise bewirkte, daß viele Wissenschaftler davon absahen, ihre Publikationen online zu veröffentlichen. Diese Situation wurde von Gersmann folgendermaßen beschrieben: "Die immer wieder zu beobachtenden Hauptprobleme lassen sich am prägnantesten mit den drei Schlagworten Mangel an Beständigkeit, Mangel an inhaltlicher Qualität und Mangel an methodischem Know-how umreißen." ${ }^{98}$ Mit welchen Problemen sich die Herausgeber von Online-Dokumenten zum damaligen Zeitpunkt konfrontiert sahen, hat Duncan im Jahre 1998 ausführlich dargelegt. ${ }^{99}$

Aus den oben dargelegten Gründen bestand dringender Entscheidungsbedarf hinsichtlich der Zitierweise sowie der Beschreibung des Umfangs eines Dokuments und seiner Plazierung im World Wide Web. Im Verlaufe der vergangenen Jahre wurden diese Punkte ausführlich thematisiert, woraus unterschiedliche Vorschläge zu Zitiervorschriften für WWW-Ressourcen hervorgingen. Wenn diese auch in ihrer Form noch sehr uneinheitlich und durchaus erweiterungsbedürftig erscheinen, so bilden sie doch eine akzeptable Ausgangsbasis für eine den individuellen Bedingungen von Anglistik und Amerikanistik angepaßte Zitierform. Eine kurze Abwägung der gegenwärtig diskutierten Leitlinien und eine für die deutsche Sprache sowie den literaturwissenschaftlichen Kontext modifizierte und für vorliegende Arbeit verbindliche Liste mit praktischen Beispielen findet sich am Ende dieses Kapitels.

Hat ein Nutzer eine Online-Ressource ausgewählt, so sieht er sich zunächst mit der Frage nach der Authentizität sowie der Aktualität des Dokuments konfrontiert. Beide Punkte stellen nach wie vor ein unumgängliches Hindernis im Umgang mit Web-Ressourcen dar. Noch immer scheint ungeklärt, ob und auf welchem Wege es gelingen kann, dem Nutzer alle für ihn wichtigen Informationen über ein Online-Dokument in aktueller Form zu übermitteln. Als ebenso ungelöst beziehungs-

98 Gersmann, "Schöne Welt der bunten Bilder", 109f.

99 Bonnie Duncan, "Citing Hypermedia: Solving the Indexing Dilemma”, (Re)Soundings 2.1 (1998), $<$ http://www.millersv.edu/ resound/*vol2iss1/topframe.html $>, 01.10 .2002$. Teilweise haben Duncans Ausführungen auch im Jahre 2004 noch Aktualität. 
weise vorläufig nur mangelhaft aufgearbeitet erweist sich das Problem des Urheberrechts. Ein Überblick über alle wichtigen Punkte im Umgang mit Online-Ressourcen soll Aufschluß über die gegenwärtige Sachlage geben. ${ }^{100}$

\section{Dokumenten-Integrität}

Ruft der Nutzer eine Seite im World Wide Web auf, so kann er nicht sicher sein, ob es sich bei dem ihm vorliegenden Dokument um jene Fassung handelt, die der Autor ursprünglich für die Veröffentlichung vorgesehen hatte. Das Dokument kann nämlich durch das Betreuungspersonal des Servers, jenes Rechners, der die Daten für andere Netzteilnehmer zur Verfügung stellt, jederzeit verändert werden. Ebenso unterliegt es während des Datentransfers vom WWW-Server zum Endnutzer der Einflußnahme und kann "abgehört, verändert, ausgetauscht oder neu eingespielt" Sicherheitsmaßnahmen, die auf dem Prinzip von Ver- und Entschlüsselung beruhen, wie beispielsweise kryptisches Verschlüsseln ${ }^{102}$, sind nur bedingt einsetzbar, da diese Methoden nur nach Absprache zwischen Daten-Sender und Daten-Empfänger durchführbar sind. Ihre Anwendung würde verlangen, daß der Nutzer zunächst mit dem Betreiber des jeweiligen WWW-Servers in Kontakt träte - ein Verfahren, das sich nur in Einzelfällen durchführen ließe. Zudem könnten diese Verschlüsselungsmaßnahmen keine uneingeschränkte Garantie auf Integrität des Dokumentes gewähren, da sich schon in der Phase vor der Kodierung Manipulationen durchführen ließen. ${ }^{103}$

Solange die Integrität der Dokumente nicht hundertprozentig gewährleistet werden kann, ist Online-Literatur im wissenschaftlichen Bereich in strengem Sinne eigentlich nicht zu verwenden. Zwei Wissenschaftler, die in ihren Ausführungen auf ein und dieselbe Dokumentadresse verweisen, beziehen sich unter Umständen auf vollkommen unterschiedliche Dokumente. Dieser Umstand muß nicht durch Mani-

100 Nach Ansicht von Reif ist die Datensicherheit im WWW an vier Voraussetzungen gebunden: Authentizität, Integrität, Vertraulichkeit und Nachweisbarkeit der Daten. Aus Reifs Ausführungen geht jedoch hervor, daß sich die Punkte Authentizität, Integrität und Nachweisbarkeit nur unwesentlich voneinander unterscheiden. In vorliegender Arbeit sollen die technischen, rechtlichen und dokumentarischen Probleme unter den Stichworten Integrität, Aktualität, Identität und Kontinuität als den wichtigsten Voraussetzungen für Datensicherheit abgehandelt werden. Holger Reif, "Datensicherheit in vernetzten Systemen", in: Feldmann, Neumann und Rommel, Hg., Anglistik im Internet, 85-98, hier: 86f.

101 Rico Apitz, Andreas Guther und Gero Hoffmann, Wissenschaftliches Arbeiten im World Wide Web: HTML - Style-Guide - Sicherheit (Bonn, 1996), 250.

102 Ebd., 246.

103 Vittorio E. Klostermann, Verlegen im Netz: Zur Diskussion um die Zukunft des wissenschaftlichen Buches (Frankfurt a.M., 1997), Punkt 4.d.) des Textes. 
pulation verursacht sein; bereits die Aktualisierung einer WWW-Seite führt zu veränderten Voraussetzungen. Im Unterschied zum Print-Bereich läßt sich bei OnlineLiteratur stets nur das gerade aktuelle Dokument aufrufen, zurückliegende Versionen sind für den Nutzer nicht mehr zugänglich. Gerade aus diesem Grunde sind bei Bezugnahme auf Web-Dokumente Angaben zum Zeitpunkt des Zugriffs auf das Dokument sowie zum Datum der letzten Aktualisierung der Seiten unentbehrlich. Nicht selten fehlt jedoch der Hinweis auf die letzte Bearbeitung, so daß sich keinerlei Rückschlüsse auf mögliche zwischenzeitliche Veränderungen ziehen lassen und der Aktualitätsgrad der Ressource ungeklärt bleibt.

Ein vorbildliches Beispiel für den korrekten Umgang eines Autors mit seinen Dokumenten geben die Herausgeber des Humboldt Forum Recht, kurz HFR, einer von Studierenden und wissenschaftlichen Mitarbeitern der Humboldt-Universität Berlin herausgegebene juristische Online-Publikation. ${ }^{104}$ HFR ist ein zitierfähiges Medium, da alle Beiträge unter ihrer Beitragsnummer des jeweiligen Jahres veröffentlicht und auch unter dieser archiviert werden, so daß sie dauerhaft zugänglich sind.

\section{Aktualität der Dokumente}

Ein weiteres Problem, mit dem der WWW-Besucher ${ }^{105}$ konfrontiert wird, betrifft die Unbeständigkeit der Web-Adressen.

Wie bereits ausgeführt, muß sich jeder Nutzer darüber im klaren sein, daß Adressenangaben keineswegs immerwährende Gültigkeit haben, vielmehr muß stets einkalkuliert werden, daß Dokumenten jederzeit neue Plätze zugewiesen oder aber

104 Humboldt-Forum Recht, Hg., Humboldt-Forum Recht, < $\underline{\text { http://www.humboldt-forum- }}$ recht.de/index 2.html >, 14.11.2002.

105 Diese Bezeichnung wird in Anlehnung an den allgemeinen Sprachgebrauch verwendet, der gelegentlich der Anschein erweckt, beim World Wide Web handele es sich um eine gegenständliche Einrichtung, die tatsächlich zu betreten sei. Ein solcher Eindruck wird vermittelt durch die Verwendung von Worten wie zum Beispiel Zugang, siehe Neumann, "Geisteswissenschaften im Internet", 64, oder Rudolf Seising, "Internet - neue Kulturtechnik zwischen den Kulturen”, in: Gross, Langer und Seising, Hg., Studieren und Forschen im Internet, 11-50, 34, oder eben das Wort Besucher, siehe August Wegmann, "Elektronische Bücher im Internet: Modell und standardisiertes Werkzeug zur Präsentation sequentiell abfolgender Seiten", in: Weber und Maier, Hg., Digitale Archive und Bibliotheken, 287-296, hier: 291, oder Reinhard Keil-Slawik, Werner Beuschel, Birgit Gaiser, Michael Klemme, Cornelia Pieper und Harald Selke, "Multimedia in der universitären Lehre: Eine Bestandsaufnahme an deutschen Hochschulen", in: Ingrid Hamm und Detlef Müller-Böling, Hg., Hochschulentwicklung durch neue Medien: Erfahrungen - Projekte - Perspektiven. Mit einer Bestandsaufnahme über Multimedia-Projekte an deutschen Hochschulen (Gütersloh, 1997), 73-122., hier: 106, oder Dörr, Marianne, "Bibliotheken im Internet - Auf dem Weg zur virtuellen Bibliothek?”, in: Gross, Langer und Seising, Hg., Studieren und Forschen im Internet, 163-172, hier: 167. 
daß sie gänzlich aus dem Netz genommen werden können. Gelegentlich findet sich nach einem Adressenwechsel über einen gewissen Zeitraum hinweg ein kurzer Hinweis auf den neuen Platz des Dokuments; oftmals jedoch erscheint lediglich die Mitteilung, die eingegebene Adresse sei nicht korrekt. In diesen Fällen muß der Nutzer selbst recherchieren, ob das gewünschte Dokument überhaupt noch existiert und wo es gegebenenfalls nunmehr abgelegt ist. ${ }^{106}$

Unabhängig davon, aus welchem Grunde der Zugriff auf ein Dokument nicht mehr erfolgen kann - die Unbeständigkeit der URLs schmälert den Nutzen des World Wide Web für den wissenschaftlichen Gebrauch und disqualifiziert das Online-Medium vorläufig noch erheblich gegenüber den Print-Medien. Als Beispiel mag in diesem Zusammenhang jene URL-Sammlung dienen, die im Anhang des eingangs bereits erwähnten Buches Anglistik im Internet ${ }^{107}$ aufgeführt ist und als Link-Liste auch online auf den Seiten der Erfurt Electronic Studies in English zur Verfügung steht. ${ }^{108}$ Das im Jahre 1997 aufgelegte Dokument war bereits im Juli 1998 nur noch bedingt gültig, da sich genau ein Drittel der aufgeführten WWW-Seiten nämlich exakt 95 von insgesamt 288 Links - zu jenem Zeitpunkt schon nicht mehr aufrufen ließ. Nachdem die Liste der Links bis Oktober 2002 aufgrund von Überarbeitungen um weitere sechs Positionen angewachsen war, führten nur noch $142 \mathrm{der}$ nunmehr 294 Verknüpfungen den Nutzer zum angegebenen Ziel, das heißt mehr als die Hälfte der Links ist außer Kraft gesetzt. Diese Größenordnung ist leider durchaus repräsentativ und beweist anschaulich, wie ernst das Problem der Unbeständigkeit von URLs tatsächlich zu nehmen ist. Auch daß sich die Verantwortlichen dieser Umstände durchaus bewußt sind und ihre WWW-Seiten oftmals mit dem Zusatz versehen, eine immerwährende Aktualität der aufgelisteten Verknüpfungen könne nicht gewährleistet werden, vermag hieran nichts zu ändern. ${ }^{109}$ Da eine vergleichbare

106 Da die Wahrscheinlichkeit besteht, daß sich das Dokument noch auf seinem angestammten Server befindet und lediglich das Verzeichnis gewechselt hat, empfiehlt es sich, als erstes unter der Serveradresse nachzuforschen. Andernfalls muß die Recherche mittels einer Suchmaschine fortgesetzt werden. Bleibt auch diese Suche erfolglos, muß dies nicht zwangsläufig bedeuten, daß das Dokument nicht mehr online ist. Möglicherweise wurden nur die falschen Suchparameter verwendet.

107 Feldmann, Neumann und Rommel, Anglistik im Internet.

108 Dies., "Anglistik im Internet - Proceedings of the Erfurt Conference on Computing in the Humanities, April 1996. Web Sites - Uniform Resource Locators”, Erfurt Electronic Studies in English, $<$ http://webdoc.gwdg.de/edoc/ia/eese/urls.html $>, 01.10 .2002$.

109 Die Link-Liste zu Anglistik im Internet wurde daher mit folgendem Hinweis versehen: "Even if some of these URLs will be obsolete in the foreseeable future, Thomas Rommel as well as the team running the Erfurt Web site will do their best to keep up with changes and new servers." Feldmann, Neumann und Rommel, "Anglistik im Internet", <http://webdoc.gwdg.de/edoc/ia/eese/urls.html $>$, 01.10.2002. 
Konstellation im Print-Bereich undenkbar wäre, bleibt festzuhalten, daß die elektronischen Medien gegenwärtig in der zugriffgewährleistenden Vorhaltung der Daten noch nicht konkurrenzfähig sind.

Gerade weil Wahrung und permanente Aktualisierung des ständig anwachsenden Datenbestandes im World Wide Web so kompliziert und zeitaufwändig sind, muß an dieser Stelle auch über die auslösenden Faktoren und denkbare Wege zur ihrer Beseitigung gesprochen werden. Möglicherweise liegt die Ursache für die desolate Situation auf dem Gebiet elektronischer Veröffentlichungen in der Prägung der Herausgeber durch die Verhältnisse im Bereich der Print-Medien. Dort ist eine Nachbearbeitung von Publikationen nur im Rahmen einer neuen Auflage möglich, was allgemein akzeptiert und berücksichtigt wird. Veröffentlichungen im WWW hingegen erwecken bei der Leserschaft die Erwartung, sie unterlägen steter Überarbeitung und seien somit immer aktuell. Dies ist in Wirklichkeit nicht der Fall. Hinsichtlich der Pflege von Online-Veröffentlichungen muß den Herausgebern zwar Nachlässigkeit vorgeworfen werden, doch ist dies vor dem Hintergrund nach wie vor geringer oder gar fehlender Erfahrungen im Bereich elektronischen Publizierens zu sehen. Die Forderung an die Herausgeber muß aus diesem Grunde dahin gehen, mehr Verantwortung für ihre Veröffentlichungen zu zeigen und sie im Interesse aller Online-Nutzer kontinuierlich $\mathrm{zu}$ überarbeiten und - wie es bei den Print-Medien üblich ist - mit Angaben zur Auflage und zum Erscheinungsdatum zu versehen. Mit dieser Vorgehensweise ließe sich zumindest eine der Problematiken elektronischen Publizierens mehr oder weniger bewältigen.

\section{Autoren-Identität und Urheberrechtsschutz}

Eine weitere Schwierigkeit bildet oftmals die Klärung der Identität der an der Erstellung der Online-Werke beteiligten Personen.

Im Gegensatz zum Print-Bereich lassen sich Werke aus dem World Wide Web oftmals keinem konkreten Autor oder einer bestimmten Autorengemeinschaft zuordnen. Der Nutzer wird zumeist nur darüber informiert, wer für Konzept und Gestaltung der WWW-Seiten verantwortlich zeichnet. Zu beachten ist jedoch, daß im Hinblick auf Online-Präsentationen zwischen mehreren Zuständigkeitsbereichen differenziert werden muß: Zum einen geht es um den dargebotenen Inhalt und deren geistige Schöpfer, zum anderen handelt es sich um die äußere Präsentation eines 
WWW-Angebots und den hierfür autorisierten Personenkreis. Neben diesen Verantwortlichen, die kurz als Autoren und Designer bezeichnet werden sollen, stehen oftmals noch technische Bearbeiter, die nicht selten auch als die einzigen Kontaktpersonen auf den Seiten aufgelistet sind, und die Betreiber der Web-Seiten, wobei alle erwähnten Aufgaben nicht selten von einem beziehungsweise einer Verantwortlichen in Personalunion ausgeübt werden.

Für den Leser von Web-Literatur steht - unter anderem im Hinblick auf eine spätere Auflistung der bibliographischen Angaben - in erster Linie der Autor als geistiger Urheber im Vordergrund des Interesses, Bearbeiter und Betreiber der WebSeiten sind lediglich von zweitrangiger Bedeutung. Angesichts oftmals lückenhafter Online-Dokumentationen ist der Nachweis der Urheberschaft jedoch vielfach nicht in der gewünschten Weise zu führen, so daß die bibliographischen Angaben oftmals unvollständig bleiben müssen.

Gleichfalls problematisch erscheint die Situation im Hinblick auf die Identität jener Personen, die sogenannte interaktive literarische Online-Werke in kreativer Mitwirkung und somit gewissermaßen in Koautorschaft erweitern. Da sie ebenso wie der Autor der literarischen Ausgangsform an der Entstehung des Werkes beteiligt sind und unter Umständen maßgeblich zu seiner Erweiterung beitragen, müßten sie gegebenenfalls unter entsprechendem Hinweis auf den Umfang ihrer Beteiligung namentlich erwähnt werden. Tatsächlich gelten sie laut deutschem Urheberrechtsgesetz als Miturheber des Werkes. ${ }^{110}$ Bleiben der oder die einzelnen Mitarbeiter jedoch unerwähnt, weil die Namen vom Herausgeber zurückgehalten werden oder aber die Beiträge anonym eingegangen sind, so liegt die Verantwortung für den Inhalt der Dokumente auf Herausgeberseite, wobei fraglich ist, wie dann das Urheberrecht an diesen Werken gehandhabt wird. So ist beispielsweise der Fall denkbar, daß ein Gedicht, das durch kooperative Zusammenarbeit im World Wide Web entstanden ist, in die Geschichte der Literatur eingeht, woraufhin sich vermutlich nicht nur auf seiten der Leser, sondern in erster Linie bei den Autoren der Wunsch nach Klärung des geistigen Eigentums an diesem Werk ergäbe.

Kist hat im Jahre 1988 festgestellt, daß die Gesetzgebung weit hinter der Entwicklung im Bereich elektronischer Medien zurückstehe, und gleichzeitig auf die bedenkliche Tendenz zu wachsender Indifferenz gegenüber Urheberrechten verwie-

${ }^{110}$ H. Jochen Krieger, Verantw., Das deutsche Urheberrechtsgesetz - Teil 1 Urheberrecht, $\S 8$ Miturheber (14.08.2002), <http://transpatent.com/gesetze/urhg1.html\#8>, 22.10.2002. 
sen. ${ }^{111}$ Diese Neigung scheint nach wie vor ungebrochen zu sein, und so drängt sich der Eindruck auf, daß die derzeitige unbefriedigende Lage auf dem Gebiet des Urheberrechts nicht allein auf ein etwaiges Fehlen eindeutiger Rechtsvorschriften zurückzuführen ist, sondern daß vielmehr eine - von der Existenz aller wirksamen Gesetze anscheinend unbeeindruckte - allgemeine Einstellung vorherrscht, das World Wide Web als rechtsfreien Raum zu betrachten. Kist merkte hierzu kritisch an, es sei ganz offensichtlich dem "Fehlen einer öffentlichen Moral" zuzuschreiben, daß der Umgang mit fremdem geistigem Eigentum im WWW die angemessene Sorgfalt und das notwendige Rechtsbewußtsein vermissen lasse. ${ }^{112}$

Da die ganz überwiegende Zahl der Urheberrechtsverletzungen eher harmloser Natur sein dürfte, scheint der von Kist erhobene Vorwurf fehlender öffentlicher Moral allerdings zu hoch gegriffen. Statt dessen sind es möglicherweise die Internationalität des WWW mit seinen vielen fremden Netzteilnehmern in (Wohn-)Sitzstaaten fernab der Jurisdiktion des eigenen Landes und das Fehlen einer länderübergreifenden, omnipräsenten Überwachungsinstanz, gleich in wessen Trägerschaft, die in vielen Fällen bei dem Schädiger den Gedanken, es könne eine Urheberrechtsverletzung begangen werden, gar nicht erst aufkommen lassen.

Entgegen Kist stellt zumindest die deutsche Rechtsprechung durchaus eindeutige Vorschriften zur Regelung von Urheberrechtsfragen bereit. Wiewohl diese zum Teil erst innerhalb der vergangenen Jahre aufgrund der verstärkten Nutzung des WWW geschaffen beziehungsweise den veränderten Bedingungen angepaßt worden sind, scheint die Situation aber auch vorher nicht so ungeregelt gewesen zu sein, wie sie oftmals beschrieben und kritisiert wurde. Hierzu findet sich beispielsweise bei Cyberlaw folgende Ausführung:

Im Internet besteht kein rechtsfreier Raum. Es gelten dieselben Regeln wie sonst, nur mit internationalen Konsequenzen. Insbesondere das Urheberrecht wie auch der Wettbewerb sind detailliert festgelegt und auch im Internet gültig. ${ }^{113}$

Ein weiterer Textauszug untermauert die These, daß das Hauptproblem bei der Debatte um Urheberrechte für Online-Werke nicht im Mangel an konkreten Rechts-

\footnotetext{
111 Kist, Elektronisches Publizieren, 86.

112 Ebd.

113 Wilfried Gaiser, Hg., "Urheberrechte im Internet", in: Ders., Verantw., Cyberlaw ([ $\left.\left.{ }^{1} 2002\right]\right)$, $<\underline{\text { http://www.cyberlaw.de/Recht/Urheberrecht/ReUr025.pdf }>, 04.11 .2002 . ~}$
} 
vorschriften, sondern in der ungenügenden Durchsetzung der bereits bestehenden Gesetze liegt:

Das Internet ist kein rechtsfreier Raum. [...] die staatlichen Regeln hierzu sind bereits vorhanden. Auch wenn die Rechtsdurchsetzung durch den Staat derzeit noch schwierig ist. ${ }^{114}$

Welche Voraussetzungen ein Werk im World Wide Web erfüllen muß, damit dessen geistiger Schöpfer Urheberrechtsansprüche anmelden kann, ist dem folgenden Zitat zu entnehmen:

Um einen urheberrechtlichen Anspruch für Inhalte im Internet geltend machen zu können, müssen die Anforderungen des $\S 2$ Abs.2 Urheberrechtsgesetz (UrhG) erfüllt sein. Die Internetseite muss eine persönliche geistige Schöpfung des Urhebers sein, also ein Werk darstellen. Ist dies der Fall, stehen dem Urheber insbesondere die Verwertungsrechte der $\S \S 15 \mathrm{ff}$ UrhG zu, mit deren Hilfe er eine Vergütung für sein Werk erlangen kann. Um den Anforderungen des $\S 2$ Abs.2 UrhG zu genügen, muss das Werk das Ergebnis individuellen Schaffens sein und eine gewisse Gestaltungshöhe erreichen. Dabei ist jedoch kein zu hoher Maßstab anzulegen, weshalb die Mehrzahl der Homepages im Internet diese Anforderung erfüllen dürfte.

Da auch frei zugängliche Texte im Netz in einer sogenannten HTML-Datei der Computersprache des Internets - veröffentlicht werden müssen, um an den Bildschirmen der Nutzer lesbar zu sein, können diese Texte trotz nicht schutzwürdiger Inhalte als Datei gem. § 2 UrhG Schutz genießen. Außerdem kann der in einer Internetseite enthaltene Text als Sprachwerk gem. § 2 Abs. 1 Nr. 1 UrhG geschützt sein, Grafiken als Werke bildender Kunst nach $\S 2$ Abs. 1 Nr. 4 UrhG und Fotos nach $\S 2$ Abs. 1 Nr. 5 UrhG. ${ }^{115}$

Es wäre nun allerdings zu kurz gegriffen anzunehmen, bei der Forderung nach einer rechtlichen Grundlage gehe es ausschließlich um materielle Vergütung. Der Schutz des geistigen Eigentums soll vielmehr gleichzeitig auch verhindern, daß ein Werk einen Re- oder Dekontextualisierungsprozeß erfährt. ${ }^{116}$ Ist die rechtliche Grundlage nicht vorhanden, so kann "ohne den Schutz des Urheberrechts das zerbrechliche und vielfältig strahlende System des Schaffens und Verbreitens informationeller Inhalte, das die Basis unserer kulturellen Kommunikation bildet, schließlich zusammenbrechen."117

\footnotetext{
114 Ders., Hg., "Warum Info zum Internet-Recht?", in: Cyberlaw ([ $\left.\left.{ }^{1} 2002\right]\right)$, $<$ http://www.cyberlaw.de/Recht/Onlinerecht/ReOn049.pdf $>$, 04.11.2002.

115 Ralf Möbius, "Urheberrechte im Internet", in: Ralf Möbius, Hg., Internet-Recht-online, $<\underline{\text { http://mitglied.lycos.de/InternetRechtOnline/urhr.htm }>, 04.11 .2002 .}$

116 Steven Jones, "Kommunikation, das Internet und Elektromagnetismus", in: Stefan Münker und Alexander Roesler, Hg., Mythos Internet (Frankfurt a.M., 1997), 131-146, hier: 139.

117 Kist, Elektronisches Publizieren, 87.
} 
Grundsätzlich sollte gewährleistet sein, daß sich wissenschaftliche Ressourcen im World Wide Web korrekt zitieren lassen, ohne daß der Nutzer befürchten muß, daß die angegebenen Seiten ihre Gültigkeit verlieren. So wurde Anfang 1998 in weltweiter Übereinkunft ein neues Identifikationssystem eingeführt, das sich auf eine Identifizierungsnummer für digitale Objekte gleich welcher Art stützt, den sogenannten Digital Objects Identifier $(D O I),{ }^{118}$ der etwa der International Standard Book Number $(I S B N)$ für gebundene Bücher entspricht. ${ }^{119}$ Dieses Angebot der $\mathrm{Zu}$ weisung und Verwaltung einer Identifikationsnummer richtet sich derzeitig hauptsächlich an Unternehmen, doch auf lange Sicht könnte dieses System auch für Einzelpersonen realisierbar und hilfreich sein.

Einen weiteren Dienst zum Schutz von Autoren bietet die Clearingstelle Multimedia für Verwertungsgesellschaften von Urheber- und Leistungsschutzrechten $G m b H$, kurz $C M M V$. Diese zentrale Anlaufstelle bietet Multimedia-Produzenten die Möglichkeit, die Rechteinhaber all jener urheberrechtlich geschützten Werke oder Inhalte zu ermitteln, die sie im Rahmen einer Multimedia-Produktion verwenden möchten. ${ }^{120}$ Allerdings kann die Lizenzierung bislang noch nicht von der CMMV durchgeführt werden, sondern muß unmittelbar durch die Rechteinhaber selbst erfolgen.

Unabhängig von der Diskussion, ob und wie Urheberrechtsansprüche durchgesetzt werden können, bleibt fraglich, ob kostenpflichtige Literatur im World Wide Web zukünftig überhaupt die Chance hat, vom Nutzer angenommen zu werden. Nachweislich stehen die meisten Befragten dem Lesen am Bildschirm eher ablehnend gegenüber. ${ }^{121}$

Und noch ein weiteres Problem tut sich auf: Sollte Online-Literatur weiterhin größtenteils kostenfrei angeboten werden, so ist ungewiß, ob dies nicht letztlich zu

118 Tremmel Verlag, Hg., Urheberrechte im Netz, <http://www.tremmel.de/html/urheberrecht.htm>, 23.10.2002. Der elektronische Datenbestand erhält einen individuellen Identifizierungscode, den sogenannten DOI, der in einer zentralen Datenbank gespeichert ist. Aufgabe der Betreiber dieser Datenbank ist es, jederzeit die aktuelle Web-Adresse des Datenbestandes bereitzuhalten. $\mathrm{Zu}$ dessen Aufruf bedient sich der Nutzer anstelle eines URL des DOI; er wird sodann automatisch an den aktuellen Speicherplatz der Daten weitergeleitet. Marcel Schellong, “'how to ...': DOI - Digital Object Identifier", in: Martin Huber u.a., Hg., Zentrum Elektronisches Publizieren in den Literaturwissenschaften ([127.08.2003]), <http://www.zepl.uni-muenchen.de/reports/how to doi.htm>, 09.12.2003.

119 ISBN-Agentur für die Bundesrepublik Deutschland, Hg., Erste Informationen für Erzeuger, Verlage und Informationsanbieter zur Anwendung des Digital Object Identifier, <http://www.germandoi.org/LEITFADE.HTM>, 23.10.2002.

120 Clearingstelle Multimedia für Verwertungsgesellschaften von Urheber- und Leistungsschutzrechten GmbH, Hg., CMMV-Info, <http://www.cmmv.de/cmmv-info.htm>, 04.11.2002.

121 Boesken, “Lesen am Bildschirm”, 141. 
Lasten der Qualität des Dargebotenen geht. Es bleibt abzuwarten, ob es auch langfristig Autoren geben wird, die ihre Arbeiten frei zugänglich im WWW präsentieren und somit auf eine materielle Vergütung verzichten. Unbestritten besteht kein notwendiger Zusammenhang zwischen der Qualität literarischer Online-Werke und einem Anspruch auf Kompensation; es dürfte jedoch außer jedem Zweifel sein, daß es in der Regel ein hohes Maß an Idealismus, Altruismus und finanzieller Unabhängigkeit erfordert, persönliches Gedankengut kostenfrei für die Allgemeinheit zur Verfügung zu stellen. Dies gilt zumindest für jene Autoren, die sich hohen Qualitätsansprüchen verpflichtet fühlen, sich intensiv engagieren und als Verfasser von Werken hoher Qualität anerkannt sind.

\section{Kontinuität der Dokumente}

Außer Papyri und Pergamenten geben vor allem Schriften auf Papier Zeugnis von unserer Vergangenheit; allerdings müssen wir in neuerer Zeit zunehmend negative Erfahrungen mit der Haltbarkeit des Mediums Papier machen. Wertvolle und einzigartige Bestände drohen sich infolge ihres Säuregehalts allmählich zu zersetzen und damit für immer verloren zu gehen. Aufgrund dieser Erkenntnis im Bereich der Print-Medien laufen angestrengte Versuche, Computer zur Wahrung des Druckbestandes einzusetzen. Da zur Zeit jedoch noch keine gesicherten Erkenntnisse hinsichtlich der Beständigkeit dieses elektronischen Mediums vorliegen, ist ungewiß, ob dieser Schritt nicht seinerseits trotz der Multiplizierbarkeit der Daten ebenfalls in absehbarer Zukunft den unwiderbringlichen Verlust von Datenmaterial zur Folge hat.

$\mathrm{Da}$ elektronische Texte aus dem ausgehenden zwanzigsten Jahrhundert auch nach einem vergleichsweise kurzem Zeitraum von hundert Jahren noch gelesen werden können, ist durchaus nicht sicher, denn gerade der Computer in seiner Doppelfunktion als Speichermittel und notwendiger Lesehilfe setzt gewisse technische Gegebenheiten sowohl auf Seiten des Datenarchivars als auch des Lesers voraus. Angesichts des raschen technischen Fortschritts im Soft- und Hardware-Bereich läßt sich zum gegenwärtigen Zeitpunkt noch nicht absehen, welchen Verlauf diese Entwicklungen nehmen werden. Soll das World Wide Web tatsächlich dazu dienen, Vergangenheit und Gegenwart für die Zukunft zu bewahren, müssen zum einen internationale Vereinbarungen zu einer planvollen und übersichtlich strukturierten Verteilung der Materialien getroffen werden, um dem Nutzer das Auffinden der Daten- 
materialien weitestgehend zu erleichtern; zum anderen ist dafür Sorge zu tragen, daß diese Daten kontinuierlich gepflegt werden und jederzeit für den Nutzer präsent sind.

Zimmer, der den Seiten im World Wide Web eine durchschnittliche Lebensdauer von nicht mehr als vierundvierzig Tagen zuschreibt ${ }^{122}$ und künftigen Generationen in der Frage der Bewahrung menschlichen Wissens desolate Perspektiven bescheinigt - "Das Gedächtnis der Menschheit wird so löchrig sein wie ein Alzheimergehirn." 123 -, erörtert drei Möglichkeiten zur Lösung des Problems der DatenFragilität. Leider kann keiner der Vorschläge wirklich überzeugen. So ist das "Modell Technikmuseum" $" 124$ schon deshalb nicht geeignet, weil die Aufbewahrung und Wartung der Daten sowie der für ihre Wiedergabe erforderlichen Geräte und Programme wegen Raummangels und hoher Personalkosten faktisch nicht zu gewährleisten ist. Auch das Modell der Emulation stellt keine vertretbare Maßnahme dar: Die Möglichkeit der Reproduktion veralteter Hard- und Software auf Computern des jeweils aktuellen Standards würde die Sicherung der entsprechenden technischen Metainformationen bedingen, was wiederum Konservierungs- und Lagerungsprobleme zur Folge hätte. Einzig das Modell der Migration böte durch kontinuierliches Umkopieren des gesamten Datenmaterials von einem Speichermedium auf das technisch nächstfolgende eine, wenn auch immer wieder sehr zeitaufwendige, so aber doch akzeptable und realistische Alternative zur langfristigen Datensicherung. ${ }^{125}$

Für den Nutzer sollte so oder so gewährleistet sein, daß er jederzeit von zentralen Punkten aus öffentlichen Zugang zu den elektronischen Daten erhält. Denn auch er - der Nutzer - wird seine Soft- und Hardware zwangsläufig zu gewissen Zeitpunkten aufrüsten müssen; auch für ihn wird es sich als zeit- und kostenintensiv erweisen, auf dem Stand der Technik zu bleiben. Daher erscheint es fraglich, ob das World Wide Web bezüglich der dauerhaften Archivierung von Datenmaterial den allgemeinen Erwartungen gerecht werden kann.

\footnotetext{
${ }^{122}$ Zimmer, Die Bibliothek der Zukunft, 184.

123 Ebd., 186.

124 Ebd., 181.

125 Vgl. ebd.
} 


\section{Dokumentation von WWW-Materialien}

\subsection{Präsentation}

Damit jeder Verfasser eines elektronischen Dokuments auf ein verbindliches Regelwerk zurückgreifen kann, das ihn dazu befähigt und anleitet, sein Werk ausreichend zu kennzeichnen, bedarf es verbindlicher Präsentations-Richtlinien. Als Anhaltspunkt für eine detaillierte Beschreibung von WWW-Dokumenten mag das Dublin Core Metadata Element Set, kurz Dublin Core, herangezogen werden, anhand dessen Online-Materialien nach einem festgelegten Schema mit Informationen versehen werden. ${ }^{126}$ Das seit dem Jahre 1995 kontinuierlich in interdisziplinärer Übereinstimmung weiterentwickelte Verfahren ermöglicht die gezieltere Lokalisierung von Web-Ressourcen durch Suchmaschinen. Zu diesem Zwecke werden die elektronischen Dokumente mit einer strukturierten Kurzbeschreibung ihres Inhalts ausgestattet, den sogenannten Metadaten. Diese "Daten über Daten"127 werden allerdings nicht in den Dokumenten selbst aufgelistet, sondern in ihrem Programmier-Code. Sinnvoller wäre es jedoch, sie in Form einer tabellarischen Auflistung an einer festzusetzenden Stelle dokumentintern zu präsentieren und vergleichbar der CIPKurztitelaufnahme der Deutschen Bibliothek als Einheitsbeschreibung zu etablieren. Von dieser Regelung würden sämtliche Online-Nutzer profitieren, indem sie jene Informationen als Orientierungshilfe im Netz verwenden könnten; Geisteswissenschaftlern - und innerhalb dieser Gruppe speziell den Literaturwissenschaftlern könnte ein solches Schema als Basis für ihre bibliographischen Tätigkeiten dienen.

126 Das Erschließungsmodell wurde im März 1995 im Rahmen eines interdisziplinären MetadatenWorkshops in Dublin/Ohio entworfen und erfährt seitdem eine kontinuierliche Weiterentwicklung und Anpassung an die Gegebenheiten elektronischen Publizierens. Das Dublin-Core-Schema enthält 15 Grundelemente zur Spezifizierung von Titel, Verfasser/Urheber, Thema, Inhalt, Verleger/ Herausgeber, inhaltlich verantwortlichen Personen, Publikationsdatum, Ressourcenart, Format, WebAdresse, Quelle, Sprache, formalem Zusammenhang zu anderen Ressourcen, räumlichem oder zeitlichem Umfang und Rechtsgrundlagen. Staats- und Universitätsbibliothek Göttingen, "SSG-FI Metadatenkonzept (Template)", in: Dies., Hg., SSG-Fachinformation (SSG-FI) (28.04.1999 [11997]), $<$ http://www.sub.uni-goettingen.de/ssgfi/projekt/doku/templ-de.html $>, 03.12 .2002$. Im Gegensatz zu anderen Schemata empfiehlt sich dieses Konzept aufgrund seiner Anwendungsfreundlichkeit und seiner Applikationsvielfalt. Vgl. auch Staats- und Universitätsbibliothek Göttingen, "Dublin-CoreMetadata-Element-Set", in: Dies., Hg., Metadata Server - Einführung in Metadaten (27.03.2001 $\left.\left[{ }^{1} 1997\right]\right),<$ http://www2.sub.uni-goettingen.de/intrometa.html $>, 03.12 .2002$.

127 Staats- und Universitätsbibliothek Göttingen, "Was sind Metadaten?", in: Dies., Hg., Metadata Server - Einführung in Metadaten (27.03.2001 [ $\left.\left.{ }^{1} 1997\right]\right),<$ http://www2.sub.unigoettingen.de/intrometa.html>, 04.12.2002. 
Das Metadatenkonzept des Dublin Core beinhaltet im Dezember 2002 insgesamt 46 Kategorien. ${ }^{128}$ Diese dienen nicht allein der Erfassung ressourceneigener Daten, wie zum Beispiel Titel, Verfasser, Mitarbeiter und Verleger, Zielgruppe, Inhaltsverzeichnis, Zusammenfassung, Kategorisierung, Kurzbeschreibung und Schlagwörter, Version, Umfang, Format, Norm und Sprache, Datum der Erstellung, der Vorlage, der Annahme, des urheberrechtlichen Schutzes, der Veröffentlichung, der Überarbeitung und der Verfügbarkeit, sondern sie sollen den Nutzer darüber hinaus umfänglich über alle mit der Ressource in Zusammenhang stehende Quellen informieren sowie auf über- und untergeordnete Seiten und themenverwandte Querverbindungen hinweisen.

Natürlich ist eine solche Aufstellung viel zu umfangreich, um sie als verbindliche Vorlage für die Erstellung von Metadaten-Verzeichnissen zu etablieren. Zudem sind zahlreiche Angaben für literarische und literaturwissenschaftliche OnlinePublikationen irrelevant. Dennoch läßt sich anhand oben genannten Katalogs eine Liste jener Daten zusammenstellen, mit denen eine Ressource bereits von Autorenseite aus versehen werden sollte:

- Titel der Ressource

- Titel der übergeordneten Ressource (zum Beispiel eines Periodikums oder einer Fakultät)

- Autor beziehungsweise inhaltlich Verantwortlicher

- Herausgeber der Ressource

- Verantwortlicher Vertreiber der Ressource (zum Beispiel ein Verlag oder eine Fakultät)

- Schlagwörter zum Inhalt der Ressource

- Erscheinungsverlauf der Ressource (zum Beispiel eines Periodikums)

- URL der Ressource

- Datum der ersten Veröffentlichung im WWW

- Datum der letzten Überarbeitung

- Bezüge zu Druckpublikationen (vorher/gleichzeitig/nachträglich erschienen ${ }^{129}$ )

128 Dublin Core Metadata Initiative, Hg., Dublin Core Metadata Initiative Elements and Element Refinements - a current list (06.10.2002), <http://dublincore.org/documents/2002/10/06/currentelements/>, 03.12.2002.

129 Die hier zu verwendenden Begriffe werden in Kapitel 5.2.3. näher erläutert. 
Diese Metadaten sollten den Ressourcen entweder vorangestellt werden oder aber an ihrem Ende stehen, wobei eine Platzierung im Dokument-Kopf den Vorteil böte, daß der Nutzer bereits vor Sichtung des Online-Materials über seinen Inhalt informiert würde.

\subsection{Zitation}

Obgleich im World Wide Web mittlerweile zahlreiche Vorschläge für korrektes bibliographisches Arbeiten vorliegen, darunter die ersten allgemein anerkannten, von der Modern Language Association, kurz MLA, autorisierten Richtlinien zur Dokumentation von WWW-Ressourcen ${ }^{130}$, bleibt die Forderung Duncans nach einem einheitlichen Maßstab für die Zitierung von Online-Dokumenten nach wie vor aktuell. ${ }^{131}$ Die derzeitigen Richtlinien sind in ihrer Gesamtheit uneinheitlich und im einzelnen teilweise lückenhaft oder umgekehrt viel zu ausführlich. Hierfür sind die jeweiligen Verfasser teilweise selbst verantwortlich, da sie ihre Richtlinien in bezug auf Kurztitelangaben im allgemeinen nur mangelhaft erläutern und auf diese Weise eine unterschiedliche Interpretation ihrer Anweisungen provozieren. Eine einheitliche Dokumentationsform kann somit nach wie vor nicht gewährleistet werden.

Schuld trifft aber auch die Herausgeber von Online-Dokumenten. Viele WebSeiten enthalten nur unzureichende Angaben, so daß oftmals allein der Titel des Werkes, der Name der Seite, das Zugriffsdatum sowie der URL aufgeführt werden können. Daher wäre es wünschenswert, wenn sich die Herausgeber von OnlinePublikationen künftig an Zitierrichtlinien orientierten und entsprechend zitierfähige Dokumente veröffentlichten. Bei diesen Überlegungen darf allerdings nicht vergessen werden, daß das World Wide Web ein Medium ist, das sich vermutlich erst in der Anfangsphase seiner Entwicklung befindet. Rotermund gab im Jahre 1997 folgende Umstände zu bedenken: "Das Internet, speziell das World Wide Web, befindet sich in bezug auf seine Techniken und vor allem in bezug auf seine Inhalte noch in den Kinderschuhen. Die meisten seit 1800 entstandenen technischen Medien haben ihre inhaltliche Bestimmung, die ihnen ihre Durchsetzung und ihren Erfolg sicherte, erst

130 Modern Language Association, Hg., MLA Style: Citing Sources from the World Wide Web (14.05.1998 [ $\left.\left.{ }^{1} 07.04 .1998\right]\right),<$ http://www.mla.org/main_stl.htm>, 11.11.2002.

131 Duncan hatte sich bereits im Jahre 1998 zur Problematik des Online-Publizierens geäußert und einen einheitlichen Maßstab für die Zitierung von Web-Dokumenten gefordert. Duncan, "Citing Hypermedia", <http://www.millersv.edu/ resound/*vol2iss1/topframe.html $>$. Siehe hierzu auch die Ausführungen zu Beginn des Kapitels. 
nach manchen Umwegen gefunden."132 Mittlerweile scheint es allerdings an der Zeit, diese vielen Umwege, die oftmals in einer Sackgasse münden, zusammenzuführen, zu begradigen und gewissermaßen zur Hauptstraße auszubauen.

Vor der genaueren Erörterung und Abwägung der bestehenden Normen ist zunächst kurz zu klären, welchen Anforderungen Zitierrichtlinien grundsätzlich entsprechen sollten. Walker und Taylor haben fünf Kriterien herausgearbeitet, die der Wahrung von Autoren- und Nutzerrechten gleichermaßen dienen sollen. Die Five Principles of Citation Style lauten:

- The Principle of Access

- The Principle of Intellectual Property

- The Principle of Economy

- The Principle of Standardization

- The Principle of Transparency ${ }^{133}$

Prinzip 1 soll den Leser dazu befähigen, schnell und mit minimalem Aufwand Zugang zu dem zitierten Dokument zu erhalten; Prinzip 2 soll dem Schutz des geistigen Eigentums an dem betreffenden Dokument dienen; gemäß Prinzip 3 sollten die bibliographischen Angaben zugunsten der Zeit- und Platzersparnis keine über die erforderlichen Angaben hinausgehenden Informationen enthalten; Prinzip 4 unterstützt die Vereinheitlichung im Sinne der Förderung einer uneingeschränkten, globalen Verständigung, und Prinzip 5 fordert - in einem gewissen Widerspruch zu Prinzip 3 - die Einbeziehung zusätzlicher wertvoller Angaben zum unmißverständlicheren und rascheren Verständnis der bibliographischen Daten. ${ }^{134}$

Die genannten fünf Grundsätze, die ursprünglich für gedruckte Veröffentlichungen entwickelt worden sind, lassen sich allerdings nicht ohne weiteres auf Online-Publikationen übertragen. So hat der Nutzer - wie bereits ausgeführt derzeit noch keine Garantie, daß die von ihm gewünschten Dokumente in unveränderter Form dauerhaft zugänglich sind, sei es, weil sie überarbeitet oder aber

132 Rotermund, "Warten auf den Online-Ulysses", 78.

133 Janice R. Walker und Todd Taylor, The Columbia Guide to Online Style (New York, NY; Chichester, West Sussex, 1998), 11-15.

134 Prinzip 3 und 5 sollten gegeneinander abgewogen werden, wobei Erstellung und Verständnis der Angaben in Punkt 5 kein Fachwissen erfordern sollten. Payer beschreibt in ihren Grundlagen der Formalerschließung den Umfang der aufzuführenden Daten als so groß, daß "das Objekt identifiziert und wiedergefunden werden kann”, wobei zusätzliche, ergänzende Informationen der sachdienlicheren Veranschaulichung und dem besonderen Sachverständnis der "Benutzer spezieller Einrichtungen", wie zum Beispiel Galerien, dienen sollen. Margarete Payer, Grundlagen der Formalerschließung: Skript - Kap. 1.3.: Welche Informationen sollen erfaßt werden? (18.10.1999), $<$ http://www.payer.de/grundlagenfe/fegscr01.htm> 11.11.2002. 
weil sie gänzlich gelöscht werden. Auch wurde bereits daraufhingewiesen, daß die Umsetzung von Prinzip 2 derzeit noch mit Problemen behaftet ist. Ebenso verhält es sich mit Punkt 4, da eine einheitliche Terminologie und Schreibweise erwähntermaßen noch aussteht.

\subsubsection{Typographie und Sprache}

Die in der Praxis bestehende Uneinheitlichkeit des Dokumentierens beziehungsweise Zitierens beginnt bereits bei der äußeren Gestaltung der bibliographischen Angaben. So wird der Titel der Web-Seite beziehungsweise der Titel des übergeordneten Werkes, Textes oder Dokuments nach einigen Richtlinien unterstrichen ${ }^{135}$, während er anderen Bestimmungen zufolge kursiv gesetz ${ }^{136}$ oder aber in keiner Weise hervorgehoben wird ${ }^{137}$. Um diesem unerfreulichen Durcheinander zu begegnen, erscheint es empfehlenswert, stets in Übereinstimmung mit den bibliographischen Angaben für gedruckte Literatur zu zitieren. ${ }^{138}$

Weitere Diskrepanzen ergeben sich aus einer unterschiedlichen typographischen Anordnung der bibliographischen Angaben. Üblicherweise wird der URL direkt an die Angaben zu Verfasser und Werkstitel angeschlossen, wobei die Länge der Adresse oftmals einen Zeilenumbruch erfordert. Trennstriche weisen jedoch eine sehr hohe Ähnlichkeit zu Bindestrichen auf, die ihrerseits fester Bestandteil des Zeichensatzes für URLs sind, weshalb ihre Verwendung unter Umständen zu einer Veränderung der Online-Adresse und daher zu Mißverständnissen führen kann. ${ }^{139}$ Aus diesem Grunde schlägt Marx beispielsweise vor, den URL stets in einer neuen Zeile beginnen zu lassen, um Trennungen möglichst zu vermeiden. ${ }^{140}$ Dieser Vorschlag ist durchaus sinnvoll, da er ein hohes Fehlerpotential bereits im voraus beseitigen

135 Joseph Gibaldi, MLA Handbook for Writers of Research Papers (New York, 1999 [ $\left.\left.{ }^{1} 1977\right]\right), 180 \mathrm{ff}$.

136 Walker und Taylor, The Columbia Guide to Online Style, 45.

137 Jens Runkehl und Torsten Siever, Das Zitat im Internet: Ein Electronic Style Guide zum Publizieren, Bibliografieren und Zitieren (Hannover, 2001 ['2000]), 99.

138 Alle nachfolgend entwickelten Regeln sollen in vorliegender Arbeit Anwendung finden. In diesem speziellen Falle sollen Titel von Web-Seiten beziehungsweise übergeordneten Werken, Texten oder Dokumenten in Anlehnung an den zugrundeliegenden Zitierstandard und somit in Übereinstimmung mit den Angaben für gedruckte Literatur kursiv gesetzt werden.

139 Vgl. hierzu auch Runkehl und Siever, Das Zitat im Internet, 109.

140 Thomas Christoph Marx, "Zur Problematik des Zitierens aus dem Internet: Anforderungen, Möglichkeiten und Grenzen - Übersicht 2: Problemfelder bei Nachweisen aus dem Internet”, in: AlbertLudwigs-Universität Freiburg, Historisches Seminar: Forum, < http://www.geschichte.unifreiburg.de/histsem/forum/zitieren.html>, S. 12, 06.11.2002. In Kurzfassung als Vorabversion erschienen: Thomas Christoph Marx, "Zur Problematik des Zitierens aus dem Internet: Anforderungen, Möglichkeiten und Grenzen", Geschichte in Wissenschaft und Unterricht (GWU), 52 (2001), 238245. 
könnte; fraglich ist jedoch, ob sich eine solch grundlegende Veränderung des Fußbeziehungsweise Endnotenformats wird durchsetzen können. ${ }^{141}$

Anders liegt der Fall bei der zunehmenden Verwendung englischsprachiger Begriffe. Vor dem Hintergrund wachsender Globalisierung schlagen Runkehl und Siever vor, die Abkürzung Hg. (für Herausgeber) durch Ed. (für Editor) zu ersetzen, nicht ohne gleichzeitig einzuräumen, daß die Entscheidung für das eine oder andere letztlich abhängt von der jeweiligen Zielgruppe und der Sprache, in der das Dokument verfaßt ist. ${ }^{142}$ Diese Argumentation scheint überzeugend; somit würde es keinen Sinn machen, einen deutschen Text im bibliographischen Teil anders als mit deutschen Bezeichnungen und Abkürzungen zu versehen.

\subsubsection{Zitatinhalt}

Das eigentliche Problem bei der Zusammenstellung der bibliographischen Angaben für Online-Publikationen ergibt sich aus der Frage nach der Relevanz der aufzunehmenden Informationen. Einigkeit herrscht zwischen den Verfassern bestehender Zitiervorschriften offenbar lediglich in bezug auf die Nennung des beziehungsweise der Autoren und des Titels der Web-Seite sowie - falls vorhanden - des Werktitels. Allen anderen Daten wird sehr unterschiedliche Bedeutsamkeit zugewiesen; die Bandbreite reicht von der Beschränkung auf das Publikationsdatum, die WebAdresse und das Zitationsdatum ${ }^{143}$ bis hin zur Nennung aller mit der jeweiligen Online-Publikation in Verbindung stehenden Informationen. So wären gemäß den Richtlinien der MLA beispielsweise - sofern verfügbar und relevant - folgende Punkte zu nennen:

- Name of the author, editor, compiler, or translator of the source (if given), reversed for alphabetizing and, if appropriate, followed by an abbreviation, such as $e d$.

- Title of an article, poem, short story, or similar short work in the Internet site (enclosed in quotation marks). Or title of a posting to a discussion list or forum (taken from the subject line and put in quotation marks), followed by the description Online posting

- Title of a book (underlined)

- Name of the editor, compiler, or translator of the text (if relevant and if not cited earlier), preceded by the appropriate abbreviation, such as $E d$.

${ }^{141}$ In vorliegender Arbeit soll dieser Vorschlag keine praktische Umsetzung finden. Das erwähnte Fehlerpotential besteht zwar durchaus und könnte mit dieser Vorgehensweise vermieden werden, mittlerweile aber dürfen bei der angesprochenen Zielgruppe ein ausreichendes Bewußtsein und die nötige Aufmerksamkeit im Umgang mit diesem Problem vorausgesetzt werden.

${ }_{142}$ Runkehl und Siever, Das Zitat im Internet, 93.

143 Ebd., 99. 
- Publication information for any print version of the source

- Title of the Internet site (e.g., scholarly project, database, online periodical, or professional or personal site (underlined) or, for a professional or personal site with no title, a description such as Home page

- Name of the editor of the site (if given)

- Version number of the source (if not part of the title) or, for a journal, the volume number, issue number, or other identifying number

- Date of electronic publication, of the latest update, or of posting

- For a work from a subscription service, the name of the service and - if a library or a consortium of libraries is the subscriber - the name and geographic location (e.g., city, state abbreviation) of the subscriber

- For a posting to a discussion list or forum, the name of the list or forum

- The number range or total number of pages, paragraphs, or other sections, if they are numbered

- Name of any institution or organization sponsoring the site (if not cited earlier)

- Date when the researcher accessed the source

- URL of the source or, if the URL is impractically long and complicated, the URL of the site's search page. Or, for a document from a subscription service, the URL of the service's home page, if known; or the keyword assigned by the service, preceded by Keyword; or the sequence of links followed, preceded by Path. ${ }^{144}$

Der bibliographische Beleg für ein ursprünglich in gedruckter Form veröffentlichtes Gedicht, das dann auch online publiziert wird, sähe demnach beispielsweise folgendermaßen aus:

Richtlinien-Vorgabe:

Author. "Title of the poem." Title of the book. Publication information for the print version of the source. Title of the scholarly project. Name of the editor of the scholarly project. Date of electronic publication. Name of the institution associated with the Web site. Date when the researcher accessed the source. $<$ Electronic address $>$.

\section{Beispiel:}

Nesbit, Edith. "Marching Song." Ballads and Lyrics of Socialism. London. 1908. Victorian Women Writers Project. Ed. Perry Willett. Apr. 1997. Indiana U. 26

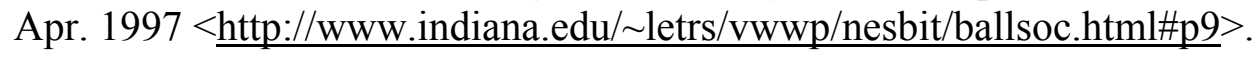

Vergleicht man die hier genannten Informationen mit der Menge der Angaben, die bei Bezugnahme auf gedruckte Publikationen aufgeführt zu werden pflegen, so wird die wesentlich größere Zahl an aufgelisteten Daten deutlich. Dies ist nicht zwingend, sondern teilweise bedingt durch Hinweise, die beim Zitieren gedruckter Veröffentlichungen üblicherweise nicht erscheinen. Gemäß den Zitierrichtlinien für schriftliche Publikationen, auf die sich vorliegende Arbeit stützt, wären beispiels-

\footnotetext{
144 Modern Language Association, Hg., MLA Style: How do I document sources from the World Wide Web in my list? (04.12.2003), $<\underline{\text { http://www.mla.org/publications/style/style_faq/style_faq4 }>, 11.02 .2004 .}$
} 
weise der Titel des wissenschaftlichen Projekts, sein Herausgeber und der Name der Institution, die in Verbindung mit der Publikation steht, unerwähnt geblieben. Dieser Umstand ist damit zu erklären, daß bei Print-Veröffentlichungen in der Regel die Angabe des Verlages eine Qualitätseinschätzung gestattet, wohingegen bei OnlinePublikationen das Projekt von höherer Relevanz ist. Dessen ungeachtet scheint es wünschenswert, die bestehenden Zitiernormen für Online-Publikationen in höherem Maße an die Richtlinien für gedruckte Veröffentlichungen anzulehnen, denn eine Angleichung könnte dem Leser nach dem Prinzip, daß weniger manchmal mehr ist, unter Umständen einen wesentlich klareren Überblick verschaffen.

Die Einführung konkreter Richtlinien sollte zudem auch im Interesse der Herausgeber liegen, denn nur ein zitierfähiges Dokument wird langfristig Akzeptanz und Resonanz in der Wissenschaftsgemeinde erfahren. Liegen nämlich keine eindeutigen oder nur unvollständige Angaben des Autors beziehungsweise Herausgebers der Seiten vor, so ist nach Runkehl und Siever die Abhängigkeit P-B-Z nicht gegeben. Diese besagt, daß die Zitierung $(Z)$ und die bibliographische Verzeichnung (B) von Texten, deren Quellen im World Wide Web liegen, sinnvollerweise nur dann erfolgen können, wenn die formale Qualität der Publikationen (P) selbst dies zuläßt. ${ }^{145}$ Infolgedessen müssen Quellen, die dieser Interdependenz P-B-Z nicht genügen, im Hinblick auf die Untersuchungen zu vorliegender Arbeit - ungeachtet ihrer möglicherweise hohen literaturwissenschaftlichen Relevanz - als ungeeignet angesehen werden; sie gelten demgemäß nach Willamowski als nicht zitierfähig ${ }^{146}$.

Obwohl oder vielleicht gerade weil das ausgewogene Maß an bibliographischen Angaben also offensichtlich eine notwendige Voraussetzung für Rezeption und Anerkennung ist, erweist es sich nach wie vor als gravierendes Hindernis bei der Publikation im World Wide Web. Um hier Abhilfe zu schaffen, mag der nachfolgende abwägende Vergleich zwischen Online- und Offline-Publikationen zeigen, welche Informationen wirklich Erwähnung verdienen.

145 Runkehl und Siever, Das Zitat im Internet, 8.

146 Marcus Willamowski, “Zitierfähigkeit von Internetseiten”, JurPC Web-Dok. 78 (2000), Abs. 3, 22.05.2000, <http://www.jurpc.de/aufsatz/20000078.htm>, 06.11.2002. Gemäß Willamowski gilt eine Seite aus dem World Wide Web genau dann als zitierfähig, wenn ihr Inhalt und gleichzeitig auch das Dokument als solches als zitierfähig gelten können. Voraussetzung hierfür muß sein, daß das Dokument "auch zitiert werden könnte, wenn es nicht im Internet veröffentlicht worden wäre". Ebd. 


\subsubsection{Neuе Zitierrichtlinien für literaturwissenschaftliche Arbeiten}

Der Name des Autors, der Titel der betreffenden Veröffentlichung sowie der Titel des übergeordneten Werkes sind standardisierte Angaben für Druckpublikationen, die deshalb konsequenterweise auch bei Online-Veröffentlichungen - sofern vorhanden - grundsätzlich genannt werden sollten. Für das Datum der letzten Überarbeitung des Online-Dokuments, gewissermaßen als Pendant zum Datum des Erscheinens der gedruckten Version, empfiehlt sich entsprechend den Zitierrichtlinien für gedruckte Publikationen die Verwendung runder Klammern, für das Datum der Erstbearbeitung als Pendant zum Datum der Erstausgabe die Verwendung eckiger Klammern. Anschließend sollte der Publikationsort, das heißt der URL, aufgeführt werden. Dies widerspricht zwar den Richtlinien für Druckpublikationen, wonach der Publikationsort vor dem Publikationsdatum genannt wird, ist aber im Hinblick auf die abschließende Erwähnung des Datums der Dokumentsichtung sinnvoll; auf diese Weise folgen nicht drei Daten aufeinander, so daß eine übersichtliche Darstellung gewährleistet ist. Ist das Web-Dokument in zitierfähiger Weise untergliedert, so sollte der entsprechende Hinweis im Anschluß an den URL erfolgen, da er gewissermaßen eine Ergänzung der Ortsangabe darstellt. Auch diese Regelung bildet eine Abweichung von den Zitiervorschriften für gedruckte Publikationen, wonach die Seitenangabe am Ende des bibliographischen Hinweises erscheint, ist aber durch die Besonderheiten bei Online-Veröffentlichungen gerechtfertigt. Aus ihnen ergibt sich auch, daß im Falle einer Untergliederung des Web-Dokuments in einzelne Seiten die Seitenangabe zugunsten einer besseren Überschaubarkeit ausnahmsweise durch ein "S." ergänzt werden sollte. Da der Aspekt der Unübersichtlichkeit in den nachfolgenden Kurztitelangaben nicht mehr gegeben ist, kann der Hinweis "S." dort entfallen.

Allerdings ist üblicherweise keine Seitenangabe vorhanden. Infolgedessen muß in einer Kurztitelangabe, sofern es sich nicht um eine der ausführlichen bibliographischen Information unmittelbar folgende, mit ebd. zu kennzeichnende Angabe handelt, stets die vollständige Web-Adresse wiederholt werden. In jedem Falle ist das Datum des Abrufs der Online-Veröffentlichung fester Bestandteil einer Literaturangabe.

Was die Ausführlichkeit der bibliographischen Daten betrifft, so können grundsätzlich alle Angaben zu einzelnen Werken - vergleichbar der Nennung von Reihentiteln - durch die Erwähnung des Titels der übergeordneten Web-Seite ergänzt werden. Bei wissenschaftlichen Projekten oder Datenbanken erscheint es 
zudem empfehlenswert, im Anschluß an den Titel des Projekts oder der Datenbank den Namen der übergeordneten verantwortlichen Institution zu vermerken.

Diese ist auch zu nennen, wenn einem Online-Werk weder ein Autor beziehungsweise eine Autorengruppe noch ein oder mehrere Herausgeber zugeordnet werden können. Sofern keine gegenteiligen Informationen darauf hinweisen, ist davon auszugehen, daß eine übergeordnete verantwortliche Institution den Inhalt aller Beiträge auf ihren WWW-Seiten mitträgt. Zum Zwecke der Differenzierung zwischen Herausgebern und Verantwortlichen sollte der Nennung - entsprechend dem Hinweis Hg. - die Information Verantw. beigefügt werden.

Existiert neben der elektronischen Version des Dokuments auch eine gedruckte Publikation, so ist der Verweis auf sie ebenfalls anzubringen. Gerade im Hinblick auf die erwähnte Unbeständigkeit des Mediums muß der Leser die Möglichkeit besitzen, den Text in Druckform nachzulesen. Zudem sollte er auf diesem Wege darüber informiert werden, ob die Druckpublikation der elektronischen Version vorausgeht, parallel dazu oder aber erst im Anschluß daran veröffentlicht wurde. Der Hinweis sollte jedoch nicht innerhalb der Informationen zur elektronischen Publikation erfolgen, sondern als eigenständige bibliographische Angabe an deren Ende angegliedert werden. Eine Online-Version, die inhaltlich identisch mit einer Druckausgabe ist, sollte in ihrem Verhältnis zu dieser gedruckten Veröffentlichung unabhängig von ihrem zeitlichen Erscheinen als analoge Publikation bezeichnet werden. ${ }^{147}$ Gilt die Druckversion als die ursprüngliche Ausgabe, der erst im nachhinein eine Online-Publikation gefolgt ist, so ist sie als Ursprungsversion zu bezeichnen; geht die Druckveröffentlichung der eigentlichen, digitalen Publikation nur als vorläufige und eventuell gekürzte Variante voraus, so handelt es sich dabei um eine Vorabversion. Im Falle einer gleichzeitigen Veröffentlichung von On- und OfflineDokument ist der Ausdruck Parallelpublikation zu gebrauchen. Erscheint eine Online-Publikation nachträglich auch in Papierform, so ist von einer Folgeversion zu sprechen. Alle davon abweichenden Konstellationen sollten gesondert gekennzeichnet werden.

Auf der Basis obiger Erwägungen lassen sich nunmehr neue Zitierrichtlinien formulieren, die auch bei der vorliegenden Arbeit zur Anwendung kommen.

147 Dieser Begriff soll signalisieren, daß es sich zwar um eine inhaltliche Entsprechung, nicht jedoch um eine Zeitgleichheit handelt: eine Analogie bezeichnet eine Entsprechung von Verhältnissen, eine Übereinstimmung, wohingegen bei einer Parallelität ein Gleichlauf von Geschehnissen vorliegt. 


\section{Web-Seiten in ihrer Gesamtheit}

\section{Einzelner Autor}

Name, Vorname, Titel der Web-Seite (Datum der letzten Dokument-Überarbeitung $\left[{ }^{1}\right.$ Datum der Erstveröffentlichung]), <URL $>$, Datum des Abrufs. Ggf. Hinweis auf Druckversion.

\section{Autorengruppe}

Autorengruppe, Titel der Web-Seite (Datum der letzten Dokument-Überarbeitung $\left[{ }^{1}\right.$ Datum der Erstveröffentlichung]), <URL>, Datum des Abrufs. Ggf. Hinweis auf Druckversion.

\section{Ungenannter Autor}

Name, Vorname, Hg./Name, Verantw., Titel der Web-Seite (Datum der letzten Dokument-Überarbeitung [ ${ }^{1}$ Datum der Erstveröffentlichung]), $<\mathrm{URL}>$, Datum des Abrufs. Ggf. Hinweis auf Druckversion.

\section{Herausgeber}

Name, Vorname. Hg., Titel der Web-Seite (Datum der letzten Dokument-Überarbeitung $\left[{ }^{1}\right.$ Datum der Erstveröffentlichung]), $<\mathrm{URL}>$, Datum des Abrufs. Ggf. Hinweis auf Druckversion.

\section{Einzelne Werke}

\section{Autor genannt}

Name, Vorname, "Titel des Werkes", in: Vorname Name, Hg., Titel des übergeordneten Werkes (Datum der letzten Dokument-Überarbeitung $\left[{ }^{1}\right.$ Datum der Erstveröffentlichung]), Titel der Web-Seite, des Projekts o. ä., <URL>, Datum des Abrufs. Ggf. Hinweis auf Druckversion.

\section{Autor ungenannt}

Name, Vorname, Hg./Name, Verantw., "Titel des Werkes", in: Titel des übergeordneten Werkes (Datum der letzten Dokument-Überarbeitung $\left[{ }^{1}\right.$ Datum der Erstveröffentlichung]), Titel der Web-Seite, des Projekts o. ä., <URL>, Datum des Abrufs. Ggf. Hinweis auf Druckversion.

\section{Monographien}

Name, Vorname, Titel der Monographie (Datum der letzten Dokument-Überarbeitung [ ${ }^{1}$ Datum der Erstveröffentlichung]), $<$ URL $>$, Datum des Abrufs. Ggf. Hinweis auf Druckversion. 


\section{Werke innerhalb wissenschaftlicher Projekte}

\section{Einzelnes Werk innerhalb eines wissenschaftlichen Projekts}

Name, Vorname, "Titel des Werkes", in: Vorname Name, Hg., Titel des wissenschaftlichen Projekts, Name der übergeordneten, verantwortlichen Institution (Datum der letzten Dokument-Überarbeitung [ $\left[{ }^{1}\right.$ Datum der Erstveröffentlichung]), $<$ URL $>$, Datum des Abrufs. Ggf. Hinweis auf Druckversion.

\section{Monographie innerhalb eines wissenschaftlichen Projekts}

Name, Vorname, Titel der Monographie, in: Vorname Name, Hg., Titel des wissenschaftlichen Projekts, Name der übergeordneten, verantwortlichen Institution (Datum der letzten Dokument-Überarbeitung $\left[{ }^{1}\right.$ Datum der Erstveröffentlichung]), $<\mathrm{URL}>$, Datum des Abrufs. Ggf. Hinweis auf Druckversion.

\section{Werke in Foren}

\section{Einzelnes Werk innerhalb eines Forums}

Name, Vorname, "Titel des Werkes", in: Name der übergeordneten, verantwortlichen Institution, Titel des Forums (Datum der letzten Dokument-Überarbeitung $\left[{ }^{1}\right.$ Datum der Erstveröffentlichung]), <URL $>$, Datum des Abrufs. Ggf. Hinweis auf Druckversion.

\section{Monographie innerhalb eines Forums}

Name, Vorname, Titel der Monographie, in: Name der übergeordneten, verantwortlichen Institution, Titel des Forums (Datum der letzten Dokument-Überarbeitung $\left[{ }^{1}\right.$ Datum der Erstveröffentlichung]), <URL $>$, Datum des Abrufs. Ggf. Hinweis auf Druckversion.

\section{Artikel in Online-Periodika}

\section{Zeitschriftenartikel}

Name, Vorname, “Titel des Artikels”, Zeitschriftentitel Ausgabennummer (Erscheinungsjahr), $<$ URL $>$, Datum des Abrufs. Ggf. Hinweis auf Druckversion.

\section{Zeitungs- oder Magazin-Artikel}

Name, Vorname, “Titel des Artikels”, Zeitungs- oder Magazin-Titel, Erscheinungsdatum, $<\mathrm{URL}>$, Datum des Abrufs. Ggf. Hinweis auf Druckversion. 
Erläuternde Angaben vor der Nennung des URL wie zum Beispiel "Retrieved [...] from [...]"148, "Online unter URL: [...]"149, "Online in Internet: URL: [...]"150 oder "[online] Available: [...]"151 beziehungsweise "Available from World Wide Web: [...]"152 können immer dann vernachlässigt werden, wenn ausschließlich auf elektronische Ressourcen aus dem World Wide Web zurückgegriffen wird. Bei allen bibliographischen Angaben für Web-Publikationen geht klar aus dem URL hervor, daß die Quelle im WWW vorliegt. Daß alle aufgeführten Ressourcen zum Zeitpunkt ihrer Erfassung zugänglich waren, sollte vorauszusetzen sein, so daß sich weitere Hinweise auf die Gültigkeit des URL von selbst ergeben.

Abschließend ist hinzuzufügen, daß jede Web-Seite ihre eigenen Charakteristika aufweist und somit die bibliographischen Angaben nicht immer exakt nach den aufgeführten Schemata zu erstellen sind. Solange sich das WWW noch in statu nascendi befindet, unterliegen auch die Richtlinien einer steten Entwicklung und Anpassung. Im Einzelfalle liegt es daher im persönlichen Ermessensspielraum, wie die Online-Dokumente zitiert werden.

148 American Psychological Association, Hg., Reference Examples for Electronic Source Materials, $<$ http://www.apastyle.org/elecsource.html $>, 12.11 .2002$.

149 Thomas Christoph Marx, "Zur Problematik des Zitierens aus dem Internet: Anforderungen, Möglichkeiten und Grenzen - Tabelle 2: Zitier-Muster und Beispiele”, in: Albert-LudwigsUniversität Freiburg, Historisches Seminar: Forum, <http://www.geschichte.unifreiburg.de/histsem/forum/zitieren.html\#tabelle2>, S. 9f., 12.11.2002. Als Vorabversion erschienen: Thomas Christoph Marx, "Zur Problematik des Zitierens aus dem Internet: Anforderungen, Möglichkeiten und Grenzen”, Geschichte in Wissenschaft und Unterricht (GWU), 52 (2001), $238-245$.

150 Jens Bleuel, Online Publizieren im Internet: Elektronische Zeitschriften und Bücher. Mit einem Vorw. von Norbert Mundorf (Pfungstadt; Bensheim, 1995), 62f. Angesichts des heutigen Bekanntheitsgrades des World Wide Web und der einschlägigen allgemeinen Kenntnisse werden hier gleich zwei überflüssige Informationen geliefert: Der Hinweis auf das Internet und die Erwähnung, es handele sich um einen URL. Laut Definition des World Wide Web Consortiums existiert lediglich eine Technik der Web-Adressierung, nämlich diejenige mittels URL. World Wide Web Consortium, Hg., Naming and Addressing: URIs, URLs,... (09.07.2002), $<\underline{\text { http://www.w3.org/Addressing/> }, 13.11 .2002}$. Man sollte bei der Erstellung bibliographischer Angaben stets davon ausgehen können, daß der künftige Leser hierzu über gewisse Grundkenntnisse verfügt; übertragen auf Druckpublikationen müßten die bibliographischen Daten gemäß Bleuel konsequenterweise durch den Hinweis Titelangabe ergänzt werden.

151 The University of Missouri-Kansas City Libraries, Hg., Guide to Evaluating Resources on the World Wide Web (18.09.2002), <http://www.umkc.edu/lib/Resources/webeval.htm>, 12.11.2002.

152 National Library of Canada, Hg., ISO 690-2: Information and documentation - Bibliographic references - Part 2: Electronic documents or parts thereof - 5: Outline of Bibliographic References (22.08.2002), < http://www.nlc-bnc.ca/iso/tc46sc9/standard/690-2e.htm\#5>, 12.11.2002. 


\section{Die Begriffsproblematik - Übersicht, Diskussion und Festlegung}

\section{Standortbestimmung}

Wie bereits in der Einleitung dargelegt, weckt das World Wide Web offensichtlich vielfach die Erwartung, das ultimative Präsentationsmedium für eine - bislang noch nicht realisierte - künstlerische Bestleistung zu sein, nämlich eben jenes eingangs zitierte "hypermediale Großwerk" $" 153$, das Text-, Bild-, Ton-, Animations- und Interaktionselemente miteinander verbindet.

Wenngleich die Entdeckung des sogenannten "Online-'Ulysses""154 vorläufig noch ein reines Zukunftsprojekt ist, so existiert doch bereits eine gewisse Zahl an speziell für dieses elektronische Medium konzipierten literarischen Werken, die zumeist unter der Bezeichnung "Digitale Literatur” präsentiert und eingeordnet werden. Angesichts des von Rezipienten und Produzenten gleichermaßen erhobenen Anspruchs, diese moderne Form des literarischen Kunstwerks habe eine völlig andersgeartete Struktur aufzuweisen, die sowohl dem Autor als auch dem Leser grundlegend neue Möglichkeiten und Erfahrungen eröffne, drängt sich die Frage auf, ob die Bezeichnung Literatur hier überhaupt noch angemessen ist. Sollte es sich nämlich tatsächlich um eine Spezies handeln, die nicht den traditionellen Literaturkriterien entspricht, so wäre zwangsläufig darüber nachzudenken, ob die bestehenden Begriffe, das heißt die gedanklichen Konzepte zur Klassifizierung herkömmlicher Literaturformen, auch der Einteilung digitaler Literatur zugrunde liegen können.

Cramer erachtet in dieser Hinsicht jede Diskussion als überflüssig, denn seiner Meinung nach ist das Internet ein durch und durch literarisches Medium:

Das Internet ist das erste neue Massenmedium des zwanzigsten Jahrhunderts, das auf einem Code basiert, der - wie Morsecode - der Logik eines Alphabets folgt. Das Internet basiert also auf Text. Nicht nur die Daten, die in ihm übertragen werden - E-Mail-Nachrichten, Web-Seiten und selbst Töne und Bilder - sind textuell codiert. Auch die Programme, die für diese Datenübertragung sorgen, sind Texte, die Computer mit Maschinenbefehlen ansteuern. ${ }^{155}$

153 Rotermund, "Warten auf den Online-Ulysses", 78.

154 Ebd.

155 Florian Cramer, "Warum es zuwenig interessante Netzdichtung gibt. Neun Thesen", in: Johannes Auer, Christiane Heibach und Beat Suter, Hg., netzliteratur.net ([ $\left.\left.{ }^{1} 27.04 .2000\right]\right)$, $<\underline{\text { http://www.netzliteratur.net/cramer/karlsruher_thesen.html }>, ~ 25.07 .2004 . ~}$ 
Bereits zu einem früheren Zeitpunkt hatte er die Eigenschaften des Internet folgendermaßen beschrieben:

Indem das Internet Telegraph und Textspeicher zugleich ist und Algorithmen ausführt, vereint es die Funktionen von Buch, Bibliothek, Salon und poetischer Maschine. ${ }^{156}$

Dementsprechend lautete sein Fazit:

Das Internet ist eine Literatur, ein Buchstabenwesen. ${ }^{157}$

Diese Argumentation klingt durchaus schlüssig und überzeugend. So gesehen dürfte keinerlei Zweifel bestehen, daß im Zusammenhang mit allen Online-Publikationen narrativen Charakters von Literatur gesprochen werden kann.

Es gibt jedoch auch Stimmen, die in die entgegengesetzte Richtung gehen. Rotermund beispielsweise äußerte bereits im Jahre 1997 die Hoffnung, es möge eine neue Benennung erfolgen, und er stellte die Frage:

Gibt es überhaupt Internet-Literatur? Muß nicht von vorneherein ein neuer Name für eine dem neuen Medium adäquate Kunstform gefunden werden, die Schrift und Text zwar einbezieht, aber nicht notwendig auf ihnen basiert? ${ }^{158}$

In einem Interview ging Rotermund sogar noch weiter und meinte, daß nicht allein eine neue Bezeichnung für diese literarische Gestaltungsweise gefunden werden müsse, sondern die gesamte literaturwissenschaftliche Terminologie einer Überarbeitung bedürfe: "Es gibt keine Literaturwissenschaftlichen Kategorien, die einen Kanon bilden, mit dem man die spezifischen Dinge der Netzliteratur bewerten kann." ${ }^{159}$ Dieser Kanon müsse gewissermaßen erst gemeinschaftlich geschaffen werden. $\mathrm{Zu}$ einer vergleichbaren Einschätzung gelangt auch Daiber; seiner Ansicht nach müssen die bestehenden sprachlichen Mittel durch zusätzliche Elemente ergänzt werden, die speziell auf das neue Online-Medium zugeschnitten

\footnotetext{
156 Ders., “Literatur im Internet”, in: netzliteratur.net ([101.12.1999]), $<\underline{\text { http://www.netzliteratur.net/cramer/alg-literatur im internet.html }>, ~ 25.07 .2004 . ~}$

157 Ders., "Warum es zuwenig interessante Netzdichtung gibt", 1.

158 Ebd.

159 Wolfgang Tischer, “Anfangserscheinungen und Kinderkrankheiten - Interview mit Dr. Hermann Rotermund über den Zeit-Literaturpreis '97 und die Lage der Netzliteratur", in: Das Literatur-Café: Der literarische Treffpunkt im Internet, <http://www.literaturcafe.de/bf.htm?/berichte/roterm.shtml>, 25.11.2002.
} 
sind. ${ }^{160}$ In diesem Sinne urteilt auch Schröder: "Der Begriff Internet-Literatur ist zu eng, zu wenig."161

Die Forderungen nach neuen Fachausdrücken scheinen folgerichtig und nachvollziehbar. Angesichts dessen, was mit den neuen technischen Gestaltungs- und Präsentationsmitteln offensichtlich möglich ist, könnte die traditionelle Terminologie durchaus überholt und unzureichend anmuten. Werden die vorhandenen Mittel aber wirklich in der Weise ausgeschöpft, daß etwas umstürzend Neuartiges entstünde? Gelingt es tatsächlich, eine neue Kunstform zu schaffen und "die Tür zu kühnen, lichteren Räumen des Geistes" "162 aufzustoßen? Oder ist es nicht vielmehr so, daß auch im Web "nur die alten Lesefunzeln brennen"? ${ }^{163}$

Etwas ernüchternd, aber vielleicht gerade deshalb hilfreich ist in diesem $\mathrm{Zu}$ sammenhang der Hinweis von Bolter auf die ersten Jahrzehnte des Buchdrucks. Damals wurden die Druckerzeugnisse den handgeschriebenen Manuskripten ganz bewußt in deren Individualität und Mangelhaftigkeit nachgearbeitet, und erst nach längerer Zeit etablierte sich die moderne Druckweise. ${ }^{164}$

Es scheint, daß wir uns momentan noch in einer Übergangsphase des elektronischen Publizierens befinden, die der Orientierung und der Gewöhnung an das neue Medium dient und die zwangsläufig erst überwunden werden muß, bevor sich der Schwerpunkt des Interesses vom Medium auf die Präsentationsform und den Inhalt verlagert. $\mathrm{Zu}$ vage sind momentan noch die Vorstellungen, was Web-Literatur ausmacht, beziehungsweise ausmachen sollte, zu unklar die Ideen, welchen Stellenwert sie aus literaturwissenschaftlicher Sicht einnehmen kann und viel zu uneinheitlich die in diesem Zusammenhang verwendeten Begriffe. So läßt sich die gegenwärtige Lage nur mit den Worten Rotermunds zusammenfassen: "Hier dürfen wir nicht so

160 Jürgen Daiber, “Literatur und Nicht-Linearität: Ein Widerspruch in sich?”, Jahrbuch für Computerphilologie - online, 1 (1999), <http://computerphilologie.uni-muenchen.de/jahrbuch/jb1/daiber.html>, 26.11.2002. Als Folgeversion erschienen: Jürgen Daiber, "Literatur und Nicht-Linearität: Ein Widerspruch in sich?”, Jahrbuch für Computerphilologie 1 (1999), 21-38.

161 Schröder, "Der Link als Herme und Seitensprung", 45.

162 Dieter E. Zimmer, "Die digitale Bibliothek: Eine fünfteilige Artikelserie für Nutzer und Verächter der Computernetze", in: Georg Ruppelt, Hg., Bibliothekspolitik in Ost und West: Geschichte und Gegenwart des Deutschen Bibliotheksverbandes. [Zeitschrift für Bibliothekswesen und Bibliographie: Sonderheft 72] (Frankfurt a.M., 1998), 265-317, hier: 285.

163 Ebd.

164 Jay D. Bolter, "Das Internet in der Geschichte der Technologien des Schreibens”, in: Münker und Roesler, Hg., Mythos Internet, 37-55, hier: 47. 
tun, als wüßten wir, was die Kunst oder die Literatur des Internet sei. Wir wissen es nicht." 165

Auf der Basis des Existierenden sowie des Denk- und Realisierbaren soll daher nachfolgend erörtert werden, wie neu das Neue wirklich ist, das heißt ob der Unterschied der neuen Literaturformen zu gedruckter Literatur tatsächlich so groß ist, daß die Notwendigkeit einer grundlegenden Überarbeitung der bestehenden Terminologie oder gar der Schaffung vollkommen neuer Begriffe zwingend gegeben ist.

\subsection{Online-Literatur zwischen Tradition und Moderne}

Eine der wichtigsten Eigenschaften des Menschen ist seine Fähigkeit, Erfahrungen der Vergangenheit für den Umgang mit Neuem zu nutzen. Wird er mit Unbekanntem konfrontiert, ist er intuitiv oder bewußt bestrebt, alle Erkenntnisse, die er im Verlaufe seines Lebens gewonnen hat, auf die fremde Situation zu transferieren und diese so zu bewältigen. Allerdings kann diese Fähigkeit nicht nur zu Orientierung verhelfen, sondern sie kann umgekehrt auch blockierend wirken, indem sie einen unbeeinflußten, neutralen Blick auf die Dinge verhindert.

Genau so mag es sich im Falle dessen verhalten, was hier vorläufig noch als elektronische Literatur oder Online-Literatur bezeichnet werden soll. Aus dem vom Umgang mit gedruckter Literatur geprägten Blickwinkel mag das, was seit geraumer Zeit im World Wide Web veröffentlicht wird, gelegentlich nicht die Wirkung entfalten, die von den Publizierenden ursprünglich intendiert war. Die Erfahrungen mit Druckwerken haben im Laufe der Vergangenheit zur Formung einer besonderen Sichtweise und einer diesem Medium entsprechenden Herangehensweise beigetragen, die nun auf die neuen Formen der Literatur angewandt werden. Diese neuen Formen wiederum rufen bei den Lesern - und dieser Begriff ist bewußt gewählt, um die genannten Vorprägungen und Rezeptionserfahrungen der Nutzer deutlicher hervorzuheben - bestimmte Vorstellungen und Wünsche hervor, wobei die Erwartungshaltungen vermutlich selten indifferent sind, sondern im allgemeinen zu einer von zwei gegensätzlichen Auffassungen tendieren: Entweder sollte das, was im WWW als Literatur angeboten wird, in möglichst hohem Maße den Kriterien von gedruckter Literatur entsprechen, oder aber der Leser erwartet genau das Gegenteil, nämlich daß sich das Online-Angebot grundlegend von der herkömmlichen Form der

165 Ebd. 
Literatur unterscheidet. In ersterem Falle mißt der Leser alles Dargebotene an den der gedruckten Literatur zugrundeliegenden Maßstäben und empfindet starke Abweichungen als befremdlich und somit als negativ. Umgekehrt verspürt der Leser jede Ähnlichkeit mit gedruckter Literatur als störend und dem Charakter des Neuen abträglich.

Wenn das Denken in Kategorien auch in der Natur des Menschen zu liegen scheint, so muß sich doch mancher Leser den Vorwurf gefallen lassen, daß er Neuem gegenüber nicht genügend Toleranz walten läßt. Ob er nun Herkömmlichem oder aber Revolutionärem entgegensieht - in beiden Fällen liegen seine Erwartungen zu hoch. "Die Wirklichkeit der virtuellen Welten" ${ }^{166}$ liegt - zumindest zum augenblicklichen Zeitpunkt - noch irgendwo dazwischen. Das, was derzeit auf künstlerischliterarischem Gebiet im World Wide Web zur Verfügung steht, 'erzählt' durchaus Geschichten, wenn dies auch zum Teil mit nicht-sprachlichen Mitteln geschieht. "Literatur hatte und hat stets nur dieses eine Ziel: Die Bevölkerung des inneren Theaters des Lesers." ${ }^{\prime 167}$ Eben dies vermag Online-Literatur grundsätzlich zu bewirken. Im Großen und Ganzen kann man den Eindruck gewinnen, daß es sich die Autoren des Dargebotenen zum Ziel gesetzt haben, die herkömmliche Vorgehensweise, die Phantasie des Lesers zu binden, mit den neuen Medien zu verknüpfen: "Erzählen wie seit tausend Jahren, aber mit den modernsten Mitteln"168. Damit besteht eindeutig ein Bezug zu gedruckter Literatur, gleichzeitig aber existieren auch deutliche Abweichungen:

Und einiges an Differenz zum Bekannten müssen wir schon in Kauf nehmen: Es wäre leichtfertig, die Erwartungen an die 'Erzählmaschine' zu nah an das zu binden, was wir entweder von der herkömmlichen Erzählung zwischen Buchdeckeln oder der 'Zählmaschine' und ihren mehr oder weniger geglückten Verwendungen zur Produktion ästhetischer Effekte schon kennen. ${ }^{169}$

166 Deutscher Titel des Werkes Virtual Worlds. Benjamin Wooley, Die Wirklichkeit der virtuellen Welten, übers. von Gabriele Herbst, (Basel; Boston; Berlin, 1994 [Virtual Worlds (Oxford, 1992)]).

167 Peter Berlich, "Das Internet als innerer Ort - 'Das digitale Kloster'”, in: Suter und Böhler, Hg., Hyperfiction, 137-148, hier: 146.

168 Michael Charlier, "Von der Armenbibel zu Myst - Zurück zu einer Literatur ohne Worte?", in: Suter und Böhler, Hg., Hyperfiction, 125-136, hier: 130.

169 Ebd. 
Auch wenn Charlier damit die Unterschiede zwischen Traditionellem und Neuem hervorhebt, scheint er mit nachfolgender Anregung eine Brücke schlagen zu wollen zwischen zwei Medienformen, denen die öffentliche Meinung extremere Unterschiede zuschreibt, als tatsächlich bestehen:

Warum sollten, wenn das Geschriebene als nicht mehr ausreichend empfunden wird, nicht auch Bilder wieder verstärkt in die Kommunikationsebenen einbezogen werden, aus denen sie im Laufe der Jahrhunderte verdrängt wurden? Und wo sollten diese Bilder stattfinden, wenn nicht auf dem Bildschirm? ${ }^{170}$

Die Fragen scheinen berechtigt, zumal die Arbeit am Computer in hohem Maße schriftliche Tätigkeiten beinhaltet; vor allem die neue Form der elektronischen Korrespondenz scheint dem Schreiben wieder eine unverhoffte Popularität zu verschaffen. Dennoch wird vielfach bezweifelt, daß der Computer neben der Nutzung für vorwiegend praktische Tätigkeiten auch für den Genuß künstlerischer Präsentationen tauglich ist. Birkerts beispielsweise beschreibt seine Beweggründe folgendermaßen:

Meine zweite grundlegende Annahme bezieht sich auf die Literatur und lautet dahin, daß elektronische Schaltkreise und Monitore zwar die idealen Kanäle für bestimmte Daten sind - für Zahlen, Bilder, querverkettete Informationen jeglicher Art -, daß sie sich aber durchaus nicht vertragen mit dem subjektiveren Material, das seit eh und je der Stoff ist, aus dem die Kunst gemacht wird. Will sagen: Sie stehen der Innerlichkeit entgegen. Das hat mehrere Gründe, und der wichtigste davon liegt in dem Faktor Zeit. ${ }^{171}$

Die noch weiter ausgreifende, insgesamt sehr ausführliche Darstellung wirkt zwar möglicherweise konstruiert und spiegelt überdies eine recht enge, vorgefaßte Ansicht von Literatur wieder, verdient aber gleichwohl Aufmerksamkeit, weil Birkerts sich dem Thema ernsthaft und aufrichtig widmet. Seine Argumente lassen sich nichtsdestoweniger entkräften: Birkerts Kritik an Literatur auf einem Computer-Monitor betrifft die Begleitumstände in Form unausgesetzter visueller oder akustischer Signale, die seiner Meinung nach eine völlige, Zeit und Raum entrückte Hingabe an das Kunstwerk verhindern. Birkerts ist offensichtlich der Ansicht, schon aufgrund seiner Beschaffenheit gemahne das elektronische Medium den Nutzer an die Flüchtigkeit des Dargestellten.

\footnotetext{
170 Ebd.

171 Birkerts, Die Gutenberg-Elegien, 259.
} 
Wenn dem wirklich so wäre, dürfte der Zustand des absoluten Eintauchens ausschließlich Kunstwerken vorbehalten sein, die unabhängig von weiteren technischen Hilfsmitteln genutzt werden können, wie beispielsweise Büchern oder Gemälden. Sobald ein Präsentationsmedium vonnöten wird, wie es bei dem Genuß von Musik durch das Abspielen eines Tonträgers oder beim Ansehen eines Films auf einem Bildschirm oder einer Leinwand der Fall ist, müßte es - wenn man die Gedanken Birkerts' zu Ende verfolgt - nicht mehr möglich sein, einen Zustand völliger Entrückung zu erreichen. Daß es jedoch auch unter diesen Umständen und somit auch vor einem Computerbildschirm durchaus möglich ist, Zeit und Raum zu vergessen, sollte eigentlich jeder bestätigen können, der irgendwann einmal die Bereitschaft aufgebracht hat, sich auf das dort Präsentierte einzulassen. Genau dieses Entgegenkommen ist ein wichtiger Schlüssel zur Einsicht in die gedankliche Welt anderer. Der Grad der Hingabe an ein Kunstwerk ist in hohem Maße abhängig von der inneren Einstellung dazu.

\subsection{Das Problem der Linearität}

Während Birkerts den Computer-Bildschirm als ein grundsätzlich ungeeignetes Präsentationsmedium für Literatur ansieht, wird die Existenz von Online-Literatur von anderer Seite als gegeben und berechtigt vorausgesetzt. Dort betrifft die Kritik in erster Linie die Konzeption des Dargebotenen. Die im Laufe der Zeit durch die Rezeption von Druckwerken geprägten Betrachtungsweisen und Interpretationstechniken - so wird gesagt - erschwerten oder verhinderten gar den Zugang zu digitaler Literatur $^{172}$. Der Wert der Online-Werke werde anhand jener Maßstäbe ermittelt, die üblicherweise auf gedruckte Literatur angewandt würden, weshalb der Leser oftmals $\mathrm{zu}$ der Auffassung gelange, Web-Literatur weise keine Gemeinsamkeiten mit gedruckter Literatur auf ${ }^{173}$; im Extremfall werde ihre Lesbarkeit sogar grundsätzlich in Frage gestellt. ${ }^{174}$

Eine zentrale Rolle spielt bei dieser Argumentation die Linearität im Hinblick auf die Darstellungs- und Rezeptionsweise. Die Tatsache, daß Geradlinigkeit untrennbar mit dem Begriff Literatur verbunden zu sein scheint, ja gewissermaßen synonym dafür verwendet wird, ist eine weitere Bestätigung der obigen Ausfüh-

\footnotetext{
172 Vgl. ebd.

173 Schröder, "Der Link als Herme und Seitensprung", 44.

174 Todesco, "Hyperkommunikation”, 121.
} 
rungen: Das Denken in festgelegten Strukturen steht einer angemessenen Betrachtungsweise entgegen. Auch bei der strengen Einteilung von Literatur in lineare (in gedruckter Form) und nicht-lineare Publikationen (auf dem Computer) wird nämlich oftmals übersehen, daß eine solche Abgrenzung nicht nur die Sichtweise beengt, sondern letztlich gar nicht möglich ist. Gedruckte Literatur müßte demzufolge stets geradlinig ohne Rückblick, Vorschau oder Exkurs zu Hintergrundinformationen gelesen werden, wohingegen der Leser bei der Literaturrezeption am Computer gezwungen wäre, grundsätzlich alle Verknüpfungsangebote wahrzunehmen. Tatsächlich aber beinhalten beide Literaturformen sowohl Linearität als auch NichtLinearität. Zudem hat Zimmer völlig richtig erkannt: prinzipiell ist es unerheblich, ob es sich um ein gedrucktes Werk mit einem einzigen, vorgegebenen Lesestrang oder aber um ein Online-Werk mit unzähligen Verknüpfungen und somit verschiedenen möglichen Leseweisen handelt. Hier wie da kann der Rezeptionsvorgang nur aufeinanderfolgend ablaufen; "kreisförmig oder Verschiedenes simultan kann der Mensch nicht lesen". ${ }^{175}$

Daß die individuellen Leseerfahrungen auch in Hypertext-Systemen linear verlaufen, wird von Nestvold bestätigt:

Beim Lesen der gleichen Hyperfiction werden zwar zwei verschiedene Leser selten den gleichen Text in der gleichen Reihenfolge lesen, aber beide werden einen Bildschirm nach dem anderen rezipieren und sich so in ausgesprochen linearer Weise durch den Text bewegen. ${ }^{176}$

Diesen Standpunkt vertritt auch Bart:

We live and think and perceive and act in time, and time implies sequence, and sequence is what gives rise to narrative. This happened and then that and then that, and if we want to recount what happened, to share it with others and even with ourselves, we have to proceed in narrative sequence - the story of our day, the stories of our lives. Those stories are linear, even when their subject is often not, and they remain linear even when the order of narration is dischronological. ${ }^{177}$

$\mathrm{Da}$ Erzählungen - und im Grunde genommen nur diese - linear sind, glaubt Kuhlen mit dem Hinweis belegen zu können, daß Texte - bei Kuhlen verstanden als schriftlich Niedergelegtes - bereits aufgrund ihres physikalischen Zustands zweidimen-

175 Zimmer, Die Bibliothek der Zukunft, 56.

176 Nestvold, "Das Ende des Buches", 23.

177 John Barth, "The State of the Art", WQ-The Wilson Quarterly 20 (1996), 36-45, hier: 43. 
sional sind. ${ }^{178}$ Tatsächlich läßt sich gedruckte Literatur auch in nicht-linearer Weise rezipieren: Klostermann erinnert daran, daß Bücher sich auf mannigfaltigem Wege erschließen lassen, wie zum Beispiel durch ihr Inhaltsverzeichnis, anhand der Fußnoten, mit Hilfe des Registers usw.; auch könne der Leser mehrere Publikationen parallel bearbeiten und außerdem seine Finger als "nicht-elektronische 'bookmarks" einsetzen. ${ }^{179}$

Auch Aarseth nimmt Abstand von den Begriffen der Linearität und der NichtLinearität. Seiner Überzeugung nach vereinbart jede Form der Literatur, ob sie nun online oder offline präsentiert wird, beiderlei Aspekte. So sei jeder Lesevorgang bis $\mathrm{zu}$ einem gewissen Grade nicht-linear und lasse somit ein Werke bei jeder neuen Rezeption unterschiedlich erscheinen. Eine konsequent nicht-lineare Wahrnehmung sei nun allerdings nicht möglich, da der Leser - wie oben bereits ausgeführt - nicht mehrere Sequenzen gleichzeitig lesen könne. ${ }^{180}$

178 Kuhlen, Hypertext, 27.

179 Klostermann, “'Von Gutenberg zum Internet' - Wie bitte?!”, 25. Tatsächlich könnte man sagen, daß sich ein Inhaltsverzeichnis aus Hypertext-Verweisen zusammensetzt, die den Leser zu den unterschiedlichen Knoten des Gesamttextes, das heißt den einzelnen Kapiteln, lotsen. Hier handelt es sich eigentlich um Hypertext-Verknüpfungen, mittels derer der Leser das Dokument auf nichtlineare Weise erschließen könnte, die aber oftmals nur dazu dienen, dem Leser einen Überblick über die Inhalte einer Publikation zu verschaffen. Vergleicht man nun elektronische und gedruckte Dokumente, so wird deutlich, daß der Ursprung elektronischer Hypertext-Links in Print-Dokumenten liegt. Schreiber bringt diesen Zusammenhang auf eine simple Formel und kommentiert Interaktivität, die eigentlich so oft als Novum moderner digitaler Medien bezeichnet wird, folgendermaßen: "Es ist im Grunde lediglich eine Art elektronische Blätterhilfe." Gerhard Andreas Schreiber, Neue Wege des Publizierens: Das Handbuch zu Einsatz, Strategie und Realisierung aller elektronischen Medien (Braunschweig, 1997), 15. - Wie aus obigem Zitat hervorgeht, vertritt Klostermann die These, auch Bücher ließen sich nicht-sequentiell rezipieren. Mit seiner Veröffentlichung Verlegen im Netz bricht er eine Lanze für das Buch und scheint mit der strukturellen Konzeption dieser Publikation schon vorweg den Beweis für seine These führen zu wollen. Der Drucktext ist ebenso gestaltet wie ein Online-Dokument: Kapitelüberschriften, einzelne Begriffe und Hinweise auf Beiträge anderer Autoren sind farblich hervorgehoben und als Verknüpfungen gekennzeichnet. Am Ende fast aller Kapitel finden sich Verweise, die zur Kapitelübersicht oder zum nächsten Abschnitt leiten. Diese für Drucktexte ungewöhnliche Konzeption vermittelt in der Tat zunächst das Gefühl, man bewege sich in einem Hypertext. Bei der Rezeption des Textes wird aber auch schnell deutlich, wo die Schwachpunkte der gedruckten Publikation im Hypertext-Format liegen - nämlich im Suchen und Blättern - und man gelangt zu der Erkenntnis, daß elektronische Hypertexte eine sehr hilfreiche und komfortable Navigationshilfe darstellen. Klostermanns Werk läßt zudem einen weiteren Nachteil von Druckpublikationen deutlich werden: die Abgeschlossenheit des Textes. Wenn Klostermann davon spricht, es handele sich bei seiner Veröffentlichung um einen offenen Beitrag zu dem behandelten Thema, und er daraufhin betont, er nehme gerne neue Links auf, entsteht für den Leser der gedruckten Textversion das Gefühl, wichtige Beiträge eventuell nicht zu Gesicht zu bekommen. Hingegen läßt sich die Web-Version jederzeit erweitern oder unter Umständen auch reduzieren, wenn Aspekte wieder verworfen werden. Allerdings stellt diese Tatsache gleichzeitig auch wieder einen Nachteil dar: Klostermann selbst weist darauf hin, daß das Werk aufgrund dieser Veränderbarkeit "somit nach den üblichen Kriterien nicht zitierfähig" ist. Klostermann, Verlegen im Netz, Punkt 1.d.) des Textes.

${ }^{180}$ Espen J. Aarseth, Cybertext: Perspectives on Ergodic Literature (Baltimore; London, 1997), 2. 
Nicht im Hinblick auf die Rezeption, sondern vielmehr vor dem Hintergrund der physikalischen Struktur eines Werkes verwendet Costanzo die Begriffe von Linearität und Nicht-Linearität:

Books are linear because their pages are physically bound in a fixed sequence. For the most part, we read them as continuous stream of words. By contrast, electronic texts have no set boundaries. ${ }^{181}$

Vermutlich ist dieser konzeptionsorientierte Ansatz von Costanzo noch am ehesten $\mathrm{zu}$ akzeptieren. Zwar scheint es nicht angeraten, eine strikte Trennung zwischen linearen Texten $=$ Ducktexten und nicht-linearen Texten $=$ elektronischen Hypertexten vorzunehmen; es ist jedoch als vertretbar anzusehen, zwischen linear beziehungsweise nicht-linear konzipierten Texten zu unterscheiden: Alle Texte, die als konventionelle Drucktexte abgefaßt sind, gelten demnach als linear konzipierte Texte, wohingegen diejenigen, die als elektronische Hypertexte gestaltet sind, als nichtlinear konzipiert zu verstehen sind.

Sobald die Frage nach Linearität beziehungsweise Nicht-Linearität unter dem Aspekt der Textrezeption erörtert wird, beginnen die Begriffe dagegen ineinander zu fließen und verhindern somit eine klare Trennung voneinander. Drucktexte lassen durchaus eine nicht-lineare Lesart $\mathrm{zu}$ und die Tatsache, daß eine Geschichte im World Wide Web präsentiert wird, wo sie gegebenenfalls Verknüpfungen aufweist, die über die eigene Erzählung hinausgehen und den Leser mit Zusatzinformationen oder anderen Online-Werken verbinden, ändert nichts an der Gegebenheit, daß diese Geschichte unabhängig von dem Weg, den der Leser jeweils wählt, dennoch immer einen linearen Verlauf nimmt. So sollten die Texte der jeweiligen informationellen Einheiten stets "kohäsiv geschlossen, kontextoffen und untereinander relationiert sein"182. Die Schwierigkeit liegt dabei in der Verständnissicherung, die durch ein aufeinander abgestimmtes Arrangement der informationellen Einheiten sichergestellt sein muß. Die einzelnen Einheiten müssen nicht nur für sich genommen verständlich sein, sondern auch in den situativen Kontext passen. ${ }^{183}$ Allerdings muß eingeräumt werden, daß ein Zusammenhang des gesamten Hypertextes, das heißt eine

181 William Costanzo, "Reading, Writing, and Thinking in an Age of Electronic Literacy", in: Cynthia L. Selfe und Susan Hilligoss, Hg., Literacy and Computers: The Complications of Teaching and Learning with Technology (New York, 1994), 11-21, hier: 12.

182 Kuhlen, Hypertext, 333.

183 Vgl. ebd., 81. 
"kohärente Ganzheit aus sinnvoll angeordneten Untereinheiten" ${ }^{184}$ nur schwer zu gewährleisten ist. Der inhaltliche Hergang muß aber für den Leser schlüssig und nachvollziehbar sein, da dieser andernfalls zu demselben Urteil gelangen könnte wie Klostermann:

"Die Ideologie des elektronischen Hypertextes gibt dagegen dem nicht-linearen Lesen den Vorrang; sie serviert nur noch Häppchen."185 Es darf nicht unerwähnt bleiben, daß diese Auffassung zum Teil durchaus begründet ist: Im World Wide Web finden sich viele Dokumente, die relativ kurz, oftmals noch unvollständig und teilweise zusammenhanglos sind. Eine These, Online-Texte bestünden generell ausschließlich aus Bruchstücken, wäre allerdings nicht haltbar.

Sieht man ab von der sehr stark eingeschränkten Bedeutung von Linearität, wie Costanzo sie versteht, so zeigt sich, daß Linearität der Übertragung des Literaturbegriffes auf den WWW-Bereich keineswegs entgegensteht. Andererseits legt sie eine solche Begriffserweiterung aber auch nicht nahe. So interessant der Aspekt der Linearität zunächst auch anmutet: Für die Lösung des Terminologieproblems, der sich der vorliegende Abschnitt III. widmet, ist er letztlich ohne Bedeutung.

\section{Begriffsdiskussion}

Als was also ist das Ergebnis der in das WWW eingestellten multimedialen Gestaltungen zu bezeichnen?

Wie bereits ausgeführt, besteht eine starke Tendenz, den Begriff Literatur durch ein aliud zu ersetzen, das den Nutzer sofort erkennen läßt, daß Kunstwerke jener Bezeichnung Eigenschaften wie Verknüpfung und Interaktivität aufweisen. Gesucht wird in diesem Sinne ein Begriff, der unmißverständlich zum Ausdruck bringt, daß es sich um "webpublizierte, für das Web geschaffene und dort möglichst lebendige (zum Beispiel wachsende) Werke narrativen Charakters" ${ }^{\text {186 }}$ handelt, oder wie Simanowski das Verhältnis von Literatur und Internet beschreibt - um "Literatur, die unmittelbar in und aus diesem Medium entstanden ist"187.

184 Ebd., 89.

185 Klostermann, “'Von Gutenberg zum Internet' - Wie bitte?!”, 25.

186 Schröder, "Der Link als Herme und Seitensprung”, 48.

187 Roberto Simanowski, “Autorschaft in digitalen Medien. Eine Einleitung”, in: Ders., Hg., Digitale Literatur (München, 2001) [Text + Kritik: Zeitschrift für Literatur 152 (2001)], 3-21, hier: 3. 


\section{1. "Textwucherungen, Rhizome, Konglomerate" 188}

Auf der Suche nach einem geeigneten Begriff können die Betrachtungen von Krahberger, der sich für die Bezeichnung Hypertext verwendet, eventuell einen kreativen Einstieg darstellen. In seiner Analyse der neuen Literaturform erhält das Präfix hyper- nämlich eine geradezu programmatische Bedeutung. Seiner Auffassung nach charakterisiert hyper- eine Zustandssteigerung, "eine über den Normzustand hinausgehende Entwicklung"189, wobei aber nicht ganz deutlich wird, ob Krahberger dieses Resultat als durchweg fortschrittlich ansieht, da er in diesem Zusammenhang auch von Übertreibung spricht. Auch seine Begriffsvorschläge geben in dieser Hinsicht keinen Aufschluß, vermeidet er doch jegliche terminologische Festlegung, indem er Hypertexte in seinen Ausführungen vieldeutig als Textwucherungen beziehungsweise Rhizome oder Konglomerate bezeichnet. ${ }^{190}$

Zimmer, der sich ausführlich dem Thema Text und Schrift in den Zeiten des Internet widmet, wird in dieser Hinsicht wesentlich konkreter. Er schlägt die Bezeichnung "intermediale Online-Bezugsgenerierung" "191 vor, womit er offensichtlich ganz bewußt einen abstrakten und nüchternen Begriff konstruieren will, der - wie er wohl hofft - geeignet ist, die tiefe Kluft zwischen Literatur in ihrem ursprünglichen Sinne und dem neuerlichen "besonders leichtfertigen Umgang"192 mit ihr zu signalisieren.

188 Franz Krahberger, "Hypertext, Hyperraum, Kulturentwicklung”, in: Franz Krahberger, Hg., Das Babylonprojekt: Ein Textcompendium zur Computerkultur, Electronic Journal Literatur Primär, $<$ http://ezines.onb.ac.at:8080/ejournal/pub/ejour-97-II/buecher/babyl/hypertext.html $>, 31.01 .2003$.

189 Ebd., 31.01.2003.

190 Allen drei Phänomenen ist eines gemeinsam: die Unübersichtlichkeit. Innerhalb eines Konglomerats, das heißt einer Zusammenballung oder eines Gemisches läßt sich nur wenig oder gar keine Systematik erkennen; Wucherungen erweisen sich im allgemeinen als unbeherrschbar, als unkontrollierbar, was gleichermaßen für Wurzelwildwuchs (Rhizome) gilt. Gerade das Bild von den Rhizomen wird häufig im Zusammenhang mit Hypertext verwendet: Unterirdisch verlaufend verbinden sie zwar alle Elemente miteinander, doch widerstehen Rhizome jedem Versuch einer gliedernden Ordnung. Vgl. Schröder, "Der Link als Herme und Seitensprung", 53. - Im Unterschied zum geläufigen Baummodell sehen Deleuze und Guattari die Merkmale der nichthierarchischen und nonlinearen Struktur eines Rhizoms in der "Verkettung bzw. Vernetzung, Heterogenität und irreduziblen Vielheit der Elemente". Lévy, "Cyberkultur", 62f. Bollmann und Heibach erläutern das Modell der Internet-Struktur von Deleuze und Guattari in ihrem abschließenden Beitrag zum Kursbuch Internet anhand seiner fünf Prinzipien wie folgt: 1. Nach dem Grundsatz der Konnexion (Vernetzung) müssen alle Punkte eines Rhizoms/des Internet miteinander zu verbinden sein. 2. Das Gesetz der Heterogenität basiert auf den verschiedenartigen Zeichensystemen des Internet, wohingegen 3. das Prinzip der Vielheit auf den unterschiedlichen Dimensionen von Rhizom und Internet beruht. 4. Die Regel des asignifikanten Bruchs impliziert, daß keine Zerstörung einen Weiterwuchs aufhalten kann. 5. Das System der Kartographie stützt sich auf die Karten-Form von Rhizom und Internet, die von allen Seiten zugänglich und beliebig zusammensetzbar ist. Stefan Bollmann und Christiane Heibach, "Sucht keine Wurzeln, folgt dem Kanal: Die frohen Botschaften der französischen Zahnärzte", in: Dies., Hg., Kursbuch Internet, 466-474, hier: 470 .

191 Zimmer, Die Bibliothek der Zukunft, 59.

192 Ebd., 58. 
Mit dieser Bezeichnung liegt Zimmer nicht weit entfernt von Kuhlen, der für das, was mittels Computer generiert wird, die Formulierung "Medium der nicht-linearen Organisation von Informationseinheiten" ${ }^{\prime 193}$ am treffendsten empfindet und mit ihrer Hilfe eine systematische Abgrenzung von Texten und Hypertexten anstrebt. Beide Vorschläge sollen zwar eine ebenso nüchterne wie präzise Charakterisierung der neuen Literaturformen geben, haben allerdings in Wirklichkeit keinen tatsächlichen Aussagewert; beide Formulierungen lassen wohl erahnen, was gemeint ist, von gebrauchsfähigen Termini kann man jedoch keinesfalls sprechen. Zudem sind beide Versionen und damit hat zumindest Zimmer möglicherweise sein Ziel erreicht - so wortaufwendig abgefaßt, daß sie sich schon aus diesem Grunde nicht für eine praktische Verwendung empfehlen.

Eine andere Richtung schlagen Böhle, Riehm und Wingert auf ihrer Suche nach einem neuen Namen für das Angebot der digitalen Texttechnologie ein. Auch sie stützen ihren Begriff auf das Merkmal Interaktivität, legen den Schwerpunkt aber eindeutig auf eine durch den Computer geprägte neue Spielkultur und bezeichnen die modernen "Leseerlebnisse" als "Interaktive Textspiele". ${ }^{194}$ Ihre Definition nimmt allerdings keinerlei Bezug auf spielerische Elemente, vielmehr verstehen Böhle, Riehm und Wingert unter Interaktiven Textspielen einfach ein Textangebot, auf dessen Form und Inhalt der Nutzer mittels interaktiver Softwarefunktionen situativ innerhalb eines bestimmten Rahmens Einfluß nehmen kann, wodurch er dann zu einem “"einmaligen', 'individuellen' Rezeptionserlebnis" ${ }^{195}$ kommt. Im Unterschied zu den ehemals in Form und Inhalt vorbestimmten, starren, unveränderbaren Texten kann der Nutzer nun zumindest die Anordnung, wenn nicht unter Umständen sogar den textuellen Inhalt mitbestimmen. So kreativ die Wortschöpfung Interaktive Textspiele auch sein mag, eine offizielle Aufnahme in die Terminologie der Literaturwissenschaft ist auch im Falle dieser Formulierung nicht zu befürworten, da sie keinen begrifflichen Bezug zu dem Trägermedium World Wide Web erkennen läßt, vor allem aber die spielerische Komponente zu stark akzentuiert.

Ebenfalls ungewollte Assoziationen weckt die bei Schröder auftauchende Bezeichnung "Komposition"196, die vorwiegend im musikalischen Bereich verwendet

\footnotetext{
193 Kuhlen, Hypertext, 27.

194 Knud Böhle, Ulrich Riehm und Bernd Wingert, Vom allmählichen Verfertigen elektronischer Bücher: ein Erfahrungsbericht (Frankfurt a.M.; New York, 1997), 325.

195 Ebd.

196 Schröder, "Der Link als Herme und Seitensprung”, 50f.
} 
wird und somit zunächst die Vorstellung von Klangwelten hervorruft. Zwar denkt Schröder bei diesem Begriff vor allem an die neuen Möglichkeiten des Lesers, der sich nun seinen eigenen Text aus vielen einzelnen Einheiten zusammensuchen und diesen somit gewissermaßen 'komponieren' kann, doch legt er den Schwerpunkt damit eindeutig auf den Leser. Für das WWW konzipierte Literatur sollte jedoch nicht nur aktiven, sondern vielmehr interaktiven Charakter haben, ${ }^{197}$ das heißt von Autoren und Lesern gleichermaßen geprägt sein. Folglich kann auch die Bezeichnung Komposition keine adäquate begriffliche Alternative darstellen.

Während der von Schröder favorisierte Begriff zu eng und einseitig erscheint, ist die von Blank verwendete Bezeichnung "Netart"198 eindeutig zu weit gefaßt. Sowohl die Verwendung von Netart als auch der Rückgriff auf die deutsche Entsprechung "Netzkunst" führen zu einer deutlichen Akzentverschiebung. Hauptsächlich dürften beide Ausdrücke nämlich als Oberbegriffe verstanden werden: als zusammenfassende Bezeichnungen für alle Werke im World Wide Web, die dem Bereich der sogenannten Schönen Künste zuzuordnenden sind, worunter gemeinhin außer Dichtkunst, Musik und darstellenden Künsten wie Theater, Tanz und Film, auch die bildenden Künste wie Architektur, Bildhauerei und Malerei sowie reproduzierende Künste fallen, Kunstformen also, die offensichtlich weit über das hinausgehen, wofür hier der treffende Begriff gesucht wird. ${ }^{199}$ Daher können auch diese Termini keine Anerkennung finden.

Gleiches gilt für die Bezeichnung "Hyper-Kunst"200, die Todesco den Texten im World Wide Web aufgrund Wegfalls jeglicher Rezeptionsorientierung zuschreibt. Seiner Ansicht nach dient Kunst dort nicht der Mitteilung, sondern dem persönlichen Ausdruck des Künstlers. Im Hypertext-Medium des WWW ist der Textproduzent Todescos Ansicht nach um seiner selbst willen tätig, nicht um einem Publikum zu dienen, beziehungsweise sich seiner Gunst zu vergewissern. Aus diesem Grunde - so

197 Wie sich später noch herausstellen wird, beschränkt sich die Interaktion zwischen Autor und Leser häufig auf eine reine Reaktion des Lesers auf die Vorgaben des Autors.

198 Joachim Blank, What is netart ;-)?, <http://www.hgb-leipzig.de/theorie/netlag.htm>, 17.02.2003.

199 Auch eine geringfügige Abwandlung der Bezeichnung Netart in Netarts ließe keinen größeren Interpretationsspielraum. The arts sind nur zu übersetzen als "die schönen Künste", und auch im universitären Bereich sind mit arts die Geisteswissenschaften gemeint, die als Abgrenzung zu den Naturwissenschaften verstanden werden und damit eindeutig auch Musikwissenschaften und Kunstgeschichte miteinbeziehen.

200 Todesco, "Hyperkommunikation", 122. 
Todesco - sollen seine Werke nicht mehr als Texte wahrgenommen werden, sondern als autonome Kunstwerke. ${ }^{201}$

Todescos Argumentation kann nicht unwidersprochen bleiben; vielmehr muß gefragt werden, ob die Rezeptionsorientierung im Hypertext-Medium tatsächlich wegfällt. Zum einen unterliegt auch die Selbstdarstellung eines Künstlers einer gewissen Rezeptionsorientierung, da auch sie eine bestimmte Wirkung beim Leser erzielen soll (Rezeption $=$ Aufnahme, Textverständnis; Orientierung $=$ Ausrichtung): nach Todescos These nämlich die des Verständnisses als autonomes Kunstwerk. Möglich ist hier allerdings, daß das Leserverständnis von der Intention des Autors beziehungsweise Künstlers abweicht.

Zum anderen macht der Autor/Künstler auch in einem Hypertext-System Rezeptionsvorgaben, indem er vorab festlegt, welche Informationseinheiten dem Leser zur Verfügung stehen sollen beziehungsweise indem er den Leser möglicherweise aufgrund seiner Hypertext-Konzeption sogar lenkt.

Ganz bestimmt liegt eine Rezeptionsorientierung aber im Falle jener Werke im World Wide Web vor, die auf Mitarbeit des Lesers ausgelegt sind. Der Mitwirkung muß nämlich notwendigerweise eine Beschäftigung mit der Materie und somit eine Rezeption und eine Interpretation vorausgegangen sein.

Im Gegensatz zu vielen anderen Autoren schenkt Ziegfeld dem Aspekt der Interaktion in seiner Abhandlung "Interactive Fiction: A New Literary Genre?"202 zweifellos die gebührende Beachtung. Dennoch spricht ein entscheidendes Argument gegen eine Verwendung der Bezeichnung Interactive Fiction: Sowohl eine Übersetzung in die deutsche Sprache als auch ein Blick auf die traditionellen, auf Konvention basierenden Gattungsbegriffe Lyrik (engl. poetry), Drama (engl. drama) und Erzählliteratur (engl. prose) zeigen, daß der Begriff Fiction auch falsche Assoziationen wecken kann: Übersetzt als Erzähl- und Prosaliteratur würde Fiction die Gattungen Lyrik und Drama ausgrenzen. Zwar dient der Begriff Fiction auch allgemein zur Bezeichnung der "Phantasie als Kraft dichterischer Weltgestaltung"203, besonders im Englischen und Amerikanischen jedoch ist damit meist die erzählende Prosa gemeint. Ziegfeld selbst nimmt in seinen Ausführungen Bezug auf die herkömmliche

201 Ebd., $122 \mathrm{f}$.

202 Richard Ziegfeld, “Interactive Fiction: A New Literary Genre?”, New Literary History: a Journal of Theory and Interpretation 20 (1989), 341-372, hier: 341ff.

${ }^{203}$ Gero von Wilpert, Sachwörterbuch der Literatur, 7., verb. u. erw. Aufl. (Stuttgart, 1989 [ $\left.{ }^{1} 1955\right]$ ), 298. 
Einteilung und verwendet dabei sogar den Begriff Fiction anstelle von Prose. ${ }^{204}$ Wie auch immer jedoch der Begriff Fiction von Ziegfeld selbst interpretiert werden mag, und wie auch immer er anderorts verwendet zu werden pflegt, als Oberbegriff für die gesamte Bandbreite der Online-Literatur kann er nicht dienen, denn eine präzise, unmißverständliche Charakterisierung dieser Literaturform ist nicht wirklich gewährleistet. $^{205}$

Aus demselben Grunde kann auch die zweite von Schröder vorgeschlagene Bezeichnung "Webfiction"206 die Kriterien für einen allumfassenden Terminus nicht erfüllen. Schröder wollte bewußt eine "Unterscheidung von nicht-multimedialer Hyperfiction ohne externe Verknüpfungen, von kollaborativen Projekten konventioneller Schriftlichkeit und von MUDs" treffen. ${ }^{207}$ Und es ist positiv hervorzuheben, daß diese Überlegung Grundlage seiner Begriffsfindung war. Leider vermag aber die Prägnanz des ersten Wortbestandteiles die Mehrdeutigkeit des zweiten Teiles nicht auszugleichen.

Auch der von Simanowski geprägte Begriff "Interfiction"208 ist deshalb letztlich nicht sinnvoll. Simanowski will sich damit eindeutig von allen nichtfiktionalen Werken distanzieren und gleichzeitig eine gewisse künstlerische Komponente sowie den multimedialen Aspekt des World Wide Web begrifflich zum Ausdruck bringen. Es ist jedoch fraglich, ob dieses Wort tatsächlich geeignet ist, sich gegen die existierenden "begrifflichen Fehlbesetzungen und Mißverständlichkeiten"209 abzusetzen. Die von Simanowski gewünschte Einbindung der Kunst läßt sich nicht ohne weiteres herstellen.

Auch die beabsichtigte Assoziation zu Interaktivität, Intermedialität und Internet erscheint nicht ganz so offensichtlich wie beabsichtigt; immerhin wäre der Gedanke, es könnte sich um eine Art literarische 'Zwischenform' handeln (lat. inter = zwischen), durchaus nicht abwegig. Daher wirft der Terminus Interfiction zu viele Fragen auf, um als unmißverständlich und präzise gelten zu können. Zugegebener-

204 Ziegfeld, "Interactive Fiction”, 368.

205 Welche unterschiedlichen Lesarten ein Begriff hervorrufen kann, beweist Costanzo mit seiner persönlichen Interpretation von Interactive Fiction. Er bezeichnet diese als "the textual incarnation of adventure games" und läßt damit wieder an eine eher spielerischen Komponente von OnlineLiteratur denken. Constanzo, "Reading, Writing, and Thinking in an Age of Electronic Literacy", 16.

206 Schröder, "Der Link als Herme und Seitensprung", 48.

207 Ebd.

208 Roberto Simanowski, "Interfictions. Vom Schreiben im Netz”, in: Ders., Hg., dichtung-digital,

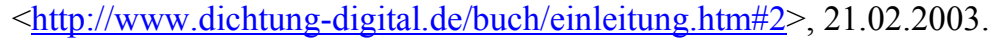

209 Ebd., 21.02.2003. 
maßen findet der Begriff Interfiction bereits seit geraumer Zeit innerhalb bestimmter Personengruppen und auf Fachtagungen Verwendung, doch ist er schwerlich geeignet, auch außerhalb von Fachkreisen die gewünschten Vorstellungen hervorzurufen.

\subsection{Grundsätzliche Tauglichkeit des Begriffes Literatur}

Die Unzulänglichkeit aller bisher betrachteten Termini führt $\mathrm{zu}$ dem vorläufigen Schluß, daß die geforderte Abkehr vom Begriff Literatur wahrscheinlich nicht gerechtfertigt ist. Es ist davon auszugehen, daß das Wort Literatur auch für Werke zu verwenden ist, deren Schwerpunkt eindeutig nicht auf einem schriftlichen Zeichensystem, sondern auf Bild-, Ton-, Animations- und Interaktionselementen liegt. Das daraus resultierende Erscheinungsbild führt allerdings zu der Frage, ob die Beschäftigung mit einem (literarischen) Online-Werk als Lesen bezeichnet werden kann. Käme man zu der Überzeugung, daß hier durchaus von einem Lesevorgang gesprochen werden kann, ließe sich unter Inanspruchnahme eines gewissen Interpretationsspielraumes auch in bezug auf das World Wide Web ohne weiteres der Begriff Literatur verwenden.

Ein lebhaftes Plädoyer g e g e n den Begriff des Lesens hält Kress. Ehemals gedruckt vorliegender Text präsentiert sich auf einem Computerbildschirm - so Kress - in gänzlich veränderter Gestalt, weshalb er ihn dort als Ressource bezeichnen möchte; da diese Ressource nicht mehr in herkömmlicher Weise rezipiert werden kann, spricht Kress auch nicht mehr von einem Lese-, sondern von einem Nutzungsvorgang: Sei das herkömmliche Buch noch von Anfang bis Ende durchgelesen worden, so werde es in seiner neuen Erscheinungsform nicht mehr gelesen, sondern genutzt. ${ }^{210}$ Die herkömmlichen Begriffe von Text und Lesen sind also durch die Einführung der neuen Medien angeblich zu speziell geworden, weshalb sie nach Meinung von Kress durch unspezifischere Bezeichnungen ersetzt werden müssen.

Kress leistet ein offenes Bekenntnis zum Zeitgeist, der den Aspekt der Zweckgebundenheit menschlichen Handelns akzentuiert. Er ist reformbereit und bedient sich gängiger Bezeichnungen, die sowohl kurzgefaßt als auch aussagekräftig sind. Bei konsequenter Weiterführung seiner Gedanken müßte man zu der Schluß-

${ }^{210}$ Gunther Kress, "Visual and verbal modes of representation in electronically mediated communication: the potentials of new forms of text", in: Ilana Snyder, Hg., Page to Screen, 53-79, hier: 66. 
folgerung gelangen, daß im Hinblick auf elektronische Publikationen auch der Ausdruck Literatur keine weitere Verwendung mehr finden kann.

Dagegen stehen andere Argumente, die für eine Weiterverwendung beziehungsweise für einen großzügigeren Gebrauch des Lesebegriffs sprechen. Einen Einstieg in diese Frage liefert uns die Analyse des Lesevorganges. Daß der Begriff des Lesens nämlich nicht notwendigerweise an Schrift gebunden ist, wird deutlich, wenn man sich vergegenwärtigt, was sich während des Leseprozesses im Kopf eines Lesers abspielt. "Lesen ist ausdeutendes Bemerken differenzierender Merkmale." 211 Dieses Urteil beruht auf der Tatsache, daß der Leser bei der Rezeption eines Textes nicht allein die Schrift dechiffriert, sondern vielmehr den dahinterliegenden Gedankengang erschließt. "Lesen heißt also nicht nur, Buchstaben und Wörter zu verlautlichen, Text zu entziffern, sondern das darin Eingekleidete so zu erkennen, als sei der Text gar nicht da." ${ }^{212}$ Genau darum geht es beispielsweise auch, wenn man vom Lesen einer Tierspur spricht: Gemeint ist in diesem Zusammenhang eigentlich, daß die Fährte interpretiert, das heißt der Hintergrund des Abdrucks ausgedeutet wird.

Losgelöst von Schrift taucht der Begriff des Lesens noch in einem weiteren Zusammenhang auf: Auch bei Karten und Stadtplänen spricht man in der Regel vom Lesen. Gerade dieser Umstand legt einen Vergleich mit dem vernetzten System des World Wide Web nahe und führt zu einer weiteren Assoziation: Indem sich der Nutzer im WWW auf der Suche nach Informationen von einer Verknüpfung zur nächsten bewegt, sammelt er gewissermaßen alle relevanten Daten ein; so wie der Winzer, der die Trauben einsammelt, Wein le s e hält, betreibt auch der Nutzer Lese.

Es wird deutlich, daß der Begriff des Lesens vielfältige Interpretationen zuläßt. Damit sollte eigentlich auch der Begriff der Literatur, dem die Lesbarkeit immanent ist, grundsätzlich über die für eine Verwendung im Bereich des World Wide Web nötige Flexibilität verfügen. Unerklärlich bleibt, warum sich so viele Autoren mit einer Übernahme des Begriffes - unmittelbar oder in abgewandelter Form - so schwer tun. Auch Birkerts sucht zu erfahren, wie die Widerstände zu erklären sind:

\footnotetext{
211 Wingert, "Kann man Hypertexte lesen?", 189.

212 Ebd.
} 
Wer da behauptet, daß wir nur lesen, solange unser Blick über die Zeilen gleitet, solange wir unmittelbar im Bann der Sprache stehen, die Worte stumm in unserem Innern nachsprechen, der behauptet nach derselben Logik auch, daß der Schriftsteller nur schreibt, solange er damit beschäftigt ist, das Papier mit Schriftzeichen zu bedecken. Welcher Schriftsteller würde sich über ein so borniert wörtliches Verständnis seines Tuns nicht mokieren? ${ }^{213}$

An dieser Stelle wird endgültig deutlich, daß es aus Gründen der allenthalben wünschenswerten Flexibilität, Dynamik und Farbigkeit geboten erscheint, sämtlichen hier behandelten Begriffen mit mehr Großzügigkeit zu begegnen: dem Wort Lesen, dem Ausdruck Autor, dem Begriff Literatur und ebenso der Bezeichnung Text, denn auch dieser stellt sich nicht mehr als "klassische typographische Differenz von Schriftbild und Hintergrund" ${ }^{14}$ dar, sondern präsentiert sich nunmehr als "Anzahl seiner Verknüpfungsmöglichkeiten zwischen Datenmengen"215, was bedeutet, daß der Text zu einem Datengeflecht wird.

So enthalten die neuen Werke im World Wide Web zwar die bekannten Elemente, die Literatur üblicherweise ausmachen, doch alles scheint in erweiterter Form aufzutreten: Es handelt sich nicht mehr nur um reinen Text, sondern zusätzlich auch um multimediale Elemente; es gibt nicht mehr nur e in e Leseweise, sondern zahlreiche Versionen; oftmals zeichnet nicht nur e in Autor, sondern eine Autorengemeinschaft für ein Werk verantwortlich.

Ungeachtet ihrer Andersartigkeit weisen die neuen Formen von Literatur im Wesentlichen noch zu große Ähnlichkeit mit den traditionellen Literaturformen auf, als daß eine trennende Grenze gezogen werden sollte. Mit einem Verständnis von Online-Literatur als einer weiterentwickelten, gewissermaßen gesteigerten Variante von gedruckter Literatur sollte man sowohl dem innovativen Charakter der neuen Literaturform als auch dem traditionellen Literaturbegriff gerecht werden können. Ein Auseinanderdividieren beider Varianten sollte hinter einem Verständnis von Vielfalt in der Einheit zurücktreten.

${ }^{213}$ Birkerts, Die Gutenberg-Elegien, 131.

${ }^{214}$ Karin Wenz, "Die unerträgliche Leichtigkeit des Textes", in: Bildschirmfiktionen, 245-254, hier: 248.

${ }^{215}$ Ebd., 249. 


\section{Abstimmung auf die Besonderheiten des WWW}

Das vorstehende Plädoyer für eine Verwendung des Begriffes Literatur bedeutet allerdings nicht zwingend eine Art Eins-zu-eins-Übernahme in den Web-Sprachgebrauch. Denkbar sind durchaus Zusätze und Abwandlungen, die die Besonderheiten des World Wide Web signalisieren.

\subsection{Der Feinabstimmung erster Schritt}

So könnte beispielsweise Hyperliterature in Anlehnung an Selfe und Hilligoss ${ }^{216}$ die begriffliche Synthese von Altem und Neuem bilden. Nicht ausreichend zum Ausdruck kommen in dieser Bezeichnung allerdings die entscheidenden Merkmale des World Wide Web wie globale Zugriffsmöglichkeit, Interaktivität und Kooperationsmöglichkeit, die dieses Online-Medium von anderen elektronischen Datenträgern unterscheiden. Eine CD-ROM beispielsweise bildet ein in sich geschlossenes Werk, das auch auf Interaktivität, Kooperativität und Zugriff auf das WWW ${ }^{217}$ ausgelegt sein kann, aber niemals die Dynamik des Web aufzuweisen vermag. Da es in vorliegender Arbeit ausschließlich um Online-Literatur geht, erscheint die Bezeichnung Hyperliterature als nur bedingt geeignet.

Ebenfalls weitgehend unberücksichtigt bleiben die besonderen Eigenschaften des WWW bei dem Terminus Digitale Literatur. Zu Recht differenziert Heibach in ihrem Artikel zwischen der vernetzten und der unvernetzten digitalen Literatur ${ }^{218}$, weshalb Simanowski Digitale Literatur folgerichtig als "Dachbegriff" 219 bezeichnet. Tatsächlich ist das Netz "nur eines der digitalen Medien"220; daher kommt auch dieser Terminus als Alternative nicht in Frage.

216 Selfe und Hilligoss verwenden den Ausdruck Hyperliteracy und erfassen damit wesentliche Charakteristika von Online-Literatur in einem einzigen Wort. Cynthia L. Selfe und Susan Hilligoss, "Introduction", in: Selfe und Hilligoss, Hg., Literacy and Computers, 1-7, hier: 5.

217 Bei dieser Zugriffsmöglichkeit wird durch das Anklicken eines Verknüpfungshinweises auf der CD-ROM automatisch der Zugang zum World Wide Web aktiviert. Bei diesem Automatismus gehen allerdings gewisse Veränderungen wie beispielsweise ein Wechsel der WWW-Adresse sofern keine automatische Weiterschaltung zur neuen Adresse erfolgt - notwendigerweise ins Leere.

${ }^{218}$ Christiane Heibach, "Ins Universum der digitalen Literatur. Versuch einer Typologie", in: Digitale Literatur [Text + Kritik: Zeitschrift für Literatur 152 (2001)], 31-42, hier: 33.

219 Simanowski, "Autorschaft in digitalen Medien ", 4.

220 Ebd. 
Eine völlig neue Dimension eröffnet Aarseth mit seinem Terminus "Ergodic Literature", ${ }^{221}$ Ergodisch ist ein aus der Physik stammender Fachausdruck, der auf den griechischen Worten ergon und hodos basiert und damit die Begriffe Arbeit und Weg in einer Wendung zusammenführt. ${ }^{222}$ Aarseth weist dem Lesevorgang in computergesteuerten Umgebungen nämlich einen neuen, handlungsorientierten Aspekt $\mathrm{zu}$, indem er zwischen dem Lesen eines herkömmlichen Werkes als einem hauptsächlich geistigen Vorgang und der Rezeption eines Cybertextes als einer vorwiegend manuellen Tätigkeit unterscheidet. Letztere erklärt sich aus der Konzeption dieser Texte, aufgrund derer der Leser mittels Auf- und Abwärtsbewegen der Seiten sowie durch Anklicken von Links und aktive Kooperation körperliche Leistung verrichtet:

The cybertext reader is a player, a gambler; the cybertext is a game-world or world-game; it is possible to explore, get lost, and discover secret paths in these texts, not metaphorically, but through the topological structures of the textual machinery. ${ }^{223}$

Die physische Arbeit des Cybertext-Lesers stellt Aarseth als einen "nontrivial effort" dar, während er das Lesen nicht-ergodischer Literatur als "trivial effort" bezeichnet. $^{224}$

So neuartig und progressiv der Ansatz von Aarseth auch erscheint, vermag er dennoch nicht zu überzeugen. Zum einen wird der Umgang mit digitaler Literatur zu stark auf die manuelle Betätigung des Lesers beschränkt, wodurch die Literatur auf eine reine "Klickeratur",225 und der Leser auf einen "Knöpfchendrückenden Affen”226 reduziert wird. Tatsächlich geht mit der Beschäftigung mit Cybertexten jedoch oft eine erhebliche Kopfarbeit einher; fast könnte man so weit gehen zu behaupten, der Leser müsse sogar eine höhere geistige Flexibilität aufbringen, als es bei gedruckter Literatur der Fall ist: Er muß nicht nur passiv den Inhalt seiner Lektüre aufnehmen, sondern muß sich gleichzeitig auch aktiv durch den

\footnotetext{
221 Aarseth, Cybertext, 1.

222 Ebd.

223 Ebd., 4.

224 Ebd., 1

225 Schröder, "Der Link als Herme und Seitensprung", 50.

226 Rena Tangens und padeluun, "Auf dem Weg zu einer Informationsgesellschaft", in: Enquête-Kommission 'Zukunft der Medien in Wirtschaft und Gesellschaft; Deutschlands Weg in die Informationsgesellschaft' Deutscher Bundestag, Hg., Medienkompetenz im Informationszeitalter (Bonn, 1997), 147-161, hier: 151.
} 
vernetzten Text bewegen und verlaufsrelevante Entscheidungen treffen. ${ }^{227}$ Zum anderen kann Aarseth deshalb nicht überzeugen, weil seine Kriterien für ergodische Literatur generell auf alle digitalen Texten zutreffen. Im Rahmen vorliegender Arbeit sollen die Begrifflichkeiten jedoch - wie bereits betont - nur in bezug auf OnlineLiteratur hinterfragt werden. Da die Bezeichnung Ergodische Literatur also zu weitgefaßt ist, kann der Vorschlag von Aarseth ebenfalls nicht aufgegriffen werden.

Eine treffendere Benennung findet sich bei Zimmer, der in seinen Ausführungen zu Literatur in Verbindung mit Computern den Begriff "Web-Literatur" ${ }^{\text {"228 }} \mathrm{zu}$ verwenden pflegt. Zimmer, der sich intensiv und - wie vorangehend erörtert - durchaus kritisch mit dem Thema Text und Schrift im Zeitalter des World Wide Web auseinandersetzt, bedient sich hier eines Ausdrucks, der alle vorangehend aufgestellten Forderungen für einen neuen Terminus in sich vereint: Es geht eindeutig aus ihm hervor, um welches Präsentationsmedium es sich handelt, und es ist gleichzeitig auch klar zu erkennen, was genau dort dargeboten wird.

Dasselbe gilt offenbar für die bereits vielfach verwendete Bezeichnung Netzliteratur $^{229}$. Bei einer Gegenüberstellung der beiden Ausdrücke hätte der Terminus Netzliteratur sogar den Vorteil, daß er keine zusammengesetzte, mehrsprachige Verbindung aus der englischen Kurzfassung für das WWW und dem deutschen Wort Literatur darstellt. Für den deutschen Sprachraum sollte sich diese Bezeichnung daher eigentlich als die überzeugendere Wortschöpfung empfehlen. Der Begriff Web-Literatur genießt aber vermutlich eine höhere internationale Akzeptanz. Weit schwerer fällt jedoch ins Gewicht, daß Web-Literatur und Netzliteratur eben doch nicht völlig gleichbedeutend sind.

227 Diese Einschätzung widerspricht zwar auf den ersten Blick den in Kapitel I.3. erwähnten Untersuchungsergebnissen, die eindeutig eine Verkümmerung entscheidender geistiger Fähigkeiten durch Bildschirmnutzung aufzeigen; so bemerkten beispielsweise Bischof und Heidtmann bei Jugendlichen eine deutliche Verschlechterung der Fähigkeit, mehrschichtige Texte zu verstehen. Bischof und Heidtmann, "Warum sind Film- und Fernsehbücher so erfolgreich?", 414. Diese Verschlechterung muß allerdings nicht notwendigerweise auf Computer-Nutzung zurückzuführen sein. Im übrigen ist davon auszugehen, daß sich die bei Bischof und Heidtmann aufgeführten Erkenntnisse offenbar nicht auf die Rezeption komplexer literarischer Hypertexte stützen, da deren Bearbeitung geistige Kompetenzen eher fördert als beeinträchtigt.

228 Zimmer, "Die digitale Bibliothek", 285ff. und ders., Die Bibliothek der Zukunft, 59.

229 Berlich, "Das Internet als innerer Ort", 146. Reinhold Greter, "Versuch über Welttexte”, in: Suter und Böhler, Hg., Hyperfiction, 85-100, hier: 86. Martina Kieninger, "Vom Schreiben auf glatten Oberflächen: Zur Geschichte des zweisprachigen Mehrautorenprojekts Tango", in: Suter und Böhler, Hg., Hyperfiction, 183-199, hier: 199. Schröder, "Der Link als Herme und Seitensprung", 43. Hautzinger, Vom Buch zum Internet?, 17ff. Wolfgang Tischer, "AAnfangserscheinungen und

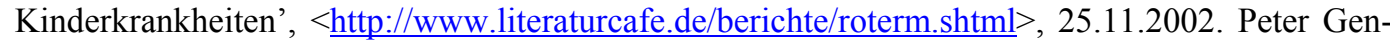
dolla und Jörgen Schäfer, "Auf Spurensuche. Literatur im Netz, Netzliteratur und ihre Vorgeschichte(n)", in: Digitale Literatur [Text + Kritik: Zeitschrift für Literatur 152 (2001)], 75-86, hier: 75ff. Simanowski, "Interfictions", <http://www.dichtung-digital.de/buch/einleitung.htm\#2>, 18.02.2003. 
Simanowski definiert Netzliteratur unter Berücksichtigung der besonderen Dynamik des Netzes als "das, was sich der spezifischen Eigenschaft des Internet in ästhetischer Absicht bedient". ${ }^{230}$ Simanowski bezieht sich damit ausdrücklich auf das Internet. Als synonyme Begriffe werden oftmals auch die Bezeichnungen NetLiteratur und Internet-Literatur verwendet. Letztere nimmt bereits seit mehreren Jahren einen festen Platz im Sachwörterbuch zur deutschen Literatur ein und definiert sich dort als "durch das neue elektronische Medium verbreitete beziehungsweise eigens für dieses Medium geschriebene Literatur", die als spezifische Net- oder Internet-Literatur "die Möglichkeiten des Mediums selbst fruchtbar macht und dadurch zu neuen Literaturformen zu gelangen sucht". ${ }^{231}$

$\mathrm{Da}$ die Begriffe Internet und World Wide Web aber immer wieder - fälschlicherweise - synonym gebraucht werden, hat Cramer zu folgender Klarstellung bewogen:

Die Gleichsetzung von Netzdichtung und Hyperfiction ist so falsch wie die Gleichsetzung des Internets mit dem World Wide Web. Neben zum Beispiel EMail, Newsgroups, IRC, ICQ und Napster ist das World Wide Web nur einer von unzähligen Internetdiensten, $[\ldots]^{232}$

Zimmer begeht genau diesen Fehler. Einerseits scheint ihm an einer sehr präzisen Sprachverwendung gelegen $\mathrm{zu}$ sein, andererseits aber bleiben seine eigenen Aussagen ungenau und widersprüchlich: “'Internetliteratur' $[\ldots]$ - das ist die eigens fürs Internet, genauer fürs World Wide Web geschriebene Literatur". 233

Wenn das WWW als ein eigener Bereich mit einer eigenen, ganz spezifischen Charakteristik zu behandeln ist, muß das dort Präsentierte konsequenterweise auch einen eigenen Namen erhalten. ${ }^{234}$

230 Ebd., 18.02.2003.

231 Volker Meid, Sachwörterbuch zur deutschen Literatur (Stuttgart, 1999), 252.

${ }^{232}$ Cramer, Warum es zuwenig interessante Netzdichtung gibt, 4.

233 Zimmer, Die Bibliothek der Zukunft, 52.

234 Die hier eingeforderte Genauigkeit mag als ein Widerspruch zu der bisher immer wieder angemahnten Großzügigkeit im Umgang mit dem Begriff Literatur erscheinen. Warum sollte es gestattet sein, gewisse Aspekte mit Toleranz zu behandeln, bei anderen hingegen für Exaktheit zu plädieren? - Diese Vorgehensweise ist keineswegs ein Widerspruch. Solange sich ein bereits etablierter Begriff als tragfähig erweist und als ausbau- und entwicklungsfähig angesehen werden kann, besteht keine Notwendigkeit, ihn durch einen neuen Begriff zu ersetzen. Diese Erfordernis ist erst recht nicht gegeben, wenn sich das Zielobjekt der Neubenennung noch im Anfangsstadium seiner Entwicklung befindet. Daher scheint es angeraten, den Terminus Literatur als begriffliche Ausgangsbasis weiterzuverwenden und ihn durch Wortergänzungen genauer zu spezifizieren und damit den Gegebenheiten des WWW anzupassen. Hier sei auf Grether verwiesen, der die Verwendung des Literaturbegriffes mit folgendem Argument rechtfertigt: "Wenn es Oral-, Manuskript- und Druckliteratur gibt, dann kann es auch Netzliteratur geben." Grether, "Versuch über Welttexte", 86. - Warum sollte es also nicht auch Web-Literatur geben? 
Im Hinblick auf den Untersuchungsgegenstand vorliegender Arbeit muß der Ausdruck Web-Literatur eindeutig für die präzisere Bezeichnung erklärt werden. Thema ist die englischsprachige Literatur im World Wide Web, womit eine eindeutige Abgrenzung zu dem übergeordneten Medium Internet intendiert ist. Das Internet umfaßt als weltweites Computernetz die verschiedensten Netzwerke, die beispielsweise zur Übertragung von Dateien, zur Übermittlung elektronischer Nachrichten oder eben für den Zugriff auf das WWW genutzt werden. Daher ist der Begriff Netzliteratur nicht spezifisch genug, könnte es sich doch auch um eine Literaturform handeln, die i n einem, ü b e r ein oder fü $r$ ein Chat-Forum - ebenfalls ein Internetdienst - konzipiert wird. Der Ausdruck Web-Literatur hingegen signalisiert eindeutig, daß es sich um das Präsentationsmedium World Wide Web handelt. Zwar gibt es auch dort unterschiedliche Dienste, wie E-Mail oder Kommunikationsforen, aber die terminologische Eingrenzung auf das World Wide Web ist zumindest gegeben.

So müßte die Entscheidung an dieser Stelle ungeachtet der Sprachmischung eigentlich zugunsten des Kompositums Web-Literatur fallen, da es ausdrucksstark, unmißverständlich, eingängig, international und eben konkret auf das WWW bezogen ist.

\subsection{Der Feinabstimmung zweiter Schritt}

Vordergründig scheint der Ausdruck Web-Literatur bislang am ehesten den vorgegebenen Anforderungen zu entsprechen. Gleichwohl ist zu kritisieren, daß er für sich alleine genommen mißverständlich ist. Er signalisiert zwar deutlich, daß es sich bei einer solchermaßen bezeichneten Kunstform um Literatur handelt und diese in Beziehung zum World Wide Web steht. Weitestgehend ungeklärt bleibt aber, ob diese Art der Literatur auch unabhängig von Computer und WWW existieren könnte oder ob ihre Rezeption zwingend die Nutzung dieser Hilfsmittel bedingt.

Da es nunmehr darum geht, den Ausdruck Web-Literatur gegebenenfalls als festen Begriff im literaturwissenschaftlichen Sprachgebrauch zu verankern, ist eine sprachliche Nachbearbeitung zwangsläufig notwendig. 
Literatur im Web $=$ Web-Literatur - diese einfache Gleichung mag auf der Hand liegen ${ }^{235}$, sie deckt sich aber keineswegs mit der Sachlage. Literatur im Web umfaßt auch alle literarischen Werke, die auch unabhängig vom World Wide Web bestehen könnten. Dazu zählen sämtliche linear-konzipierte Werke und all jene Dokumente, für deren Konzeption und Rezeption es nur des Computers als Hilfsmittel bedarf. Web-Literatur hingegen ist ausschließlich auf das WWW ausgerichtet, insofern sowohl ihre Erstellung als auch ihre Nutzung allein in diesem Medium möglich ist.

Um eine Verwechslung der beiden Ausdrücke grundsätzlich auszuschließen, muß der als möglicher alternativer Terminus im Raum stehende Begriff WebLiteratur demzufolge spezifiziert werden. Ursprung und Bestehen der neu zu benennenden Kunstform basieren auf der Existenz des World Wide Web. Da diese Abhängigkeit entscheidend ist für Charakter und Dynamik des neuen Literaturtypus, sollte sie auch in der Bezeichnung zum Ausdruck kommen. An die Stelle von WebLiteratur sollte daher die präzisere Bezeichnung Webbasierte Literatur treten.

${ }^{235}$ Vgl. Zimmer, "Die Digitale Bibliothek", 285 und ders., Die Bibliothek der Zukunft, 52. 


\section{Formen webunabhängiger digitaler Literatur im WWW}

Elektronische Computer-Medien vereinen Horizonte, die bislang zwei unterschiedlichen Medienbereichen vorbehalten waren: sie kombinieren Aspekte der PrintMedien mit denen nicht-interaktiver elektronischer Medien, zu denen Hörfunk, Fernsehen und der Sektor der Audio-CDs zählen, und brechen bisher bestehende Grenzen auf. In elektronischen Publikationen lassen sich somit schwerpunktfreiere Medienpräsentationen realisieren als in gedruckten Veröffentlichungen. Dort besteht lediglich die Möglichkeit, Text mit Bildern und Graphiken zu kombinieren, da sich weder Ton noch Bewegtbilder integrieren lassen. In elektronischen Medien hingegen kann diese Dominanz des Statischen zugunsten einer Medienvielfalt und -parallelität aufgebrochen werden. Text läßt sich mit Bewegtbildern verbinden und gleichzeitig mit Ton unterlegen. ${ }^{236}$ Elektronische Computer-Medien erlauben dem Leser eine multimediale, interaktive und gelegentlich sogar kooperative Rezeption.

Historisch betrachtet war elektronisches Publizieren zunächst nicht viel mehr als die Digitalisierung gedruckter Vorlagen, so daß sich das Lesen am Bildschirm ausschließlich dadurch von der herkömmlichen Leseweise unterschied, daß es des Hilfsmittels Computer bedurfte. Diese Form des elektronischen Publizierens ist nach wie vor weit verbreitet. Durch die spätere Integration von Video- und Audiosequenzen und durch Interaktivität sowie Kooperativität steigerte sich die Akzeptanz der elektronischen Veröffentlichungen seitens der Computer-Nutzer. Alsdann etablierte sich ein System, auf das der heutige Nutzer wie selbstverständlich zurückgreift: Das Schlüsselwort für diese neuen Möglichkeiten lautet Hypertext. ${ }^{237}$ Bei Hypertexten handelt es sich um Systeme zur Verwaltung assoziativ miteinander verknüpfter informationeller Einheiten. Diese Einheiten, auch Knoten genannt, präsentieren Informationen in textueller, graphischer sowie audiovisueller Form, deren Verknüpfungspunkte untereinander jeweils als Verweise oder sogenannte Links kenntlich gemacht

236 Vgl. Schreiber, Neue Wege des Publizierens, 15.

237 Die Idee, ein Informationssystem im Hypertextformat zu gestalten, ist allerdings nicht neu: Bereits in den Jahren 1932-1933 hat sich Vannevar Bush (1890-1974) gedanklich mit einem HypertextSystem befaßt, das er 1939 zu Papier brachte. 1945 veröffentlichte er seine Ausführungen und präsentierte die theoretische Konzeption einer Maschine namens Memex (memory extender), die alle interessanten Informationen mit Hilfe einer Kamera, gleich dem menschlichen Auge, aufnehmen und dauerhaft verfügbar machen sollte. Obwohl sein System niemals in die Praxis umgesetzt wurde, gilt Bush gewissermaßen als der Ahnherr des Hypertextes. Siehe Jakob Nielsen, Multimedia and Hypertext: The Internet and beyond (Boston, 1995), 33. Vgl. ebenso Kuhlen, Hypertext, 67. 
sind. ${ }^{238}$ Der Leser kann die Verknüpfungsangebote wahrnehmen und den Links nachgehen $^{239}$, wobei er sich entweder innerhalb eines geschlossenen Hypertext-Systems bewegen kann, wie zum Beispiel bei der Nutzung einer CD-ROM ${ }^{240}$, oder aber auf ein offenes Hypertext-System wie beispielsweise das World Wide Web zugreift.

Die drei Kategorien, die sich auf dem Gebiet literarischer Werke im WWW herausgebildet haben, lassen sich folgendermaßen charakterisieren:

Erstens: Diejenigen Werke, die nicht auf einem Hypertext-System basieren, da sie entweder die digitalisierte oder digitale Version eines Druckwerkes darstellen beziehungsweise in digitaler Form erzeugt, dennoch aber linear konzipiert sind und daher als Konventionelle, linear-konzipierte Literatur im Web, kurz Literatur im Web, bezeichnet werden sollen.

Zweitens: Alle literarischen Werke, die ihrer Konzeption nach der Nutzung eines Computers bedürfen, weil sie beispielsweise durch Multimediasequenzen ergänzt sind: Sie lassen sich weder der ersten Kategorie zuordnen - da sie nicht computerunabhängig bestehen könnten beziehungsweise vor ihrer Digitalisierung bestanden haben - noch gehören sie der webbasierten Literatur an - weil ihre Existenz und Nutzung nicht auf das WWW angewiesen ist. Aus diesem Grunde sind sie der computergestützten, webunabhängigen Literatur zuzurechnen, der Kürze halber Computergestützte Literatur genannt. Dieser Begriff ist bewußt in Abgrenzung zu Bezeichnungen wie Computer-Literatur ${ }^{241}$ gewählt, um zum einen stärker hervorzuheben, daß diese zweite Literaturform abhängig vom Computer und unabhängig vom Web ist, und zum anderen den Eindruck zu vermeiden, Literatur dieser Kategorie sei computergeneriert.

238 Die Bezeichnung Hypertext kann strenggenommen nur für Systeme verwendet werden, die ausschließlich mit Textdokumenten operieren. Im folgenden soll indes die Vorgehensweise von Nielsen aufgegriffen werden, der trotz der wachsenden Verbreitung multimedialer Hypertext-Systeme keine Veranlassung sieht, einen neuen Begriff zur Abgrenzung gegenüber reinen Textversionen einzuführen. Vgl. Nielsen, ebd., 5. Der vielfach verwendete Terminus Hypermedia legt die Betonung zuungunsten des Textes zu sehr auf Medien. Die nachfolgend verwendeten Bezeichnungen implizieren demzufolge stets, daß Multimedia-Anwendungen möglich sind, es sei denn, es wird konkret von einem Text im Hypertextformat gesprochen.

239 Vgl. diesbezüglich Martin Hofmann und Lothar Simon, Problemlösung Hypertext: Grundlagen, Entwicklung, Anwendung (München, 1995), 2. Siehe ebenso Rolf Assfalg, Udo Goebels und Heinrich Welter, Internet-Datenbanken: Konzepte, Modelle, Werkzeuge (Bonn, 1998), 373. Vgl. außerdem Kuhlen, Hypertext, 13.

240 Es muß allerdings angemerkt werden, daß auch von einem geschlossenen Hypertext-System Verknüpfungen zum World Wide Web bestehen können. Das System an sich ist jedoch vorgegeben und somit für den Nutzer unveränderbar.

241 Ortmann beispielsweise bedient sich dieser Bezeichnung in Abgrenzung zu Literatur im Netz und Netz-Literatur innerhalb ihrer Kategorisierung digitaler Literatur. Sabrina Ortmann, netz literatur projekt: Entwicklung einer neuen Literaturform von 1960 bis heute (Berlin, 2001), 48. 
Drittens: Die aus Sicht der vorliegenden Arbeit wichtigste Kategorie der Werke mit Kooperationsmöglichkeit, die hier gemäß dem vorangehenden Kapitel als Webbasierte Literatur bezeichnet wird.

Zur Veranschaulichung obiger Einteilung gibt nachfolgende Darstellung die Zusammenhänge schematisch wieder:

\begin{tabular}{|c|c|c|}
\hline Ebene & Ebene 2 & Ebene 3 \\
\hline \multirow{7}{*}{ 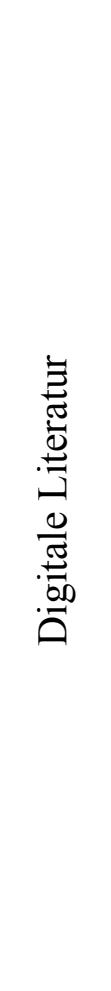 } & \multirow{3}{*}{$\begin{array}{l}\text { Konventionelle, linear- } \\
\text { konzipierte Literatur im } \\
\text { Web }\end{array}$} & $\begin{array}{l}\text { Digitalisierte Druckwerke in Datenbanken } \\
\text { und Archiven }\end{array}$ \\
\hline & & $\begin{array}{l}\text { Analoge Publikationen von Monographien, } \\
\text { Periodika und Einzelwerken }\end{array}$ \\
\hline & & $\begin{array}{l}\text { Linear-konzipierte, in digitaler Form } \\
\text { erzeugte zeitgenössische Werke }\end{array}$ \\
\hline & \multirow{3}{*}{$\begin{array}{l}\text { Computergestützte, } \\
\text { webunabhängige Literatur } \\
\text { im Web }\end{array}$} & $\begin{array}{l}\text { Programmgesteuerte Literatur ohne } \\
\text { Interaktionsmöglichkeit }\end{array}$ \\
\hline & & $\begin{array}{l}\text { Programmgesteuerte Literatur mit } \\
\text { Interaktionsmöglichkeit }\end{array}$ \\
\hline & & Geschlossene Hypertext-Systeme \\
\hline & Webbasierte Literatur & $\begin{array}{l}\text { Offene Hypertext-Systeme mit } \\
\text { Kooperationsmöglichkeit }\end{array}$ \\
\hline
\end{tabular}

Die Gliederung des im World Wide Web präsentierten literarischen Angebots in drei Kategorien mit zusätzlichen Unterkategorien zur genaueren Differenzierung eröffnet die Möglichkeit, eine relativ präzise Einteilung des vorhandenen Materials vorzunehmen. Der Spielraum innerhalb der jeweiligen Kategorien ist so groß, daß es keine Grenzfälle geben sollte.

So ist jedes literarische Werk im World Wide Web für sich genommen einem der Elemente aus Ebene 3 obiger Auflistung zuzuordnen. Neben jenen WWW-Seiten, die ausschließlich Literaturformen aus dieser Spalte anbieten, existieren jedoch auch Web-Angebote, die dem Nutzer den Zugang zu einer gemischten Sammlung von Dokumenten ermöglichen. Dort werden nicht nur Werke aus der gesamten Bandbreite digitaler Literatur präsentiert, sondern der Nutzer erhält zusätzlich Zugriff auf externe Verknüpfungen sowie gegebenenfalls die Gelegenheit zu eigener 
Mitarbeit. Diese Angebote sollen nachfolgend als Konglomerate bezeichnet und an gegebener Stelle anhand ausgewählter Beispiele skizziert werden.

\section{Konventionelle, linear-konzipierte Literatur im Web (Literatur im Web)}

Literatur im Web mit ihrer Gliederung in die drei Unterkategorien

a) digitalisierte Druckwerke in Datenbanken und Archiven, z.T. als Ergebnis von Digitalisierungsprojekten,

b) analoge Publikationen von Monographien, Periodika und Einzelwerken sowie

c) linear-konzipierte, in digitaler Form erzeugte zeitgenössische Werke

benötigt definitiv in keinem Stadium ihrer Entstehung oder ihrer Darstellung das World Wide $\mathrm{Web}^{242}$, sondern ist in der gedruckten Ausgabe inhaltlich wie darstellerisch identisch mit der Online-Version; das WWW dient hier ausschließlich zu Präsentations-, Verbreitungs- und Archivierungszwecken und liefert dem Nutzer gewisse Suchfunktionen.

\subsection{Digitalisierte Druckwerke in Datenbanken und Archiven}

Zur ersten Gruppe zählen all jene Werke, die ursprünglich ausschließlich in gedruckter Form vorlagen und für die Präsentation im World Wide Web digitalisiert wurden. Ortmann verwendet in diesem Zusammenhang die Bezeichnung "Klassiker"243; gemeint sind alle Werke, die keinen Verwertungsrechten mehr unterliegen beziehungsweise ausdrücklich für die Digitalisierung freigegeben sind. Sie stellen vermutlich noch den größten Anteil an elektronischen literarischen Dokumenten im

242 Damit distanziert sich die vorliegende Untersuchung bewußt von Ortmanns Definition von Literatur im Netz, nach der auch die Ergebnisse kreativer Literaturprojekte der Kategorie digitaler Literatur zuzurechnen sind. In ihren Ausführungen stützt sich Ortmann dabei auf ein Projekt, dessen Resultate zwar größtenteils vordefiniert sind, für dessen Entstehung und in gewissem Maße auch für dessen Umfang das WWW allerdings unabdingbar ist. Überraschenderweise zieht Ortmann sogar einen Auszug aus Charliers Laudatio zu diesem Projekt als Beweis für ihre Kategorisierung heran. Charlier bescheinigt aber gerade, daß hier "die größte Beweglichkeit des Inhalts [...] ein denkwürdiges Spiel [spielt], wie es so nur im Netz möglich ist”. Ortmann, netz literatur projekt, 50f. Nach Meid fiele diese Art der Literatur damit eindeutig dem Zweig der Web-Literatur zu, da sie zu verstehen ist als "durch das neue elektronische Medium verbreitete beziehungsweise eigens für dieses Medium geschriebene Literatur [...], die die Möglichkeiten des Mediums selbst fruchtbar macht und dadurch zu neuen Literaturformen zu gelangen sucht". Keinesfalls kann hier von Literatur im Internet beziehungsweise Literatur im Web gesprochen werden, denn auch hier liefert Meid eine eindeutige Definition, nach der "die Differenz zur traditionellen Literatur letztlich nur in der neuen Publikationsform liegt”. Meid, Sachwörterbuch zur deutschen Literatur, 252.

243 Ebd. 
WWW, lassen aber dessen gestalterische Möglichkeiten in den seltensten Fällen zum Tragen kommen. In der Regel werden die Texte nach ihrer Digitalisierung in elektronischen Archiven oder Datenbanken gesammelt, um sie der Allgemeinheit oder oftmals auch nur einem eingeschränkten Nutzerkreis schnell, zu jeder Zeit und theoretisch von jedem Ort aus zugänglich zu machen. Oftmals werden sie im Rahmen von Digitalisierungsprojekten durch geisteswissenschaftliche Institute bearbeitet, aber auch durch Verlage, Wissenschaftler oder Laien veröffentlicht, die Material allgemeinen Interesses zu einer bestimmten Literaturgattung, einer Epoche oder einem einzelnen Autor beziehungsweise einer Autorengruppe anzubieten haben. Dabei läßt sich die Tendenz feststellen, daß umfangreichere Archive oder Datenbanken größtenteils von Institutionen angeboten werden, wohingegen Einzelpersonen vorzugsweise bestimmte Autoren oder Forschungsthemen vertreten. Ob die angebotenen Web-Seiten von wissenschaftlichem Interesse sind, muß im Einzelfall entschieden werden.

In Abgrenzung zum herkömmlichen Sprachgebrauch, der den Eindruck erweckt, als seien die Übergänge zwischen Archiv und Datenbank fließend, soll hier gelten: Archive sind Sammlungen von elektronischen Texten, auf die der Nutzer direkt zugreifen kann; die Betreiber von Online-Archiven müssen die präsentierten Dokumente nicht notwendigerweise selbst erstellen, sondern können sich auf ihre Bereitstellung beschränken. Datenbanken hingegen sind Verzeichnisse von WebDokumenten, das heißt der Nutzer erhält den Zugang zu den jeweiligen Dokumenten nur indirekt über eine Verknüpfung; damit verzichten die Betreiber von Datenbanken grundsätzlich auf eine eigene Erstellung der digitalen Dokumente.

Sowohl Archive als auch Datenbanken stehen oftmals unter dem Grundsatz, das elektronisch gespeicherte Material für jeden Nutzer frei zugänglich und kopierbar zu machen. Dieses Prinzip wirkt befreiend und einengend zugleich, da das Bestreben, Dokumente allgemein und unbeschränkt zur Verfügung zu stellen, unvereinbar ist mit Urheberrecht und Kopierschutz. Online-Texte können nur dann frei zugänglich präsentiert werden, wenn das Copyright abgelaufen oder aber das Urheberrecht auf diese Texte freigegeben ist. ${ }^{244}$ Aus diesem Grunde sind viele neuere Editionen noch nicht in den elektronischen Archiven und Datenbanken zu finden.

Nachfolgend sollen für die genannten Präsentationsformen ausgewählte Beispiele vorgestellt werden, die aufgrund ihrer Konzeption und ihrer Maßstäbe rich-

244 Vgl. Vera Schäffer, Hg., Deutsche Klassiker von Andersen bis Wieland im Internet und auf CD$R O M,<$ http://www.leu.bw.schule.de/beruf/projektg/online/news4/tips/cdtip.htm>, 26.03.2003. 
tungsweisenden Charakter hatten und deshalb das Erscheinungsbild des World Wide Web in entscheidendem Maße geprägt haben; sie alle eint die Tatsache, daß sie trotz der hohen Veränderungsfrequenz des Datennetzes nunmehr seit vielen Jahren eine feste Größe im WWW darstellen. Hinzu kommen als dritte Gruppe verschiedene Beispiele von Digitalisierungsprojekten.

Zwar wäre es durchaus möglich gewesen, Digitalisierungsprojekte zusammen mit Archiven abzuhandeln, denn der einzige konzeptionelle Unterschied liegt darin, daß die Texte in Digitalisierungsprojekten per definitionem Resultate eigener Erstellung sind. Damit wäre aber der innovative Charakter der Projekte nicht angemessen zum Tragen gekommen. Alle Initiatoren zeichnen sich nämlich dadurch aus, daß sie mit ihren Projekten neue Maßstäbe setzten, indem sie etwas Neues schufen, das in dieser Form zuvor noch nicht existiert hatte oder nach besonderen wissenschaftlichen Ansprüchen in richtungweisendem Stil realisiert wurde. Daher soll im Anschluß an die Ausführungen über die Archive ein eigener Abschnitt ausschließlich für jene Digitalisierungsprojekte reserviert sein.

\subsubsection{Archive}

\subsubsection{Oxford Text Archive}

Das im Jahre 1976 gegründete Oxford Text Archive ${ }^{245}$, kurz OTA, enthält gegenwärtig mehr als 2500 elektronische Texte in verschiedensten Sprachen und zu unterschiedlichsten Themenbereichen. Die Dokumente, zu denen neben Chroniken, Genealogien und Handbüchern auch literarische Texte, unterteilt nach Autoren und Gattungen, sowie Standardnachschlagewerke, wie beispielsweise ein- beziehungsweise zweisprachige Wörterbücher, Dissertationen, Biographien, Grammatiken, Zeitungen und Periodika zählen, werden von den Mitarbeitern des OTA nicht selbst erstellt, sondern lediglich archiviert und präsentiert. Ziel ist, die Akzeptanz elektronischer Dokumente in Wissenschaftskreisen durch die Präsentation sorgfältig edierten, zitierfähigen Materials zu fördern:

Easy access to well-documented digital resources, adhering to recognized standards, is essential if they are to be accepted by the academic community as a whole. The Oxford Text Archive works to identify, collect, and preserve high-quality, well-documented electronic texts and linguistic corpora, which it then makes available to others. ${ }^{246}$

${ }^{245}$ Michael Popham, Hg., The Oxford Text Archive (12.02.2003), <http://ota.ahds.ac.uk/>, 05.03.2003.

${ }^{246}$ Ders., Hg., The Oxford Text Archive - About the Oxford Text Archive (12.02.2003), $<$ http://ota.ahds.ac.uk/>, 05.03.2003. 
Die Dokumente liegen im allgemeinen in unterschiedlichen Dateiformaten vor und sind jeweils mit bibliographischen Angaben zur Digitalisierungsvorlage sowie mit Informationen zu Größe, Format, Sprache und Verfügbarkeit der elektronischen Ressource versehen. Der Zugang zu denjenigen Texten, die nicht direkt über das WWW aufzurufen sind, kann brieflich oder per Fax in Oxford erbeten werden und ist vom Zeitpunkt der Freigabe an für einen beschränkten Zeitraum möglich.

Die Betreiber des Oxford Text Archive werden ihrem Anspruch, die Akzeptanz digitaler Ressourcen in Wissenschaftskreisen zu fördern, mit dem derzeitigen Angebot durchaus gerecht. Im Verlaufe der Zeit hat sich das OTA zu einem ebenso verläßlichen wie festen Bestandteil des WWW entwickelt. Gleichwohl wären noch Ergänzungen denkbar, die eine Steigerung der Integrität des OTA-Bestandes bewirken könnten: So wäre es wünschenswert, auf Informationen über den Zeitpunkt der Digitalisierung, den Bearbeiter und gegebenenfalls die Umstände der Bearbeitung zurückgreifen zu können.

\subsubsection{Alex Catalog of Electronic Texts}

In 1993/94 ursprünglich konzipiert als Hilfsmittel zur Online-Recherche, präsentiert Alex Catalog of Electronic Texts gegenwärtig Texte zu englischsprachiger Literatur und abendländischer Philosophie. ${ }^{247}$ Im März 2003 bestand Zugang zu 679 Büchern und kürzeren Texten, die alle als Kopie im Katalog von Alex Catalog of Electronic Texts vorlagen; der Zugriff auf die elektronischen Originaltexte, die in diversen anderen Online-Archiven abgelegt sind, ist offensichtlich durchgängig nicht mehr möglich. Damit nimmt Alex Catalog of Electronic Texts entgegen seiner eigentlichen Konzeption keine Doppelfunktion mehr als Archiv u n d Datenbank ein.

Alle Texte lassen sich zusätzlich auch in unterschiedlichen eBook-Formaten auf den eigenen Computer herunterladen. Leider sind die Dokumente nur mit unzureichenden editorischen Angaben versehen. In den meisten Fällen bleibt unklar, auf welcher Grundlage und nach welchen Maßstäben die elektronischen Dokumente erstellt wurden. Auch fehlen Informationen zu den Daten der Bearbeitung beziehungsweise eventuellen Überarbeitungen. Dennoch hat Alex Catalog of Electronic Texts in der wechselvollen Zeit seines Bestehens Wegmarken gesetzt und verdient aus diesem Grunde Erwähnung.

${ }^{247}$ Eric Lease Morgan, Hg., Alex Catalogue of Electronic Texts, $<$ http://www.infomotions.com/alex/>, 06.03.2003. 


\subsubsection{Digitalisierungsprojekte}

En effet le but d'une encyclopédie est de rassembler les connaissances éparses sur la surface de la terre; d'en exposer le système général aux hommes avec qui nous vivons, \& de la transmettre aux hommes qui viendront après nous; afin que les travaux des siècles passés n'aient pas été des travaux inutiles pour les siècles qui succéderont $[\ldots] .{ }^{248}$

Die Intention der Enzyklopädisten, die Diderot in einem Artikel seiner Encyclopédie ou Dictionnaire raisonné des sciences, des arts et des métiers Mitte des 18. Jahrhunderts darlegt, liegt in nur unwesentlich abgewandelter Form auch den modernen Initiativen zur elektronischen Sammlung von literarischen Texten zugrunde: Digitalisierungsprojekte des 20. und 21. Jahrhunderts dienen der Sammlung und allgemeinen Verbreitung von Texten zu gewissen Themengebieten, womit sie von ihrer Zielsetzung her an die bedeutendste Bibliothek der Antike erinnern, die Alexandrinische Bibliothek, die fast die gesamte bis in ihre Zeit verfaßte Literatur enthalten haben soll.

\subsubsection{Project Gutenberg}

Das im Jahre 1971 ins Leben gerufene Project Gutenberg ${ }^{249}$ entstand aus dem Grundgedanken, allen Menschen klassen- und altersübergreifend eine frei zugängliche und unbeschränkt geöffnete Bibliothek zu liefern, die jene Literatur präsentiert, die die Menschheit interessiert und bewegt. ${ }^{250}$ In den Ausführungen zu Geschichte und Philosophie des Project Gutenberg heißt es hierzu: “[...] we choose etexts we hope extremely large portions of the audience will want and use frequently." ${ }^{251}$ Diese Initiative zur elektronischen Sammlung klassischer Werke in englischer Sprache oder englischer Übersetzung wird hauptsächlich von freiwilligen Mitarbeitern aus dem WWW getragen. Nachdem in der US-amerikanischen Rechtsordnung festgelegt

248 Denis Diderot, Encyclopédie ou Dictionnaire raisonné des sciences, des arts et des métiers, par une Société de Gens de lettres III. Tome VII, (Paris, 1976), 174.

249 Michael Hart, Hg., Project Gutenberg (12.02.2003 [ $\left.\left.{ }^{1} 1971\right]\right),<$ http://gutenberg.net/>, 12.03.2003.

250 Diese Zielsetzung ist begrüßenswert, basiert aber unter anderem auf der Voraussetzung, daß der Zugang zum WWW uneingeschränkt von allen Bevölkerungsgruppen finanziert werden kann. Zwar schaffen die Anbieter elektronischer Texte theoretisch eine zu allen Tageszeiten und von allen Punkten dieser Welt aus zugängliche digitale Bibliothek, praktisch aber besteht in keiner Weise ein gleichberechtigter und einheitlicher Zugang zu dieser Bibliothek. Hier erfolgt keine Förderung der Demokratie, sondern es werden im Gegenteil neue soziale Barrieren geschaffen. Nur ein verhältnismäßig geringer Prozentsatz der bundesdeutschen Bevölkerung und ein Bruchteil der Weltpopulation besitzt einen Computer, geschweige denn einen Internet-Anschluß. Zudem ist eine Kluft zwischen den Generationen entstanden, die diejenigen Altersgruppen, die keinerlei Erfahrung im Umgang mit Computern haben, aus der Welt der neuen elektronischen Medien ausgrenzt.

251 Hart, "What is Project Gutenberg? - The Beginning of the Gutenberg Philosophy", in: Ders., Hg., Project Gutenberg (13.05.2002 [ $\left.\left.{ }^{1} 1971\right]\right),<$ http://gutenberg.net/history.html\#beginningphil>, 12.03.2002. 
worden ist, daß ein Copyright erst 50 Jahre nach Ableben des Autors erlischt, ${ }^{252}$ hat sich die Zahl der frei verfügbar werdenden Werke beträchtlich verringert. Dennoch belief sich die Zahl der erfaßten Dokumente im November 2002 auf 6267 Texte $^{253}$ mit der Aussicht, im Verlaufe des Jahres 2003 eine Gesamtzahl von 10.000 Dokumenten zu erreichen ${ }^{254}$.

Das Corpus der Sammlung gliedert sich in drei Sektionen und unterscheidet zwischen Literatur mit Unterhaltungscharakter, anspruchsvollerer Literatur und Referenzmaterial:

1) Light Literature - hierzu zählen beispielsweise Alice in Wonderland, Through the Looking-Glass, Peter Pan oder Aesop's Fables. ${ }^{255}$

2) Heavy Literature - zum Beispiel die Bibel oder andere Dokumente religiösen Inhalts, Shakespeare-Texte, Moby Dick, Paradise Lost oder ähnliche Werke.

3) References - zu dieser Gruppe gehören Rogets Thesaurus, Enzyklopädien, Wörterbücher oder Almanache.

Die Projektmitglieder erstellen die Editionen jedoch nicht nach den Grundsätzen von Verbindlichkeit und Zuverlässigkeit:

Also in the same vein, Project Gutenberg has avoided requests, demands, and
pressures to create 'authoritative editions.' We do not write for the reader who
cares whether a certain phrase in Shakespeare has a ':' or a ';' between its clauses.
We put our sights on a goal to release etexts that are $99.9 \%$ accurate in the eyes of
the general reader.

Dieser Tatsache muß sich jeder Nutzer bewußt sein: das präsentierte Material ist nicht zitierfähig und somit für den wissenschaftlichen Gebrauch nicht verwendbar. Da das Projekt jedoch nunmehr über einen Zeitraum von mehr als 30 Jahren geführt und kontinuierlich ausgebaut worden ist und sein Herausgeber Hart damit gewisser-

252 Ebd., 12.03.2002.

253 Ders., "Welcome to Project Gutenberg", in: Ders., Hg., Project Gutenberg (12.02.2003 [ $\left.\left.{ }^{1} 1971\right]\right)$,

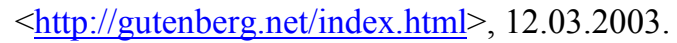

254 David Moynihan, "Distributed Proofing site goes through the roof: One Page Per Day", the inquirer, 11.11.2002, < http://www.theinquirer.net/?article=6167> $>$ 12.03.2003.

${ }^{255}$ Die Zuordnung zur ersten Sektion wird nach Hart vorrangig durch den Wunsch bestimmt, alle Altersgruppen gleichermaßen an den Gebrauch des Computers heranzuführen. Es schaffe stets ein befriedigendes Gefühl zu hören, daß Kinder oder Großeltern durch die Filmversion eines literarischen Werkes an die elektronische Textversion herangeführt worden seien. Hart, "About Project Gutenberg? - The Selection of Project Gutenberg Etexts", in: Ders., Hg., Project Gutenberg (28.10.2003 [ $\left.\left.{ }^{1} 1971\right]\right),<$ http://www.gutenberg.net/about.shtml $>, 13.02 .2004$.

256 Ders., "What is Project Gutenberg?", < $\underline{\text { http://gutenberg.net/history.html\#beginningphil> }} 26.03$. 2003. 
maßen als Pionier auf diesem Gebiet bezeichnet werden kann, verdient Project Gutenberg unbedingt Erwähnung.

\subsubsection{Bartleby.com: Great Books Online}

Im Gegensatz zum Project Gutenberg sehr sorgfältig ediert sind die Texte bei Bartleby.com: Great Books Online ${ }^{257}$. Gegründet im Jahre 1993, veröffentlichte das zunächst noch unter dem Titel Project Bartleby Archive laufende Forschungsprojekt nach einem Jahr Laufzeit Whitman's Leaves of Grass als erstes klassisches Buch im WWW. ${ }^{258}$ Aufgrund der Qualität des präsentierten Materials sowie dessen kontinuierlich wachsenden Umfangs konnte sich das Archiv zu einer festen und zuverlässigen Referenzquelle im Web etablieren. Gemäß seinen Prinzipien gewährleistet es nach wie vor den freien öffentlichen Zugang zu Erzählprosa und Versdichtung, stets präsentiert nach dem jeweils aktuellen Stand der Technik:

Our ever-expanding list of great books - currently thousands of works by hundreds of authors - provides millions of students, educators and the intellectually curious with unparalleled access to online reference books and classics and forms the preeminent electronic publishing enterprise of the twenty-first century. ${ }^{259}$

Aufgrund seiner sorgfältig bearbeiteten elektronischen Editionen empfiehlt es sich in jeder Hinsicht für die wissenschaftliche Nutzung: Die elektronischen Texte entsprechen genau dem gedruckten Original und sind durch ausführliche bibliographische Angaben zu Titel, Verfasser, Erscheinungsort, Verlag, Erscheinungsjahr und Auflage ergänzt. Oftmals sind die Seiten mit Autorenporträts oder Abbildungen, handschriftlichen Anmerkungen oder Textauszügen aus den Originaltexten illustriert, bei einigen Autoren werden zusätzlich ein Titelindex und ein Verzeichnis mit alphabetischer Auflistung der ersten Zeilen aller Werke dieser Autoren angeboten. ${ }^{260}$ Die Literatur wird sorgfältig und gezielt im Sinne der Autoren, der Nutzer und der Literatur selbst ausgewählt.

${ }^{257}$ Steven H. Van Leeuwen, Hg., Bartleby.com: Great Books Online ([ ${ }^{1}$ Dez. 1995]), $<$ http://www.bartleby.com/>, 26.03.2003.

258 Ders., Hg., Welcome to Bartleby.com: Great Books Online ([ ${ }^{1}$ Dez. 1995]), $<$ http://www.bartleby.com/sv/welcome.html $>$, 26.03.2003.

259 Ders., Hg., Bartleby.com: Great Books Online: Mission Statement ([ ${ }^{1}$ Dez. 1995]), $<$ http://www.bartleby.com/press/profile.html $>, 26.03 .2003$.

${ }^{260} \mathrm{Zu}$ kritisieren wäre dieses nützliche und gut gestaltete Archiv nur insofern, als die Illustrationen leider in den seltensten Fällen erläutert werden. 
Bartleby.com: Great Books Online ist jedoch bemerkenswert nicht allein wegen seines hochwertigen und umfangreichen Textmaterials; in Verbindung mit seinem weiteren reichhaltigen Angebot an Sachbüchern und Nachschlagewerken, wie beispielsweise Enzyklopädien, Thesauri, Zitatensammlungen oder Wörterbüchern, präsentiert es sich dem Nutzer als eine herausragende und innovative Informationsquelle.

\subsubsection{Victorian Women Writers Project}

Das im Jahre 1995 gegründete Victorian Women Writers Project ${ }^{261}$ der Universitätsbibliothek von Indiana verdient Aufmerksamkeit aufgrund seiner besonderen Thematik: Hier werden Romane, Werke aus Versdichtung und -drama, politische und religiöse Schriften sowie Kinderbücher vergleichsweise wenig beachteter Schriftstellerinnen des Viktorianischen Zeitalters in den Vordergrund gerückt. ${ }^{262}$

Alle Dokumente sind sehr sorgfältig im Hinblick auf Vollständigkeit und Übereinstimmung mit dem Original ediert und weisen alle notwendigen bibliographischen Angaben wie Titel, Verfasser, Erscheinungsort, Verlag sowie Erscheinungsjahr auf. Darüber hinaus geben sie genauen Aufschluß darüber, aus welcher Quelle das Dokument von welcher Person zu welchem Zeitpunkt in die digitalisierte Form umgesetzt wurde, nach welchen Regeln die Digitalisierung erfolgte und welche Veränderungen mit ihr einhergingen. Die Gestaltung der Dokumente basiert auf der ursprünglichen Seitenaufteilung der Texte, das heißt Seitenumbrüche im Originaltext werden innerhalb des fortlaufenden elektronischen Dokuments deutlich mit Seitenzahlen gekennzeichnet und optisch hervorgehoben. Eingescannte Bilder einiger Autorinnen sowie vereinzelter Buchseiten ergänzen das Archivangebot.

${ }^{261}$ Perry Willett, Hg., Victorian Women Writers Project (19.01.2001 [ $\left.\left.{ }^{1} 1995\right]\right)$, $<$ http://www.indiana.edu/ letrs/vwwp > 29.03.2003.

262 Das Archivangebot ist so konzipiert, daß vor allem Dokumente jener Autorinnen Verbreitung finden, die zu Lebzeiten Popularität genossen, heute hingegen zu den weniger bekannten Verfasserinnen zählen, wie zum Beispiel Marie Corelli mit The Mighty Atom, Flora Annie Webster Steel mit The Potter's Thumb oder Augusta Webster mit Daffodil and the Croäxaxicans. Im selben Zuge dient das Projekt der Erhaltung vom Zerfall bedrohter Bestände. In seinem Artikel über Inhalt und Ziele des Victorian Women Writers Project erläutert Willett die Digitalisierungsgründe: "The breadth of writing by women in this period seems staggering, and much of it was either printed in limited press runs, not collected by libraries, or has disintegrated along with so many other works from the late nineteenth century (many of which were printed on acid-based paper)." Perry Willett, "The Victorian Women Writers Project: The Library as a Creator and Publisher of Electronic Texts", The Public-Access Computer Systems Review 7 (1996), $<$ http://info.lib.uh.edu/pr/v7/n6/will7n6.html>, 29.03.2003. 
Hier erweist sich das World Wide Web einmal mehr als sinnvoller Datenträger, da durch die Online-Präsentation unbekanntes und schwer zugängliches Material weltweite Verbreitung und wissenschaftliche Nutzung finden kann.

\subsubsection{Datenbanken}

\subsubsection{Banned Books On-Line}

Einem umstrittenen Thema widmet sich der Herausgeber der Web-Seiten Banned Books On-Line ${ }^{263}$. In Kooperation mit anderen Gleichgesinnten wendet er sich im Sinne der Kampagne Free Speech Online - Blue Ribbon Campaign gegen die Zensur elektronischer Bücher durch öffentliche Bibliotheken. In einer repräsentativen Auswahl werden jene Werke vorgestellt, die zu irgendeinem Zeitpunkt der Zensur oder Zensurbestrebungen unterlagen. Zu diesen Texten zählen beispielsweise Ulysses von Joyce, Canterbury Tales von Chaucer, Moll Flanders von Defoe, Leaves of Grass von Whitman, Twelfth Night und The Merchant of Venice von Shakespeare oder Tom Sawyer und Huckleberry Finn von Twain. Die zu allen aufgeführten Werken mitgelieferten kurzen Hintergrundinformationen geben jeweils Aufschluß darüber, warum ein Werk als anstößig angesehen wurde; da diese Ausführungen sehr knapp gehalten sind, bleiben indes einige Fragen offen. Als vergleichsweise ausführlich erscheinen hingegen die Informationen zur Banned Books On-Line-Initiative oder ähnlichen Kampagnen sowie die Literaturhinweise zum Thema Zensur. Leider bleibt hierbei eine Option unrealisiert, nämlich eine Textaufbereitung für das Thema Zensur, bei der die als anstößig erachteten Passagen besonders hervorgehoben sind. Da es sich hier allerdings um eine Datenbank handelt - die Werke selbst sind auf diversen Servern archiviert und nur über eine Verknüpfung zugänglich -, ist diese Funktion vermutlich nicht umzusetzen.

$\mathrm{Ob}$ es Web-Nutzern freigestellt sein sollte, Anstoß erregende Dokumente mittels spezieller Software-Programme herauszufiltern, oder ob die Publikation bestimmter Dokumente generell verboten werden sollte - die Seiten von Banned Books On-Line geben Anstoß zur Auseinandersetzung mit der noch immer ungeklärten Frage des Ob und Wie einer Zensur von Publikationen im World Wide Web.

263 John Mark Ockerbloom, Hg., The Online Books Page - Banned Books On-Line ([ $\left.\left.{ }^{1} 1993\right]\right)$, $<$ http://digital.library.upenn.edu/books/banned-books.html $>, 07.03 .2003$. 


\subsubsection{Prize-Winning Books On-Line}

Vom Herausgeber der Banned Books On-Line stammt auch die Auswahl an online zur Verfügung stehenden elektronischen Versionen jener Bücher, die mit bedeutenden Literaturpreisen bedacht wurden, wie beispielsweise dem Newbery Award für herausragende Kinderbücher, dem Literaturnobelpreis für Werke mit außergewöhnlich idealistischem Grundgedanken oder dem Pulitzer-Preis für amerikanische Literatur in Lyrik, Drama, Prosa, Biographie und Geschichte. ${ }^{264}$ Die Veröffentlichung dieser Werke setzt grundsätzlich voraus, daß sie entweder nicht mehr dem Urheberrecht unterliegen oder per Genehmigung freigegeben sind, weshalb die Liste der mit dem Nobelpreis ausgezeichneten Werke nicht über das Jahr 1953 hinausgeht und die Aufstellungen in den Kategorien Pulitzer-Preis und Newbery Award bereits in 1923 beziehungsweise in 1924 enden. ${ }^{265}$ Zumindest für die wissenschaftliche Nutzung wäre hier eine lückenlose Version wünschenswert. Gegebenenfalls müßte diese kostenpflichtig angeboten werden, auch wenn es dem idealistischen Grundgedanken vieler Herausgeber im WWW widersprechen mag, den Zugang zu Online-Publikationen zu beschränken. Neben dem inhaltlichen Wert rechtfertigt aber gerade die Unvollständigkeit der Auflistungen die Erwähnung von Prize-Winning Books OnLine; führt sie doch vor Augen, daß die Frage einer Anpassung des Urheberrechts an die Gegebenheiten des WWW und der Regelung des Zugangs zu Online-Dokumenten dringend einer einheitlichen Klärung bedarf.

\subsection{Analoge Publikationen von Monographien, Periodika und Einzel- werken}

Zur zweiten Gruppe der Literatur im Web zählen jene Veröffentlichungen, die im WWW als analoge Publikationen, das heißt entweder als Vorabversion, als Parallelpublikationen oder aber als Folgeversion eines Druckwerkes auftreten. Sie entwickeln als Spiegel der Papierausgaben keine eigene Dynamik im Web, sondern ermöglichen dem Nutzer a) einen vorzeitigen Zugriff im Falle einer Vorabversion, b) die Möglichkeit schnellen, effektiven Recherchierens durch spezielle Such-

\footnotetext{
${ }^{264}$ Ders., Hg., The Online Books Page - Prize-Winning Books On-Line ([ $\left.\left.{ }^{1} 1993\right]\right)$, $<$ http://digital.library.upenn.edu/books/prize.html $>$, 07.03.2003.

265 An dieser Stelle sei betont, daß der Herausgeber von Banned Books On-Line von der Entscheidung hinsichtlich der Freigabe eines Werkes für eine Veröffentlichung im World Wide Web nur indirekt betroffen ist, insofern er in seiner Datenbank selber keine Dokumente archiviert, sondern dem Nutzer nur eine Verknüpfung zu ihnen anbietet.
} 
funktionen ${ }^{266}$ sowie c) - im Falle von Periodika - gegebenenfalls den Zugriff auf ein Archiv vorangegangener Ausgaben. Das Online-Angebot analoger Zeitschriften ist allerdings nicht immer uneingeschränkt und kostenfrei zugänglich. Ein Beispiel ist Connotations - A Journal for Critical Debate, eine parallel publizierte internationale Fachzeitschrift zur Förderung der kritischen wissenschaftlichen Diskussion auf dem Gebiet der englischen Literatur vom Mittelenglischen bis zur Gegenwart. Der Nutzer hat hier lediglich Zugriff auf eine Auswahl der Beiträge; zur uneingeschränkten Nutzung bedarf es eines Abonnements. ${ }^{267}$

Auch wenn eine Zugangsbeschränkung einen schwerwiegenden Nachteil für den Nutzer darstellt, ist die parallele Publikation von gedruckten und digitalen Dokumentversionen dennoch als zukunftsweisend anzusehen. Gerade mit Blick auf einen möglichst regen wissenschaftlichen Austausch stellt das World Wide Web einen außerordentlichen Fortschritt dar. Die Vorteile liegen unter anderem in der kostengünstigen und vor allem raschen allgemeinen Verbreitung von Forschungspublikationen, die Verlegern, Leserschaft und Autoren gleichermaßen zugute kommt. ${ }^{268}$ In seiner Beitragsfolge für Gegner und Befürworter der elektronischen Netzwerke zeichnet Zimmer den langen und zähen Weg einer wissenschaftlichen Arbeit von ihrer Entstehung bis zur Publikation in einer gedruckten Zeitschrift nach. Danach veranschlagt er für den Veröffentlichungsprozeß eine Dauer von 3 bis 36 Monaten,

${ }^{266}$ Die Vorteilhaftigkeit der Suchfunktionen erweist sich zum Beispiel bei der gezielten Suche nach speziellen Themen oder auch einzelnen Begriffen. Suchprogramme erlauben, innerhalb kürzester Zeit bestimmte Daten zusammenzustellen und anschließend zu speichern oder auf Papier auszudrucken. Auf diese Weise lassen sich Recherchen in Datenbanken durchführen oder Texte nach bestimmten Begriffen durchsuchen. Dies eröffnet beispielsweise völlig neue Perspektiven im Hinblick auf Untersuchungen zu Worthäufigkeiten oder zur Frequenz morphologischer Elemente, wie zum Beispiel Präfixen oder Suffixen.

${ }^{267}$ Inge Leimberg und Matthias Bauer, Hg., Connotations - A Journal for Critical Debate, $<$ http://www.connotations.de/>, 22.05.2003. Parallelpublikationen: Connotations - A Journal for Critical Debate 1 (1991)-11 (2002). Connotations mag nicht nur als Beispiel für Zugriffsbeschränkungen bei Periodika dienen, sondern auch für eine Eins-zu-eins-Übernahme der Seitenangaben. Um eine korrekte Zitierung der Aufsätze zu gewährleisten und zudem sicherzustellen, daß die bibliographischen Angaben zu den Online-Ausgaben deckungsgleich sind mit den Bezugnahmen auf die gedruckten Ausgaben von Connotations, sind alle Online-Texte mit den Seitenzahlen der Print-Versionen versehen.

${ }^{268}$ So können die Autoren sicher sein, daß die Ergebnisse ihrer Arbeit auf schnellstmöglichem Wege an die Öffentlichkeit gelangen; für die Verlage wiederum entfällt der kostspielige Marketing-Prozeß - ihre Einnahmen sind ihnen auch im Internet durch Lizenzierung ihrer Zeitschriften garantiert; die Leserschaft schließlich erhält schnellen und direkten Zugriff auf die neuesten Forschungsergebnisse. In zeitschrifteninternen Kommunikationsforen besteht zudem oftmals die Möglichkeit zu Nachfragen, zu spontanen Meinungsäußerungen und zum Gedankenaustausch mit Gleichgesinnten. 
was die Aktualität einer Arbeit deutlich verringern kann und zudem noch hohe Verlags- und Versandkosten verursacht. ${ }^{269}$

Bei den Peer-reviewed electronic journals liegt eine gewisse Verzögerung des Publikationsvorganges gleichsam in der Natur der Sache. Diese Zeitschriften, die ein Auswahlverfahren durchlaufen, können im akademischen Bereich am ehesten den Anspruch auf Wissenschaftlichkeit erheben. Obwohl der Prüfungsprozeß keine Garantie für inhaltliches Niveau darstellen kann, bietet er dem Leser doch einen gewissen Schutz vor unfundierten oder unwissenschaftlichen Ausführungen. Die Verleger vorgenannter Zeitschriften gewährleisten einen Mindest-Qualitätsstandard, indem sie alle Artikel vor ihrer Veröffentlichung durch einen Gutachterstab prüfen lassen. Dieser Vorgang der peer review, das heißt der Prüfung durch Gleichrangige, wird anonym ${ }^{270}$ von einem Gremium durchgeführt, das dem jeweiligen Fachgebiet entsprechend ausgewählt wird. ${ }^{271}$

Während sich die Peer-reviewed electronic journals ohne weiteres für den (geistes-)wissenschaftlichem Gebrauch empfehlen, setzt die Nutzung anderer elektronischer Zeitschriften ${ }^{272}$ eine kritische Prüfung voraus. So erheben E-zines gemäß

269 Zimmer, "Die digitale Bibliothek", 279f. Allerdings stellen die Veränderbarkeit und Kopierbarkeit der Dokumente nach wie vor eine Publikationshürde dar. Die Ungewißheit, ob die eigenen Forschungsergebnisse auch nach einigen Jahren noch unverändert an zuverlässiger Stelle vorzufinden sind, läßt viele Wissenschaftler gedruckten Zeitschriften den Vorteil geben. Eine Ausnahme bilden - siehe oben - analoge Publikationen. Hier gewährleistet das zusätzliche Erscheinen in gedruckter Form gewissermaßen die dauerhafte Beurkundung der Forschungsarbeit. Vgl. ebd, 282.

270 Man spricht in diesem Falle von blind peer review. Die Identität des Autors bleibt dem Gutachter unbekannt; umgekehrt wird dessen Name nicht an den Autor weitergegeben. Auf diese Weise soll eine vorurteilsfreie Bewertung durch den Gutachter gewährleistet werden, der seinerseits vor einer möglichen Einflußnahme der Autoren geschützt bleiben soll. Siehe hierzu auch Zimmer, Die Bibliothek der Zukunft, 78.

271 Obwohl Online-Periodika einen nicht unerheblichen Anteil der Informationsträger im WWW darstellen, sollen sie im weiteren Verlauf vorliegender Arbeit keine exponierte Stellung einnehmen. Dies liegt darin begründet, daß der thematische Schwerpunkt der Untersuchung in erster Linie auf den Charakteristika webbasierter Literatur liegt und nicht auf den Träger- beziehungsweise Präsentationsmedien.

272 Die Bezeichnung Elektronische Zeitschrift wird oftmals für die Gesamtheit aller elektronischen Periodika sowohl im World Wide Web als auch auf anderen elektronischen Datenträgern verwendet. Diese breite Auslegung des Begriffes findet sich beispielsweise bei Möbius, der sowohl E-zines als auch Electronic Newsletters, E-journals und Peer-reviewed electronic journals unter der Bezeichnung Elektronische Zeitschrift zusammenfaßt. Eine Unterteilung nimmt Möbius nur insofern vor, als er all jene Periodika, die mittels eines beliebigen elektronischen Mediums (CDROM, Magnetband, WWW oder ähnliches) verbreitet werden, als Elektronische Zeitschriften im weiteren Sinne von Elektronischen Zeitschriften im engeren Sinne abgrenzt, worunter Möbius ausschließlich jene Zeitschriften versteht, die über das Netz vertrieben werden. Michael Uwe Möbius, "Elektronische Zeitschriften über Internet", in: Jüngling, Hg., Internet und Bibliotheken, 136-169, hier: 139. 
einer Einteilung von Möbius ${ }^{273}$ offensichtlich keinen wissenschaftlichen Anspruch und widmen sich oftmals bizarren, esoterischen oder auch ausgesprochen fragwürdigen Themen. Je nach inhaltlicher Ausrichtung des Periodikums kann die Bezeichnung zine entweder für fanzine oder magazine stehen. Electronic Newsletters hingegen widmen sich teilweise auch wissenschaftlichen Themen; sie enthalten aber hauptsächlich Kurzinformationen, aktuelle Nachrichten und Reviews. Electronic Journals (E-journals) schließlich dienen der Förderung bestimmter Fachgebiete durch die Veröffentlichung fachbezogener Artikel und Forschungsberichte; vom Themenspektrum her stehen sie auf gleicher Stufe wie die Peer-reviewed electronic journals, mit dem Unterschied, daß sie eben nicht dem beschriebenen Prüfungsvorgang unterliegen.

\subsection{Linear-konzipierte, in digitaler Form erzeugte zeitgenössische Werke}

Zur dritten Gruppe zählen zeitgenössische Werke, die in ihrer linearen Konzeption das WWW nur als Publikations- und Verbreitungsmedium nutzen, bei denen also keinerlei Veränderungs-, Interaktivitäts- oder Verknüpfungsmöglichkeiten bestehen. ${ }^{274}$ Der Leser hat hier nur Zugang zu jeweils einer Text- oder Text-Bild-Ebene. Gelegentlich liefern die Autoren bereits in der Entstehungsphase gewisse Zusatzinformationen, die normalerweise erst nach Abschluß eines Werkes zugänglich zu sein pflegen; diese sind jedoch nicht vom jeweiligen Werk heraus abrufbar, das heißt die Werke selbst könnten in ihrer Form grundsätzlich auch unabhängig vom WWW existieren.

Im Falle der Kurzgeschichte Double Crossings ${ }^{275}$ von Lee White beispielsweise liegt das Dokument im WWW als abgeschlossener Fließtext vor, der dem Leser in dieser linearen Konzeption keinerlei Nutzungsvorteile gegenüber gedruckten Publikationen eröffnet.

Die Geschichte zeichnet den Leidensweg deutscher Auswanderer nach und präsentiert sich in ihrer Mischung aus Historiendarstellung und Gefühlsbeschreibung

273 Die nachfolgende Unterscheidung basiert auf der Einteilung von Möbius, der sich bei seiner Kategorisierung englischer Begriffe bedient, da diese seiner Ansicht nach internationaler und geläufiger sind als deutsche Bezeichnungen. Möbius, "Elektronische Zeitschriften über Internet", 139f.

274 In vereinzelten Fällen nutzen die Autoren das WWW gleichzeitig als Vertriebsmedium und geben ihre Werke ausschließlich gegen Entrichtung einer Gebühr zur Lektüre frei.

275 Lee White, "Double Crossings", in: Gutenberg Litegraphic Society, Hg., Pictures and Stories - The Online Community of Creative Minds, $<\mathrm{http}$ //www.picturesandstories.org/doublecrossings.html $>$, 02.06.2003. 
als eine ebenso schlüssige und überzeugende wie auch ergreifende Erzählung, die auf Autorenseite eine tiefe Leidenschaft für die Menschen und ihre Schicksale erkennen läßt.

Double Crossings erstreckt sich über zwölf relativ kurze Kapitel, zuzüglich eines Prologs und eines Epilogs. Der Leser erfährt vom Schicksal des deutschen Jungen Frederich Jacob Walter, der im Alter von sieben Jahren mit seinen Eltern aus seinem Heimatdorf nach Amerika auswandert. Die Schiffsreise, die sich scheinbar unendlich in die Länge zieht und die Familie an den Rand ihrer knappen finanziellen Möglichkeiten bringt, erweist sich angesichts drangvoller Enge an Bord und erbärmlicher hygienischer Verhältnisse als zu kräftezehrend für die Eltern, so daß Frederich schließlich als mittelloser Waisenjunge in Amerika ankommt. Dort tritt er in den Dienst eines Kaufmannes, um die Kosten der Schiffspassage abzuarbeiten. Viel später erfährt Frederich durch Zufall, daß sich der grausame und gefürchtete Kapitän an den zahlreichen Todesfällen während der Schiffspassage auf schamlose Art und Weise bereichert hat. ${ }^{276}$

Die Veröffentlichung dieser Kurzgeschichte erfolgte in Ausgabe 2 (2003) der elektronischen Zeitschrift Pictures and Stories - Ezine of Literature \& Art. ${ }^{277}$ Herausgeber ist die gemeinnützige Gutenberg Litegraphic Society, die es sich zum Ziel gesetzt hat, mit ihrer Initiative Pictures and Stories - The Online Community of Creative $M_{i n d s^{278}}$ im World Wide Web ein ausgewähltes Forum zur Förderung, Präsentation und Wahrung künstlerischer Kreativität zu etablieren. Die Herausgeber verhelfen Künstlern nicht nur zur Publikation ihrer Werke, die einer der zehn Untergruppen der Kategorien Pictures und Stories zuzurechnen sein müssen ${ }^{279}$, sondern bieten darüber hinaus Gelegenheit zu Gemeinschaftsprojekten sowie Informationen und Links zu anderen künstlerischen oder kunstbezogenen Institutionen. Regelmäßig werden Wettbewerbe ausgeschrieben, über deren Ausgang entweder die Preisrichter der Gutenberg Litegraphic Society oder der Forumssponsoren entscheiden beziehungsweise per Sta-

${ }^{276}$ Auf dieses falsche Spiel des Kapitäns (engl. double-crossing).; darüber hinaus nimmt der Titel der Kurzgeschichte vermutlich ebenso bezug wie auf den Übertritt in eine neue Welt, der zugleich einen Scheideweg, das heißt eine Kreuzung im Leben der Familie darstellt (engl. crossing).

277 White, "Double Crossings", Pictures and Stories - Ezine of Literature \& Art 2 (2003), $<$ http://www.picturesandstories.org/vol2issue2.html > , 02.06.2003.

278 The Gutenberg Litegraphic Society, Hg., Pictures and Stories - The Online Community of Creative Minds, $<\underline{\mathrm{http}}: / / \mathrm{www}$.picturesandstories.org/>, 02.06.2003.

279 Die Kategorien umfassen folgende Themenbereiche: Fiction, Poetry, Essay, Biographical Story, Script, Picture-Story, Color Photography, Black \& White Photography, Traditional Art, Computer-Generated Art. The Gutenberg Litegraphic Society, Hg., Pictures and Stories: Submission Guidelines ([12002]), <http://www.picturesandstories.org/submission.html> , 02.06.2003. 
tistik die höchsten Zugriffszahlen den Ausschlag geben. Double Crossings beispielsweise wurde im Rahmen der Literature \& Art Open Competition des Winters 2003 in der Kategorie Fiction mit dem ersten Preis ausgezeichnet. ${ }^{280}$

Alle Werke sind lediglich mit Namen und Wohnorten der jeweiligen Künstler versehen, weisen jedoch keine Entstehungsdaten auf. Auch erhält der Rezipient zumindest in der Kategorie Literature - keinerlei Hintergrundinformationen zu den Werken oder den Motivationen der Schöpfer.

\subsection{Drucktexte im Web - cui bono?}

Wie die aufgeführten Beispiele zeigen, zeichnen sich digitalisierte Drucktexte in erster Linie durch ihre globale Verbreitung aus. Sie gestattet den jederzeitigen Zugriff vom heimischen Rechner aus und ist infolgedessen gleichbedeutend mit hoher Zeit- und Kostenersparnis. Diese Art der Verfügbarkeit ist aber auch vom wissenschaftlichen Standpunkt aus zu befürworten und zu fördern. Zum einen können im World Wide Web auch jene Materialien präsentiert werden, die nur in wenigen Bibliotheken vorhanden sind oder $\mathrm{zu}$ denen der Zugang ungeachtet ihrer hohen wissenschaftlichen Relevanz aus Konservierungsgründen nicht zu verantworten wäre; zum anderen können auch jene Themenbereiche ein Forum für Präsentation, Verbreitung und wissenschaftlichen Gedankenaustausch erhalten, die normalerweise nur im Fokus einer kleinen Interessengruppe stehen. Ferner lassen sich den Werken alle denkbaren Zusatzinformationen beifügen, so daß im Laufe der Zeit elektronische Spezialausgaben entstehen können, die eine Zuhilfenahme weiterer Sekundärliteratur erübrigen.

Nachstehend eine Auflistung an Materialien und Dienstleistungen, die allgemein wünschenswert wären und daher eine Digitalisierung von Drucktexten zusätzlich rechtfertigen:

- Digitalisierte Originaltexte

- Bibliographien

- Kommentierte zeitgenössische Illustrationen

- Literatur- und zeitgeschichtliche Ausführungen

- Biographien

${ }^{280}$ Dies., Hg., Pictures and Stories: Awards, <http://www.picturesandstories.org/awards.html $>$, 02.06.2003. 
- Glossare

- Rezensionen, ggf. als Text- und Wortbeiträge

- Editionsgeschichtliche Informationen

- Sequenzen aus Werkverfilmungen

- Vorlesefunktionen für Sehgeschädigte

- Garantien für regelmäßige Nachbearbeitungen

- Individuell einstellbare Seiten- beziehungsweise Textgrößen für Sehgeschädigte

- Kopier- und Druckfunktionen für Illustrationen, Abschnitte, Kapitel und Gesamttexte

- Suchmaschinen

- Verknüpfungen zu themennahen Web-Seiten

Allerdings werden derzeit nur wenige dieser Dienste tatsächlich angeboten; andererseits wird ein nicht unerheblicher Teil der aufgeführten Leistungen bereits von PrintPublikationen gewährleistet, die sich im allgemeinen außerdem wesentlich übersichtlicher und augenschonender darstellen.

Werden 'lineare' Texte delinearisiert, das heißt mit zusätzlichen, aus den Texten heraus aufrufbaren Verknüpfungen versehen, so gehören sie nicht mehr zur Literatur im Web, sondern zählen schon zur Kategorie der Computerliteratur; eine alle Informationsangebote ausschöpfende Rezeption ist nur unter Zuhilfenahme eines Computers möglich. Der Nutzer befindet sich in einem Hypertextsystem, innerhalb dessen er per Links zwischen den einzelnen Informationseinheiten wechseln kann. Im Falle der Literatur im Web hingegen befindet sich der Nutzer stets nur auf jeweils einer Ebene; die einzelnen Informationseinheiten sind in sich abgeschlossen und weisen keine Verknüpfungen auf.

\section{Computergestützte, webunabhängige Literatur im Web (Computergestützte Literatur im Web)}

Diese Gattung der im Web präsentierten Literatur zeichnet sich dadurch aus, daß sie auch unabhängig vom WWW ausschließlich mit Hilfe eines Computers existieren und rezipiert werden könnte. Sie läßt sich in drei Gruppen einteilen, deren Verschiedenheiten sich an dem Grad der jeweiligen Interaktionsmöglichkeiten festmachen lassen: 
So kann sich der Unterschied zu Print-Literatur ausschließlich auf den Aspekt der Multimedialität ohne Eingriffsmöglichkeit seitens der Nutzer beschränken, wie es bei Darstellungen der Fall ist, die in der Art eines Kurzfilms in unausgesetzter Folge vor dem Auge des Betrachters ablaufen und keine Interaktions- oder Kooperationsmöglichkeiten bieten.

Die Besonderheit kann sich aber auch in einer eingeschränkten Interaktivität manifestieren, bei der der Nutzer mittels Eingabe von Stichworten ein Programm in Gang setzt, das nach den Vorgaben seines Programmierers ein literarisches Werk auf der Basis der eingegebenen Wörter erstellt.

Beiden Literaturformen fehlt es an einem System miteinander verknüpfter Informationseinheiten, weshalb sie - zwecks deutlicher Abgrenzung zur dritten Gruppe - der computergesteuerten Literatur ohne beziehungsweise mit Interaktionsmöglichkeiten zugeordnet werden sollen (siehe nachfolgend 2.1. beziehungsweise 2.2.).

Die dritte Gruppe computergestützter, webunabhängiger Literatur im Web nämlich präsentiert sich als geschlossener Hypertext. Die Eigenschaft der Geschlossenheit ist gleichbedeutend mit Unabhängigkeit von anderen WWW-Dokumenten; die Existenz und volle Funktionsfähigkeit eines solchen HypertextSystems wäre auch offline, das heißt ausschließlich mit Hilfe eines Computers gegeben, da aus dem Hypertext-System heraus keine Verknüpfungen zu systemexternen Dokumenten angeboten werden. Die Interaktionsfreiheit des Rezipienten liegt also ausschließlich innerhalb der Grenzen des jeweiligen Hypertext-Systems (siehe nachfolgend 2.3.).

\subsection{Programmgesteuerte Literatur ohne Interaktionsmöglichkeiten - Dargestellt am Beispiel von "easter offering"}

Das für diese Form der Literatur ausgewählte Beispiel vereinbart sowohl optische als auch inhaltliche Aussagekraft und ist auf den Web-Seiten des $W r$-eye- tings Scratchpad ${ }^{281}$ ausgestellt. Dieses dient seit seiner Gründung im Februar 1997 der Präsentation visueller und medienübergreifender literarischer Werke, wobei es weniger als Museum denn als eine Art Labor begriffen werden soll. Der Titel verkündet das Programm: $W r$-eye-tings Scratchpad - ein Notizblock für visuelle Literatur; ein

\footnotetext{
${ }^{281}$ Luigi-Bob Drake, $W r$-eye- tings Scratchpad (21.05.1999 [ ${ }^{1}$ Februar 1997]), $<$ http://www.burningpress.org/wreyeting/>, 01.04.2003.
} 
Wortspiel, das sich in ähnlich markanter Weise unter Eingrenzung auf den Bereich Lyrik etwa folgendermaßen in die deutsche Sprache übertragen ließe: Notizblock für Poe-sieh!

Das Werk, das an dieser Stelle vorgestellt werden soll, stammt von Komninos Zervos und trägt den Titel "easter offering". ${ }^{282}$ Es besteht aus sechs bunten Buchstaben, die anfänglich das Wort easter darstellen und sich innerhalb einer Art Filmsequenz von ca. 10 Sekunden Dauer verändern und fünfmal in anderer Reihenfolge zusammengesetzt werden, so daß insgesamt sieben verschiedenartige Bilder beziehungsweise fünf unterschiedliche Worte entstehen. Ziel des Künstlers ist die Verdeutlichung der diversen Auffassungen, die mit dem Osterfest verbunden sind: In christlichem Sinne bedeutet Ostern das Fest der Auferstehung; diese religiöse Bedeutung spiegelt sich in dem Kreuz wider, das als das Symbol des Glaubens gilt. Das Wort tears steht im Zusammenhang mit den Tränen, die am Grabe Christi vergossen wurden. Das Dollarzeichen repräsentiert den finanziellen Faktor, den das Osterfest in unserer wirtschaftlich orientierten Zeit ebenfalls darstellt. Hauptsächlich aber ist es das Fest der Liebe zu Christus und der Liebe der Menschen untereinander, worauf das Herz am Ende der Sequenz hinweist. Das Wort arts schließlich erinnert daran, daß es sich bei diesem Arrangement um ein Kunstwerk handelt.

Dieses Beispiel zeigt, daß sich Literatur im World Wide Web auf neue und bereichernde Weise entfalten kann. In Verbindung mit visuellen Effekten oder Videosequenzen ergeben sich bislang gänzlich unbekannte Darstellungsformen, die ihrerseits neuartige Sichtweisen produzieren. Gerade "easter offering" veranschaulicht deutlich die neuen Dimensionen, die Literatur mittels Computereinsatzes gewinnen kann. Diese moderne, visuelle Literaturform erweitert die Grenzen des Literaturbegriffes und fordert damit die im Verlaufe dieses Kapitels immer wieder so eindringlich angemahnte großzügige Begriffsauslegung heraus.

${ }^{282}$ Komninos Zervos, "easter offering", in: Drake, Hg., Wr -eye- tings Scratchpad ([ ${ }^{1}$ Oktober 1997]), <http://www.burningpress.org/wreyeting/zervos/easter.html>, 01.04.2003. 


\subsection{Programmgesteuerte Literatur mit Interaktionsmöglichkeiten - Dargestellt am Beispiel des Heretical Rhyme Generator}

Hatte der Nutzer bei der Literaturform zu vorstehend 2.1. keinerlei Einflußmöglichkeiten, so ist er im Falle des Heretical Rhyme Generator ${ }^{283}$ ausdrücklich zur Mitarbeit aufgerufen. Nach Vorgabe der ersten Gedichtzeile erstellt die Gedichtmaschine binnen weniger Sekunden einen Vierzeiler. Oftmals erscheinen die maschinengesteuerten Kreationen wenig schlüssig, doch der Nutzer beziehungsweise Leser wird vom Herausgeber ausdrücklich darauf hingewiesen, daß das poetische Ergebnis möglicherweise keinen Sinn mache. Für diesen Fall wird empfohlen, einen erneuten Versuch zu starten oder einen anderen Satz vorzugeben.

Ein Test dieser Gedichtmaschine führte unter Vorgabe der Zeile "A new kind of poetry" unter anderem zu folgenden vier Ergebnissen:

1. A new kind of poetry swimming in the freshness of my solitude when by shapes my words are known, I try Reality is a staircase leading nowhere.

2. A new kind of poetry Hello, My name's Earth. Have we met? With an exercise in mass poetry.

To buy the lotto or place a bet.

3. A new kind of poetry

Sit on it. Jump on it. Push it and prod it. when by shapes my words are known, I try and forgetteth where exactly i put it.

4. A new kind of poetry Relent, and harbor your time along the shores of tranquility Pundit plots of putrid poetry towards the syntax of your romance and divinity. ${ }^{284}$

Anhand dieser Ergebnisse wird deutlich, nach welchem System der Reimgenerator funktioniert: Das Computerprogramm stellt für einen Vierzeiler nach dem Reimschema $\mathrm{ABAC}$ oder $\mathrm{ABAB}$ aus einem Repertoire 'poetischer' Versatzstücke drei weitere Zeilen zusammen ${ }^{285}$, wobei im Ergebnis offensichtlich eine Klanggleichheit der jeweils letzten Silbe von Zeile eins und drei vorgesehen ist. Allerdings berück-

${ }^{283}$ Chris Seidel, Heretical Rhyme Generator, <http://www.pangloss.com/seidel/Poem/>, 02.04.2003.

284 Ders., Heretical Rhyme Generator, <http://www.pangloss.com/seidel/Poem/poem.cgi>, 02.04.2003.

285 Testversuche der Autorin haben ergeben, daß das Programm über ein begrenztes Spektrum vorgefertigter Zeilen verfügt, die von diesem nach einem Zufallsprinzip ausgewählt und in das Gedicht eingestellt werden. 
sichtigt das Programm offensichtlich nur rein lexikalische Ähnlichkeiten der Endsilben, nicht jedoch ihre lautlichen Ausformungen. Schon aufgrund dieser Tatsache kann der Heretical Rhyme Generator nur ein kurzweiliger Zeitvertreib sein. Dennoch ist er ein gutes Beispiel für programmgesteuerte Literatur mit Interaktionsmöglichkeiten, da er eine allgemeine Tendenz treffend wiedergibt, die neben der ernsthaften Beschäftigung mit den neuen Literaturformen zu beobachten ist: den Spaß an humorvollem, experimentellem Umgang mit Worten. Die Möglichkeit zur Interaktion regt zum Experimentieren an und fördert die Kreativität. Unter Umständen mag diese Literaturform sogar zu einer ernsthafteren Beschäftigung mit Literatur anzuregen.

Programmgesteuerte Literatur zielt in der Regel nicht darauf ab, einen Sinn nach herkömmlichem Verständnis zu erzeugen; vielmehr geht es um ein modernes Bewußtsein von signifiant vs. signifié, das heißt im Vordergrund steht der Formaspekt des sprachlichen Zeichens, während der inhaltliche Aspekt zurücktritt.

\subsection{Geschlossene Hypertext-Systeme - Dargestellt am Beispiel von The Benefactor}

Weitergehende Interaktionsmöglichkeiten eröffnen sich für den Nutzer im Falle des geschlossenen Hypertext-Systems. Dort bietet sich ihm eine Rezeptionsform, die sich weder auf rein textueller noch auf audio-visueller Ebene umsetzen läßt, sondern nur mit Hilfe eines Computers zu realisieren ist. Das Hypertext-System eröffnet den Zugang zu einer neuen Welt, die zwischen den herkömmlicherweise angebotenen Alternativen liegt, nämlich zwischen der rein textuellen Leseerfahrung und dem audio-visuellen Miterleben in Kino, Theater oder Fernsehen. Hypertext bricht die starre Struktur der Literatur auf und bewirkt einen Übergang von dem abgeschlossenen Werk als einem Endprodukt literarischen Schaffens zu einem Entwicklungsprozeß, das heißt von Statik zu Dynamik. In Begriffen der englischen Sprache ausgedrückt, findet eine Umgestaltung von form zu forming statt. Hypertext macht Literatur zu einem Ereignis. Es entsteht Bewegung und Wechsel, den der Leser unmittelbar miterleben kann. Er wird nicht mehr mit einem abgeschlossenen Werk konfrontiert, sondern kann während des Rezeptionsprozesses die Entstehung dieses Werkes nachvollziehen.

Literatur im Hypertextformat ist dergestalt konzipiert, daß mehrere Handlungsstränge simultan ablaufen und der jeweilige Handlungsverlauf durch den Leser bestimmt wird, der seinen persönlichen Weg durch das literarische Werk 
finden muß. Er kann also bei jedem Lesevorgang einen unterschiedlichen Handlungsverlauf erleben. Um alle Handlungsstränge verfolgen zu können, muß der Leser einen Hypertext demnach mehrmals lesen beziehungsweise erleben.

Damit der Lesevorgang für den Leser tatsächlich zu einem Ereignis wird, bedarf es einer flexiblen Hypertextstruktur, wobei im Idealfall jedes Wort oder zumindest jeder Satz einen Link darstellt. Die Rezeption, vor allem aber die Konzeption eines Hypertextes erfordert daher sehr viel Aufmerksamkeit und Mühe. Dies mag auch die Erklärung dafür sein, warum die Auswahl an Werken, die den beschriebenen Anforderungen entsprechen, relativ gering ist.

Besonders gut eignet sich geschlossener Hypertext für die Präsentation von Dramen. Der Rezipient erhält die Möglichkeit, unterschiedliche Rückschlüsse aus den verschiedenen Handlungssträngen zu ziehen und dergestalt sein persönliches Bild bei jedem Lesevorgang zu erweitern, bis letztlich ein Gesamteindruck entsteht, der sich aus den unterschiedlichsten Facetten mosaikartig zusammensetzt und in dieser Ausprägung durch Lektüre eines Drucktextes oder im Theater niemals entstehen könnte. Während dem passiven Theaterbesucher ein Theaterstück lediglich vorgeführt wird, kann ein Drama im Hypertextformat aktiv miterlebt werden. Befindet sich der Zuschauer im Theater in der Rolle des Voyeurs, so wird er im Hypertext gewissermaßen zum Mitspieler des Stückes. Treten in einem Theaterstück im allgemeinen Haupt- und Nebendarsteller auf, so kann das Hypertext-System mittels der simultan ablaufenden Handlungsstränge eine Aufhebung dieser Trennung bewirken und alle Darsteller durchgehend präsent sein lassen. Nach Ansicht von Deemer demonstriert das Hypertext-Drama im Gegensatz zum traditionellen Theater, wie das reale Leben abläuft und wirkt aus diesem Grunde wesentlich überzeugender:

Hypertext is a web of possibilities, a web of reading experiences. Hypertext is like life itself, full of choices and consequences, full of forks in the road. Hypertext is the language of exploration and discovery - and therefore is the perfect language to become the mother tongue of the Information Age.

For writers and readers alike, hypertext may well define what it means to be literate in the 21 st century. ${ }^{286}$

${ }^{286}$ Charles Deemer, "What is Hypertext?", in: Center for the Public Domain/University of North Carolina - Chapel Hill, Hg., ibiblio - the public's library and digital archive ([ $\left.\left.{ }^{1} 1994\right]\right)$,

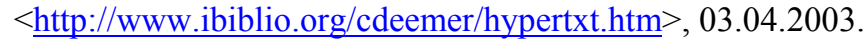


Ein sehr gutes Beispiel bietet das Drama The Benefactor. ${ }^{287}$ Die Handlung findet an fünf verschiedenen Orten statt, für die das Skript sechs verschiedene Ebenen mit einer erzählten Gesamtzeit von ungefähr 45 Minuten vorsieht. Alle Charaktere und Örtlichkeiten sind gleichgewichtig. Eine schematische Darstellung aller Szenen gibt einen Überblick über das gesamte Drama. ${ }^{288}$ Der Nutzer kann entweder die Erlebnisse eines einzelnen Charakters teilen und ihn das gesamte Drama hindurch begleiten, oder aber er kann zwischen den einzelnen Räumlichkeiten wechseln beziehungsweise alle Skripte in Folge lesen. Wie immer er sich entscheidet, er erfährt jeweils nur Teilaspekte, und so erschließt sich der wahre Hintergrund des Dramas erst nach Ende der Lektüre. Nur nach und nach eröffnen sich neue Einblicke, bis sich letztlich alle Erkenntnisse wie bei einem Puzzle zusammenfügen. ${ }^{289}$

The Benefactor versetzt den Leser in das Jahr 1912 und führt ihn in ein englisches Landhaus viktorianischen Stils, wo er Einblick erhält in das Leben des Kaufmannes und Parlamentsmitgliedes Mr. Sutherland, eines Mannes Anfang Sechzig, seiner Ehefrau und seiner vier Kinder. Nachdem der Leser bereits am Ende des

287 Billy Smart und Hannah E. Rudman, The Benefactor - a Short Hyperdrama (30.05.1998), $<$ http://www.rhul.ac.uk/drama/research/benefactor/>, 07.04.2003.

288 Dies., Table of Scenes (30.05.1998), <http://www.rhul.ac.uk/drama/research/benefactor/tabler.html\#table>, 07.04.2003.

${ }^{289}$ Mr. Sutherlands Leben wird bestimmt durch die Arbeit in seinem Familienbetrieb, den er an seine Söhne weitergeben möchte. Mrs. Sutherland, 55, die überzeugt ist, sich unter ihren Verhältnissen verehelicht zu haben, pflegt ihre Krankheiten, versucht, Familienskandale zu vertuschen und die Bediensteten zu bespitzeln. Zu ihren vier Kindern Harley, 28, Herbert, 18, Georgina, 26 und Vita, 22, unterhält sie ein gespanntes Verhältnis. Harley führt ein zügelloses Leben, Georgina, die den Namen George bevorzugt, tritt engagiert und kämpferisch für die Rechte der Frauen ein und zeigt nach Auftreten und Erscheinungsbild eher männliche Züge. Herbert und Vita dagegen verhalten sich zwar unauffällig und zeigen gutes Benehmen, können aber den Ansprüchen ihrer Mutter ebenfalls nicht genügen, da Herbert ihr noch zu kindlich erscheint und Vita ohne Selbstvertrauen und von Depressionen geplagt ist. - Das Drama beginnt mit dem Auftreten von vier Farmern, die Mr. Sutherland aufsuchen, um unterschiedliche Anliegen vorzutragen. Mr. Sutherland läßt alle Farmer zu Wort kommen, geht allerdings in sehr ungleicher Weise auf ihre Bitten ein, indem er sich drei Farmern gegenüber außerordentlich großzügig verhält, während er einem nur den Bruchteil des erbetenen Geldbetrags zugesteht. - Ebenfalls zu Beginn treten zwei von Harley bestellte Prostituierte auf, Molly, 17, und Nell, 39, die nach Erfüllung ihres Auftrags die Familie bestehlen. Während Molly die Flucht ergreifen kann, ist Nell gezwungen, sich im Haus zu verstecken, wo sie von George entdeckt wird. Im Verlaufe eines Gesprächs entwickelt George gewisse Gefühle für Nell. - Um Harley vor gesellschaftlichem Abstieg und der Heranziehung zum Wehrdienst zu bewahren, trägt Mr. Sutherland ihm einen sehr gut bezahlten Posten mit geringem Arbeitsaufwand in Tonga und eine neue Identität an, unter der Voraussetzung, nie wieder nach England zurückzukehren. An Stelle des ältesten Sohnes möchte er die betriebliche Nachfolge dem jüngeren Herbert antragen, der allerdings beabsichtigt, als Missionar nach Abessinien zu gehen. Da Harley nicht gewillt ist, sich dem Willen des Vaters zu fügen, weist ihn dieser aus dem Haus. - Als ihm, Harley, der Teufel in weiblicher Gestalt erscheint und ihm anbietet, ihn mit in die Hölle zu nehmen, fühlt er sich angezogen von der geschilderten Perspektive und geht auf das Angebot ein. - Der von Mr. Sutherland finanziell benachteiligte Farmer greift diesen sowohl verbal als auch tätlich an, woraufhin Mr. Sutherland einen Herzanfall erleidet. Während seiner letzten Lebensminuten gelingt es ihm, sein Testament aufzusetzen und Butler Arthur zu dessen großem Erstaunen zum Erben zu bestimmen. 
ersten Aktes Zeuge von Mr. Sutherlands Tod wird, sieht er sich im weiteren Verlauf des Dramas vorwiegend mit den Problemen menschlichen Daseins und Miteinanders konfrontiert, die sich in unrealistischen Wunschvorstellungen, innerer Zerrissenheit und Zügellosigkeit manifestieren.

The Benefactor vermittelt dem Rezipienten das Gefühl, alle Geschehnisse im Haus hautnah mitzuerleben. Liest der Nutzer das Web-Drama zunächst, ohne einer Verknüpfung zu folgen, so hat er bis zum Schluß Mrs. Sutherland und ihren Söhnen und Töchtern das Wissen um das Schicksal des Hausherrn voraus. Im Gegensatz zur restlichen Familie besitzt der Leser Kenntnis von dem kurz bevorstehenden Tod und beginnt bald zu erkennen, welche Ereignisse Mr. Sutherland zu seiner Testamentsentscheidung bewogen haben. Allerdings muß der Leser geistige Flexibilität beweisen, da das Drama bei sequentieller Rezeption einen recht sprunghaften Verlauf nimmt.

Dank des vorangestellten Übersichtsschemas kann der Nutzer stets eine gute Orientierung bewahren. Würde er nämlich nicht über die Gesamtstruktur des Hypertextes in Kenntnis gesetzt, könnte er sich leicht innerhalb des Textgefüges verlieren. Der Leser befände sich dann in der dauernden Ungewißheit, "ob er wichtige Elemente 'verpaßt' oder ob er bereits alle Möglichkeiten durchgespielt hat" 290.

\subsection{Computergestützte Literatur - Spielerei oder Bereicherung?}

Im Gegensatz zu konventioneller, linear-konzipierter Literatur im Web vermitteln die Formen computergestützter, webunabhängiger Literatur im Web dem Nutzer eher das Gefühl, sich eines der Druckliteratur überlegenen, innovativen oder gar revolutionären Mediums zu bedienen. Unendlich ablaufende Bilderfolgen werden via World Wide Web auf den heimischen Computer-Bildschirm transportiert und verschaffen dem Betrachter damit tatsächlich ein neuartiges Literaturerlebnis, das in dieser Form vor der Verbreitung des WWW nur im Rahmen einer Ausstellung zu erleben war. Auch die unbegrenzt wiederholbare Möglichkeit der Interaktion, die dem Nutzer den Eindruck verschafft, er könne seine ganz individuelle Literatur selbst kreieren, ist nur mit Hilfe des Computers zu gewährleisten. Anders formuliert: Die Chance, einen literarischen Text nach eigenem Gusto zu rezipieren und die traditionelle, lineare Konzeption aufzubrechen, bestand zwar - wie ausgeführt ${ }^{291}$ - schon vor Einführung des elektronischen Hypertext-Systems, ist

\footnotetext{
${ }^{290}$ Hautzinger, Vom Buch zum Internet?, 59.

291 Siehe Kapitel III.1.2.
} 
jedoch mit der diesem System eigenen Schnelligkeit und Unmittelbarkeit nur bei Verwendung eines Computers gegeben.

Die Tatsache, daß programmgesteuerte Literatur sowohl ohne als auch mit Interaktionsmöglichkeiten aufgrund ihrer zeit- und ortsunabhängigen Realisierbarkeit als innovativ angesehen werden muß, ändert allerdings nichts daran, daß sie oftmals nur eine spielerische Abwechslung darstellt. Literatur im geschlossenen Hypertext hingegen birgt tatsächlich das Potential einer "Revolution des Lesens"292. Voraussetzung sind selbstverständlich eine professionelle Gestaltung und inhaltliches Niveau.

Wie ein Hypertext-System gestaltet sein, welche Möglichkeiten es eröffnen sollte und ob es diese Voraussetzungen tatsächlich erfüllt, ist Thema zahlreicher Untersuchungen, die in ihrer Menge in keinem Verhältnis zu der Anzahl der angeboten Hypertexte stehen. Bereits im Jahre 1996 konstatierte Nestvold, die Theorie des elektronischen Kunstwerks sei der praktischen Umsetzung weit voraus: "Die Zahl der kritischen Studien zur Hyperfiction übertrifft die der erschienenen Werke bei weitem." 293 Dies glaubte Nestvold zum damaligen Zeitpunkt noch damit erklären zu können, daß die Ausarbeitung eines anspruchsvollen Romans mit einem Umfang von ungefähr dreihundert Seiten im Regelfall eine Dauer von fünf bis sechs Jahren beanspruche; Autoren könnten aber realiter erst seit ebenso vielen Jahren auf Hypertext-Systeme zur Erstellung ihrer literarischen Werke zurückgreifen. ${ }^{294}$

Inzwischen ist das Argument, es könnten schon aus zeitlichen Gründen noch gar keine Werke im Hypertextformat vorliegen, durch den Gang der Historie überholt. Doch auch nach abermals fünf bis sechs Jahren hat sich am zahlenmäßigen Mißverhältnis zwischen Primär- und Sekundärliteratur kaum etwas geändert. Worin dies begründet liegt und welche Positionen die Autoren der zahlreichen Abhandlungen vertreten, soll nachfolgend in einem Exkurs über das Medium Hypertext analysiert werden.

\footnotetext{
${ }^{292}$ Nestvold, "Das Ende des Buches", 23.

293 Ebd., 16f.

294 Ebd., 16.
} 


\section{Das Phänomen Hypertext}

Schon immer haben einzelne Autoren die Konvention der linearen Textgestalt experimentell durchbrochen, wie zum Beispiel Laurence Sterne mit seinem Werk Tristram Shandy. Dennoch zählen derartige Dokumente im Print-Bereich zu den Ausnahmen. Das elektronische Informationssystem World Wide Web hingegen scheint nunmehr die Perfektionierung der nicht-linearen Konzeption darzustellen. Hier liegen die Vorteile in dem schnellen und direkten Zugriff auf Zusatzinformationen sowie der Präsentations- und Zugangsvielfalt, was die Feststellung nahelegen könnte, Hypertext-Dokumente seien gedruckten Dokumenten grundsätzlich vorzuziehen. ${ }^{295}$ Woraus aber erwächst diese Präsentations- und Zugangsvielfalt, aufgrund derer der Nutzer Hypertexten gegenwärtig den Vorrang geben sollte?

$\mathrm{Da}$ die moderne Hypertext-Systematik nicht so neu ist, wie oft gepriesen, zeigt ein vergleichender Blick auf die Struktur elektronischer und gedruckter Dokumente. Dabei wird deutlich, daß der Ursprung elektronischer Hypertext-Verknüpfungen in Print-Dokumenten liegt. Schreiber bringt diesen Zusammenhang auf eine simple Formel und kommentiert die digitale Vernetzung, die eigentlich immer wieder als Novum moderner Medien bezeichnet wird, folgendermaßen: "Es ist im Grunde lediglich eine Art elektronische Blätterhilfe." ${ }^{296}$ Auch Gilster setzt dem vermeintlich Neuem einen Hinweis auf bereits Bestehendes entgegen, indem er auf antike Texte verweist, die ursprünglich auf Papyrusrollen fortlaufend ohne Interpunktion, Seitenzählung oder Inhaltsverzeichnis niedergeschrieben wurden. ${ }^{297}$ Gilster darf sich mit diesem Hinweis insofern bestätigt sehen, als das sogenannte Scrolling, dt. Scrollen, das mittlerweile als etablierte Bezeichnung für den Vorgang des sich Auf- und Abbewegens auf dem Computerbildschirm angesehen werden kann, von dem englischen Nomen scroll, dt. Schriftrolle abgeleitet ist. Dieser Umstand erklärt, weshalb Gilster Hypertext nicht als ein r e volutionäres, sondern vielmehr als ein e volutionäres Konzept

\footnotetext{
${ }^{295}$ Gegen eine Weiterführung dieses Gedankens spricht allerdings von vornherein die Tatsache, daß der Literaturrezeption an einem Computerbildschirm Grenzen gesetzt sind, die sowohl mit der Belastbarkeit der Sehorgane als auch damit zu tun haben, daß die Lesebereitschaft bei elektronischen Dokumenten im allgemeinen stark begrenzt ist. Nach den Angaben von Zimmer geht sie je Rezeptionsprozeß nicht über 1.500 Wörter hinaus, was etwa vier bis fünf Buchseiten entspricht. Zimmer zieht aus diesem Sachverhalt die Konsequenz, der Computerbildschirm eigne sich weniger zum Lesen als in erster Linie zum schnellen Nachschlagen. Zimmer, "Die digitale Bibliothek", 272. Es ist zudem fraglich, ob überhaupt alle Texte dazu geeignet sind, in einen Hypertext konvertiert zu werden.

296 Schreiber, Neue Wege des Publizierens, 15.

297 Paul Gilster, Digital Literacy (New York, 1997), 137.
} 
bezeichnet wissen möchte. ${ }^{298}$ So liegt der Aspekt des Modernen bei ComputerHypertexten eigentlich nur in der Reduzierung auf Bildschirmgröße und der unmittelbaren Verknüpfung mit anderen Texten oder Medienformen. ${ }^{299}$

Im Grunde genommen ist Hypertext nichts weiter als elektronischer Text, der durch Verzeichnisse oder Verbindungen (links) zwischen einzelnen Wörtern und weiterführenden Abschnitten den nicht-linearen Zugang zu Informationen ermöglicht. ${ }^{300}$

Durch den direkten Zugriff wird allerdings eine völlig neue Form der Disponibilität ermöglicht, die eine permanente und umfassende Verfügbarkeit themenbezogener Materialien erlaubt und Begriffen wie Interdisziplinarität eine völlig neue Dimension verleiht: "Hypertext heißt Integration." 301 Informationen, die bislang üblicherweise aus unterschiedlichen Quellen zusammengetragen werden mußten, können nun theoretisch in ein einziges digitales Dokument eingegliedert werden. Barth demonstriert auf anschauliche Weise anhand des Beispielsatzes The quick brown fox jumps over the lazy dog, welche Möglichkeiten dieser Satz in Form eines Hypertextes eröffnen würde: Von der Flinkheit der Vierfüßler über die Geschichte der Fuchsjagd und ihrer Darstellung in der Kunst bis hin zu kommentierten Tonbeispielen und Abhandlungen über die Rechte von Tieren liefere das Anklicken einzelner Worte dem Leser detaillierte Angaben, aus denen vermutlich wiederum neue Verknüpfungen zu weiteren Informationsquellen hervorgingen:

[...] and every one of those associated "lexias" similarly loaded, another ring of keys with which one may open yet further doors, and on and on and on $[\ldots]^{302}$

Für den Nutzer bedeutet Hypertext Arbeitsvereinfachung sowie Zeit- und Kostenersparnis. Gleichzeitig entbindet es ihn aber nicht von einem verantwortungsbewußten, selektiven Umgang mit dieser Art von 'Materialsammlungen', denn die Angebotsfülle allein garantiert weder Zuverlässigkeit noch Vollständigkeit der Informationen.

\footnotetext{
298 Ebd.

299 Vgl. ebd., 136f.

300 Nestvold, "Das Ende des Buches", 14.

301 Neumann, "Geisteswissenschaften im Internet", 58.

302 Barth, "The State of the Art", 40.
} 


\section{Strukturierung von Hypertexten}

Je nach Art der angebotenen Informationen erfordert der Hypertext eine bestimmte Strukturierung. Dabei stellt sich die Frage, wie ein Hypertext - bzw. im Falle vorliegender Arbeit genauer: ein literarischer Hypertext - idealerweise aussehen sollte und welche Konzeption ihm zugrunde liegen müßte, damit er Autoren und Lesern gleichermaßen entgegenkäme. Allgemeingültige, verbindliche Vorgaben oder Maßstäbe zur Erstellung von Hypertext-Systemen liegen gegenwärtig nicht vor und scheinen auch wenig sinnvoll, denn zu Recht bemerkt Kuhlen: "Es ist offensichtlich, daß es keinen besten Weg der Strukturierung von Verknüpfungstypen gibt, zu unterschiedlich sind die möglichen Sichten. ${ }^{, 303}$ Kuhlens Erkenntnis basiert vermutlich auf eigener Erfahrung, hat er doch selbst einen Vorschlag zur Typologisierung von Verknüpfungen auszuarbeiten versucht. ${ }^{304}$

Grundsätzlich sind zwei Typen zu unterscheiden, die an dieser Stelle als zielpunktorientiert und funktionsorientiert bezeichnet werden sollen.

Die Einteilung nach Zielpunktorientierung soll der Unterscheidung zwischen systeminternen und systemexternen Vernetzungen dienen. Verweist ein Link auf eine Informationseinheit innerhalb ein und desselben Hypertext-Systems, ist er als systeminterner Verweis zu bezeichnen, wohingegen eine über das Hypertext-System hinausgehende Verknüpfung als systemextern anzusehen ist.

Diese Einteilung verzichtet bewußt auf eine zu starke Differenzierung und grenzt sich damit von den Unterteilungen Kuhlens und Storrers ab, nach denen jeweils drei Untergruppen vorgesehen sind. Kuhlen beispielsweise trennt a) intrahypertextuelle Verknüpfungen innerhalb ein und derselben Einheit eines HypertextSystems von b) interhypertextuellen Verknüpfungen zwischen unterschiedlichen Einheiten eines Systems und c) extrahypertextuellen Verknüpfungen zwischen verschiedenen Hypertext-Systemen. ${ }^{305}$ Storrers Kategorisierung basiert auf denselben Interdependenzen, die Verknüpfungstypen werden jedoch a) mikrostrukturelle, b) makrostrukturelle und c) intertextuelle Verweise genannt. ${ }^{306}$

${ }^{303}$ Kuhlen, Hypertext, 107.

${ }^{304}$ Ebd., 104ff. Hier ist allerdings unklar, wie der Widerspruch aufzulösen ist, daß Kuhlen die Festsetzung bestimmter Richtwerte für wenig zweckmäßig erachtet, selber aber konkrete Strukturierungsvorschläge ausarbeitet.

305 Ebd., $107 \mathrm{f}$.

306 Angelika Storrer, "Vom Text zum Hypertext: Die Produktion von Hypertexten auf der Basis traditioneller wissenschaftlicher Texte", in: Knorr und Jakobs, Hg., Textproduktion in elektronischen Umgebungen, 121-139, hier: 130. 
Der Komplementärbegriff der Zielpunktorientierung, die Funktionsorientierung, stellt in den Vordergrund die Bestimmung der jeweiligen Verknüpfungen. Entscheidend soll sein, welchem Zweck die Links dienen, d.h. welche Angaben sich dahinter verbergen. Dabei sei zwischen zwei Kategorien differenziert, die der Einteilung in Primärliteratur und Sekundärliteratur entsprechen. So seien

a) die Verknüpfungen zwischen den Handlungselementen (ausschließlich systemintern)

von

b) den Verknüpfungen zu weiterführenden Informationen wie z.B.

- inhaltlichen Informationen zu Thema oder Gegenstand des jeweiligen Werkes (systemintern und -extern),

- kontextuellen Hinweisen zum Hintergrund des Werkes und seines Autors (systemintern und -extern),

- Orientierungsangaben, wie beispielsweise Inhaltsverzeichnis oder Übersichtsschema (systemintern) oder

- stichwortbezogenen enzyklopädischen Informationen (systemintern) abgegrenzt.

Diese obige Klassifikation nach dem Verknüpfungszweck findet sich ansatzweise wieder in einer von Kuhlen vorgenommenen Unterteilung; der hauptsächliche Unterschied besteht darin, daß die Kategorisierung nach Kuhlen auf HypertextSysteme im allgemeinen und nicht speziell auf literarische Hypertexte ausgerichtet ist.

Laut Kuhlen können die einzelnen Informationseinheiten eines HypertextSystems zum einen durch referentielle Verknüpfungen verbunden sein, die nicht genauer spezifiziert sind, sondern lediglich Verkettungsfunktion haben. Diese ermöglichen die assoziative Navigation, weshalb sie auch Navigationsverknüpfungen genannt werden. ${ }^{307}$ In diese Gruppe ließen sich durchaus die in a) genannten Verbindungen zwischen Handlungselementen eingliedern.

Gemäß Kuhlen kann ein Hypertext-System jedoch auch aus typisierten Verknüpfungen bestehen, die genau spezifiziert sind. ${ }^{308}$ Dieser Gruppe ließe sich die

\footnotetext{
307 Kuhlen, Hypertext, $105 \mathrm{f}$.
}

308 Ebd. 
obige Kategorie b) der Verknüpfungen zu weiterführenden Informationen untergliedern.

Etwas differenzierter und ausschließlich auf die Gegebenheiten des World Wide Web ausgerichtet präsentiert sich die Kategorisierung der Firma Matterform: Navigationshinweise führen von einer Verknüpfung zur nächsten, Multimediahinweise leiten $\mathrm{zu}$ eigenständigen Abbildungen und Filmdateien, Seitentypenhinweise verbinden den Nutzer entweder mit interaktiven Formularen, E-Mail-Programmen, Suchseiten, erläuternden Seiten oder Informationsseiten; Netzdiensthinweise schließlich verweisen auf ein Ftp-Archiv bzw. einen Telnet-Server. ${ }^{309}$

Im Vergleich der beiden funktionsorientierten Kategorisierungen scheint diejenige von Kuhlen zunächst eher geeignet, Verknüpfungen in Hypertext-Systemen literarischer Art zu beschreiben. Allerdings machen der technische Fortschritt sowie die zunehmende Verbreitung nicht-linear konzipierter Literatur im World Wide Web eine Anpassung an dieses Medium wünschenswert, so daß auch die Einteilung der Firma Matterform im Hinblick auf Multimedialität und Kooperativität eine Basis für Erstellung einer literaturspezifischen Link-Typologisierung bilden könnte.

Angesichts der bereits existierenden Vielfalt an Verknüpfungsarten und des gestiegenen Bedarfs aufgrund der veränderten technischen Gegebenheiten wird deutlich, daß es grundsätzlich nicht sinnvoll erscheint, eine Strukturierung vorzugeben, da sie letztlich immer der Thematik und Dynamik des jeweiligen Hypertext-Systems angepaßt sein muß. Daher ist man geneigt, sich Kuhlens Einschätzung der Gleichwertigkeit von Strukturierungsformen anzuschließen. Im Hinblick auf Wert und Möglichkeiten von Hypertexten bleibt die Frage nach der Verknüpfungsstruktur letztlich zweitrangig und so ist Böhle, Riehm und Wingert beizupflichten, daß “die Hypertext-Debatte um Netzstrukturen und Verknüpfungsarten eine für die praktische Entwicklung und Anwendung von Hypertexten [...] wenig ergiebige Debatte darstellt" ${ }^{\prime 310}$.

${ }^{309}$ Schröder, "Der Link als Herme und Seitensprung", 54f. Grafiken sowie eine Bedienungsanleitung werden auf den Seiten der Firma Matterform Media kostenlosen zur Verfügung gestellt. Matterform Media, Hg., Qbullets, <http://www.matterform.com/qbullets/index.php>, 03.07.2003.

310 Böhle, Riehm und Wingert, Vom allmählichen Verfertigen elektronischer Bücher, $306 \mathrm{f}$. 


\section{Inhaltliche Gestaltung - Auf dem schmalen Grat zwischen Leselust und Lesefrust}

Wenngleich es nebensächlich sein dürfte, welche Systematik der äußeren Strukturierung eines Hypertext-Systems zugrunde liegt, so besteht mit Sicherheit kein Zweifel daran, daß seine inhaltliche Konzeption von ganz entscheidender Bedeutung ist. Dabei wird sowohl der Autor als auch der Leser vor ungewohnte Herausforderungen gestellt. Die Abkehr von der herkömmlichen Schreib- und Leseweise eröffnet eine völlig neue Dimension:

Diese Loslösung von den narrativen Schemata der gedruckten Literatur führt zu einer neuen Ästhetik, die das geschriebene Wort transzendieren will: Chronologie schlägt um in Topographie. Das Wort wird zum Ort. Orte und Netze lösen Worte und Sätze ab. ${ }^{311}$

Wesentlich größer als für den Leser ist die Umstellung vermutlich für den Autor, wenn er sich auf eine Konzeption im Hypertext-Format einläßt. Nachstehend soll überblicksartig dargelegt werden, welche Charakteristika ein Hypertext-System aufweisen muß, um den Leseerwartungen gerecht $\mathrm{zu}$ werden, welche neuen Perspektiven sich damit eröffnen und welche Grenzen daraus gleichzeitig auch für den Leser erwachsen.

\subsection{Anforderungen an die literarische Arbeit}

Bei der Erstellung eines literarischen Hypertext-Werkes fühlt sich der Autor einerseits herausgefordert, sich der neuartigen Verknüpfungsstruktur zu unterwerfen, zugleich ist er aber auch gehalten, sich an den traditionellen Rezeptionsbedingungen auszurichten. Der Leser wird dem literarischen Werk in der Regel mit einer ausgeprägten Erwartungshaltung hinsichtlich der äußeren wie inhaltlichen Struktur begegnen:

With hypertext we focus, both as writers and as readers, on structure as much as on prose, for we are made aware suddenly of the shapes of narratives that are often hidden in print stories. ${ }^{312}$

${ }^{311}$ Hilmar Schmundt, "Strom, Spannung, Widerstand: Hyperfictions - die Romantik des elektronischen Zeitalters", in: Klepper, Mayer und Schneck, Hg., Hyperkultur, 54.

312 Robert Coover, "The End of Books", New York Times Book Review, 21.06.1992, 1, 23-25, hier: 24. 
Unter Berücksichtigung dieser Tatsache sollte das Resultat der literarischen Arbeit letztlich folgende Eigenschaften aufweisen:

\section{a) Plausibilität}

Um der genannten Erwartung zu entsprechen, hat eines bei der Erstellung eines Hypertext-Systems im Vordergrund zu stehen: die Plausibilität, d.h. die inhaltliche Schlüssigkeit. Ein literarischer Hypertext muß stets in der Weise aufgebaut sein, daß jede mögliche Aneinanderreihung der einzelnen Text-Elemente einen plausiblen $\mathrm{Zu}$ sammenhang ergibt. Handelt es sich um einen rein literarischen Hypertext, so sollte gewährleistet sein, daß der Leser die erzählte Geschichte trotz ihrer Fragmentierung zu jedem Zeitpunkt als eine schlüssige Schilderung wahrnimmt.

\section{b) Attraktivität}

Zusätzlich wird der Leser eine Konzeption erwarten, die sein Interesse auch dann noch bindet, wenn er die Erzählung mehrmals hintereinander auf unterschiedlichen Lesewegen durchläuft.

Dieser Anspruch stellt für den Autor vermutlich die größte Herausforderung dar, hat er es doch im Gegensatz zu einem linear-konzipierten Werk nicht mit einem durchgehenden Erzählstrang zu tun; vielmehr muß er sich gleichzeitig auf mehreren Erzählebenen bewegen, die alle möglichst das gleiche Spannungsniveau aufweisen sollten. Andernfalls könnte der Leser das Interesse an der Lektüre verlieren und den Rezeptionsvorgang vorzeitig abbrechen.

c) Abgeschlossenheit

Ist der Autor geneigt, sich auf die Erwartungshaltung des potentiellen Leser einzurichten und ihr entgegenzukommen, so muß er ihr auch noch in einem weiteren Punkt Rechnung tragen: Er muß sein Werk an irgendeiner Stelle zum Abschluß kommen lassen. Auch wenn die Hypertext-Struktur dem Autor strenggenommen verbietet, ein 'offizielles' Ende seines Werkes auszusprechen, so muß für den Leser gleichwohl ein gewisser Abschluß erkennbar sein, bildet die Aussicht darauf doch offensichtlich den entscheidenden Motor für die Bereitschaft, sich dem Werk zu widmen: 
Even in interactive narratives, where we as readers never encounter anything quite so definitive as the words The End, or the last page of a story or novel, our experience of the text is not only guided but enabled by our sense of the 'ending' awaiting us. ${ }^{313}$

Die Erfahrung eines abgeschlossenen Kunstwerks, die im übrigen nicht nur durch die Literatur, sondern auch durch künstlerische Werke anderer Art, wie beispielsweise Musik, Film oder Theater geprägt ist, schafft beim Rezipienten jedoch nicht nur die innere Bereitschaft, sich darauf einzulassen, sondern sie bedeutet für ihn vor allem einen wesentlichen Genuß: "[...] the experience of narrative closure numbers among the principle pleasures of reading narratives - the one thing that both prompts and enables us to read." 314

Zudem verschafft die Abgeschlossenheit eines Werkes dem Rezipienten Befriedigung. In dem Augenblick, da er sich auf ein künstlerisches Werk einläßt, geht sein Bestreben in der Regel dahin, das Ende zu erreichen, d.h. er 'arbeitet' gewissermaßen ein bestimmtes Pensum 'ab'. Gleichgültig, ob der Schluß seinen persönlichen Vorstellungen oder Interpretationen entspricht oder der Autor ihn mehrdeutig gestaltet - wichtig ist allein die Tatsache, das Werk in seiner Gesamtheit wahrgenommen zu haben. ${ }^{315}$

\subsection{Perspektiven ...}

In Kapitel I. wurde bereits angedeutet, daß die Auswirkungen der Verbreitung des World Wide Web teilweise recht unterschiedlich beurteilt werden. Was Kritikern als ernsthafte Bedrohung der Buchkultur und der damit verbundenen traditionellen Werte erscheint ${ }^{316}$, wird von Anhängern des WWW als Befreiung von der Autorität des Autors gefeiert ${ }^{317}$. Mit Hilfe des Hypertext-Systems soll der Leser nunmehr in der Lage sein, seine Leseerfahrungen selbstbestimmt zu erleben, ohne sich strengen und einengenden Autorenvorgaben unterwerfen zu müssen.

Dieses Ideal des modernen Lesers kann bei offenen Hypertext-Systemen in der Tat Gestalt annehmen. Dort nämlich reicht der Bewegungsspielraum über die Grenzen des vom Autor vorgegebenen Werkes hinaus, der Leser kann mithin tatsächlich selbst bestimmen, wohin ihn seine 'Lesereise' führt. Er kann aus der Lektüre seines Werkes

\footnotetext{
313 Douglas, The End of Books, 121.

314 Ebd., 92.

315 Vgl. Hautzinger, Vom Buch zum Internet?, 60.

316 Nestvold, "Das Ende des Buches", 15. Birkerts, Die Gutenberg-Elegien, 216.

317 Hautzinger, Vom Buch zum Internet?, 31.
} 
hinaus umgehend auf themenbezogene Dokumente zugreifen und sich damit möglicherweise wirklich ein einzigartiges Leseerlebnis schaffen. ${ }^{318}$

\section{3. ... und Grenzen des Hypertextes}

Bei geschlossenen Hypertext-Systemen hingegen muß stark bezweifelt werden, daß der Leser freier ist als bei Print-Literatur. Hier scheint die angebliche Selbstbestimmung eher nur ein Wunschbild zu sein, das sich bei näherer Betrachtung als reine Täuschung erweist. Was gemeinhin als Befreiung von "der Bevormundung durch den Autor" 319 (ein-)geschätzt wird, ist in Wirklichkeit nur eine andere Art der Führung durch den Text, denn das Material, innerhalb dessen der Leser seine Freiheit ausleben soll, muß ja zunächst einmal von einem Autor erdacht und arrangiert worden sein. ${ }^{320}$

Was die mehr oder weniger spürbare Lenkung des Lesers anbetrifft, glaubt Todesco gar, die Macht des Autors trete in den neuen Literaturformen besonders deutlich zu Tage. Todesco vermag nach eigener Aussage weder den Sinn noch den Wert eines Computers im Hinblick auf die Literaturproduktion zu erkennen, und die neue Kunstform Hyperfiction bleibt in seinen Augen auch nicht mehr als ein mangelhafter literarischer Versuch; doch sieht er durch die Beschaffenheit des Hypertextes immerhin offen bestätigt, was unterschwellig bereits seit langem allgemein bekannt war: "Literatur beruht auf der Bestimmung des Autors, was der Leser wann zu lesen hat." 321

Der Leser wird also weiterhin - möglicherweise nun sogar in ausgeprägterem Maße - durch den Text geleitet, denn auch im Hypertext beeinflußt eine vorgegebene Struktur seinen Leseverlauf. Mit Hilfe sichtbar hervorgehobener Links wird dem Leser signalisiert, daß an jenen Stellen Leseoptionen bestehen. Seinen Weg durch den Text bestimmt der Leser also nicht durch eigene Motivationen und Assoziationen, sondern er folgt im Grunde nur den Vorgaben des Autors. Die feste Konfigurierung ist vom Leser nicht aufzubrechen, was allerdings nicht zwangsläufig bedeuten muß, daß ihm die “'gesteuerte' Freiheit der Navigation” keine Freude

318 Einzigartig wird dieses Erlebnis im Zweifel schon deshalb oftmals sein, weil der Leser nicht in der Lage sein wird, seinen Weg durch das World Wide Web bis ins Detail zurückzuverfolgen: “[...] no two routes through the maze ever likely to be the same, and every venturer thereinto not only a Theseus but a Daedalus, remodeling the labyrinth at will en route through it." Barth, "The State of the Art", 40.

319 Birkerts, Die Gutenberg-Elegien, 221.

320 Vgl. Zimmer, Die Bibliothek der Zukunft, 58.

321 Todesco, "Hyperkommunikation", 121. 
bereitet; allein das Spiel mit dem Text kann bereits suggerieren, er bewege sich "autonom durch einen (endlosen) Text". 322

Die Beeinflussung des Lesers aufgrund der optischen Hervorhebung der Verknüpfung wäre gegebenenfalls zu vermeiden, wenn diese Kennzeichnung wegfiele, der Leser sich also aus eigener Motivation und Neugier auf die Suche nach möglichen Verknüpfungen im Text begeben müßte. ${ }^{323}$ Dann stünde allerdings zu befürchten, daß sich das Bewegen in einem Hypertext auf ein "erratisches Umherirren und 'Zusammenklauben' " ${ }^{\prime 324}$ reduziert oder in einer rein sequentiellen Leseweise endet.

Von dieser "Klickeratur" 325 - wie er es nennt -, die das Interesse des Nutzers nicht zu fesseln vermöge und ihn deshalb zum unsteten (Weg-)Klicken veranlasse, will Schröder Hypertexte strikt abgegrenzt wissen. Hypertext-Komposition berge das Potential, "Texteinheiten zu einem Mehrwert des Nichtlinearen"326 zu strukturieren.

Wenn Schröder damit impliziert, dem Leser sei nun nicht mehr nur eine einzelne, sequentiell zu lesende Textversion vorgegeben, sondern eine Vielzahl individuell kombinierbarer Textelemente, bleibt doch weiterhin folgende Frage ungelöst: worin liegt der Mehrwert des Nichtlinearen? Liegt er nur im Handlungsspielraum des Lesers, sich gewissermaßen seinen eigenen Text zusammenzustellen? Daß dieser Mehrwert nicht selten zum Minderwert werden kann, ist eine von Todescos Erfahrungen mit dieser Literaturform: "Man muss sich vom 'federführenden' Autor ans Gängelband nehmen lassen und in Kauf nehmen, dass man immer nur interpretiert, was in diesem Kontext zwangsläufig heisst, falsch interpretiert." ${ }^{\text {327 }}$ Zudem fühlt sich der Leser angesichts kompliziert strukturierter und schwer durchschaubarer Hypertexte oftmals schon mit der Bedienung dieser Systeme überfordert.

Zwar könnte man einwenden, diese Verwaltung von Informationseinheiten befinde sich derzeit noch im Entwicklungsstadium, es bestehe berechtigter Grund zur Annahme, die aufgezeigten Grenzen der Hypertext-Systematik könnten früher oder später abgebaut werden. Es ist aber nicht anzunehmen, die technische oder inhaltliche Weiterentwicklung werde alle Einschränkungen beseitigen; die folgenden beiden Probleme scheinen nämlich systeminhärent zu sein:

\footnotetext{
322 Suter und Böhler, Hyperfiction, 19.

${ }^{323}$ Hautzinger, Vom Buch zum Internet?, 72f.

324 Wingert, “Kann man Hypertexte lesen?”, 200.

325 Schröder, "Der Link als Herme und Seitensprung", 50.

326 Ebd.

327 Todesco, “Hyperkommunikation”, 121.
} 


\section{a) Kein konkreter Anfang/Schluß}

Während gedruckte Texte und auch viele webunabhängige digitale Dokumente den Leser in der Regel in einer einzelnen einführenden Sequenz mit Thematik und Protagonisten vertraut machen, weisen die meisten Hypertext-Systeme - gleichgültig ob geschlossen oder offen - mehrere Einstiegsmöglichkeiten auf. ${ }^{328}$ Sie besitzen also im allgemeinen keinen konkreten Anfang und - wie bereits ausgeführt - keinen definitiven Schluß, weshalb Neumann beispielsweise Hypertext als einen Raum "ohne Anfang und Ende, nur mit einer 'ewigen Mitte" ${ }^{, 329}$ sieht.

Gleich zu Anfang muß der Leser sich festlegen, welche Richtung er einschlagen möchte und welche Interessen er zu verfolgen gedenkt. Somit steht er von Anbeginn an unter dem Zwang, Entscheidungen fällen zu müssen. Douglas nennt dies "the burden of interactivity", die den Leser zu keinem Zeitpunkt vergessen läßt, daß er sich in einem virtuellen, dreidimensionalen Raum bewegt. ${ }^{330}$ Der Leser befindet sich also in einer permanenten Anspannung, die vermutlich einem 'Versinken' in der Lektüre, wie dies bei Drucktexten zweifellos der Fall sein kann, entgegensteht. Sicherlich vermag ein Hypertext den Leser zu fesseln, doch es ist fraglich, ob der Zustand der vollständigen Entrückung von Zeit und Raum auch hier erlangt werden kann.

Zudem ist aufgrund der Orientierungslosigkeit, die bei allen Hypertexten ohne Übersichtsschema aufkommen kann, einzukalkulieren, daß der Leser den Leseprozeß vorzeitig abbricht. Er wird sich niemals sicher sein, wirklich alle vom Autor vorgegebenen möglichen Textelemente gelesen zu haben, was dazu führen kann, daß bei der Rezeption dieser Art von Literatur stets ein Gefühl der Unsicherheit oder sogar des Verdrusses mitschwingt. ${ }^{331}$ So geht er womöglich fälschlicherweise davon aus, alle Verknüpfungen verfolgt zu haben, oder aber er wird der Angelegenheit überdrüssig. ${ }^{332}$

328 Douglas, The End of Books, 40.

329 Neumann, "Geisteswissenschaften im Internet", 58.

330 Douglas, The End of Books, 52.

331 Siehe hierzu auch Hautzinger, Vom Buch zum Internet?, 59-61.

332 Letzteres wirft die Frage auf, ob das Hypertext-System einen Autor nicht in besonders hohem Maße dazu zwingt, möglichst fesselnd zu schreiben, da er andernfalls seine Leser verlieren könnte. Offensichtlich sind die Wechselwirkungen zwischen Text, Autor und Leser in bezug auf Hypertexte besonders extrem ausgeprägt. 


\section{b) Flüchtigkeit des Textes}

Das zweite Problem betrifft die Vergänglichkeit der vom Leser gewählten Lesewege: Ein einmal gelesener Text ist in der Regel nicht bzw. nicht ohne weiteres in genau derselben Form reproduzierbar. ${ }^{333}$ Damit erhält jeder Lesevorgang zwar seinen ganz spezifischen Charakter sowie seine individuelle Dynamik, jedoch bringt diese Flüchtigkeit signifikante Nachteile für den Leser mit sich. Nicht nur, daß ein Text auf diese Weise an Authentizität einbüßt, er verkörpert - im Gegensatz zu gedruckten Werken auch niemals etwas Beständiges, an dem der Rezipient sich zu orientieren und festzuhalten vermöchte. Vor allem aber ist der Leser nicht mehr in der Lage, sein Rezeptionserlebnis zu teilen. Die Flüchtigkeit des Textes führt zu einem Verlust der Kommunizierbarkeit; es fehlt der sogenannte common point of reference.

\section{Hypertext-Rezeption}

Was nun bedeuten alle die genannten Vor- und Nachteile für den Leser? Auf welche Weise bewegt er sich durch ein Hypertext-Dokument?

Hypertexte - seien sie in geschlossener oder offener Form konzipiert - können auf unterschiedliche Weise bearbeitet werden. Zum einen ist eine Leseweise möglich, die alle innerhalb des Dokuments offerierten zusätzlichen Informationseinheiten unbeachtet läßt und sich ausschließlich auf den Hauptstrang des Hypertext-Systems konzentriert. In diesem Zusammenhang wird oftmals von linearer Leseweise gesprochen, was allerdings oben bereits als oberflächliche und unpräzise Formulierung charakterisiert wurde, da der Rezeptionsvorgang eigentlich per se eine Beschränkung auf jeweils einen Sachverhalt beinhaltet und somit eine bewußte, gleichzeitige Wahrnehmung zweier Handlungsstränge ausschließt. Vielmehr sollte diese Leseweise eines Hypertextes als widerkonzeptionelle Rezeption bezeichnet werden, da sie die eigentliche Bestimmung dieser Textform ignoriert.

\subsection{Formen der konzeptionsentsprechenden Rezeption}

Bei einer konzeptionsentsprechenden Rezeption ${ }^{334}$ - anders als bei der widerkonzeptionellen - reagiert der Leser flexibel und läßt sich auf die Gestaltung des

333 Douglas, The End of Books, 49.

${ }^{334}$ Der ebenfalls als Alternative in Frage kommende Begriff konzeptionsbejahend trifft nicht den eigentlichen Sinn, da die Bejahung der Normalzustand ist; daher bilden die Bezeichnungen konzeptionsentsprechend oder konzeptionsgemäß die korrektere Variante. 
Textes ein. Diese Bearbeitungsform eines elektronischen Hypertext-Systems kann sequentiell, gezielt oder mehr oder weniger wahllos erfolgen.

Bei einer sequentiellen Verfahrensweise werden alle offerierten Textangebote systematisch nacheinander abgearbeitet. Diese Methode garantiert, daß alle Informationen ausgeschöpft werden, gleichzeitig verlangt sie aber dem Leser die Disziplin ab, sich nicht durch neue Verweise vom eigentlichen Text fortleiten zu lassen.

Bei gezieltem Vorgehen kann die Wahl des Leseweges entweder autorengelenkt oder aber gänzlich autorenunabhängig sein. Dementsprechend werden entweder planmäßig nur jene Informationen weiterverfolgt, die zur Ergänzung eines angestrebten Wissensstandes notwendig sind, wobei die Entscheidungen des Lesers auf Hinweisen des Autors basieren, oder aber der Leser bewegt sich zwar ebenfalls mit Bedacht und intentional durch den Text, ohne hierbei jedoch der "Leseeinladung des Autors" ${ }^{\prime 35}$ nachzukommen. Auch im letzteren Falle kann der Leser auf die Erreichung eines bestimmten Wissensstandes ausgerichtet sein, doch erlangt er sein Ziel unbeeinflußt von Autorenempfehlungen. ${ }^{336}$ Bei Wingert taucht diese bestimmt-unabhängige Leseweise unter der Bezeichnung "strong reading" 337 auf, was die Selbstbestimmtheit des Vorgehens auf recht anschauliche Weise unterstreicht.

Bei mehr oder weniger wahllosen Bewegungen innerhalb eines HypertextSystems schließlich liegt kein konkretes Ziel vor; vielmehr läßt sich der Leser in der Hoffnung, zufällig auf interessante Informationen zu stoßen, oder auch nur zum reinen Zeitvertreib von seiner Intuition leiten:

Instead of a linear, page-by-page, line-by-line, book-by-book approach, the user connects information in an intuitive, associative manner. Hypertext fosters a literacy that is prompted by jumps of intuition and association. ${ }^{338}$

Andere Systematisierungen ergeben zum Teil ebenfalls eine Dreiteilung der Leseweisen.

335 Bernd Wingert, "Der Leser im Hypertext: Im Weinberg oder im Steinbruch?”, in: Suter und Böhler, Hg., Hyperfiction, 159-172, hier: 170.

336 Natürlich bewegt sich der Leser stets innerhalb des vom Autor vorgegebenen Systems und kann insofern strenggenommen niemals gänzlich frei und unbeeinflußt handeln. Gemeint ist damit der systembedingt größtmögliche Entscheidungs- und Aktionsspielraum des Rezipienten.

337 Wingert, "Der Leser im Hypertext", 170.

338 Michael Heim, The Metaphysics of Virtual Reality (New York, 1993), 30. 
Wingert beispielsweise differenziert nach 1.) thematischer Folge, die sich z.B. an der Anordnung der Handlungsträger orientieren kann, 2.) traversaler Leseweise auf der Basis einer autorengelenkten Tour oder der eigenen Inspiration folgend sowie 3.) montierender, d.h. die Abfolge selbst zusammenstellender Rezeptionsform, die auf der leserbestimmten Anordnung der Textelemente beruht. ${ }^{339}$

Wesentlich sprachkreativer präsentiert sich die Unterteilung von Flusser: Er bezeichnet die drei Formen der Textrezeption - nämlich "das vorsichtige Auseinanderfalten, das hastige Überfliegen und das mißtrauische Nachschnüffeln" - als

1. die "kommentierende",

2. die "folgsame" und

3. die "kritische" Leseweise. ${ }^{340}$

1. Unter kommentieren versteht Flusser das Mitdenken des Lesers, der das vom Schreiber gewissermaßen 'An'-Gedachte in seiner Vorstellung verlängert, d.h. weiter- und schließlich zu Ende denkt. ${ }^{341}$

Orientiert man sich am Aspekt des Mitdenkens, dann ließe sich Flussers Form der Textrezeption gegebenenfalls mit gezieltem, autorengelenktem Vorgehen im Hypertext-System vergleichen. Dort werden - den Hinweisen des Autors folgend - nur jene Informationen weiterverfolgt, die zur Ergänzung eines angestrebten Wissensstandes notwendig sind. Damit wird in gewisser Weise auch das vom Autor Gedachte und Intendierte vervollständigt.

2. Das folgsame Lesen richtet sich nach Flusser an der zeilenförmigen Struktur der Texte aus. Der Inhalt erschließt sich dem Leser nur, wenn er dem Text Zeile für Zeile folgt, was allerdings - so Flussers Aussage - nicht nur bei hastigem Überfliegen, sondern auch bei langsamem "Kriechen der Zeile entlang"342 der Fall ist. Im Hinblick auf die Hypertext-Systematik ist diese Art des Lesens mit der sequentiellen Verfahrensweise vergleichbar, nach der alle offerierten Informationen systematisch

\footnotetext{
339 Wingert, "Der Leser im Hypertext", 162.

340 Vilém Flusser, Die Schrift: Hat Schreiben Zukunft? (Göttingen, 1987), 88.

341 Flusser interpretiert den Terminus sehr großzügig und setzt sich damit über dessen etymologische wie gegenwärtige Bedeutung hinweg. Im 17. Jh. wurde kommentieren aus dem Lateinischen entlehnt: commentari = etwas überdenken, Betrachtungen anstellen; auslegen. Mittlerweile hat kommentieren die Bedeutung von erläutern, zu etwas Stellung nehmen, einen Text mit kritischen Anmerkungen versehen. Flusser hingegen beruft sich bei seiner Auslegung auf die Bedeutung von mens (= Denktätigkeit, Verstand; Vorstellung), zu dessen Wortfamilie das lateinische commentari zählt. Flusser, Die Schrift, 89. Günther Drosdowski, Hg., Duden Etymologie: Herkunftswörterbuch der deutschen Sprache, 2., völl. neu bearb. u. erw. Aufl. (Mannheim; Wien; Zürich, 1989 [ $\left.\left.{ }^{1} 1963\right]\right), 365 f$.

342 Flusser, Die Schrift, 89.
} 
nacheinander abgearbeitet werden. Auch dort erschließt sich der komplette Zusammenhang nur dann, wenn der gesamte Text Verknüpfung für Verknüpfung verfolgt wird.

3. Die kritische Leseweise stellt das genau Gegenteil des folgsamen Lesens dar. Dieses Vorgehen macht den Leser sozusagen zum Detektiv. Er deckt die Lügen des Schreibenden auf, indem er wider die Zeilenrichtung liest.

Wollte man auch hier eine Querverbindung zur Hypertext-Systematik herstellen, so müßte man das sogenannte strong reading, d.h. die gezielte, autorenunabhängige Vorgehensweise als Pendant heranziehen. Diese bestimmt-unabhängige Leseweise ließe sich möglicherweise mit kritischem Lesen gleichsetzen, denn der Leser läßt sich dort nicht von den Empfehlungen des Autors beeinflussen, sondern folgt nur seinen eigenen Vorstellungen. Damit liest er ebenfalls gleichsam gegen den vorgesehenen Ablauf und deckt auf diese Weise eventuell auch die Wahrheit auf, die der Autor zu verdecken suchte.

Für welche Form der konzeptionsentsprechenden Rezeption der Leser sich auch immer entscheidet, er stellt sich grundsätzlich einer höheren geistigen Herausforderung, als dies bei widerkonzeptioneller Rezeption der Fall wäre. Anstatt sich wie in der Regel im linear-konzipierten Text - vorwiegend auf den Inhalt zu konzentrieren, muß er zudem die besondere Zeichensprache eines Hypertext-Systems beherrschen und zusätzlich Kenntnis der Softwarefunktionen besitzen. Wie bereits in Kapitel III.3.1. angedeutet, muß der Leser bei der Bearbeitung von Hypertexten also eine höhere geistige Flexibilität aufbringen als bei gedruckten Texten. Genauer gesagt muß er $\mathrm{d}$ re i heterogene, kognitive Leistungen in e in e m Leseproze $ß$ erbringen: Passive Aufnahme des Inhalts, aktive Bewegung durch den Text und Bestimmung verlaufsrelevanter Entscheidungen. ${ }^{343}$

\subsection{Entfaltungsmöglichkeiten des Nutzers}

\subsubsection{Interaktivität}

An dieser Stelle sei der Leser daran erinnert, daß im bisherigen Verlauf des Kapitels V.3. vorläufig nur von geschlossenen literarischen Hypertext-Systemen gesprochen wurde. Diese lassen nur e i n e der beiden möglichen Formen der Nutzeraktivität zu, nämlich die der Interaktivität. Geschlossene Hypertext-Systeme basieren auf einer

343 Böhle, Riehm und Wingert, Vom allmählichen Verfertigen elektronischer Bücher, 321. 
festgelegten Struktur, die sich vom Nutzer nicht verändern läßt, innerhalb der er sich aber frei bewegen kann.

Interaktion ist in unterschiedlichen Ausprägungen möglich. Hesse und Mandl differenzieren hier zwischen vier Formen, nämlich

a) der direkten Navigation, bei der der Nutzer anhand des Zeichensystems durch den Hypertext gelenkt wird,

b) der aktiven Exploration, bei der er auf weiterführende Informationen zurückgreift, wie zum Beispiel Tabellen,

c) der Einbindung von Simulationen zum anschaulicheren Verständnis und als Hilfe zur Problemlösung sowie

d) der direkten Rückmeldung auf Problemlösungsvorschläge des Nutzers ${ }^{344}$.

Zwar orientierten sich Hesse und Mandl bei der Auflistung dieser Formen der Interaktivität an multimedialen Lehr- und Lernumgebungen, es besteht aber kein grundsätzlicher Unterschied zu literarischen Hypertext-Systemen: Dort verlaufen die Fortbewegung durch den Text, das Auskundschaften der Zusatzinformationen und die Ergebniskontrolle ${ }^{345}$ nach denselben Prinzipien; einzig das Anschauungsmaterial hat vorzugsweise audiovisuellen Charakter.

Grundsätzlich beziehen interaktive Hypertexte den Rezipienten in das Werk mit ein, weshalb die Kunst der Hyperkultur nicht als 'Leser'-Kunst bezeichnet zu werden pflegt, sondern als ein Erlebnis, das sich durch die Interaktion von Kunstwerk und Leser ergibt. ${ }^{346}$ Das individuelle - da immer wieder andersartig erscheinende - Gesamtwerk ergibt sich aus der gegenseitigen Beeinflussung von Werk und Rezipient; der Leser bestimmt durch Anklicken der textinhärenten Verknüpfungen,

344 Friedrich W. Hesse und Heinz Mandl, unter Mitarbeit von Gabi Reinmann-Rothmeier und Steffen-Peter Ballstaedt, "Neue Technik verlangt neue pädagogische Konzepte: Empfehlungen zur Gestaltung und Nutzung von multimedialen Lehr- und Lernumgebungen”, in: Bertelsmann Stiftung und Heinz Nixdorf Stiftung, Hg., Studium Online, 31-49, hier: 44. Diese Form der Interaktion bezieht sich in erster Linie auf Lehr- und Lernprogramme mit Aufgaben und Übungen. Dort wird der Nutzer in der Regel durch direkte Rückmeldungen über die Richtigkeit seiner Lösungen informiert. Im besten Falle erhält er sogar eine individualisierte Rückmeldung mit speziellen Hinweisen zu seinen einzelnen Fehlern sowie konkreten Vorschlägen zu ihrer Beseitigung.

345 Die in Kapitel IV.2.2. dargestellte Gedicht-Maschine von Seidel illustriert als ein Beispiel für programmgesteuerte Literatur mit Interaktionsmöglichkeiten anschaulich die Funktionalität unmittelbarer Rückmeldung auf die Eingaben des Nutzers. Seidel, Heretical Rhyme Generator, $<$ http://www.pangloss.com/seidel/Poem/>, 05.07.2003.

346 Martin Klepper, Ruth Mayer und Ernst-Peter Schneck, “Glossar”, in: Dies., Hg., Hyperkultur, 267-285, hier: 277. 
welchen weiteren Verlauf das Leseerlebnis nehmen soll, wird seinerseits gleichzeitig aber auch durch den Inhalt der jeweiligen Textelemente beeinflußt.

Wie bereits an anderer Stelle, muß auch hier betont werden, daß der Entscheidungsfreiheit des Lesers klare Grenzen gesetzt sind, da er sich notwendigerweise immer innerhalb des vom Autor vorgegebenen Rahmens bewegt. In Kapitel III.2.1. wurde bereits angedeutet, was hier und jetzt unübersehbar wird: die In t e raktion zwischen Kunstwerk - besser gesagt dessen Gestalter, also dem Autor - und Leser beschränkt sich auf eine reine R e aktion des Lesers auf die Vorgaben des Autors.

Dies hat offensichtlich dazu geführt, daß - wie auch bei dem Wort Literatur Bestrebungen dahin gehen, den Begriff Interaktivität nicht vorbehaltlos weiterzuverwenden, sondern ihn durch eine treffendere Bezeichnung zu ersetzen. Aarseth beispielsweise schlägt vor, anstelle von interaction künftig Bezeichnungen wie participation, play oder auch use zu gebrauchen. ${ }^{347}$ Diesen Vorschlägen entsprächen im Deutschen vermutlich die Bezeichnungen Teilnahme, Spiel oder Nutzung.

Auch wenn der Begriff Interaktion nicht uneingeschränkt überzeugen kann, so stellen die Vorschläge von Aarseth doch keine sachangemessenen Alternativen dar, denn bei den Vorgängen innerhalb eines (geschlossenen ebenso wie offenen) Hypertext-Systems handelt es sich tatsächlich in gewisser Weise um eine Art Wechselbeziehung zwischen Kunstwerk und Leser. Aus diesem Grunde scheint es angeraten, die bereits bestehende und allgemein gebräuchliche Bezeichnung beizubehalten.

\subsubsection{Kooperativität}

Für eine Beibehaltung der Bezeichnung Interaktivität spricht zudem die Notwendigkeit der Abgrenzung von einer weiteren Form des Hypertext-Lesens, für die der Begriff der Kooperativität verwendet wird. ${ }^{348}$ Er steht für die Idee der aktiven und befruchtenden Zusammenarbeit zwischen Autoren bzw. Herausgebern und Lesern und bezeichnet eine Form der Nutzeraktivität, die nur bei offenen Hypertext-Systemen stattfinden kann. Diese sind in der Regel so konzipiert, daß sie nicht nur Zugriffe auf system-

\footnotetext{
347 Aarseth, Cybertext, 49.

348 Heiko Idensen, "Schreiben/Lesen als Netzwerk-Aktivität. Die Rache des (Hyper-)Textes an den Bildmedien", in: Klepper, Mayer und Schneck, Hg., Hyperkultur, 81-107, hier: 104. Christiane Heibach, “'Creamus, ergo sumus' - Ansätze zu einer Netz-Ästhetik”, in: Suter und Böhler, Hg., Hyperfiction, 101-112, hier: 110. Hesse und Mandl, "Neue Technik verlangt neue pädagogische Konzepte”, 48.
} 
interne und -externe Verknüpfungen zulassen, sondern ihr wesentliches Merkmal liegt darin, daß sie Benutzereingriffe in die bestehenden Systemstrukturen gestatten. ${ }^{349}$

Heibach unterscheidet sechs verschiedene Umgebungen, die eine Kooperation seitens des Nutzers erlauben: ${ }^{350}$

1. Feedback und Diskussion

2. Kontrolliert-partizipatives Projektnetz (redaktionell geleitete Gestaltungsfreiheit in vorgegebenen Themenrahmen)

3. Non-partizipatives Work-in-Progress (kontinuierlich fortgeschriebenes Werk als Ergebnis aus Künstler-Dialogen)

4. Mitschreibeprojekt (Fortschreiben bereits existierender Werke)

5. Explorativ-partizipatives Werk (der Nutzer als werkveränderndes Moment)

6. Partizipative Kommunikationsumgebung (der Nutzer als integraler Mitgestalter)

Sie nimmt damit eine sehr differenzierte Unterteilung vor, innerhalb derer sie - unter Punkt 3. - sogar eine ausschließlich Autoren vorbehaltene Form der Mitwirkung in Ansatz bringt.

Die vorgenannten sechs Formen des Benutzereingriffs werden von Heibach ausdrücklich als Gegenpol zur Interaktivität verstanden; sie möchte damit ihren Wert deutlicher hervorheben und sie absetzen von jenen (vorgeblichen) Möglichkeiten der Mitgestaltung, die nicht mehr zulassen als unkreatives Anklicken von Verknüpfungen.

In ihrem Anliegen ist Heibach uneingeschränkt zu unterstützen. Nicht mitzutragen ist jedoch ihre Verwendung der Bezeichnung Partizipation. Heibach greift

${ }^{349}$ Kooperativität ist also als eine Art Weiterentwicklung von Interaktivität anzusehen. Gilster hingegen vertritt eine abweichende Auslegung der grundlegenden Eigenschaften des WWW. Seiner Ansicht nach stellt Interaktivität eine der drei Hauptmerkmale des Web dar: "All three start with the letter I: interactivity, immediacy, and integration. Interactivity, because a welldesigned site lets you talk to the players and conceivably influence the way a particular situation is handled; interactivity also means being able to choose your own path through the site. Immediacy, because a frequently updated Web site can put you on the scene of a continuing story, just as I check in every day to track the progress of a cyclist through the Australian outback. And finally, integration, because a good Web site exploits varied forms of media to support its message." Gilster, Digital Literacy, 154. Hinsichtlich der Attribute Unmittelbarkeit und Integration ist Gilster uneingeschränkt zuzustimmen, dagegen ist Widerspruch angebracht, was seine Definition von Interaktivität betrifft. Die Aktivitäten eines Nutzers dürfen sich nicht auf selbstbestimmtes Agieren beschränken, vielmehr sollte das Web als offenes Hypertext-System ausdrücklich auch die Möglichkeit zu mitgestalterischer Kreativität eröffnen. Genau darin unterscheidet es sich nämlich von webunabhängigen, geschlossenen Hypertext-Systemen.

${ }^{350}$ Heibach, "“Creamus, ergo sumus"”, $107 \mathrm{ff}$. 
damit auf dasselbe Wort zurück, das Aarseth bei seiner Suche nach einer möglichen Alternative für den Terminus Interaktivität favorisiert. Wie im vorangehenden Abschnitt bereits festgestellt wurde, ist der Ausdruck Partizipation nicht geeignet, die Bedeutung der - eingeschränkten - Form der Nutzeraktivität (im geschlossenen Hypertext-System) überzeugend zum Ausdruck zu bringen; noch viel weniger taugt die Bezeichnung Partizipation zur Erfassung strukturverändernder Benutzereingriffe (im offenen Hypertext-System). Die 'Teilnahme' (Partizipation) eines Nutzers an einem Hypertext-'Ereignis' kann sich auch auf reines Miterleben ohne aktive Mitgestaltung beschränken; bei offenen Hypertext-Systemen sollte der Grad der Aktivität jedoch eindeutig darüber hinausgehen und entsprechend im Terminus zum Ausdruck kommen. ${ }^{351}$

Aus diesem Grunde scheint das Wort Kooperation geeigneter zu sein, die kreativen Möglichkeiten des Nutzers in den offenen, digitalen Literatursystemen angemessen darzustellen. ${ }^{352}$

\subsection{Text - Kontext - Hypertext}

Im bisherigen Verlauf dieser Arbeit wurde versucht, gewisse Kategorien bei der Strukturierung und Gestaltung von Hypertexten sowie deren Wirkung auf den Rezipienten zu unterscheiden. Sie werden - wie die Autorin hofft - eine solide Basis für die detaillierter angelegten Ausführungen zu ausgewählten Werken der webbasierten Literatur in Kapitel VI. sein. Zum besseren Verständnis der modernen

351 Dies ist bei der Bezeichnung Kollaboration durchaus gegeben. Sie wird beispielsweise verwendet von Schröder, "Der Link als Herme und Seitensprung", 46 und 48, von Ruth Nestvold, "Der neue Autor. Die elektronische Gemeinschaft und das Ende des einsamen Schriftstellers", in: Griem, Hg., Bildschirmfiktionen, 205-221, hier: 213ff. oder von Heiko Idensen, "Hyper-Scientifiction", in: Suter und Böhler, Hg., Hyperfiction, 61-83, hier: 76. Was die Nutzer-Aktivität im Hypertext-System betrifft, gibt es keinen Bedeutungsunterschied zwischen Kooperation und Kollaboration, so daß an dieser Stelle durchaus auch für die Verwendung des Ausdrucks Kollaboration hätte plädiert werden können. Aufgrund des (primären) Sinngehaltes dieses Begriffs (Kollaboration = Zusammenarbeit mit dem Feind) soll hier der Bezeichnung Kooperation (= Mitwirkung, Zusammenarbeit) der Vorzug gegeben werden.

352 Hesse und Mandl gehen der Frage nach, welche Fähigkeiten einem Lernenden auf der allgemeinen, medienunspezifischen Ebene dienlich sein können, und bezeichnen schließlich vier Attribute als wesentlich: Neben a) Eigenverantwortlichkeit, b) Selbststeuerung und Selbstkontrolle sowie c) selbstkonstruierendem Vorgehen zählt ihrer Meinung nach d) interaktives und kooperatives Arbeiten zu den erfolgbestimmenden Kompetenzen. Hesse und Mandl, "Neue Technik verlangt neue pädagogische Konzepte", 48. Interessant ist, daß Hesse und Mandl Interaktivität und Kooperativität nebeneinander stellen und damit gleichsam als (Gegensatz-)Paar präsentieren. Diese Art der Darstellung hebt die unterschiedliche Wertigkeit der beiden Formen der NutzerAktivität deutlich hervor und legt unmißverständlich dar, daß die Bezeichnungen Interaktivität und Kooperativität keinesfalls austauschbar sind. 
Literaturform seien jedoch zuvor noch einige abrundende Bemerkungen zur Beziehung zwischen Kontext und (Hyper-)Text gestattet.

Wie in Kapitel IV.1. beschrieben wurde, verfügen die beiden Darbietungsformen analoger Veröffentlichungen per definitionem über einen identischen Inhalt, d.h. die gedruckte Ausführung eines Textes ist absolut wortgleich mit der elektronischen Version. Der Unterschied zwischen beiden Darstellungsvarianten wird umso weniger wahrgenommen, als die elektronische Fassung oftmals auf Papier ausgedruckt wird. Beide Publikationsformen können aber ungeachtet ihrer inhaltlichen Übereinstimmung auch unterschiedliche Empfindungen beim Leser hervorrufen.

Birkerts gibt den Zusammenhang zwischen Form und Inhalt mit folgenden Worten wieder: "Übermittlungsweise bestimmt Empfangsweise bestimmt Reaktionsweise." ${ }^{353}$ Unabhängig von der Gewichtigkeit des Inhalts kann das Wort auf dem Computerbildschirm nicht die gleiche Unabänderlichkeit und Verläßlichkeit entwickeln wie das gedruckte Pendant. Sein Status wird vielmehr zwangsläufig geprägt durch die technischen Spezifika des Trägermediums. Dessen als positiv empfundene Eigenschaften der Anpassungsfähigkeit und hohen Aktualität, die auf der schnellen Veränderbarkeit des Dargestellten beruhen, bilden zugleich einen negativen Kontext. Die Wahrnehmung des elektronischen Wortes wird nämlich mitbestimmt von dem unterbewußten Wissen, daß es Teil einer labilen Umgebung ist. Auf dem Bildschirm existiert das Wort immer nur "potentialiter", während das gedruckte Wort "actualiter" an seiner ihm zugewiesenen Stelle immer wiederzufinden ist. ${ }^{354}$ Sobald der Bildschirm erlischt, verliert das Dargestellte gewissermaßen seinen Raum und mithin auch sein Gewicht. Auch wenn dieser Effekt möglicherweise nicht bewußt wahrgenommen wird, so ist doch davon auszugehen, daß das Darstellungsmedium die Rezeption beeinflussen kann. ${ }^{355}$ Es ist somit davon auszugehen, daß elektronische Texte aufgrund ihrer Flüchtigkeit und Vergänglichkeit grundsätzlich eine andere Wirkung ausüben als Print-Texte.

Das Wissen um diese Umstände ist notwendig, um zu verhindern, daß sich die nachfolgende Analyse webbasierter Literatur ausschließlich auf die inhaltliche Aussage konzentriert und dabei den Einfluß des Präsentationsmediums unberücksichtigt läßt. Existenz und Wirkungsfähigkeit von Hypertext-Werken - und ganz

\footnotetext{
353 Birkerts, Die Gutenberg-Elegien, 207.

354 Ebd., 210.

355 Vgl. hierzu auch Birkerts, Die Gutenberg-Elegien, $173 \mathrm{f}$.
} 
besonders jener in offenen Systemen - stehen in direkter Abhängigkeit zu ihrem Trägermedium. Sie dürfen demzufolge also auch nur als untrennbares Paar gesehen und interpretiert werden.

Umgekehrt darf die verstärkte Beschäftigung mit der Vielfalt der neuen multimedialen Hypertext-Systeme nicht grundsätzlich $\mathrm{zu}$ einer geringeren oder oberflächlicheren Beschäftigung mit den ehemals - und möglicherweise eigentlich wichtigen inhaltlichen Fragen eines Werkes führen. Wird nämlich die Aufmerksamkeit durch die Präsentationsform gebunden, kann damit die inhaltliche Aussage leicht in den Hintergrund treten. 


\section{Webbasierte Literatur - Analyse und Bewertung anhand konkreter Fallbeispiele}

Alles 'umsonst'?

Einer der Vorzüge von webbasierter Literatur ist in der Regel der freie, kostenlose Zugang. Allerdings ließ die Suche nach diskussionsgeeigneten Werken von Anfang an immer wieder die Befürchtung aufkommen, das Projekt Webbasierte Literatur im Rahmen vorliegender Arbeit werde sich letztlich als ergebnislos herausstellen. Was eigentlich als kostenbezogene Aussage über den Zugang zu webbasierten literarischen Werken gedacht war, erwies sich auf weite Strecken als umgangssprachliche Beschreibung für ausgesprochen ernüchternde Rechercheergebnisse:

Alles 'umsonst'!

Das Fehlen fester Wertmaßstäbe auf dem Gebiet der webbasierten Literatur erschwert die Auswahl und Analyse einzelner Werke oder Schreibprojekte in nicht $\mathrm{zu}$ unterschätzender Weise. Zwar wurde in Kapitel III. festgestellt, daß webbasierte Literatur trotz ihrer mannigfachen Andersartigkeit prinzipiell so große Ähnlichkeit mit den traditionellen Literaturformen aufweist, daß sie durchaus noch als Literatur $\mathrm{zu}$ bezeichnen ist; demzufolge sollten Text und Textaussage eines HypertextSystems zwangsläufig auch mit den Mitteln und Methoden der Literaturwissenschaft $\mathrm{zu}$ erschließen sein. Bislang völlig ungeklärt ist aber, inwieweit die Dynamik des Web sowohl die Auswahl als auch die anschließende Interpretation beeinflußt.

So ist beispielweise fraglich, welche Kriterien im Rahmen der vorliegenden Arbeit die Entscheidung für ein Werk oder Projekt bestimmen sollten. Ginge es etwa darum, nach dem jeweiligen literarischen Werte auszuwählen, so müßte zunächst geklärt werden, wie und anhand welcher Merkmale dieser Wert festzusetzen wäre. Rezensionen stehen oftmals nicht zur Verfügung, die Gestaltung der Web-Seiten läßt keine Rückschlüsse auf den literarischen Gehalt zu, der persönliche Eindruck ist rein subjektiv und Zahlen zur Nutzerfrequenz - sofern sie denn vorhanden sind - haben keinen Aussagewert, da eine hohe Zugriffszahl nicht notwendigerweise auch eine herausragende Qualität bedeuten muß. Ebenfalls unergiebig ist die Frage nach dem eventuellen repräsentativen Charakter eines Werkes, da sich bislang noch kein Kanon herausgebildet hat und die Werke beziehungsweise Projekte zudem einen derart unterschiedlichen Charakter und solch individuelle Dynamik aufweisen, daß das Kriterium der Repräsentativität schon aus diesem Grunde nicht greifen kann. 
Ferner besteht Unklarheit, bis zu welchem Grad die Charakteristika des Hypertext-Mediums World Wide Web in die Analyse miteinfließen sollten. Gerade in dieser Hinsicht müssen mehrere Punkte berücksichtigt werden:

1. Wie bedeutsam ist das Ausmaß der Vernetzung innerhalb eines HypertextSystems?

2. Wie entscheidend ist der Anteil von Bild und Ton?

3. Inwieweit erhält der Leser Spielraum und Möglichkeit zur Kooperation?

Unbestimmt ist ebenfalls, ob die Untersuchungen ausschließlich auf das literarische Ergebnis zielen oder ob das Interesse vielmehr dem experimentellen Vorgang der kooperativen Zusammenarbeit gelten sollte. Der Durchführung eines Mitschreibprojekts können nämlich sehr unterschiedliche Motive zugrundeliegen:

\section{a) Erlebnisorientierte Kooperation}

Hier dient das Projekt ausschließlich der experimentellen Erfahrung. In diesem Falle ist das Ergebnis der kooperativen Zusammenarbeit nebensächlich; statt dessen ist gewissermaßen der Weg das Ziel. Hier geht es in erster Linie um das Miteinander, um das Gemeinschaftserlebnis, den erlebten Augenblick. Was zählt, ist die Momentaufnahme und nicht das Gesamtwerk. ${ }^{356}$

\section{b) Zielorientierte Kooperation}

Hier ist das Konzept zielgerichtet und ergebnisorientiert. Das Bestreben der Initiatoren geht dahin, zu irgendeinem Zeitpunkt ein abgeschlossenes literarisches Gesamtwerk vorliegen zu haben. Nicht selten gelangt dieses Werk nach seiner Fertigstellung in den Druck, womit oftmals auch die Beendung der Online-Präsentation verbunden ist, das heißt die jederzeitige und kostenfreie Verfügbarkeit ist fortan nicht mehr gegeben.

Innerhalb der zielorientierten Kooperativität lassen sich abermals zweierlei Vorgehensweisen unterscheiden:

356 So wird beispielsweise von einem Projekt kooperativer Zusammenarbeit berichtet, in dem es offensichtlich nur um Augenblicks-Erfahrungen ging: "[...] the Hivemind project did not attempt to produce a final product of any merit. Participating in the Hivemind was entirely about the ephemeral experience - not about producing a lasting work of literary merit." Dementsprechend werden die Schreibhandlungen im Rahmen dieses Projekts mit "experimental free jazz sessions" verglichen. Chris Holstrom, "Collaborative Fiction Writing as an Example of Online Community", in: Ders., Hg., The Mahoney Project ([ $\left.\left.{ }^{1} 2002\right]\right),<\underline{\text { http://www.cholstro.net/mahoney/thesis3.shtml }>, 09.07 .2003 .}$ 


\section{a. Eingeschränkte Textkenntnis}

Hier ist das Projekt dergestalt konzipiert, daß immer nur der zuletzt eingegebene Beitrag für die Allgemeinheit sichtbar ist. Das Gesamtwerk bleibt verborgen und steht ausschließlich den Initiatoren zur Verfügung. Jeder Bearbeiter kann sich also nur an der Eingabe seines Vorgängers orientieren.

So ist das Endprodukt zwar das Werk vieler Koschreiber, aber es kann immer nur jeweils eine einzige Person an dem Text arbeiten. Erst wenn diese ihren Beitrag fertiggestellt hat, kann das nächste Teilstück folgen.

Die Kenntnis des Lesers beziehungsweise potentiellen Koautors auf den jeweils letzten Textabschnitt zu reduzieren, bleibt allerdings zwangsläufig nicht ohne Einfluß auf das Gesamtwerk: So kann dieses leicht zu einem bruchstückhaften Zufallsprodukt geraten, das Zusammenhang und Folgerichtigkeit vermissen läßt. ${ }^{357}$

Ungeachtet des experimentellen Charakters und der Zufälligkeit der Ergebnisse ist diese Art der Konzeption gleichwohl ziel- und ergebnisorientiert, insofern sie darauf ausgerichtet ist, letztlich zu einem - irgendeinem! - Ergebnis zu führen.

\section{ß. Uneingeschränkte Textkenntnis}

Ist das literarische Werk allerdings zu jedem Zeitpunkt seines Entstehens uneingeschränkt einsehbar, so sind zumindest alle Voraussetzungen für ein schlüssiges Resultat gegeben. Jeder potentielle Mitschreiber kann sich alle notwendigen Kenntnisse hinsichtlich des Inhalts und der Charaktere verschaffen und seinen Beitrag demzufolge kontextentsprechend und folgerichtig konzipieren. Das Ergebnis stellt im Idealfall ein harmonisches Gesamtkunstwerk darstellen, das von der Befruchtung gemeinschaftlichen Schreibens profitiert, jedoch ohne Werteinbuße durch unterschiedliche Schreibstile und verschiedenartige Intentionen hinsichtlich Inhalt und Aussage des Werkes.

357 Die Musikgruppe Wilco bediente sich dieser Methode, um auf dem Wege der verdeckten kooperativen Zusammenarbeit sogenannte Zufallsdichtung - "accidental poetry" - zu erschaffen. Nicht selten flossen die Ergebnisse dieser Experimente in die Texte ihrer Lieder ein. Holstrom, "Collaborative Fiction Writing as an Example of Online Community”, <http://www.cholstro.net/mahoney/thesis3.shtml>, 09.07.2003. 
Falls ein webbasiertes Werk gleichermaßen auf ein literarisch akzeptables Ergebnis wie auf den experimentellen Vorgang der kooperativen Zusammenarbeit hin angelegt ist, müßte daher zunächst eine Norm festgelegt werden, die sowohl die Gewichtigkeit der beiden Modi als auch die Reihenfolge ihrer Bewertung sowie die Art und Weise der Analyse vorgibt.

\section{c) Gelenkte zielorientierte Kooperation}

Dieses Konzept basiert auf einer gezielten Arbeitsteilung. Zu diesem Zwecke wird das Kooperationsprojekt während des gesamten Verlaufs von einem festgelegten Teilnehmerkreis bearbeitet. Jeder Mitstreiter bekommt einen bestimmten Teilbereich zugewiesen, den er - möglicherweise seinen Kompetenzen entsprechen - gestaltet. Dabei ist es durchaus möglich, daß sich mehrere Personen die Verantwortung für einen Bereich teilen. Unabdingbar ist in jedem Falle die Absprache aller Mitarbeiter untereinander, um auch bei dieser Art der Projektgestaltung eventuelle Brüche in Zusammenhang und Folgerichtigkeit zu vermeiden.

Für die nunmehr gebotene Analyse und Auswertung anhand konkreter Fallbeispiele sei folgendes Arbeitsschema zugrunde gelegt:

1. Die Analyse der webbasierten Werke erfolgt in Anlehnung an die traditionellen Gattungsbegriffe in den drei Bereichen Erzählliteratur, Lyrik und Drama. Dabei soll untersucht werden, ob diese Gattungen, die sich per Konvention herausgebildet haben, mit den ihnen zugeschriebenen kennzeichnenden Merkmalen auch in webbasierter Form zu finden sind.

2. Alle Untersuchungen werden eingeleitet durch eine kurze Präsentation der WebSeite, in die das jeweilige Werk eingebettet ist. In diesem Zusammenhang werden auch der oder die Herausgeber vorgestellt sowie die Hintergründe und Ziele des Online-Projektes dargelegt.

3. Es folgen die Vorstellung des Werkes und eine Analyse unter Einbeziehung nachstehender Fragen:

- Wie und in welchem Maße kommt die Hypertext-Systematik zum Tragen und wie hoch ist der Anteil der hypermedialen Elemente?

- Welchen Bewegungs- und Kooperationsspielraum hat der Leser?

- Welche Wirkungen erzielen diese Möglichkeiten, und erweisen sich die Aktionsformen als vorteilhaft oder behindern sie den Leser eher während seines Rezeptionsvorganges? 
4. Abschließend wird jede der drei Literaturformen webbasierte Erzählliteratur, webbasierte Lyrik und webbasiertes Drama im Hinblick auf ihre spezifischen Eigenschaften und deren Entfaltungsmöglichkeiten im Web kritisch bewertet:

- Bringen die Charakteristika des Trägermediums WWW die Spezifika des Dargebotenen in überzeugender und vor allem bereichernder Weise zum Ausdruck?

- Inwieweit erweisen sich die Präsentationsmöglichkeiten des elektronischen Hypertext-Systems im Hinblick auf die genannten Literaturformen als nützlich und vorteilhaft gegenüber den Möglichkeiten des Print-Systems?

\section{Webbasierte Prosa}

Hypertext-Systematik und World Wide Web besitzen das Potential, eine Erzählung durch Bewegung, Wechsel und Nutzereingriffe zu einem Ereignis zu machen, das der Web-Besucher unmittelbar miterleben und mitgestalten kann. Auf diese Weise sollte die vergleichsweise starre Struktur einer Erzählung aufbrechen, es sollte mit anderen Worten ein Übergang von der Erzählung als einem Endprodukt literarischen Schaffens zu einem Entwicklungsprozeß stattfinden. Der Herausgeber webbasierter Prosa muß seine Erzählung folglich mit einer besonders sorgfältig durchdachten und flexiblen Hypertext-Struktur ausstatten, um dem Interagierenden nicht das Gefühl zu vermitteln, mit einer bloßen Aneinanderreihung von "Datenclustern" ${ }^{358}$ konfrontiert zu sein.

Aus diesem Grunde erfordert webbasierte Prosa in der Regel allein aufgrund ihres Umfangs besondere Weitsicht, fundierte Programmierkenntnisse und vor allem Ausdauer. Allerdings kann es geschehen, daß sich der Initiator nach einer gewissen Zeit der Werkpräsenz im World Wide Web hinter den Kooperierenden, das heißt seinen Koautoren $\mathrm{zu}$ verlieren beginnt; bereits in Kapitel I. dieser Arbeit wurde Woolleys These angesprochen, im Web hebe sich der Unterschied zwischen Autoren und Lesern auf. ${ }^{359}$

\footnotetext{
358 Krahberger, "Hypertext, Hyperraum, Kulturentwicklung", $<$ http://ezines.onb.ac.at:8080/ejournal/pub/ejour-97-II/buecher/babyl/hypertext.html $>, 31.01 .2003$.

359 Woolley, Die Wirklichkeit der virtuellen Welten, 178.
} 
$\mathrm{Ob}$ es zu einer Gleichstellung oder gar einer Vormachtstellung der Kooperierenden kommt, sei dahingestellt, zumindest stellt sich das Verhältnis zwischen Verfasser und Rezipient online anders dar, als dies offline der Fall ist. Beispielsweise wird der Leser beziehungsweise Nutzer webbasierter Prosa häufig direkt angesprochen und zu einer Entscheidung herausgefordert. Fragen im Sinne von "What do you think happened next?" sollen ihn aktiv in das Geschehen einbinden und seine Identifikation mit der einen oder anderen Figur forcieren. ${ }^{360}$ Die direkte Anrede ist zwar auch ein Element der gedruckten Prosa, doch dient sie im Web als Movens für Aktivität - entweder in Form von Interaktivität oder Kooperativität -, denn der Fortgang der Erzählung wird allein durch die Entscheidung beziehungsweise Kreativität des Nutzers bestimmt. ${ }^{361}$

Gelegentlich kann Literatur im Web - was manchmal übersehen wird - auch eine Bevormundung des Interagierenden bedeuten, zum Beispiel wenn sie in "eine Art Konkurrenzkampf mit dem Leser" ${ }^{\prime 32}$ tritt und ihm durch automatischen Seitenwechsel nicht genügend Zeit zur Entscheidung läßt. Nestvold kommt in diesem Zusammenhang ähnlich wie Krahberger zur Definition von hyper als einer Zustandssteigerung $^{363}$, hier allerdings mit unmißverständlich kritischem Unterton:

Dieser 'lebendige' Text, der der umgangssprachlichen Bedeutung des Wortes 'hyper' entspricht und leicht 'hyperaktiv' wirkt, stellt auch für geübte Leser von HypertextWerken eine Herausforderung dar. ${ }^{364}$

Anders die webbasierte Literatur. Im Gegensatz zur computergestützten, webunabhängigen Literatur im Web eröffnet sie dem Rezipienten deutlich erweiterte Kompetenzen; durch ihre Mitgestaltungsmöglichkeiten liefert sie ihn der Willkür des Autors in weit geringerem Maße aus. Sache des Nutzers ist es, die erweiterten Kompetenzen nicht nur als eine Offerte zu begreifen, sondern als persönliche Herausforderung. Damit werden dem Rezipienten gesteigerte Aufmerksamkeit und Experimentierfreudigkeit abverlangt.

\footnotetext{
360 Nestvold, "Der neue Autor", 211.

361 Vgl. Ebd., 211 f.

362 Ebd., 212.

363 Siehe auch Kapitel III.2.1. vorliegender Arbeit. Krahberger, "Hypertext, Hyperraum, Kulturentwicklung", <http://ezines.onb.ac.at:8080/ejournal/pub/ejour-97-II/buecher/babyl/hypertext.html>, 31.01.2003.

364 Ebd., 31.01. 2003.
} 
Welche Dimensionen Hypertext in Literaturkonzeption und -rezeption en détail eröffnet, sei am Beispiel zweier Projekte untersucht, die aufgrund ihrer ausgefallenen Thematik, ihrer innovativer Konzeption und der professionellen Gestaltung bestechen. Beide Projekte sind noch im Verlauf begriffen und gewähren damit Einblick in den Entstehungsprozeß von Prosaliteratur, die im, durch und über das Web erstellt wird.

Im Falle des ersten Beispiels - Mahoney the Cat - handelt es sich um eine Erzählung der Gegenwart, deren Veröffentlichung im World Wide Web zum Zwecke der allgemeinen Verbreitung und gemeinschaftlichen Ergänzung durch Herausgeber und Kooperierende erfolgt ist.

Das zweite Beispiel - The Diary of Samuel Pepys - verfolgt ein grundlegend anderes Ziel: Der Herausgeber des Projekts präsentiert bereits abgeschlossene Literatur in Form von Teilen des bekannten Tagebuchs aus dem 17. Jahrhundert, die eine Fortentwicklung der Handlung weder fordern noch gestatten. Hier geht es vielmehr darum, die Sekundärliteratur zum Tagebuch von Samuel Pepys fortzuschreiben. Was in The Diary of Samuel Pepys der Zusammenarbeit von Herausgeber und WebBesuchern entspringt, kann in dieser Form ausschließlich mit Hilfe von Globalität und Kooperativität des WWW entstehen. Da die Publikation die wesentlichen Voraussetzungen für eine webbasierte Veröffentlichung erfüllt, soll auch sie als wertvolles Beispiel webbasierter (Prosa-)Literatur Eingang in vorliegende Arbeit finden.

\subsection{Ausgewählte Beispiele webbasierter Lyrik}

\subsubsection{Mahoney the Cat}

Mahoney the $\mathrm{Cat}^{365}$ ist ein privates Projekt kooperativer Zusammenarbeit im World Wide Web, das im Februar 2002 von Chris Holstrom initiiert wurde. Es dient der Wahrung einer Familiengepflogenheit, anknüpfend an eine zauberhafte Tiergeschichte, die für Holstrom so eigentümlich und untrennbar mit glücklichen Kindheitserinnerungen verbunden ist, daß er sie zur allgemeinen Verbreitung und Fortführung im Web veröffentlichte. Das Projekt bildet eine Art Hommage an Holstroms verstorbenen Vater Peter Holstrom, der zur abendlichen Unterhaltung seiner beiden Söhne die wundersamen Abenteuer eines Katers erdachte: "The purpose of the

\footnotetext{
365 Chris Holstrom, Mahoney the Cat ([ $\left.\left.{ }^{1} 08.02 .2002\right]\right), \quad<$ http://www.mahoneythecat.org/>,
} 15.09.2003. 
Mahoney the Cat project is to bring my father's stories to a wide audience." ${ }^{\text {366 }} \mathrm{Im}$ Gedenken an den Vater und in Fortsetzung der von ihm bereits begonnenen Aufzeichnung der zunächst nur mündlich überlieferten Geschichten soll Mahoney the Cat einem breiten Publikum zugänglich gemacht werden: "His stories, however, shouldn't be forgotten. They are magical." 367

\subsubsection{Präsentation}

Mahoney the Cat ist ein webbasiertes Forum, das dem Nutzer sowohl Interaktivität als auch Kooperativität erlaubt. Diese Aktionsmöglichkeiten klingen programmatisch im ausführlichen Titel des Projekts an - Mahoney the Cat ... And What Do You Think Happened Next? - und signalisieren dem Nutzer, daß sich seine Aktivität innerhalb des Forums nicht auf bloße Rezeption beschränken sollte. Vielmehr ist der Nutzer dazu eingeladen, die Geschichte des Katers Mahoney über diverse Einzelkapitel hinweg $\mathrm{zu}$ verfolgen und gegebenenfalls eine eigene Fortsetzung $\mathrm{zu}$ verfassen. Notwendige Voraussetzung ist lediglich eine intensive Vertrautheit mit dem vorliegenden Kontext und den beteiligten Charakteren, so daß ein schlüssiger und logischer Handlungsverlauf gewährleistet ist. Zudem ist der Nutzer gehalten, das Vermächtnis von Peter Holstrom zu respektieren und zu wahren: "All that I ask is that you write with the essence of the story and my father's legacy in mind." 368 Darüber hinaus wird von den Kooperierenden ganz allgemein erwartet, daß sie mit ihren Beiträgen keinen Anstoß erregen. Um sicher zu gehen, äußert Holstrom in seinen Vorbemerkungen die dezente Bitte: "Just make sure that your additions are in good taste." 369

Die Web-Seiten sind übersichtlich und ansprechend gestaltet und lassen Liebe und Begeisterung für die Thematik erkennen. Die Einstiegsseite ist gemäß der Struktur des gesamten Forums in drei Teilbereiche gegliedert: 1. den Lese- und Kooperationsbereich Read \& Write, 2. die Rubrik About mit Verknüpfung zu Hintergrundinformationen und 3. das Portal zum internen Nutzerabschnitt Sign-In beziehungsweise das Nutzerkonto Welcome.

\footnotetext{
366 Ders., "How Mahoney started", in: Ders., Hg., Mahoney the Cat ([ $\left.\left.{ }^{1} 2002\right]\right)$, $<$ http://www.mahoneythecat.org/creator.asp $>$, 15.09.2003.

367 Ebd., 15.09.2003.

368 Ebd., 15.09.2003.

369 Ders., "About the story", in: Ders., Hg., Mahoney the Cat ([ 2002$])$, $<\underline{\text { http://www.mahoneythecat.org/aboutstory.asp }>, 15.09 .2003 . ~}$
} 


\section{Read \& Write:}

Dieser Bereich kann als der zentrale Anlaufpunkt des Forums bezeichnet werden. Er ist in fünf Segmente untergliedert, die dem aktuellen Textgefüge der Geschichte über Mahoney the Cat sowie seiner Weiterentwicklung gewidmet sind. Hier findet auch der Dialog über Inhalt und Hintergründe der Erzählung statt.

1. Beginning Of Story: Unter diesem Stichwort werden alle existierenden Kapitel der Abenteuer des Katers Mahoney präsentiert. Am 15.09.2003 bestand die Geschichte aus insgesamt neun Einzelelementen: Sechs aufeinanderfolgende Teile basieren auf den Erzählungen von Peter Holstrom und bilden den Hauptstrang der Geschichte; dieser wurde durch die Beiträge dreier verschiedener Nutzer in seinem Ablauf variiert und erhielt dadurch interaktiven Charakter.

2. Outline View: Die Verknüpfung zu einem Überblicksschema ist von Holstrom zwar bereits installiert worden, gegenwärtig liegen jedoch noch keine Daten vor, anhand derer sich die komplette Struktur des Hypertext-Systems rekonstruieren ließe. Die Funktionsfähigkeit eines sogenannten Outline View-Elements wird von Holstrom aber für die nähere Zukunft in Aussicht gestellt. Genaue Daten sind in diesem Zusammenhang allerdings nicht genannt. ${ }^{370}$

3. Chat Room: Der Chat Room - ein Begriff, der sich im deutschen Sprachgebrauch mittlerweile etabliert hat - besteht hier aus insgesamt fünf Sektionen, die jeweils unterschiedlichen Themen zugeordnet sind:

- Enchanted Forest

- The Cottage

- St. James Academy

- Hypertext

- Site Issues

Diese einzelnen Sektionen, die als Räume ${ }^{371}$ bezeichnet werden, sind jeweils auf bestimmte Themenbereiche der Mahoney-Erzählungen ausgerichtet. Während es innerhalb der ersten drei Räume um bestimmte Handlungsorte geht, die eine maßgebliche Rolle in den Mahoney-Geschichten spielen, dienen die beiden letzten

370 Ders., "Mahoney Outline View", in: Ders., Hg., Mahoney the Cat ([ $\left.\left.{ }^{1} 2002\right]\right)$, $<\underline{\text { http://www.mahoneythecat.org/outline.asp }>, 15.09 .2003 . ~}$

371 Für die Bezeichnung dieses webbasierten Treffpunkts zum kommunikativen Austausch wird die Metapher des Raumes bemüht, wie auch das gesamte Web als ein real begehbarer Ort wahrgenommen wird. Gerade im Falle der Chat Rooms wird wieder deutlich, wie stark englische Begriffe sich mittlerweile im alltäglichen deutschen Sprachgebrauch etabliert haben. 
Themenbereiche der Erörterung allgemeiner Fragen zu Hypertext und Forumskonzeption.

Eine Teilnehmerliste der jeweils gerade im Chat Room anwesenden Nutzer erlaubt eine konkrete und unmittelbare Kontaktaufnahme mit diesen Personen. Die Gespräche laufen somit nahezu in Echtzeit ab. Der Vorteil dieses direkten Gedankenaustausches liegt in einer wesentlich effizienteren Problembewältigung, die ein unmittelbares Nachfassen und Korrigieren von Sachverhalten erlaubt; als nachteilig erweist sich hingegen die Flüchtigkeit der Kommunikation, da in Chat Rooms - anders als in Discussion Boards - keine Speicherungsfunktion vorgesehen ist, die alle geschriebenen Äußerungen festhält und somit eine spätere Rekonstruktion der Gesprächsabläufe ermöglicht.

4. Discussion Board: Im Gegensatz zu einem Chat Room erlaubt ein Diskussionsforum zwar ebenfalls - je nach Zufall oder Absprache - einen unmittelbaren Austausch, seine eigentliche Bestimmung liegt jedoch in der Speicherung der eingegebenen Beiträge. Auf diese Weise können Nutzer Anfragen und Nachrichten hinterlassen, die in der Regel zeitversetzt beantwortet werden.

Wie allgemein üblich, ist auch das Diskussionsforum von Mahoney the Cat thematisch untergliedert und gewährleistet auf diese Weise eine thematische Vorsortierung und eine gewisse Ordnung der eingehenden Beiträge:

- General

- Newcomers

- Characters

- Places

- Site Issues

Die Rubrik Newcomers ist Erstnutzern des Diskussionsforums vorbehalten, die sich dort den etablierten Gesprächsteilnehmern vorstellen können. Die anderen Rubriken sind allgemeinen Fragen der Mahoney-Geschichten und der Forumskonzeption oder ganz speziell den Handlungsträgern oder -orten der Erzählungen gewidmet. Die eingehenden Beiträge sind mit Verfassernamen und Einstellungsdatum gekennzeichnet und können mit Hilfe der Funktion Reply unmittelbar beantwortet werden. Wahlweise besteht die Möglichkeit, sich per E-Mail direkt an den Verfasser der Nachricht zu wenden oder auch einen gänzlich neuen Beitrag zu konzipieren. 
5. The Writers: Wie dem noch folgenden Abschnitt Sign-In genauer zu entnehmen sein wird, sind alle Besucher des Forums gehalten, sich als Nutzer registrieren zu lassen. Sofern eine kooperative Mitarbeit angestrebt wird, ist die Anmeldung sogar zwingend erforderlich. Die hierfür anzugebenden personellen Daten sind anschließend im Bereich Read \& Write über die Verknüpfung The Writers in Form von Nutzerprofilen allgemein einsehbar; lediglich die Wiedergabe der EMail-Adresse kann auf Wunsch unterdrückt werden. Die Profile geben unter anderem Aufschluß über die Dauer der Mitgliedschaft im Forum Mahoney the Cat und die Anzahl der innerhalb dieses Zeitraums eingestellten kooperativen Beiträge. Eine hilfreiche Funktion für regelmäßige Nutzer bilden die Verknüpfungen zu dem zuletzt eingestellten sowie dem am besten bewerteten Beitrag des jeweiligen Kooperierenden. ${ }^{372}$

About:

In diesem Bereich werden Hintergrundinformationen zur Projektinitiierung und -gestaltung präsentiert. Hier erhält der Nutzer detaillierte Informationen über die Hintergründe der Veröffentlichung sowie einen Überblick über die Motive der Abenteuer des Katers Mahoney. Fünf Verknüpfungspunkte stehen dem Nutzer zur Verfügung:

1. The Story: Die webbasierte Veröffentlichung der Abenteuer des Katers Mahoney basiert auf den Erzählungen Peter Holstroms. Die Grundzüge der Ursprungsgeschichten werden im Abschnitt The Story dargelegt und allen Kooperierenden als Ausgangs- und Bezugspunkt für mögliche Fortsetzungskapitel anempfohlen. Da die Geschichten von Peter Holstrom grundsätzlich denselben Schemata folgten, im Detail aber bei jedem Erzählvorgang variierten, betrachtet Chris Holstrom die Überlieferungen seines Vaters gewissermaßen als unvollendete Fortsetzungsgeschichte: "Each time the story has been told it has been a little different. These are some of the most consistent plot points, but the story is very open-ended. You can add to the story as you wish." ${ }^{373}$

372 Ein Profil beinhaltet darüber hinaus noch weitere Informationen über den jeweiligen Nutzer; welche Angaben nach der Registrierung zur Ansicht bereitstehen, wird in der Rubrik Sign-In erörtert werden.

${ }^{373}$ Holstrom, “About the story”, < $<$ ttp://www.mahoneythecat.org/aboutstory.asp $>, 15.09 .2003$. 
Mahoney the Cat weist die charakteristischen Merkmale eines Märchens auf: Es handelt sich um mündlich überlieferte kürzere Prosaerzählungen, die von frei erfundenen Begebenheiten ohne Raum-Zeit-Bindung berichten. Vor dem Hintergrund einer schwarz-weißen Weltordnung widerfahren dem Protagonisten zahlreiche Abenteuer, die durch das Walten einer bösen Macht ausgelöst werden. $\mathrm{Zu}$ den unglaubhaften Erscheinungen, die kennzeichnend sind für die Literaturgattung Märchen, zählen sprechende Tiere und Pflanzen sowie die Aufhebung von Naturgesetzen.

Konkret handelt es sich um die magischen Abenteuer des Katers Mahoney, der mit seinem Besitzer, einem liebenswürdigen und großherzigen Arzt, in einem Häuschen am Rande eines verwunschenen Waldes lebt. Dank spezieller Begabungen vermag Mahoney das Verhalten anderer positiv zu beeinflussen und mit bestimmten Wesen zu kommunizieren, zu denen die Tiere und Pflanzen des verwunschenen Waldes sowie der junge Schüler Peter der nahegelegenen St. James Academy zählen. Die bedrohliche Macht tritt in diesem Märchen in Form eines Zauberers auf, der alle Waldbewohner in Angst und Schrecken versetzt. Mahoney versucht, alle Lebewesen des Waldes hinter sich zu bringen, um dem bösen Zauberer gemeinschaftlich entgegentreten zu können.

2. The Characters: Hier werden insgesamt acht Hauptfiguren des Märchens vorgestellt, zu denen neben Mahoney unter anderem sein Besitzer "The Good Doctor" und der Antagonist der Geschichten, "The Evil Wizard”, zählen. Alle Figuren werden ausführlich beschrieben und in ihrer Rolle dem Kater Mahoney zugeordnet. Sollte die Einstellung weiterer Fortsetzungen zusätzliche Figurenbeschreibungen erforderlich machen, sichert Holstrom die Ergänzung dieses Abschnitts zu.

3. The Places: Fünf Handlungsorte des Märchens sind an dieser Stelle bereits dargestellt und können im Bedarfsfalle um weitere Schauplätze ergänzt werden.

4. How Mahoney Started: Die Ausführungen dieses Abschnitts verdeutlichen dem Leser das Anliegen Holstroms, der mit der Veröffentlichung der Mahoney-WebSeiten an einen Familienbrauch anknüpft und diesen gewissermaßen fortsetzt. Nicht genannt sind in diesem Zusammenhang die Gründe für die Wahl des World Wide Web als Publikationsmedium. Lediglich aus einer später noch zu diskutierenden ausführlichen Darstellung, auf die sich von den Projektseiten aus aber 
nicht zugreifen läßt, ist $\mathrm{zu}$ folgern, daß die Veröffentlichung der MahoneyGeschichten zunächst auf eine lineare Darstellung des Märchens ausgerichtet war. ${ }^{374}$ Erst nach mehrfachen gescheiterten Versuchen, sich für einen einzigen sukzessiven Handlungsablauf zu entscheiden, wurde offenkundig, wie sehr die persönlichen Vorstellungen Holstroms und die Darstellungsmöglichkeiten des Web einander entsprachen. Es wurde deutlich, daß die Wiedergabe der zahlreichen Versionen der Mahoney-Geschichten, die nur zu einem geringen Teil schriftlich fixiert worden waren und überwiegend in einer allmählich verblassenden Erinnerung existierten, eines flexiblen Präsentationsmediums bedurften. Holstrom schildert diesen Entwicklungsprozeß folgendermaßen:

The humble, wrinkled sheets and my fading memories felt like an inadequate tribute to what had once been. The Mahoney stories were a definitive part of my childhood and one of my fondest memories of my father. I wanted to write the Mahoney stories to preserve my father's memory, but each time I began I was frustrated by having so many plotlines in my head from the many different times I heard the ever-changing and evolving stories. I could not conceive of a single, authoritative, and linear story; and writing efforts stalled.

It seemed that I could not translate the stories; I needed a nontraditional and flexible environment for writing the story and publishing the text. ${ }^{375}$

Die Entscheidung für die Technologie des WWW festigte sich mit der allmählichen Erkenntnis, daß Holstrom nicht in der Lage sein würde, die Komplexität der Aufgabe ohne fremde Hilfe zu überwinden. Ebenso wie sein Vater gelegentlich einer Inspiration bedurfte und in solchen Fällen seine Zuhörer um Anregungen zu bitten pflegte, meinte auch Chris Holstrom, die Aufbereitung der Geschichten von Mahoney nur in gemeinschaftlicher Arbeit bewältigen zu können. Einzig das Web als Medium der Kommunikation und der hypertextuellen Darstellung konnten diesen Notwendigkeiten gerecht werden. Aus diesem Grunde wurde das Mahoney-Projekt als Hypertext-System mit den Möglichkeiten von Interaktion und Kooperation konzipiert und vereinbart nun die Vorteile einer Synergie von Tradition und Moderne: "[The Mahoney Project] is the logical result of a marriage between the traditional (my father's oral story telling) and the modern (networked computing and hypertext).",376

\footnotetext{
${ }^{374}$ Ders., "Introduction", in: Ders., Hg., The Mahoney Project ([ $\left.\left.{ }^{1} 2002\right]\right)$, $<$ http://www.cholstro.net/mahoney/thesis1.shtml $>, 19.09 .2003$.

375 Ebd., 19.09.2003.

${ }^{376}$ Ebd., 19.09.2003.
} 
5. Mahoney Home Page: Dieser Link ist nicht themenrelevant, sondern erfüllt ausschließlich die Funktion, den Nutzer von jedem beliebigen Punkt des HypertextSystems direkt zur Einstiegsseite des Forums, der sogenannten Homepage, zurückzuführen.

Die ersten vier Verknüpfungspunkte des Bereiches About sind als Kontrollfaktoren für Kontinuität und Seriosität der Nutzerbeiträge konzipiert. Holstrom lehnt es ab, strenge und verbindliche Auflagen zu erteilen und setzt daher auf eine unterschwellige und unaufdringliche Steuerung durch detaillierte Hintergrundangaben. Seine Beschreibungen sollen die Kooperierenden so eng mit der Materie vertraut machen, daß ihre Beiträge gewissermaßen automatisch den kontextuellen Vorgaben folgen: "This description gives writers enough context and detail to guide them as they write about Mahoney the cat." ${ }^{377}$

Diese Methode der diskreten Unterweisung gilt als passive Kontrolle, da es letztlich dem Nutzer überlassen bleibt, ob er sein Verhalten tatsächlich nach den Vorgaben ausrichtet. Die Entscheidung über die inhaltliche wie auch die stilistische Weiterentwicklung von Mahoney the Cat liegt in erster Linie auf Seiten der Nutzergemeinschaft. $^{378}$

Sollten die kooperativen Beiträge dennoch in intolerabler Weise von den Vorgaben abweichen - Holstrom bezeichnet Divergenzen dieser Art als "tendencies toward off-color material”,379 -, behält sich der Herausgeber zensorische Maßnahmen vor, die von Ermahnungen bis zur unwiderruflichen Entfernung der betreffenden Texte reichen können.

Sign-In:

Einen zusätzlichen Schutz vor unerwünschten Beiträgen verspricht sich der Herausgeber auch von der Registrierungsverpflichtung für Kooperierende. Vor der Einstellung eines neuen Textelements ist eine Erstanmeldung mit Angabe der E-MailAdresse obligatorisch. Holstrom ist damit gegebenenfalls in der Lage, in direkten Kontakt zu einem Nutzer zu treten. Darüber hinaus schreibt er der Übermittlung persönlicher Daten einen Einhalt gebietenden, befriedenden Effekt zu. Die schützende Anonymität, die einem Kooperierenden plein pouvoir suggeriert, sollte im Forum

377 Ders., "Collaborative Fiction Writing as an Example of Online Community”, in: Ders., Hg., The Mahoney Project ([ $\left.\left.{ }^{1} 2002\right]\right),<$ http://www.cholstro.net/mahoney/thesis3.shtml $>, 19.09 .2003$.

378 Ebd., 19.09.2003.

379 Ebd., 19.09.2003. 
Mahoney the Cat aufgrund der Registrierungspflicht somit eigentlich nicht gegeben sein. $^{380}$

Allen Nutzern, denen Mahoney the Cat ausschließlich als Lektüreforum dient, ist eine Anmeldung grundsätzlich freigestellt. Im Unterlassungsfall entstehen dem Leser keine Nachteile im Hinblick auf das Verständnis der Geschichten, allerdings bleiben ihm die Zutritte zu Chat Room und Diskussionsforum sowie alle weiteren Vorteile einer Registrierung versagt.

Entschließt sich der Nutzer zu einer Registrierung, erfolgt eine Abfrage obligatorischer Daten für die Einrichtung eines Nutzerkontos und fakultativer Informationen für die Erstellung des Nutzerprofils. Bindend vorgegeben ist die Wahl eines Nutzernamens sowie eines Paßworts als Identifikationsmittel für den internen Nutzerbereich des Forums. Ebenfalls verpflichtend ist die Angabe der E-Mail-Adresse, um die Aufhebung der Anonymität und eine etwaige direkte Kontaktaufnahme zu gewährleisten. Die Nennung von Vor- und Zuname, Geschlecht, Geburtsdatum und Nationalität ist hingegen ebenso freigestellt wie die Aufzählung der Hobbies. Der eigenen Entscheidung des Nutzers überlassen bleibt auch eine persönliche Stellungnahme, die ein Lebensmotto wiedergeben oder Bezug auf das Mahoney the CatProjekt nehmen kann.

Sobald die Registrierung des Antragstellers erfolgt und ein Nutzerprofil erstellt ist, besteht die Zugangsberechtigung zum internen Bereich des Forums. Nach jeder Anmeldung mit Nutzername und Paßwort erscheint anstelle des Eingabefeldes Sign-In das Navigationselement Welcome für das persönliche Nutzerkonto, das zur Verwaltung von Bookmarks, eigenen Beiträgen, der Überarbeitung der Profil-Daten und der Speicherung persönlicher Favoriten aus der Gruppe der Kooperierenden dient.

380 Daß Kontrollmaßnahmen dieser Art zwar routinemäßig durch ein Programm durchgeführt werden können, daß es aber gleichwohl der zusätzlichen manuellen Überprüfung durch den Herausgeber bedarf, hat ein praktischer Registrierungsversuch der Autorin bei Mahoney the Cat bewiesen. Anstelle der E-Mail-Adresse wurde der Hinweis eingegeben, eine solche Adresse sei nicht vorhanden. Das System akzeptierte diese Angabe und führte die Registrierung durch. Während manche Programme automatisch erkennen, daß der eingegebene Text aufgrund seiner BuchstabenZeichen-Kombination nicht einer E-Mail-Adresse entsprechen kann und den Zugang zum internen Nutzer-Bereich verweigern, konnte die Anmeldung in diesem Falle problemlos erfolgen. Es wäre also theoretisch möglich gewesen, die Mahoney-Geschichten um einen wider alle Vorgaben konzipierten Beitrag zu ergänzen, der zumindest solange einen Teil des Forums gebildet hätte, bis er durch Holstrom entfernt worden wäre. Insofern ist sein Versuch der Kontrollausübung letztlich doch nur eine sehr unvollkommene Maßnahme und nur bedingt geeignet, unerwünschte Kooperierende fernzuhalten. 
Jeder Antragsteller sollte sich bei seiner Bewerbung um die Mitgliedschaft im Mahoney the Cat-Forum dessen bewußt sein, daß Holstrom sich die weitere Verwendung aller eingestellten Texte für die Konzeption der Geschichten über Kater Mahoney vorbehält. Eine Registrierung erfolgt nur bei Anerkennung der Verzichterklärung, womit der Antragsteller alle Rechte an seinen Beiträgen abtritt und demzufolge im Falle einer kommerziellen Verwertung der Geschichten keinerlei Anspruch auf Gewinnbeteiligung oder finanzielle Entschädigung hat. Dies betrifft nicht nur die webbasierte Veröffentlichung, sondern auch eine mögliche Print-Publikation:

By signing up to take part in Mahoney the Cat, you are agreeing that your submissions are part of a story developed by the creators of the website. These creators make your stories available to the public online and may use your story ideas in developing a hardbound version of the Mahoney the Cat stories. All information on the site is the property of the Mahoney the Cat developers regardless of its origin. If the Mahoney the Cat stories are ever sold for a profit, private contributors are not guaranteed any financial benefit, but you will be thanked for taking part in the effort. ${ }^{381}$

Bis zum Zeitpunkt der Recherche für vorliegende Arbeit hat Holstrom allerdings offensichtlich noch keinen finanziellen Nutzen aus den Beiträgen der Kooperierenden gezogen.

Verzichtet der Nutzer auf eine Registrierung, begibt er sich damit, wie oben bereits angedeutet, gewisser Vorteile einer Forumsmitgliedschaft. So erlaubt das System beispielsweise nur registrierten Nutzern das Setzen von Lesezeichen, um eine Rückkehr zu ausgewählten Beiträgen zu erleichtern. Ferner besitzen nur die Forumsmitglieder die Befugnis der inhaltlichen Bewertung aller eingestellten Texte durch Vergabe von bis zu fünf Sternen.

Die Bekanntgabe der Bewertungen ist von Holstrom als eine weitere Vorsichtsmaßnahme zur Vermeidung unliebsamer Tendenzen gedacht. Holstrom setzt damit auf mehrere miteinander verbundene Faktoren: einen gewissen Ehrgeiz der Kooperierenden sowie die Kritikfähigkeit und -freudigkeit der Leser. ${ }^{382}$ Die Verfasser neuer Beiträge kooperieren unter anderem aus Streben nach Bestätigung und Anerkennung und versuchen ihre Texte schon aus diesem Grunde, zumindest aber aus Furcht vor Kritik möglichst hochwertig zu gestalten. Das Bewertungssystem soll allen

\footnotetext{
381 Holstrom, "Sign Up", in: Ders., Hg., Mahoney the Cat ([ $\left.\left.{ }^{1} 2002\right]\right)$, $<$ http://www.mahoneythecat.org/signup.asp >, 20.09.2003.

382 Ders., "Collaborative Fiction Writing as an Example of Online Community", $<$ http://www.cholstro.net/mahoney/thesis3.shtml $>, 19.09 .2003$.
} 
Kooperierenden als Korrektiv und damit im Sinne der Nutzergemeinschaft und des literarischen Wertes des Hypertext-Werkes als Mittel der Qualitätssicherung dienen.

Das Bewertungssystem ist zweifellos nicht ganz unproblematisch. Holstrom erhofft sich von einem gewissen Wettbewerbsdenken einerseits zwar positive Effekte wie Sicherung und Wahrung eines hohen literarischen Standards, fürchtet andererseits jedoch auch, Konkurrenzverhalten und persönliche Animositäten zwischen besonders ehrgeizigen Kooperierenden auszulösen beziehungsweise langfristig zu fördern. Um derartige Tendenzen zu minimieren und gleichzeitig ein Klima der fruchtbaren Zusammenarbeit und gegenseitigen Inspiration entstehen $\mathrm{zu}$ lassen, verleiht Holstrom seinem Bewertungssystem uneingeschränkte Transparenz. Die Benotungskriterien sind eindeutig und allgemein nachvollziehbar. Die Notenskala umfaßt fünf Zensuren - poor, fair, good, great und outstanding - und erlaubt damit eine vergleichsweise nuancierte Bemessung. Darüber hinaus ist bei allen Bewertungen mit angegeben, auf wievielen Beurteilungen die jeweilige Note basiert. Es obliegt der Gemeinschaft der registrierten Nutzer, das Bewertungssystem verantwortungsvoll einzusetzen. Sie allein bestimmt die Benotungsqualität und damit letztlich auch das inhaltliche Niveau des Forums. Da jeder Kooperierende Mitglied dieser Gemeinschaft ist, sollte jeglicher Verdacht von Unsachlichkeit, Ungerechtigkeit oder Mißgunst ausgeschlossen sein. Ungeachtet dessen gewährt das System aber immer noch genug Spielraum für Subjektivität oder gar gezielte Unbotmäßigkeiten. Die Tatsache, $\mathrm{da} ß$ in dieser Hinsicht bislang offensichtlich noch keine Probleme aufgetreten sind, läßt auf die Effektivität der Holstromschen Vorsichtsmaßnahme schließen.

\subsubsection{Analyse und Auswertung}

Gemessen am Umfang des literarischen Angebots, befand sich das Mahoney-Projekt auch nach einer Laufzeit von gut eineinhalb Jahren noch immer in statu nascendi. Am 20.09.2003 beschränkte sich das Ergebnis der kooperativen Mitarbeit der Nutzer auf drei Beiträge, was mehr oder weniger einer Entwicklungsstagnation gleichkommt. Das Projekt wurde zur Etablierung einer Web-Gemeinschaft ins Leben gerufen und sollte Anziehung und Abschöpfung kreativen literarischen Potentials bewirken. Aufgrund seiner Intention und Konzeption verlangt das Projekt zwar von vornherein eine längere Laufzeit und ist somit noch immer in der Entwicklung begriffen, die geringe Beteiligung seitens der Web-Nutzer dokumentiert jedoch, daß sich das Projekt noch nicht als fester Treffpunkt im World Wide Web etablieren konnte und 
sich daher weiterhin in der Anlaufphase befindet. Die begrenzte Resonanz läßt entweder auf einen geringen Bekanntheitsgrad oder eine möglicherweise mangelnde Attraktivität schließen - beide Alternativen kommen gleichermaßen als Erklärung für den zögerlichen Etablierungsprozeß in Betracht.

Holstrom scheint sich dieser Tatsache durchaus bewußt zu sein. Nach insgesamt acht Hypertext-Elementen befand er noch im Jahr der Projektinitiierung, das Projekt sei weit von der Qualität eines bedeutenden literarischen Werkes entfernt. Er begründete die Unzulänglichkeit des Zwischenergebnisses mit dem Mangel an Beiträgen seitens der Nutzergemeinschaft, bekräftigte gleichzeitig aber auch seine Zufriedenheit über die Qualität sowie die Motiv- und Konzeptnähe der eingegangenen Beiträge: "These few nodes are, however, carefully written and in the spirit and context of the original story." 383

Was das Mahoney-Projekt ungeachtet seines geringen Umfangs aus der Masse der webbasierten Publikationen hervorhebt, sind die Konzeption des Forums und das aktuelle literarische Ergebnis. Mahoney the Cat überzeugt bereits optisch durch seine übersichtliche und logische Oberfläche. Die Gestaltung der Bedienungsknöpfe auf der Einstiegsseite in Form stilisierter Katzenköpfe lassen Verbundenheit mit der Thematik und Liebe zum Detail erkennen. Die augenfällige und klare Aufteilung der drei Forumsbereiche garantiert eine hohe Funktionalität, die der raschen Orientierung und gezielten Verwendung der Bedienungselemente zugute kommt. Die praktische Nutzung bestätigt den ersten Eindruck: Die Handhabung erweist sich als zweckmäßig und anwenderfreundlich.

Überzeugend und ganz offensichtlich effizient sind Holstroms Maßnahmen zur Vermeidung obszöner oder themenfremder Beiträge. Die Zweiteilung des Forums in einen öffentlichen und einen internen Bereich scheint ein wirkungsvolles Zugangshindernis für unliebsame Besucher zu bilden. Gleichzeitig trägt Holstrom mit dieser Gestaltung auch zur Förderung des Teamgeistes bei. Das Bewußtsein, sich mittels Nutzernamens und Paßwortes für die Nutzung eines Bereichs legitimieren zu können, der lediglich einem eingeschränkten Nutzerkreis vorbehalten ist, schafft bereits ein intensives Gemeinschaftsgefühl, das durch Angebote wie Chat Room und Diskussionsforum nachhaltig verstärkt wird. Diese Konzeptionselemente bilden die Basis für eine harmo-

383 Ders., “Conclusions", in: Ders., Hg., The Mahoney Project ([ $\left.\left.{ }^{1} 2002\right]\right)$, $<\underline{\text { http://www.cholstro.net/mahoney/thesis5.shtml }>, 20.09 .2003 . ~}$ 
nische und kreative Zusammenarbeit, denn langfristig kann nur ein Klima der Zusammengehörigkeit die von Holstrom angestrebte fruchtbare Gruppendynamik erzeugen.

Begrüßenswert sind auch Konzeption und Gestaltung des Bewertungssystems. Den Kooperierenden dient es als Maßstab und Korrektiv und hilft vermutlich auch, nachlässig verfaßte oder gar kontraproduktive Beiträge zu verhindern. Aufgrund der non-verbalen Beurteilung durch Vergabe einer bestimmten Anzahl von Sternchen garantiert es eine unmißverständliche Benotung, ohne doch die Betroffenen durch allzu deutliche Worte zu verletzen.

Allerdings ist festzustellen, daß eine negative Bewertung bislang noch nicht vorgenommen wurde. Zum Zeitpunkt der Erhebung am 20.09.2003 waren fünf der insgesamt neun Beiträge durch Forumsmitglieder mit zweimal fünf, zweimal vier und einmal drei Sternchen zensiert worden. Dabei ist zu beobachten, daß es die zuletzt eingestellten Texte sind, die eine Beurteilung vermissen lassen. Dies könnte darauf hindeuten, daß das Interesse der Kooperationsgemeinschaft an der Fortentwicklung des Projekts nicht mehr zureichend ist für eine kritische Auseinandersetzung. ${ }^{384}$

Insgesamt weicht keiner der eingestellten Texte qualitativ oder inhaltlich gravierend von der Vorgabe des Projekts ab. Der Leser gewinnt den Eindruck, daß die Beiträge im Sinne der gemeinschaftlichen Sache und folgerichtig konzipiert sind. Sie fügen sich aus seiner Sicht inhaltlich alle zu einem homogenen Gesamtwerk zusammen, wobei aber dennoch jeder Beitrag für sich genommen eine ganz individuelle Geschichte erzählt, die der Handlung mitunter eine völlig neue Richtung gibt. So erfährt der Leser in insgesamt sechs 'Kapiteln' von dem Wunsch des Katers, Erkundungsgänge zu unternehmen, von seinen Plänen für ein heimliches Entschwinden sowie der späteren Planverwirklichung; in einem anderen Beitrag tritt ein kleiner Junge in das Geschehen, in einem dritten Abschnitt beschäftigt sich der Kater gedanklich mit dem Alltag der Mädchen in einem nahegelegenen Weiler.

Da sich die webbasierte Veröffentlichung aufgrund der unterschiedlichen Handlungsversionen an mehreren Stellen verzweigt, erfüllt sie neben der Bedingung der allgemeinen und zeitlich unbegrenzten Zugänglichkeit sowie der Kooperativität auch die Voraussetzung der Interaktivität. Wegen der geringen Anzahl von Beiträgen stößt der Nutzer allerdings spätestens nach sechs Entscheidungsvorgängen an die

384 Einer der unbewerteten Nutzerbeiträge weist im Vergleich zu den übrigen Texten des Forums eine eher geringere literarische Qualität auf. Es könnte also auch sein, daß Rücksichtnahme seitens der Gemeinschaft ursächlich für die Zurückhaltung ist. 
Grenzen des Hypertext-Systems. Auch inhaltlich erschöpft sich die Spannung der Handlung nach sehr kurzer Zeit in der Vorstellung der Handlungsträger und weitschweifiger Ausblicke auf die - wann immer auch folgenden - Abenteuer des Protagonisten. Stilistisch bewegen sich die Autoren vorwiegend auf durchschnittlichem Niveau. Nachstehender Textausschnitt aus Mahoney the Cat wird dem Leser einen flüchtigen Eindruck vom Stil der Beiträge vermitteln:

The doctor's stories made Mahoney wonder what the world was like outside the cozy cottage. He thought that the village folk lived peculiar and intriguing lives. Lives that were much more interesting than Mahoney's days of chasing an occasional mouse and waiting for the doctor to come home and start the fire in the fireplace. Mahoney wanted to see the village folk and how they lived, but even more he wanted to explore the dark forest that loomed outside the cottage windows. ${ }^{385}$

Einige Episoden beeindrucken durch ausgesuchte Wortwahl und intelligenten Handlungsaufbau, während andere durch kindlich-naive Darstellung enttäuschen. Indes hat der Herausgeber selbst mit seinen Texten einen relativ schlichten Stil vorgegeben, der zwar bei diesem Genre und der vergleichsweise anspruchslosen Handlung durchaus akzeptiert werden kann, der aber keineswegs konform geht mit Holstroms persönlichen qualitativen Ansprüchen. Dabei ist es keineswegs so, daß das Sujet nur begrenzten Freiraum für Tiefgang und Sprachgewandtheit ließe. Der überzeugende Beitrag einer Kooperierenden, mit dem der schmächtige Schüler Joshua, ebenfalls Schüler der nahegelegenen St. James Academy, in die Erzählung eingeführt wird, mag als Beweis für die Realisierbarkeit hervorragender inhaltlicher wie stilistischer Qualität dienen. ${ }^{386}$

Welcher Maßstab letztlich bei der Beurteilung der literarischen Qualität der Texte anzulegen ist, hängt unter anderem davon ab, um welche Literaturgattung es sich bei Mahoney the Cat handelt beziehungsweise welche Zielgruppe angesprochen ist. Aufgrund der Konzeption, die die Nutzung des internen Bereiches einem ausgewählten Nutzerkreis vorbehält, und seiner Intention, die den Aufbau einer Autorengemeinschaft und die Herausbildung eines hohen literarischen Standards anstrebt, scheint sich das Forum vorwiegend an erwachsene Nutzer zu wenden. Dieser Ein-

385 Holstrom, "Mahoney considers the world outside the cottage", in: Ders., Hg., The Mahoney Project ([ $\left.\left.{ }^{1} 18.04 .2002\right]\right),<$ http://www.mahoneythecat.org/node.asp?Node=23>, 19.02.2004.

386 Alison Cline, "Joshua", in: Holstrom, Hg., The Mahoney Project ([18.04.2002]), $<$ http://www.mahoneythecat.org/node.asp?node=66>, 20.09.2003. 
druck festigt sich angesichts des durchschnittlichen Alters der Kooperierenden, das bei schätzungsweise 30 Jahren liegt ${ }^{387}$.

Es gibt allerdings einen Aspekt, der sich gegen eine derartige Zielgruppenannahme ins Feld führen ließe. Wie dem vorangehenden Kapitel zu entnehmen, weisen die kurzen, mündlich überlieferten Prosaerzählungen frei erfundener Begebenheiten Merkmale eines Märchens ${ }^{388}$ auf. Ebenso wie diese, die traditionell von Generation zu Generation überliefert wurden, entstammen auch die Geschichten von Mahoney the Cat den Erzählungen, die ein Vater an seinen minderjährigen Sohn weitergab. Die Tatsache, daß sie also ursprünglich auf eine kindliche oder jugendliche Zielgruppe ausgerichtet waren, läßt Raum für die Vermutung, das Forum könnte durchaus auch für junge Rezipienten konzipiert sein.

Der Widerspruch zwischen gewollter und tatsächlicher Zielgruppenausrichtung ist vermutlich unter den gegenwärtigen Umständen nicht aufzulösen. Das äußere Erscheinungsbild des Forums ist derzeit ebensowenig mit Holstroms literarischem Anspruch in Einklang zu bringen, wie die Struktur des Forums als eines Mediums des gehobenen kommunikativen Austausches tauglich ist, heranwachsende Nutzer mit einzubeziehen. Das Dilemma des Forums besteht darin, daß es gemessen an seiner äußeren Konzeption für Kinder weder gedacht noch geeignet, gleichwohl aber aufgrund seines literarischen Angebots sehr wohl auf diese Zielgruppe ausgerichtet ist, daß andererseits die erwachsenen Nutzer, die Holstrom ansprechen möchte, wenig Resonanz zeigen. Offenbar bietet ihnen das Forum nicht das erforderliche Identifikationspotential.

Auch wenn die Möglichkeiten der webbasierten Publikation Mahoney the Cat sowohl qualitativ als auch quantitativ noch lange nicht ausgeschöpft sind, rechtfertigen doch Intention und Konzeption des Forums allein bereits die Einbindung in die vorliegende Arbeit. In diesen Punkten wird das Projekt den in Kapitel III. herausgearbeiteten Ansprüchen an eine qualitativ hochwertige webbasierte Veröffentlichung gerecht. Darüber hinaus erhält das Mahoney-Projekt aus zwei Gründen besondere wissenschaftliche Relevanz:

\footnotetext{
387 In einem Falle fehlt die Angabe des Geburtsdatums, so daß hier kein exakter Durchschnitt errechnet werden kann.

388 Diese Form der Prosaliteratur ist allerdings nicht notwendigerweise identisch mit Jugendliteratur. Die Differenzierung von Kinder- und Jugendliteratur ist der deutschen Terminologie vorbehalten; in der Diskussion über englischsprachige Literatur wird ausschließlich der Begriff Jugendliteratur verwendet.
} 
- aufgrund seines theoretischen Anhangs zu allgemeinen Fragen webbasierten Publizierens und

- dank der projektbegleitenden Abhandlung, die alle Hintergründe, Umstände und Konsequenzen des Projekts offenlegt und ihm dadurch eine in der webbasierten Literatur seltene Transparenz verleiht.

Der dem Thema Hypertext gewidmete Teil der theoretischen Ausführungen Holstroms ist für den Nutzer des Mahoney-Forums vor allem deshalb von Bedeutung, weil er die Entscheidung des Herausgebers für das Hypertext-Medium World Wide Web erklärt und insbesondere seine Gründe für die Wahl einer einfachen Hypertext-Struktur darlegt.

Die Diskussionen über die potentiellen Auswirkungen verstärkter Computer-Nutzung waren zu Anfang stark geprägt von der spekulativen Annahme, die neuen elektronischen Medien könnten die herkömmlichen Print-Medien verdrängen. Dabei wurde durch Titel wie Von Gutenberg zum Internet ${ }^{389}$ oder Am Endedas Buch ${ }^{390}$ suggeriert, der Computer - und damit die Hypertext-Systematik - sei selbstverständlich die Weiterentwicklung der Druckkultur. Wie in Kapitel I. vorliegender Arbeit ausgeführt, wurde der Hypertext nicht selten als Befreiung von der Bevormundung linearer Darstellungsweise gepriesen. Der enge Bezug zwischen beiden Publikationsformen wird nach Ansicht Holstroms jedoch eindeutig überbewertet. Die engere Verwandtschaft bestehe eigentlich zwischen mündlicher Überlieferung und Hypertext-Darstellung und nicht - wie aufgrund der chronologischen Entwicklung oftmals angenommen - zwischen Print- und HypertextMedium. ${ }^{391}$ Zur Untermauerung seiner These bedient sich Holstrom eines über vier Schritte verlaufenden Vergleichs der Eigenschaften von Buch, mündlicher Erzählung und Hypertext.

Der erste Vergleich zielt auf den zeitlichen Aspekt: Während sich die mündliche Erzählung als Vorgang innerhalb eines ganz bestimmten Zeitrahmens vollziehe und damit ein singuläres und flüchtiges Ereignis darstelle, sei das Buch scheinbar unvergänglich, etwas Konstantes, jederzeit Einsehbares. Hypertexte vereinbarten die

\footnotetext{
389 Sabine Wefers, Hg., Von Gutenberg zum Internet/7. Deutscher Bibliothekskongreß, 87. Deutscher Bibliothekartag in Dortmund 1997, Zeitschrift für Bibliothekswesen und Bibliographie: Sonderheft 68 (Frankfurt a.M., 1997).

390 Jochum und Wagner, Hg., Am Ende - das Buch.

391 Holstrom, "Hypertext", in: Ders., Hg., The Mahoney Project ([ $\left.\left.{ }^{1} 2002\right]\right)$, $<\underline{\text { http://www.cholstro.net/mahoney/thesis4.shtml }>, ~ 25.09 .2003 . ~}$
} 
Charakteristika beider Darstellungsformen, indem sie zum einen Verfügbarkeit aufwiesen, zum anderen aber dank ihrer Multidirektionalität jedem Lesevorgang Einzigartigkeit verleihen könnten.

In einem zweiten Schritt untersucht Holstrom den Faktor Beständigkeit. Während sich ein Buch in der Regel durch Unveränderbarkeit auszeichne, liege ein wesentliches Merkmal mündlicher Überlieferung in ihrer Singularität; jeder Erzählvorgang habe seine individuelle Prägung. ${ }^{392}$ Auch eine Hypertext-Erzählung weise bei jedem Rezeptionsvorgang eine unterschiedliche Dynamik auf, so daß kein Handlungsverlauf als maßgebend oder verbindlich angesehen werden könne. Insofern bestehe eindeutig eine Ähnlichkeit zwischen oraler und hypertextueller Übermittlungsform.

Was den räumlichen Gesichtspunkt betrifft, geht der Vergleich Holstroms ausnahmsweise zugunsten des Buches. Das Bild des isolierten Computernutzers läßt sich für Holstrom eher mit der Vorstellung eines einsamen Buchlesers in Einklang bringen als mit einer Gruppe von Zuhörern, die sich um einen Erzähler schart. Auch wenn gerade das Hypertext-Medium Mahoney the Cat auf eine Zusammenführung möglichst vieler Nutzer abziele und somit von Abgeschiedenheit nicht die Rede sein könne, sitze der Computeranwender in concreto doch meistens allein vor seinem Bildschirm.

Der vierte und letzte Vergleich Holstroms betrifft die Kooperativität der drei Darstellungsformen. Auch hier sei die Ähnlichkeit zwischen mündlicher Erzählung und Hypertexten evident: In beiden Fällen würden die Adressaten mit einbezogen, so daß sich die Geschichten entlang von Publikumsreaktion und -kooperation entwickelten. Das Buch hingegen erlaube in der Regel keinerlei Beeinflussung durch den Leser.

Die Ergebnisse seiner vergleichenden Analyse bestätigen Holstrom in seiner Annahme:

Hypertext and oral stories are similar in that they are events dependent on time, they are impermanent and changing, and they can facilitate an involved community - be it geographically centered or virtually created across wires and networks. 393

392 Die Singularität mündlicher Überlieferung dürfte allerdings nicht unumstritten sein. In der Tat geht Holstrom in keiner Weise darauf ein, daß gewisse Inhalte gelegentlich wortgetreu überliefert werden, wie zum Beispiel Tischgebete.

393 Holstrom, "Hypertext", <http://www.cholstro.net/mahoney/thesis4.shtml> , 25.09.2003. 
Holstroms Ausführungen bilden einen wertvollen Beitrag für die HypertextForschung im allgemeinen und das Verständnis der Hintergründe von Mahoney the Cat im besonderen.

Vor der praktischen Umsetzung seines Vorhabens mußte Holstrom aber noch ein weiteres Problem überwinden: Wieviel Spielraum und Mitbestimmungsrecht sollte den Nutzern gewährt werden, ohne Transparenz und Bedienungsfreundlichkeit des Hypertext-Systems zu gefährden? Bei den vielen Gelegenheiten, da Peter Holstrom sein Söhne mit Geschichten unterhielt, führten die jeweiligen Begleitumstände, Kreativität, Zuhörerbeiträge und mitunter auch eingeschränktes Erinnerungsvermögen $\mathrm{zu}$ unterschiedlichen Varianten der Mahoney-Abenteuer. Resultat war schließlich ein abwechslungsreiches Corpus von Episoden, die ohne Verlust ihres individuellen Charakters nicht in einem Medium sequentieller Darstellung wiederzugeben waren.

Holstroms Anspruch an die Verschriftlichung der Mahoney-Abenteuer sah Beteiligung und Mitbestimmung der Rezipienten vor. Das sogenannte "participatory behavior" 394 der Nutzer sollte sich in Interaktion und Kooperation niederschlagen, wobei soviel Freiheit wie möglich und sowenig Kontrolle wie notwendig den Handlungsrahmen bestimmen sollten. Anstatt in seiner Funktion als Herausgeber und Projektleiter uneingeschränkte Verfügungsgewalt auszuüben, sah Holstrom eine Kompetenzverlagerung auf die Kooperierenden vor, die gemeinschaftlich über die Struktur des Hypertextes zu entscheiden hatten. Gleichzeitig sollten die freiwilligen Autoren die Freiheit zu uneingeschränkter kreativer Entfaltung erhalten. Diese Zielsetzung war allerdings nicht ohne weiteres mit bedienungsfreundlicher Hypertext-Nutzung und inhaltlicher Plausibilität in Einklang zu bringen. Holstroms Bestrebungen, Freiheit und Kontrolle in ein ausgewogenes Verhältnis zu bringen, mündeten schließlich in der Entscheidung für einen einfach strukturierten Hypertext. Dieser erlaubt eine unbegrenzte Zahl von Verzweigungen, gestattet aber keine Querverbindungen. Neue Elemente können den bereits vorhandenen Texten nur unmittelbar angehängt werden, so daß sequentielle Abfolgen entstehen, das heißt dem Beitrag A folgt Beitrag $B$, dem seinerseits Beitrag $C$ angehängt werden kann. Eine Verknüpfung von A und $\mathrm{C}$ ist somit nicht möglich. Diese Strukturierungsform reduziert den Hypertext auf ein einfaches Verzweigungsgefüge und läßt das eigentliche

394 Ebd., 25.09.2003. 
Potential nicht-linearer Darstellungsweise unausgeschöpft. Damit erhält jeder Kooperierende minimale Kontrolle über die Textstruktur, die ihm lediglich an den Knotenpunkten Entscheidungsgewalt zugesteht, ihm aber keine weiterreichende Kompetenzen einräumt. Organisation und Änderung der Gesamtstruktur obliegen allein dem Herausgeber und Projektleiter.

Nur wenige privat veröffentlichte Projekte im World Wide Web sind so ausführlich dokumentiert wie Mahoney the Cat. In der ausführlichen projektbegleitenden Abhandlung The Mahoney Project ${ }^{395}$ legt Holstrom die Hintergründe, Ziele und Maßstäbe seines Hypertext-Systems dar. Aufgrund seiner sachlichen Einschätzung der Projektergebnisse erlauben die umfassenden Darlegungen auch einen Abgleich zwischen theoretischer Zielsetzung und praktischer Ausführung und gestatten somit einen realistischen Einblick in Chancen und Grenzen webbasierter Veröffentlichungen.

Holstrom hatte es sich zur Aufgabe gemacht, mit der Realisation seines Projekts fünf Zielsetzungen zu entsprechen:

1. Schaffung einer literarisch hochwertigen zusammenhängenden Erzählung

2. Vorbereitung und Förderung multipler Lesewege

3. Einbeziehung einer unbegrenzten Anzahl von Autoren

4. Vermittlung eines Gemeinschaftsgefühls, das Leser und Autoren langfristig an das Projekt bindet

5. Nutzung der technischen Möglichkeiten des World Wide Web im Sinne eines grundlegenden Wandels in Literaturkonzeption und -rezeption, Kommunikationsund Diskussionskultur sowie in Denk- und Lebensweise

Holstrom war sich von Beginn an bewußt, daß die Veröffentlichung von mündlich Überliefertem in Form einer webbasierten Publikation, an deren Entstehung eine möglichst große Anzahl von Autoren beteiligt sein sollte, zahlreiche Schwierigkeiten aufwerfen würde. Wie sollte es gelingen, mit einer unbegrenzten Anzahl von Autoren einen durchgehend überzeugenden, logisch strukturierten Hypertext zu erzeugen? Ebenfalls unklar schien, wie das Verhältnis von Selbstbestimmung jedes einzelnen Kooperierenden zur Kontrolle des Herausgebers zu definieren war.

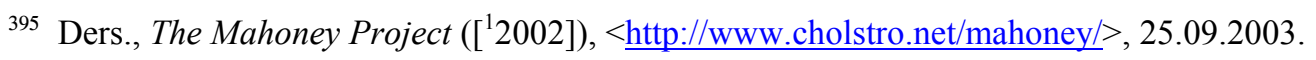


Trotz aller Bedenken ist es Holstrom gelungen, seinen Vorgaben weitestgehend gerecht zu werden:

1. Holstroms langfristiges Ziel war und ist nach wie vor die Veröffentlichung der Mahoney-Geschichten durch einen Verlag, wobei das Publikationsmedium eine untergeordnete Rolle spielt; entscheidend ist für Holstrom lediglich, daß das Gesamtwerk durch ausgefeilten Stil und gewählte Ausdrucksform sowie Logik und Folgerichtigkeit der Handlung überzeugen kann.

Von diesem Ziel sieht sich Holstrom derzeit wie gesagt noch weit entfernt. Qualität und Interdependenz der Hypertext-Elemente entsprechen zwar seinen Vorstellungen, doch räumt er selbst ein, das literarische und das hypertextuelle Potential des Werkes werde aufgrund der geringen Anzahl kooperativer Beiträge nicht in angemessener Weise ausgeschöpft.

Welche Wirkungsmöglichkeiten das Projekt in sich birgt und welche Vorteile jemals zum Tragen kommen werden, läßt sich momentan noch nicht sagen. Die (bislang) korrekte Umsetzung der Projektziels läßt lediglich darauf schließen, daß die Kooperierenden alle Vorgaben verstanden und verinnerlicht haben.

2. Die schriftliche Umsetzung der mündlich überlieferten Geschichten ließ sich angesichts der variierenden Handlungsverläufe nur in einem offenen HypertextSystem in befriedigender Weise realisieren. Nur dort konnte gleichzeitig auch der Unabgeschlossenheit der Erzählungen in adäquater Weise entsprochen werden.

Wiewohl die Hypertext-Systematik die Anlage multipler Lesewege gestattet und Holstrom von dieser nicht-linearen Erzählweise auch Gebrauch macht, sieht er selbst sich noch weit von der Verwirklichung seiner Ziele entfernt. Das Defizit an Interaktivität erklärt Holstrom nicht - wie vielleicht zu vermuten gewesen wäre - mit der geringen Zahl von Beiträgen, sondern mit der Einfachheit der Hypertext-Architektur, die - wie vorangehend ausgeführt - kein komplexes, multidirektionales Verknüpfungssystem erlaubt. Die technischen Voraussetzungen hierfür sind gegeben, allein Holstroms Programmierfähigkeiten bestimmen hier die Grenzen des Machbaren. Allerdings gibt er sich in dieser Hinsicht zuversichtlich und sieht das Mahoney-Projekt als einen entscheidenden ersten Schritt in Richtung einer effizienten Implementierung kreativer Schreibprojekte in das 
World Wide Web. ${ }^{396} \mathrm{Ob}$ seine Planungen in Richtung auf eine grundsätzliche Überarbeitung beziehungsweise Modernisierung des Mahoney-Forums gehen, oder ob er alle Kompetenzgewinne voll zugunsten neuer Projekte einzusetzen gedenkt, geht aus der Stellungnahme nicht hervor. Die vielversprechende Konzeption des Forums läßt jedoch vermuten, daß die Nutzer von Mahoney the Cat von den voraussichtlichen Fortschritten Holstroms profitieren werden. ${ }^{397}$

3. Holstroms Entscheidung für das World Wide Web als Publikationsort wurde maßgeblich durch die Kooperativität dieses Mediums beeinflußt. Das Web empfahl sich als ideales Instrument, die väterliche Tradition der gegenseitigen Inspiration und des gemeinschaftlichen Schaffens fortzuführen und $\mathrm{zu}$ perfektionieren. Holstrom mußte allerdings erfahren, daß es nicht ausreicht, nur die Möglichkeit $\mathrm{zu}$ kooperativer Zusammenarbeit anzubieten, sondern daß die eigentliche Herausforderung in der Überzeugungskraft dieses Angebots liegt. Das Mahoney-Forum ist über das Web allgemein zugänglich und zeichnet sich durch ein nutzerfreundliches Anwendungssystem aus. Insofern konnte Holstrom seiner Zielsetzung der Zusammenführung einer unbegrenzten Anzahl von Autoren gerecht werden. Der Erfolg des Projekts mißt sich jedoch in erster Linie an seiner Publikumsresonanz, die sich in kreativer und vor allem umfangreicher Mitarbeit äußert. In dieser Hinsicht müssen Holstroms Bemühungen angesichts der ernüchternd geringen Zahl von Nutzerbeiträgen letztlich als gescheitert betrachtet werden.

Fraglich ist, ob die Ursache für den Mißerfolg des Projekts tatsächlich mit der unzureichenden Bekanntheit des Forums und seiner vorläufigen technischen wie gestalterischen Unzulänglichkeit zu erklären ist. Holstrom mag richtig liegen, wenn er das Fehlen effizienter Werbung für die Zurückhaltung der Nutzer verantwortlich macht. Mit hoher Wahrscheinlichkeit könnte das Projekt durch Plazierung von Werbebannern und Back Links langfristig wachsende Besucherzahlen verzeichnen; damit wäre aber noch kein Anstieg der Kooperationsbereitschaft garantiert. Ein Blick auf die Statistik des Forums legt vielmehr die gegenteilige

396 Ders., "Conclusions", <http://www.cholstro.net/mahoney/thesis5.shtml>, 25.09.2003.

397 Ein weiterer Hinweis hierauf findet sich auch in einer Stellungnahme, die Holstrom am 12.06.2003 im Diskussionsforum veröffentlicht hat und die Überarbeitung und Ausbau des Forums in Aussicht stellt. Holstrom, "Message in 'Mahoney Discussion Topics"”, in: Ders., Hg., The Mahoney Project ([ $\left.\left.{ }^{1} 12.06 .2003\right]\right), \quad<$ http://www.mahoneythecat.org/Discussion/forum/start.asp? forumid=3\&select=491\&>, 25.09.2003. 
Annahme nahe: Nur drei von insgesamt 22 eingetragenen Nutzern waren während einer gut eineinhalbjährigen Projektlaufzeit zur Kooperation bereit. Die Scheu vor kreativem Engagement muß demzufolge noch auf andere Umstände zurückzuführen sein. Die Bedeutung technischer Perfektion wird von Holstrom offenbar auch deutlich überschätzt; die vorstehend erwähnte Unzulänglichkeit sollte einen bereitwilligen Nutzer nicht wirklich an der Einstellung eines Beitrags hindern. Vielmehr scheint - wie bereits vermutet - das geringe Identifikationspotential der Literaturform Märchen das zurückhaltende Interesse zu begründen.

4. Eng verbunden mit vorstehendem Punkt 3 war Holstroms Anliegen, ein intensives, die Nutzer langfristig emotional an das Forum bindendes Gemeinschaftsgefühl zu erzeugen. Holstrom war davon überzeugt, nur ein Gefühl der Zusammengehörigkeit könne persönliches Engagement hervorbringen, dieses wiederum werde dann zu einer Identifikation mit dem Projekt und damit langfristig zu innerer Verbundenheit und Treue führen.

Generell gilt, daß die eigentliche Herausforderung in der Maximierung der Attraktivität und Überzeugungskraft des Projekts besteht. Sollte es gelingen, die Kooperationsbereitschaft des Nutzers zu wecken, stiege damit die Wahrscheinlichkeit einer längerfristigen Verbundenheit mit dem Forum: Der Nutzer opfert mit der Erstellung eines eigenen Beitrags Zeit und Kreativität; er bringt sich mit seinem ganz persönlichen Werk als Individuum in das Forum mit ein. Gleichzeitig wird er dadurch auch Teil eines großen Ganzen, was seine Solidarität mit den übrigen Forummitgliedern weckt. Zwei Faktoren bewirken daher eine zumindest vorläufige Verbundenheit mit dem Projekt: 1. Der emotionale Bezug zum eigenen Werk. In der Regel dürfte Selbstgefälligkeit primärer Auslöser für die Wiederkehr des Nutzers sein: Er möchte sein Werk veröffentlicht und im weiteren Verlauf auch durch die Gemeinschaft positiv bewertet sehen. 2. Das persönliche Opfer für die Sache. Der Nutzer hat mit seinem Beitrag zeitlichen und kreativen Aufwand erbracht, den er durch Fortbestehen des Forums gerechtfertigt und gewahrt wissen möchte. Demzufolge versucht er, durch weiteres Engagement die Kontinuität des Projekts als eines festen Bestandteils des Web zu sichern.

Für einen gewissen Zeitraum konnte Holstrom laut Serverstatistik ${ }^{398}$ tatsächlich ein treues Publikum binden. Langfristig ist es ihm allerdings nicht gelun-

398 Ders., "Conclusions", <http://www.cholstro.net/mahoney/thesis5.shtml $>, 25.09 .2003$. 
gen, sein Forum als festen Anlaufpunkt für eine feste Nutzergemeinschaft zu etablieren. Bezeichnend ist in diesem Zusammenhang die im Diskussionsforum gestellte Anfrage eines Mitglieds vom 24.04.2003, in der Holstrom um Stellungnahme zur vermeintlichen Stagnation des Projekts gebeten wird. ${ }^{399}$

5. Das anspruchsvollste und ehrgeizigste aller von Holstrom verfolgten Ziele war und ist unverkennbar jenes, mit dem Projekt einen eigenen, selbständigen Beitrag zur Revolutionierung menschlichen Denkens und Handelns zu leisten: “[...] users can know, however, that the Mahoney Project was designed with the intention of using the Web to change the way they lived and thought." 400 Holstrom schreibt dem World Wide Web somit das ungeheure Potential $\mathrm{zu}$, einen grundlegenden Wandel in entscheidenden Bereichen des Lebens auszulösen; Aufgabe eines Herausgebers sei es, dieses Potential zu aktivieren. Für sich persönlich verzeichnet Holstrom schon einen spürbaren Wandel seiner Schreib-, Lese- und Denkgewohnheiten; was die Nutzer seines Forums angeht, gibt er sich in diesem Punkt indes zurückhaltend und überläßt den Lesern selbst die Beurteilung, inwieweit er seiner Zielsetzung gerecht werden konnte.

Zu eigener kritischer Beurteilung aufgefordert, erkennt der Nutzer, daß er durch die Konzeption des Forums an Möglichkeiten herangeführt wird, die traditionellerweise nicht in vergleichbarer Unmittelbarkeit, Effizienz und Internationalität, vor allem aber nicht in dieser Konstellation zur Verfügung standen. So vermag Holstrom unkonventionelle Methoden der Literaturkonzeption und -rezeption aufzuzeigen und den Weg zu einer neuen Kommunikations- und Diskussionskultur zu bereiten. Daß er mit Mahoney the Cat unsere Denk- und Lebensweise wirklich nachhaltig verändern könnte, ist allerdings kaum anzunehmen. Nachdem sich das World Wide Web mittlerweile als nahezu selbstverständlicher Bestandteil des Alltags etabliert hat, scheint der Anspruch des Revolutionären ein wenig zu hoch gegriffen. Das Mahoney-Forum überzeugt als Beispiel für vorbildliche Intention und Konzeption; ein umwälzender

399 lizzard, "Message in "Mahoney Discussion Topics", in: Holstrom, Hg., The Mahoney Project ([ $\left.\left.{ }^{1} 24.04 .2003\right]\right), \quad<$ http://www.mahoneythecat.org/Discussion/forum/start.asp?forumid=3\&select=490\&>, 25.09.2003. Am 12.06.2003 gibt Holstrom in bereits erwähnter Erklärung bekannt, trotz geringer Kooperationsbereitschaft laufe das Projekt kontinuierlich weiter. Er habe bereits zahlreiche Verbesserungen vorgesehen und werde auch nach außen hin wieder mehr Engagement zeigen, sobald er sich in seinem neuen beruflichen Tätigkeitsbereich etabliert habe. Seine Planungen richteten sich weiterhin auf ein umfangreiches Textangebot. Ders., "Message in "Mahoney Discussion Topics", $<$ http:/www.mahoneythecat.org/Discussion/forum/start.asp?forumid=3\&select=491\&>, 25.09.2003.

400 Ders., "Conclusions", <http://www.cholstro.net/mahoney/thesis5.shtml $>, 25.09 .2003$. 
Charakter kann dem Projekt hingegen nicht zugeschrieben werden. Um als bahnbrechend, zumindest aber progressiv gelten zu können, müßte das Forum wirkliche Neuerungen beinhalten, die den Nutzer verblüffen und ihm gänzlich unbekannte Möglichkeiten aufzeigen. Allein die technischen Gegebenheiten verhindern jedoch bereits die Entstehung eines epochalen literarischen Werkes.

Eine anspruchsvollere Hypertext-Systematik wäre demzufolge unabdingbare Voraussetzung für die Herausbildung komplexerer Handlungsverläufe und differenzierterer Zusammenhänge. Nur eine vielschichtigere Strukturierung ließe neben einfachen Verzweigungen auch Querverbindungen $\mathrm{zu}$ und trüge damit $\mathrm{zu}$ einer fruchtbaren Ausnutzung des eigentlichen Potentials nicht-linearer Darstellungsweise bei.

Eine höhere Ausnutzung der Ressourcen des World Wide Web ergäbe sich durch die Bereitstellung zusätzlicher Informationsquellen innerhalb des Forums. Beispielsweise käme der Ursprung der Geschichten - die Erzählungen Peter Holstroms im Kreise seiner Söhne - durch die Einbindung von Audiosequenzen stärker zum Tragen. Eine akustische Darbietung der Texte unterstriche den Aspekt des Märchen e rzäh lens und schlüge eine Brücke zwischen Oralität und Hypertextualität, das heißt zwischen Tradition und Moderne. Bedeutend mehr Anschaulichkeit und Authentizität erhielte die Entstehungsgeschichte des Projekts darüber hinaus durch eingescannte Manuskripte der verschiedenen Varianten von Mahoney the Cat, die Peter Holstrom bis seinem Tode noch handschriftlich niederlegen konnte. Nach Aussage von Chris Holstrom ging der Wunsch nach allgemeiner Verbreitung vom Vater selbst aus ${ }^{401}$, so daß Zurückhaltung aus Pietät nicht geboten ist.

Neben internen Ressourcen sollten aber auch Anlaufpunkte im World Wide Web aufgelistet werden. Bislang enthält das Forum noch keine einzige externe Verknüpfung. Im Gegensatz zu vielen Herausgebern im WWW, deren Entscheidung für das Web als Publikationsmedium sichtbar beeinflußt ist durch allgemeine Trends oder Gegebenheiten und Zwänge des Zeitgeistes beziehungsweise des Marktes, verbindet Holstrom nach eigener Aussage ein besonderes Anliegen mit seinem Projekt. Im Abschnitt About gibt er aber unter der Überschrift How Mahoney started lediglich einen kurzen Einblick in die Entstehungsgeschichte. Ausführ-

401 Ders., "Introduction", $<\underline{\text { http://www.cholstro.net/mahoney/thesis1.shtml }>, 25.09 .2003}$. 
lich werden die Zusammenhänge dagegen in seiner projektbegleitenden Abhandlung The Mahoney Project dargestellt, doch findet sich innerhalb des Forums keinerlei Hinweis auf die Existenz dieses aufschlußreichen Dokuments; eine Verknüpfung besteht nur umgekehrt vom theoretischen Teil zur praktischen Umsetzung. Die Gründe hierfür lassen sich nicht nachvollziehen, zumal Holstrom vor dem Hintergrund seiner intensiven Bemühungen zur Vermeidung unerwünschter Tendenzen eigentlich ein ausgeprägtes Interesse an der Verbreitung des theoretischen Hintergrundes haben sollte.

Angesichts der Verknüpfung zu seiner eigenen Web-Seite drängt sich die Einbindung weiterer externer Links geradezu auf. Holstrom vertritt in der Frage der Beziehung zwischen mündlicher Überlieferung, Gedrucktem und Hypertexten eine ausdrückliche Gegenposition zur überwiegenden Meinung von der chronologischen Abfolge dieser drei Übermittlungsformen. Die Nachvollziehbarkeit seiner These ließe sich signifikant steigern, wenn Holstrom durch entsprechende Verknüpfungen auch die Vertreter der gegenläufigen Meinung zu Wort kommen ließe. Ebenfalls verständnisfördernd wären weiterführende Informationen zur HypertextTheorie beziehungsweise Links zu entsprechenden Web-Seiten. Auch eine Verbindung mit theoretischen Abhandlungen zum Thema Märchen sowie mit ähnlichen Projekten würde die tiefergehende Auseinandersetzung mit der Materie eleichtern.

Im Rahmen der vorliegenden Arbeit erfolgten die Auswahl und Vorstellung des Forums Mahoney the Cat in erster Linie aufgrund seines überzeugenden praktischen wie theoretischen Hintergrundes und seiner gelungenen Konzeption. Die Beschäftigung mit ihm, sowohl die bloße Lektüre als auch die eigene aktive Mitarbeit, ist mit Sicherheit für alle Beteiligten von großem Gewinn. Was die Qualität der Texte anbetrifft, besteht die Hoffnung, das Projekt Mahoney the Cat werde durch intensive und themenkonforme Kooperation eine Qualitätssteigerung und Weiterentwicklung erfahren, die das Forum seinem anspruchsvollen Ziel der Revolutionierung menschlichen Denkens und Handelns näherbringt. 


\subsubsection{The Diary of Samuel Pepys}

\subsubsection{Präsentation}

Ein Beispiel für eine hochgradige Ausnutzung der Möglichkeiten von Hypertext und World Wide Web ist das von Phil Gyford als private Initiative auf uneigennütziger Basis $^{402}$ durchgeführte Projekt The Diary of Samuel Pepys ${ }^{403}$. Das Thema dieses Vorhabens ist die Präsentation der Tagebücher des Engländers Samuel Pepys in einem täglich aktualisierten Forum. Dem Umfang der Tagebucheintragungen von Pepys entsprechend, die sich über knappe neuneinhalb Jahre erstrecken ${ }^{404}$, ist das Projekt auf eine Dauer von genau 113 Monaten angelegt. Der erste Tagebucheintrag, der mit dem ersten Januar 1659/60 ${ }^{405}$ datiert ist, wurde anläßlich des dreihundertsten Todesjahres von Pepys am ersten Januar 2003 veröffentlicht. Seitdem wird an jedem Tag ein neuer Eintrag im WWW hinzugefügt. Da Pepys seine Tagebucheintragungen stets am Ende eines Tages vorzunehmen pflegte, werden auch alle neuen Einträge im virtuellen Tagebuch erst am Abend ins World Wide Web eingestellt. Dieser Aktualisierungsvorgang findet nach britischer Zeit um 11pm, nach US-amerikanischer Zeit um 6pm EST beziehungsweise um 3pm PST statt. ${ }^{406}$ Durch die täglich neu hinzukommenden Aufzeichnungen und die Übereinstimmung des aktuellen Tages mit dem von Pepys angegebenen Tag des Monats entsteht für den Leser der Eindruck, er könne das Leben von Samuel Pepys gewissermaßen mit(er)leben.

402 Phil Gyford, "Support this site", in: Ders., Hg., The Diary of Samuel Pepys (05.08.2003 $\left.\left[{ }^{1} 01.01 .2003\right]\right),<$ http://www.pepysdiary.com/about/support/>, 06.08.2003.

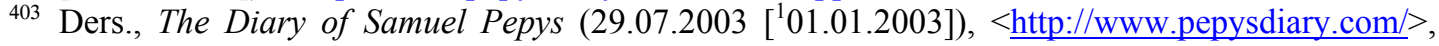
30.07.2003.

404 Das Ende der Tagebuchaufzeichnungen war bedingt durch eine zunehmende Verschlechterung der Sehfähigkeit von Pepys. Bereits im Jahre 1664 schrieb er in seinem Tagebuch von einer Einschränkung seines Sehvermögens, das zunächst durch Verwendung spezieller Augengläser korrigiert werden sollte. Seine Sehkraft verschlechterte sich jedoch zunehmend, und da Pepys den Verlust seines Augenlichts befürchten mußte, sah er sich schließlich gezwungen, seine Aufzeichnungen am 31. Mai 1669 zu beenden. Sein letzter Tagebuch-Eintrag dokumentiert seine physische wie psychische Verfassung: "And thus ends all that I doubt I shall ever be able to do with my own eyes in the keeping of my journal, I being not able to do it any longer, having done now as long as to undo my eyes almost every time that I take a pen in my hand." Gyford, "Particulars of the life of Samuel Pepys", in: Ders., Hg., The Diary of Samuel Pepys (29.07.2003 $\left.\left[{ }^{1} 01.01 .2003\right]\right),<$ http://www.pepysdiary.com/intro/pepys/>, 30.07.2003.

405 Während der drei Monate Januar, Februar und März nannte Pepys in den Datierungen seiner Tagebuch-Eintragungen stets sowohl das aktuelle Jahr als auch das jeweils vorangehende. Mit dieser Doppel-Nennung berücksichtigte er sowohl den offiziellen Beginn des Kalenderjahres als auch die inoffizielle Zeitrechnung, denn obwohl der Jahrsanfang auf den 25. März festgelegt war, wurde vielfach bereits der erste Januar als der Beginn des Jahres angesehen. Gyford, "Frequently Asked Questions", in: Ders., Hg., The Diary of Samuel Pepys (29.07.2003 $\left.\left[{ }^{1} 01.01 .2003\right]\right),<$ http://www.pepysdiary.com/about/faq/> 30.07.2003.

406 Ebd., 30.07.2003. 
Die Aufzeichnungen von Pepys gestatten aufschlußreiche Einblicke in die politischen, kulturellen und sozialen Verhältnisse der Restaurationsepoche. Pepys wurde am 23. Februar 1633 vermutlich in Brampton ${ }^{407}$ als fünftes von elf Kindern geboren und verstarb am 26. Mai 1703 nach langer Krankheit in London. Durch seine Tagebucheintragungen, die aller Wahrscheinlichkeit nach nicht zur Veröffentlichung gedacht waren, gibt er detaillierte Einblicke in das öffentliche Leben seiner Zeit; gleichzeitig gestatten seine Ausführungen Rückschlüsse auf sein Privatleben. In den Tagesrückblicken werden die besonderen Ereignisse des Privatlebens, aber auch die Alltagstrivialitäten dargelegt, wobei Pepys sogar intimste Details seiner Ehe mit der um sieben Jahre jüngeren Elizabeth preisgibt. ${ }^{408}$ Auch aufgrund dieser Tatsache kommt den Tagebüchern ein erheblicher kulturhistorischer Wert zu.

Die Tagebücher dokumentieren daneben einen bemerkenswerten beruflichen Werdegang, der Pepys zu Erfolg und Einfluß führte. Durch persönliche Beziehungen und Protektion, doch zweifelsohne auch durch Leistung und Bedachtsamkeit gelang es ihm, zum hochgestellten Staatsdiener aufzusteigen, der in enger Verbindung zum königlichem Hof und zum Hochadel stand. Pepys besaß weitreichende Kenntnisse der politischen Geschehnisse seiner Zeit, an denen er oftmals auch persönlich beteiligt war.

Grundpfeiler des Projekts The Diary of Samuel Pepys bilden die vier wesentlichen Eigenschaften des World Wide Web:

a) Unmittelbarkeit

b) Aktualität

c) Interaktivität

d) Kooperativität

407 Ders., "Particulars of the life of Samuel Pepys", <http://www.pepysdiary.com/intro/pepys/>, 04.08.2003.

408 Die Eheschließung zwischen Samuel Pepys und Elizabeth St. Michel erfolgte im Jahre 1655 in der St. Margaret's Church in Westminster/London. Der Heiratsurkunde zufolge fand die Trauung am ersten Dezember 1655 statt, wohingegen Pepys - in Übereinstimmung mit seiner Frau Elizabeth - in seinen Aufzeichnungen den zehnten Oktober desselben Jahres als Hochzeitstag nennt. - Die 14 Jahre währende Ehe zwischen Samuel und Elizabeth Pepys bleibt kinderlos. Elizabeth verstirbt im November 1669 im Alter von 29 Jahren an einem heftigen Fieber, das sie nach der Rückkehr von einer längeren Reise mit ihrem Mann befallen hatte.

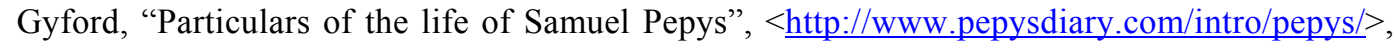
05.08.2003. 
Diese Eigenschaften hat Gyford folgendermaßen umgesetzt:

a) The Diary of Samuel Pepys ist jederzeit, von jedem Punkt der Welt aus kostenfrei zugänglich.

b) Der Inhalt der Seiten wird täglich aktualisiert, überarbeitet und angepaßt. So ergänzt Gyford das Tagebuch jeden Tag um einen neuen Eintrag, gleicht diesen an die Präsentationsform des bereits vorliegenden Datenbestandes an und fügt neue beziehungsweise neugewonnene Informationen hinzu.

Für diejenigen Nutzer des World Wide Web, die auf ihrem Computer einen sogenannten RSS news reader installiert und in diesem Informationsübermittlungsprogramm den URL des Tagebuchforums eingetragen haben, bietet Gyford die aktuellen Tagebucheinträge in zwei unterschiedlichen RSS-Formaten an. ${ }^{409}$ Die Nutzer erhalten automatisch jede Aktualisierung der Seiten entweder in der ausführlichen Version mit dem gesamten Tagebucheintrag und allen enthaltenen Verknüpfungen zu Hintergrundinformationen, ausgenommen Nutzeranmerkungen, oder aber in der verkürzten Version, die auf die Angabe der ersten 20 Worte eines jeden Eintrags beschränkt ist und keine Verknüpfungen enthält. ${ }^{410}$

c) Gyford veröffentlicht The Diary of Samuel Pepys nicht als linear-konzipierten, abgeschlossenen Fließtext, wie er beispielsweise im Project Gutenberg ${ }^{411}$ aufzurufen ist, sondern präsentiert das Tagebuch als Hypertext mit einer Vielzahl teilweise mehrfach verzweigter Verknüpfungen. Zahlreiche farbig gekennzeichnete Links weisen aus dem Text heraus auf weiterführende Informationen innerhalb des Tagebuchforums oder extern im World Wide Web. In welcher Weise genau die Hypertext-Systematik von Gyford umgesetzt wird, soll an späterer Stellen anhand eines einzelnen Tagebucheintrags exemplarisch dargestellt werden.

409 Gyford verwendet RSS als Abkürzung für Rich Site Summary. Gelegentlich kann RSS auch für RDF Site Summary oder Really Simple Syndication stehen; diese Uneinheitlichkeit hängt mit der Entstehungsgeschichte der verschiedenen RSS-Versionen zusammen. Es handelt sich dabei aber immer um ein Programm, das Informationen so aufbereiten kann, daß sie durch andere Programme interpretiert werden können. Auf diese Weise lassen sich Aktualisierungen von Web-Seiten automatisch übermitteln. RSS-Reader überwachen gewissermaßen die ihnen vorgegebenen Web-Adressen und zeigen Veränderungen umgehend an, was dem Anwender überflüssige Besuche auf unveränderten Web-Seiten erspart. Gyford, "What is RSS?", in: Ders., Hg., Writing, <http://www.gyford.com/phil/writing/2003/01/05/an introduction_php $>$, 06.08.2003. Alp Uçkan, Was ist RSS?, in: Marianne Bunyan und Alp Uçkan, Hg., windhunde.de - Das Windhunde-Portal (12.07.2003 [ $\left.\left.{ }^{1} 09.03 .2003\right]\right),<\underline{\text { http://www.windhunde.de/wasistrss.php }>, 06.08 .2003 . ~}$

${ }^{410}$ Gyford, "Other formats", in: Ders., Hg., The Diary of Samuel Pepys (05.08.2003 [ $\left.\left.{ }^{1} 01.01 .2003\right]\right)$, $<$ http://www.pepysdiary.com/about/formats/>, 06.08.2003.

411 Hart, Hg., Project Gutenberg (12.02.2003 [ $\left.\left.{ }^{1} 1971\right]\right),<$ http://gutenberg.net/>, 30.07.2003. 
d) Nach eigenem Eingeständnis ist Gyford kein Experte auf dem Gebiet "Leben und Werk von Samuel Pepys". ${ }^{412}$ Sein Tagebuchprojekt ist vielmehr ganz offensichtlich so konzipiert, daß die Gemeinschaft vom Wissen aller profitiert. Daher ist jeder Nutzer, der zu einem besseren Verständnis der Tagebucheintragungen beitragen kann, dazu eingeladen, ihren Inhalt zu kommentieren. Für diesen Zweck ist ein Verknüpfungspunkt mit der Bezeichnung annotations vorgesehen, der sich am Ende aller Tagebucheinträge befindet und zu allen hierzu bereits eingegangenen Nutzerkommentaren sowie einem Eingabeformular für eigene Anmerkungen weiterleitet. Die kooperativen Beiträge lassen sich anschließend nicht nur über den entsprechenden Tagebucheintrag aufrufen, sondern sind für die Dauer einiger Tage zusätzlich auch über eine Liste aller zuletzt eingegangenen Kommentare zugänglich, zu der die Verknüpfung Recent annotations im Kopf der Tagebuchseiten weiterführt. ${ }^{413}$

Bezieht sich der Kommentar eines Leser nicht direkt auf eine einzelne, ganz spezielle Tagebucheintragung, sondern betrifft er vielmehr ein immer wiederkehrendes Element der Tagesrückblicke von Pepys, zum Beispiel eine bestimmte, regelmäßig erwähnte Person, oder liefert der Kommentar allgemeine Hinweise zur Zeitgeschichte oder zur Biographie des Autors, so besteht die Möglichkeit, die von Gyford bereitgestellten Hintergrundinformationen zu ergänzen. Im Bereich Background information finden sich 15 Verknüpfungspunkte, die sich ihrerseits wieder mehrfach verzweigen und dem Nutzer Zugang zu detaillierten Hinweisen über das Leben der damaligen Zeit schaffen. Folgende Themenbereiche stehen dort zur Verfügung: ${ }^{414}$

- Art and Literature

- Entertainment

- Fashion

${ }^{412}$ Gyford, “About this site", in: Ders., Hg., The Diary of Samuel Pepys (03.08.2003 [101.01.2003]), $<$ http://www.pepysdiary.com/about/>, 04.08.2003.

${ }_{413}$ Jeder Verfasser einer Anmerkung ist dazu angehalten, diese kritisch auf ihre Relevanz, ihren Neuigkeitswert und ihren Bezug zu dem jeweiligen Tagebucheintrag zu überprüfen. Wortgetreue Wiedergaben sollten korrekt zitiert und als Zitate optisch hervorgehoben sein sowie mit einem Hinweis auf ihre Quellen versehen werden. Grundsätzlich sollte ein Kommentar eine Länge von zwei bis drei Absätzen nicht überschreiten, es sei denn, das Thema erfordert zwingend eine ausführlichere Darstellung. Gyford, "Annotation guidelines", in: Ders., Hg., The Diary of Samuel Pepys (03.08.2003 [ $\left.\left.{ }^{1} 01.01 .2003\right]\right),<$ http://www.pepysdiary.com/about/annotation/>, 04.08.2003.

${ }^{414}$ Ders., "Background information", in: Ders., Hg., The Diary of Samuel Pepys (03.08.2003 $\left.\left[{ }^{1} 01.01 .2003\right]\right),<$ http://www.pepysdiary.com/background/>, 04.08.2003. 
- Food and Drink

- General Reference

- Government and Law

- Health

- Holiday and Events

- Jobs and Professions

- Money

- People

- Places

- Religion

- Science and Technology

- Travel and Vehicles

Eine weitere Hilfestellung für den Nutzer, die ebenfalls durch kooperative Beiträge ergänzt werden kann, ist das Glossar im Bereich Background information. Obgleich Anregungen zu einer solchen Informationsquelle seit längerer Zeit vorgelegen hatten, zeichnete sich die Notwendigkeit hierfür erst im Verlaufe des Projekts verstärkt ab. Das Anfang August des Jahres 2003 eingerichtete Glossar soll exakt jene Worte aufgreifen, die relativ häufig im Tagebuchtext erscheinen und erläuterungsbedürftig sind, sich aber keiner der übrigen Kategorien zuordnen lassen. ${ }^{415}$ Das Glossar enthielt am 05.08.2003 nur einen einzigen Eintrag und soll durch die Nutzer im Laufe der Zeit - angeregt durch bereits eingestellte oder neu hinzukommende Tagebucheinträge - erweitert werden. ${ }^{416}$

Alle Nutzer, die sich über Anmerkungen zum Text oder zu den Hintergrundinformationen beziehungsweise über Ergänzungen im Glossar hinaus persönlich äußern möchten, erhalten in einem Diskussionsforum die Gelegenheit dazu. Die Notwendigkeit der Bereitstellung eines zusätzlichen, informelleren Bereichs zur Kommunikation hatte sich erst einige Zeit nach Start des Projekts ergeben, nachdem zunehmend textferne Anmerkungen, beispielsweise zu Konzeption und Gestaltung des Projekts eingegangen waren. ${ }^{417}$ So dient der Bereich Pepys Diary Discussion seit dem 06. März 2003 dem allgemeinen - gleichwohl aber dennoch themenbezogenen - nicht moderierten Meinungsaustausch. Die Teilnahme an der Diskussion ist unbeschränkt, unverbindlich und kostenfrei, setzt

415 Ders., "New glossary in Background Info", in: Ders., Hg., The Diary of Samuel Pepys (04.08.2003 [ [01.01.2003]), <http://www.pepysdiary.com/about/archive/2003/08/04/1168.php >, 05.08.2003.

416 Ders., "Glossary”, in: Ders., Hg., The Diary of Samuel Pepys (04.08.2003 [0101.01.2003]), $<$ http://www.pepysdiary.com/background/?c=glossary $>, 05.08 .2003$.

417 Ders., “Join the discussion forum!", in: Ders., Hg., The Diary of Samuel Pepys (03.08.2003 [ $\left.\left.{ }^{1} 01.01 .2003\right]\right),<$ http://www.pepysdiary.com/about/archive/2003/07/27/1137.php >, 04.08.2003. 
aber eine einmalige Registrierung und eine Anmeldung vor jeder Benutzung voraus. Am 04.08.2003 umfaßte die Gruppe der Gesprächsteilnehmer 49 Personen. $^{418}$

Die soeben dargestellten vier Merkmale, die das Projekt von Gyford auszeichnen - Aktualität, Unmittelbarkeit, Interaktivität und Kooperativität - werden entscheidend ergänzt und bereichert durch einen weiteren Faktor, der gerade im Hinblick auf das WWW und seine heterogenen Nutzergruppen von besonderer Bedeutung ist: Interdisziplinarität. Gyfords Initiative stützt sich auf ein literarisches Werk, das Einblick in die unterschiedlichsten Bereiche des Lebens in der Zeit von 1660 bis 1669 gewährt: Kunst und Literatur, Staatsgewalt und Justiz, Gesundheit, Zahlungsmittel, Religion, Wissenschaft und Technologie, um nur einige Gebiete zu nennen. Gyford bietet dem Leser ausführliche Informationen zu allen denkbaren verwandten Themen. Allein das Informationsmaterial zum Stichwort Geld deckt mehrere Themenbereiche gleichzeitig ab. Währungen, Preise, Gehälter und Besteuerung, vor allem aber das damalige Preis-Leistungsgefüge sind gleichermaßen für Historiker wie für Juristen beziehungsweise Wirtschaft- oder Sozialwissenschaftler von Interesse.

Der Tagebuchtext von Samuel Pepys ist im Laufe der Jahre mehrfach veröffentlicht und zu diesem Zwecke teilweise erheblichen Veränderungen in Umfang und Inhalt unterzogen worden. Da einige der Maßnahmen bis heute nachhaltigen Einfluß haben, erscheint ein Blick auf die wechselvolle Publikationsgeschichte aufschlußreich. ${ }^{419}$

Pepys hatte den größten Teil seiner Eintragungen in der von Thomas Shelton ausgearbeiteten Kurzschrift vorgenommen; nur einige wenige Worte wie beispielsweise Eigennamen von Personen oder Ortsnamen hatte er in Langschrift notiert. ${ }^{420}$

418 Ders. (Verantw.), Pepys Diary Discussion, in: Freeserve Plc, Hg., SmartGroups.com, $<$ http://www.smartgroups.com/groups/pepysdiary $>, 04.08 .2003$.

419 Sofern nicht gesondert vermerkt, siehe zu allen nachfolgenden Informationen Gyford, "About the text", <http://www.pepysdiary.com/about/text/>, 04.08.2003.

${ }^{420} \mathrm{Da}$ das Manuskript von Pepys nur sehr schwer zu entziffern war, wurde lange Zeit angenommen, er habe seine Tagebücher in einer Geheimschrift verfaßt. Wie sich später herausstellte, handelte es sich dabei jedoch um eine von John Shelton ausgearbeitete Kurzschrift. Gyford, "About the text", in: Ders., Hg., The Diary of Samuel Pepys (03.08.2003 [ $\left.\left.{ }^{1} 01.01 .2003\right]\right)$, $<$ http://www.pepysdiary.com/about/text/>, 04.08.2003. Siehe auch Walter Jens, Hg., Kindlers Neues Literaturlexikon, Bd. 13 (München, 1991), 93. Seit dem Jahre 1588, in dem Timothy Bright sein Werk Characterie: an Art of Shorte, Swifte, and Secret Writing by Character veröffentlichte, entwickelten sich während der darauffolgenden 50 Jahre 13 Kurzschrift-Systeme, von denen Thomas Sheltons Short Writing aus dem Jahre 1626, das später von Pepys verwendet wurde, als das bekannteste gilt. The New Encyclopcedia Britannica in 32 volumes, 15. Aufl. (Chicago, 2002). Bd. 9, s.v. "Samuel Pepys". 
John Smith, ein Cambridge-Absolvent, legte im Jahre 1822 eine Transkription des Tagebuchtextes vor, der daraufhin erstmals im Jahre 1825 von Richard Neville, dem späteren Lord Braybrooke, und Henry Colburn in einer zweibändigen Ausgabe veröffentlicht wurde. Braybrooke nahm hierbei starke Kürzungen im Umfange von ungefähr drei Viertel des Textes vor; auf der anderen Seite fügte er einige Fußnoten hinzu, die jedoch offensichtlich nur sehr kurz und nicht immer korrekt waren. Trotz dieser wesentlichen Veränderungen unterließ Braybrooke es, sein Vorgehen zu dokumentieren, weshalb die Veröffentlichung als "a travesty of the original" gilt. ${ }^{421}$ Im Jahre 1828 wurde die Ausgabe nochmals aufgelegt. In einer dritten Ausgabe der Jahre 1848/49 versuchte Braybrooke, seine drastischen Kürzungen etwas zurückzunehmen, was den Umfang der Publikation auf 40 Prozent des Originaltextes erhöhte. Braybrooke veröffentlichte im Jahre 1854 auch noch eine vierte, überarbeitete und korrigierte Ausgabe, die sich allerdings nicht wesentlich von der vorangegangenen Version unterschied.

Im Jahre 1872 begann Reverend Mynors Bright mit einer völlig neuen Transkription von Pepys' Kurzschrift, die er als eine sechsbändige neue Ausgabe des Tagebuchs zwischen 1875 und 1879 herausgab. Darin enthalten waren ungefähr 80 Prozent des gesamten Manuskripts sowie Braybrooks Fußnoten und eine einleitende Kurzbiographie von Pepys aus dem Jahre 1828.

Die sechste Ausgabe umfaßte acht Bände und wurde zwischen 1893 und 1896 von Henry Benjamin Wheatley veröffentlicht. Dieser fügte zahlreiche Fußnoten ein, die eine große Bandbreite an Themen abdeckten. Erstmals wurden annähernd alle Eintragungen von Pepys abgedruckt und die wenigen verbliebenen Auslassungen größtenteils kenntlich gemacht. Doch wurde der Originaltext nicht ausnahmslos in seiner ursprünglichen Fassung abgedruckt; wie oft bei viktorianischen Editionen älterer Texte, wurden einige anstößig erscheinende Worte oder Passagen durch schicklichere Ausdrücke ersetzt wurden; bei anderen Veränderungen des Textes ist bis heute ungeklärt, aus welchem Grund sie durchgeführt worden sind. Zusätzlich zu den acht Bänden publizierte Wheatley im Jahre 1899 zwei Ergänzungsbände nebst einem Register und den sogenannten Pepysiana, einer Sammlung weiterführender Informationen über Pepys und dessen Tagebuch.

${ }^{421}$ Gyford, "Previous editions of the diary", in: Ders., Hg., The Diary of Samuel Pepys (05.08.2003 [ $\left.\left.{ }^{1} 01.01 .2003\right]\right),<$ http://www.pepysdiary.com/about/text/>, 06.08.2003. 
Im Jahre 1971 wurde eine weitere Ausgabe des Tagebuchs von Robert Latham und William Matthews ${ }^{422}$ veröffentlicht, die sehr viel genauer und vollständiger ist als die Publikation von Wheatley aus dem Jahre 1893 und ausführlichere Anmerkungen enthält. Der erste Band beinhaltet außerdem eine umfassende Einleitung sowie einen Lageplan, während der Begleitband Zusatzinformationen zu unterschiedlichen Themen bereithält. ${ }^{423}$

In neuerer Zeit hat David Widger die Ausgabe Wheatleys aus dem Jahre 1893 in ein elektronisches Dokument umgewandelt und erstmals im Dezember $2001 \mathrm{im}$ Rahmen des Project Gutenberg kostenfrei zur Verfügung gestellt. ${ }^{424}$ Vermutlich waren es Urheberrechtsgründe, die Widger veranlaßten, auf die Ausgabe von Wheatley zurückzugreifen. Widger hat vereinzelt Fußnoten hinzugefügt, die aufgrund der Initialen "D.W." als seine eigenen Anmerkungen zu erkennen sind.

Die elektronische Tagebuchausgabe Widgers, die vom Project Gutenberg aus kostenfrei zugänglich ist, bildet wiederum die Vorlage für den Text, den Gyford - mit einigen Änderungen seinerseits - in seinem Forum zur Verfügung stellt.

Die zahlreichen Fußnoten in Gyfords Text sind verschiedenen Herausgebern des Tagebuchs zuzuordnen. So stehen die Initialen “D.W.” für David Widger, den oben genannten Editor der elektronischen Version des Project Gutenberg. Hinter dem Kürzel "B” vermutet Gyford den Herausgeber der früheren Ausgaben des Tagebuchs, Lord Braybrooke, während alle Fußnoten, die keiner bestimmten Person zugeordnet sind, offensichtlich von Henry Benjamin Wheatley stammen. Gyford seinerseits verwendet bei seinen eigenen Anmerkungen im Text die Buchstaben "P.G.”, wobei er nur in Ausnahmefällen, wenn der Tagebuchtext zu mißverständlich und aus diesem Grunde erläuterungsbedürftig erscheint, Fußnoten in den Text einbindet. Soweit es das Textverständnis nicht zwingend erfordert, pflegt Gyford seine Anmerkungen ebenso wie alle anderen Kommentatoren aus dem Web in den

${ }^{422}$ Einige Nutzer beziehen sich in ihren Kommentaren mit dem Kürzel "L\&M" auf die beiden Herausgeber Latham und Matthews. Gyford, "Frequently Asked Questions", $<$ http://www.pepysdiary.com/about/faq/>, 06.08.2003.

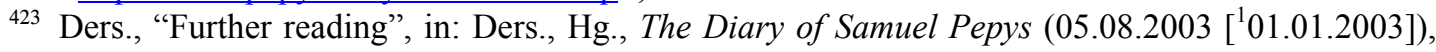
$<$ http://www.pepysdiary.com/about/further/>, 06.08.2003.

424 David Widger, "Samuel Pepys - Unabridged Diary", in: Hart, Hg., Project Gutenberg, $<$ http://www.gutenberg.net.au/widger/home.html\#pepys $>, 06.08 .2003$. Ders., "Entire Gutenberg Edition of the Diary of Samuel Pepys", in: Center for the Public Domain/University of North Carolina - Chapel Hill, ibiblio - the public's library and digital archive (Juni 2003 $\left.\left[{ }^{1} 12.12 .2001\right]\right),<$ http://www.ibiblio.org/gutenberg/etext03/sp85g10.txt>, 06.08.2003. 
offiziellen Rubriken für Erläuterungen zu veröffentlichen, das heißt entweder am Ende eines jeden Eintrags von Pepys über die Verknüpfung annotations oder übergeordnet in einer der Kategorien des Bereichs Background information. Gyford versucht auf diese Weise, den Tagebuchtext möglichst unverändert in genau der Form wiederzugeben, in der er bei Project Gutenberg präsentiert wird. ${ }^{425}$

An der dort vorgegebenen Konzeption hat Gyford dementsprechend nur wenige Veränderungen vorgenommen. Dazu gehört unter anderem, daß alle Anmerkungen von Pepys, die sich nicht auf den Inhalt eines konkreten Tagebucheintrags beziehen, wie beispielsweise Angaben zur Biographie einer Person oder Ortsbeschreibungen, aus dem Text herausgenommen und im Abschnitt Background information eingeordnet wurden. Wo immer die betreffende Person oder der betreffende Ort im weiteren Verlauf des Tagebuchs erneut erwähnt wird, hat Gyford Verknüpfungen zu den jeweiligen Hintergrundinformationen hergestellt. ${ }^{426}$ Diese Vorgehensweise ist ein Beispiel dafür, wie das elektronische Medium zu einer übersichtlichen Darstellung und damit zum Vorteil des Lesers genutzt werden kann. Ohne die Möglichkeit der Präsentation im Hypertext-Format hätte Gyford alle immer wiederkehrenden Elemente an jeder Stelle ihres Erscheinens im Text gesondert kommentieren müssen, um alle Tagebucheinträge kontextunabhängig verständlich erscheinen zu lassen und damit auch für die im Verlaufe des Projekts neu hinzukommenden Leser ohne Hintergrundwissen nachvollziehbar zu machen.

Gyford hat nicht nur den Computer, sondern auch das Medium World Wide Web zum Vorteil des Projekts eingesetzt. Einige Straßen oder Gebäude, die im Tagebuch von Pepys eine Rolle spielen, sind auf aktuellen, im WWW kostenfrei zugänglichen Stadtplänen oder Straßenkarten durch einen Pfeil markiert. ${ }^{427}$ Dies erweist sich vor allem dann als hilfreich, wenn sich Namen geändert haben oder bestimmte Gebäude bereits seit langer Zeit nicht mehr existieren.

Teilweise mußte sich Gyford allerdings auch den aktuellen technischen Gegebenheiten beugen, selbst wenn die Konzeption des Tagebuchs eine andere Gestaltung erfordert hätte. Beispielsweise pflegte Pepys grundsätzlich zum (inoffiziellen) Jahresbeginn die Ereignisse des vorausgegangenen Jahres resümierend zusammenzufassen.

\footnotetext{
425 Gyford, "Previous editions of the diary", <http://www.pepysdiary.com/about/text/> , 06.08.2003.

426 Ebd., 06.08.2003.

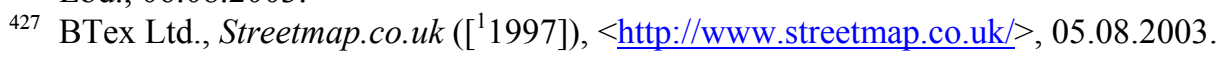


Die Tatsache, daß die ursprünglich selbständigen Jahresrückblicke nun jeweils dem ersten Januar eines jeden Jahres zugeordnet sind, erklärt Gyford mit den Unzulänglichkeiten der von ihm verwendeten Software. Sie erlaube es nicht, dem ersten Januar eines jeden Jahres gewissermaßen einen Tag “ 0 ” voranzustellen, da Daten wie 1660-01-00 regulär nicht vorgesehen seien und damit den reibungslosen Ablauf eines Programms behinderten. Aufgrund dieser technischen Zwänge seien die ursprünglich selbständigen Jahresrückblicke nun jeweils dem ersten Januar eines jeden Jahres zugeordnet. ${ }^{428}$

Eine andere Änderung betrifft die Überschriften der Tagebucheintragungen. Während Pepys auf seinen Manuskripten nur den Monatsnamen in der Kopfzeile und die Ziffern für den Wochentag jeweils am linken Seitenrand aufgeführt hatte, wurde die gedruckte Ausgabe von 1893 bereits durch Anhängen von Suffixen an die Tagesangaben erweitert. Da sich die Schreibweise von Pepys nicht für eine Wiedergabe im WWW eignete, verzichtete Gyford auf eine Kopfzeile, ersetzte die Numerierungen durch vollständige Datumsangaben und stellte sie den Tagebucheinträgen als Titelzeile voran. ${ }^{429}$ Auf Bitten einiger Nutzer und zur anschaulicheren Darstellung der Eintragungen fügte Gyford schließlich auch die Namen der Wochentage hinzu, was bei Pepys nur gelegentlich an Sonntagen der Fall gewesen war. ${ }^{430}$

Wie im Falle der Datierung verfuhr Gyford auch in einigen anderen Punkten anders als frühere Herausgeber. So wurden die von Pepys verwendeten Zeichen für das englische Pfund pound - nämlich "l." oder "li." - für die Ausgabe von 1893 in das Zeichen “ $£$ ” umgewandelt. Die Herausgeber der Veröffentlichung im Project Gutenberg gingen noch einen Schritt weiter und verwendeten nur noch den Buchstaben "L". Statt eine der beiden moderneren Schreibweisen zu übernehmen, kehrte Gyford im Einklang mit der modernen Editionspraxis zu der bei Pepys verwendeten, ursprünglichen Form der Währungsangabe zurück und gebraucht nunmehr das Zeichen "l.". 431

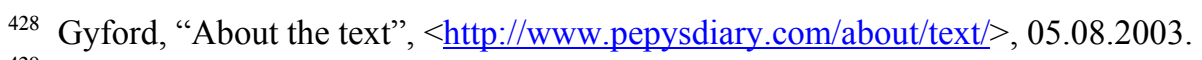

429 Ebd., 05.08.2003.

430 Gyford, "Should entries display the weekday?", in: Ders., Hg., The Diary of Samuel Pepys (04.08.2003 [ $\left.\left.{ }^{1} 01.01 .2003\right]\right),<$ http://www.pepysdiary.com/about/archive/2003/01/13/200.php >, 05.08.2003.

431 Ders., "About the text", <http://www.pepysdiary.com/about/text/>, 05.08.2003. 
In Kapitel II. dieser Arbeit wurde bereits darauf hingewiesen, die Frage des Urheberrechtsschutzes im World Wide Web sei gegenwärtig noch nicht international in einer die Autoren wie Leser beziehungsweise Nutzer gleichermaßen zufriedenstellenden Weise geklärt worden. In diesem Zusammenhang wurde ferner auf die Beobachtung von Kist verwiesen, es sei eine bedenkliche Tendenz zu wachsender Indifferenz gegenüber Urheberrechten festzustellen. ${ }^{432}$

Die Seiten The Diary of Samuel Pepys bilden in dieser Hinsicht eine beispielhafte Ausnahme. Hier wird der Nutzer vom Herausgeber über die derzeit gültige Rechtslage informiert und zusätzlich auf weiterführende Informationen im World Wide Web verwiesen. Gyford zufolge sind alle Tagebuchtexte von Pepys sowie die dazugehörigen Fußnoten, der Einbandtext ${ }^{433}$ und der hauptsächliche Text der Kategorien People und Places aus dem Bereich Background information der auf den Seiten des Project Gutenberg veröffentlichten Ausgabe des Tagebuchs entnommen und unterliegen aus diesem Grunde keinen Urheberrechtsbeschränkungen. ${ }^{434}$

Sämtliche Materialien, die von den Nutzern im Zusammenhang mit ihren Anmerkungen zum Tagebuch von Pepys in Form eines Zitats in die Seiten eingefügt werden, behalten ihren urheberrechtlichen Status. ${ }^{435}$ Die Nutzeranmerkungen selbst sowie alle Darstellungen von Gyford unterliegen speziellen Vorschriften, die von der Creative Commons Corporation verfaßt worden sind. ${ }^{436}$

\subsubsection{Handhabung des Hypertextes}

Wie bereits angekündigt, soll nunmehr ein einzelner Tagebucheintrag beispielhaft näher vorgestellt werden. Ausgewählt wurden Pepys' Ausführungen von Mittwoch, dem 18. Juli $1660 .{ }^{437}$ Die detaillierte Schilderung und die teilweise vollständige Erwähnung der Nutzerkommentare erfolgen dabei im Sinne eines umfassenden Ein-

432 Kist, Elektronisches Publizieren, 86.

${ }^{433}$ Gyford, “Cover text”, in: Ders., Hg., The Diary of Samuel Pepys (04.08.2003 [ 01.01 .2003$\left.]\right)$, $<$ http://www.pepysdiary.com/intro/>, 05.08.2003.

434 Ders., "About the text", <http://www.pepysdiary.com/about/text/>, 05.08.2003. Wie bereits in Kapitel IV. in den Ausführungen zu Project Gutenberg erwähnt, werden dort ausschließlich Texte veröffentlicht, die nicht mehr unter Urheberrechtsschutz fallen. Hart, "What books will I find in Project Gutenberg?", in: Ders., Hg., Project Gutenberg (20.06.2002 [ $\left.\left.{ }^{1} 1971\right]\right)$, $<\underline{\text { http://promo.net/pg/helpex.html\#What-books }>, 05.08 .2003 . ~}$

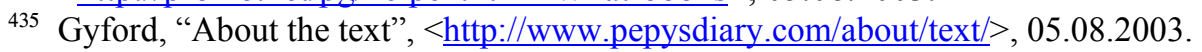

436 Creative Commons Corporation, Hg., Creative Commons - Commons Deed: Attribution - Noncommercial - Share Alike - Key License Terms, $<$ http://creativecommons.org/licenses/by-nc-sa/1.0/>, 05.08.2003.

437 Gyford, “Archive for July 1660”, in: Ders., Hg., The Diary of Samuel Pepys (05.08.2003 $\left.\left[{ }^{1} 01.01 .2003\right]\right),<\underline{\text { http://www.pepysdiary.com/archive/1660/07/index.php }>, 06.08 .2003 .}$ 
blicks in die Qualität der Publikation und den (teilweise zweifelhaften) Wert der kooperativen Beiträge.

Auf der Einstiegsseite des Forums stehen jeweils die letzten sieben Einträge zur Verfügung. Alle älteren Aufzeichnungen sind über das virtuelle Archiv der Seite zugänglich, wo die Einträge nach Jahr und Monat sortiert sind. In welchem Abschnitt des Forums der Nutzer sich gerade befindet, wird ihm in der Navigationsleiste am oberen Rand der Web-Seite angezeigt, auf der immer der aktuelle der vier Aufenthaltsbereiche hell markiert ist: The Diary, Background info, Diary introduction oder About this site.

Den Einstieg in das Forum erhält der Nutzer über The Diary. In diesem Abschnitt sind alle Informationen zu finden, die zu den aktuellen Tagebucheinträgen in Beziehung stehen: die Neueinstellungen der letzten sieben Tage mit Verknüpfungen zu den jeweiligen Anmerkungen der Nutzer, Zugänge zu den Hintergrundinformationen, ein Hinweis für Erstbesucher der Seite, der zu einer kurzen Darstellung der Person Samuel Pepys und der Geschehnisse vor Beginn des Tagebuchs führt ${ }^{438}$, sowie eine Verknüpfung mit allen neuesten von Gyford veröffentlichten themenbezogenen Meldungen. Ferner enthält dieser Abschnitt eine gesonderte Verknüpfung zu den neuesten Nutzeranmerkungen und einen Link zu dem erwähnten virtuellen Archiv. Dieses bietet eine Jahresübersicht sowie eine monatsweise Ansicht der Tagebucheinträge, wobei die Navigationselemente (Links) im oberen Teil der Seite Zugriffe auf den jeweils vorangehenden beziehungsweise den nachfolgenden Monat erlauben. Jedem Tagebucheintrag ist eine Menüleiste mit Verknüpfungen zu den Hintergrundinformationen an der rechten Seite beigegeben, was die Navigation bedeutend erleichtert; andernfalls hätte der Zugang zu weiterführenden Angaben über die oberste Navigationsleiste erfolgen müssen und dies wiederum hätte ein ständiges Auf- und Abscrollen der bisweilen recht langen Seiten erfordert.

Die ausgewählte Eintragung vom 18. Juli 1660 ist von mittlerer Länge und weist eine durchschnittliche Verknüpfungsdichte auf. Der Text enthält eine Fußnote von David Widger, der die elektronische Version des Tagebuchs für Project

\footnotetext{
438 Das Kapitel "Events preceding the diary" bedarf einer dringenden Aktualisierung. Aus dem entschuldigenden Hinweis von Gyford ist aber zu schlußfolgern, daß in absehbarer Zeit mit einer Ergänzung des unvollständigen Textes gerechnet werden darf: "Apologies for this not being up to date... we're working on it!" Gyford, "The story so far", in: Ders., Hg., The Diary of Samuel Pepys (06.08.2003 [ $\left.\left.{ }^{1} 01.01 .2003\right]\right),<$ http://www.pepysdiary.com/about/history/>, 07.08.2003.
} 
Gutenberg zur Verfügung gestellt hat, und wird in vier Anmerkungen durch Nutzer kommentiert. Folgendes schreibt Pepys an jenem Mittwoch in sein Tagebuch:

This morning the carpenter made an end of my door out of my chamber upon the leads. ${ }^{1}$ This morning we met at the office: I dined at my house in Seething Lane,

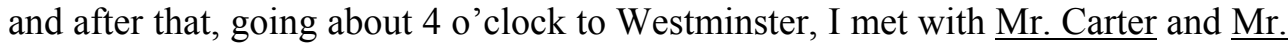
Cooke coming to see me in a coach, and so I returned home. I did also meet with Mr. Pierce, the surgeon, with a porter with him, with a barrel of Lemons, which my man Burr sends me from sea. I took all these people home to my house and did give them some drink, and after them comes Mr. Sheply, and after a little stay we all went by water to Westminster as far as the New Exchange. Thence to my Lord about business, and being in talk in comes one with half a buck from Hinchinbroke, and it smelling a little strong my Lord did give it me (though it was as good as any could be). I did carry it to my mother, where I had not been a great while, and indeed had no great mind to go, because my father did lay upon me continually to do him a kindness at the Wardrobe, which I could not do because of my own business being so fresh with my Lord. But my father was not at home, and so I did leave the venison with her to dispose of as she pleased. After that home, where $\underline{W}$. Hewer now was, and did lie this night with us, the first night. My mind very quiet, only a little trouble I have for the great debts which I have still upon me to the Secretary, Mr. Kipps, and Mr. Spong for my patent. ${ }^{439}$

1. Jacques Casanova's narration of his escape from prison in Venice refers to "under the leads" in the attic of the Palace of Doges. (The roof being plates of lead.) Perhaps Pepys "door" was an access to the roof? A few thousand pages may clear this. D.W.

Innerhalb des Textes sind zahlreiche Worte farblich als Verknüpfungen gekennzeichnet und führen den Leser direkt zu Informationen über das jeweilige Stichwort. In der vorstehenden Textwiedergabe sind diese Verknüpfungen durch Unterstreichungen als Links kenntlich gemacht.

Wie bei Gyford üblich, befindet sich auch der obige Fußnotentext unmittelbar unterhalb des Tagebucheintrags. Er bezieht sich hier gleich auf dessen ersten Satz und beschäftigt sich mit der Frage, welchen Zweck die von Pepys erwähnte Tür möglicherweise habe und wo sie sich befinde. Allerdings vermag Widger an dieser Stelle des Tagebuchs keine Antwort zu geben und stellt eine Klärung der Umstände im weiteren Verlauf von Pepys' Ausführungen in Aussicht. Dabei geht aus Widgers Fußnote nicht hervor, ob er die Motive für den Einbau besagter Tür zum Zeitpunkt der Digitalisierung des Eintrags tatsächlich nicht kennt oder ob er den Leser möglicherweise absichtlich im Unklaren läßt. ${ }^{440}$ Ebensowenig hilfreich ist in diesem $\mathrm{Zu}$ sammenhang die anachronistische Analogie zu Casanova.

439 Ders., “Archive for July 1660”, < http://www.pepysdiary.com/archive/1660/07/index.php $>$, 07.08.2003.

${ }^{440}$ Immerhin gibt er sich recht zuversichtlich, daß der Leser im weiteren Verlauf des Tagebuchs Klarheit erhalten werde. 
So sehr auch die Unbestimmtheit des ersten Satzes - sei sie nun gewollt oder nicht - zur Hebung der Neugier beitragen mag, so will sich doch beim Leser angesichts der noch zu bewältigenden "few thousand pages" keine rechte Vorfreude auf die weitere Lektüre einstellen. Daher wäre wünschenswert gewesen, wenn Gyford hier eine zusätzliche Anmerkung eingefügt hätte.

Mehr Aufklärung erhält der Leser durch die vier Anmerkungen zu dem Tagebuchtext. ${ }^{441}$ Dort wird unter anderem dargelegt, der erste Satz sei am Ende um das Wort roof $\mathrm{zu}$ ergänzen, woraus $\mathrm{zu}$ folgern sei, Pepys habe sich eine Art Aussichtsturm auf seinem Haus errichten lassen. In einer anderen Anmerkung wird die Mutmaßung geäußert, Pepys habe die Installation eines Zugangs zu seinem Dach veranlaßt, um von dort aus seine Taubenhaltung leichter bewältigen zu können.

Die beiden weiteren Anmerkungen beschäftigen sich mit der Frage, wie Pepys' Einstellung zu seiner Ehefrau und seinen Eltern zu beurteilen sei, da er dem Leser in seiner Schilderung nicht den Eindruck vermittele, als ob die Fürsorge für seine Familie für ihn an erster Stelle stehe.

Im nachfolgenden Teil des Tagebucheintrages berichtet Pepys zunächst von einem office, das ihm am Morgen des 18. Juli als Treffpunkt gedient hatte. Das Wort ist von Gyford als Link gekennzeichnet und führt den Leser in eine tiefere Ebene des Hypertextes, auf eine Seite zum Thema Navy Office. Hier sind alle wissenswerten Informationen $\mathrm{zu}$ dieser Institution gesammelt und können jederzeit durch weitere Angaben ergänzt werden. Die Seite umfaßt drei Bereiche:

1. Eine Ausführung von Gyford darüber, worum es sich bei besagtem Navy Office handelt. Seine geographische Lage und sein Zweckdienlichkeit werden dort folgendermaßen beschrieben:

The Navy Office was erected on the site of Lumley House, formerly belonging to the Fratres Sancta Crucis (or Crutched Friars), and all business connected with naval concerns was transacted there till its removal to Somerset House. - The ground was afterwards occupied by the East India Company's warehouses. The civil business of the Admiralty was removed from Somerset House to Spring Gardens in $1869 .{ }^{442}$

441 Ders., "Wednesday 18 July 1660 - Annotations", in: Ders., Hg., The Diary of Samuel Pepys (06.08.2003 [01.01.2003]), <http://www.pepysdiary.com/archive/1660/07/18/index.php\#annotations $>$, 07.08.2003.

${ }^{442}$ Ders., "Navy Office", in: Ders., Hg., The Diary of Samuel Pepys (06.08.2003 [101.01.2003]), $<$ http://www.pepysdiary.com/p/483.php $>, 07.08 .2003$. 
Zur anschaulicheren Darstellung bedient sich Gyford darüber hinaus eines visuellen Elements, indem er die Verknüpfung zu einem neueren Stadtplan von London bereitstellt, auf dem ein gelber Pfeil den ehemaligen Standort des Gebäudes markiert. ${ }^{443}$ Zusätzlichen Wert gewinnt diese Karte für den Nutzer, weil aus ihr auch hervorgeht, daß sich heute an besagter Stelle die Pepys Street befindet, deren Parallelstraße an die Crutched Friars erinnert, die Gyford in seiner obigen Erläuterung als Fratres Sancta Crucis (or Crutched friars) erwähnt. Ebenfalls erkennbar ist auf der Karte die Seething Lane, die quer zu beiden Straßen verläuft und im zweiten Satz des Tagebucheintrags vom 18. Juli als Link hervorgehoben ist.

Ebenso wie der Tagebuchtext beinhaltet auch die Erläuterung zu Navy Office Verknüpfungen, die auf weiterführende Informationen verweisen, nämlich Somerset House und the Admiralty. Bevor diese aber weiterverfolgt werden, sollen zunächst nach sequentieller Verfahrensweise alle Angaben dieser Ebene des Hypertext-Systems ausgeschöpft werden.

2. Der zweite Bereich dieser Seite ist den Kommentaren gewidmet. Unter dem Stichwort Annotations werden alle Angaben aufgelistet, die speziell das Thema Navy Office betreffen. Hier können nicht nur Nutzer ihre Kenntnisse zur Verfügung stellen, gelegentlich dient dieser Bereich auch Gyford selbst zur Ergänzung seiner Informationen. Die Eingabe der Kommentare erfolgt auf derselben Seite mit Hilfe eines kurzen Formulars, das sich unterhalb der Anmerkungen befindet. Zum Zeitpunkt der Erhebungen für diesen Teil der vorliegenden Arbeit, am 07.08.2003, lagen sechs Beiträge vor:

- Die erste Anmerkung stammt offensichtlich von Gyford selbst, der darin erklärt, das Navy Office habe sich von 1654 bis 1673 am Ort von Lumley House befunden, und dem Nutzer die Möglichkeit gibt, per Verknüpfung die Erklärung zu Navy Board aufzurufen. ${ }^{44}$

- Auf anschauliches Material im WWW wird in der zweiten Anmerkung verwiesen, in der ein Nutzer den Link zu einer Karte aus dem Jahre 1746 zur Verfügung

443 BTex Ltd., Streetmap.co.uk, < $\underline{\mathrm{http}}$ ://www.streetmap.co.uk/newmap.srfx=533441\&y=180852\&z=1>, 07.08.2003.

${ }^{444}$ Gyford, "Navy Board”, in: Ders., Hg., The Diary of Samuel Pepys (06.08.2003 [ $\left.\left.{ }^{1} 01.01 .2003\right]\right)$, $<$ http://www.pepysdiary.com/p/482.php>, 07.08.2003. 
stellt; ${ }^{445}$ auf ihr ist das Navy Office in dem Dreieck zwischen Crutched Friars und Seething Lane deutlich zu erkennen. ${ }^{446}$

- In der dritten Anmerkung schildert eine Nutzerin ihre persönlichen Erfahrungen auf der Suche nach Hinweisen zu Pepys während eines Aufenthalts in London. ${ }^{447}$ Auch Informationen dieser Art können durchaus bereichernd sein; in diesem Fall ist beispielsweise von einer Ausstellung zu Pepys die Rede, allerdings wären hier unbedingt detailliertere Angaben zu Veranstaltungsort und -dauer wünschenswert.

- Auch die vierte Anmerkung enthält einen Besichtigungshinweis für Besucher der Stadt London, ${ }^{448}$

- ebenso der fünfte Nutzerbeitrag, der allerdings auf eine Web-Seite verweist, die den gewünschten Inhalt zum oben genannten Zeitpunkt nicht bereithielt. ${ }^{449}$

- Die letzte Anmerkung eines Nutzers ist wieder hilfreich: sie gibt einen Hinweis darauf, wie das Wort Crutched in diesem Falle auszusprechen ist, nämlich nicht [, kr $\left.\Lambda t \int t\right]$, sondern als Adjektiv mit zweiter Silbe $\left[, k r \Lambda t \int I d\right]$.

3. Der untere Bereich der Seite bietet dem Nutzer unter dem Stichwort Track Back eine Sammlung von Links zu allen bereits vorhandenen Tagebucheinträgen, in denen eben diese Institution erwähnt wird. Am 07.08.2003 ist das Thema Navy Office in den Ausführungen von Pepys bereits neunzehnmal zur Sprache gekommen.

445 language hat, "Annotation on 'Navy Office'”, in: Gyford, Hg., The Diary of Samuel Pepys $\left(\left[{ }^{1} 09.03 .2003\right]\right),<$ http://www.pepysdiary.com/p/483.php $>, 07.08 .2003$.

446 MOTCO Enterprises Limited, "London, Westminster and Southwark by John Rocque 1746", in: Dies., Hg., MOTCO UK Directory and Image Database - Antique Maps, Prints \& Books ([ $\left.\left.{ }^{1} 2001\right]\right),<$ http://www.motco.com/map/81002/>, 07.08.2003. Ruft man allerdings diese WebAdresse auf, so erscheint nicht der gewünschte Ausschnitt, sondern zunächst die Titelseite der Karte. Erst über die Verknüpfung Place Name Index und von dort aus über Seething Lane gelangt der Nutzer schließlich zu dem gewünschten Kartenausschnitt. Richtigerweise hätte der URL folgendermaßen angegeben werden müssen: MOTCO Enterprises Limited, "John Rocque London, Westminster and Southwark, First Edition 1746 (26 inches to a mile); Segment 87”, in: Dies., Hg., MOTCO UK Directory and Image Database - Antique Maps, Prints \& Books ([ $\left.\left.{ }^{1} 2001\right]\right)$, $<$ http://www.motco.com/map/81002/SeriesSearchPlatesFulla.asp?mode=query\&title=Seething + La ne\&artist $=384 \&$ other $=321 \& \mathrm{x}=11 \& \mathrm{y}=11>, 07.08 .2003$.

447 Martha Wishart, "Annotation on 'Navy Office'", in: Gyford, Hg., The Diary of Samuel Pepys ([ $\left.\left.{ }^{1} 31.03 .2003\right]\right),<$ http://www.pepysdiary.com/p/483.php>, 07.08.2003.

448 Arbor, "Annotation on 'Navy Office'”, in: Gyford, Hg., The Diary of Samuel Pepys ([101.07.2003]), $<$ http://www.pepysdiary.com/p/483.php>, 07.08.2003.

449 Vincent, "Annotation on 'Navy Office”", in: Gyford, Hg., The Diary of Samuel Pepys ([101.07.2003]), $<$ http://www.pepysdiary.com/p/483.php >, 07.08.2003. Ebenso wie im Falle des Kartenausschnitts wird auch hier eine übergeordnete Einstiegsseite angezeigt. Hier ist es allerdings nicht möglich, zur empfohlenen Seite zu gelangen, da alle Recherchen - trotz Eingabe unterschiedlichster Stichworte - ergebnislos bleiben. 
Wie erwähnt, besteht das Hypertext-System The Diary of Samuel Pepys aus mehreren Ebenen. Besonders anschaulich wird diese Struktur in allen dem Bereich Background info (bei Gyford mit Top bezeichnet) untergeordneten Informationsschichten. Jedem erläuterten Element ist eine Übersicht über die verschiedenen Ebenen des Hypertext-Systems beigefügt, anhand derer der Nutzer die Einordnung dieser Elemente bis hinauf zur obersten Ebene der Background info zurückverfolgen kann. Wählt man dort beispielsweise den Punkt Places, so erhält man eine Liste unterschiedlicher Kategorien von Gebäuden, Straßen und Gegenden innerhalb und außerhalb Londons und Großbritanniens ${ }^{450}$ :

- Churches and cathedrals

- Coffee houses

- London streets and areas

- Other London buildings

- Outside Britain

- Outside London

- Taverns

In der Kategorie Other London buildings, zu der unter anderem das oben erwähnte Navy Office zählt, sind wiederum 22 verschiedene Gebäude Londons aufgelistet. Hinter jeder Bezeichnung läßt eine Ziffer in Klammern die Anzahl der zu diesem Stichwort eingegangenen Anmerkungen erkennen; zum Zeitpunkt der Recherchen für vorliegende Arbeit zeigt die Ziffer (6) bei Navy Office beispielsweise an, daß sechs Anmerkungen vorliegen. Diese Kommentare waren vorangehend bereits Gegenstand der Untersuchung; zumindest in diesem Punkt darf festgestellt werden, daß die Elemente des Hypertext-Systems von Gyford aufeinander abgestimmt und aktualisiert sind.

Die oben angesprochene Übersicht über die verschiedenen Ebenen des Hypertext-Systems stellt sich dem Nutzer am Beispiel von Navy Office also folgendermaßen dar und signalisiert ihm anhand der Link-Kennzeichnung, daß die ersten drei Elemente der Kette von seinem aktuellen Standort aus jeweils direkt aufgerufen werden können:

$$
\underline{\text { Top }}>\underline{\text { Places }}>\underline{\text { Other London buildings }}>\text { Navy Office }
$$

\footnotetext{
${ }^{450}$ Gyford, "Places", in: Ders., Hg., The Diary of Samuel Pepys (06.08.2003 [0101.01.2003]), $<$ http://www.pepysdiary.com/background/?c=places $>, 07.08 .2003$.
} 
Wie bereits angekündigt, seien nun auch die beiden Verknüpfungen innerhalb des Erläuterungstextes zu Navy Office weiterverfolgt: Zum einen der Hinweis auf Somerset House, zum anderen der Link zu the Admiralty.

Innerhalb des Bereiches Somerset House ${ }^{451}$ präsentiert Gyford zwar keine eigenen Hintergrundinformationen $\mathrm{zu}$ diesem Gebäude, gestattet dem Nutzer aber wiederum einen Blick auf den gegenwärtigen Londoner Stadtplan. ${ }^{452}$ In Ergänzung dazu verweist eine Nutzerin in ihrem Kommentar auf ein weiteres Segment des von MOTCO UK Directory and Image Database zur Verfügung gestellten historischen Kartenmaterials aus dem Jahre 1746, auf dem Somerset House ebenfalls verzeichnet ist. ${ }^{453}$ Wie aus dem Hinweis eines anderen Nutzers hervorgeht, handelt es sich bei dem derzeitigen Gebäude an der Themse nicht um das ursprüngliche Bauwerk, auf das sich Pepys in seinen Ausführungen bezogen hat, da dieses - offensichtlich an derselben Stelle - in der Zeit von 1776 bis 1786 durch eines neues Gebäude ersetzt wurde. ${ }^{454}$ Das ehemalige sogenannte Old Somerset House war einer dritten Anmerkung zufolge im Jahre 1547 errichtet worden und diente Königin Elizabeth sowie der Gemahlin James' I. als Wohnsitz. Eine Zeichnung auf den Seiten von Britannia vermittelt dem Nutzer einen Eindruck von Stil und Größe dieser Residenz. ${ }^{455}$ Den Angaben des Bereiches Track Back ist zu entnehmen, daß Somerset House zuvor bereits in zwei Tagebucheintragungen aus dem Monat Februar erwähnt wurde.

Ganz anders das Stichwort Admiralty, das bis zu Pepys' Niederschrift des Tagesgeschehens vom 18. Juli 1660 bereits fünfzehnmal gefallen ist. ${ }^{456}$ Gyford selbst hält hierzu keine Informationen bereit, zwei Anmerkungen von Nutzern geben jedoch nähere Aufschlüsse. ${ }^{457}$ Der erste Kommentar beschreibt ausführlich die Ge-

451 Ders., "Somerset House", in: Ders., Hg., The Diary of Samuel Pepys (06.08.2003 [ 01.01 .2003$])$, $<$ http://www.pepysdiary.com/p/243.php $>, 07.08 .2003$.

452 BTex Ltd., Streetmap.co.uk, $<$ http://www.streetmap.co.uk/newmap.srf? $\mathrm{x}=530756 \& \mathrm{y}=180770 \& \mathrm{z}=1>$, 07.08.2003.

453 MOTCO Enterprises Limited, "John Rocque London, Westminster and Southwark, First Edition 1746 (26 inches to a mile); Segment 81", in: Dies., Hg., MOTCO UK Directory and Image Database ([12001]), $<$ http://www.motco.com/Map/81002/SeriesSearchPlatesFulla.asp?mode=query\&artist=384\&other=315\& $\mathrm{x}=11 \& \mathrm{y}=11>, 07.08 .2003$.

454 Gyford, "Somerset House", <http://www.pepysdiary.com/p/243.php>, 07.08.2003.

455 Britannia.com, LLC, Hg., Britannia - British History, Life \& Travel, s.v. "Old Somerset House", $<$ http://www.britannia.com/history/londonhistory/oldsomhse.JPG>, 07.08.2003.

456 Gyford, “Admiralty”, in: Ders., Hg., The Diary of Samuel Pepys (06.08.2003 [ 01.01 .2003$])$, $<$ http://www.pepysdiary.com/p/297.php>, 07.08.2003. Zum Zeitpunkt der Erfassung für vorliegende Arbeit hat der Nutzer bereits Zugriff auf insgesamt 18 Verweise.

457 Ebd., 07.08.2003. 
schichte der britischen Admiralität seit $1649 ;{ }^{458}$ Pepys war in den sechziger Jahren des 17. Jahrhunderts im Range eines Offiziers Mitglied dieser Institution. Der zweite Kommentar enthält die Namen der fünf Offiziere des Jahres 1660 - darunter auch Pepys selbst -, wie sie von ihm am 31. Mai desselben Jahres handschriftlich niedergelegt worden sind. ${ }^{459}$

Bewegt sich der Nutzer zurück zum Tagebucheintrag vom 18. Juli 1660, erfährt er in dessen weiterem Verlauf zunächst von Pepys' Haus in der Seething Lane sowie von acht Personen, mit denen Pepys offensichtlich regelmäßigen Kontakt unterhielt.

In dem verlinkten Abschnitt Seething Lane verweist Gyford abermals nur auf den Londoner Stadtplan: auf ihm ist zu erkennen, daß besagte Straße quer zur heutigen Pepys Street und zu Crutched Friars verläuft. In der Rubrik Annotations sind fünf Kommentare aufgelistet, wovon der erste indes wenig hilfreich ist: Der dort angegebene Link zu einer Photographie jenes Grundstücks in der Seething Lane, auf dem Pepys ab Juli des Jahres 1660 wohnhaft war, führt tatsächlich nicht zu der gewünschten Seite. ${ }^{460}$ Der zweite Kommentar enthält einen Literaturhinweis zur Geschichte der Seething Lane in der Zeit von 1303 bis 1673, also einschließlich der Zeit, in der Pepys dort lebte. ${ }^{461}$ Der dritte Kommentar informiert den Nutzer in einer detaillierten Ausführung über die ebenso zielstrebige wie ungenierte Art und Weise, mit der es Pepys seinerzeit gelang, sich die gewünschte Immobilie in der Seething Lane zu sichern, obgleich er auf sie zum damaligen Zeitpunkt keinen Nutzungsanspruch hatte. ${ }^{462}$ Der vierte und der fünfte Kommentar befassen sich in einer Reaktion auf den dritten Beitrag mit der Frage, wie dessen Verfasserin persönlich zu Pepys' Vorgehensweise steht. ${ }^{463}$

458 Helena Murphy, “Annotation on 'Admiralty",, in: Gyford, Hg., The Diary of Samuel Pepys $\left(\left[{ }^{1} 15.03 .2003\right]\right),<$ http://www.pepysdiary.com/p/297.php $>, 07.08 .2003$.

459 Vincent, "Annotation on 'Admiralty", in: Gyford, Hg., The Diary of Samuel Pepys ([104.07.2003]), $<$ http://www.pepysdiary.com/p/297.php $>, 07.08 .2003$.

460 Ders., "Annotation on 'Seething Lane",, in: Gyford, Hg., The Diary of Samuel Pepys ([105.07.2003]), <http://www.pepysdiary.com/p/1023.php $>, 07.08 .2003$.

461 Graham Travis, "Annotation on 'Seething Lane"”, in: Gyford, Hg., The Diary of Samuel Pepys ([111.07.2003]), <http://www.pepysdiary.com/p/1023.php >, 07.08.2003.

462 Pauline, "Annotation on 'Seething Lane", in: Gyford, Hg., The Diary of Samuel Pepys ([11.07.2003]), <http://www.pepysdiary.com/p/1023.php > 07.08.2003.

463 Chip, "Annotation on 'Seething Lane'”, in: Gyford, Hg., The Diary of Samuel Pepys (['12.07.2003]), $<$ http://www.pepysdiary.com/p/1023.php>, 07.08.2003. language hat, "Annotation on 'Seething Lane”,

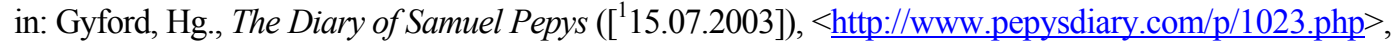
07.08.2003. 
Die im Tagebucheintrag vom 18. Juli genannten Herren Carter, Cooke, Pierce (beziehungsweise Pearse), Burr, Shepley, Hewer, Kipps und Spong werden von Gyford entweder gar nicht oder nur äußerst knapp vorgestellt. Hier ist der interessierte Leser in erster Linie auf die Nutzeranmerkungen angewiesen, die einige aufschlußreiche Angaben zu Charakter und beruflicher Position der genannten Personen sowie ihrer Beziehungen zu Samuel Pepys enthalten.

Die Tatsache, daß Gyford hier nur recht spärliche Informationen zur Verfügung stellt, ist nicht - wie man meinen könnte - damit zu erklären, daß diese Personen lediglich aus Pepys' weiterem Umfeld stammen. Auch zu den Menschen aus Pepys' engerem Kreis hält Gyford offenbar keine ausführlicheren Beschreibungen bereit. Bei Vater und Mutter des Tagebuchschreibers, John und Margaret Pepys, die in der Eintragung vom 18. Juli 1660 gleichfalls Erwähnung finden, wird ebenso wie bei Ehefrau Elizabeth Pepys nur kurz das verwandtschaftliche Verhältnis dargestellt. ${ }^{464}$ Verließe sich der Nutzer in dieser Hinsicht einzig auf die genannten Personenangaben, die auch direkt über die Rubrik Background Information zugänglich sind, bliebe ihm ein wesentlicher Teil der von Gyford zur Verfügung gestellten Informationen verschlossen, denn entscheidende Auskünfte über die Biographien der wichtigsten Personen im Leben von Samuel Pepys sind nicht über die Hintergrundinformationen zugänglich, sondern über den Abschnitt Diary introduction in der Navigationsleiste am oberen Rand der Web-Seite. Dort finden sich im Bereich Particulars of the life of Samuel Pepys detaillierte biographische Beschreibungen. ${ }^{465}$

Im Tagebuchtext vom 18. Juli 1660 verbleiben noch fünf Stichworte, die von Gyford als Verknüpfungen gekennzeichnet sind: Lemons, New Exchange, Hinchinbroke, the Wardrobe und venison. Zum Teil erweisen sich die Zugriffe auf die entsprechenden Abschnitte erneut als wenig ergiebig. Der Rubrik Lemons zum Beispiel sind keinerlei weiterführende Informationen zu entnehmen; weder von Gyford

${ }^{464}$ Gyford, "Pepys, John (father)", in: Ders., Hg., The Diary of Samuel Pepys (06.08.2003 [101.01.2003]), $<$ http://www.pepysdiary.com/p/154.php >, 07.08.2003. Ders., "Pepys, Margaret (mother)", in: Ders., Hg., The Diary of Samuel Pepys (06.08.2003 [101.01.2003]), <http://www.pepysdiary.com/p/282.php>, 07.08.2003. Ders., "Pepys, Elizabeth (wife)", in: Ders., Hg., The Diary of Samuel Pepys (06.08.2003 [101.01.2003]), < $\underline{\text { http://www.pepysdiary.com/p/150.php }>, 07.08 .2003 . ~}$

465 Ders., "Particulars of the life of Samuel Pepys", <http://www.pepysdiary.com/intro/pepys/>, 07.08.2003. Auch wenn die an dieser Stellen präsentierten Informationen nicht von Gyford selbst zusammengestellt, sondern nur der Tagebuch-Veröffentlichung aus dem Jahre 1893 entnommen worden sind, wäre es im Interesse der Vollständigkeit der Hintergrundinformationen sinnvoll gewesen, wenn Gyford jenen Teil der Angaben, der die Personen aus Pepys' privatem Umkreis betrifft, in diese Rubrik eingearbeitet hätte. 
noch von Kooperierenden werden einschlägige Hinweise zur Verfügung gestellt. ${ }^{466}$ $\mathrm{Zu}$ New Exchange findet der Leser neben einem kurzen Nutzerkommentar zur Historie dieses Gebäudes nur die von Gyford üblicherweise zur Verfügung gestellte Verknüpfung zum Stadtplan von London. ${ }^{467}$ Detaillierte Informationen hingegen erhält der Leser im Abschnitt Hinchinbroke. ${ }^{468}$ Dort wird nicht nur ein Erläuterungstext von Gyford nebst dem obligatorischen Stadtplan-Link präsentiert, vielmehr erhält der Leser zahlreiche Kommentare von Nutzern, die sich mit der 800-jährigen Geschichte sowie der gegenwärtigen Nutzung dieses Gebäudes auseinandersetzen. Das im Jahre 1830 fast gänzlich durch einen Brand zerstörte, jedoch nach überlieferten Bildern im selben Stile wiederaufgebaute Anwesen beherbergt seit dem Jahre 1970 unter dem heutigen Namen Hinchingbrooke wieder - wie bereits mehrfach in der Vergangenheit - eine Schule. Verbindungen zu Web-Seiten mit Photographien des Gebäudes in seinem derzeitigen Zustand tragen dazu bei, daß die Berichte von Pepys konkrete Gestalt annehmen und dadurch lebendig werden. Unter anderem wird der Leser durch eine der Verknüpfungen zu einer Seite mit dem Titel Samuel Pepys Diary geleitet, die von einem ehemaligen Lehrer besagter Schule veröffentlicht worden ist; sie gestattet einen interessanten Vergleich mit dem Tagebuchforum von Gyford und stellt eine aufschlußreiche Ergänzung zu den vom ihm zusammengestellten Materialien dar. ${ }^{469}$ Dort kann der Nutzer der sukzessiven Veröffentlichung von Gyford vorgreifen und das komplette Tagebuch von Pepys einsehen.

Im Abschnitt the Wardrobe stehen dem Nutzer etwas weniger ausführliche Erläuterungen zur Verfügung. Der Zugriff auf drei Karten - den bereits bekannten Stadtplan von London ${ }^{470}$, einen Plan aus dem 18. Jahrhundert ${ }^{471}$ sowie einen Ausschnitt des von MOTCO Enterprises im Web präsentierten Kartenmaterials aus dem

466 Ders., "Lemons", in: Ders., Hg., The Diary of Samuel Pepys (31.08.2003 [101.01.2003]), $<$ http://www.pepysdiary.com/p/1084.php $>, 01.09 .2003$.

467 Ders., "New Exchange", in: Ders., Hg., The Diary of Samuel Pepys (31.08.2003 [ 01.01 .2003$])$, $<$ http://www.pepysdiary.com/p/1085.php $>, 01.09 .2003$.

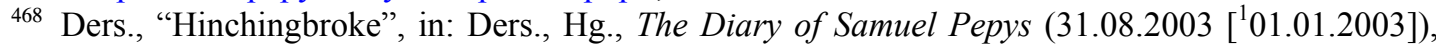
$<$ http://www.pepysdiary.com/p/104.php $>, 01.09 .2003$.

469 Duncan Grey, Samuel Pepys Diary (27.05.2003), <http://www.pepys.info/>, 01.09.2003.

470 BTex Ltd., Streetmap.co.uk, <http://www.streetmap.co.uk/newmap.srf?x=531893\&y=181025\&z=1>, 01.09.2003.

471 London Ancestor, Hg., Genealogy, Local \& Family History in London, England, s.v. "Plan of Baynards Castle Ward \& Faringdon Ward Within", <http://www.londonancestor.com/maps/baynards.htm>, 01.09.2003. 
Jahre $1746^{472}$ - verschafft dem Nutzer dennoch einen kleinen Einblick in die Historie. ${ }^{473}$

In der letzten der fünf verbliebenen Rubriken (venison) sind alle sechs Nutzerhinweise der Bedeutung und Zubereitung von Rehfleisch gewidmet. ${ }^{474}$ Ein Kommentator zum Beispiel verweist auf zwei Web-Seiten zu schottischer und irischer Tradition, die sich ausführlich mit dem Vorkommen, der Verfügbarkeit und der Verarbeitung von Rotwild beschäftigen. ${ }^{475}$ Ein anderer Nutzer stellt in zwei Beiträgen je eine Anweisung für geschmortes und eingekochtes Fleisch nach Rezepten aus dem 18. Jahrhundert zur Verfügung.

Aus Gründen der Straffung wurde darauf verzichtet, die Verweise zu allen im Tagebucheintrag vom 18. Juli 1660 erwähnten Personen weiterzuverfolgen. In erster Linie ging es darum, einen Einblick in die Vielfalt des Hypertext-Angebots zu geben und die unterschiedlichen Bereiche der Hintergrundinformationen vorzustellen. Die beschriebene intensive Nutzung des ausgewählten Eintrags hat dabei deutlich gezeigt, welche Vorteile, aber auch welche Schwächen das von Gyford konzipierte, gestaltete und verwaltete Forum aufweist. $\mathrm{Zu}$ nennen sind beispielsweise die zahlreichen Informationslücken oder die Beschränkungen auf den Londoner Stadtplan. Weitere - schwererwiegende - Nachteile werden im Verlaufe der Auswertung zur Sprache kommen.

\subsubsection{Auswertung}

Es ist festzustellen, daß Gyford die Gegebenheiten des neuen Mediums World Wide Web zu nutzen wußte und sie zugunsten einer facettenreichen Präsentation von Pepys' Tagebuch in sein Forum integriert hat. Obwohl er ein über 300 Jahre altes Werk im Web veröffentlicht, vermag Gyford dem Nutzer aufgrund der täglichen Aktualisierung der Einträge das Gefühl des Miterlebens zu vermitteln. Es ist

472 MOTCO Enterprises Limited, "John Rocque London, Westminster and Southwark", in: Dies., Hg., MOTCO UK Directory and Image Database ([ $\left.\left.{ }^{1} 2001\right]\right)$, $<$ http://www.motco.com/MapImages/81002/81002113.jpg>, 01.09.2003.

473 Gyford, "King's Great Wardrobe", in: Ders., Hg., The Diary of Samuel Pepys (31.08.2003 $\left.\left[{ }^{1} 01.01 .2003\right]\right),<$ http://www.pepysdiary.com/p/946.php $>, 01.09 .2003$.

474 Ders., "Venison", in: Ders., Hg., The Diary of Samuel Pepys (31.08.2003 [ ${ }^{101.01 .2003]), ~}$ $<$ http://www.pepysdiary.com/p/383.php>, 01.09.2003.

475 Scotland On Line, "Venison Haunch", in: Dies., Hg., scotland online.com, $<$ http://www.scotlandonline.com/heritage/cookery august recipe two.cfm $>, 03.09 .2003$. John McClelland, "'Grace Neill's' - John McClelland experiences the tradition of Ireland's oldest bar", Countrysports and Countrylife.com, <http://www.countrysportsandcountrylife.com/sections/restaurants/grace$\underline{\text { neills/>, 03.09.2003. }}$ 
Gyford zudem gelungen, sein Forum weitestgehend übersichtlich und logisch strukturiert zu gestalten. Alle Bedienungselemente und Menüpunkte sind so angeordnet, daß sie praktisch zu bedienen sind, wie es beispielsweise bei den Rubriken des Bereichs Background info der Fall ist, die nicht nur über die Navigationsleiste am oberen Rand der Web-Seite, sondern auch über jeden einzelnen Tagebucheintrag zugänglich sind.

Im Gegensatz zu vielen anderen Herausgebern präsentiert Gyford sein Forum nicht nur als offenes Hypertext-System, sondern er bedient sich auch tatsächlich der damit gegebenen Möglichkeiten und bietet dem Nutzer so diverse Gelegenheiten zur Aktivität. Deutlich erkennbar signalisieren die Verknüpfungen potentielle Wege zur Interaktion: Der Nutzer findet eine Vielzahl von Einstiegspunkten vor, die ihn zu zahlreichen weiterführenden Angaben leiten. Diese reichen von den Umständen der Tagebuchentstehung über detaillierte biographische Angaben zu Pepys und seiner Familie bis hin zu vielfältigen sonstigen Hintergrundinformationen.

Das Forum ist dergestalt konzipiert, daß sich Erstnutzer schnell mit der Tagebuchthematik vertraut machen, daß gleichzeitig aber auch alle regelmäßigen Besucher der Seite innerhalb des Hypertext-Systems agieren können, ohne allzu häufiger Wiederholungen bestimmter Informationen überdrüssig zu werden. Sind die Hintergründe des Tagebuchs sowie die wiederkehrenden Elemente bekannt, empfiehlt sich die widerkonzeptionelle Rezeption, bei der alle innerhalb des Dokuments offerierten zusätzlichen Informationseinheiten unbeachtet bleiben und der Leser sich ausschließlich auf den Hauptstrang des Hypertext-Systems konzentriert. $^{476}$

Hinsichtlich der Konzeption ist außerdem die Rubrik Recent news hervorzuheben, in der Gyford über alle themenbezogenen Geschehnisse sowie foruminterne Veränderungen informiert. Neben der täglichen Ergänzung des Tagebuchs prägt auch dieser Bereich aktueller Meldungen in entscheidendem Maße den Web-Charakter des Projekts.

${ }^{476}$ Siehe hierzu Kapitel V.3. vorliegender Arbeit. 
Neben den Möglichkeiten zur Interaktion bestehen vor allem auch Gelegenheiten zur Kooperation. Hier ist positiv anzumerken, daß sich die Kooperativität nicht auf eine (Alibi-)Mitarbeit beschränkt, sondern der Nutzer gleich an mehreren Stellen aufgefordert ist, die Tagebucheintragungen von Pepys anhand persönlicher Kenntnisse zu erläutern. Er kann seinen Hinweis entweder als unmittelbare Ergänzung zu Pepys' Aufzeichnungen verfassen oder ihn im gesonderten Bereich der Hintergrundinformationen einstellen. Aufgrund dieser Trennung sind die allgemeingültigen Kommentare auch nach längerer Zeit noch problemlos wiederzufinden. Alle darüber hinausgehenden allgemeinen Anmerkungen können in einem speziellen Diskussionsforum veröffentlicht werden. Die Einrichtung dieses Kommunikationsbereichs ist ebenfalls als eine durchdachte Entscheidung hervorzuheben, da auf diese Weise einerseits Gelegenheit zur Meinungsäußerung gegeben wird, andererseits die themenbezogenen Kommentare nicht mehr von themenfernen Anmerkungen durchsetzt sind.

Der Vorteil, den die Nutzergemeinschaft aus den Kooperationsmöglichkeiten ziehen kann, ist außerordentlich. Die Nutzerkommentare im Bereich venison beispielsweise veranschaulichen besonders deutlich die Bandbreite von Informationen, die sich durch ein derart offenes und zur Kooperation einladendes Medium wie das World Wide Web zu einem bestimmten Thema bündeln lassen. Die allgemeine Zugänglichkeit und Unmittelbarkeit des Web sowie - vermutlich zu einem nicht geringen Maße - auch seine Anonymität tragen dazu bei, daß von Nutzerseite in großer Zahl und Themenbreite ideenreiche und bisweilen sogar ausgefallene bis kuriose Hinweise bereitgestellt werden. Für den Unterhaltungswert der Tagebucheinträge erweist sich dies in der Mehrzahl der Fälle als vorteilhaft. Insofern wird der Charakter des Forums entscheidend durch die Möglichkeit zur Kooperation mitbestimmt.

Neben den genannten Vorteilen dürfen jedoch auch die Nachteile des Tagebuchforums nicht unerwähnt bleiben.

Der erste Kritikpunkt betrifft den Rückgriff auf die Tagebuchausgabe von Widger. Vermutlich aufgrund von Urheberrechtsgründen verwendet Gyford nicht die genauere und vollständigere Veröffentlichung von Latham und Matthews, sondern gebraucht als Vorlage die digitalisierte Version aus dem Jahre 2001, die auf der alten Ausgabe Wheatleys von 1893 basiert. 
Ein weiterer Kritikpunkt bezieht sich auf Gyfords Zensurmaßnahmen. In seiner Funktion als Herausgeber und Gestalter der Web-Seite verfolgt er ein konkretes Konzept mit bestimmten Regeln für die Kooperation, deren Einhaltung er konsequent verfolgt und notfalls auch durch persönliche Intervention $\mathrm{zu}$ sichern weiß. ${ }^{477}$ Wenngleich die Gestaltung des Forums nicht willkürlich, sondern anhand einer bestimmten Zielsetzung erfolgt, bleibt doch anzumerken, daß die Festlegung allzu strenger Maßstäbe und allzu nachdrückliches Beharren auf ihrer Befolgung dem eigentlichen Anliegen unter Umständen auch abträglich sein können. Sicherlich ist eine Zensur aufgrund der Offenheit und allgemeinen Zugänglichkeit des Forums unumgänglich. Wie aber das Problem der Kontrolle in Projekten kooperativer Zusammenarbeit zu lösen wäre - durch Nutzerregistrierung, gegenseitige Kontrolle der Kooperierenden oder aber besagte Eingriffe seitens des Herausgebers - ist bislang noch ungelöst.

Gyfords Regeln für die Veröffentlichung von Kommentaren sehen zwei bis drei Absätze als durchschnittliche Beitragslänge vor. ${ }^{478}$ Auch wenn Ausnahmefälle einen größeren Umfang gestatten, ist diese Regelung grundsätzlich zu beanstanden. Sofern der Hinweis eines Nutzers eine Bereicherung beziehungsweise Belehrung der Mehrheit der anderen Nutzer sein kann, ist nicht nachvollziehbar, weshalb gerade im World Wide Web eine Längenbeschränkung vorgenommen werden sollte. Während sich Begrenzungen bei Print-Veröffentlichungen durchaus mit technischen Vorgaben, zum Beispiel Layout-Bestimmungen, mit finanziellen Aspekten wie Druck- oder Versandkosten oder aber auch mit Rücksichtnahme auf die Benutzerfreundlichkeit erklären lassen, muß sich der Herausgeber einer Web-Seite keiner dieser Restriktionen unterwerfen. Im World Wide Web können allenfalls die Mietkosten des Speicherplatzes eine Begründung für derartige Einschränkungen seitens des Herausgebers darstellen. Allerdings wäre zu prüfen, ob sich die Länge der

\footnotetext{
477 Am 6. Mai 2003 spricht Gyford das Veröffentlichungsverbot für einen bestimmten, auffällig gewordenen Nutzer aus und trägt damit seinem persönlichen Anliegen sowie den ihm offensichtlich privat zugegangenen Beschwerden zahlreicher Nutzer Rechnung. Der Grund für diese Maßnahme wird in der Meldung nur sehr vage dargelegt, doch ist den Andeutungen und Verweisen Gyfords zu entnehmen, daß sich besagter Nutzer wiederholt über die Vorschriften zum Umfang von Kommentaren hinweggesetzt hat. Gyford, "Banned user", in: Ders., Hg., The Diary of Samuel Pepys (02.09.2003 [ $\left.\left.{ }^{1} 01.01 .2003\right]\right),<\underline{\text { http://www.pepysdiary.com/about/archive/2003/05/06/748.php }>, 03.09 .2003 .}$

478 Ders., "Annotation guidelines", < $<$ http://www.pepysdiary.com/about/annotation/> , 05.09.2003.
} 
Nutzerkommentare längerfristig tatsächlich einschneidend auf die Steigerung des Mietpreises auswirkte. ${ }^{479}$

Sollte die Regelung hingegen in der Sorge um die Geduld der Leser begründet sein, wäre dies zwar rücksichtsvoll, aber in Zeiten der Computernutzung nicht notwendig; bei Nichtgefallen lassen sich lange Textpassagen schnell mit Hilfe der Computer-Maus 'wegscrollen'. Das Teilnahmeverbot eines Nutzers vom 6. Mai 2003 erscheint daher völlig unverständlich, zumal Gyford in seiner Erklärung freimütig einräumt, daß die zahlreichen Kommentare des zurückgewiesenen Nutzers für die Nutzergemeinschaft bereichernd waren. ${ }^{480}$ Für einen verantwortungsbewußten Herausgeber sollten Fortschritt und Ausbau seines Projekts sowie Interesse und Bildungsbereitschaft der Nutzergemeinschaft vor einer einseitigen Durchsetzung seiner persönlicher Vorstellungen stehen.

Ein weiterer Kritikansatz sind die bibliographischen Angaben des Herausgebers. Die Veränderungen, die seit der Entschlüsselung des Originaldokuments im Laufe der Zeit durch verschiedene Editoren vorgenommen worden und in die vorliegenden Tagebuchtexte eingeflossen sind, lassen sich für den Leser der WebVersion von Gyford nicht mehr nachvollziehen. Angesichts der bewegten Vergangenheit des Dokuments, die durch Textkürzungen und deren teilweise Rücknahmen, durch Zensurbestimmungen und Hinzufügung von Anmerkungen geprägt ist, wäre es nicht nur aufschlußreich, sondern für einen Literaturwissenschaftler geradezu unabdingbar, Auskunft über Ursprung und Entstehung der aktuellen Tagebucheinträge zu erhalten. Jede einzelne Aufzeichnung müßte ihre individuelle Historie anhand von Quellenangaben eindeutig nachvollziehbar offenbaren. Anmerkungen dürften nicht mehr wahlweise den Hintergrundinformationen zugeordnet werden, sondern müßten grundsätzlich dem Text unmittelbar angeschlossen sein. ${ }^{481}$ Allerdings handelt es sich bei dem Tagebuchforum nicht um ein wissenschaftliches Projekt, das vorgenannten Maßstäben entsprechen müßte. Gyford weist selbst darauf hin, daß sein Forum

479 Je nach Anbieter variieren die Preise für einen Speicherplatz im World Wide Web teilweise signifikant, so daß an dieser Stelle kein Urteil hinsichtlich der Auswirkung längerer Nutzer-Kommentare auf den beanspruchten Speicherplatz und dessen Mietpreis getroffen werden kann. Es steht aber zu vermuten, daß die Länge der Hinweise im Verhältnis zur Gesamtgröße des Forums unerheblich ist.

${ }^{480}$ Gyford, "Banned user", <http://www.pepysdiary.com/about/archive/2003/05/06/748.php $>$, 05.09.2003.

481 Wie in Punkt 1.1.2.1. erwähnt, kürzt Gyford die Tagebuch-Einträge um alle Fußnoten, die nicht direkten Bezug auf den Inhalt des jeweiligen Tagebuch-Eintrags nehmen, wie zum Beispiel biographische Erläuterungen oder Ortsbeschreibungen, und ordnet diese Angaben im Abschnitt Background information ein. 
keinerlei Anspruch auf Wissenschaftlichkeit erhebe. Wäre ihm daran gelegen gewesen, dem Leser alle verfügbaren Informationen zu präsentieren, hätte Gyford - da er die genauere und vollständigere Ausgabe von Latham und Matthews vermutlich aus Urheberrechtsgründen nicht verwenden konnte - eine eigene Neuausgabe des Tagebuchs vornehmen müssen; diese Maßnahme hätte allerdings ein hohes Maß an Kompetenz vorausgesetzt und wäre darüber hinaus zeit- und kostenaufwendig gewesen.

Abschließend noch zwei kritische Anmerkungen im Sinne aller Nutzer, nicht nur der wissenschaftlich Interessierten: Gyford bedient sich zwar der Gestaltungsmittel von Hypertext und World Wide Web, doch hat er in dieser Hinsicht die derzeitigen Möglichkeiten noch nicht ausgeschöpft. Das Tagebuchforum läßt in seiner aktuellen Präsentationsform weiteres anschauliches Material vermissen. Ein Nutzer bringt die Situation in einem Verbesserungsvorschlag auf den Punkt, in dem er bittet: "more spice please". ${ }^{482}$ Die offensichtlich unter anderem gewünschte Unterhaltungsfunktion kann Gyfords Forum also nur unzulänglich erfüllen. In der Tat bleibt die Darstellung gegenwärtig noch etwas farblos und ließe sich durch verstärkte Einbindung zeitgenössischen Materials noch ausdrucksvoller und authentischer gestalten.

Einer Ergänzung bedarf aber vor allem der Bereich Background information. Die Handhabung des Hypertextes am Beispiel des Tagebucheintrags vom 18. Juli 1660 hat ergeben, daß die Hintergrundinformationen eindeutig zu wenig Angaben enthalten. Oftmals setzen sich die Auskünfte ausschließlich aus Nutzerkommentaren zusammen, die dem Leser zwar größtenteils durchaus interessante und bereichernde Informationen, aber keine knappe und übersichtliche Auskunft bieten. Das Material zur Ergänzung liegt teilweise sogar bereits vor, müßte jedoch aus den Angaben zum Leben von Samuel Pepys ${ }^{483}$ extrahiert und in die einzelnen Kategorien der Background information einsortiert werden. Vermutlich ist aber auch dies ein Vorgang, der nicht mehr im Rahmen einer privaten Einzelinitiative zu bewerkstelligen wäre.

${ }^{482}$ Hhomeboy, "Annotation on 'Annotation length and errors", in: Gyford, Hg., The Diary of Samuel Pepys ([102.05.2003]), < http://www.pepysdiary.com/about/archive/2003/04/30/742.php>, 05.09.2003.

${ }^{483}$ Gyford, "Particulars of the life of Samuel Pepys", <http://www.pepysdiary.com/intro/pepys/, 05.09.2003. 


\subsubsection{Verbesserungsansätze}

1. Herausgebergremium: Um eine Arbeitsentlastung zu erreichen, könnte Gyford auf die Unterstützung eines Herausgebergremiums zurückgreifen und damit viele Tätigkeiten ohne weiteres delegieren. So ließe sich beispielsweise mit technischer und gegebenenfalls mit fachlicher Hilfe das Projekt einer Neuedition des Tagebuchs realisieren.

2. Wissenschaftsorientierte Richtlinien für Nutzerkommentare: Zensurmaßnahmen des Herausgebers sollten sich weniger gegen die überdurchschnittliche Länge mancher (inhaltlich fundierter) Nutzerkommentare richten, sondern vielmehr der Einschränkung neben der Sache liegender, überflüssiger Hinweise oder Fragen seitens der Kommentierenden dienen. Nicht selten stellen mehrere Nutzer inhaltlich identische Kommentare in das Forum ein; diese Deckungsgleichheit könnte durch das Eingreifen des Herausgebers aufgehoben werden. Umgekehrt sollten alle wirklich relevanten Informationen willkommen sein und ungekürzt Eingang in das Forum finden.

3. Visuelle Elemente: Gyford schöpft die sich anbietenden Möglichkeiten keineswegs aus. Denkbar sind beispielsweise visuelle Elemente wie eingescannte Tagebuchmanuskripte, zeitgenössische Porträts, Landschaftsbilder, Landkarten oder Stadtpläne, Presseausschnitte zu politischen Ereignissen der Zeit, Karikaturen oder auch aktuelle Photos noch existierender Bauwerke beziehungsweise ihrer Umgebungen sowie neuzeitliches Kartenmaterial. Gerade der Abschnitt zu Pepys' Biographie ließe sich mit Hilfe der genannten Mittel illustrieren; auf diese Weise könnte der Leser nicht nur literarisch, sondern auch optisch auf den Spuren von Pepys wandeln.

4. Audio-Elemente: Neben visuellen könnten auch akustische Elemente zur Steigerung der Authentizität und des Web-Charakters der Tagebuchpräsentation beitragen. In diesem Zusammenhang sei der Vorschlag eines Forumnutzers aufgegriffen:

I strongly recommend that the inclusion of streaming audio of diary passages would be a superb way of getting the language and inferences right, as well as an amusing and instructive mode of imparting the verve and dialect of Pepys and his era. ${ }^{484}$

\footnotetext{
484 Hhomeboy, "Annotation on "Annotation length and errors",
} <http://www.pepysdiary.com/about/archive/2003/04/30/742.php>, 05.09.2003. 
Die Idee, Textpassagen in der Sprechweise der Zeit von Samuel Pepys in das Forum einzubinden, ließe sich zwar vermutlich nur durch Experten in korrekter Weise umsetzen, akustisches Material würde aber in hohem Maße zur Intensivierung der Anschaulichkeit des Dargestellten beitragen und damit unter Umständen auch den kulturhistorischen Wertes des Forums steigern. Darüber hinaus ergäbe sich - wie der Nutzer in seinem obigen Vorschlag betont - auch ein didaktischer Effekt.

5. Pepys-Links: Für alle Nutzer, die sich tiefergehend mit dem Thema Samuel Pepys auseinandersetzen möchten, wäre es hilfreich, wenn sie direkt von Gyfords Seiten auf Verknüpfungen mit anderen Web-Seiten zugreifen könnten. Gyford berücksichtigt in der Kategorie Background info bereits den Wunsch nach thematischer Vertiefung, indem er eine Liste von Buchtiteln und -rezensionen zur Verfügung stellt, die teilweise auch kostenlos online abrufbar sind. ${ }^{485}$ Außerdem informiert er die Forumnutzer in der Rubrik Recent news regelmäßig über alle themenverwandten Neuigkeiten. Beide Bereiche bilden eine ausbaufähige Ausgangsbasis für einen neuen Forumsabschnitt, der unter dem möglichen Titel Links präsentiert werden könnte. Die dort aufgelisteten Verknüpfungen könnten folgende Zielpunkte im Web aufführen:

- weitere Tagebuchprojekte im World Wide Web ${ }^{486}$

- Web-Seiten zum Tagebuch von Samuel Pepys ${ }^{487}$

- Web-Seiten zur Biographie von Samuel Pepys ${ }^{488}$

- Web-Seiten zu Gebäuden, die in einer Beziehung zum Leben von Pepys stehen, wie beispielweise Hinchingbrooke House ${ }^{489}$

- Web-Seiten zu Geschichte und Literatur der Restaurationsepoche ${ }^{490}$

485 Gyford, "Further reading", <http://www.pepysdiary.com/about/further/>, 09.09.2003.

486 Beispielsweise das bereits erwähnte Tagebuch-Forum eines ehemaligen Lehrers der Hinching-

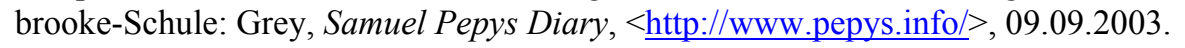

487 Zugänglich zum Beispiel über das erwähnte Project Gutenberg: Hart, Hg., Project Gutenberg, $<$ http://gutenberg.net/>, 09.09.2003.

488 Siehe beispielsweise Microsoft Corporation, Hg., Microsoft Encarta Online Encyclopedia (2003 [ $\left.\left.{ }^{1} 1997\right]\right)$, S.v. "Samuel Pepys", <http://encarta.msn.com/encnet/refpages/refarticle.aspx?refid=761552401>, 09.09.2003. Siehe auch Paul Hehn und R.F. Holznagel, Hg., Who2?, s.v. "Samuel Pepys", $<$ http://www.who2.com/samuelpepys.html $>, 09.09 .2003$.

489 Duncan Grey, "Samuel Pepys at Hinchingbrooke", in: Ders., Hg., The History of Hinchingbrooke House, <http://www.hinchbk.cambs.sch.uk/historical/hinchhistory/spepys/spepys.html>, 09.09.2003.

490 Eine sehr umfangreiche Liste von Verknüpfungen zu diesem Thema liefert beispielsweise Voice of the Shuttle: Alan Liu, Hg., Voice of the Shuttle - Restoration \& $18^{\text {th }}$ Century, $<\underline{\text { http://vos.ucsb.edu/browse.asp?id=2738 }>, 09.09 .2003 .}$ 
- Informationen zu John Evelyn, Zeitgenosse von Samuel Pepys und seinerseits Tagebuchschreiber ${ }^{491}$

- Bildungsinstitutionen, die nach Samuel Pepys benannt sind, zum Beispiel Museen oder Bibliotheken, wie die zum Magdalene College gehörige Bibliothek Pepys Library ${ }^{492}$, in der sich unter anderem das Originalmanuskript der Tagebücher von Pepys befindet

- Vereinigungen zur Wahrung des Andenkens von Samuel Pepys, wie zum Beispiel der Samuel Pepys Club $b^{493}$

Sicherlich sind noch verschiedene weitere Anlaufpunkte denkbar. Das Konzept des Tagebuchforums würde allerdings bereits an Profil gewinnen, wenn Gyford sich zunächst zu einer Einarbeitung obiger Punkte verstehen würde.

6. Veranstaltungskalender: Dieser Bereich sollte nicht in die Rubrik Pepys-Links (siehe Punkt 5) integriert sein, sondern eine eigenständig Kategorie bilden. Wie bei den Meldungen des Bereichs Recent news handelt es sich bei allen hier angesprochenen Informationen um termingebundene Daten, denen in der Menge der übrigen Verknüpfungen nicht die gebührende Beachtung zukäme. Gerade im Jahr 2003 finden anläßlich des dreihundertsten Todestages von Pepys bedeutende Veranstaltungen statt, über die ein interessierter Leser des Forums regelmäßig in Kenntnis gesetzt werden sollte. Die Angaben müßten alle notwendigen Hinweise zu Veranstaltungsorten und -terminen, Öffnungszeiten und Eintrittspreisen sowie möglichst auch eine Anfahrtskizze enthalten. Gleichzeitig böte es sich an, die Unmittelbarkeit des World Wide Web zu nutzen und die Web-Adressen der jeweiligen Institutionen mit aufzuführen, um dem potentiellen Besucher die Möglichkeit zur Vorabinformation zu geben. ${ }^{494}$

491 Siehe hierzu beispielsweise Guy de la Bédoyère, Who was John Evelyn?,

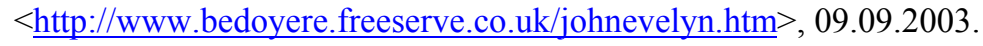

492 Magdalene College, Hg., Pepys Library, <http://www.magd.cam.ac.uk/pepys/>, 09.09.2003.

493 Samuel Pepys Club, Samuel Pepys Club, <http://www.pepys-club.org.uk/>, 09.09.2003. Dieser Club wurde anläßlich des zweihundertsten Todestages von Samuel Pepys gegründet. Er setzt sich bislang ausschließlich aus Fachleuten zusammen, die sich in bedeutenden wissenschaftlichen Beiträgen mit Leben und Werk des Tagebuchautors auseinandergesetzt haben.

494 Vorbildlich ist hier ein Bereich auf den Seiten des Samuel Pepys Club, in dem alle bedeutenden Veranstaltungen und Ausstellungen anläßlich des dreihundertsten Todestages von Samuel Pepys im Jahre 2003 einschließlich aller notwendigen Hinweise für Besucher aufgeführt sind. Samuel Pepys Club, Samuel Pepys Club - Major tercentenary events and exhibitions, <http://www.pepysclub.org.uk/events.html>, 09.09.2003. 
Die Bewertung der gegenwärtigen Präsentationsform ergibt gleichwohl ein positives Resümee: Das Tagebuchforum von Gyford bildet eine Mischung aus Fortsetzungsgeschichte, Autobiographie, Zeitgeschichte und Familienhistorie, die durch die Anmerkungen der Nutzer zusätzliche Würze und Brisanz erhält und in einer postmodern zu nennenden Weise wissenschaftliche Erschließung mit literarischer Unterhaltung verbindet.

\subsection{Prosa \& WWW - Zettels Albtraum Ein Resümee}

Experimente mit neuen Erzählformen sind keine Erscheinung des Web-Zeitalters, sondern haben Schriftsteller immer wieder beschäftigt. Dabei zielten die Versuche nicht nur auf eine unkonventionelle inhaltliche Struktur, sie bezogen sich auch auf das äußere Arrangement der Prosaerzählung, wie es beispielsweise Laurence Sterne in seinem Werk Tristram Shandy und Arno Schmidt in seinem Roman Zettels Traum $^{495}$ praktiziert haben. Infolge der ungewöhnlichen dreispaltigen Textanordnung sowie des mannigfaltigen illustrativen Materials widersetzt sich dieses Werk herkömmlichen Lesegewohnheiten. Die in der mittleren Spalte ausgeführte Haupthandlung beziehungsweise der Rahmen des Romans wird vermöge der Erläuterungen der linken Spalte analysiert, wohingegen sich in der rechten Spalte Assoziationen und Kommentare enzyklopädischen Charakters finden. Insofern kann Zettels Traum aufgrund seiner Verweisstruktur als gedruckter Hypertext bezeichnet werden.

Eine dem Roman Zettels Traum ähnliche, wenngleich nicht ganz so komplexe Sammlung bildet auch die Basis von Matthias Polityckis schriftstellerischer Arbeit. Über einen Zeitraum von mehreren Jahren trägt auch er Gedanken und Ideen auf Zetteln zusammen, um sie dann zu einer schlüssigen Geschichte, seinem Roman Marietta, aneinanderzufügen, üblicherweise ein sukzessiver, vor allem aber privater Vorgang, der keinen öffentlichen Einblick gewährt und bis zum Abschluß der Arbeit von Veränderungen, Umstellungen und neuen Eingebungen geprägt ist. Die Erfah-

\footnotetext{
495 Zwei Jahre lang sammelte Schmidt auf insgesamt etwa 120.000 Zetteln das Material zu seinem opus magnum, in das er sich während der Ausarbeitungsphase in so exzessiver und gesundheitsbedrohender Weise hineinsteigerte, daß das Werk allein aus diesem Grunde, aber auch wegen seines Umfangs von 1.330 Seiten im DIN-A3-Format als "das Hauptwerk Schmidts" bezeichnet wird. Wolfgang Albrecht, Arno Schmidt (Stuttgart; Weimar, 1998), 72.
} 
rungen, die Politycki während der Abfassung seines Romans durchleben muß, lassen sein System allerdings schließlich zu Zettels Albtraum werden. ${ }^{496}$

Politycki hatte sich entschlossen, sein Werk online entstehen zu lassen, was seiner Arbeit schließlich jedoch mehr Transparenz verlieh, als er sich ausgemalt hatte. Das Schreiben unter öffentlicher Beobachtung entwickelte sich zunehmend zu einem Zwang, der seine Kreativität entscheidend hemmte. Nach einigen Monaten der Ausarbeitung legte er in einem Interview seine entmutigenden Erfahrungen dar:

Ich sammele über Jahre Notizen mit Ideen für einen Roman, und mit diesen langwierig hin- und hersortierten Schnipselhaufen lege ich dann los. Diesmal steht die Gliederung bereits im Netz, seit einigen Monaten gebe ich die Schnipsel ins Internet ein. [...] Einerseits sind meine Schnipsel nun so gut geordnet wie sonst nie, andererseits lege ich mich zu einem Zeitpunkt fest, an dem ich das eigentlich noch gar nicht will. [...] Und auch die Tatsache, daß ich jetzt schon, obgleich erst die Hälfte der Schnipsel im Internet steht, angefangen habe zu schreiben, macht mich nicht recht glücklich. ${ }^{497}$

Die Nähe zu seinem Publikum und die Transparenz seiner Arbeit begann einen Schaffensdruck auszulösen, der dem Fortschritt seines Werkes nicht unbedingt zuträglich war. Bereits in der Phase des Ideensammelns war Politycki zu sehr sorgfältigen und verständlichen Formulierungen gezwungen; zudem legte er sich in einem viel zu frühen Stadium auf eine inhaltliche Gliederung seines Romans fest, was den Handlungsverlauf übereilt in bestimmte Bahnen zwang und dazu führte, daß Politycki seine Kreativität nicht weiter herausforderte. Der Schaffensprozeß verlief

496 Marietta war das dritte Romanprojekt im World Wide Web, das die Online-Redaktion der ZDFSendung Aspekte in Zusammenarbeit mit namhaften Autoren und den Nutzern des WWW durchführte. Ende des Jahres 1997 begann Politycki, seinen Roman Ein Mann von vierzig Jahren im Web als sogenannte Novel in Progress zu erstellen. In satirisch genauen Milieubeschreibungen schildert Politycki den Gefühlstaumel seines Protagonisten Gregor Schattschneider und das ewige Katz-und Maus-Spiel der Geschlechter. Der mäßig erfolgreiche Werbetexter Schattschneider gerät anläßlich seines vierzigsten Geburtstages in die Midlife-crisis. Sein Leben mit der Table-Dancerin Mascha bietet scheinbar keine neue Reize mehr und so erliegt er der Faszination der geheimnisvollen Marietta, eines Mitglieds der Münchner Intellektuellen-Schickeria. Politycki verfaßte sein Werk über einen Zeitraum von zwei Jahren unter dem Arbeitstitel Marietta gewissermaßen coram publico und dokumentierte auf diese Weise die Entwicklungsstufen einer Romanentstehung. Parallel dazu wurde für die Web-Nutzer ein Mitschreibeprojekt angeboten, in dem aus der Grobgliederung der Handlung zu Marietta ein eigener Web-Roman entstehen sollte. Ziele des ZDFProjekts waren die Entwicklung neuer Ausdrucksformen, die Heranführung von Autoren an das WWW und die Erprobung neuer Kommunikationsformen im Web mit Hilfe von Literatur. Max Sterz, "marietta - novell [sic!] in progress", in: Ders., Hg., publizieren im internet (08.03.2001 [ $\left.\left.{ }^{1} 23.11 .2000\right]\right),<$ http://www.lrz-muenchen.de/ p piiseminar/2311nlmarietta.htm $>, 11.10 .2003$. Tilman Urbach, "Matthias Politycki: Ein Mann von vierzig Jahren", in: BR-online, Hg., \{literatur + \} Lesezeichen, <http://www.br-online.de/kultur/literatur/lesezeichen/20000220/20000220_1.html>, 11.10.2003.

497 Politycki, Matthias, “Auch ein Schock. Autoren online: der Zukunftsroman? - Hanna-Sophia Bucher im Interview mit dem Autor Matthias Politycki”, Spiegel special 3 (1999), 118. 
dementsprechend schleppend und brachte es mit sich, daß Politycki nach einiger Zeit durch die Web-Gemeinschaft überholt wurde, die den Roman in ihrem Parallelforum fortsetzte und ausschmückte. ${ }^{498}$ Politycki zog angesichts dieser Nachteile die Konsequenz, es diesbezüglich bei einer einmaligen Erfahrung bewenden zu lassen und seine Arbeiten nur noch fernab von jeglicher Publikumskontrolle durchzuführen: "[...] auch die Einsicht, daß ich beim Schreiben eigentlich in jeder Hinsicht wieder offline sein muß, ist durch dieses Projekt verstärkt worden." 499 So läßt Politycki seinen Protagonisten einen symbolischen Schlußstrich vollziehen: "Deshalb meldet sich mein Held Gregor am Ende des Romans auch von seinem Internet-Server ab. ${ }^{, 500}$

Nicht nur Politycki zieht das private Schreiben dem transparenten Schaffensprozeß im WWW vor. ${ }^{501}$ Daß bislang weder im englischen noch im deutschen Sprachraum ein überzeugendes Gesamtkunstwerk der Erzählprosa gefunden oder gar eine Revolution der Denk-, Kommunikations-, Schreib- und Lesekultur heraufbeschworen wurde, könnte somit an einer Scheu vor der Preisgabe von Privatem liegen, eng verbunden mit einer Angst vor dem Verlust der Kontrolle über das eigene Werk. Hinzu kommt, daß die Komplexität der Hypertext-Systeme nicht nur technische Fähigkeiten, sondern auch Geduld, Ausdauer und Abschätzungsvermögen erfordern, um eine vielschichtige, gleichzeitig aber schlüssige Handlung gewährleisten zu können. Die Erstellung webbasierter Prosa erweist sich als zeit- und arbeitsintensiver Vorgang, der mit der Bereitstellung des Werkes keineswegs abgeschlossen ist. Nur durch kontinuierliche Beobachtung und Nachbearbeitung ist sicherzustellen, daß sich das Werk nicht entgegen die Zielrichtung entwickelt. Dies impliziert nicht, daß der Herausgeber wie im Print-Medium die gesamte Hypertext-Struktur vorgeben sollte, da damit das Prinzip der Kooperativität ad absurdum geführt würde. Seine Aufgabe liegt vielmehr ausschließlich in der Überprüfung inhaltlicher und technischer Übereinstimmung.

498 Politycki hatte es zwar abgelehnt, der Web-Gemeinschaft direkten Zugriff auf seinen Roman zu gewähren - "Literatur ist kein demokratisches Medium, von Teamwork auf dieser Ebene halte ich wenig." -, doch bestand die Möglichkeit der indirekten Einflußnahme durch E-Mail-Kontakt. Der Autor gewährte damit nicht nur Einblick in seinen Schaffensprozeß, sondern zeigte sich auch offen für Anregungen und Fragen. Gelegenheit zur eigenen schriftstellerischen Entfaltung erhielten die Web-Nutzer in erwähntem Parallelforum, in dem sie den Roman bald wieter vorangetrieben hatten, als Politycki selbst. Viktor Schlawenz, "Novel in Progress: Matthias Polityckis Roman 'Ein Mann von vierzig Jahren' entstand im Internet”, literaturkritik.de 4 (2000), <http://www.literaturkritik.de/public/rezension.php?rez id=957> , 11.10.2003.

499 Bucher, “Auch ein Schock. Autoren online: der Zukunftsroman?”, 118.

500 Ebd.

501 Matthias Politycki, “Das war's: Matthias Politycki im Gespräch mit Gerald Giesecke”, literaturkritik.de, 4 (2000), <http://www.literaturkritik.de/public/rezension.php?rez_id=961>, 11.10.2003. 
Ohne nachhaltige Unterstützung kann ein privater Herausgeber den Ansprüchen webbasierter Prosa als Einzelperson im Grunde nicht entsprechen. Neben Kreation und Koordination des Hypertextes sowie aller internen Ressourcen und Multimediaelementen obliegen ihm zusätzlich noch die Moderation von Chat Rooms oder Diskussionsforen und die Pflege und Aktualisierung der externen Verknüpfungen. Angesichts dieser Belastung wird deutlich, daß webbasierte Prosaliteratur weitaus mehr Engagement seitens des Herausgebers voraussetzt als eine entsprechende Print-Publikation.

Ob Autoren der Gegenwart dieses Engagement nicht erbringen können oder es möglicherweise gar nicht erbringen wollen, sei dahingestellt. Fakt ist, daß die Suche nach einem epochemachenden Kunstwerk im Web bislang kein positives Ergebnis geliefert hat. Die Recherche zu vorliegender Arbeit hat gezeigt, daß das gegenwärtige Angebot seine Einstellung in das WWW schwerlich rechtfertigt. Die ausgeführten Analysen ebenso wie zahlreiche Stichproben haben ergeben, daß es nicht ausreicht, einzelne Textelemente aneinanderzureihen, gelegentliche Verknüpfungen herzustellen und diese 'Bricolage' als innovative Literatur im World Wide Web anzubieten. Um zumindest den derzeit gegebenen Möglichkeiten in angemessener Weise gerecht zu werden - von weiteren theoretisch denkbaren Realisierungsformen ganz zu schweigen - müßten die Herausgeber ihre webbasierten Prosaerzählungen wesentlich komplexer gestalten. Diese Literaturform birgt

\begin{tabular}{|c|c|}
\hline das Potential von: & Dazu bedürfte es (im Idealfall) eines \\
\hline Unmittelbarkeit, & regelmäßigen, direkten Austausches unter den Kooperierenden \\
\hline Globalität, & in einer multikulturell zusammengesetzten Gruppe, \\
\hline Dynamik, & $\begin{array}{l}\text { die wächst und sich durch regelmäßigen Wechsel der Koope- } \\
\text { rierenden erneuert, }\end{array}$ \\
\hline Kontinuität, & mit täglich überarbeiteten und \\
\hline Aktualität, & fortlaufend aktualisierten Textelementen, \\
\hline Multidirektionalität, & mit dem Ziel zahlloser untereinander verbundener Beiträge \\
\hline $\begin{array}{l}\text { Vielschichtigkeit } \\
\text { und }\end{array}$ & $\begin{array}{l}\text { bei möglichst unterschiedlichen parallelen Handlungsverläu- } \\
\text { fen/Ebenen }\end{array}$ \\
\hline Bewegung. & mit maximaler Interaktionsfreiheit für den Rezipienten. \\
\hline
\end{tabular}


Statt dessen reduziert sich das Angebot webbasierter Prosa - sofern denn eine solche, die diese Bezeichnung verdient, überhaupt existiert - mehrheitlich, wie z.T. bei Mahoney the Cat, auf Eindimensionalität, Überschaubarkeit, Trägheit, Stagnation, und Gleichförmigkeit. Die meisten Dokumente, die Anspruch auf den Titel Webbasierte Prosa erheben, bestechen allenfalls in optischer Hinsicht durch technische Raffinessen, nicht aber durch inhaltliche Qualität. Oftmals offenbaren sie sich auch schlicht als konventionell linear-konzipierte Literatur, zumindest aber als webunabhängige Literatur im WWW.

Angesichts der Perspektiven, die dem Nutzer einst von zahlreichen selbsternannten Visionären euphorisch in Aussicht gestellt wurden ${ }^{502}$, ist das gegenwärtige Ergebnis außerordentlich enttäuschend. Ernüchterndes Resultat einer fast zehnjährigen Entwicklung ist die Erkenntnis, daß bislang kein Werk - noch nicht einmal in Unvollkommenheit - alle potentiellen Elemente einer webbasierten Erzählung in überzeugender Weise vereinbaren konnte. Entweder erlaubte das System keine Interaktivität oder keine Kooperativität, gestattete es keine strukturverändernden Eingriffe, gewährte es keinen Zugriff auf externe Ressourcen oder ließ es die Möglichkeit zu kommunikativem Austausch vermissen. Was sich dem Leser beziehungsweise Nutzer respektive Anwender darbot, waren mehrheitlich eine bloße sukzessive Abfolge, also eine Aneinanderreihung von Textelementen, die in der Regel auf nur einen einzigen Verfasser zurückgingen, Textbaustellen mit einer unübersichtlichen Anzahl von 'Kürzest'-Geschichten oder aber hoffnungsvoll begonnene, dann jedoch stagnierende Kooperationsprojekte.

Wenn gerade die Recherchen zu webbasierter Prosaliteratur so mangelhafte Ergebnisse geliefert haben, stellt sich die Frage, ob diese Literaturgattung gegebenenfalls eine Ausnahmestellung einnimmt. Die offensichtlich nicht zu meisternden Mängel webbasierter Publikationen könnten in der starken Prägung durch die lineare Darstellungsweise begründet liegen. Möglicherweise trifft die in Kapitel III.1. gestellte Vermutung zu, daß Print-Medien unverrückbare Maßstäbe gesetzt haben und es bislang einfach noch nicht gelungen ist, eine gedankliche Loslösung von diesen Vorgaben zu erwirken. Doch drängt sich damit die Frage auf, ob es grundsätzlich möglich ist, Linearität zu überwinden. Da der Leser nicht mehrere Handlungsstränge gleichzeitig lesen kann, bleibt sein Lesen immer linear - gleichgültig, ob er nun über

502 Siehe hierzu Kapitel I.2. vorliegender Arbeit. 
B oder über C von A nach D gelangt. Im Kopf des Lesers läuft die Handlung in jedem Falle sequentiell ab.

Weil dies so ist und sich auch niemals ändern wird, sei hier nochmals darauf hingewiesen, daß es bei webbasierter Literatur und vor allem bei webbasierter Prosa nicht darum geht, wie der Leser jeden einzelnen Lesestrang verarbeitet - natürlich kann dieser Vorgang nur sequentiell verlaufen -, sondern welche Auswahlmöglichkeiten und neuen Rezeptionsformen die Gesamtheit eines Hypertext-Systems bietet. Das heißt, es geht in dieser Arbeit in erster Linie um den Vorteil webbasierter Veröffentlichungen gegenüber Print-Publikationen. Für die Beurteilung ist entscheidend, wieviele der charakteristischen Merkmale dieser Literaturform ein webbasiertes Prosawerk enthält und bis zu welchem Grad sie den Nutzer überzeugen können.

Unter diesem Blickwinkel verliert gleichzeitig mit der Frage nach dem Einfluß der Linearität auch das Problem der Rollenverteilung von Autor und jenem Leser, der wirklich nur Leser und nicht zugleich auch Kooperierender ist, an Bedeutung. Grundsätzlich gilt zwar, daß der Leser einer Illusion erliegt, wenn er im Hinblick auf webbasierte Prosa glaubt, er könne den Autor durch Selbstbestimmung des Handlungsablaufs entmachten. In Wirklichkeit hat nämlich keine Demokratisierung stattgefunden, wie sie von manchem herbeigewünscht worden sein mag; nach wie vor bleibt der Autor Herr seines Werkes und vermittelt dem Leser lediglich das Gefühl von Selbstbestimmung. Im Hinblick auf webbasierte Prosa, bei der der Leser zum Kooperierenden wird, spielt die Autorität des Autors allerdings keine Rolle mehr, da sich diese Form der Literatur im Regelfall als das Ergebnis kooperativer Zusammenarbeit präsentiert, bei der die Kontrolle - so wie im Projekt Mahoney the Cat praktiziert - der Nutzergemeinschaft obliegt. Jeder Kooperierende trägt durch Hinzufügen seines individuellen Hypertext-Elements zur Gestaltung des Prosawerkes bei und übt durch gezieltes Plazieren der neuen Informationseinheit an der von ihm präferierten Stelle innerhalb des Gesamtwerkes für einen kurzen Moment Kontrolle und Macht aus.

Wenn auch festgestellt werden mußte, daß webbasierte Prosa bis heute noch keine adäquate Darstellungsform finden konnte und allenfalls in ihren Ansätzen überzeugt, empfiehlt es sich doch unbedingt, die weitere Entwicklung im Auge zu behalten und abzuwarten, ob sich die Erzählprosa im WWW etablieren kann und zu einer ernstzunehmenden Literaturgattung entwickelt. Vorläufig lautet das Fazit, daß 
diese Literaturgattung deutlich hinter ihren potentiellen inhaltlichen wie technischen Möglichkeiten zurücksteht und in dieser Reduzierung auf eine technische Spielerei in der Regel nur Ermüdung und Resignation hervorruft: "Das Medium ist eben nicht die Botschaft. Es ist die pure Langeweile, wenn seine Botschaft nur es selbst ist." ${ }^{\circ 03}$

503 Zimmer, "Die digitale Bibliothek”, 289. 


\section{Webbasierte Lyrik}

Das Angebot an webbasierter Literatur ist derzeit vermutlich im Bereich der Lyrik am größten und auch vielfältigsten. ${ }^{504}$ Dies ist nicht verwunderlich, da diese Literaturgattung der vorherrschenden Vorliebe der WWW-Leserschaft für kurze Texte sehr weit entgegenkommt; wie bereits ausgeführt, wird ja am Bildschirm hauptsächlich nachgeschlagen und nicht gelesen. Als Lesehilfe wird der Computer im allgemeinen lediglich dann genutzt, wenn die gewünschte Literatur in gedruckter Form nicht mehr erhältlich ist oder es sich um elektronische Dokumente mit interessanten Anmerkungen und Erläuterungen handelt.

Vermutlich vermag webbasierte Lyrik die Aufmerksamkeit des Lesers aufgrund ihrer Kürze und Prägnanz besonders zu fesseln, denn im allgemeinen lassen sich Gedichte in ihrem gesamten Umfang auf dem Computerbildschirm erfassen und haben damit die beste Form der Anpassung an das neue Medium erreicht. Dabei stellt sich übrigens auch die Frage, ob diese Annäherung der Literatur an das darstellende Element nicht dazu führt, daß jenes - vor allem im Bereich Lyrik allzusehr in den Vordergrund rückt. Auf diese Frage wird später noch gesondert einzugehen sein. Fakt ist vorläufig, daß webbasierte Lyrik einerseits die Konzentration nur für relativ kurze Zeit bindet, andererseits aber gleichzeitig das Verlangen nach hoher Aussagekraft durch vergleichsweise knappe Texte befriedigt.

Die thematische Vielfalt des Dargebotenen bewirkt zudem, daß sich jeder Leser angesprochen fühlen kann. Dabei entsteht allerdings auch der Eindruck, daß webbasierte Lyrik dem Online-Publikum oftmals nicht mehr als 'Literatur-Häppchen' serviert, die bereitwillig zwischendurch und zur Zerstreuung konsumiert werden. Dies soll keineswegs bedeuten, daß zwischen banalen Wortspielereien nicht auch anspruchsvolle Kreationen $\mathrm{zu}$ finden wären. Jedoch muß man gerade im Bereich Lyrik viel Zeit auf das 'An'-Lesen einer großen Zahl von Gedichten verwenden, um die Spreu vom Weizen trennen zu können.

504 Sehr augenfällig erscheint diese Diskrepanz in dem Literatur-Forum Prosebush - Collaborative Fiction Community, das nachfolgend noch ausführlich besprochen wird. Dort befindet sich auf der Einstiegsseite eine Übersicht über die verschiedenen Literatur-Gattungen und die Anzahl der Werke, durch die sie innerhalb des Forums vertreten sind. Am 30.07.2003 ist die Gattungen Lyrik dort mit einer Zahl von 74 Beiträgen mit weitem Abstand am häufigsten repräsentiert. Zum Vergleich: Comedy ist 40 Mal und damit am zweithäufigsten vertreten, gefolgt von Fantasy mit 24 Nutzer-Veröffentlichungen. Prosebush.com, Hg., Prosebush - Collaborative Fiction Community, $<$ http://www.prosebush.com/>, 18.07.2003. 
Aus diesem Grunde kann es zum Einstieg hilfreich sein, die Literaturrecherche in Foren oder Projekten wissenschaftlicher Einrichtungen durchzuführen. Wie in Kapitel IV. bereits angekündigt, bieten diese Web-Angebote dem Nutzer nicht nur Zugang zu Datenbanken und/oder Archiven. Im allgemeinen erhält der Nutzer zusätzlich Zugriff auf themenbezogene Auskünfte und Hilfsmittel sowie Verknüpfungen zu externen Informationsquellen. Gegebenenfalls besteht zudem die Gelegenheit $\mathrm{zu}$ wissenschaftlichem Austausch oder auch zu eigener Mitarbeit.

Auf eine solche Form des Online-Angebots, die ein Themengebiet mit allen dazugehörigen Hilfs- und Kommunikationsmitteln präsentiert, wurde ebenfalls in Kapitel IV. hingewiesen. Dort wurde diesbezüglich die Bezeichnung Konglomerat eingeführt, da es sich bei einem solchen Forum um eine mannigfaltige - teilweise durchaus auch geballte und etwas ungeordnete - Zusammenstellung unterschiedlichster Informationselemente handelt.

Um dem vielfältigen Angebot an webbasierter Lyrik gerecht zu werden, sollen in dieser Gattung insgesamt drei unterschiedliche Initiativen vorgestellt werden. Da die relative Kürze dieser Literaturform es zuläßt, soll zudem jeweils ein Werk oder Projekt herausgegriffen werden. An erster Stelle wird ein wissenschaftliches Forum für webbasierte Lyrik stehen. Neben diesem Projekt eines universitären Herausgebers sollen noch zwei Privatinitiativen präsentiert werden.

\subsection{Vorstellung dreier Foren zur webbasierten Lyrik}

\subsubsection{Electronic Poetry Center}

Das Electronic Poetry Center ${ }^{505}$ der University at Buffalo/The State University of New York ist konzipiert als ein zentrales Tor zu Lyrik-Ressourcen im World Wide Web. In seiner Gestaltung folgt es dem Konzept der Doppelfunktion als Datenbank und Archiv: Zum einen dient es als zentraler Zugang zu elektronischen Dokumenten im World Wide Web und fungiert aufgrund dieser externen Links als Online-Datenbank; zum anderen bietet das Electronic Poetry Center, kurz EPC, aber auch interne Verknüpfungen und stellt sich somit gleichzeitig auch als Online-Archiv dar. Dabei sind alle Formen digitaler Literatur vertreten: Das EPC soll dazu dienen, eine möglichst große Bandbreite an Websites zum Thema Lyrik zur Verfügung zu stellen, weshalb nicht nur webbasierte Werke sondern auch konventionell linear-konzipierte

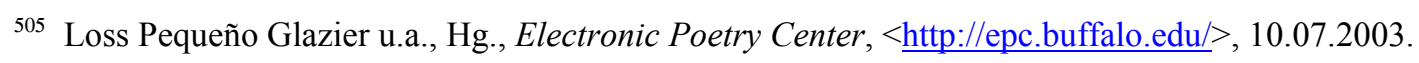


und computergestützte, webunabhängige Veröffentlichungen präsentiert werden. Der Schwerpunkt liegt allerdings eindeutig auf experimenteller und innovativer, das $\mathrm{Me}$ dium WWW kreativ nutzender Gegenwartslyrik.

Gegründet im Jahre 1994, wird das Center zudem nicht nur als Literaturquelle, sondern auch als Orientierungshilfe, Informationszentrum, Kommunikationsmedium und Treffpunkt für Wissenschaftler, Studenten und Poeten geführt. So erhält der Nutzer neben Verknüpfungen zu Gedichten außerdem Zugriff auf Bibliographien, Rezensionen, Zeitschriften und Magazine, Interviews, Veranstaltungskalender, Diskussionsforen, auf je eine Gallerie für visuelle und audiovisuelle Lyrik sowie auf andere WebSeiten des Themas Lyrik.

Den Zugang zu den internen und externen Ressourcen des $E P C$ erhält der Nutzer über sieben verschiedene Einstiegspunkte:

1. New: Dieser Bereich eröffnet den Zugriff auf Veröffentlichungen neueren Datums. Allerdings waren einige Publikationen bereits Anfang 2002 und somit eineinhalb Jahre vor Abfassung dieser Zeilen (im Sommer 2003) in die Seiten des Electronic Poetry Centers integriert worden; der zweite Abschnitt dieser Kategorie bezog sich zum Zeitpunkt der letzten Recherche der Verfasserin sogar explizit auf die Zeit bis Ende 2001. ${ }^{506}$

2. Authors: Die Verknüpfung führt den Nutzer zu einer alphabetischen Liste von Autoren, zu denen neben Verfassern konventioneller, linear-konzipierter Lyrik und Dichtern innovativer computergestützt-webunabhängiger beziehungsweise webbasierter Werke auch Kritiker dieser Genres zählen. Bei diesen Verknüpfungen handelt es sich ausschließlich um interne Links, das heißt alle Autorenseiten sind auf dem Server des EPC gespeichert. Während das Center hiermit als Archiv fungiert, steht es zusätzlich auch als Datenbank zu Verfügung: Eine gesonderte Verknüpfung auf dieser Seite verbindet den Nutzer mit externen Autorenseiten, das heißt allen privaten Initiativen außerhalb des EPC.

506 Bei diesen schon etwas älteren Veröffentlichungen noch von News zu sprechen, ist einigermaßen unverständlich. Die Beobachtung ist auch nicht damit zu erklären, daß die letzte Überarbeitung der Seiten eventuell schon länger zurückläge, denn einige Dokumente wurden tatsächlich erst im Juni 2003 eingestellt wurden. Vielmehr ist hier Nachlässigkeit in Pflege und Überarbeitung der Seiten zu verzeichnen. 
3. E-poetry: Der Teilbereich ist ausschließlich der digitalen, speziell für das elektronische Medium konzipierten Dichtung gewidmet. Neben einer alphabetischen Autorenliste finden sich hier Verknüpfungen zu themenbezogenen Sonderbeiträgen, einigen Web-Seiten zum Thema Lyrik, elektronischen Magazinen sowie Hilfsmitteln zu Gestaltung und Programmierung von Web-Seiten. Diese Liste beinhaltet sowohl interne als auch externe Links.

4. Links-Alpha: Führt zu einer alphabetischen Liste von ausschließlich externen Links, das heißt vom EPC ausgehenden Verknüpfungen mit anderen Lyrikseiten im WWW. Private Autorenseiten sind hier allerdings ausgenommen, da diese bereits in der Link-Liste im Abschnitt Authors erfaßt sind.

5. Links-Subj: Hier eröffnet eine thematische Auflistung interner wie externer Links den Zugang zu Web-Seiten von Zeitschriften- und Buchverlagen sowie Lyrikinitiativen und -organisationen, zu audiovisueller Lyrik, Volltextarchiven und (Hand-)Schriftensammlungen und zu Kunst, Kultur und Literatur in Buffalo.

6. UB Poetics: An dieser Stelle wird das Poetikprogramm der Universität von Buffalo ausführlich vorgestellt. Die in 1991 gegründete Initiative ist hier mit allen Informationen zu ihren Zielen, den Lehrenden und der Berichterstattung in den Medien vertreten. Daneben werden Verknüpfungen zu Lehrmaterialien, eigenen Veröffentlichungen und studentischen Web-Seiten ebenso angeboten wie eine Vielzahl themenbezogener Links.

7. Sound: Dieser letzte Einstiegspunkt ermöglicht dem Nutzer den Zugriff auf Tonund Radiokunst. Hierbei handelt es sich um eine Sammlung von Klangpoetik, Audiokunst, Audio-Hypermedien und Rundfunkübertragungen zu lyrischen Themen.

Was die thematische Abgrenzung der sieben Einstiegspunkte betrifft, so möchte man bei näherer Betrachtung meinen, daß der Wissenschaftscharakter des Electronic Poetry Centers als einer universitären Einrichtung stärker hätte zum Ausdruck kommen sollen. Daß dies nicht geschehen ist, liegt möglicherweise durchaus in der Intention der Herausgeber, doch wäre für dieses Portal zur Lyrik im WWW jedenfalls ein überzeugenderes und vor allem logischer strukturiertes Konzept denkbar.

Gleichwohl empfiehlt sich das EPC aufgrund seiner Vielzahl von Ressourcen und seiner Auswahlkriterien. Besonders hervorzuheben ist die Zusammenstellung 
der präsentierten Werke. So widmen sich die Herausgeber des EPC nicht nur etablierten Autoren, sondern sie sehen ihre Aufgabe offenbar auch darin, jene Werke zu präsentieren, deren Publikation von anderen Institutionen abgelehnt wurde. Diese Auswahlkriterien tragen dazu bei, daß der Nutzer einen Eindruck von der bunten und mannigfaltigen Mischung literarischer Kreativität im beginnenden 21. Jahrhundert gewinnen kann.

Sogar zu jenen Texten, die unter Urheberrechtschutz stehen, besteht uneingeschränkter und unentgeltlicher Zugang. Alle Texte, die auf den Seiten des EPC archiviert sind, können außerdem von den Nutzern als endgültig und verbindlich angesehen werden, da ihre Veröffentlichung von den Autoren beziehungsweise den Verantwortlichen offiziell genehmigt wurde..$^{507}$

Die $E P C$-internen Dokumente sind teilweise aufwendig gestaltet und präsentieren mitunter nicht nur Textmaterial, sondern auch multimediale Elemente in Form von Bild-, Ton- oder Filmsequenzen. Ein Großaufgebot an Autorenporträts fördert den persönlichen Charakter des Centers und trägt zur Anschaulichkeit bei. Allerdings wäre zu wünschen, daß Hintergrundfakten und bibliographischen Daten intensivere Beachtung geschenkt würde. So gehen die Informationen teilweise nicht über die Nennung der Verfassernamen hinaus und lassen damit wichtige Angaben wie beispielsweise Entstehungsdaten, etwaige Hinweise auf Zusammenhänge mit Print-Publikationen und Hintergrundberichte vermissen.

Dennoch sei das Electronic Poetry Center für die Beschäftigung mit lyrischen Werken im World Wide Web nachdrücklich empfohlen, da es sich seinem Grundsatz entsprechend als Tor zur Online-Lyrik etabliert und qualifiziert hat. Die Tatsache, daß dieses Forum seit nunmehr (im Sommer 2003) fast einem Jahrzehnt Bestand hat, spricht zudem für die Akzeptanz dieser Verbreitungsform. Es ist den Herausgebern gelungen, ein Forum für Lyrik zu schaffen, das bislang durch die Print-Medien nicht in befriedigender Weise umgesetzt wurde. Die gleichzeitige, unmittelbare und jederzeitige Verfügbarkeit der unterschiedlichen Materialien rund um das Thema Lyrik ist in keinem anderen Medium in vergleichbarer Weise gegeben.

Das Electronic Poetry Center wird betrieben als "central gateway to resources in electronic poetry and poetics at the University at Buffalo and on the

${ }^{507}$ Glazier, "Intro", in: Ders., Hg., Electronic Poetry Center, <http://epc.buffalo.edu/intro.html>, 10.07.2003. 
Web at large" ${ }^{908}$. Damit liegt das Ziel allein darin, den Zugang zu einer möglichst großen Bandbreite an Online-Ressourcen bereitzustellen. Außer der Möglichkeit, einen persönlichen Kommentar an die Herausgeber des EPC zu senden, bestehen im Rahmen des Forums für den Nutzer keine Gelegenheiten zur Interaktion oder Kooperation. Erwähnt wird das Zentrum in diesem Kapitel ausschließlich aufgrund seines Stellenwertes im wissenschaftlichen Bereich, seines visionären Weitblicks im Hinblick auf digitale Literatur, seiner Vielzahl von Ressourcen sowie seiner besonderen Berücksichtigung innovativer multimedialer Lyrik.

\subsubsection{Prosebush - Collaborative Fiction Community}

Eine grundlegend andere Konzeption liegt dem Lyrikforum Prosebush - Collaborative Fiction Community ${ }^{509}$ zugrunde. Hierbei handelt es sich um eine private Gruppeninitiative zur gemeinschaftlichen Nutzung des Mediums World Wide Web im Sinne literarischer Aktivität und Kreativität. Prosebush ist deshalb erwähnenswert, weil es sich mit seinem Konzept in Übereinstimmung mit den entscheidenden Kriterien für webbasierte Literatur befindet: Diese kooperative Gemeinschaft kann in genau der Form, in der sie sich gegenwärtig präsentiert, nur im World Wide Web existieren. Sie lebt von der Hypertext-Systematik und der allgemeinen Verfügbarkeit dieses Mediums. Jeder Nutzer hat die Möglichkeit, innerhalb der einzelnen Werke zu interagieren, gleichzeitig kann er vor allem aber auch kreativ mitarbeiten.

Prosebush ist andererseits deshalb interessant, weil es sich bereits aufgrund seiner optischen Gestaltung von anderen Initiativen dieser Art und Thematik abhebt. Hinzu kommt, daß Prosebush nicht von einer verantwortlichen Einzelperson oder Herausgebergruppe moderiert ${ }^{510}$ wird. Die Initiative existiert vielmehr, was die inhaltliche Ebene betrifft, eigenständig und basiert allein auf der Kooperationsbereitschaft der Web-Nutzer.

Wie der Titel bereits andeutet, ist Prosebush nicht auf ein einzelnes Genre spezialisiert, sondern widmet sich der Literatur in ihrer Gesamtheit. In zusammen 23 verschiedenen Kategorien sind nicht nur die zentralen traditionellen Literaturgattungen Lyrik, Drama und Erzählliteratur vertreten, es finden sich dort auch Literaturformen wie beispielsweise Kinderliteratur und diverse Sonderthemen wie Tages-

\footnotetext{
508 Ebd., 10.07.2003.

509 Prosebush.com, Hg., Prosebush, <http://www.prosebush.com/>, 18.07.2003.

510 Ebd., 18.07.2003.
} 
politik und Sport. Allerdings soll uns hiervon nachfolgend - entsprechend dem Thema dieses Abschnitts - allein die Gattung Lyrik interessieren.

Innerhalb des Lyrikbereiches sind alle Hypertext-Werke jeweils dreimal klassifiziert: 1. alphabetisch, 2. nach dem Datum ihrer Einstellung in die Seiten von Prosebush und 3. nach ihrer Beliebtheit bei den Lesern. Diese wird anhand der Zugriffshäufigkeit gemessen; erhoben werden ferner Zahlen zur prozentualen Verteilung der gerade beginnenden, der im Entstehen begriffenen und der bereits beendeten Werke, zur Geschlechterverteilung aller Nutzer, zu Präferenzen der Leser, zur Produktivitätsfrequenz der Autoren sowie zur Wort- und Buchstabenhäufigkeit in Prosebush.

Hinter jedem Werk ist vermerkt, aus wievielen Beiträgen es sich nach dem Stand des jeweiligen Tages zusammensetzt. ${ }^{511}$ Ein Verlaufsschema in Form eines Baumdiagramms gibt Überblick, in welcher Reihenfolge und Ordnung und von welchem Autor die einzelnen Werkelemente eingegangen sind. Derjenige Teil, von dem aus der Nutzer das Schema aufruft, ist stets rot markiert, so daß er jederzeit in der Lage ist, seine Position in dem Gesamtwerk zu bestimmen. Diese Orientierungshilfe beugt dem Effekt des sogenannten "lost in hyperspace" 512 vor.

Zusätzlich besteht von jedem Teilstück aus die Option, sich alle vorangegangenen Elemente anzeigen zu lassen. Auf dem Bildschirm erscheint dann in zusammenhängender Form das Gedicht mit allen seinen bisherigen Strophen, wobei diese jeweils am rechten Rand mit Angaben zum Autor und zum Erstellungsdatum versehen sind. Von dort aus hat der Nutzer auch die Möglichkeit, die Strophe seiner Wahl direkt aufzurufen.

Beginnt der Leser mit der Rezeption eines Werkes, so steht er am Ende des ersten Abschnitts - und gleichermaßen auch am Ende aller nachfolgenden Einzelelemente - vor der Wahl, entweder nur zu interagieren oder aber zu kooperieren. Entscheidet er sich für Interaktion, so kann er einer der aufgelisteten Verzweigungen folgen; sofern er jedoch aktiv mitarbeiten möchte, kann er an dieser Stelle wie nach allen weiteren Teilabschnitten seinen eigenen Beitrag schaffen. Diesen kann er in ein gesondertes Feld eingeben, in dem er gleichzeitig auch die Option erhält, das Werk

\footnotetext{
511 Am 18.07.2003 belief sich das umfassendste Werk im Bereich Lyrik auf 47 Beiträge. Insgesamt lagen zu diesem Zeitpunkt 74 lyrische Gesamtwerke vor, wobei das neueste zwei Monate zuvor begonnen worden war. Ca. 10 Prozent aller Werke waren neu eingestellt, 86 Prozent waren im Werden begriffen und 4 Prozent bereits abgeschlossen.

512 Schmundt, "Strom, Spannung, Widerstand", 54.
} 
nach Eingabe seines Textes zu beenden. Er muß sich als Autor nicht mit seinem eigenen Namen zu erkennen geben, sondern kann einen Decknamen verwenden beziehungsweise anonym bleiben.

Eine Anmeldung ist nicht zwingend erforderlich. Die Vorteile einer (freiwilligen) Registrierung liegen hauptsächlich auf seiten der Herausgeber von Prosebush, die auf diese Weise wichtige Daten für die Erstellung ihrer Statistiken erhalten. Im Gegenzug wird der Nutzer regelmäßig per E-Mail über Neuigkeiten und Überarbeitungen der Web-Seite informiert.

Prosebush ist ein Lyrikforum für alle Nutzer, die eine gelegentliche, weitestgehend unverbindliche Beschäftigung mit dieser Literaturgattung suchen und mit Freude an Interaktion den nötigen Elan für kooperative Mitarbeit aufbringen. Dieses Forum ist ferner jenen Lesern zu empfehlen, die Übersichtlichkeit und Unkompliziertheit in Gestaltung und Handhabung schätzen. Prosebush ist ein gutes Beispiel für eine private Initiative, die zwar von einer Personengruppe zur Verfügung gestellt wird, ansonsten aber weitestgehend selbständig abläuft und offensichtlich ohne tiefgreifende zensierende Maßnahmen auskommt.

\subsubsection{Interactive Poetry Pages}

Als private Initiative einer Einzelperson verdienen die Interactive Poetry Pages ${ }^{513}$ Erwähnung wegen ihres ausgefallenen Aufbaus und ihrer besonderen Zielsetzung.

Die Seiten sind gestaltet als Forum zur kooperativen Teilnahme. Ausdrücklich wird der Leser dazu aufgefordert, mitzuarbeiten und eine Zeile zu einem im Entstehen begriffenen Gedicht hinzuzufügen oder die Werke auf diesen Web-Seiten zu kommentieren. Die Möglichkeiten zur Interaktion sind hingegen minimal und zu vernachlässigen.

Ann Cantelow, die für die Seiten verantwortlich ist, hat ihr Forum in neun Kategorien mit jeweils unterschiedlicher Anzahl sogenannter Salons unterteilt, um damit den heterogenen Bedürfnissen aller freiwilligen Mitarbeiter gerecht zu werden. Jede Kategorie ist einem besonderen Thema gewidmet und stellt entsprechende Forderungen an Kreativität, Niveau sowie Urteils- und Kritikfähigkeit. ${ }^{514}$

\footnotetext{
513 Ann Cantelow, "Welcome to the Interactive Poetry Pages", in: Dies., Hg., Interactive Poetry Pages, $<$ http://www.csd.net/ cantelow/poem_welcome.html $>, 16.07 .2003$.

514 Ebd., 16.07.2003.
} 
1. General Poems: Hier findet der Besucher fünf Lyriksalons, die jeden Gedichtstil zulassen und daher ein Forum für all jene Beiträge darstellen, die keiner der nachfolgenden acht Kategorien zuzuordnen sind. ${ }^{515}$

2. High Critique Poems: Die fünf Salons sind als Plattform für kooperative Zusammenarbeit vorgesehen, wobei die Herausgeberin der Interactive Poetry Pages zu verstärkter Kritik einlädt.

3. High Critique Solo Works: Alle Kreativen, die eine Bewertung ihrer Einzelarbeiten auf gehobenem Niveau erwarten, erhalten hier in fünf verschiedenen Salons die Gelegenheit dazu.

4. Rhyming Poems: Gereimte Gedichte sollten vorzugsweise in einen dieser fünf Salons eingestellt werden, wenngleich auch alle anderen Kategorien durchaus offen für Reimlyrik sind.

5. Haiku Poems: Fünf weitere Salons sind der aus Japan stammenden Gedichtform Haiku vorbehalten. Aus drei Zeilen mit insgesamt 17 Silben bestehend ${ }^{516}$, entsprechend dem Schema $5+7+5$, bildet diese Lyrikart eine Art Epigramm ${ }^{517}$. Einem Hinweis der Herausgeberin Cantelow zufolge wird die Aussage eines Haiku-Gedichtes gewissermaßen unausgesprochen vermittelt, also ohne den Sachverhalt konkret zu nennen. Wie diese, vom Zen-Buddhismus inspirierte Lyrikgattung zu interpretieren sei, müsse allerdings letztlich jeder Leser für sich selbst entscheiden. ${ }^{518}$

6. Gothic/Surreal Poems: Drei Salons sind ausschließlich Schauer- und surrealistischen Themen gewidmet.

7. Song Lyrics: Ebenfalls drei Salons geben Raum für Liedertexte.

515 Nach welchem System eine Kategorie in verschiedene Salons gegliedert ist, geht aus den sehr knappen Ausführungen Cantelows nicht hervor. Eine kurze Anweisung in der 9. Kategorie (Rough Allowed), welche Beiträge in den einen und welche in den anderen Salon einzustellen sind, weist darauf hin, daß möglicherweise eine gewisse Ordnung zugrunde liegt, für den Nutzer ist allerdings kein System erkennbar.

516 Im Englischen werden Haiku-Gedichte Ann Cantelow zufolge oftmals etwas freier mit geringerer Silbenzahl verfaßt, was sie darauf zurückführt, daß die japanische Sprache teilweise mehr Silben zur Informationsübermittlung benötigt. Auf den Seiten der Interactive Poetry Pages wird allerdings überwiegend das 17-Silben-Prinzip praktiziert, was nach Cantelow mit der Gruppendynamik innerhalb des Forums zusammenhängt. Cantelow, "Welcome to the Interactive Poetry Pages", < $<$ http://www.csd.net/ cantelow/poem_welcome.html>, 16.07.2003.

517 Das Wort Haiku ist offenbar aus einer Verkürzung von Haihai no Hokku entstanden, der Bezeichnung für ein scherzhaftes Kettengedicht; es entspricht in etwa dem Epigramm (= kurzes, meist geistvolles, oft spottendes Gedicht).

518 Ann Cantelow, “A Very Brief Definition of Haiku”, in: Dies., Hg., Ann Cantelow's Homepage, $<\underline{\text { http://www.csd.net/ cantelow/poem_haikudef.html }>, 16.07 .2003 . ~}$ 
8. Teen Poems: Nutzer im Teenageralter können ihre Kreativität hier in eigens für sie eingerichteten fünf Salons unter Beweis stellen. ${ }^{519}$

9. Rough Allowed: Die letzte Kategorie mit zwei Salons ist für jene Personen vorgesehen, die aus den Salons aller vorhergehenden Bereiche verwiesen wurden. Hier ist derber Stil gestattet, sofern der Beitrag nicht gegen ethnische oder soziale Gruppen gerichtet ist.

Weder für den gesamten Datenbestand noch für die einzelnen Kategorien existieren Personen- oder Werkregister. Auch ist es nicht möglich, eine Titelrecherche durchzuführen, da ein Großteil der Werke unbetitelt ist. Einzig eine Autorenrecherche ist möglich, die allerdings nicht von der Einstiegsseite der Interactive Poetry Pages aus durchgeführt werden kann; entsprechende Suchmaschinen befinden sich nur in den einzelnen Salons.

Innerhalb der einzelnen Salons sind die Werke in numerischer Reihenfolge aufgelistet. Dabei besteht entweder die Möglichkeit, sie in ihrer Gesamtheit wahrzunehmen oder aber alle Autoren der jeweiligen Beiträge mit anzeigen zu lassen. Im letzteren Falle sind die einzelnen Zeilen durch die Verfasserangaben voneinander getrennt.

Jedes Werk besteht immer nur aus einer Ebene, das heißt es ist stets das gesamte Werk sichtbar. Der Nutzer kann somit nicht werkintern interagieren, sondern lediglich kooperieren. Hierfür besteht am Ende jeder Gemeinschaftsarbeit die Möglichkeit zur Eingabe einer Folgezeile, wobei das Konzept des Forums vorsieht, daß immer nur einzelne Zeilen eingegeben werden. Unabhängig davon, ob ein Nutzer kooperiert oder nicht, kann er jedes Werk beenden, sofern er den Eindruck hat, es sei lange genug online gewesen und eigentlich abgeschlossen. Der Nutzer bewirkt mit seinem Befehl die umgehende Entfernung des betreffenden Werkes aus seinem Salon; es wird damit automatisch Bestandteil des Bereiches für abgeschlossene Arbeiten und erhält dort eine laufende Nummer. Die Numerierung aller anderen noch unvollendeten Gedichte ändert sich nicht. ${ }^{520}$

519 Cantelow kommt mit der Schaffung dieser Kategorie jugendlichen und erwachsenen Nutzern gleichermaßen entgegen: Die jüngere Generation kann in eigenen Salons unter Gleichaltrigen ihre Werke präsentieren, ohne Kritik oder gar Häme älterer Generationen befürchten zu müssen; diese wiederum sind nicht gezwungen, sich mit den Werken und somit dem Schreib-Stil sowie den Problemen Heranwachsender auseinanderzusetzen.

520 Cantelow, Hg., Interactive Poetry Pages - Completed Poems, $<\underline{\text { http://atlas.csd.net/ cantelow/cgi- }}$ bin/poem_history.pl/crit4/1/>, 17.07.2003. 
Eine zweite Möglichkeit der Nutzerkooperation ist die der Kommentierung und Bewertung. So kann jede Gemeinschaftsarbeit mit einer Anmerkung versehen werden, wobei Angaben zur Identität auch in diesem Falle fakultativ sind. Nicht selten sind diese Beiträge selbst lyrischen Charakters, so daß sie durchaus als Ergänzungen zu den eigentlichen Werken verstanden werden können, obwohl sie von ihren Verfassern offensichtlich bewußt nicht als solche eingeordnet worden sind.

Wie auch in vielen anderen Foren, in denen die Beiträge der kooperierenden Nutzer nicht durch eine höhere Instanz geprüft und gegebenenfalls zensiert werden, laufen hier alle Vorgänge unmittelbar, das heißt umgehend und direkt ab. Ein derartiger Geschehensablauf wird als Echtzeitvorgang bezeichnet; auch Cantelow verwendet diesen Ausdruck zur Kurzbeschreibung ihres Konzepts der kooperativen Zusammenarbeit: "Real time poetry collaboration." 521

Die Interactive Poetry Pages sind ein anschauliches, positives Beispiel für Kooperativität im World Wide Web, da sich gerade auf diese unverbindliche Art im Bereich Lyrik der eine oder der andere Leser dazu ermuntert fühlen mag, kreativ zu werden und an einem Gedicht mitzuarbeiten. Möglicherweise werden durch derartige Initiativen auf spielerische und unverbindliche Art Talente geweckt oder gefördert, die ansonsten aus Unwissenheit, persönlicher Unterschätzung oder mangelnder Gelegenheit niemals zur Geltung kämen; schließlich sollte es nicht allzu viel Überwindung kosten, eine Zeile zu einem Gedicht hinzuzufügen, das ebenfalls von Laien verfaßt worden ist.

Die Interactive Poetry Pages stellen gleichzeitig auch eine Fundgrube an poetischen Werken der Gegenwart dar, die einen realistischen Einblick in die Gedanken- und Traumwelt, aber auch in die Ängste der Menschen im beginnenden 21. Jahrhundert eröffnen. Obwohl die Probleme und Wünsche des Alltags in den hier aufgeführten Gedichten freimütigen Ausdruck finden, werden alle Gefühlsäußerungen und poetischen Beiträge respektvoll behandelt. Möglicherweise ist dies darin begründet, daß die Herausgeberin Ann Cantelow nicht zögert, gelegentlich zensierend einzugreifen. Als deutlichen Hinweis hat sie ihre Seiten zudem mit folgendem höflichen Hinweis versehen:

521 Dies., "Welcome to the Interactive Poetry Pages", $<$ http://www.csd.net/ cantelow/poem welcome.html $>$, 16.07.2003. 
In the interests of making this a safe space for all participants, your web host here reserves the right to remove any material she subjectively feels challenges that safety, however mildly. ${ }^{52}$

Der gegenseitige Respekt mag aber auch einem unausgesprochenen Toleranzkodex zuzuschreiben sein, dem sich alle Autoren verpflichtet wissen, die an den Gedichten mitarbeiten. Cantelow ist es jedenfalls gelungen, in Eigeninitiative seit 1996 ein Online-Forum zu führen, das sich - ebenso wie das Electronic Poetry Center nunmehr über mehrere Jahr behauptet und regen Zuspruch hat. Mit der differenzierenden Einteilung ihres Lyrikangebots in neun unterschiedliche Kategorien gelingt es Cantelow, allen Altersgruppen sowie allen Wünschen, Fähigkeiten und Ansprüchen gerecht zu werden. So bilden die Interactive Poetry Pages ein durchaus nachahmenswertes Beispiel privater Initiativen im WWW. Originell, aber dennoch seriös gestaltet, eröffnen sie dem Nutzer die Möglichkeit, sich unverbindlich dem Thema Lyrik zu nähern.

\subsection{Ausgewählte Beispiele webbasierter Lyrik}

Die Reihenfolge, in der nunmehr verschiedene Lyrikbeispiele vorgestellt und analysiert werden, entspricht dem Grad der Komplexität der einzelnen webbasierten Gedichte sowie ihren Angeboten zur Interaktion und Kooperation, die in dem ersten Gedicht am eingeschränktesten und im letzten Beispiel am vielfältigsten sind. Es ergibt sich eine genaue Umkehrung der Reihenfolge, in der oben die entsprechenden Präsentationsforen dargestellt worden sind.

\subsubsection{Poem Number 334}

\subsubsection{Präsentation}

Das Gedicht "Poem Number 334" findet sich in der Kategorie High Critique Poems, Salon Vier der Interactive Poetry Pages. ${ }^{523}$ Bis auf wenige Ausnahmen tragen die Gedichte dieses Forums keinen Titel, weshalb zur Benennung des vorliegenden Beispiels die von Cantelow angegebene laufende Nummer verwendet wird.

522 Ebd., 16.07.2003.

523 Bei der Suche nach einem repräsentativen Beispiel für die Beiträge des Lyrikforums Interactive Poetry Pages bot sich die Kategorie High Critique Poems an, da nur in ihren Salons Gedichte veröffentlicht werden, die das Ergebnis kooperativer Zusammenarbeit darstellen und gleichzeitig auf gehobenem Niveau verfaßt sind. Im Bereich High Critique Solo Works wäre zwar vermutlich der Anspruch, den die Autoren an ihre Gedichte stellen, höher gewesen als in der Kategorie High Critique Poems, doch beschränkt sich die Auswahl in High Critique Solo Works auf Einzelarbeiten, während hier die Resultate gemeinschaftlicher Arbeit im Vordergrund stehen sollen. 
"Poem Number 334" besteht aus nur einer einzigen Strophe mit lediglich drei Zeilen. Diese könnten durchaus von insgesamt drei unbekannten Verfassern stammen, doch ist zu vermuten, daß ein Autor allein für alle drei Zeilen verantwortlich zeichnet:

Where's a star when you need a wish?

--- Anonymous

behind the darkness of a frown

--- Anonymous

look around...

--- Anonymous ${ }^{524}$

Leider weist dieses Gedicht wie auch die anderen Veröffentlichungen auf den Seiten der Interactive Poetry Pages keine Erstellungs- oder Einstellungsdaten auf. Angaben hierzu werden nur in den Ergebnislisten für Autorenrecherchen aufgeführt. Aus ungeklärten Gründen beinhaltet die Trefferliste der Suche nach Anonymous jedoch nicht das Gedicht Nummer 334, weshalb unklar bleibt, wie lange es der Allgemeinheit schon zur Verfügung steht. Es ist daher nicht möglich, die Anzahl der Kommentare zu diesem Gedicht in ein Verhältnis zur Dauer seiner bisherigen Verfügbarkeit zu setzen und daraus Schlußfolgerungen hinsichtlich seiner Akzeptanz und Beliebtheit zu ziehen.

Innerhalb des Gedichts hat der Leser keinerlei Möglichkeiten zur Interaktion. Alle eingegangenen Beiträge bilden zusammengenommen die aktuelle Version des Gedichts, die nicht mehr verändert oder ergänzt werden kann. Die kooperative Mitarbeit beschränkt sich ausschließlich auf das Fortschreiben des Gedichts. Hierfür kann jeweils eine neue Zeile an den letzten Vers angehängt werden.

Multimediale Elemente sind in "Poem Number 334" ebenso wenig enthalten wie Verknüpfungen zu themenrelevanten Web-Ressourcen. Diese Möglichkeiten der Hypertext-Systematik werden somit nicht genutzt.

Die Analyse der formalen Struktur des Gedichts läßt metrische Gesetzmäßigkeiten nicht erkennen. Die Schlußsilben der drei unterschiedlich langen Zeilen korrespondieren nicht miteinander, das heißt sie weisen keinen eindeutigen Gleichklang der Endsilben auf, sind also nicht gereimt. Unklar ist, ob der Verfasser der letzten Zeile eventuell eine Klangähnlichkeit von around mit frown intendiert hat.

524 Cantelow, Hg., The Interactive Poetry Pages - Salon of Poetry for Critique - Four,

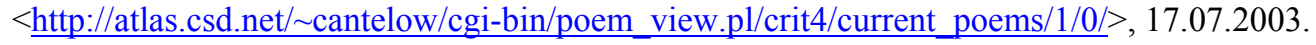


Die formal-optische Gliederung unterstützt sowohl die syntaktische als auch die dem Gedicht immanente semantische Dimension. Die Zeilenbrüche stellen gewissermaßen kurze Pausen dar, wodurch sich die einzelnen Zeilen zu Einheiten formen und sogenannte Verse bilden. Die formale Minimalbedingung für einen lyrischen Text scheint somit gegeben. ${ }^{525}$

\subsubsection{Analyse und Auswertung}

Hintergrund der einleitenden Frage in "Poem Number 334" ist der Volksglaube, jeder stumme Wunsch angesichts einer Sternschnuppe gehe irgendwann in Erfüllung. Der Sprecher hat aber offensichtlich erfahren müssen, daß im entscheidenden Moment zumeist keine hilfreiche Sternschnuppe am Himmel auszumachen ist. Dementsprechend schwermütig erscheint die Atmosphäre in der zweiten Zeile: "behind the darkness of a frown" - hier mag die Düsternis und Aussichtlosigkeit gemeint sein, die sich in einem sorgenvollen Gesichtausdruck niederschlägt. Dies kann eine Anspielung auf einen Menschen sein, dessen Stimmung durch Hoffnungslosigkeit und Dunkelheit geprägt ist, weil er durch unlösbare Probleme belastet wird.

In "Poem Number 334" verleiht ein fiktiver Sprecher, das sogenannte lyrische Ich, seiner persönlichen, subjektiven Stimmung Ausdruck. Seine Gefühlsbekundungen vermitteln eine Stimmung, die zwischen Mutlosigkeit und Hoffnung zu schwanken scheint. Der Sprecher wirkt niedergeschlagen und desillusioniert; fast anklagend wirken seine Worte: “Where's a star when you need a wish?” Seine Äußerungen offenbaren seine Erlebnisse und die Konsequenzen, die er anscheinend aus ihnen gezogen hat.

Ob diese gedrückte Atmosphäre in der dritten und letzten Zeile eine positive Wendung erfährt, liegt in der individuellen Interpretation des Lesers. Die Punkte, die dem Gedicht ein offenes Ende verleihen, lassen zweierlei Auslegungen zu: Zum einen kann die Aufforderung "look around..." als Ausdruck noch nicht erloschener Hoffnung erscheinen. Wenn man sich nur umsieht, - so möchte man glauben - wird vielleicht doch noch eine Sternschnuppe auftauchen, auf die der dringlichste Wunsch projiziert werden kann. Die Aufforderung kann zum anderen aber auch die Resignation widerspiegeln, die den Sprecher angesichts seiner Umwelt befällt. So kann die Aufforderung, sich umzusehen, auch als Anklage gemeint sein, die dem Angesprochenen die Unbarmherzigkeit und Perspektivlosigkeit der Realität vor Augen führen

${ }^{525}$ Vgl. Burdorf, Einführung in die Gedichtanalyse, 58. 
soll. Diese Interpretation wird verstärkt durch die abfallende Länge der drei Gedichtzeilen. Die immer kürzer werdenden Aussagen intensivieren den Eindruck der Resignation und lassen daher vermuten, daß die gedrückte Atmosphäre keinen Hoffnungsschimmer zuläßt.

"Poem Number 334" enthält keinerlei Hinweise, die eine geographische Zuordnung oder eine Einordnung in die Historie erlauben. Auch ist das Thema des Gedichts kein spezifisches Problem einer bestimmten Situation, sondern zeitlos. Das Gedicht erhält damit eine universelle Dimension: Sein Thema ist über alle Kontinente, über alle Menschengeschlechter und über alle Kulturen hinweg von immerwährender Aktualität. Daß das Gedicht nur aus drei Zeilen besteht und somit sehr kurz ist, wirkt sich nicht etwa nachteilig auf die Leserwirkung aus; vielmehr verleiht gerade die Kürze dem Text mehr Gewicht, er wird dadurch geheimnisvoll und bedeutungsschwer.

Was “Poem Number 334” zusätzlich interessant macht, ist der nachfolgende Text, den ein Leser dem Gedicht als Kommentar beigefügt hat:

\author{
in this city they ${ }^{526}$ are no stars that shine bright \\ just the flashes and blurs of the big city lights \\ ellucidating the miserable streets \\ that eat the souls of the wretched and rich \\ and without thought \\ leave them in a flooded ditch \\ No smile on my face \\ not for the place I am. \\ New York City... \\ the city of dreams, hopes, aspirations \\ the city of death, drugs, and aggrivations ${ }^{527}$. \\ The city that devoured my soul, \\ yep the city ate it whole, \\ and destroyed my many loves. \\ This city has millions of stars, \\ Just none to make a wish upon... \\ So I hide behind this frown. ${ }^{528}$
}

${ }^{526}$ Hier handelt es sich um die authentische Wiedergabe des Originaltextes. Wie bereits erwähnt, hat die Verfasserin vorliegender Arbeit keinerlei Veränderungen an den vorgestellten Werken vorgenommen. Es steht aber zu vermuten, daß es sich hier um einen Rechtschreibfehler der Autorin dieses Kommentars handelt und diese Verszeile statt dessen folgendermaßen lauten müßte: in this city there are no stars that shine bright.

527 Auch hier handelt es sich um einen Rechtschreibfehler: Statt aggrivations müßte das Wort aggravations lauten. Möglicherweise ist diese Schreibweise aber von der Autorin des Kommentars aus Reimgründen beabsichtigt.

${ }^{528}$ Cantelow, Hg., The Interactive Poetry Pages - Salon of Poetry for Critique - Four,

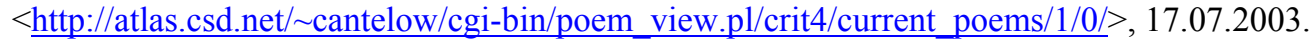


Dieser Leserbeitrag ist unbetitelt, trägt keine Datierung und läßt keinerlei Rückschlüsse auf den Verfasser zu. Der Text umfaßt 17 Zeilen, die ebenfalls in Gedichtform abgefaßt sind. Dies ist eher ungewöhnlich, da der Text gewissermaßen eine Fortsetzung von "Poem Number 334" darstellt, offensichtlich aber für sich alleine stehen und wirken soll. Durch die inhaltliche Ähnlichkeit bekräftigt er in seiner Einzelstellung die Aussage von "Poem Number 334" in höherem Maße, als wenn er als Fortsetzung an den Dreizeiler angehängt worden wäre. Auf diese Weise wird die Stimmung des Ausgangsgedichts gebannt und in den Leserkommentar hinübertransportiert.

$\mathrm{Da}$ es sich hierbei um Lyrik handelt, geht nicht nur aus den Zeilenbrüchen hervor. So sind gewisse metrische Regelmäßigkeiten zu beobachten, die allerdings nur vereinzelt auftreten, so daß nicht von einer einheitlichen metrischen Regulierung gesprochen werden kann.

Die einzelnen Zeilen sind von unterschiedlicher Länge und füllungs- und hebungsfrei. Das Gedicht zeigt keine innere Strukturierung durch Strophen, läßt sich aber in fünf verschieden lange Abschnitte gliedern. Diese Einteilung orientiert sich an der syntaktischen Gestaltung des Gedichts. Es ist zu beobachten, daß im ersten Abschnitt gänzlich auf Interpunktion verzichtet wird, während der restliche Teil des Gedichts durch Satzzeichen in vier abgeschlossene Sätze unterteilt ist. Da es die inhaltliche Gestaltung des Gedichts nahelegt, sollen die syntaktischen Einheiten je für sich behandelt werden.

In den insgesamt fünf Abschnitten ist kein sich wiederholendes bestimmtes Schema zu beobachten:

Im Abschnitt (1), der sechs Zeilen umfaßt, reimen sich die Endsilben des vierten und des sechsten Verses in einem unterbrochenen Kreuzreim; die letzten Wörter des ersten und des zweiten Verses sind klangähnlich.

Abschnitt (2) ist ungereimt und umfaßt lediglich zwei Zeilen. Beide Verse beginnen mit einer Verneinung, das heißt es liegt eine herausgehobene Negation vor. Die beiden Verse sind zudem vergleichsweise kurz, was ihrem Inhalt mehr Gewicht verleiht und zusätzlich zur Betonung der Negation beiträgt.

Abschnitt (3) ist drei Zeilen lang, wovon die letzten beiden eine Klanggleichheit in den Endsilben aufweisen, also einen Paarreim bilden. 
Abschnitt (4) besteht ebenfalls aus drei Zeilen und beginnt mit einem Paarreim.

Auch der letzte Abschnitt (5) ist dreizeilig, enthält aber keine Klanggleichheiten.

Inhaltlich vollzieht sich mit jedem neuen Abschnitt ein Wechsel von Beschreibung und persönlicher Stellungnahme:

Abschnitt (1) veranschaulicht die Anonymität und das Elend einer Stadt und schildert die destruktive Wirkung, die sie auf viele Menschen ausübt.

In Abschnitt (2) äußert der Sprecher seine depressiven Gefühle für die betreffende Stadt, die offensichtlich sein Wohnort ist.

In Abschnitt (3) folgt wieder eine Charakterisierung der Stadt, aus der nun aber konkret hervorgeht, daß es sich um New York handelt. Der Sprecher akzentuiert in diesem Abschnitt die Diskrepanz von Traum und Realität, indem er die Wünsche, Hoffnungen und Ziele, die die Menschen auf diese Stadt projizieren, gegen die ernüchternde Wirklichkeit von Tod, Frust und Drogen stellt.

In Abschnitt (4) beschreibt der Sprecher, welche zerstörerische Wirkung die Stadt New York auf ihn persönlich ausgeübt hat.

Abschnitt (5) schließlich greift in seinen drei Zeilen die entscheidenden, themenbestimmenden Worte aus "Poem Number 334", nämlich stars, wish und frown, auf und stellt damit den direkten Bezug zu diesem Gedicht her.

Ebenso wie in "Poem Number 334" legt auch hier ein fiktiver Sprecher seine persönliche, subjektive Stimmung dar und verleiht seinen individuellen Erfahrungen mit der Stadt New York Ausdruck. Anders als bei "Poem Number 334" läßt der Sprecher hier allerdings keinen Interpretationsspielraum mit Hoffnung auf ein positives Ende; vielmehr scheint dem Sprecher, sein Schicksal sei besiegelt, und so fügt er sich mit einer Geste der Resignation in das Unabänderliche. ${ }^{529}$

Die Gefühlsbekundungen des Sprechers versetzen den Leser direkt in den Moloch der Großstadt. Dort ist kein Platz für Menschlichkeit. Erbarmungslos macht

529 Die Zeilen des Kommentars mit ihrer eindringlichen, aber durchaus nicht moralisierenden Beschreibung der Begleiterscheinungen großstädtischer Zivilisation erinnern an "The Waste Land" von T.S. Eliot. Dort werden Eindrücke wiedergegeben, die den Rezipienten nicht zu einer neuen Denkart oder Verhaltensweise bekehren sollen, sondern ausschließlich als "Symptome der Entwirklichung und Sinnentleerung zeitgenössischen Daseins" beschrieben werden. Hans Egon Holthusen, "Vorwort", in: T.S. Eliot, Das Wüste Land, übers. von Ernst Robert Curtius, (Frankfurt a.M., 1975 [The Waste Land (New York, 1922)]), 27. 
die Stadt jeden Bewohner zu ihrem Opfer, indem es ihm seine Seele raubt. New York, die Metropole, die Träume und Hoffnungen weckt wie keine andere Stadt, verbirgt hinter einer glitzernden Fassade nur Leere und Dunkelheit, die keinen Hoffnungsschimmer und keine Sternschnuppe bereithält.

Das Gedicht sowie der dazugehörige Kommentar - nachfolgend unter dem Titel "Poem Number 334" zusammengefaßt - behandeln grundlegende gesellschaftliche Probleme wie Gefühlskälte, Vereinsamung und Gewalt in Großstädten und verdienen allein aufgrund dieses sozialen Themas Erwähnung. Dennoch geht es nachfolgend nicht um eine inhaltliche Bewertung, sondern um die Frage der techniknutzenden Darstellung und der Berücksichtigung des Aspektes des gemeinschaftlichen Gestaltens. In dieser Hinsicht wurde einleitend festgestellt, daß "Poem Number 334" keine Möglichkeiten zur Interaktion gebe und Kooperation nur in eingeschränktem Maße zulasse, da ausschließlich Fortsetzungen und diese auch nur zeilenweise eingegeben werden könnten. Gut umgesetzt ist die Idee der Kooperation hier lediglich in den Abschnitten für persönliche Kommentare. Themenbezogene Ressourcen stehen nicht zur Verfügung, so daß die entscheidenden Vorteile der Hypertext-Systematik nicht zum Tragen kommen. Insofern unterscheidet sich "Poem Number 334" von gedruckter Lyrik ausschließlich aufgrund seiner Allgegenwärtigkeit und seines Angebots zur immediaten Kooperation. Was den Aspekt des gemeinschaftlichen Dichtens anbelangt, so ist dies durchaus auch im Print-Bereich umzusetzen, so zum Beispiel in Zeitschriften, und muß dort nicht notwendigerweise wesentlich zögerlicher ablaufen als im World Wide Web. Einer engagierten Redaktion kann es durchaus gelingen, in wöchentlichem Rhythmus die Reaktionen und Beiträge der Leser zu berücksichtigen. Der Versand der Kommentare und Fortsetzungen kann per E-Mail erfolgen und ist damit für den Leser ebenso unkompliziert wie eine direkte Eingabe im WWW.

Somit stellt sich die Frage, ob das verbleibende Argument der jederzeitigen Zugriffsmöglichkeit im Falle des "Poem Number 334" ein entscheidender Grund für die Konzeption als webbasiertes Gedicht sein könnte. Jederzeitige Zugriffsmöglichkeit heißt soviel wie rund um die Uhr online zu sein. Dies ist schon aus Zeit- und Kostengründen alles andere als selbstverständlich und bei den Nutzern vermutlich die ganz große Ausnahme. Doch selbst wenn man den Zeit- und Kostenaufwand unberücksichtig läßt, bleibt festzustellen, daß "Poem Number 334” keinen grundsätzlichen Unterschied zu in Print-Medien verbreiteten Gedichten aufweist. Allge- 
meine Verfügbarkeit und Zugriffsschnelligkeit sind zwar durchaus praktische Vorteile, sie bieten jedoch keinen wesentlichen literarischen Mehrwert.

\subsubsection{Verbesserungsansätze}

Gerade bei "Poem Number 334" wären zahlreiche Wege denkbar, die Möglichkeiten des World Wide Web umfassend auszuschöpfen.

Zunächst ließe sich an eine Ausweitung des Kooperationsspielraums der Koautoren denken. Dabei versteht es sich von selbst, daß es den Teilnehmern möglich sein muß, dem Gedicht vollständige Strophen anzufügen oder einzelnen bereits bestehenden Strophen neue zuzuordnen, und zwar parallel oder alternativ. Die Ausweitung könnte aber zum Beispiel auch darin bestehen, daß den Kooperierenden gestattet würde, an beliebiger Stelle einer Strophe eine oder mehrere neue Verszeilen hinzuzufügen, und zwar auch hier - ähnlich wie bei den Strophen - entweder als neue (eingeschobene) Zeile(n) oder aber als Parallele(n) ${ }^{530}$ oder Alternative(n) zu einem bereits bestehenden Vers beziehungsweise $\mathrm{zu}$ mehreren schon existenten Versen. ${ }^{531}$ Auf diese Weise stünden den Koautoren Ansatzpunkte im Umfange bis zum Mehrfachen der Zahl der zu dem betreffenden Zeitpunkt bereits verfaßten Verse für ihren kreativen Anteil zur Verfügung.

Aus der Sicht des Rezipienten gewönne das Gedicht auf diese Art deutlich an Vielschichtigkeit und Variationsbreite, denn einmal eingestellte Beiträge wären nicht mehr unveränderlich, vielmehr bliebe das gesamte Gedicht bis zu seiner Beendigung offen für Veränderungen.

Im Interesse eines erweiterten Interaktionsspielraums empfiehlt es sich darüber hinaus, den Werdegang des Gedichtes transparent zu machen. Dies könnte entweder dadurch gewährleistet werden, daß jeder neu eingestellte Beitrag mit genau der Gedichtversion verknüpft wird, die vor seiner Einfügung vorlag, es wäre aber auch denkbar, an zentraler Stelle eine Chronologie des Entstehungsprozesses anzubieten.

Zur besseren Übersicht könnte ferner eine schematische Darstellung der Struktur von "Poem Number 334" zur Verfügung stehen, die dem Nutzer anzeigte, wie das Gedicht aufgebaut ist und wieviele Alternativen die Verse oder Strophen gegebenenfalls aufweisen.

${ }^{530}$ Als Beispiel für eine parallele Anordnung von Strophen siehe nachfolgendes Kapitel 2.2.2.

531 Hier wäre es außerdem dringend erforderlich, das Datum der Einstellung in das Forum mit aufzuführen. 
Ferner wäre die Einbindung folgender foruminterner Links möglich:

1. Autoren-Links: Sofern der Autor von "Poem Number 334" mehrere Beiträge auf den Seiten der Interactive Poetry Pages veröffentlicht hat, sollten alle seine Gedichte miteinander vernetzt werden, das heißt in diesem Falle von "Poem Number 334" aus zugänglich sein.

2. Audiosequenzen: Besitzt der Autor die Fähigkeit, Audiosequenzen herzustellen, so sollte er die Möglichkeit haben, diese dem Gedicht beizufügen und so seine individuelle Form der Darbietung akustisch zu unterstreichen. Dabei kann es sich entweder um die Rezitation des eigenen Gedichts handeln, aber auch um persönliche Erläuterungen oder die Einspielung von themenbezogenen musikalischen Beiträgen.

3. Visuelle Elemente: Um das Gedicht anschaulicher zu gestalten, könnten zusätzlich visuelle Elemente beigefügt werden. Zur optischen Ergänzung wären beispielsweise Animationen oder eine Bildergalerie denkbar. Gerade zu den Themen New York und Großstadt dürfte hier genügend Material zu finden sein.

4. Audio-visuelle Elemente: Bei dem Thema New York würde es sich zweifellos anbieten, kurze Filmsequenzen über diese Stadt zur Verfügung zu stellen. Gerade die Probleme der Großstädte wie Verelendung und Vereinsamung sind vielfach durch Künstler in Kurzfilmen verarbeitet worden und könnten an dieser Stelle zur Verdichtung der Atmosphäre beitragen.

Um alle Vorteile des Präsentationsmediums World Wide Web auszuschöpfen, müßte auch ein Zugang zu externen Ressourcen geschaffen werden. Hier wären theoretisch mehrere Anknüpfungspunkte vorstellbar, wie zum Beispiel - sofern vorhanden - die private Web-Seite des Autors, die offizielle Homepage der Stadt New York oder Web-Seiten zum Thema Astronomie, aber auch die Homepage der MakeA-Wish Foundation ${ }^{532}$, die schwerkranken Kindern hilft, ihren Herzenswunsch zu erfüllen. Allerdings würde eine Verbindung solcher Informationseinheiten die kulturelle Funktion von Lyrik ad absurdum führen.

${ }^{532}$ Make-A-Wish Foundation, Hg., Make A Wish, <http://www.wish.org/>, 25.07.2003. 


\subsubsection{PERL Poetry}

\subsubsection{Präsentation}

Im Gegensatz zu Poem Number 334 erlaubt das dem Forum Prosebush entnommene Werk nicht nur kooperative Mitarbeit, sondern auch Interaktion. Um einen Eindruck von seiner Konzeption und Gestaltung zu vermitteln, seien die drei Elemente, aus denen das Werk besteht, nachfolgend so wiedergegeben, wie sie im WWW in die Lyrikseiten von Prosebush eingebettet sind.

Die Lektüre beginnt mit dem Beitrag eines Verfassers mit dem Pseudonym Toddius Maximus ${ }^{533}$ :

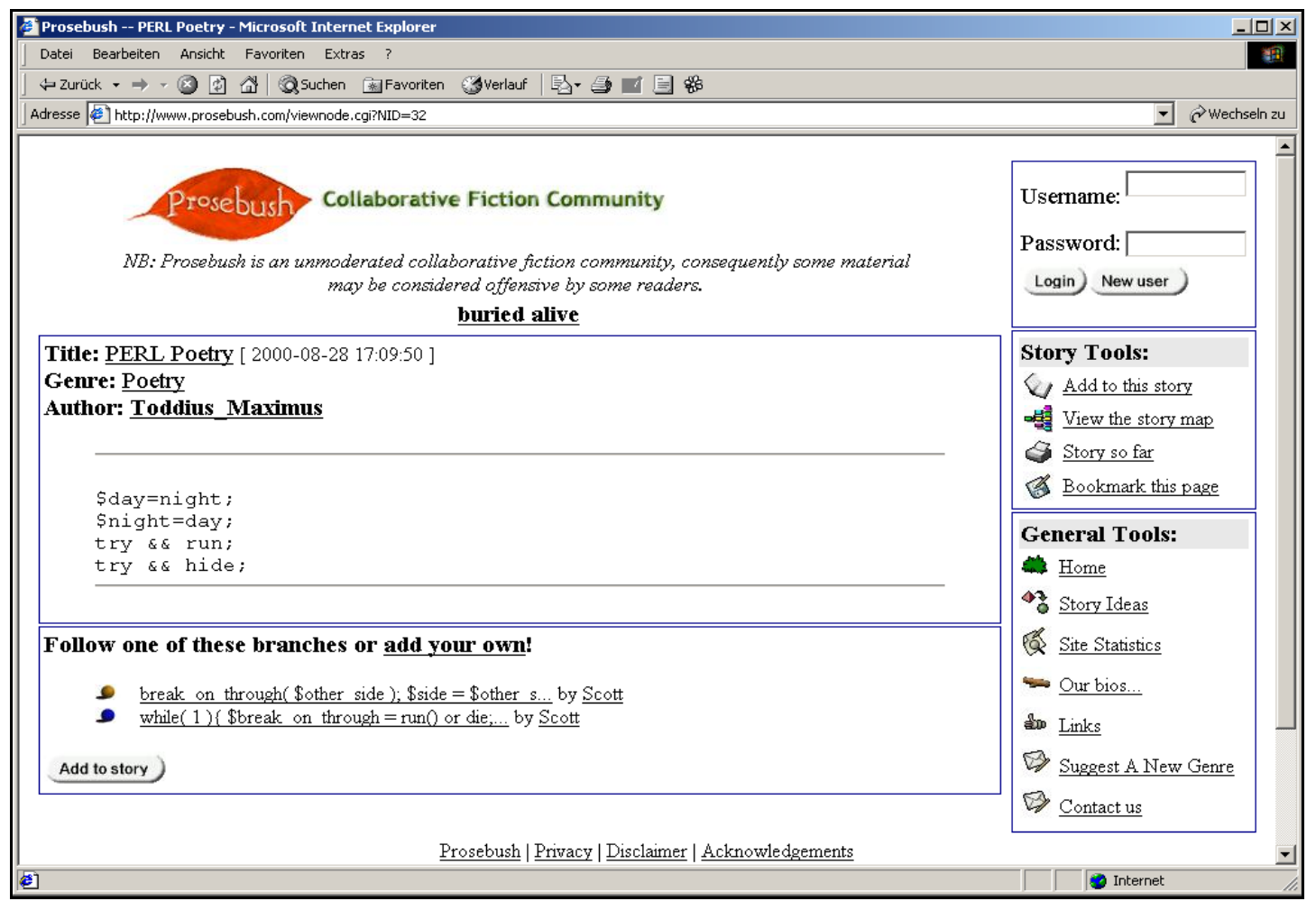

Abb. 1: "PERL Poetry" - Strophe 1

Aus dem vorstehenden Beitrag heraus ergeben sich zwei alternative Verknüpfungen, die beide einem Verfasser namens Scott zugeschrieben sind, nämlich zum einen: ${ }^{534}$

533 Toddius Maximus, "PERL Poetry" [Strophe 1], in: Prosebush.com, Hg., Prosebush ([ 28.08 .2000$])$, $<$ http://www.prosebush.com/viewnode.cgi?NID=32>, 22.07.2003.

${ }^{534}$ Scott, "PERL Poetry" [Strophe 2a], in: Prosebush.com, Hg., Prosebush ([128.08.2000]), $<$ http://www.prosebush.com/viewnode.cgi?NID=36>, 22.07.2003. 


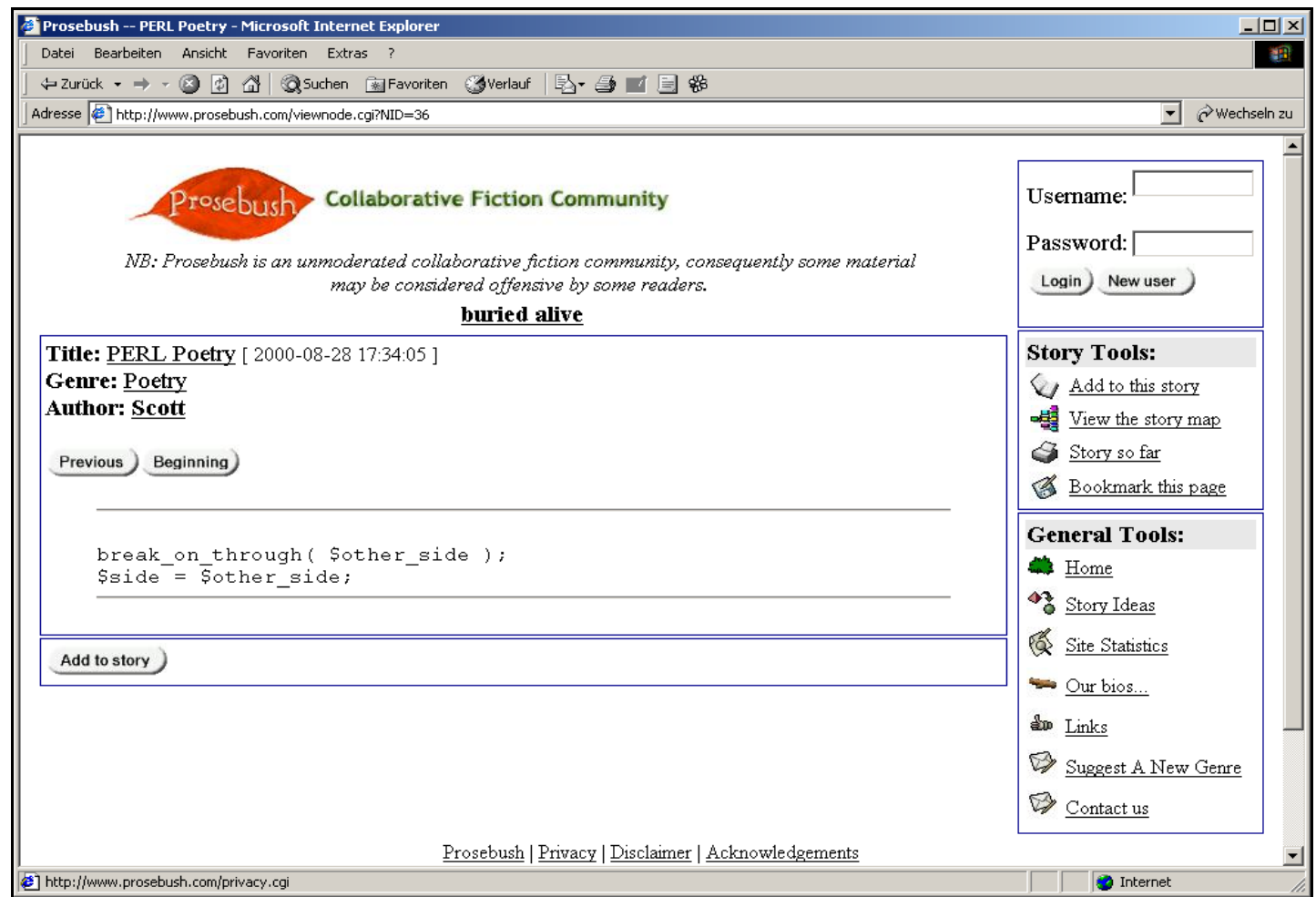

Abb. 2: "PERL Poetry"- Strophe 2a

Zum anderen: ${ }^{535}$

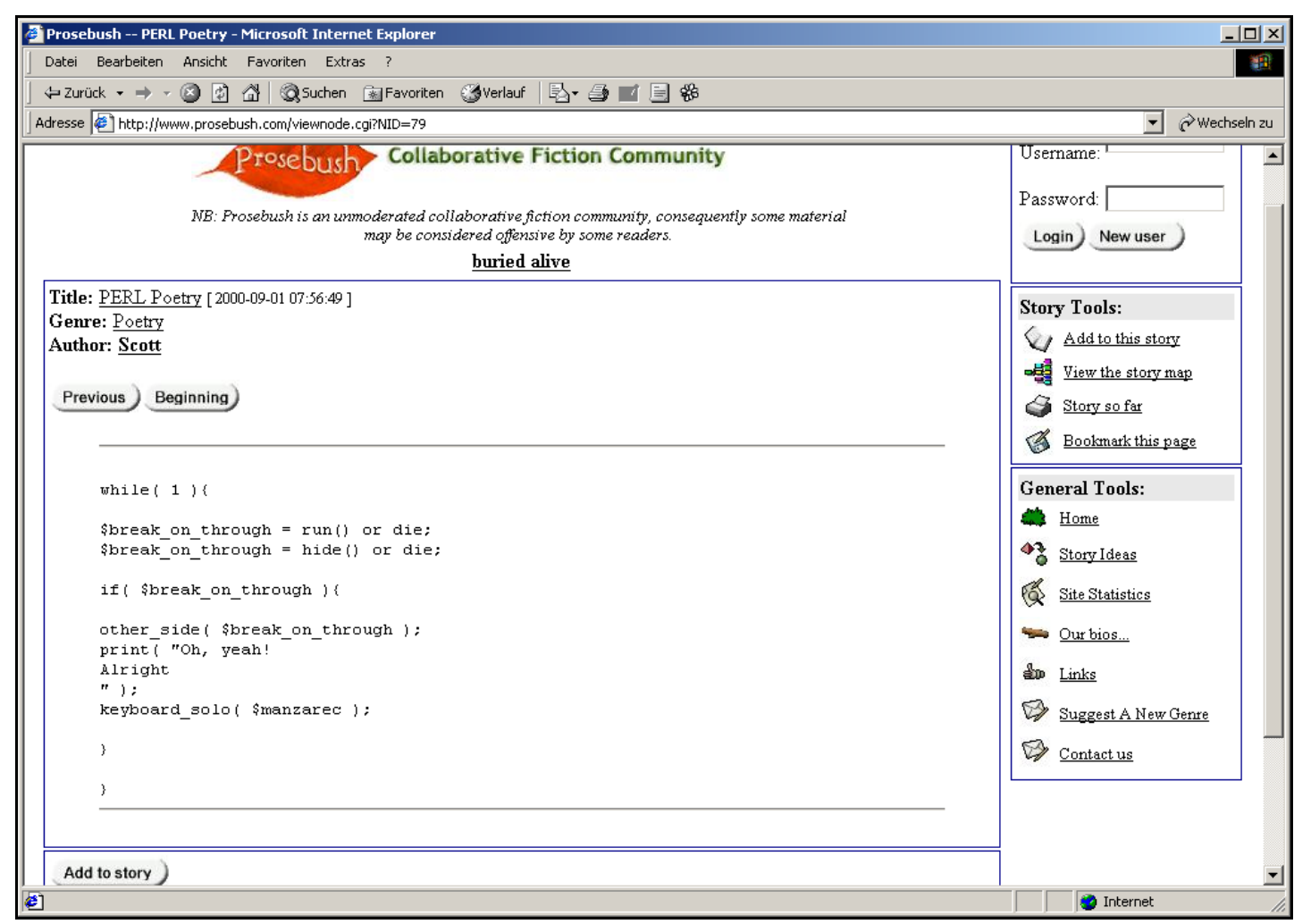

Abb. 3: "PERL Poetry" - Strophe $2 b$

${ }^{535}$ Ders., "PERL Poetry" [Strophe 2b], in: Prosebush.com, Hg., Prosebush ([101.09.2000]), $<$ http://www.prosebush.com/viewnode.cgi?NID=79>, 22.07.2003. 
Beide Beiträge von Scott stellen je für sich eine Fortsetzung des Vierzeilers von Maximus dar, beide Weiterführungen der ersten Strophe befinden sich also auf derselben Ebene des Hypertextgedichts "PERL Poetry". Damit bewegt sich der Leser während des Rezeptionsvorganges auf exakt zwei verschiedenen Ebenen: Von der ersten Gedichtstrophe gehen zwei Verknüpfungen aus, die jedoch ihrerseits nicht miteinander verlinkt sind, so daß der Leser nicht die Möglichkeit hat, von der ersten Fortsetzung direkt zur zweiten zu gelangen, sondern zunächst wieder zur ersten Strophe zurückkehren muß, bevor er die zweite Fortsetzung aufrufen kann. Nachstehendes Übersichtsschema verdeutlicht die Struktur des Gesamtwerks: ${ }^{536}$

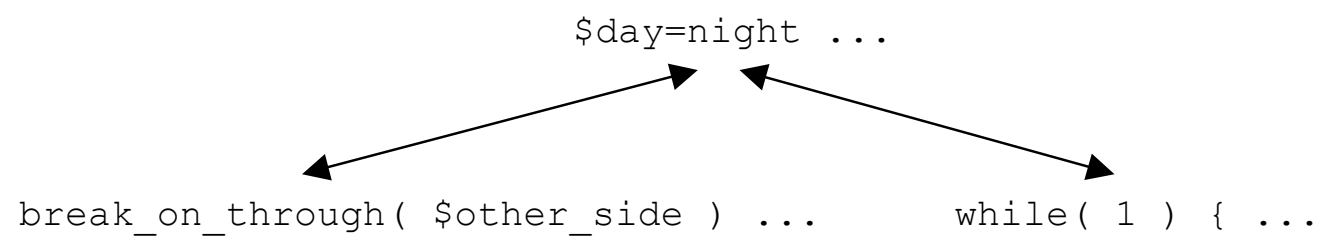

Angesichts dieser Konstellation ist fraglich, wie das Verhältnis der einzelnen Strophen zueinander sprachlich wiedergegeben werden sollte. Würde man Scotts zweite Veröffentlichung als die dritte Strophe des Gedichts bezeichnen, implizierte dies, jener zweite Beitrag Scotts sei die Fortsetzung des anderen. Damit würde jedoch die Gleichwertigkeit seiner beiden Strophen vernachlässigt, denn tatsächlich stellen beide Beiträge von Scott gleichzeitig die zweite Strophe des Gedichts dar. Es bietet sich demzufolge an, sie als Strophe 2a und Strophe $2 \mathrm{~b}$ zu bezeichnen.

Das Gedicht "PERL Poetry" besteht also insgesamt aus drei Strophen, die von zwei verschiedenen Autoren verfaßt sind. Der Autor mit dem Pseudonym Toddius Maximus hat das Werk begonnen, es mit dem Titel "PERL Poetry" versehen und auf den Seiten von Prosebush zur Fortsetzung freigegeben. Kurze Zeit später wurden ihm zwei Beiträge von einem anderen Autor namens Scott beigefügt.

Was "PERL Poetry" von anderen Werken des Lyrikforums unterscheidet, ist seine Durchbrechung formaler Konventionen. Zudem experimentieren die Autoren in ihren Versen mit einer Sprache, von der anzunehmen ist, daß sie für einen Großteil der Rezipienten nicht verständlich ist. Bereits der Titel des Gedichts weist auf die Form hin, in der die Autoren den Inhalt ihrer Verse vermitteln: Maximus und Scott greifen hierfür auf Elemente der Programmiersprache PERL zurück. PERL ist die

\footnotetext{
536 Prosebush.com, Hg., "PERL Poetry" $\quad-\quad$ Story Map $\quad\left(\left[{ }^{1} 01.09 .2000\right]\right)$, $<$ http://www.prosebush.com/storymap.cgi?NID=79>, 22.07.2003.
} 
Abkürzung für Practical Extraction and Report Language und bezeichnet eine Skriptsprache. ${ }^{537}$ Ein Skript wiederum ist ein kleines Programm, das die Kommunikation zwischen einem Browser und einem Web-Server ermöglicht.

"PERL Poetry" setzt sich aus Wörtern und Zeichen zusammen, wobei in der Programmiersprache mehrere Zeichen hintereinander eine Zeichenkette bilden, die auch String genannt wird. Die Zeichen, die Maximus und Scott verwenden, haben unterschiedliche Bedeutung, werden allerdings von den Autoren nicht immer ihrem eigentlichen Sinn und Zweck entsprechend verwendet, so daß es sich bei dem vorliegenden Code nicht um ein lauffähiges Programm handelt. In einer Stellungnahme hierzu räumt Maximus ein, daß bei ihm selbst Nachlässigkeit im Spiel gewesen sei:

Please excuse some of my posts. They were for the beta. I could not come up with anything and just put down some pure crap. Then They did not clear them and released PB to the masses. ${ }^{538}$

Nichtsdestoweniger ist "PERL Poetry" nun ein fester Bestandteil von Prosebush und bildet mittlerweile Ausgangs- und Verbindungspunkt für die beiden anderen Strophen. Die erste Strophe von "PERL Poetry” umfaßt vier Programmzeilen:

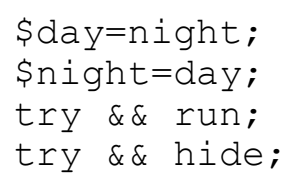

Sie ist nicht in Reimform abgefaßt, weist jedoch aufgrund der Längengleichheit der einzelnen Zeilen und der parallelen Gestaltung jeweils zweier Zeilen eine metrische Ordnung auf. So unterscheiden sich Zeile eins und zwei lediglich durch die umgekehrte Stellung der Worte day und night. Bei dieser kreuzweisen Gegenüberstellung zweier gleicher Begriffe handelt es sich um das Stilmittel des Chiasmus:

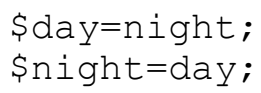

${ }^{537}$ PERL gilt als eine Programmiersprache, die "einfache Dinge einfach und komplizierte Dinge möglich" macht. Larry Wall, Tom Christiansen und Randal L. Schwartz, Programmieren mit Perl, übers. von Peter Klicman, (Köln, 1997 [Programming Perl (Cambridge, 1996)]), 2.

${ }^{538}$ Maximus, "Biography", in: Prosebush.com, Hg., Prosebush, $<\underline{\mathrm{http}}$ ://www.prosebush.com/userinfo.cgi?user=Toddius_Maximus $>, 22.07 .2003$. "PB" steht hier als Abkürzung für Prosebush. 
Allerdings ergibt sich durch die Programmiersprache eine unterschiedliche Bedeutung beider Zeilen. Bei dem Dollarzeichen "\$” handelt es sich um eine Variable, das heißt gewissermaßen um einen Speicherplatz für beliebig oft veränderliche Werte, die über den Zuweisungsoperator "=" unumkehrbar zugewiesen werden. So erhält die Variable auf der linken Seite durch den Zuweisungsoperator den Wert der rechten Seite. In Zeile eins bekommt day auf diese Weise den Wert night. Das Semikolon am Ende der Zeile zeigt dem Interpreter ${ }^{539}$ an, daß die Zeile abgeschlossen ist. In Zeile zwei erfolgt wieder eine Zuweisung an eine Variable, wobei dort night den Wert day erhält.

In den Zeilen drei und vier läuft ein anderer Prozeß ab. Bei dem doppelten sogenannten Ampersand "\&\&" handelt es sich um einen logischen Operator, der als and zur Verknüpfung zweier Aussagen verwendet wird. Die Zeilen drei und vier werden demnach folgendermaßen gelesen: "Try and run" beziehungsweise "Try and hide":

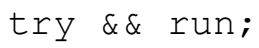

Da das Hypertextwerk seit nunmehr drei Jahren nicht ergänzt wurde, ist fraglich, ob es jemals weitere Hinzufügungen geben wird. Die Interaktionsmöglichkeiten sind demzufolge bis auf weiteres gering: Der Nutzer kann nur auf zwei Ebenen agieren und kooperieren; zusätzliche hypermediale Elemente wie Bild oder Ton sind in dem Hypertextsystem von "PERL Poetry" nicht enthalten.

Entscheidet sich der Nutzer bei Fortsetzung seiner Lektüre für die erste alternative Verknüpfung, gelangt er wie gesagt zu dem sehr kurzen ersten Beitrag von Scott, der lediglich zwei Zeilen umfaßt:

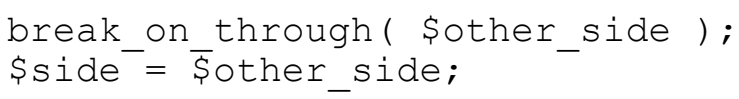

Der Unterstrich “_" steht für ein Leerzeichen und dient nur dazu, den Programmtext lesbarer zu machen. Die runden Klammern "(" und “)" sind sogenannte Metazeichen, die dazu verwendet werden, Zeichen oder Ausdrücke zu gruppieren. Hier ergibt der Programmcode der ersten Zeile folgenden Ablauf: Führe break on through mit other side aus, wobei - entsprechend dem Code der zweiten

539 Damit ein Programm, das mit Hilfe von PERL erstellt worden ist, ausgeführt werden kann, bedarf es eines Übersetzungsprogramms, des sogenannten Interpreters, der das in PERL erstellte Programm interpretiert und damit lauffähig macht. Zu diesem Zweck überträgt der Interpreter den Programmcode zu dessen Laufzeit Befehl für Befehl in den rechnerspezifischen Maschinencode und veranlaßt damit seine Ausführung. Vgl. Joachim Ziegler, Programmieren lernen mit Perl (Berlin, 2002), 44. 
Zeile - die Variable des Wertes side gleich der Variablen des Wertes other side ist.

Wählt der Nutzer die zweite von Scott bereitgestellte Fortsetzung des Werkes "PERL Poetry", gelangt er zu einem Programmabschnitt, der eine Endlosschleife darstellt. ${ }^{540}$ In sogenannten Bedingten Schleifen werden Anweisungen so oft durchlaufen, bis eine Abbruchbedingung erfüllt ist oder der Vorgang künstlich abgebrochen wird. ${ }^{541}$ Ein in runden Klammern angegebener Bedingungsausdruck wird ausgewertet und die nachfolgende, in geschweiften Klammern " $\{$ ” und " $\}$ " enthaltene Anweisung ausgeführt, sofern die entsprechende Bedingung erfüllt ist. Hinter der Anweisung kann sich optional eine zweite Bedingung befinden, die dann zur Ausführung kommt, wenn die wahr-Bedingung nicht erfüllt wird. Erfolgt kein Abbruch des Vorgangs, wird die Anweisung unendlich oft durchlaufen.

Strophe $2 b$ des Gedichts "PERL Poetry" enthält eine solche sogenannte Endlosschleife. Der besseren Übersicht halber sollen Text und Zeichen dieser Strophe hier ausnahmsweise wie folgt angeordnet werden:

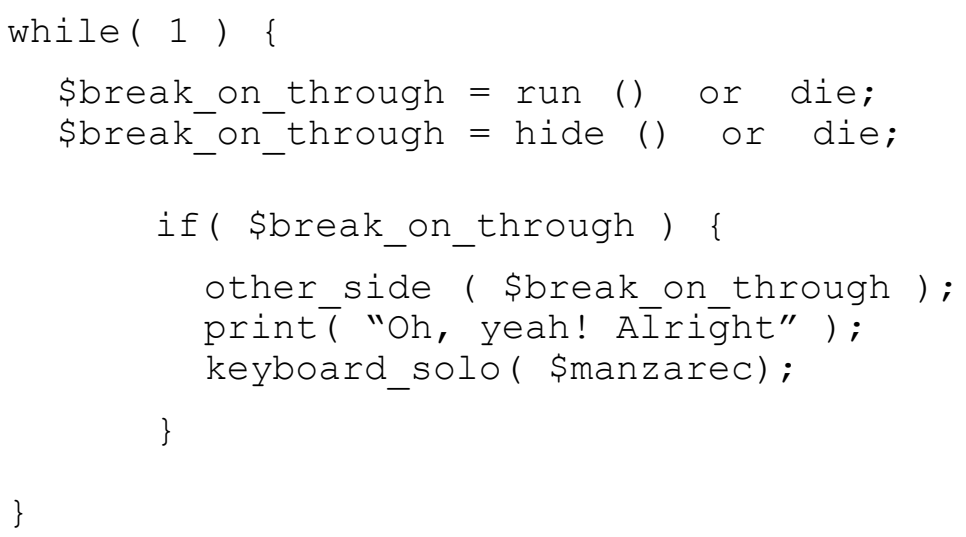

Die erste der obigen Zeilen beinhaltet zunächst die Anweisung mit dem Schlüsselwort while, wobei die runden Klammern allerdings keine Bedingung enthalten, die erfüllt werden könnte. Der Autor setzt sich damit über die übliche $P E R L$ Syntax hinweg. Statt dessen enthalten die Klammern eine "1", die den Wahrheitswert festsetzt. Die Zeile kann also nur so interpretiert werden, daß nachfolgende Anweisungen auszuführen sind, solange ein wahr-Wert geliefert wird:

540 Mit Endlosschleifen können bestimmte Anweisungen unendlich oft durchlaufen werden. Das Programm läuft dabei automatisch solange immer wieder an den Anfang der Schleife, bis der Vorgang abgebrochen wird.

541 Hermann Engesser, Hg., Duden Informatik: Ein Sachlexikon für Studium und Praxis, 2., vollst. bearb. u. erw. Aufl. (Mannheim; Wien; Zürich, 1993 [ $\left.\left.{ }^{1} 1988\right]\right), 623$. 
- Anweisung 1: break on through gleich run oder die

- Anweisung 2: break on through gleich hide oder die

Daß die beiden runden Klammern nach run und hide nicht gefüllt sind, hat keine Auswirkungen auf den Programmablauf. Möglicherweise hat der Autor diese Funktion hier vorgesehen, weil die Computersprache PERL viele Verben als Befehle verwendet, die dem PERL-Interpreter bestimmte Anweisungen geben. ${ }^{542} \mathrm{Im}$ vorliegenden Falle wurde allerdings keine Anweisung definiert; dies ist kein Fehler, da die Funktion nicht notwendigerweise einen Wert erfordert, die Eingabe also optional ist.

Konsequenterweise müßten dem Verb die in Anweisung 1 und 2 ebenfalls runde Klammern folgen. In $P E R L$ ist dieses Wort jedoch reserviert für die Funktion, die den Abbruch des Programmes bewirkt.

Auch sogenannte if-Anweisungen dienen der Auswertung eines Bedingungsausdrucks und der Ausführung der nachfolgenden Anweisung, sofern die vorgegebene Bedingung erfüllt ist. In dieser Strophe wird vorgegeben, daß drei Anweisungen umgesetzt werden sollen, wenn die Bedingung break on through gegeben ist:

- Anweisung 1: Führe die Funktion other side mit break on through aus

- Anweisung 2: Gebe auf dem Bildschirm oder Drucker aus: "Oh, yeah! Alright"

- Anweisung 3: Führe keyboard solo mit manzarec aus

\subsubsection{Analyse und Auswertung}

Day soll den Wert night erhalten, damit also zu night werden und night soll seinerseits den Wert day erhalten und dadurch zu day werden.

Diese Befehle, denen die Aufforderungen "Try and run" und "Try and hide" folgen, sind unverkennbar eine Anspielung auf das Lied Break On Through der Rockband The Doors. Dessen erste Strophe lautet:

You know the day destroys the night

Night divides the day

Tried to run

Tried to hide

${ }^{542}$ Wall, Christiansen und Schwartz, Programmieren mit Perl, 9. 
Break on through to the other side

Break on through to the other side

Break on through to the other side, yeah $[\ldots]^{543}$

Ganz deutlich bildet diese Liederstrophe die Grundlage für die Gedichtstrophen 1 und $2 \mathrm{a}$.

Darüber hinaus ergibt sich die Bezugnahme der Autoren aus dem letzten Befehl der Gedichtstrophe 2b. Dort lautet die Anweisung: "Führe keyboard solo mit manzarec aus". Raymond Daniel Manzarek, kurz Ray Manzarek genannt, war im Jahre 1965 zusammen mit Jim Morrison, Robby Krieger und John Densmore Mitbegründer der Band The Doors, die von 1967 bis 1971 zu den populärsten Rockbands der USA gehörte. ${ }^{544}$ Aufgrund seines außergewöhnlichen musikalischen Talents gilt Manzarek vielen seiner Anhänger als Genie, das die Fähigkeiten aller Bandmitglieder in sich vereinte. ${ }^{545}$ Offensichtlich spielt Scott in der letzten Zeile seines Gedichts auf Manzareks herausragende Beherrschung seiner Instrumente an, wenn er die Anweisung zu einem Keyboard-Solo von Manzarek gibt. Der Song Break On Through, der auf der allerersten Single der Doors im Januar 1967 erschien, war ein "explosive white hot statement of purpose" 546 und bildete den Auftakt einer musikalischen Form der Rebellion.

Maximus versucht in der von ihm verfaßten Strophe des Gedichts den unaufhörlichen Wechsel von Tag und Nacht wiederzugeben, der immer als eine Art Zerstörung empfunden wird. Keine der beiden Tageszeiten ist von längerer Dauer, stets wird sie nach einiger Zeit von der jeweils anderen verdrängt. Auch wenn sich beispielsweise die Zeiten des Wachseins über den Tag hinweg verschieben und damit im Extremfall die Nacht zum Tag und der Tag zur Nacht wird, ist man stets dem zwangsläufigen und unaufhaltsamen 24-Stunden-Rhythmus ausgesetzt. Letztlich ist es gleichgültig, welcher Abschnitt des Tages gerade vorherrscht: Tag und Nacht gehen ineinander über; Tag ist Nacht und Nacht ist Tag. Angesichts dieser Tatsache

${ }^{543}$ Laciefae, "Break on through", in: Dies., Hg., Waiting for the Sun - The Spirit of Jim Morrison, $<$ http://archives.waiting-forthe-sun.net/Pages/Legacy/Albums/TheDoors/Songs/BreakOnThrough.html $>$, 19.07.2003.

544 Uwe Huhn, "Die Band", in: Ders., Hg., The Doors, <http://www.doors-online.de/Bandframe.htm>, 19.07.2003.

545 Markus Kock, “Biographie Raymond Daniel Manzarek”, in: Ders., Hg., The Doors - Die inoffizielle Fanpage, $<$ http://private.addcom.de/schafi/raybio.html $>, 19.07 .2003$.

546 Laciefae, "Break on through", < http://archives.waiting-forthesun.net/Pages/Legacy/Albums/TheDoors/Songs/BreakOnThrough.html > , 19.07.2003. 
scheint es nur einen einzigen Ausweg aus diesem Kreislauf zu geben: Der Versuch, fortzulaufen und sich zu verstecken.

Bemerkenswert ist an dem Gedicht vor allem die kooperative Zusammenarbeit der beiden Autoren. Maximus hat gewissermaßen als Initiator des Projekts "PERL Poetry" mit einer nur vier Zeilen umfassenden Strophe seine Intentionen so ausreichend $\mathrm{zu}$ erkennen gegeben, daß sie von Personen mit gleichem Wissenshintergrund und gleichen Interessen $\mathrm{zu}$ identifizieren waren. Scott wiederum hat offensichtlich alle Hinweise von Maximus richtig gedeutet. Er hat nicht nur verstanden, welches Thema das Gedicht behandelt, sondern er hat auch realisiert, daß es sich um einen experimentellen Umgang mit der Programmiersprache PERL handelt. Für seinen ersten Beitrag hat er dementsprechend das Thema aufgegriffen und den zweiten Teil der ersten Strophe von Break On Through in Skriptsprache umgesetzt. Diese Strophe ist allerdings mit zwei Zeilen nur sehr kurz; sie war vermutlich eine spontane Reaktion auf die Veröffentlichung von Maximus, denn die beiden Strophen wurden mit einem Abstand von nur 25 Minuten in das Prosebush-Forum eingestellt. $^{547}$

Scotts zweiter Beitrag folgt erst einige Tage später und ist wesentlich umfangreicher und komplexer. In dieser Strophe $2 \mathrm{~b}$ werden die Iterationen in dem Lied Break On Through durch die Endlosschleife der while-Anweisung simuliert. Bei den Iterationen des Liedes geht es zum einen um Wiederholungen auf thematischer Ebene. So kommt das Gefühl der Endlosigkeit nicht nur in dem Hinweis auf den unausgesetzten Wechsel von Tag und Nacht zum Ausdruck, es wird vor allem in der letzten Strophe des Liedes noch einmal wieder aufgegriffen:

Made the scene
Week to week
Day to day
Hour to hour
The gate is straight
Deep and wide $[. . .]^{548}$

Ebenfalls iterativ sind die Refrains der einzelnen Strophen, in denen der Titel des Liedes ein ums andere Mal wiederholt wird.

547 Maximus mußte allerdings mit Hilfe des Gedicht-Titels einen zusätzlichen, unmißverständlichen Hinweis auf die Programmiersprache PERL geben, da die von ihm gebrauchten Zeichen und Befehle auch in anderen Skriptsprachen verwendet werden.

548 Laciefae, "Break On Through", <http://archives.waiting-forthesun.net/Pages/Legacy/Albums/TheDoors/Songs/BreakOnThrough.html>, 19.07.2003. 
So wie Tag und Nacht zwangsläufig aufeinander folgen und gewisse Dinge sich Woche für Woche, Tag für Tag und Stunde für Stunde wiederholen, setzt auch die while-Anweisung einen Prozeß in Gang, der immer und immer wieder abläuft und somit zu einer Endlosschleife wird, sofern sich die Gegebenheiten nicht ändern. Da auch sonst vieles ebenso unabänderlich erscheint wie der Wechsel von Tag und Nacht, entsteht der Wunsch, aus diesem Kreislauf der Zwangsläufigkeiten auszusteigen. Eine Möglichkeit des Ausbrechens verrät der Liedertext von Break On Through:

The gate is straight

Deep and wide

Break on through to the other side

Break on through to the other side $[\ldots]^{549}$

"Das Tor ist vor uns, groß und breit. Brich durch zur anderen Seite ..." ${ }^{, 550}$.Die Biographie der Rockband The Doors gibt uns konkrete Hinweise darauf, wie diese Aufforderung interpretiert werden könnte. Offensichtlich basieren die Songtexte mit Todesträumen, Schreckensvisionen und Zaubersymbolen auf persönlichen Erfahrungen der Gruppe mit Meskalin, Methedrin und LSD. ${ }^{551}$ Es ist anzunehmen, daß mit einem Durchbrechen zur anderen Seite des Lebens entweder eine (scheinbare) innere Befreiung durch eine Einnahme von Drogen oder aber eine endgültige Flucht aus dieser Welt mit Hilfe einer Überdosis an Rauschgift beziehungsweise durch Suizid gemeint ist.

Das Gedicht "PERL Poetry" wird dem Leser in Form eines Hypertextes präsentiert, der sowohl Interaktion als auch Kooperation erlaubt. Insofern kommt im Forum Prosebush die Hypertext-Systematik stärker zum Tragen als auf den Seiten der Interactive Poetry Pages, wo die Gedichte lediglich Kooperationsmöglichkeiten eröffnen. Auch bietet "PERL Poetry" den Zugang zu foruminternen Informationsquellen. Jeder Gedichtstrophe ist ein Hinweisfeld mit dem Datum ihrer Veröffentlichung in Prosebush sowie dem Namen ihres Autors beigefügt. Von dort aus kann der Nutzer grundsätzlich detailliertere Auskünfte über den Autor sowie gegebenenfalls dessen weitere Veröffentlichungen abrufen.

549 Ebd., 19.07.2003.

550 Huhn, "Break On Through", in: Ders., Hg., The Doors, <http://www.doors-online.de/1.htm>, 19.07.2003. Möglich wäre auch folgende Übersetzung: Das Tor vor uns ist groß und breit. So brich doch durch zur anderen Seit'.

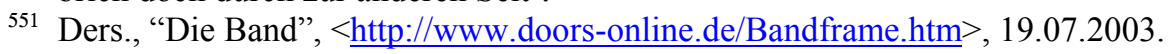


Gleichwohl ist der Aktionsspielraum innerhalb des Gedichts recht eingeschränkt, da es aus reinen Textelementen besteht, die keine multimedialen Sequenzen und keine Verknüpfungen mit Prosebush-internen oder -externen themenbezogenen Ressourcen beinhalten. Insofern unterscheidet sich "PERL Poetry" von seiner Präsentationsform her nicht in überzeugender Weise von einer Druckpublikation.

Der Vorteil der Gestaltung von "PERL Poetry" als webbasiertem Hypertext liegt so gesehen in erster Linie in der Unmittelbarkeit und der globalen, jederzeitigen Zugänglichkeit, die theoretisch eine weltweite kooperative Zusammenarbeit ermöglicht. Praktisch blieb die allgemeine Resonanz allerdings eher zurückhaltend. Bedenkt man, daß nur zwei Personen an der Schaffung von "PERL Poetry" beteiligt waren, drängt sich der Schluß auf, daß die Zusammenarbeit bisher keineswegs erfolgreich war und keine befriedigenden Ergebnisse hervorbrachte. Ein entscheidender Nachteil von "PERL Poetry" ist nämlich beispielsweise seine Kürze. Zwar ist die Möglichkeit der Interaktivität gegeben, aber bei einer Auswahl von nur drei Strophen scheint dieser Begriff beinahe nicht mehr gerechtfertigt. Anders verhielte es sich bei einer größeren Auswahl an Verknüpfungen. Da das Konzept von Prosebush gestattet, an jeder beliebigen Stelle Ergänzungen einzufügen, könnte "PERL Poetry" mit einer höheren Anzahl von Beiträgen ein ausgesprochen vielschichtiges und für den Nutzer spannendes Hypertext-Gedicht ergeben. Unter Umständen sind der geringe Umfang des Gedichts und der nunmehr dreijährige Stillstand seit der Einstellung von Strophe 2b am 01.09.2000 aber auch weniger auf einen konzeptionellen Mangel als auf die Besonderheit des Themas zurückzuführen. Es wäre durchaus denkbar, daß die Verwendung der Programmiersprache PERL der Grund dafür ist, weshalb sich keine weiteren Kooperierenden gefunden haben.

"PERL Poetry" bleibt aber insofern ein interessantes Studienobjekt, als seine Autoren den Computer und das WWW dazu nutzen, mit einer Sprache zu experimentieren, die dazu dient, den Umgang mit diesen elektronischen Medien möglich und vor allem einfacher zu machen. Auch die stille Übereinstimmung der Autoren in dieser Sache sowie die Art und Weise, wie Scott sich mit dem Song von The Doors auseinandersetzt, heben "PERL Poetry" aus der Gruppe der übrigen Gedichtbeiträge im Forum von Prosebush heraus. Dennoch bleibt festzustellen, daß die Vorteile des Trägermediums World Wide Web, die dem Gedicht ganz wesentlich hätten zugute kommen können, nur in geringem Umfange genutzt wurden. 
Gewiß ließe sich einwenden, ein Gedicht solle für sich alleine wirken und nicht durch zusätzliche visuelle oder akustische Elemente von der eigentlichen Aussage ablenken. Auch solle es jedem Leser selbst überlassen bleiben, wie er die einzeInen Strophen interpretiere. Dem ist jedoch entgegenzuhalten, daß es an dieser Stelle nicht darum geht, den grundsätzlichen Wert von "PERL Poetry" in Frage zu stellen, sondern daß es das Ziel ist, den Vorteil eines webbasierten Gedichts gegenüber konventionell-linear-konzipierten und computergestützt-webunabhängigen Gedichten herauszuarbeiten. In dieser Hinsicht befinden sich die Herausgeber des Forums Prosebush durchaus auf dem richtigen Weg, doch sind sie, was die Möglichkeiten der Hypertext-Systematik anbelangt, noch weit davon entfernt, an die Grenzen des Machbaren zu stoßen.

\subsubsection{Verbesserungsansätze}

Ähnlich wie bei "Poem Number 334" setzen die Vorschläge zur Verbesserung bei der Konzeption des Hypertext-Systems an. So wäre es denkbar, den Interaktionsspielraum ${ }^{552}$ der Nutzer auszuweiten. Zu diesem Zwecke müßten neben den Verknüpfungen zwischen den drei Strophen auch Verbindungen zu Prosebush-internen Links sowie zu themenbezogenen externen Ressourcen im World Wide Web angelegt werden. Als interne Verknüpfungen würden beispielsweise folgende Angebote eine Bereicherung für den Leser bedeuten:

1. Audiosequenzen: Auch bei "PERL Poetry" sollten die Autoren die Möglichkeit haben, ihren Gedichtstrophen Audiosequenzen beizufügen, um die Wirkung akustisch zu betonen. Rezitationen der einzelnen Strophen sind hier vermutlich aufgrund der verwendeten Programmiersprache nicht möglich; statt dessen könnten persönliche Erläuterungen zum Verständnis der einzelnen Strophen gerade in diesem Falle, in dem sich die Autoren einer Sprache bedienen, die vermutlich nur ein geringer Teil der Leser beherrscht, äußerst hilfreich sein.

2. Visuelle Elemente: Um das Gedicht "PERL Poetry" anschaulicher und vor allem verständlicher zu gestalten, könnten die Abläufe der einzelnen dargestellten Computer-Programme in kurzen Sequenzen simuliert werden. Auf diese Weise wäre der Leser in der Lage nachzuvollziehen, welche Abläufe die Befehle der Skriptsprache PERL auslösen.

${ }^{552}$ Zur Abgrenzung der Begriffe siehe Kapitel V.3.2. vorliegender Arbeit. 
Eine weitere Möglichkeit, das Gedicht durch visuelle Elemente zu ergänzen, läge in der Bereitstellung von Photographien der Rockband The Doors. Der Leser erhielte auf diese Weise einen nachhaltigen Eindruck von den Interpreten des Liedes Break On Through.

3. Audio-visuelle Elemente: Wegen der Bezugnahme auf das Gitarrensolo von Manzarek in Strophe $2 \mathrm{~b}$ würde sich auch eine Kombination von visuellen und akustischen Elementen anbieten. Ja, es sollte sogar eine Verpflichtung sein, einen kurzen Filmausschnitt mit einem der offensichtlich legendären Gitarrensoli bereitzustellen; zusätzlich könnten einige kurze Filmsequenzen mit Ausschnitten aus den Konzerten der Doors ausgewählt werden. Auch hier dürfte reichlich Material zur Verfügung stehen.

4. Rückmeldungen: Um der Meinung der Leser Geltung zu verschaffen, sollte dem Gedicht außerdem an übergeordneter Stelle ein Forum für Anregungen und Kommentare beigefügt werden.

Um die Charakteristika des Präsentationsmediums World Wide Web erschöpfend $\mathrm{zu}$ nutzen, sollten die Organisatoren auch Zugang zu externen Ressourcen schaffen. In erster Linie kommen hier Web-Seiten zum Thema PERL in Frage, das heißt der Nutzer sollte die Gelegenheit erhalten, sich gründlicher mit dieser Programmiersprache auseinanderzusetzen. Zu diesem Zweck finden sich im World Wide Web zahlreiche Informationen, Anleitungen und Online-Kurse. In mindestens ebenso großer Zahl finden sich dort Ressourcen zum Thema The Doors, die zu größerer Anschaulichkeit und Attraktivität des Gedichts "PERL Poetry" beitragen könnten.

Auch hier sind vermutlich noch weitere Anknüpfungspunkte denkbar. Entscheidend wäre aber letztlich, daß die Herausgeber von Prosebush das innovative Konzept des Forums, das in seinen Ansätzen durchaus richtungsweisend ist, weiterentwickeln und damit den vielfältigen Möglichkeiten des World Wide Web in angemessener Weise Rechnung tragen.

\subsubsection{The Ultimate Writer}

\subsubsection{Präsentation}

Der letzte Teil vorliegender Untersuchungen zur webbasierten Lyrik betrifft ein Hypertextwerk des Autors Komninos Zervos, zu dessen Web-Seiten unter anderem das Electronic Poetry Center den Zugang vermittelt. Den Einstiegspunkt für die 
Suche nach einem geeigneten Beispiel in dem Forum EPC bildete der dortige Bereich E-Poetry, der ausschließlich der digitalen, speziell für das elektronische Medium konzipierten Dichtung gewidmet ist. ${ }^{53}$ Die Recherche wurde in der dortigen alphabetischen Autorenliste durchgeführt, wo die Wahl schließlich auf Zervos fiel. Die direkte Weiterleitung des EPC führte zu den Cyber Poetry-Sei$\operatorname{ten}^{554}$, auf denen der Autor Verknüpfungen zu verschiedenen seiner Arbeiten anbietet. $^{555}$

Die Hypertext-Werke von Zervos, eines Künstlers mit langjährigen Performance-Erfahrungen, zeichnen sich durch hohen Animationsgrad aus. Als Zervos im Jahre 1996 mit dem Aufbau einer eigenen Web-Seite begann und damit seiner Kreativität eine neue Richtung gab, hatte er sich bereits seit über zehn Jahren als Lyriker betätigt. Mit der Einbeziehung des Computers und des Web in seine dichterische Arbeit spezialisierte sich Zervos im Laufe der Zeit vom "performance poet" 556 zum selbsternannten "cyber poet" 557 . Die neuen Medien machen es ihm möglich, seinen Kunstwerken höchstmögliche Eigendynamik zu verleihen, um sie dem Online-Publikum anschließend als Gesamtwerke darbieten zu können, die gleichzeitig Spaß und Unterhaltung, aber auch Informationen vermitteln. Dementsprechend haben seine Auftritte im Web tatsächlich den Charakter von Performance oder "Kunst mit Ereignischarakter" 558 und repräsentieren damit genau das, was Suter sprachschöpferisch als "Event-ualität" mitteln den Eindruck einer großen Begeisterung für den kreativen Umgang mit dem Präsentationsmedium und wirken damit - wie von Zervos intendiert - als "celebration of words/text/sounds" 560 .

Sein erklärtes Ziel als Lyriker ist es, dem Leser die Dichtung wieder zurückzubringen, die ihm - so die Überzeugung von Zervos - durch Dichter wie T.S. Eliot oder Ezra Pound geraubt worden ist: "Komninos [...] believes in 'bringing poetry

553 Glazier u.a., Hg., Electronic Poetry Center - E-Poetry, <http://epc.buffalo.edu/e-poetry/>, 28.07.2003.

554 Komninos Zervos, cyberpoetry, <http://www.gu.edu.au/ppages/k_zervos/>, 28.07.2003.

555 Ders., The Ultimate Writer, <http://live-wirez.gu.edu.au/cyber/ultimate/>, 28.07.2003.

556 Ders., "Biography - Komninos Zervos", infLect - a journal of multimedia writing 1(2003), $<$ http://www.ce.canberra.edu.au/inflect/01/kombio.htmll $>, 28.07 .2003$.

557 Ders., "Poetry - Cyber Beginnings", in: The Thylazine Foundation, Hg., Thylazine: Australian Arts and Literature on Landscape and Animals, $<$ http://www.thylazine.org/archive1/thyla6/thyla6f.html $>$, 28.07.2003.

558 Suter, "Ein neues Literaturmilieu", <http://www.netzliteratur.net/suter/kassel.htm>, 07.07.2003.

559 Ebd., 07.07.2003.

560 Rhizome.org, “cyberpoetry 1995-1997: Komninos Zervos”, in: Dies., Hg., Rhizome.org - The New Media Art Resource ([ $\left.\left.{ }^{1} 01.01 .1995\right]\right),<$ http://www.rhizome.org/object.rhiz?14970>, 28.07.2003. 
back to the people' from whom, by implication, it was stolen by poets such as T.S. Eliot, Ezra Pound [...]." ${ }^{, 561}$ Gerade die neuen Möglichkeiten, die das Medium World Wide Web in Verbindung mit Hypertext bietet, dürften sich für Zervos als besonders effizient und zielführend erwiesen haben, denn webbasierte Literatur stellt den Leser aufgrund ihrer Angebote zur Interaktion und Kooperation zunehmend stärker in den Vordergrund. Gleichzeitig vollzieht sich ein Wandel von Starrheit zu Beweglichkeit, von Einzel- zu Gemeinschaftserlebnis und vom beständigen Kunstwerk zur Augenblickserfahrung:

We seem to have entered a phase of convergence of the writer/theorist/critic and the whole end-user experience being more of a writerly experience as well as readerly one, more of a collaborative experience than a solitary one, and more of an of-the-moment rather than universal or enduring experience. ${ }^{562}$

Genau diesem Grundgedanken entspricht das Hypertextsystem The Ultimate Writer. Nach den eher statisch wirkenden Seiten der Interactive Poetry Pages und von Prosebush - Collaborative Fiction Community überrascht das Gedichtprojekt von Zervos gleich zu Beginn mit einer animierten Einführungssequenz. The Ultimate Writer empfängt den Leser mit der Aufforderung: "Build the ultimate writer!" 563 Auf dem Bildschirm erscheint eine weibliche Gestalt, die dem Leser visuell (über eine Abfolge von fünf Sprechblasen) und akustisch das Konzept des Systems darlegt und seine Bedienung erläutert: Mit Hilfe des Manuals am unteren Bildschirmrand solle der Web-Besucher seinen eigenen Dichter erschaffen. Dies geschehe mittels einer Abfolge gestalterischer (Schreib-)Übungen, bei der jeder einzelne Handlungsschritt einen wichtigen, konstruktiven Abschnitt auf dem Weg durch den Hypertext darstelle, der seinen Höhepunkt schließlich in der Kreation des ganz persönlichen Gedichtautors finde.

${ }^{561}$ Komninos University of Queensland Press, “Articles on Komninos”, in: Komninos Zervos, Hg., cyberpoetry $\left(\left[{ }^{1} 1991\right]\right),<$ http://www.gu.edu.au/ppages/k_zervos/hsc/fascist.html $>, 28.07 .2003$.

562 Zervos, "Poetry - Cyber Beginnings", $<$ http://www.thylazine.org/archive1/thyla6/thyla6f.html $>$, 28.07.2003.

563 Ders., "Intro", in: Ders., Hg., The Ultimate Writer, $<\underline{\text { http://live- }}$ wirez.gu.edu.au/cyber/ultimate/index.html>, 28.07.2003. Alle Bereiche des Forums The Ultimate Writer tragen eine identische Web-Adresse. Dennoch soll in diesem Kapitel nicht auf bibliographische Angaben verzichtet werden, da sie auch andere wichtige Informationen enthalten. Beispielsweise zeigen sie den aufgerufenen Bereich und das Datum des Zugriffs an. 
Die siebenteilige Aufgabenreihe ist gedacht als Übung im Querdenken, als eine Übung, die darauf angelegt ist, sich von den alltäglichen, konventionellen Denkstrukturen zu lösen. "Creative writing" ${ }^{564}$ nennt Zervos diesen Prozeß, den der Ausführende auf seinem Weg bis hin zur Abfassung eines eigenen Hypertextgedichtes durchlaufen soll. Das Endergebnis der kreativen Mitarbeit bezeichnet Zervos als cyber poetry ${ }^{565}$, doch unterscheidet sich die so benannte Lyrikform in keiner Weise von webbasierter Lyrik gemäß der in dieser Arbeit formulierten Definition. Hier wie da kann die Charakterisierung von Zervos zugrunde gelegt werden, nach der cyber poetry folgende Eigenschaften aufweist:

$[\mathrm{P}]$ oetry that can not be published in the traditional print media, poetry that moves in time and space, poetry that requires new ways of reading, poetry that allows interaction, poetry that is moving digital, aural, visual, and animated. ${ }^{566}$

Der ehemals passive Leser, der sich ausschließlich auf den Rezeptionsvorgang konzentrieren konnte, ist nun dazu aufgefordert, kreativ mitzuarbeiten. Aus diesem Grunde scheint es hier nicht mehr angebracht, von einem Leser zu sprechen. War die Bezeichnung Nutzer bis zu diesem Punkt eine vertretbare Alternative, ergibt sich nunmehr im Hinblick auf die diversen Aktionsformen in The Ultimate Writer zunehmend die Notwendigkeit, einen treffenderen Begriff zu finden.

Da alle Hypertextsysteme auf Computerprogrammen basieren, läge es nahe, auf den Begriff Anwender zurückzugreifen. Ebenso wie bei dem Wort (Be-)Nutzer kommt hier jedoch nur zum Ausdruck, daß sich die betreffende Person einer Sache bedient, nicht aber daß sie an ihrer Mitgestaltung beteiligt ist. Die Begriffe Mitarbeiter, Kooperierender oder Beitragender, die diesem Aspekt der Kooperation Rechnung tragen, vernachlässigen allerdings wieder die Interaktionsmöglichkeiten eines Hypertextes. Geeignet schiene daher die Bezeichnung Agierender. Das Wort agieren hat mehrere Bedeutungen: Zum einen ist es gleichbedeutend mit "handeln" im Sinne einer sequentiellen oder aber gezielten Vorgehensweise im Hypertext, wobei entweder alle offerierten Informationen systematisch nacheinander abgearbeitet werden oder aber der Leser sich selbstbestimmt-intentional durch den Text bewegt.

\footnotetext{
564 Zervos, "Intro", < $\underline{\text { http://live-wirez.gu.edu.au/cyber/ultimate/index.html }>, ~ 28.07 .2003 . ~}$

565 Ebd., 28.07.2003.

566 Rhizome.org, “cyberpoetry 1995-1997”, <http://www.rhizome.org/object.rhiz?14970>, 28.07.2003.
} 
Zum anderen hat "agieren" auch die Bedeutung von "wirken", worin die Einflußnahme des Rezipienten auf den Text zum Ausdruck kommt. Schließlich kann agieren auch als spielen verstanden werden, das heißt als mehr oder weniger wahllose Fortbewegung im Text. Von allen in Betracht kommenden Bezeichnungen trifft Agierender somit am ehesten den Kern der Sache.

The Ultimate Writer eröffnet dem Agierenden, wie der Nutzer hier nun also genannt werden soll, alle wesentlichen Vorteile eines Hypertextes: Systemintern bietet ein umfangreiches Netz von Verknüpfungen zahlreiche Möglichkeiten zur Interaktion. Zusätzlich wird dem Agierenden vom Start des Systems bis zu seiner Beendigung immer wieder Gelegenheit zur Kooperation geboten. In einem der sieben Zugangsbereiche besteht ferner - entweder durch einen Eintrag im Gästebuch oder aber per E-Mail - das Angebot zur Meinungsäußerung. Darüber hinaus erhält der Agierende neben den internen Aktionsmöglichkeiten auch Zugriff auf diverse themenbezogene Ressourcen im World Wide Web.

Zervos hat seinem Hypertext zahlreiche visuelle und akustische Elemente beigefügt. Während die Animationen der Erläuterung oder Veranschaulichung des Dargebotenen dienen, verwendet Zervos zur akustischen Untermalung vorwiegend sehr rhythmusbetonte Musiksequenzen, die je nach Aufgabenbereich variieren.

Auf dem Weg zur Gestaltung seines ganz persönlichen virtuellen Gedichtautors muß der Besucher ${ }^{567}$ der Web-Seite The Ultimate Writer sieben verschiedene Lerneinheiten bearbeiten. Die Anlaufpunkte für die einzelnen Aufgabenbereiche sind am unteren Bildschirmrand angeordnet. Sechs von ihnen tragen die Namen der auszuwählenden Körperteile, die der Agierende dem virtuellen Gedichtautor nach und nach vor Durchführung der (Schreib-)Übungen zuweist: Mouth, Eyes, Hair, Feet, Legs und Body; der letzte, siebente Punkt führt zu einer raumgestalterischen Aufgabe und hat daher die Bezeichnung Build.

Jeder Anlaufpunkt, das heißt jede der sechs Körperpartien ist einer anderen Gedichtart zugeordnet. Diese bestimmt durch ihre strukturellen und inhaltlichen Merkmale die Aufgabenstellung der jeweiligen Schreibübung. Der Charakter der Gedichtart prägt aber auch die optische Gestaltung des Aufgabenbereichs, so daß der Agierende in jedem Abschnitt mit einer völlig andersartigen und speziellen Situation

567 Die Bezeichnung Agierender kommt erst dann zur Anwendung, wenn sich der Besucher einer Web-Seite interagierend oder kooperierend mit dem Hypertext beschäftigt. 
konfrontiert wird. Diese Verschiedenartigkeit soll es dem Agierenden ermöglichen, sein Bewußtsein auf das Unterschiedlichste zu erweitern und gleichzeitig seine Sinne für eine neue Wahrnehmungsweise zu schärfen.

Hat sich der Agierende für einen Anlaufpunkt entschieden, öffnet sich das Fenster für die Auswahl des entsprechenden Körperteils. Hier stehen jeweils vier Komponenten zur Wahl: Vier Mundpartien, vier Augenformen, vier Haarfrisuren, vier Paar Schuhe, vier Beinbekleidungen und vier Oberkörper. Gleichzeitig erscheint ein kurzer Hinweis, wie im Nachfolgenden vorzugehen ist. Sobald der Agierende eine der vier Komponenten ausgewählt hat, erhält er Zugang zum Aufgabenbereich. Dort sind - dem Charakter der jeweiligen Gedichtart entsprechend - unterschiedlich viele verschiedenartige Schreibübungen auszuführen.

Um das Endziel zu erreichen, müssen alle sieben Aufgabenbereiche durchlaufen und alle Übungen erfüllt sein. Jede Lektion kann beliebig oft wiederholt werden, wobei sowohl Veränderungen im Text als auch Wechsel der Körperteile vorgenommen werden können.

Folgende Abschnitte muß der Agierende passieren:

\section{Mouth: Rave Poetry}

Dichten bedeutet, auf dem Papier zu phantasieren. Oftmals besteht es sogar nur darin, jene Gedanken niederzuschreiben, die dem Agierenden gerade in den Sinn kommen. Einfälle tauchen in der Regel bruchstückhaft vor dem geistigen Auge auf; selten sind sie so strukturiert und klar gegliedert, wie sie beispielsweise in einer Werkinterpretation anzuordnen sind. Die Schreibübungen im Abschnitt Rave Poetry sollen dem Agierenden dabei helfen, sich von jeder erlernten, erzwungenen Ordnung freizumachen, indem er sich ganz seinen spontanen Ideen und Phantasien hingibt und nur diese schriftlich festhält. Der Agierende ist angehalten, in knappen Worten und normaler Alltagssprache zu skizzieren, was er spontan mit einem von ihm frei gewählten Thema assoziiert. Das Ergebnis bildet das erste rave poem, das sogenannte basic rave.

Ziel der zweiten Schreibübung dieses Abschnitts ist ein repetetive rave. Hier sollen die Gedankenfetzen, die im ersten Abschnitt notiert wurden, intensiv überdacht und dann immer und immer wieder ergänzt und ausgebaut werden.

In der Lektion character rave lautet die Anweisung, eine Figur mit einer eigenen Biographie zu schaffen. Aus einer ausführlichen Personenbeschreibung 
sollen Name, Alter, Geschlecht, Familienstand, Staatsangehörigkeit, soziale Herkunft, Schulbildung, Verhaltensweisen, Eigenarten usw. hervorgehen. Anschliessend soll der Agierende versuchen, sich in diese Person einzufühlen. Dazu gilt es, (in der Rolle jener Person) irgendeine beliebige Situation zu schildern und darüber so zu plaudern, daß hieraus - zwischen den Zeilen - Informationen über die betreffende Person zu entnehmen sind.

Der erste Bereich enthält keine Animationen, ist aber durchgängig von Musiksequenzen unterlegt.

2. Eyes: Picture Poems

Hier geht es darum, sich ein Ereignis aus der Vergangenheit wieder ins Gedächtnis zu rufen, bildlich vor Augen zu führen und dieses Bild mit Hilfe von fünf Worten zu beschreiben. Anschließend sollen diese Worte durch das Hinzufügen jeweils dreier Adjektive sowie eines Titels und eines Resümees zu einem Gedicht ausgebaut werden. Die Ergebnisse können mittels einer Print-Funktion ausgedruckt werden.

In diesem Bereich kommen sowohl akustische als auch optische Elemente zum Tragen. So werden beispielsweise alle Vorgänge von fanfarenähnlicher Musik begleitet. Instruktionen sowie die Eingabefelder für die Schreibübung werden auf einer Schriftrolle präsentiert. Jedes Anklicken der Bedienungsknöpfe auf dieser Rolle erzeugt ein Geräusch, das dem Agierenden das Abrollen des Papiers suggeriert. Neben diesem Effekt führen und begleiten zahlreiche weitere Animationen durch diesen Abschnitt.

\section{Hair: Surreal Poetry}

Im Abschnitt Hair betritt der Agierende eine ganz und gar neue Sphäre. Hier ist alles in Bewegung, scheint zu reagieren, dreht sich, verschwindet im Nichts oder verändert die Farbe; die unterschiedlichsten Geräusche und Musiksequenzen wechseln einander ab. Aufgabe des Agierenden ist es, verschiedene Techniken zu erlernen, mit deren Hilfe er seine persönlichen Wünsche und Vorstellungen herausarbeiten und in Gedichtform bringen kann.

Nachdem er zunächst in einem surreal wirkenden Raum mit Sturmbrausen empfangen worden ist, gelangt der Agierende als nächstes in eine Szenerie mit einem blattlosen Baum, an dem fünf Fische im - hörbaren - Wind baumeln. Jeder Fisch repräsentiert einen Teilabschnitt des Bereichs Surreal Poetry: Exquisite Corpse I, Exquisite Corpse II, One Word into Another, One List into Another und 
Known into the Unknown. Jeder Befehl per Mausklick löst irgendein Geräusch aus, zum Beispiel Klicken, Klappern, Wind, Möwengeschrei oder Wasserspülung. Der Zugang zu den Teilabschnitten erfolgt durch Anklicken der jeweiligen Bezeichnungen, woraufhin der dazugehörige Fisch von seinem Zweig herabfällt. Alle Teilabschnitte sind mit Vogelgezwitscher und Entspannungsmusik untermalt.

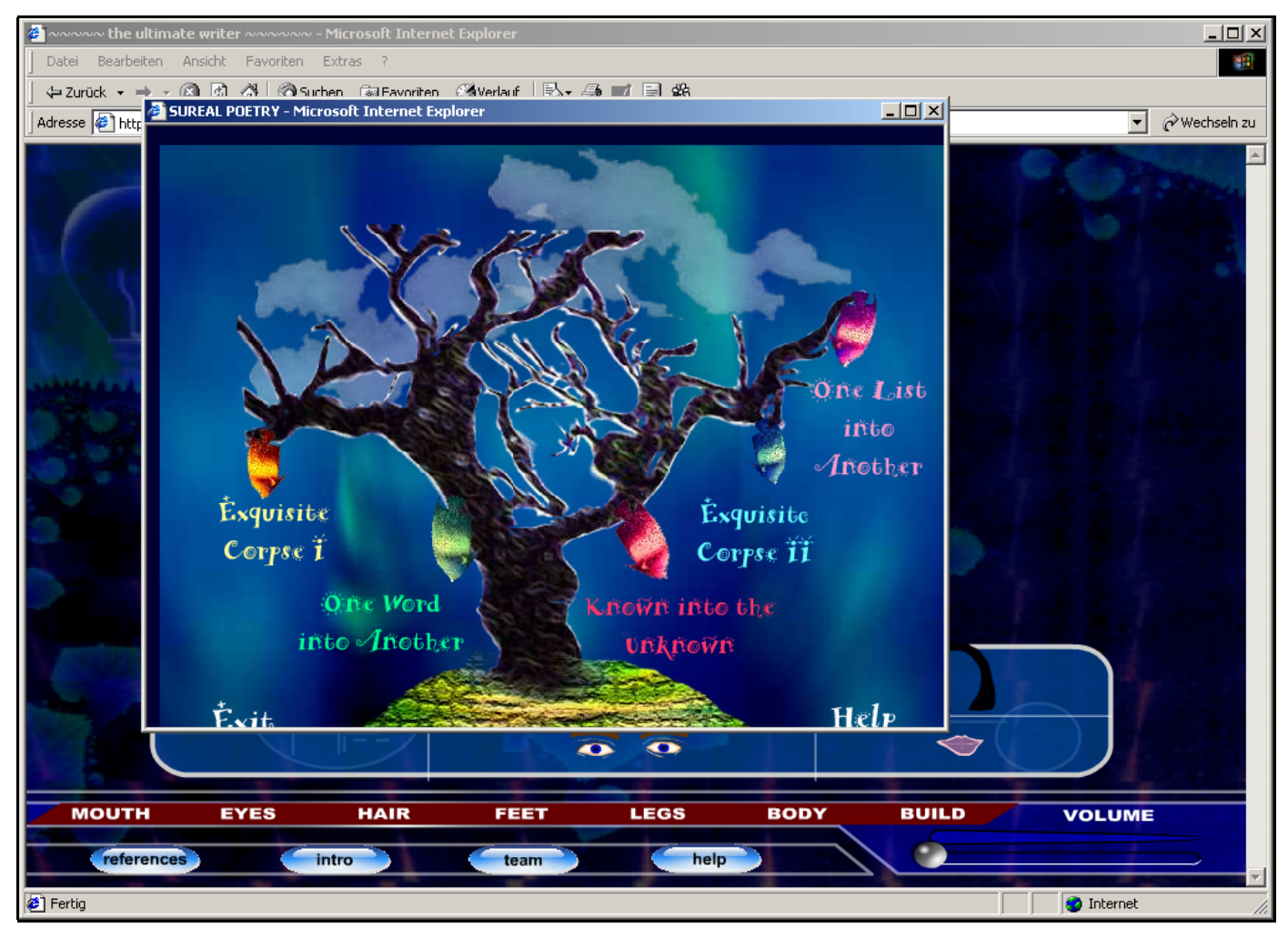

Abb. 4: 'The Ultimate Writer - Surreal Poetry'

Folgende Übungsaufgaben sind in den fünf Lernabschnitten vorgesehen:

Exquisite Corpse I: Ziel ist die zufällige Konstruktion von Sätzen, die in sechs Einzelschritten erfolgt. Mit jedem Schritt wird jeweils ein Vertreter unterschiedlicher Wortarten ausgewählt: Bestimmter oder unbestimmter Artikel + Adjektiv + Substantiv + Verb (beliebiges erstes Verb, das dem Agierenden in seinem PrintWörterbuch ins Auge fällt) + Adjektiv (ausgewählt nach derselben Methode) + Substantiv (ebenfalls beliebig ausgewählt). Der sich aus dieser Zusammenstellung ergebende (im allgemeinen sinnlose) Satz soll sodann in einem letzten Schritt durch verbindende Worte ergänzt werden, um schließlich einen flüssigen Text und damit das erste sogenannte surreal poem zu ergeben. Dieses kann der Agierende als faßbaren Beweis seiner Kreativität ausdrucken. Der Name dieses Abschnitts, Exquisite Corpse, ist dem ersten Satz entnommen, der nach dieser Methode geschaffen 
wurde. ${ }^{568}$ Zum Abschluß erhält der Agierende ein Lob für seine Kreativität: "Fantastic!"

Exquisite Corpse II ist die erweiterte Version des vorangegangenen Abschnitts, in der sich die Zahl der auszuwählenden Wörter deutlich erhöht. Der Agierende beginnt mit der Zusammenstellung von zehn Verben, die alle Bezug auf ein beliebiges von ihm gewähltes Thema haben müssen. Nachfolgend werden zehn Adjektive mit vorangehendem bestimmten oder unbestimmten Artikel, zehn Substantive, wiederum zehn Adjektive und abermals zehn Substantive hinzugefügt, alle beliebig aus dem Wörterbuch ausgewählt. Zusammengenommen ergeben diese Worte das zweite surrealistische Gedicht, das ebenfalls ausgedruckt werden kann. Auch hier erhält der Agierende lobende Worte: "Well done!"

One Word into Another: In diesem Abschnitt geht es darum, Dinge zu umschreiben und dabei Ausdrücke zu verwenden, die in dem jeweiligen Zusammenhang nicht erwartet werden. $\mathrm{Zu}$ diesem Zweck sollen fünf beliebige Wörter aufgelistet werden. Ist diese Aufgabe erfüllt, tritt ein unerwarteter Effekt ein, da sich die Wörter in steinerne Fische verwandeln, von denen der Agierende zwei auswählen muß. Die beiden 'geretteten' Wörter bilden den Anfang und das Ende des zu erstellenden Gedichts, dessen verbindenden Mittelteil der Agierende eigenschöpferisch hinzufügen muß. Was ihm merkwürdig erscheinen mag, wird von Zervos als (beabsichtigter) Effekt surrealistischer Lyrik begründet. Auch in diesem Falle ist ein Ausdruck des Gedichts möglich. "Congratulations!” lautet das Lob zum Abschluß.

One List into Another: Auch hier handelt es sich um die erweiterte Version des vorangehenden Abschnitts. Wieder sollen Dinge umschrieben werden, statt einzelner Ausdrücke werden nun allerdings immer Wortpaare verwendet. Zunächst werden zwei Wörter ausgewählt, die unterschiedliche Themenbereiche vorgeben. Diese Bereiche werden mit jeweils zehn Worten beschrieben. Daraufhin werden diese Worte miteinander vermengt und in zwei Spalten aufgeteilt. Abschließend

568 Die gemeinschaftliche Collage von Wörtern, cadavre exquis (exquisite corpse), deren Ergebnis surrealistischer Literatur ähnelt, basiert auf einem alten Gesellschaftsspiel, bei dem alle Mitwirkenden nacheinander auf einem Zettel jeweils einen Satz notierten, ohne die vorangegangenen Beiträge zu kennen. Der entstandene Text sollte versteckte Wahrheiten über die Mitspieler und die Welt im allgemeinen aufdecken. Dieses Vorgehen erhielt seinen Namen nach dem Ergebnis des ersten Spieldurchgangs: "Le cadavre exquis boira le vin nouveau." Jon Rendell, "Exquisite Corpse - Definition", in: Ders., Hg., Exquisite Corpse - Exquisite Links, <http://www.exquisitecorpse.com/definition.html>, 25.11.2003. 
müssen alle Elemente der linken Spalte mit Ausdrücken aus dem Wortfeld der rechten Spalte erklärt werden. Das Ergebnis bildet ein weiteres surrealistisches Gedicht, das der Agierende ebenfalls ausdrucken kann. Die Kreativität des Agierenden wird mit den Worten "Great effort!” bewertet.

Known into the Unknown: In diesem Abschnitt werden surreal poems gebildet, indem der Agierende bereits kreierte Gedichte in ihre gegenteilige Bedeutung übersetzt. So ändern sich beispielsweise die Worte wet saddened eyes in smiling happy faces.

Die Aufgabe verlangt zunächst die Schaffung eines eigenen Haiku-Gedichts. In dem folgenden Arbeitsvorgang soll Schritt für Schritt ein zweiter Dreizeiler erstellt werden, der das genaue Gegenteil des ersten wiedergibt. Auch hier besteht die Möglichkeit zum Ausdruck. Das abschließende Urteil: “Outstanding!”

4. Feet: Beat Poetry

Dieser Abschnitt empfängt den Agierenden mit folgendem Vierzeiler:

It's now time

To bust a rhyme

Cause writing poetry

Aint no crime ${ }^{569}$

Im Gegensatz zu den übrigen Aufgabenbereichen wird hier keine kooperative Mitarbeit erwartet. Es werden keine praktischen Übungen angeboten, statt dessen erhält der Agierende ausführliche theoretische Informationen zum Thema Beat Poetry. In drei Teilabschnitten werden die Begriffe Pentameter, Tetrameter und Rap jeweils definiert und detailliert erläutert. Die Informationen reichen vom Ursprung dieser Versformen über ihren Aufbau bis hin zu Ähnlichkeiten mit und Abweichungen von anderen Versformen. Zudem stehen in diesem Abschnitt diverse Links zu weiterführenden Informationen im World Wide Web zur Verfügung. Beispielsweise führt das Stichwort Beat Generation zu den Web-Seiten des Lyrikforums Literary Kicks, das unter anderem umfangreiches Material zur amerikanischen Beat Generation präsentiert. ${ }^{570}$

\footnotetext{
569 Zervos, The Ultimate Writer - Feet: Beat Poetry, < $\underline{\text { http://live- }}$ wirez.gu.edu.au/cyber/ultimate/index.html>, 28.07.2003.

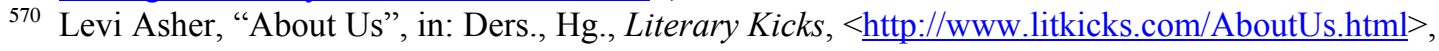
01.08.2003.
} 
Das Stichwort San Francisco öffnet dem Agierenden ein neues Fenster mit Verknüpfungen $\mathrm{zu}$ weiteren themenbezogenen Links, die zum Beispiel $\mathrm{zu}$ The Beat Page weiterleiten. Dort werden Informationen über die Geschichte der Beat Generation und ihre Hauptvertreter Allen Ginsberg, Jack Kerouac sowie Lawrence Ferlinghetti angeboten. Letzterer gründete im Jahre 1953 den City Lights Bookstore in San Francisco, der heute noch als einer der wenigen großen verlagsunabhängigen Buchhandlungen der Vereinigten Staaten gilt. ${ }^{571}$

Ein spezieller visueller Effekt ergibt sich in diesem Abschnitt, wenn der Agierende mehrere Lerneinheiten nacheinander aufruft. Mit jeder neuen Lektion wird der Text unleserlicher, da sich die einzelnen Textschichten überlagern und ineinander überzugehen scheinen, was den komplexen Inhalt der Lerneinheiten noch komplizierter erscheinen läßt.

In diesem Abschnitt entsteht ein besonderer Eindruck von Echtzeit im World Wide Web durch die Angabe des aktuellen Datums sowie der genauen Uhrzeit im rechten unteren Rand des Fensters. Die Möglichkeit der Kontaktaufnahme per E-Mail sowie des Zugriffs auf das Gästebuch intensivieren zusätzlich das Gefühl der Unmittelbarkeit.

\section{Legs: List Poems}

Ähnlich wie im Bereich Rave Poetry geht es auch in diesem Abschnitt um die Assoziationen des Agierenden. Seine Wahrnehmungen, Gefühle oder unvermittelten Gedanken zu einem bestimmten Thema bilden die Ausgangsbasis für ein Gedicht. Hat sich der Agierende für ein bestimmtes Stichwort entschieden, ist er in einem zweiten Schritt gehalten, alle gedanklichen Verknüpfungen nacheinander in einer Liste zu verzeichnen. Den Abschluß dieser Aufstellung soll ein kurzer Kommentar bilden, mit dem der Agierende seine Liste abrundet beziehungsweise zusammenfaßt. In einem dritten und letzten Arbeitsschritt werden alle einzelnen Wörter jeweils durch Sätze umschrieben und ersetzt. Der daraus entstehende Text formt das erste Gedicht dieses Aufgabenbereichs.

Die Schreibübungen im Abschnitt List Poems umfassen insgesamt fünf verschiedene Lektionen: Basic, similes, metaphors, responses und statements. Prinzipiell ist die Vorgehensweise in allen didaktischen Einheiten gleich, der Unterschied betrifft jeweils nur Art und Ausgestaltung der Assoziationen: Je nach-

571 Kenneth Rumsey, Hg., The Beat Page, <http://www.rooknet.com/beatpage/>, 01.08.2003. 
dem sind entweder Vergleiche (similes), Metaphern (metaphors), Ergänzungen (responses) oder eingängige Sätze im Stil von Werbe-Slogans, Schlagzeilen oder Liedertiteln (statements) gefordert.

\section{Body: Sound Poetry}

"Poetry is language that sounds good." von Gedichten zum Ausdruck zu bringen, kommen in diesem Abschnitt verstärkt akustische Komponenten zum Tragen. Der Bereich erinnert in seiner Gestaltung an einen Musikautomaten oder das Mischpult eines Diskjockeys. Aufgabe ist es, 15 Wörter auszuwählen, von deren Klang sich der Agierende besonders angesprochen fühlt. Diese Wörter sollen anschließend ausgeschmückt und zu einer sinnvollen Geschichte arrangiert werden. Als Klangbeispiele sind im oberen Drittel des Arbeitsbereichs, der wie eine Jukebox anmutet, 15 Wörter gruppiert, die von einer männlichen Stimme vorgetragen werden, sobald der Agierende die Felder mit der Maus anklickt. Allerdings sind für die praktische Umsetzung der Anweisung keine Eingabefelder vorgesehen. Die Ausführung dieser Aufgabe beschränkt sich in diesem Bereich ausschließlich auf theoretische Überlegungen.

\section{Build: Haiku Poetry}

Jap an ese hai ku

sev en teen syll ab les long word e con om $\mathrm{y}^{573}$

Diese Stückelung der Worte Japanese haiku seventeen syllables long word econo$m y$ dient offensichtlich einer Betonung der Silbenzahl. Haiku-Gedichte bestehen wie bereits im Kapitel Interactive Poetry Pages erwähnt - in ihrer traditionellen Form aus drei Zeilen mit insgesamt 17, das heißt $5+7+5$ Silben $^{574}$ und bilden eine Art Epigramm.

In diesem Abschnitt muß der Agierende das unordentliche Zimmer von Komninos Zervos aufräumen, indem er bestimmte Aufgaben löst. Drei Gegenstände innerhalb des Zimmers können mit der Maus angeklickt werden und stellen die Verknüpfungen zu den Aufgabenbereichen her. Die Lampe in japanischem

${ }^{572}$ Zervos, The Ultimate Writer - Body: Sound Poetry, < $\underline{\text { http://live- }}$ wirez.gu.edu.au/cyber/ultimate/index.html>, 28.07.2003.

573 Ders., The Ultimate Writer - Build: Haiku, $<$ http://live-wirez.gu.edu.au/cyber/ultimate/index.html $>$, 28.07.2003.

574 Es sei an dieser Stelle nochmals darauf hingewiesen, daß Haiku-Gedichte im Englischen oftmals etwas freier, mit geringerer Silbenzahl verfaßt werden. 
Stil führt zu dem Thema Haiku, der Fensterrahmen zu Concrete und ein langes ausgestopftes Tier zu Extended.

Der Bereich Haiku wird eingeleitet durch eine ausführliche Erläuterung von Aufbau und Bedeutung dieser Gedichtform. Hier wird vermittelt, daß ein HaikuGedicht der Ausdruck von Augenblickserfahrungen ist. Aus diesem Grunde - so die Empfehlung - sollte der Dreizeiler in einem Atemzug vorgetragen werden können, reduziert allein auf die zur Beschreibung unbedingt erforderlichen Worte. Gegenwärtig ist die Tradition dieses japanischen Kurzgedichts zunehmend westlichen Einflüssen ausgesetzt, was zu allmählichen Veränderungen im Silbenrhythmus führt. In der anschließenden Schreibübung ist der Agierende demgegenüber aufgefordert, einen Dreizeiler nach dem herkömmlichen Schema von $5+7+5$ Silben zu bilden. Der Ergebnis kann anschließend ausgedruckt werden. Kehrt der Agierende dann in das Zimmer von Zervos zurück, verschwinden die umherliegenden Kleidungsstücke im Wäschekorb.

Im Bereich Concrete Poetry geht es um Bilderlyrik beziehungsweise visuelle Poesie. Manchmal kann die Anordnung eines Wortes auf dem Papier oder dem Bildschirm diesem Wort eine besondere Bedeutung verleihen. Sie kann seine Aussage unterstreichen oder ihm auch einen völlig gegensätzlichen Sinn geben. In dem von Zervos angeführten Beispiel imitiert das vereinzelte "e" den Vorgang, den das Wort escape beschreibt:

escap e

Als weiteres Beispiel nennt Zervos das Wort Balloons, bei dem die beiden Buchstaben “o" zu Ballons werden und davonfliegen. Nach einer Reihe weiterer Darstellungen wird der Agierende aufgefordert, seinerseits Worte zu finden, deren Buchstaben sich bildhaft in einer der Wortbedeutung konformen oder auch konträren Weise arrangieren lassen. Da der augenblickliche Stand der Technik dem Agierenden noch keine praktische Umsetzung dieser Übung gestattet, beschränkt sie sich - ebenso wie im Abschnitt Sound Poetry - ausschließlich auf theoretische Überlegungen, wobei angeraten wird, sich an Beispielen aus der Werbung zu orientieren, die auf Reklametafeln, öffentlichen Verkehrsmitteln und in den Print-Medien zu finden sind. Mit der Rückkehr in das Zimmer von Zervos kehrt weitere Ordnung ein: die umherliegenden Bücher verschwinden im Regal. 
Der dritte Bereich Extended Poetry ist als Übung in freiem Dichten konzipiert. Der Agierende soll ein Gefühl dafür entwickeln, wie sich die Dinge innerhalb ein und desselben Vorgangs weiterentwickeln können. $\mathrm{Zu}$ diesem Zweck soll er sich ein Ereignis der Vergangenheit ins Gedächtnis rufen und dieses mit fünf Worten beschreiben. In einem zweiten Schritt soll jedes der fünf Worte um vier weitere ergänzt werden, die jeweils als Platzhalter für die fünf Vorgaben dienen könnten. Daraufhin soll sich der Agierende auf die Gespräche der Menschen um sich herum konzentrieren sowie auf das, was seine innere Stimme zu ihm sagt, um die entstandene Liste von 25 Worten durch die aufgegriffenen Gesprächsfetzen und seine persönlichen Gedanken erweitern zu können. Abschließend obliegt es dem Agierenden, sein nunmehr entstandenes freestyle poem zu arrangieren, so daß es schließlich in Form eines Fließtextes rezipiert werden kann. Endgültiges Ziel dieser Übung ist es, die Entwicklung eigener Kreativität zu fördern, so daß es der sogenannten extended five word formula letztlich nicht mehr bedarf.

Mit Beendigung der dritten Übung wird die Ordnung im Zimmer von Zervos endgültig wiederhergestellt, das Bett wird hergerichtet und das schiefhängende Bild zurechtgerückt. Zum Abschluß bedankt sich Zervos winkend für die geleistete Mithilfe.

Hat der Agierende alle sieben Bereiche mit den dazugehörigen Übungen durchlaufen, erwartet ihn auf dem Bildschirm das Abbild seines Dichters, zusammengesetzt aus genau jenen Körperteilen, die der Agierende zu Beginn jeder Übung ausgewählt hat; bei sechs Auswahlbereichen mit jeweils vier Möglichkeiten sind hier insgesamt $4^{6}=4096$ unterschiedliche Schriftstellertypen denkbar. Außerdem beglückwünscht Zervos den Agierenden schriftlich mit folgenden Worten: "Congratulations, you are now the ultimate writer! As a bonus you may now continue to the final section, performance poetry." 575

Zervos hat es sich offensichtlich zur Aufgabe gemacht, die Besucher seines Forums nicht nur mit der Dichtkunst, sondern auch mit den Techniken und Regeln einer gelungenen Rezitation der eigenen Gedichte vertraut zu machen. Daher erhält der Agierende vor Verlassen des Forums gewissermaßen als Anerkennung für sein Engagement und als abschließende theoretische Lektion die Gelegenheit, sich

${ }^{575}$ Zervos, The Ultimate Writer, $<$ http://live-wirez.gu.edu.au/cyber/ultimate/index.html>, 10.09 .2003$. 
anhand eines audio-visuellen Beitrags mit den Grundregeln eines überzeugenden Gedichtvortrags vertraut zu machen. Zervos spricht in diesem Zusammenhang von performance. ${ }^{576}$ Dabei ist es nebensächlich, wieviele Zuhörer der Rezitation beiwohnen; das Auditorium kann ebensogut aus lediglich einer einzigen Person wie aus tausenden von Besuchern bestehen, jeder Vortrag vor Publikum bildet eine Darbietung, die vermutlich auch im deutschen Sprachgebrauch am treffendsten mit dem Wort Performance zu bezeichnen ist:

Once there is an audience, there is a performance, there is communication or attempted communication of your ideas, thoughts, feelings, observations via the medium of poetry. ${ }^{577}$

Der hier erörterte abschließende Bereich des Forums beinhaltet sieben Lektionen für eine gelungene Performance. In kurzen Sequenzen, die aus Vorträgen, animierten Bildern und Begleittexten bestehen, werden die Fehler unerfahrener, dilettierender Rezitatoren aufgezeigt und Richtlinien für publikumswirksames Verhalten vermittelt. Auf dem Bildschirm erscheint ein im Comicstil gezeichneter Zuschauerraum mit einem schemenhaft dargestellten Auditorium und einem Vortragenden zum Thema "performing live tonight"; zuweilen tritt auch Zervos in der Funktion eines kritischen Mentors auf. Eine Stimme erläutert dem Agierenden, dem in diesem Bereich des Forums lediglich die Rolle des In t e r agierenden zukommt, welche Verhaltensmuster einer überzeugenden Rezitation abträglich sind. In kurzen animierten Sequenzen werden diese Fehler durch den Vortragenden veranschaulicht, gelegentlich durch Zervos gestisch mißbilligt und anschließend korrigiert.

Folgende Ratschläge sollte ein Vortragender beherzigen:

1. Be organised: Eine gelungene Performance zeichnet sich durch eine gute Planung und eine straffe Durchführung aus: Der Vortragende sollte nicht die Zeit des Auditoriums vergeuden, sondern durch ein ausgearbeitetes Programm einen reibungslosen Ablauf ohne ermüdende Leerläufe zwischen den einzelnen Gedichten gewährleisten. ${ }^{578}$

${ }^{576}$ Ders., "Performance", in: Ders., Hg., The Ultimate Writer - Final Section, $<\underline{\text { http://live- }}$ wirez.gu.edu.au/cyber/ultimate/index.html>, 10.09.2003.

577 Ebd., 10.09.2003.

578 Ders., "Be organised", in: Ders., Hg., The Ultimate Writer - Final Section, $<\underline{\mathrm{http}}: / /$ livewirez.gu.edu.au/cyber/ultimate/index.html>, 10.09.2003. 


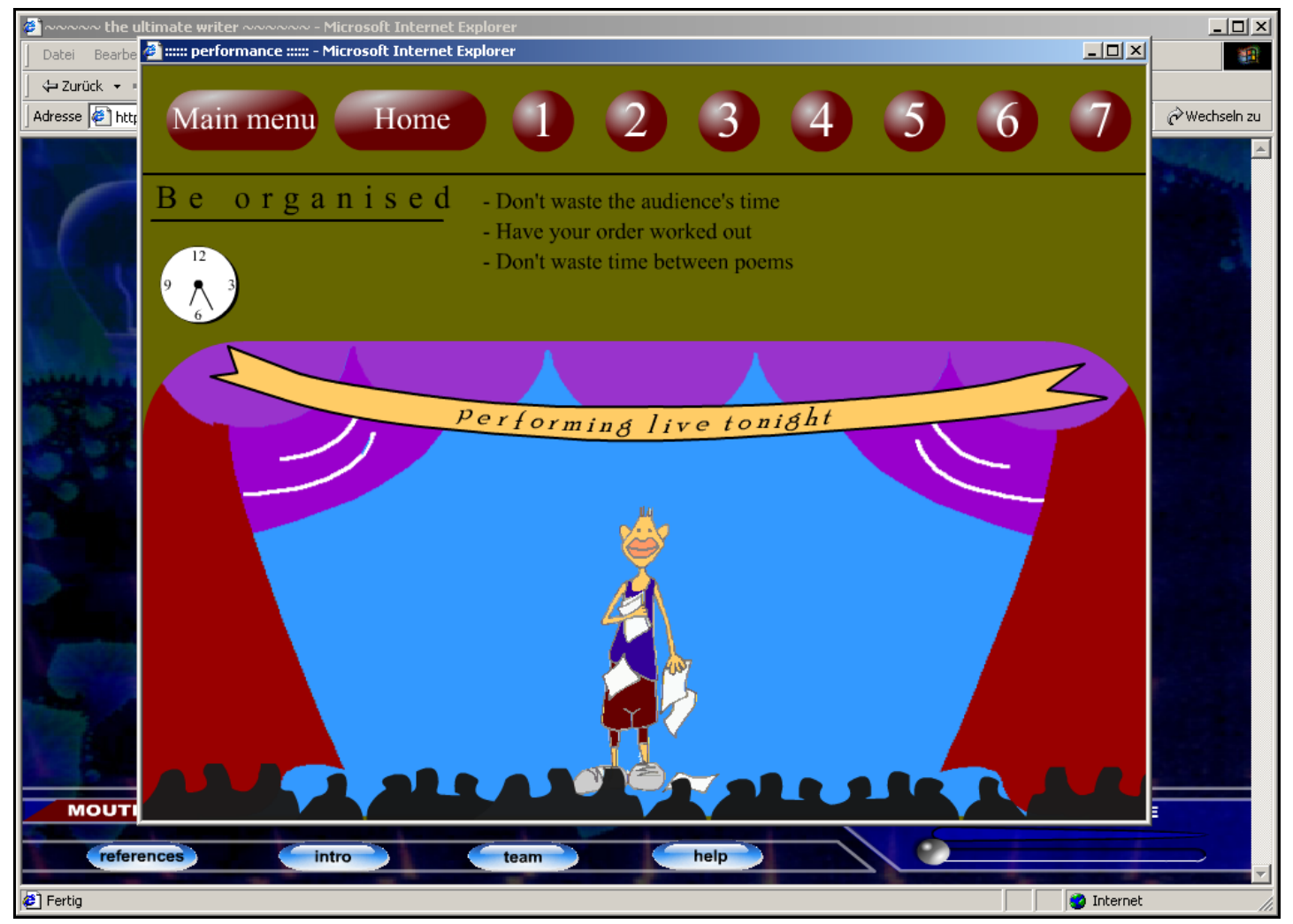

Abb. 5: 'The Ultimate Writer-Performance'

2. Avoid: Der Vortragende sollte vermeiden, sich noch vor Beginn seiner Rezitation für sein Werk oder seine Vortragsweise zu entschuldigen. Einleitungen dieser Art bewirken eine negative Einstimmung des Auditoriums und bilden daher eine schlechte Ausgangsbasis für die Performance. ${ }^{579}$

3. Don't be frightened by the microphone: $\mathrm{Zu}$ einer sorgfältigen Vorbereitung gehört auch die Justierung des Mikrophons, das auf Mundhöhe befindlich und vor allem angeschaltet sein sollte. Ferner sollte der Vortragende davon absehen, mit dem Oberkörper vor dem Mikrophonständer hin- und herzupendeln. ${ }^{580}$

4. Don't go overtime: Eine realistische Planung sollte garantieren, daß der Vortrag zeitlich nicht zu Lasten des Auditoriums, der Organisatoren und anderer Vortragender geht. Der Wunsch nach weiteren Beiträgen kann zudem nur durch eine wohldosierte Sprechdauer geweckt werden. ${ }^{581}$

579 Ders., “Avoid”, in: Ders., Hg., The Ultimate Writer - Final Section, <http://livewirez.gu.edu.au/cyber/ultimate/index.html>, 10.09.2003.

580 Ders., "Don't be frightened by the microphone", in: Ders., Hg., The Ultimate Writer - Final Section, $<$ http://live-wirez.gu.edu.au/cyber/ultimate/index.html $>, 10.09 .2003$.

581 Ders., "Don't go overtime", in: Ders., Hg., The Ultimate Writer - Final Section, < $\underline{\text { http://live- }}$ wirez.gu.edu.au/cyber/ultimate/index.html>, 10.09.2003. 
5. Establish eye contact: Wichtig ist der Augenkontakt mit dem Publikum. Der Vortragende sollte sich die Zeit nehmen, seinen Blick einige Sekunden lang über das Publikum schweifen zu lassen. ${ }^{582}$

6. How to read: Die Wirkung der Rezitation ist stark abhängig von gemäßigtem Sprechtempo und deutlicher Artikulation des Vortragenden. ${ }^{583}$

7. Control your breathing: Eine gezielte und effektivere Atmung kann in entscheidender Weise zu einer Kräftigung der Stimme beitragen. ${ }^{584}$

Nach Abschluß der sieben theoretischen Lektionen verflüchtigt sich die Menüleiste am unteren Rand des Bildschirms, und auf der Oberfläche erscheint zum letzten Mal eine Botschaft von Zervos, der sich mit folgenden lobenden und auffordernden Worten verabschiedet:

Now you have done it for yourself. You have completed all the exercises and you are now the perfect poet. By doing these exercises you have developed an appreciation of basics of poetry. Take your new knowledge, study other's [sic!] works, old and knew, and develop your own style. ${ }^{585}$

\subsubsection{Analyse und Auswertung}

Das Forum The Ultimate Writer verdient aufgrund seiner ausgesprochen phantasievollen, ausgefallenen Gestaltung und seines vielfältigen Lernangebots besondere Anerkennung und Empfehlung. Hier sind den Entfaltungsmöglichkeiten des Agierenden keine Grenzen gesetzt. Ständig lassen sich neue Besonderheiten oder Effekte entdecken; jeder Vorgang bringt neue Erkenntnisse mit sich und jede Schreibübung offenbart dem Agierenden eine neue unbekannte Seite seiner selbst. Aufgrund des umfangreichen, mannigfaltigen Angebots können die Lektionen beliebig oft wiederholt werden. Da in erster Linie der Agierende den Ablauf der Übungseinheiten bestimmt, ist jede Gleichförmigkeit aufgrund unaufhörlich wiederkehrender Sequenzen ausgeschlossen.

582 Ders., "Establish eye contact", in: Ders., Hg., The Ultimate Writer - Final Section, < $\underline{\text { http://live- }}$ wirez.gu.edu.au/cyber/ultimate/index.html> , 10.09.2003.

583 Ders., "How to read", in: Ders., Hg., The Ultimate Writer - Final Section, < http://livewirez.gu.edu.au/cyber/ultimate/index.html>, 10.09.2003.

584 Ders., "Control your breathing", in: Ders., Hg., The Ultimate Writer - Final Section, < http://livewirez.gu.edu.au/cyber/ultimate/index.html $>, 10.09 .2003$.

585 Ders., The Ultimate Writer, <http://live-wirez.gu.edu.au/cyber/ultimate/index.html>, 10.09.2003. 
Die Konzeption von The Ultimate Writer läßt eine nahezu unerschöpfliche Phantasie des Herausgebers vermuten, der die Seiten ganz offensichtlich mit großer Liebe zum Detail gestaltet hat und Spaß an Effekten und Überraschungen jeglicher Art zu haben scheint. Jede Rubrik folgt einem unterschiedlichen Konzept und führt den Agierenden in neue Sphären. Dort erwarten ihn vollendet arrangierte und aufeinander abgestimmte Erfahrungswelten. Auch im Bereich des Hauptmenüs stellt sich das Konzept als durchdacht und perfekt aufeinander abgestimmt dar, was beispielsweise die Zusammenhänge zwischen den einzelnen auszuwählenden Körperteilen sowie der dazugehörigen Hintergrundmusik beweisen. So entspricht die eingespielte Musik jeweils der Wesensart der Personentypen, die die dargestellten Körperteile vermutlich repräsentieren sollen. Die Variationen sind zwar nur geringfügig, dennoch aber deutlich erkennbar. Folgende Charaktere lassen sich ausmachen: ${ }^{586}$

- Intellektuell und lässig: Merkmale sind u.a. die Zigarette im Mundwinkel, Lesebrille, rotgefärbte Haare.

- Feminin exzentrisch: Kennzeichnend sind gepiercter Mund, gepiercte Augenbrauen, kurze struppige Haare und figurbetonte Kleidung in gedeckten Farben.

- Feminin elegant: Als ausgesprochen weibliche Elemente kommen zur Wirkung sinnlicher Mund, geschminkte, scheu blickende Augen, kinnlange, glatte, schwarze Haare.

- Erfahren, bodenständig: Als repräsentierende Elemente werden verwendet üppiger Oberlippenbart, Augen, in denen sich Lebenserfahrung und Abgeklärtheit spiegeln, Halbglatze.

Es ist Zervos' Absicht, eine möglichst große und heterogene Personengruppe anzusprechen und sie davon zu überzeugen, daß Lyrik für alle Menschen eine Bereicherung darstellen kann. Ein Ausschnitt aus den Schilderungen von Schmidt und Schmidt bestätigt das Bestreben von Zervos:

He has performed his work in schools, writers' trains, pubs, libraries, street corners, prisons, clubs and factories, his work speaks of the lives of the people in these mundane places, of life in the inner suburbs. ${ }^{587}$

586 Die Körperteile können zwar möglicherweise zu vier markanten Personentypen zusammengestellt werden, sind aber selbstverständlich auch 'typenübergreifend' zu kombinieren.

587 Mareya Schmidt und Peter Schmidt, "Komninos Constantine Zervos", in: Dies., The Ozlit Site ([ $\left.\left.{ }^{1} 26.01 .1996\right]\right),<$ http://blackdog.vicnet.net.au/ozlit/writers.cfm?id=914>, 11.09.2003. 
Mit dieser Einstellung läßt sich vermutlich auch der Rückgriff auf die Körperteile als Mittel der Darstellung erklären. Die Idee, die dem Forum zugrunde liegt, ist die einer spielerischen Heranführung an Lyrik, gleichzeitig aber auch einer intensiven Auseinandersetzung der Agierenden mit dieser Literaturform. Es ist das Ziel, den Agierenden im Verlaufe von sieben Übungseinheiten zu einem vollendeten Dichter zu formen. Als solcher bedarf er jedoch möglichst vielfältiger Erfahrungen, die Zervos in seinem Forum zu vermitteln sucht. Ein perfekter Dichter muß sich mit allen Bereichen menschlichen Daseins auseinandergesetzt haben, was bei The Ulimate Writer durch die Körperteile symbolisiert wird, die zusammengenommen einen vollständigen Menschen ausmachen. Gleichzeitig wird anhand des Ultimate Writer verdeutlicht, daß sich diese Literaturform nicht mit halbherzigem Einsatz abhandeln läßt, sondern vielmehr der uneingeschränkten Konzentration bedarf, da Lyrik nicht nur e r lebt, sondern g e lebt wird.

Die Erscheinungsvielfalt und Themenbreite der Dichtkunst werden durch die unterschiedlichen Typen des Ultimate Writer verkörpert, die der Agierende immer wieder neu zusammenstellen kann. So zahlreich die Kombinationsmöglichkeiten, so facettenreich präsentiert sich auch die Lyrik. Hinter den verschiedenartigen Personentypen, die der Agierende kreieren kann, verbirgt sich jedoch noch eine weitere Botschaft: Die Variationsvielfalt veranschaulicht, in wieviele unterschiedliche Rollen ein Dichter zu schlüpfen vermag. Je nach Stimmungslage kann er seinen Gedichten mannigfaltigen Ausdruck verleihen: Er kann dem Rezipienten gebildet, exzentrisch, romantisch oder abgeklärt gegenüberstehen - Lyrik bildet eine Literaturform, in der sich Dichter und Rezipienten unabhängig von Alter, Geschlecht oder sozialer Schicht gleichermaßen wiederfinden.

The Ultimate Writer ist unbestreitbar ein Gedichtforum, das sich in dieser Präsentationsform definitiv nicht im traditionellem Print-Medium veröffentlichen läßt. Damit erfüllt es als erstes der vorgestellten Lyrikprojekte und -foren die in Kapitel III. vorliegender Arbeit aufgestellten Bedingungen für eine Web-Veröffentlichung. Mit der Publikation von The Ultimate Writer ist Zervos aber auch seinem persönlichen Anspruch gerecht geworden, mehrdimensionale, multimediale und in gedruckter Form nicht zu reproduzierende Lyrik zu schaffen: 
my goal was to create a poetry that was computer generated, that was moving, that was two and three dimensional and that incorporated voice and sound. it was a poetry that could not be published as a traditional print publication. ${ }^{588}$

Tatsächlich sind alle genannten Elemente vorhanden - Bewegung, Mehrdimensionalität und Ton. Sie tragen dazu bei, daß der Besuch des Gedichtforums zu einem einzigartigen Erlebnis wird, das den Agierenden nicht nur unterhält, sondern gleichzeitig auch instruiert und ihn auf spielerische Weise in die Welt der Lyrik einweist.

Das Konzept fordert von dem Agierenden kontinuierliche Mitarbeit und anhaltende Kreativität, doch bietet es ihm tatsächlich nur sehr eingeschränkte Möglichkeiten zur Interaktion. Lediglich die Einstiege in die einzelnen Übungsbereiche eröffnen dem Agierenden regelmäßig Gelegenheit zur Interaktivität, indem sie ihm die Auswahl zwischen den jeweiligen Körperteilen überlassen. Ein webbasiertes Forum könnte jedoch noch weitaus zahlreichere Interaktionsmöglichkeiten bereitstellen.

Das Angebot externer Verknüpfungen beschränkt sich auf sechs Links des Bereichs Beat Poetry, von denen allein zwei Verknüpfungen kein Ergebnis lieferten, was mit dem Charakter einer webbasierten Veröffentlichung nur schwer vereinbar ist. Zudem ist es nicht möglich, von den jeweiligen Verknüpfungspunkten direkt wieder in den Bereich Beat Poetry zurückzukehren. Der Agierende ist gezwungen, diese Rubrik ganz neu zu starten. Eine entsprechende Rückkehrfunktion ist nicht integriert. In anderen Bereichen ist dieses Bedienungselement zwar vorhanden, aber nicht funktionsfähig.

Auch andere Routinen laufen nicht programmgemäß. Hat der Agierende alle Bereiche abgearbeitet, erscheint das Bild des vollendeten Dichters nicht - wie angekündigt - automatisch auf dem Bildschirm. Erst nach Aufruf der Hilfefunktion tritt dieser Automatismus in Kraft und gewährt dem Agierenden Zugang zu den abschließenden Lektionen.

Aufgrund seiner enormen Komplexität erweist sich die Beschäftigung mit dem Forum als recht zeitintensiv. Daher ist es unvorteilhaft für den Agierenden, daß Zervos keine Möglichkeit vorgesehen hat, die Ergebnisse der einzelnen Übungen auf dem Server des Forums zu speichern. Die Konzeption sieht aber vor, daß der Agierende die Abschlußsequenz erst nach Absolvierung aller Lektionen erreicht. Diese

588 Zervos, "Biography - Komninos Zervos", <http://www.ce.canberra.edu.au/inflect/01/kombio.html $>$, 11.09.2003. 
Bedingung macht daher nach jedem vorzeitigen Abbruch eine Wiederholung der bereits bearbeiteten Übungen notwendig.

Da Zervos alle Bereiche einer einzigen Web-Adresse untergeordnet hat, ist ein korrektes Zitieren dieser Seiten unmöglich. Für jede Rubrik öffnet sich ein separates sogenanntes pop-up-Fenster von ungefähr halber Bildschirmgröße, das keinen eigenen URL trägt und auch keine Menüleisten beinhaltet, sondern lediglich minimiert oder geschlossen werden kann.

\subsubsection{Verbesserungsansätze}

Folgende Möglichkeiten könnten in die Praxis umgesetzt werden, um die Charakteristika von Hypertext und World Wide Web intensiver zu nutzen:

1. Interaktionsmöglichkeiten: Im Sinne einer anschaulicheren Illustrierung der Übungslektionen wäre beispielsweise eine Erweiterung des Gedichtforums um möglichst viele Interaktionselemente - so u.a. Gedichtbeispiele - denkbar. Zervos stellt zwar bereits Anschauungsmaterial zur Verfügung, doch bleibt dieses vergleichsweise ausdruckslos und theoretisch; auch ist es nicht sehr umfangreich.

Auch die Bereitstellung detaillierterer Informationen $\mathrm{zu}$ den diversen Gedichtformen böte sich an. Diese könnten entweder jeder einzelnen Rubrik angeschlossen werden oder aber auch über ein gesondertes Lexikon zugänglich sein. In Ergänzung zu einem umfangreichen Nachschlagewerk könnte zudem ein Glossar als knappe und übersichtliche Informationshilfe an zentraler Stelle des Forums plaziert werden.

Ferner könnte auch die Einbindung von Literaturhinweisen für eine weitere Beschäftigung mit der Thematik nützlich sein. Dabei wäre Primär- und Sekundärliteratur gleichermaßen interessant, um sowohl die theoretische Beschäftigung im Sinne des abschließenden Ratschlags von Zervos - "Take your new knowledge, study other's [sic!] works, old and knew, and develop your own style" 589 - fortzuführen als auch anhand von Übungsmaterialien weitere praktische Studien zu betreiben. Interessant wären darüber hinaus auch Interpretationshilfen.

Zur Förderung des gegenseitigen Austausches wäre ferner ein Diskussionsforum sinnvoll. Im Bereich Beat Poetry liegt bereits eine Verknüpfung zu einem Gästebuch vor, die allerdings nicht aktiviert ist. Offensichtlich ist ein Medium des gedanklichen Austausches in der Konzeption des Forums vorgesehen.

589 Ders., The Ultimate Writer, <http://live-wirez.gu.edu.au/cyber/ultimate/index.html>, 11.09.2003. 
Gerade angesichts der kreativen Herausforderung, mit der der Agierende konfrontiert wird, dürfte eine berechtigtes Interesse an der Möglichkeit eines Erfahrungsaustausches bestehen.

Schließlich wäre es für den Agierenden durchaus interessant, genauere Informationen über den Herausgeber des Forums zu erhalten. Diese Angaben könnten entweder dem Menüpunkt team zugeordnet oder aber in eine neu zu schaffende Verknüpfung mit dem Titel about oder biography eingebunden werden.

2. Externe Verknüpfungen: Um den Web-Charakter des Forums zusätzlich zu intensivieren, ließe sich eine größere Anzahl externer Verknüpfungen in seine Seiten einbinden. Hier böten sich diverse Anlaufpunkte im WWW an:

- Web-Seiten zu Hintergrundinformationen über die diversen Gedichtformen, wie sie aus dem Bereich Beat Poetry bereits eingeschränkt zugänglich sind

- Web-Seiten zu anderen Gedichtforen, wie beispielsweise About Poetry ${ }^{590}$ oder das beschriebene Electronic Poetry Center ${ }^{591}$

- Web-Seiten zu Lyrik, gegliedert nach Jahrhunderten, nach Epochen und nach Autoren

- Web-Seiten zu ausgewählten Lyrikern, die Zervos geprägt haben oder deren Förderung ihm ein Anliegen ist

- Webbasierte Verlage oder Lyrikforen zur Selbstveröffentlichung, wie zum Beispiel PoetryPoem.com ${ }^{592}$ dort können Nutzer kostenlos und ohne technische Hindernisse ihre Gedichte im Web veröffentlichen ${ }^{593}$

- Lyrikverlage (Online und Print) im World Wide Web, zum Beispiel Enitharmon Press Online ${ }^{594}$, einer der führenden Literaturverlage Großbritanniens, der auch im WWW vertreten ist, oder Affected Publishing Ltd., ${ }^{595}$ ein kleines Verlags-

590 About, Inc., Hg., About Poetry, < http://poetry.about.com/>, 12.09.2003.

591 Glazier u.a., Hg., Electronic Poetry Center, $<$ http://epc.buffalo.edu/>, 12.09.2003.

592 PoetryPoem.com, Hg., PoetryPoem.com, <http://poetrypoem.com/>, 12.09.2003.

593 Auf den Seiten von PoetryPoem.com wird dem Nutzer eine komplikationsfreie Publikation der eigenen Gedichte garantiert, die keine computertechnischen Kenntnisse voraussetzt: "Poetry Poem is for poets who want to publish their poetry on the Internet for free. We offer free poetry sites to poets that do not want to bother with all the computer work that goes into making a web site. Everything is done. Poets need only add the poetry." PoetryPoem.com, Hg., PoetryPoem.com More Info, < http://poetrypoem.com/info.htm>, 12.09.2003.

594 Enitharmon Press, Enitharmon Press Online - Contemporary Poetry Publisher (21.07.2003), $<$ http://www.enitharmon.co.uk/>, 12.09.2003.

595 Affected Publishing Ltd., Affected Publishing Ltd. (27.03.2003), < $\underline{\text { http://www.affectedpublishing.co.uk/>, }}$ 12.09.2003. 
haus, das im September 2002 von Autoren gegründet wurde und sich einem hohen literarischen Anspruch verpflichtet hat ${ }^{596}$

- Web-Seiten mit sogenannter Audio Poetry, das heißt Rezitationen von Gedichten, größtenteils von den Verfassern selbst vorgetragen

- Web-Seiten mit sogenannter Video Poetry, sprich Werken der Video Performance

- Zeitschriften oder Magazine zum Thema Lyrik

- Rezensionen

- Veranstaltungskalender mit Informationen über Lesungen, Kongresse, Festivals, Workshops, Wettbewerbe, Auszeichnungen etc.

Die obige Liste erhebt keinen Anspruch auf Vollständigkeit und orientiert sich ausschließlich an den Ende 2003 realisierbaren Möglichkeiten. Sie soll lediglich aufzeigen, welche Vorteile sich für den Agierenden aus einer Überarbeitung ergeben könnten.

3. Bibliographische Angaben: Der Rezipient erhält keinerlei Daten hinsichtlich der Erstellung der Forumseiten. Es fehlen detaillierte Angaben zu Herausgeber, Datum der Erstveröffentlichung und letzter Überarbeitung der Seiten. Schließlich fehlen eigene Web-Adressen für die Unterbereiche, mit deren Hilfe ein korrektes Zitieren möglich wäre.

4. Speicherungsfunktionen: Dem Agierenden käme es sehr entgegen, wenn das Konzept des Forums eine Speicherungsfunktion für die Ergebnisse der Übungseinheiten vorsähe. Zu diesem Zweck böte es sich an, dem Agierenden die Möglichkeit der Einrichtung eines individuellen Nutzerkontos zu geben. Gleichzeitig müßte sichergestellt sein, daß der Agierende seine Ergebnisse dort nicht nur zumindest für einen vorübergehenden Zeitraum - archivieren, sondern daß er mit Hilfe der Zwischenergebnisse nahtlos an seinen letzten Besuch des Forums anschließen und seinen Weg durch die restlichen Übungseinheiten fortsetzen kann.

5. Funktionsgarantie: Das Gedichtforum von Zervos erweist sich insofern als problematisch, als - im Unterschied zu einem Print-Medium - die Kontinuität des Dargebotenen nicht gewährleistet ist.

596 "Our aim is to become a publisher who is reputed for producing works of a high, literary standard, which display originality, accessibility and a sense of humour." Affected Publishing Ltd.,

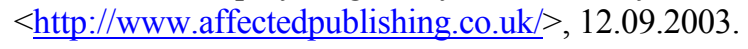


Zervos hat sich auf ganz besonders phantasievolle Weise der Mittel von Hypertext und World Wide Web bedient und damit ein außergewöhnliches, unverwechselbares und in seiner endgültigen Form und Dynamik nur im Web realisierbares Werk geschaffen. The Ultimate Writer erlaubt Interaktivität und Produktivität und gestattet einen - wenngleich eingeschränkten - Zugriff auf das Web. Auch wenn es den Agierenden bei seinen Schreibübungen jeweils sich selbst überläßt, also keine Kooperativität und damit auch keinen Austausch mit anderen Web-Besuchern vorsieht, darf es aufgrund seiner untrennbaren Verwobenheit mit dem Medium World Wide Web doch als webbasierte Publikation bezeichnet werden. Hervorzuheben ist an dieser Stelle der didaktische Anspruch von The Ultimate Writer, den Zervos auf stets originelle Art umzusetzen versteht. Angesichts der Extravaganzen, die zu Unterhaltung, geistiger Anregung und Instruktion eingesetzt werden, wird der Agierende immer wieder in ungläubiges Erstaunen versetzt, so daß die Beschäftigung mit dem Forum ihren kurzweiligen Charakter auch nach mehrmaligen Durchgängen nicht einbüßt. Ohne diese Extravaganzen hätte er aber möglicherweise eine deutlich größere Zielgruppe ansprechen können, denn es ist anzunehmen, daß The Ultimate Writer einem Web-Besucher auf den ersten Blick - völlig zu Unrecht - als Literaturforum für ein jugendliches Publikum erscheint.

\subsection{Webbasierte Lyrik - Resümee}

Die betrachteten Beispiele haben gezeigt, daß sich Lyrik im World Wide Web auf völlig neue, bereichernde Weise entfalten kann. In Verbindung mit audiovisuellen Effekten oder Videosequenzen ergeben sich bislang unbekannte Darstellungsformen, die ihrerseits neuartige Sichtweisen produzieren. In Einzelfällen werden Gedichte auf so effektive Art und Weise mit dem Darstellungsmedium World Wide Web verwoben, daß keinerlei Zweifel an dem Wert webbasierter Lyrik aufkommen kann. Lyrikseiten - vor allem, wenn sie auch Möglichkeiten zur Kooperation bieten regen zum Experimentieren an, fördern die Kreativität und erfüllen zum Teil, wie im Beispiel von The Ultimate Writer, auch einen didaktischen Anspruch. Im Gegensatz zu computergenerierter Lyrik stellt die webbasierte Form damit in der Regel mehr als einen amüsanten Zeitvertreib dar; sie fordert zu ernsthafter Beschäftigung mit Lyrik heraus, ohne dabei ihren kurzweiligen Charakter einzubüßen. 
Dennoch lassen viele Einzelgedichte, aber auch komplette Gedichtforen wie beispielsweise die Interactive Poetry Pages wirkliche Innovation vermissen. Diese Feststellung verkennt nicht, daß bereits die Möglichkeit zu unmittelbarer Kooperation den Gedichten einen ganz entscheidend neuen Charakter verleiht und diese Literaturform damit eine grundlegend andersartige Wirkung ausstrahlt als linearkonzipierte oder computergestützte Lyrik. Gerade in jenen Fällen, in denen kooperative Beiträge in Versstruktur aufeinander folgen, kommt der Einfluß verschiedener Autoren in besonderem Maße zum Tragen. Unterschiedlichste Stimmungen fließen in kooperativer Lyrik in nur wenigen Zeilen zusammen. Perspektivwechsel erscheinen in sehr kurzer Abfolge und können einem Gedicht eine Färbung verleihen, die nur diese Form der Literaturproduktion hervorbringen kann. Aufgrund der Heterogenität derartiger Gedichte qualifiziert sich kooperative Lyrik somit als eine Art Mikrokosmos der Eindrücke, Hoffnungen und Gefühle.

Trotz dieser Eigenschaften muß allerdings festgestellt werden, daß sich der Unterschied zu gedruckter Poesie gegenwärtig noch vielfach in Interaktivität und/ oder Kooperativität erschöpft. Sicherlich mag der künstlerische Wert eines Gedichtes im Web gelegentlich in der bewußten Absage an alle Präsentationsmöglichkeiten von Hypertext und WWW liegen. Die Aussage Blocks ("Digitale Poesie erzielt ästhetischen Gewinn, insofern sie nicht nur i n, sondern vor allem $\mathrm{m}$ i t ihren ganz spezifischen Medien arbeitet - oder auch g e g e n sie." ${ }^{, 597}$ ) scheint diese Formen konventioneller, linear-konzipierter Literatur im Web in gewisser Weise zu rechtfertigen. Richtig verstanden geht es hier jedoch um den Mangel an "ästhetischem Gewinn”, gegen den sich Block wendet, um den Ruf nach Qualität, die die als innovativ dargebotene Poesie oftmals nicht erzielt. Daher kann die Vorgabe für alle webbasierten Publikationen nur lauten, die darstellerischen Mittel so weit wie irgend möglich auszuschöpfen. Zu Recht warnt Block aber davor, einer Technikfaszination zu erliegen, die den schöpferischen Anteil an einem Gedicht soweit zurückdrängt, daß es zu einem reinen Technologieexperiment gerät. ${ }^{598}$ Eine solche eingangs des Kapitels VI.2. angedeutete extreme Anpassung an das Präsentationsmedium hätte zur Folge, daß die künstlerische Botschaft schließlich nur noch in dem Medium selbst läge. ${ }^{599}$

\footnotetext{
597 Friedrich W. Block, “Acht poetologische Thesen zur digitalen Poesie”, in: Johannes Auer, Christiane Heibach und Beat Suter, Hg., netzliteratur.net: Netzliteratur - Internetliteratur - Netzkunst ([ ${ }^{1}$ März 2001]), <http://www.netzliteratur.net/block/acht thesen.html >, 12.09.2003.

598 Ebd., 12.09.2003.

599 Vgl. Zimmer, "Die digitale Bibliothek", 289.
} 
Auf keinen Fall wird das WWW im Bereich Lyrik in ernsthafte Konkurrenz $\mathrm{zu}$ den Print-Medien treten wollen und können. Vielmehr wird sich allenfalls eine ganz andersgeartete Form der Dichtkunst etablieren, die nicht verdrängt, sondern ergänzt; möglicherweise wird sich webbasierte Lyrik auch als eine ganz eigenständige Kunstform durchsetzen. Eine derartige Entwicklung verlangt jedoch eine wesentlich höhere Experimentierfreudigkeit von allen Beteiligten als die bisher festgestellte. So interessant und spannend ein gemeinschaftlich erstelltes Gedicht für den Leser auch sein mag - da die kurze Abfolge der Beiträge den Kooperierenden nur vergleichsweise wenig Gelegenheit gibt, ihre Vorstellungen voll umzusetzen, fordert diese Literaturform den Autoren ein deutlich höheres Maß an Integrationsfähigkeit $\mathrm{ab}$, als es bei Prosa oder Drama der Fall ist.

Lyrikschaffenden fehlt es derzeit noch an ausreichend Innovationsgeist, Verve und Phantasie, um die Möglichkeiten des WWW auszunutzen.

Das Gleiche gilt auch für die Herausgeber: Da Lyrik tendenziell mehr noch als Prosa und Drama in Foren erstellt wird, sollte gerade die Produktivität innovativer Poesie durch aufgeschlossene Editoren gefördert werden. Eines darf hierüber jedoch nicht aus dem Blick geraten: Die Realisierung des theoretisch Möglichen verlangt neben Phantasie und Weitblick vor allem handfeste Programmierkenntnisse.

Schließlich bedarf es auch des Interesses der Rezipienten, um webbasierte Lyrik weiterzuentwickeln.

In jedem Falle ist es wichtig und ratsam, den Entwicklungsprozeß webbasierter Lyrik weiter zu verfolgen. Für Anglistik und Amerikanistik wird diese Literaturform allerdings erst dann zu einer ernstzunehmenden Gattung, wenn der Etablierungsprozeß abgeschlossen ist und sich größere Foren gebildet haben, die für die Qualität ihrer Werke bürgen. Gegenwärtig begegnen dem Leser noch oft ein beträchtlicher Dilettantismus sowie eine bedauerliche Häufung von Anzüglichkeiten. Unabhängig davon, daß es sich dabei vermutlich um ein dem WWW inhärentes Problem handelt, scheint gerade diese Literaturform verstärkt Ziel von Obszönitäten zu sein. Es ist anzunehmen, daß sich ihre Verfasser vor allem durch die Kürze der Publikationen dazu verleiten lassen, die Web-Gemeinschaft durch offenbar mehr oder weniger wahllos eingestreute Unanständigkeiten $\mathrm{zu}$ irritieren und gegeneinander aufzubringen. Im Rahmen der Recherchen für die vorliegende Arbeit ließ sich gerade auf den Seiten der Interactive Poetry Pages beobachten, wie einer 
Vielzahl von Gedichten durch einzelne eingeschobene plumpe Zeilen Ernsthaftigkeit und künstlerischer Tiefgang genommen wurde.

Das Ärgernis obszöner Beiträge ließe sich vermutlich nur begrenzen, wenn sich die Herausgeber für strikte Kontrollmaßnahmen entschieden. Dies könnte zum einen schon vor Beginn der Mitarbeit durch eine Registrierungspflicht für die Kooperierenden umgesetzt werden, was aber möglicherweise der Spontaneität und dem freigeistigen Charakter gerade dieser Literaturform abträglich wäre; es könnte zum anderen auch vor Erscheinen des kooperativen Beitrags durch sogenanntes peer review beziehungsweise erst nach Veröffentlichung durch zensorische Eingriffe oder auch vollständiges Entfernen der störenden Beiträge erfolgen. Offen bleibt, ob nicht gerade Maßnahmen der letzteren Art den Widerspruchsgeist der Verursacher wecken und dadurch eher kontraproduktiv wirken.

Für welche Form der Kontrolle sich die Herausgeber auch entscheiden, restriktive Maßnahmen bedeuten in jedem Falle eine bedauerliche Einschränkung für alle ambitionierten Kooperierenden, die bereit sind, Zeit und Kreativität in die Vielfalt webbasierter Lyrik zu investieren. 


\section{Webbasiertes Drama}

Im Vergleich zu den Literaturgattungen webbasierte Prosa und webbasierte Lyrik kommt das webbasierte Drama der hypertextuellen Struktur des World Wide Web wesentlich näher. Sein szenischer wie dialogischer Aufbau sind geradezu prädestiniert für eine Umsetzung im Web. Im Gegensatz zu Hypertext-Dramen in geschlossenen Hypertext-Systemen, wie sie in Kapitel IV.2.3. vorliegender Arbeit erläutert wurden, präsentiert sich das webbasierte Drama nicht nur als ein sogenanntes "create-your-own-play" ${ }^{\circ 00}$-Ereignis mit einem Netzwerk unterschiedlicher Leseoptionen, das dem Nutzer Interaktion erlaubt, sondern es gestattet ihm darüber hinaus auch Kooperation, die sich in aktiver Mitgestaltung des Geschehensablaufs durch strukturverändernde Eingriffe in das Hypertext-System auswirkt. Deemer sieht das Hypertext-Drama in so enger Verwandtschaft mit dem traditionellen Drama, daß er dieses als eine Untergruppe des Hypertext-Dramas einstuft. ${ }^{601}$ Vom Hypertext-Drama zum webbasierten Drama ist es für ihn daher nur ein kleiner, zwangsläufiger Schritt; er spricht in diesem Zusammenhang von einer "natural marriage of hyperdrama and the World Wide Web" und begründet diese mit ihrer "mutual foundation in hypertext". 602

Im webbasierten Drama brechen traditionelle lineare Konzeption und Darstellung in verschiedene Zweige auf, die simultan auf der virtuellen Bühne des Geschehens ablaufen. Die diversen Handlungsstränge unterliegen keiner unterschiedlichen Wertung mehr, die eine Aufteilung in Haupt- und Nebenhandlung beinhaltet; alle Darstellungen sind nunmehr gleichwertig. Dieser Simultaneität ist es zuzuschreiben, daß Skripte sowohl für Hypertext-Dramen als auch für webbasierte Dramen bei gleicher Ablaufdauer im allgemeinen etwa drei bis fünf Mal umfangreicher sind als traditionelle Dramen-Skripte. ${ }^{603}$

${ }^{600}$ Charles Deemer, "How do I number these pages", in: Center for the Public Domain/University of North Carolina - Chapel Hill, Hg., ibiblio - the public's library and digital archive, <http://www.ibiblio.org/cdeemer/howdoi.htm>, 20.10.2003.

601 Ders., "The New Hyperdrama: How hypertext scripts are changing the parameters of dramatic storytelling", in: Center for the Public Domain/University of North Carolina - Chapel Hill, Hg., ibiblio - the public's library and digital archive, <http://www.ibiblio.org/cdeemer/newhype.htm>, 20.10.2003.

602 Ders., "Hyperdrama and Virtual Development: Notes on Creating New Hyperdrama in Cyberspace", in: Center for the Public Domain/University of North Carolina - Chapel Hill, Hg., ibiblio - the public's library and digital archive, $<$ http://www.ibiblio.org/cdeemer/virtdev.htm>, 20.10.2003.

${ }^{603}$ Ebd., 20.10.2003. 
Bei jedem einzelnen Rezeptionsvorgang erschließt sich ein neuer Ausschnitt aus dem großen Gesamtwerk, das sich dem Leser als solches erst nach Verfolgung aller theoretisch möglichen Lesewege offenbart. Aufgrund der diversen Vorgehensmöglichkeiten erlebt jeder Leser sein ganz eigenes, in seinem Ablauf vermutlich einmaliges Stück, das er überdies auch aktiv mitgestalten kann. Mit dieser Entscheidungsfreiheit und diesem Mitbestimmungsrecht erfährt der Rezipient einen signifikanten Kompetenzgewinn, der ihn vom ursprünglich rein passiven Betrachter über einen interagierenden Nutzer zu einem kooperativ Mitwirkenden werden läßt.

Was hier - wie selbstverständlich - positiv gewertet wird, mag allerdings mancher auch als dem Endprodukt abträglich empfinden. Einem Rezipienten des von Deemer konzipierten Hyperdramas The Bride of Edgefield erschien die Masse der zahlreichen parallel verlaufenden Handlungsstränge als zu realistisch und daher erdrückend: “' It was too real,' he complained, 'with too much happening at the same time. " ${ }^{604}$ Üblicherweise sollte ein Leser diesen Facettenreichtum als Bereicherung ansehen. Da er anders als bei einem Theaterbesuch sowohl die Geschwindigkeit als auch die Dauer der Beschäftigung mit dem Werk selbst bestimmen kann, dürften ihn die dargestellten Ereignisse eigentlich auch nicht überfordern. Die vorstehende Aussage des Rezipienten von The Bride of Edgefield zeigt jedoch, daß es verbindliche Maßstäbe für die Beurteilung dessen, was das WWW im Bereich Drama leisten kann, nicht gibt.

Wie sich die Angebotsvielfalt im webbasierten Drama der Gegenwart tatsächlich darstellt, soll nachfolgend anhand zweier Beispiele überprüft werden.

Als Anschauungsmaterial wurden zwei Projekte ausgewählt: The Company Therapist, ein als sogenanntes Edutainment konzipiertes, privat veröffentlichtes webbasiertes Drama, und $M$ is for Nottingham?, eine Schreibübung der britischen Nottingham Trent University. Bei beiden Publikationen handelt es sich um Projekte, die mittlerweile abgeschlossen, jedoch nach wie vor mit allen Hintergrundinformationen und Ergebnissen im World Wide Web vertreten sind. Zwar ist es logischerweise nicht mehr möglich, ihre Entstehungsprozesse unmittelbar mitzuerleben oder die Projektverläufe gar aus der Position eines Kooperierenden heraus $\mathrm{zu}$ beeinflussen, dafür bieten abgeschlossene Vorhaben die Möglichkeit zu einer jedem Zeitdruck enthobenen, gründlichen Analyse. Von der Planung bis hin zur

${ }^{604}$ Ders., "How do I number these pages", <http://www.ibiblio.org/cdeemer/howdoi.htm>, 20.10 .2003 . 
Auswertung lassen sich alle Maßnahmen sowie die sich daraus ergebenden Konsequenzen Schritt für Schritt und unter Beachtung ihrer Wechselwirkungen nachvollziehen. Nur diese uneingeschränkte Transparenz gestattet eine umfassende und damit sachgerechte Bewertung.

Abgesehen davon, daß keine kooperativen Beiträge mehr eingestellt werden können, unterscheiden sich die passiven nicht von den aktiven Versionen der Projekte, so daß beide Publikationen uneingeschränkten dokumentarischen Wert besitzen. Was sie von anderen webbasierten Dramen abhebt, sind die ausgefallene Thematik und die vorbildliche nahezu vollständige Umsetzung der in Kapitel III. genannten Kriterien für webbasierte Veröffentlichungen.

\subsection{Ausgewählte Beispiele des webbasierten Dramas}

\subsubsection{The Company Therapist}

"In addition to being an entertainment and an educational venture, The Company Therapist is an innovative literary experiment [...]" ${ }^{605}$ Viele Kooperationsprojekte sind mit einem solchen Anspruch angetreten, konnten ihm aber nicht annähernd gerecht werden; The Company Therapist Project ${ }^{606}$ hingegen dürfte eines der wenigen webbasierten Werke sein, die wirklich neue Maßstäbe gesetzt haben. Initiiert im Jahre 1996, fiel das Projekt in eine Zeit des Aufbruchs, in der der Zeitgeist eine Veröffentlichung in dem neuen Medium World Wide Web quasi zur Verpflichtung machte. Dies schlug sich in einer wahren Publikationsflut nieder, weshalb dem Projekt zum Zeitpunkt seines Erscheinens angesichts der damaligen Angebotsvielfalt möglicherweise nicht die angemessene Aufmerksamkeit zuteil wurde. Mittlerweile ist die Flut abgeebbt und die Zahl der aktiven, von den Herausgebern regelmäßig gepflegten Veröffentlichungen überschaubarer geworden. The Company Therapist hat seinen Platz im Web wahren können und steht bei Recherchen zum Thema webbasierte Dramen in der Ergebnisliste gegenwärtig an vorderster Stelle.

\footnotetext{
${ }^{605}$ Dies., Hg., The Company Therapist $\left(\left[{ }^{1} 1996\right]\right),<$ http://www.thetherapist.com>, 02.10.2003.

${ }^{606}$ Ebd., 02.10.2003.
} 


\subsubsection{1. "Production \& Direction" $" 607$}

Auf den Seiten The Company Therapist betritt der Web-Besucher die virtuelle Praxis des Psychiaters Dr. Charles Balis, dessen Klientel zum größten Teil aus den gelegentlich recht exzentrischen Angestellten einer großen Computerfirma in San Francisco besteht. Dank eines uneingeschränkten Zugangs zu sämtlichen Praxisunterlagen, wie beispielsweise Patientenakten, Mitschriften von Therapiegesprächen, Kritzeleien von Patienten, medizinischen Aufzeichnungen und Korrespondenzen gewinnt der Besucher intime Kenntnis des Praxisablaufs und der Tätigkeit von Dr. Balis sowie der Lebens- und Krankengeschichten der Mitarbeiter von Silicon Impressions Inc. und kann die Entwicklung aller Beteiligten nachvollziehen.

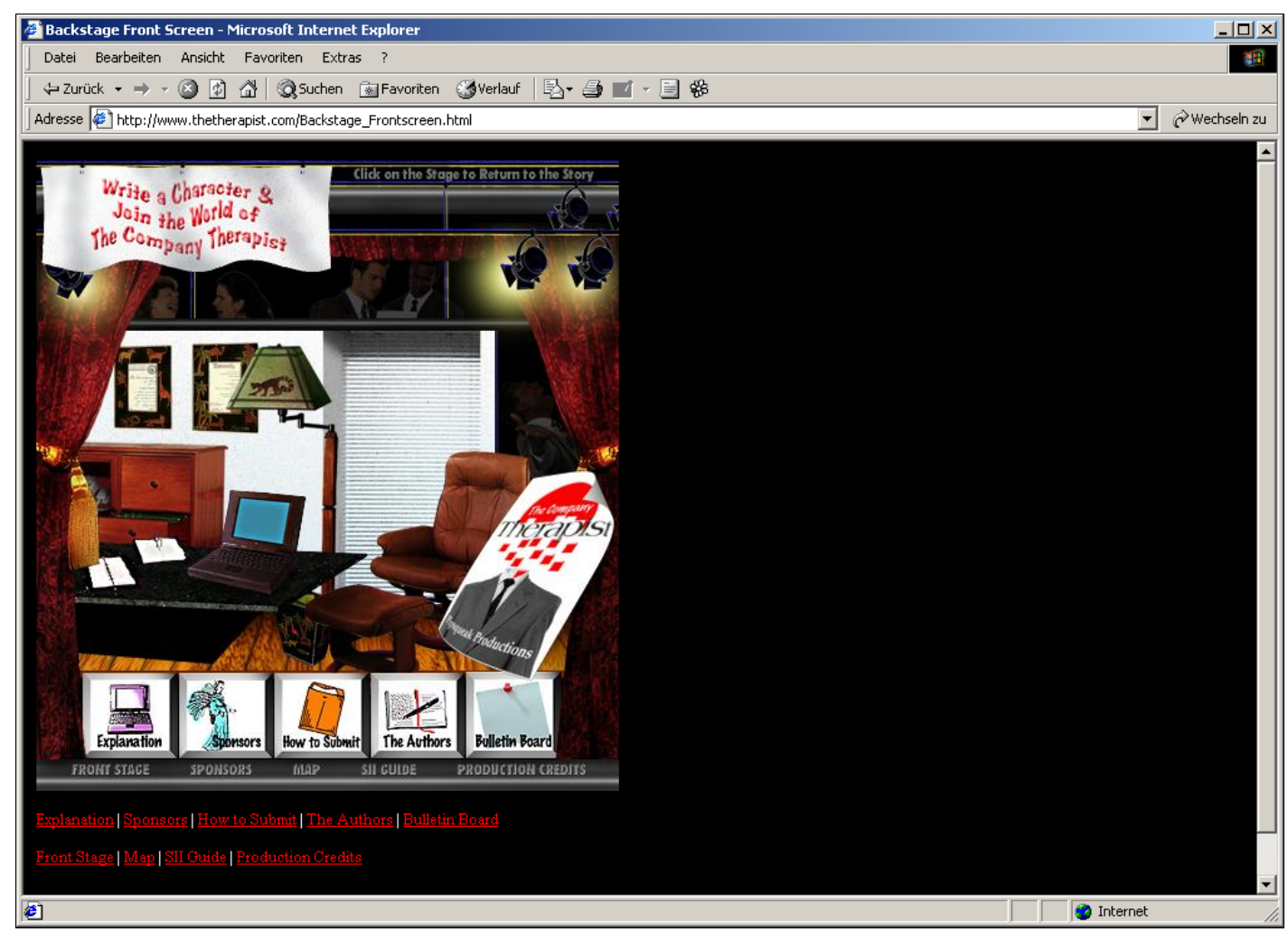

Abb. 6: 'The Company Therapist' - Sprechzimmer

Das umfangreiche Material des Company Therapist ist nicht im Rahmen eines einzigen Lesevorgangs zu bewältigen. Hat die Thematik das Interesse des Lesers geweckt, wird dieser in der Regel im Verlaufe seiner Folgebesuche sukzessive in die Thematik eingebunden. Die Vielfalt der Charaktere und Handlungs-

${ }^{607}$ In Anlehnung an die Kurzinformation über die Herausgeber des Company Therapist: "Christopher Werby, Producer \& Director; Olga Werby, Producer Pipsqueak Productions, Graphics \& Design." Pipsqueak Productions, "Credits Page", in: Dies., Hg., The Company Therapist,

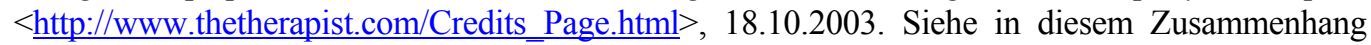
auch Fußnote 627. 
stränge vermittelt ihm die Illusion einer realen Praxis mit tatsächlich existierenden Patienten. Die Faszination dieses webbasierten Dramas beruht darauf, daß der Einblick in das Seelenleben der Patienten einen gewissen Hang zum Voyeurismus befriedigt. Der Leser erhält Kenntnis von intimen Details, die - vermeintlich - dem Psychiater Dr. Balis vorbehalten waren. Er wird dadurch zum geheimen Mitwisser, der nicht nur über die Probleme der einzelnen Angestellten von Silicon Impressions Inc. informiert ist, sondern darüber hinaus als allwissender Leser die Beziehung der Beschäftigten untereinander kennt; diese Perspektive verleiht den Geschichten zusätzliche Pikanterie.

Die Thematik des webbasierten Dramas ist von vorneherein so angelegt, daß sie Stoff und zugleich Zündstoff für unzählige kooperative Beiträge liefert. Hintergrund bilden die persönlichen Erfahrungen der Projektinitiatoren und herausgeber Christopher und Olga Werby, die diese im Verlaufe ihrer Anstellungen bei diversen Computerfirmen gesammelt haben. Die fiktive Firma Silicon Impressions Inc., kurz SII, steht stellvertretend für alle namhaften Software-Entwickler und bündelt die Schattenseiten eines hektischen Großbetriebes, dessen Belegschaft aufgrund der hohen Anforderungen des Arbeitgebers ein geregeltes (Sozial-)Leben versagt bleibt. SII erwartet, daß die Beschäftigten ihre Privatsphäre dem Erfolg der Firma opfern; um diesem Druck standhalten zu können, bedarf es der Unterstützung eines Psychiaters, verkörpert in der Person des jungen Dr. Balis. Dieser übersiedelt im Jahre 1996 - der scheinbare Realismus des Geschehens wird dadurch verstärkt, daß alle Vorgänge in der Gegenwart und in Echtzeit ablaufen - von New York nach San Francisco, um sich dort selbständig zu machen.

Das Projekt The Company Therapist lief über einen Zeitraum von insgesamt drei Jahren - von 1996 bis 1999 - und steht seit seinem Abschluß nicht mehr zur Kooperation offen. Dennoch findet der Besucher der Web-Seiten nach wie vor den gesamten Materialbestand vor, einschließlich der Richtlinien für die Eingabe neuer Beiträge. ${ }^{608}$ Intention, Konzeption und Realisation des Projekts lassen sich in allen Punkten nachvollziehen und finden durch die Ergebnisse in Form umfangreichen Textmaterials anschauliche Bestätigung. Wie der einleitende Satz belegt, erhoben

608 Dies., "Submission Guidelines", in: Dies., Hg., The Company Therapist, $<\underline{\text { http://www.thetherapist.com/Submission_Guidelines.html }>, 02.10 .2003 . ~}$ 
die Herausgeber den hohen Anspruch, Unterweisung und Unterhaltung miteinander vereinbaren und zum Vorteil eines facettenreichen und neuartigen literarischen Resultats einsetzen zu können. Unter Berücksichtigung dieses hohen Anspruchs erzielte The Company Therapist im Vergleich zu ähnlichen Initiativen eine hohe Beteiligungsquote: 28 Teilnehmer kreierten 38 Charaktere mit ebenso vielen Lebensund Krankengeschichten.

Infolge der Abgeschlossenheit des Projekts ist es zwar inzwischen nicht mehr möglich, an einem Entwicklungsprozeß teilzuhaben und diesen gegebenenfalls zu beeinflussen, dieser Umstand muß aber keineswegs als Nachteil bewertet werden. Vielmehr schafft die Unveränderbarkeit des Werkes jenen common point of reference, der es Lesern erlaubt, ein Rezeptionserlebnis miteinander zu teilen. Diese Kommunizierbarkeit ist bei webbasierten kooperativen Werken, die sich in Entstehung befinden, aufgrund der struktur- und inhaltsverändernden Mitgestaltung der Koautoren nicht gegeben. Möglicherweise liegt hier übrigens der Grund für das bisherige Ausbleiben eines sogenannten Online-Ulysses: Das Spezifikum webbasierter Literatur - nämlich ihre Veränderbarkeit - könnte der Entstehung eines Erfolgswerkes im Stile von Ulysses entgegenstehen. Daher wäre es denkbar, daß es zwar keinen O n 1 i n e- (im Sinne von webbasiert), eventuell aber irgendwann einen d i g i t a 1 e n Ulysses, das heißt ein abgeschlossenes, am Computer zu rezipierendes Werk geben wird.

Im Falle von The Company Therapist beweist die hohe Beteiligungsfrequenz, daß eine Lernumgebung niveauvoll und ansprechend zugleich sein kann. Die Beitragsinhalte wiederum zeigen, daß Engagement wie Contenance der Kooperierenden eine Frage von Projektkonzeption und -durchführung sind. Aus diesem Grunde soll The Company Therapist als Beispiel für webbasierte Dramen in vorliegende Arbeit mit aufgenommen werden. Es erlaubt sachdienliche Erkenntnisse über Möglichkeiten des Publizierens in und für das World Wide Web und fügt sich damit als weiteres außerordentliches Element in das bisher eher noch visionäre Mosaik vollkommener webbasierter Literatur ein.

Die Herausgeber verstanden The Company Therapist als erstes didaktisches kooperatives Schreibprojekt im World Wide Web und in der Tat zeichnet es sich durch anspruchsvolle Konzeption und innovative Gestaltung aus, die ihm auch heute noch - sieben Jahre nach seiner Initiierung - eine herausragende Position innerhalb webbasierter Publikationen sichern. Gemessen am gegen- 
wärtigen Standard vergleichbarer Veröffentlichungen, vor allem aber vor dem Hintergrund des Entwicklungsstandes des Jahres 1996 überzeugt The Company Therapist durch außerordentlich professionelles Design und ungewöhnlich hohe Zielsetzungen. Die ursprüngliche Einstiegsseite ${ }^{609}$ wurde inzwischen überarbeitet und gibt dem Projekt nun ein noch moderneres, progressiveres Erscheinungsbild, das der Tiefgründigkeit und Zerrissenheit der menschlichen Psyche auch optischen Ausdruck verleiht.

Die Initiatoren beabsichtigten, ein webbasiertes Drama - von ihnen mit der Bezeichnung hyperdrama ${ }^{610}$ belegt - mit sorgfältig entwickelten, facettenreichen Figuren und einer ebenso unterhaltsamen wie inspirierenden Handlung zu schaffen. Gleichzeitig planten sie, die Web-Seiten des Company Therapist als ein effektives didaktisches Hilfsmittel zur Förderung literarischen Schaffens zu etablieren. Der Unterhaltungsfaktor war dabei nach Ansicht von Werby und Werby von entscheidender Bedeutung für den Gelingen des Projekts, da eine Lernsoftware generell nur dann erfolgreich sein könne, wenn ihre Gestaltung attraktiv genug sei, um eine Reaktion bei den jeweiligen Adressaten auszulösen. ${ }^{611}$ Zielgruppe des Company Therapist waren ausschließlich erwachsene Nutzer, denen das Projekt eine Möglichkeit zur Steigerung der Schreibkompetenzen eröffnen sollte.

Die Herausgeber vermochten ihrer Intention offensichtlich zu entsprechen. Über einen Zeitraum von drei Jahren präsentierte sich The Company Therapist als eine aktive Web-Seite, auf der (angehende) Schriftsteller ihre Fähigkeiten in realistischer Umgebung umsetzen und verbessern konnten. Die imaginäre Praxis von Dr. Balis entwickelte sich zu einem Mikrokosmos mit einem komplexen Geflecht von Patientenbiographien, Ereignissen und Handlungssträngen. Die Vielschichtigkeit und Verzweigung des Hypertext-Systems verstärkten den Eindruck einer realen Atmosphäre und boten mit einer mannigfaltigen Erzähllandschaft zahlreiche Ansatzpunkte für Ergänzungen.

609 Dies., Hg., The Company Therapist - Original Front Page, $<$ http://www.thetherapist.com/Front_Screen_Old.html $>, 02.10 .2003$.

610 Dies., "The Company Therapist in General: Two Goals", in: Dies., Hg., The Company Therapist, $<$ http://www.thetherapist.com/Explanation.html\#anchorTwoGoals $>, 02.10 .2003$.

611 Dies., "Educational Philosophy", in: Dies., Hg., Pipsqueak Productions, $<$ http://www.pipsqueak.com/Educational_Philosophy-fs.html>, 02.10.2003. 
Einen ganz entscheidenden Vorteil, durch den die Initiatoren des Projekts vielen anderen Herausgebern im WWW überlegen waren, bildeten ihre Kenntnisse und Erfahrungen auf dem Gebiet elektronischen Publizierens. Ihr beruflicher Hintergrund, zu dem unter anderem Tätigkeiten als Software-Entwickler, Designer, Mediengestalter und Informatiklehrer zählen, eröffnete Werby und Werby einen immensen Spielraum in der optischen, vor allem aber technischen Gestaltung von Web-Seiten. Gleichwohl zielte ihr hauptsächliches Bestreben nicht auf die Perfektionierung von Benutzeroberflächen oder die Ausarbeitung immer ausgefallenerer Animationstechniken, sondern betraf in erster Linie die Anpassung von Lernprogrammen an die Bedürfnisse und das Leistungspotential ihrer Nutzer.

Aufgrund seiner besonderen Thematik war The Company Therapist geradezu dafür prädestiniert, sich aus gemeinschaftlicher Zusammenarbeit heraus zu entwickeln. Viele Kooperationsprojekte sind darauf ausgerichtet, die Beiträge aller Beteiligten inhaltlich wie stilistisch so aufeinander abzustimmen, daß letztlich ein möglichst homogenes Werk entsteht, das dem Leser suggeriert, es sei von einem einzigen Autor verfaßt. Allerdings verfehlen die meisten Projekte ihre Zielsetzung, da das literarische Ergebnis nur in seltenen Fällen überzeugt. In der Regel lassen sich die unterschiedlichen Einflüsse und Schreibstile der Kooperierenden eben nicht $\mathrm{zu}$ einem übereinstimmenden Ganzen zusammenfügen, sondern ergeben genau das, was die Herausgeber des Company Therapist als "Frankenstein patchwork of different writers" bezeichnen. ${ }^{612}$ Ihrer Ansicht nach unterdrücken Kooperationsprojekte dieser Art ein ganz wesentliches Merkmal von Gemeinschaftsarbeiten. Anstatt die Eigentümlichkeiten der Einzelelemente zugunsten eines wenig überzeugenden Gesamtwerks zu opfern, sollten die Initiatoren vielmehr möglichst unterschiedliche Autoren miteinbeziehen und dem Werk auf diese Weise Facettenreichtum und Unverwechselbarkeit verleihen. Daher betrachten Werby und Werby die verschiedenen Stilarten der Autoren des Company Therapist keineswegs als Nachteil, sondern im Gegenteil als entscheidende Bereicherung. Jeder Autor kreiere und entwickele seinen eigenen, individuellen Charakter, der durch seine ganz spezifische Handlungs- und Ausdrucksweise die Einzigartigkeit des Projekts steigere: "With many characters expressed through different writers, the storylines of

612 Dies., "The Company Therapist in General: A New Form”, in: Dies., Hg., The Company Therapist, $<$ http://www.thetherapist.com/Explanation.html\#anchorNewForm>, 02.10.2003. 
The Company Therapist can be rich, complex, and intriguing. ${ }^{, 613}$ Somit war es stets das Ziel, nicht nur möglichst viele Freiwillige für eine Mitarbeit zu gewinnen, sondern eine möglichst heterogene Gemeinschaft verschiedenartiger Kooperierender aus vielerlei Altersstufen, mit konträren Lebenserfahrungen und unterschiedlichem ethnischem Hintergrund aufzubauen. ${ }^{614}$

Es gehörte ebenfalls zum Konzept des Projekts, allen Kooperationswilligen unabhängig von ihren Schreibfähigkeiten - die Möglichkeit zur Mitgestaltung zu eröffnen. Eine kleine Gruppe fester Mitarbeiter bestehend aus Autoren und Herausgebern, die für die Hauptcharaktere des webbasierten Dramas verantwortlich zeichnete und die thematische Ausrichtung der Handlung vorgab, stellte zusätzlich eine spezielle Lernumgebung bereit, über die sich auch weniger erfahrene Autoren der Schreibgemeinschaft anschließen und in Nebenhandlungen ihre eigenen Charaktere entwickeln konnten. The Company Therapist sollte als Netzwerk gegenseitiger Unterstützung, Prägung und eher beiläufiger Unterweisung die allgemeine Schreibfähigkeit der beteiligten Autoren steigern. Damit bildete das Projekt zum Zeitpunkt seines Debüts im World Wide Web eine wirkliche Innovation, insofern es nicht nur eine wirklichkeitsnahe Lernumgebung bereitstellte, sondern auch den Zugang zu einem real existierenden öffentlichen Publikum schuf und gleichzeitig eine unterstützende Arbeitsgemeinschaft anbot. The Company Therapist leistete mithin einen wertvollen und außergewöhnlichen Beitrag zur Schreibförderung; es verdient dieses Urteil, weil es Schreibinteressierten einen konkreten Anlaß zur Ausübung ihrer Kreativität lieferte und im selben Zuge einen Mentor und ein Korrektiv an die Hand gab.

Bei der Ausarbeitung der Figuren und Handlungsstränge waren die Kooperierenden niemals auf sich allein gestellt, sondern konnten stets mit der Unterstützung aller Beteiligten rechnen. Die Empfehlung lautete, sich zunächst an den bereits vorhandenen Elementen des Werkes zu orientieren und etwaige Ideen für eine Fortsetzung oder Neuentwicklung gemeinschaftlich und öffentlich zu diskutieren. Zu diesem Zwecke war ein schwarzes Brett als Kommunikationsforum vorgesehen, das nicht nur den Autoren, sondern auch den Lesern die Möglichkeit zu

${ }^{613}$ Dies., "Educational Principles and Considerations: Collaborative Hyperdrama”, in: Dies., Hg., The Company

Therapist, $<$ http://www.thetherapist.com/Explanation.html\#anchorCollaborativeHyperdrama $>$, 02.10.2003.

614 Dies., "The Company Therapist in General: Two Goals", $<$ http://www.thetherapist.com/Explanation.html\#anchorTwoGoals $>, 02.10 .2003$. 
Erfahrungsaustausch und konstruktiver Kritik eröffnete. Neben dem öffentlichen Gespräch bestand außerdem Gelegenheit zu privater Kontaktaufnahme per E-Mail. Als letzte Kontrollinstanz vor der endgültigen Veröffentlichung eines Beitrags fungierte jeweils ein persönlich zugeteilter Mentor und Herausgeber aus der Reihe der ständigen Autoren. Jedes Dramenelement wurde zunächst sorgfältig redigiert, bevor es schließlich zur Veröffentlichung gelangte. Eine spezielle Software, die alle Veränderungen des Mentors farblich hervorhob, bot den Kooperierenden in diesem Verfahren eine entscheidende Hilfe. Die Herausgeber wollten mit dieser sowie mit zahlreichen anderen unterstützenden Funktionen eine möglichst effektive und fruchtbare Lernumgebung schaffen, in der angehende Autoren ihre Talente zu Geltung und Perfektion bringen konnten.

Im selben Zuge gewährleistete die intensive Betreuung der Kooperierenden Schutz vor unseriösen Beiträgen. Kein Text wurde ungeprüft zur Veröffentlichung freigegeben, stets gab der Kreis der Mentoren in Abstimmung mit den Herausgebern das Niveau und den inhaltlichen Rahmen des Werkes vor. Gerade bei der vorliegenden Thematik drohten die Grenzen des guten Geschmacks schnell überschritten zu werden. Die Verantwortlichen brachten ihre Vorstellungen in dieser Hinsicht jedoch deutlich zur Kenntnis. Sie wiesen in diesem Sinne ausdrücklich darauf hin, das Forum sei zwar mit einem "R" gekennzeichnet und daher nicht für Kinder geeignet; es sei aber andererseits eindeutig nicht mit einem " $X$ " bewertet. ${ }^{615}$ Aus diesem Grunde werde man konsequent gegen anstößiges Material vorgehen und es entweder überarbeiten oder zurückweisen: "If your material is 'over the top' it will be edited or rejected. This includes graphical material." ${ }^{\prime 616}$ Zur Veranschaulichung belegt ein ausgewählter Beitrag das nach Meinung der Verantwortlichen gerade eben noch Akzeptable ${ }^{617}$ Die strikte Entscheidung für ein gewisses Niveau des guten Geschmacks - so positiv sie grundsätzlich zu bewerten ist - bedeutet natürlich einerseits, daß die genaue Grenzziehung im Einzelfall nicht frei von Konflikten sein dürfte, andererseits daß die Thematik eine Grenzüberschreitung vermutlich geradezu herausfordert.

\footnotetext{
615 "R" wird in der US-Amerikanischen Filmbranche als Abkürzung für restricted verwendet und kennzeichnet Filme, die nicht für Jugendliche zu empfehlen sind. Ein sogenannter "X-certificate film" ist hingegen erst für Jugendliche ab 18 Jahren freigegeben.

${ }^{616}$ Pipsqueak $\quad$ Productions, "Submission $<$ http://www.thetherapist.com/Submission Guidelines.html $>, 02.10 .2003$.

617 Ebd., 02.10.2003. 
Alle einmal eingestellten Beiträge blieben fester Bestandteil des Projekts. Auf diese Weise sollten die Fortschritte der Autoren hinsichtlich Stil und Phantasie nachvollziehbar werden. Die Herausgeber versprachen sich vom Aufzeigen und Deutlichmachen der schrittweisen Talententfaltung einen hohen didaktischen Wert für jeden einzelnen Kooperierenden; über die Phase der kritischen, wenngleich von Optimismus und Vertrauen geprägten Selbsteinschätzung sollte die Entwicklung schließlich in kreativitätssteigerndem Selbstbewußtsein münden:

The abilities to review and evaluate one's own work and observe one's writing from multiple perspectives are metacognitive tools. These tools help develop selfawareness and self-assessment in writers. ${ }^{618}$

Nach Glenda Hull, Professorin der Berkeley School of Education an der Universität von Californien, entspricht das Schreiben einem Problemlösungsprozeß, zu dessen Grundprinzipien und wesentlichen Strategien Planung, Organisation, Strukturierung und Überarbeitung zählen. ${ }^{619}$ Demnach unterstützte The Company Therapist nicht nur schriftstellerische Begabungen, sondern förderte auch allgemeine Kompetenzen. Die Transparenz dieser Prozesse sollte aber nicht nur den Schreibenden zugute kommen, sondern das Projekt darüber hinaus zum Anlaufpunkt für wissenschaftliche Untersuchungen machen; der Einzelne und die Autorengruppe sollten mit anderen Worten auch als Studienobjekt für die Lern- und Entwicklungsforschung dienen.

Bei der Anpassung von Lernprogrammen an die Bedürfnisse und das Leistungspotential ihrer Nutzer orientierten sich Werby und Werby vor allem an den kognitiven Prozessen, die bei der Anwendung von Lernprogrammen ablaufen. Die Kenntnis dieser Vorgänge zur Abfrage, Umsetzung und Festigung intellektuellen Potentials sollte die beiden Software-Entwickler zur Schaffung einer neuen, lernorientierten und damit effektiveren Schulungsumgebung befähigen. Was Werby und Werby ihren Veröffentlichungen fortan als neue Lehrmodelle zugrundelegten, galt im Jahre 1996 als innovativ und richtungweisend. Heute hat sich dieser pädagogische Ansatz als Grundvoraussetzung für erfolgreiche Lern-Software eta-

618 Dies., "Educational Principles and Considerations: Conceptual Changes", in: Dies., Hg., The Company Therapist, <http:/www.thetherapist.com/Explanation.html\#anchorConceptualChanges $>$, 07.10.2003.

${ }^{619}$ Nach ebd., 07.10.2003. 
bliert und wird aufgrund seiner Kombination von Elementen aus Education und Entertainment auch als Edutainment bezeichnet. ${ }^{620}$

Mit der Abkehr vom didaktischen Standardmodell des "drill and practice"621 rückten neue Grundregeln der Programmierung in den Vordergrund. Fünf Prinzipien machten Werby und Werby daraufhin zur Basis ihrer edukativ-unterhaltenden Projekte und somit auch des webbasierten Dramas The Company Therapist:

- "Learning should be fun." - Um die höchste Wirkung zu erzielen, sollte das Lernmaterial den Anwender für sich einnehmen.

- "Learning should be authentic." - Realitätsbezug und Zweckgebundenheit sind notwendige Voraussetzungen für Aufnahmebereitschaft und -befähigung der Lernenden.

- "All people perceive and learn things differently." - Daher sollten Lernprogramme variabel gestaltet sein, so daß sie den unterschiedlichen Anforderungen der Nutzer entsprechen können. Gerade Computer und WWW erlauben im Gegensatz zu fest vorgegebenen Übungsabfolgen eine hohe Anpassungsfähigkeit.

- "Learners should be given as much freedom and control over what they're learning as reasonably possible." - Ob ein Lernender lebenslängliche Neugier und Lernbereitschaft entwickeln kann, hängt in entscheidendem Maße davon ab, ob er rechtzeitig die Gelegenheit zu eigenen Entdeckungen und Schlußfolgerungen erhält.

- "Educational projects should be constructed so that they support novice learners and allow them to act beyond their current level of ability." - Sobald die Neueinsteiger das Programm bedienen und den Stoff bewältigen können, sollten die Hilfeelemente wegfallen und das Programm auf diese Weise den Bedürfnissen der fortgeschrittenen Lerner angepaßt werden. ${ }^{622}$

Alle vorstehenden Punkte sind offensichtlich in die Konzeption des webbasierten Dramas mit eingeflossen:

Durch seine außergewöhnliche Thematik und den Facettenreichtum der Charaktere vermochte und vermag The Company Therapist den Web-Besucher für

${ }^{620}$ Doris Feldmann, "Multimedia Shakespeares", in: Dies., Neumann und Rommel, Hg., Anglistik im Internet, 129-143, hier: 133. Hannah Möckel-Rieke, "Der virtuelle Text”, in: Klepper, Mayer und Schneck, Hg., Hyperkultur, 68-80, hier: 69.

${ }^{621}$ Pipsqueak

Productions,

"Educational

Philosophy", $<$ http://www.pipsqueak.com/Educational Philosophy-fs.html $>, 07.10 .2003$.

622 Ebd., 07.10.2003. 
sich einzunehmen und an das Projekt zu binden. Der Realismus der Kulisse war und ist eindeutig gegeben, so daß sich theoretisch jeder (erwachsene) Nutzer mit der dargestellten Situation identifizieren konnte beziehungsweise kann. Dank der themenbedingten Vielzahl von Einstiegsmöglichkeiten konnte jeder Kooperationswillige einen Ansatzpunkt für einen eigenen Beitrag finden. Zudem sah das Konzept konkrete Unterstützung durch Mentoren vor, die jedem Beitragenden individuell auf ihn zugeschnittene Hilfe zukommen ließen. Damit empfahl sich The Company Therapist speziell auch für jene Web-Besucher, die keine Routine im Umgang mit Kooperationsprojekten besaßen und auf keinerlei schriftstellerische Erfahrung zurückblicken konnten. Trotz der persönlichen Betreuung erhielt jeder Kooperierende ausreichenden Spielraum für eigene Entdeckungen und Erkenntnisse.

In den Richtlinien zur Konzeption neuer Beiträge wurde allen kooperationswilligen Web-Besuchern deutlich zu verstehen gegeben, daß die Mitarbeit am Projekt mit erheblichem Zeitaufwand verbunden sein würde. Die Teilnahmebedingungen verlangten kontinuierliche Beteiligung über einen längeren Zeitraum hinweg, da sich nur auf diese Weise ein neuer Charakter mit einem eigenen Psychogramm würde entwickeln lassen. Jedem neu kreierten Patienten mußte mindestens alle zwei Wochen ein Beratungsgespräch mit Dr. Balis in Frage-und-Antwort-Stil zugeschrieben werden, wobei jede Sitzung vier bis sechs Seiten umfassen sollte, veranschlagt mit insgesamt ungefähr 2.000 Worten. Diese Arbeiten sollten spätestens 24 Stunden vor dem geplanten Erscheinungstermin per E-Mail eingereicht werden.

Von Bedeutung war auch, daß alle Kooperierenden ein sogenanntes Work for Hire Agreement unterzeichnen mußten. ${ }^{623}$ Mit dieser Art von Einverständnis-

${ }^{623}$ Dies., "Submission Guidelines", <http://www.thetherapist.com/Submission Guidelines.html $>$, 07.10.2003. - Work for Hire ist ein Terminus des United States Copyright Act, der besagt, daß sich ein Autor in bestimmten Fällen von vorneherein aller Urheberrechte an seinem Werk begibt, die ihm das Gesetz normalerweise zubilligt. Sie entstehen statt dessen in der Person desjenigen, der den Autor für die Schaffung seines Werkes unter Vertrag genommen hat. Dieser - der Auftraggeber des Autors - wird dann nach dem Gesetz so behandelt, als habe er das Werk selbst und ohne jegliches Zutun des eigentlichen Autors erstellt. Einem Work for Hire-Vertrag zufolge hat ein Autor also keinerlei Rechte an seinen eigenen geistigen Produkten. Eine solche Regelung hat ihre Berechtigung zweifellos immer dann, wenn jemand im Rahmen eines Arbeitsverhältnisses für seinen Arbeitgeber schöpferisch tätig wird. Im Falle eines Beitrags zu kooperativer webbasierter Literatur hingegen ist nur bedingt nachvollziehbar, warum ein Autor alle Rechte an seinem Werk abtreten beziehungsweise warum der Herausgeber der webbasierten Literatur das alleinige Recht an den Arbeiten der Kooperierenden erhalten sollte. Allenfalls das Problem der Feststellung der einzelnen Wertschöpfungsquote als Maßstab für die Zuweisung von Tantiemeanteilen könnte die Work for Hire-Klausel rechtfertigen. Vgl. hierzu auch Michael P. McCready, "What is a work for hire?", in: Ders., Hg., Music Law Offices, <http://www.music-law.com/workforhire.html>, 10.10.2003. 
erklärung gehen das Urheberrecht an dem Werk sowie alle damit verbundenen und nachfolgenden Rechte an allen Formen der ursprünglichen oder noch hinzukommenden Formen der Darstellung an den Werkinitiator - im vorliegenden Falle also den Webmaster - über, der diese Exklusivrechte sowohl in den USA als auch weltweit nutzen darf. Im Gegenzug erhält der Autor eine einfache, aber immerwährende Lizenz, das Werk nachzudrucken oder neu aufzulegen beziehungsweise in nachfolgenden Werken darauf aufzubauen. ${ }^{624}$ Das Work for Hire Agreement mußte den Herausgebern im Falle des Company Therapist auf postalischem Wege oder per Fax zugesandt werden.

Werby und Werby behielten sich auf diese Weise nicht nur alle weiteren Nutzungsrechte vor, sondern sicherten sich auch ein zusätzliches Mal gegen unerwünschte Beiträge ab. Diese Sicherheitsmaßnahme garantierte, daß sich der Schreibgemeinschaft tatsächlich nur jene Personen anschlossen, die zu ernsthafter und gewissenhafter Mitarbeit bereit waren. Für die Kooperierenden wiederum bedeutete diese formale Erklärung zusammen mit den Bedingungen über Inhalt, Umfang und Erscheinungsrhythmus der kooperativen Beiträge eine weitere Erschwernis ihrer freiwilligen Mitarbeit. Eine spontane Beteiligung potentieller Autoren war damit ausgeschlossen.

Die Mitarbeit am Projekt The Company Therapist erforderte zweifellos Reflexion, Disziplin, Ausdauer, Kreativität und Zeit. Dennoch wurde keiner der Kooperierenden finanziell für seinen Aufwand entschädigt, vielmehr sollte allein das Projektergebnis selbst der Lohn aller ihrer Mühen sein: "This is a labor of love." ${ }^{625}$ In der Tat bereitete es nach Aussagen von Werby und Werby allen Beteiligten Freude und Befriedigung, die 'Geburt' ihres selbsterschaffenen Charakters erleben zu dürfen. ${ }^{626}$ Auch für die Herausgeber selbst war der Erfolg des Projekts die eigentliche Entschädigung.

${ }^{624}$ Pipsqueak Productions, "Work for Hire Agreement", in: Dies., Hg., The Company Therapist, $<$ http://www.thetherapist.com/Contract.html $>, 09.10 .2003$.

625 Dies., "The Company Therapist in General: Two Goals", $<\underline{\text { http://www.thetherapist.com/Explanation.html\#anchorTwoGoals }>, 07.10 .2003 . ~}$

626 Ebd., 07.10.2003. 


\subsubsection{2. "Graphics \& Design" 627}

Die Einstiegsseite des Company Therapist - hier auch als Front Stage bezeichnet ist in den Farben Grau und Schwarz gehalten, aus denen einzelne Elemente in kräftigem Rot hervortreten. Im Hintergrund ist die Silhouette einer Großstadt angedeutet. Vorne sieht man im linken Teil des Bildschirms einen Oberkörper in eleganter Herrenbekleidung, der anstelle des Kopfes das runde Emblem der Web-Seite mit dem Schriftzug The Company Therapist trägt. Zahlreiche kleine rote Würfel lenken den Blick des Betrachters auf den linken Vordergrund. Die Bedienungselemente sind im rechten und im unteren Teil des Bildschirms arrangiert, zusätzlich aber in geordneter Form auch am unteren Bildschirmrand angeordnet.

Die Darstellungen dieser Seite, vor allem die Farben und geometrischen Muster besitzen symbolische Bedeutung und spielen auf die Thematik des Werkes an, das den Web-Besucher erwartet. Die Grau- und Schwarztöne der Skyline veranschaulichen Anonymität, Monotonie und Tristesse des Großstadtlebens. Der kopflose Oberkörper mit grauem Anzug steht als Zeichen für den Firmenpsychiater, der sich für die Personen in seinem Umfeld zumeist auf ein Medium zur Lösung innerer Spannungen reduziert; seinen Patienten dient Dr. Balis nahezu ausschließlich als Problemventil, eine eigene Identität - das heißt ein Gesicht - besitzt er aus ihrer Perspektive hingegen nicht. Die unzähligen roten Würfel könnten in Zusammenhang mit den einzelnen Elementen des Hypertextes stehen, die sich alle zu einem großen Ganzen zusammensetzen lassen. Möglicherweise versinnbildlichen sie auch die bruchstückartigen Erkenntnisse, die Dr. Balis während der Sitzungen über seine Patienten gewinnt und die sich nur nach und nach zu einem Psychogramm aneinanderfügen.

Bereits auf der Einstiegsseite signalisiert der Link mit der Bezeichnung Backstage, welcher Literaturgattung das webbasierte Hypertext-Werk zuzuordnen ist. Stellt der Nutzer, der hier gleich mit doppeltem Recht Besucher genannt werden darf, da er nicht nur den virtuellen Raum des Web, sondern auch das virtuelle Theater betritt, die Verknüpfung zum Raum hinter den Kulissen - dem sogenannten Backstage - her, blickt er in das Sprechzimmer von Dr. Balis. Werby und Werby haben die Atmosphäre einer Theaterbühne noch dadurch verstärkt, daß sie den

627 In Anlehnung an die Kurzinformation über die Herausgeber des Company Therapist: "Christopher Werby, Producer \& Director; Olga Werby, Producer Pipsqueak Productions, Graphics \& Design.” Pipsqueak Productions, "Credits Page", <http://www.thetherapist.com/Credits_Page.html $>$, 18.10.2003. Siehe in diesem Zusammenhang auch Fußnote 607. 
Betrachter durch einen geöffneten Vorhang auf die Szenerie blicken lassen. Zudem wird der Raum von oben herab durch mehrere Scheinwerfer beleuchtet.

Noch viel deutlicher kam der Eindruck eines Bühnengeschehens allerdings in der ursprünglichen Einstiegsseite zum Ausdruck. Dort nahm ein Gerüst mit zahlreichen Scheinwerfer raumgreifend die Hälfte des Bildschirms ein und ließ damit den gesamten Hintergrund zur Bühnenkulisse werden. Dort war in einer Mischung einzelner Elemente der heutigen Seiten Front Page und Backstage rechter Hand die Silhouette der Großstadt mit dem kopflosen Oberkörper abgebildet, während dem Betrachter linker Hand der Blick in zwei Geschosse eines mehrstöckigen Gebäudes gewährt wurde. Die Umgebung war in nächtliches Dunkel getaucht und wurde nur von einer schmalen Mondsichel geringfügig aufgehellt. Die Scheinwerfer waren unter anderem auf das obere der beiden Geschosse gerichtet, wo sich das beleuchtete Sprechzimmer von Dr. Balis teilweise einsehen ließ. Im Vordergrund der unteren Bildschirmhälfte war ein tafelartiges Gebilde plaziert, das aufgrund seiner Bedienungselemente an ein Regiepult erinnerte. Die zehn Tasten dieses Pultes dienten als Verknüpfungen zu weiteren Ebenen des Hypertext-Systems.

Das Regiepult erscheint in einer moderneren Variante auch auf den aktuellen Seiten von The Company Therapist. Dort befindet es sich stets am oberen Bildrand und ersetzt die üblicherweise verwendeten Menüleisten. Die Tasten des Pultes leiten den Nutzer wahlweise zu einer der zehn Rubriken des HypertextSystems, das in zwei Hauptbereiche untergliedert ist: Größe und Arrangement der Tasten verdeutlichen, daß The Company Therapist in einen erklärenden (äußeren) und einen darstellenden (inneren) Komplex aufgeteilt ist.

Folgende sechs Stichworte führen zu allgemeinen Hintergrundinformationen und jenen Projektabschnitten, die nicht das eigentliche Dramengeschehen wiedergeben:

- Home

- Map

- Authors

- Introduction

- Messages

- Search 
Hier werden alle Angaben präsentiert, die die Intention und Konzeption des Projekts beschreiben (Introduction), Auskünfte über die Kooperierenden liefern (Authors) oder dem Nutzer die Navigation erleichtern (Search und Map). Dabei erlaubt die Suchmaschine eine gezielte Recherche zu bestimmten Personen oder Charakteren, während das Überblicksschema die allgemeine Orientierung erleichtert. Das Schema, dessen Angaben gleichzeitig auch Verknüpfungen darstellen, gibt Aufschluß über die Struktur des Hypertextes und ermöglicht dem Nutzer sowohl Standortbestimmung als auch Navigation innerhalb des Systems. Es handelt sich dabei nicht um ein statisches Schema, sondern die Dynamik des Hypertext-Systems wurde zugunsten einer orientierungsfördernden Darstellung eingesetzt. Die anklickbaren Angaben erlauben einen zielgerichteten und unmittelbaren Zugriff auf die einzelnen Elemente des Projekts. Die letzte Rubrik Messages scheint allerdings nicht von Anbeginn an Teil des Hypertext-Systems gewesen, sondern erst vor kurzem eingerichtet worden $\mathrm{zu}$ sein. In der einzigen dort eingestellten Nachricht vom 26.09.2003 teilt Christopher Werby der Nutzergemeinschaft mit, daß das Bestehen des Company Therapist dank der Unterstützung des Sponsors Earthlink auch weiterhin gesichert sei. ${ }^{628}$

Nachstehende Punkte zählen zum inneren Bereich, das heißt sie verbinden den Nutzer mit allen Rubriken, die den literarischen Teil des Projekts betreffen:

- Backstage

- Doctor's Notes

- Doctor's Schedule

- Doctor's Letters

- File Cabinet

- Random Link

In diesem Bereich des Forums erhält der Nutzer hauptsächlich Zugriff auf die Praxisunterlagen und kann anhand dieser Materialien die Handlungsstränge des webbasierten Dramas und damit die Schicksale der einzelnen Patienten nachvollziehen (Doctor's Notes/Doctor's Schedule/Doctor's Letters). Die Patientenakten mit den persönlichen Daten, den Krankheits- und Therapieverläufen sind in einem virtuellen Aktenschrank unter dem Stichwort File Cabinet alphabetisch abgelegt.

${ }^{628}$ Christoper Werby, "Message Board is Back Up", in: Pipsqueak Productions, Hg., ([ 26.09.2003]), $<$ http://www.thetherapist.com/cgi/BBS2/config.pl?read=894>, 09.10.2003. 
Die Verknüpfung Backstage leitet weiter zu dem bereits erwähnten Sprechzimmer von Dr. Balis, dem zentralen Ort des Geschehens, von dem seinerseits zahlreiche Verknüpfungen ausgehen. ${ }^{629}$ Über den Link Explanation erhält der Nutzer Informationen über die biographischen Hintergründe von Dr. Balis und seinen Patienten; die Firmen- und Erfolgsgeschichte von Silicon Impressions Inc. ist im Abschnitt SII Guide ausführlich dargelegt. ${ }^{630}$

Aufbauend auf all diesen Angaben kann der Nutzer sich sodann über die Verknüpfung How to Submit mit den Regeln zur Einstellung neuer Beiträge vertraut machen. Da dieser Bereich einen geschlossenen Kontext bildet, der sämtliche für sein Verständnis notwendigen Informationen bereithält, sind in ihm auch einige Verknüpfungen eingebunden, die bereits an anderer Stelle angeboten werden, wie beispielsweise der Link zum Überblicksschema (Map) sowie zu den Autoren (The Authors) und Sponsoren (Sponsors). Auch die hier integrierte Verknüpfung Bulletin Board birgt keine unbekannten Inhalte, da sie zum Nachrichtenforum weiterleitet, das von anderer Stelle bereits unter dem Stichwort Messages zugänglich ist. Neu ist in diesem Zusammenhang einzig der Punkt Production Credits, der eine Kurzinformation über die Herausgeber des Company Therapist enthält: "Christopher Werby, Producer \& Director; Olga Werby, Producer Pipsqueak Productions, Graphics \& Design." ${ }^{631}$

629 Pipsqueak Productions, Hg., The Company Therapist - Backstage, $<$ http://www.thetherapist.com/Backstage Frontscreen.html $>, 09.10 .2003$.

${ }^{630}$ Das über den Link Explanation und im Abschnitt SII Guide bereitgestellte Begleit- und Informationsmaterial (zur Person von Dr. Balis, über die Patienten und über Silicon Impressions Inc.), aber auch das Tagebuch des Dr. Balis, von dem weiter unten im Text die Rede ist, siehe Fußnote 657, scheinen die Gattungsgrenzen in Richtung auf die Textsorte Dokumentation zu sprengen. Dieses Phänomen, das sich auch in den Bereichen webbasierte Prosa und webbasierte Lyrik wahrnehmen läßt, ist systemimmanent. Je stärker webbasierte Publikationen die Möglichkeiten von Hypertext und WWW vereinen, desto größer wird zwangsläufig der Anteil des dem literarischen Werk beigefügten Zusatzmaterials und desto stärker treten die Charakteristika der traditionellen Gattungsformen zurück. Im Sinne der in Kapitel III. gewünschten Flexibilität und Großzügigkeit im Umgang mit sämtlichen hier behandelten Begriffen scheint es aber geboten, diese Gattungsüberschreitung nicht nur als eine (systemvorgegebene) Begleiterscheinung eben gerade zu tolerieren, sondern sie vielmehr als eine Bereicherung zu begrüßen, die vielleicht erst jenes gesuchte einzigartige literarische Kunstwerk möglich macht. Diejenigen Rezipienten, denen ein bestimmtes literarisches Werk informationsüberfrachtet erscheint, mögen sich die Hypertextstruktur zunutze machen, die es gestattet, unerwünschte Informationselemente zu ignorieren.

631 Dies., "Credits Page", <http://www.thetherapist.com/Credits_Page.html>, 09.10.2003. 
Eine Funktion, die dem Nutzer bei Bedarf einen stets völlig neuen Einblick in das webbasierte Drama eröffnet, verbirgt sich hinter der Verknüpfung Random Link. Nach dem Zufallsprinzip wird bei jedem Anklicken die Verbindung zu einem beliebigen anderen Element des Hypertextes hergestellt. Auf diese Weise gelangt der Nutzer auf Seiten, die er im Zuge seiner regulären Navigation möglicherweise niemals zu Gesicht bekommen hätte. Die Funktion hebt die Neugier des Lesers und gewährleistet permanente Abwechselung. Sie unterstreicht den Charakter der Vielschichtigkeit und Unergründlichkeit des Werkes und ist daher als eine besonders innovative und originelle Bedienungsvariante hervorzuheben.

The Company Therapist zeichnet sich durch eine detaillierte Projektbeschreibung aus, die alle Hintergründe und Intentionen der Herausgeber offenlegt. Zusätzlich finden sich auf deren Homepage aufschlußreiche Informationen zur Firmenphilosophie von Pipsqueak Productions sowie biographische Angaben zu den Software-Entwicklern Werby und Werby. ${ }^{632}$ Berichte über weitere Projekte zeigen zudem, mit welcher Verve, Begeisterung und innerer Überzeugung die Herausgeber ihre Arbeit in den Dienst der Nutzer stellen. Ihre Web-Auftritte überzeugen und helfen den Menschen, sich auf unterhaltsame und anregende Weise weiterzubilden.

In der Einführung zu ihrem Projekt betonen die Herausgeber des Company Therapist vor allem den Realismus des präsentierten Materials: "The Company Therapist is creating a fictional world with many characters, events, and storylines coexisting and evolving to give an illusion of reality to its readers. ${ }^{~} 633$ In der Tat findet der Leser perfekt aufeinander abgestimmte Kreativbeiträge vor, die geeignet sind, die Illusion von Realität zu wecken. Hinzu kommt, daß das Projekt in seiner aktiven Phase in Echtzeit ablief. Dies bedeutete nicht nur, daß sich die Spielzeit des webbasierten Dramas mit der gespielten Zeit deckte, die Vorgänge folglich so konzipiert waren, wie sie im Zeitmaß ihrer Umwelt tatsächlich abgelaufen wären ${ }^{634}$, sondern daß die Dialoge zwischen Dr. Balis und seinen Patienten genau zu jenem Zeitpunkt im Web erschienen, zu dem sie sich auch

${ }^{632}$ Dies., Hg., Pipsqueak Productions - New Media Creators, < $\underline{\text { http://www.pipsqueak.com/>, }}$ 09.10.2003.

${ }^{633}$ Dies., "The Company Therapist in General: A New Form”, $<$ http://www.thetherapist.com/Explanation.html\#anchorNewForm $>, 15.10 .2003$.

634 Vgl. Hans-Jochen Schneider, Hg., Lexikon Informatik und Datenverarbeitung, 4., aktual. u. erw. Aufl. (München; Wien, 1997 [ $\left.\left.{ }^{1} 1983\right]\right), 267$. 
fiktiv zutrugen. Alle Termine waren koordiniert und fanden stets im Rahmen eines üblichen Arbeitstages statt. Mittlerweile sind sie vollständig im Kalender von Dr. Balis, dem sogenannten Doctor's Schedule, abgelegt, von wo aus die Möglichkeit des direkten Zugriffs auf jede abgewickelte Patientenberatung besteht. $^{635}$

Konzeption und Präsentation der Therapiesitzungen allein scheinen also bereits sehr lebensnah, wirklich überzeugenden Realismus verleihen dem webbasierten Drama allerdings die einführenden und begleitenden Materialien. The Company Therapist beginnt am 13.07.1996 mit einer Aufzeichnung von Dr. Balis, in der er die Ereignisse der zurückliegenden Tage reflektiert und sich mental auf die geplante Eröffnung seiner Praxis am 15.07.1996 vorbereitet. ${ }^{636}$ Er eröffnet mit diesem Eintrag ein Tagebuch, das über die Ereignisse annähernd jedes Tages der folgenden drei Jahre Rechenschaft ablegen wird. Dort sind nicht nur die Privatangelegenheiten von Dr. Balis verzeichnet, zu denen die Offenlegungen seiner physischen wie psychischen Befindlichkeiten und Hinweise auf persönliche Korrespondenzen zählen; dort sind auch alle beruflichen Vorgänge festgehalten, wie beispielsweise therapeutische Telefongespräche sowie die Protokolle der Therapiesitzungen und deren Aufarbeitungen, in denen die Unterredungen mit den Patienten reflektiert, analysiert und auf Therapieansätze hin untersucht werden. ${ }^{637}$ Jeweils nach Ablauf einer Woche werden die täglichen Schilderungen in Wochenmappen, den sogenannten Journals, abgelegt. ${ }^{638}$

Geistiger Urheber all dieser Niederschriften ist einer der Initiatoren und Herausgeber des Projekts, Christopher Werby. Ihm oblag es in der Zeit von 1996 bis 1999, das von den Kooperierenden bereitgestellte Material im Tagebuch von Dr. Balis aufzubereiten. Da die Ereignisse, wie oben erwähnt, in Echtzeit abliefen, hatte Werby die Verpflichtung, sich jeden Tag mit den aktuellen Geschehnissen zu befassen.

${ }^{635}$ Pipsqueak Productions, Hg., The Company Therapist - Doctor's Schedule, $<$ http://www.thetherapist.com/Appointments.html $>, 15.10 .2003$.

636 Dies., "Charles Balis' Journal for the Week ending 7/19/96”, in: Dies., Hg., The Company Therapist - Diaries \& Logs, <http://www.thetherapist.com/TCTSeason1/DrNotes_071996.html>, 15.10.2003.

637 Auch hier scheint das umfangreiche Informationsmaterial dem webbasierten Drama stark dokumentarischen Charakter zu verleihen und damit die Gattungsgrenzen zu sprengen. Siehe diesbezüglich auch die Ausführungen in Fußnote 650.

638 Dies., Hg., The Company Therapist - Diaries \& Logs, <http://www.thetherapist.com/Balis_Log.html>, 15.10.2003. 


\subsubsection{3. 'The Story'}

Das eigentliche Geschehen des webbasierten Dramas setzt mit dem Erscheinen des ersten Patienten ein. Bereits am Tag der Praxiseröffnung, dem 15.07.1996, erscheinen drei Angestellte von SII zu einem Termin bei Dr. Balis: Um 10.00 Uhr findet ein Gespräch mit Hal Mainor statt, um 13.00 Uhr folgt eine Unterredung mit Anna Green und um 16.00 Uhr sucht Sylvia Bows psychiatrische Hilfe. Auch diese drei Figuren sind von den Initiatoren des Projekts, Christopher und Olga Werby, konzipiert und bilden Quelle, Anstoß oder Ausgangspunkt für weitere geistige Schöpfungen. Offensichtlich ist das Konzept von The Company Therapist ansprechend und überzeugend gestaltet, denn bereits am 23. und 24.07.1996 wird die Gruppe der drei Patienten durch die Mitarbeit freiwilliger Autoren um zwei Figuren erweitert.

Gleich zu Beginn des Projekts inszenieren die Herausgeber eine Konfliktsituation. Bereits zwei Tage nach Eröffnung der Praxis am 17.07.1996 nämlich setzt ein Schreiben der Krankenversicherungsgesellschaft CalaCare Inc. ${ }^{639}$, bei der Dr. Balis unter Vertrag steht, eine rechtlich-moralische Auseinandersetzung in Gang. Um das Risiko der Selbständigkeit zu mindern und finanziell unabhängiger zu sein, hatte Dr. Balis sich einen festen Verdienst als FirmenPsychiater bei Silicon Impressions Inc. sichern wollen und war zu diesem Zweck mit 60 Prozent seiner Arbeitszeit bei CalaCare Inc. eingetreten. In einer schriftlichen Mitteilung wird er nun darüber informiert, daß man aus Rationalisierungsgründen eine langfristige Überprüfung der Versicherten vollziehe, zu denen auch die unter seiner Betreuung stehenden Patienten zählten. Um diesen Prozeß zu beschleunigen beziehungsweise eine Unterbrechung zu vermeiden, möge er umgehend Kopien aller relevanten Dokumente inklusive Tonbandaufzeichnungen, Gesprächsprotokollen, Korrespondenzen und Computerdaten an CalaCare übersenden. ${ }^{640}$

${ }^{639}$ Bei CalaCare handelt es sich um eine sogenannte HMO. Diese Abkürzung steht für Health Maintenance Organization und bezeichnet ein medizinisches Dienstleistungssystem in den USA, das vergleichbar ist mit privaten Krankenversicherungen, anders als diese jedoch keine freie Arztwahl gestattet. Die Gesellschaften stellen ihren Beitragszahlern medizinisches Personal zur Verfügung, das aus Kostendämpfungsgründen zur Förderung von Präventivmaßnahmen sowie Vermeidung unnötiger Untersuchungen und Operationen angehalten ist.

${ }^{640}$ Dies., Hg., The Company Therapist - Professional Correspondence, $<$ http://www.thetherapist.com/TCTSeason1/Letter_From_HMO_071796.html $>$, 15.10.2003. 
Dieses Anschreiben ist schon optisch durch seinen Firmenbriefkopf und die Unterschrift so realistisch gestaltet, daß der Leser geneigt ist, dem Inhalt Glauben zu schenken. Die Dramenfigur Dr. Balis reagiert entrüstet und ist aus moralischen wie standesrechtlichen Gründen nicht bereit, dem Ansinnen nachzukommen. Aus dieser Weigerung ergibt sich ein monatelanger Briefwechsel, der schließlich am 25.11.1996 damit endet, daß Dr. Balis die Drohungen und restriktiven Maßnahmen von CalaCare wie beispielsweise Honorarkürzungspläne und Umleitung potentieller Patienten an örtliche Psychologen zum Anlaß nimmt, seine Selbständigkeit unter allmählicher Distanzierung von CalaCare auszuweiten und verstärkt Patienten in Eigeninitiative anzuwerben.

Auch die Idee der Krankenversicherungsgesellschaft CalaCare stammt aus dem Familienverband der Projektinitiatoren. Mit dem Konflikt zwischen der Gesellschaft und Dr. Balis, der sich für diesen zu einer ernstzunehmenden Existenzbedrohung entwickelt, demonstrieren Andrew und Christopher Werby das inhaltliche wie illustrative Potential des webbasierten Dramas. Die Vorgaben erweisen sich als kluge, erfolgversprechende Regieentscheidung, denn gerade das Angebot an Anschauungsmaterial ist am Ende außergewöhnlich umfassend und vielgestaltig, vor allem aber ausgesprochen glaubhaft und überzeugend. Die Korrespondenzen zwischen Dr. Balis und seinem Arbeitgeber CalaCare, seinen Patienten und dem Vertragspartner SII steigern an sich bereits die Wirklichkeitsnähe des webbasierten Dramas, zusätzlich verstärken die Schreiben durch aufwendig gestaltete, personen- oder firmenspezifische Briefköpfe den Eindruck der Echtheit des gesamten eingestellten Schriftguts.

Eine eingescannte Postkarte, die Dr. Balis anonym, vermutlich von einer Patientin erhalten hat, läßt das Geschehen noch spektakulärer und für den Leser respektive Zuschauer noch greifbarer erscheinen. ${ }^{641}$ Auch die kriminellen Machenschaften eines Patienten in Verbindung mit der Visitenkarte eines FBI-Agenten sowie eines Zeitungsausschnitts zum tödlichen Mordanschlag auf den betreffenden Patienten vermitteln dem Geschehen nachhaltige Überzeugungskraft. ${ }^{642}$ Insgesamt

${ }^{641}$ Dies., "Postcard from Helen Gregory", in: Dies., Hg., The Company Therapist - Correspondence ([127.09.1996]), <http://www.thetherapist.com/TCTSeason1/Greg_Letter_092796.html>, 15.10.2003.

642 Dies., "Agent John Kline of the FBI regarding Joseph Mazurka”, in: Dies., Hg., The Company Therapist - Transcripts $\left(\left[{ }^{1} 03.12 .1996\right]\right),<$ http://www.thetherapist.com/TCTSeason1/Mazurka_Rele_120396.html $>$, 15.10.2003. Dies., 'Newspaper Article on Joseph Mazurka's Death”, in: Dies., Hg., The Company Thera-

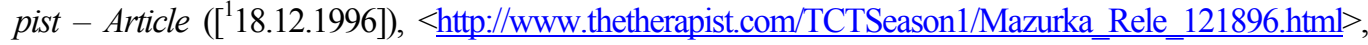
15.10.2003. 
werden von den Autoren des Company Therapist fünf Presseartikel zur Untermauerung des Dramengeschehens eingesetzt.

Ein weiterer Fundus illustrativer Materialien bilden die Kritzeleien, die von den Patienten im Verlaufe der Therapiesitzungen angefertigt worden sind. Diese Zeichnungen erweisen sich nicht nur als ein ebenso abwechslungsreiches wie zum Teil auch kurioses Anschauungsmaterial, sondern regen gleichzeitig auch zum Mitdenken an. Im Zusammenhang mit den Therapieprotokollen und der diagnostischen Aufarbeitung der Sitzungen vermag der Leser seine eigenen Interpretationen zu entwerfen. Eine besonders große Herausforderung bedeuten in dieser Hinsicht die Beiträge eines besonderen Patienten, des anonymen Fax-Versenders. Die Geschichte dieser Figur wird in erster Linie anhand zeichnerischer Darstellungen erzählt. Es handelt sich dabei um einen extrem schüchternen Mann, der sich ganz offensichtlich in seinem Körper unwohl fühlt und sich Dr. Balis nur mit Hilfe wöchentlich zugefaxter Bilder mitzuteilen vermag. Seine Zeichnungen erscheinen zwar bizarr und rätselhaft, offenbaren dem Psychiater aber sein tiefstes Innerstes.

Wie das Beispiel des anonymen Patienten erkennen läßt, bilden die Dramenfiguren eine absichtlich heterogene Gruppe. Gerade die Festlegung auf eine möglichst komplexe, perspektivreiche Handlung sowie die Schöpfung des Szenentyps 'Patientengespräch' mit den Therapiesitzungen in einer immer gleichen Struktur, die dennoch nicht eintönig wird, schaffen Raum für Kreativität und Individualismus: "In this project $[\ldots]$ the varying styles of different writers is a bonus rather than a detriment."

So verschiedenartig die Figuren sich charakterlich darstellen, so unterschiedlich sind ihre Kommunikationsweisen. Auch hier werden - sofern es Anstand und guter Geschmack erlauben - weder inhaltliche noch stilistische Einschränkungen vorgenommen. The Company Therapist vereinigt in sich somit eine Vielzahl unterschiedlichster personaler Sphären; das webbasierte Drama gestaltet sich mit anderen Worten zum Kosmos der Ängste und Schwächen, der Launen und Obsessionen, kurz des Menschlich-Allzumenschlichen.

${ }^{643}$ Dies., "Educational Principles and Considerations: Collaborative Hyperdrama", $<\underline{\text { http://www.thetherapist.com/Explanation.html\#anchorCollaborativeHyperdrama }>, 15.10 .2003 .}$ 


\begin{tabular}{|c|c|c|c|}
\hline Patientenname & Sitzungen & Erste Sitzung & Letzte Sitzung \\
\hline Anna Green (W) & 102 & 15.07 .96 & 04.03 .99 \\
\hline Alex Rozzi & 76 & 12.05 .97 & 03.03 .99 \\
\hline The Anonymous Faxer & 60 & 24.07 .96 & 12.02 .99 \\
\hline Sylvia Bows (W) & 53 & 15.07 .96 & 14.10.97 \\
\hline Katherin Lippard & 48 & 21.04 .97 & 11.12 .98 \\
\hline Sharon Lough & 41 & 07.11 .97 & 19.02.99 \\
\hline Thomas Darden & 36 & 07.03 .97 & 15.02 .99 \\
\hline Christina Herald & 25 & 27.02 .97 & 04.12 .97 \\
\hline Rachel Tanner & 19 & 10.09 .97 & 20.11 .98 \\
\hline Eliza Raven & 18 & 11.02 .97 & 12.08 .97 \\
\hline Olivia Stillwell & 16 & 09.12 .97 & 21.07 .98 \\
\hline Kester Langford & 14 & 24.06 .97 & 26.05 .98 \\
\hline Joseph Mazurka (W) & 14 & 18.07 .96 & 18.12 .96 \\
\hline Sarah Wright & 14 & 09.09 .96 & 20.01 .97 \\
\hline Cassandra Evans & 12 & 23.07 .96 & 15.07 .97 \\
\hline George Landau & 12 & 20.01 .97 & 16.06 .97 \\
\hline Peter Hossfeld (W) & 11 & 06.01.97 & 15.11.97 \\
\hline Decker Jenkins & 11 & 10.04 .97 & 10.12 .97 \\
\hline Kelly Wiseling & 11 & 29.07 .98 & 09.12 .98 \\
\hline Phylis Birch & 10 & 03.09 .96 & 02.12 .96 \\
\hline Madeline Trent & 7 & 10.08 .98 & 10.11 .98 \\
\hline Lisa Benjamin & 6 & 24.03 .98 & 16.06 .98 \\
\hline Helen Gregory & 6 & 01.08 .96 & 26.12 .96 \\
\hline Herbert Michel & 6 & 14.07 .98 & 15.02 .99 \\
\hline Jesse Trent & 6 & 27.10 .97 & 03.08 .98 \\
\hline Hal Mainor (W) & 5 & 15.07 .96 & 08.04.97 \\
\hline Nina Alvidrez & 4 & 22.06 .98 & 13.08 .98 \\
\hline Darius Booth & 4 & 05.10 .98 & 02.11 .98 \\
\hline Samuel Eldrich & 4 & 21.05 .98 & 08.07 .98 \\
\hline Jerico Freeman & 4 & 03.03 .97 & 14.04 .97 \\
\hline Logan Marcas & 4 & 19.05 .98 & 23.06 .98 \\
\hline Lloyd Major (W) & 4 & 19.05 .97 & 07.04 .98 \\
\hline Alan Kurtz & 3 & 08.08 .97 & 29.08 .97 \\
\hline Nicole Ulreich & 3 & 30.03 .98 & 23.06 .98 \\
\hline Claire Steven & 2 & 08.12 .97 & 27.02 .98 \\
\hline
\end{tabular}

Wie ausgeprägt das durchschnittliche Engagement der Mitwirkenden war, läßt sich der vorstehender Tabelle der virtuellen Patienten entnehmen, die der besseren Übersicht halber nach absteigender Anzahl der Unterredungen mit Dr. Balis erstellt wurden. Die Daten der jeweils ersten und letzten Sitzung ergeben zugleich die Dauer der Teilnahme des Autors am Projekt. Die fettgedruckten Angaben mit einem eingeklammerten "W" weisen darauf hin, daß ein Mitglied der Familie Werby Schöpfer der jeweiligen Person ist; dieser Teil der Daten fließt deshalb nicht in die statistische Auswertung mit ein.

Staffelt man die Zahl der Sitzungen in drei Schritten von bis zu 10, von 11 bis 20 und über 20 Besprechungen, wird deutlich eine abfallende Tendenz erkennbar: In der ersten Gruppe finden sich 14 Patienten, in der zweiten 9 und in der dritten nur 
noch $6{ }^{644}$ Erstaunlich ist dabei, daß sich die Beiträge der Familie Werby relativ gleichmäßig über alle drei Gruppen verteilen. Es sind also nicht notwendigerweise die Initiatoren gewesen, die den Umfang des Projekts vorangetrieben haben. In diesem Zusammenhang wäre interessant zu erfahren, ob jene Kooperierenden mit einer geringen Zahl von Beiträgen von vornherein nur eine begrenzte Teilnahme eingeplant hatten, oder ob sie sich im Verlaufe ihrer Mitarbeit zu einem vorzeitigen Abbruch ihres Engagements entschlossen haben. Die Texte lassen mehrheitlich keine Rückschlüsse zu; von 35 Patientenakten sind lediglich 5 definitiv abgeschlossen: Thomas Darden und Logan Marcas erklären die Therapie offiziell für beendet, Joseph Mazurka fällt einem Mordanschlag zum Opfer, George Landau bittet um eine Therapiepause und auch Sarah Wright möchte vorläufig keinen neuen Termin vereinbaren. Bei allen weiteren Patienten ist den jeweils letzten Sitzungen kein Hinweis auf ein bevorstehendes Therapieende zu entnehmen. In zwei Fällen (bei Olivia Stillwell und Kester Langford) sollen die Gespräche sogar über eine räumliche Distanz hinweg per E-Mail weitergeführt werden, finden tatsächlich aber keine Fortsetzung, obwohl sich die gegebenen Umstände durchaus für einen Abschluß der Therapie angeboten hätten. Im Falle des Patienten Darius Booth wird die therapeutische Beziehung zwar nicht offiziell für beendet erklärt, ein Wendepunkt im Leben des Patienten läßt den Leser aber zweifeln, ob je eine Fortsetzung der Therapie erfolgen wird.

Um dem Leser vorliegender Arbeit ein möglichst eindrucksvolles Bild des webbasierten Dramas zu vermitteln, sei hier beispielhaft eine einzelne Figur, nämlich die des eben bereits genannten Büroangestellten Darius Booth, herausgegriffen und unter Zuhilfenahme der Patientenakte, der Behandlungsprotokolle sowie Dr. Balis' Vermerken zu Diagnose und Therapie charakterlich nachgezeichnet. ${ }^{645}$

${ }^{644}$ Die Tatsache, daß einige Projektteilnehmer mehrere Figuren bereitgestellt haben, kann bei dieser Auswertung vernachlässigt werden, da nur drei Kooperierende mehrere Patienten erschaffen haben; sie verteilen sich gleichmäßig über die Gruppe aller Teilnehmer und haben zudem ihren Schwerpunkt immer deutlich auf jeweils eine ihrer Figuren gelegt. Teddy Rosen: Sharon Lough (41 Sitzungen) \& Herbert Michel (6 S.); Derek Viehman: Decker Jenkins (11 S.) \& Claire Steven (2 S.); Sharon Milano: Olivia Stillwell (16 S.), Madeline Trent (7 S.) \& Nina Alvidrez (4 S.). Dies., Hg., The Company Therapist - Authors, $<$ http://www.thetherapist.com/Authors_All.html $>$, 15.10.2003. Pipsqueak Productions, Hg., The Company Therapist - File Cabinet, $<$ http://www.thetherapist.com/File Cabinet.html $>, 15.10 .2003$.

${ }^{645}$ Grundsätzlich ist das umfangreiche Material, das zu jedem einzelnen virtuellen Patienten zur Verfügung steht, je für sich geeignet, die Systematik des Forums The Company Therapist darzulegen und zugleich die engagierte und konsequente Mitarbeit der Kooperierenden zu dokumentieren. Um jedoch eine überproportionale Ausweitung der Ausführungen von vorneherein zu verhindern, wurde eine Figur ausgesucht, für die nur vergleichsweise wenig Material zur Verfügung steht. 
Die Akte des Patienten Booth wird am 05.10.1998 angelegt und dokumentiert eine Behandlung, die bereits nach vier Therapiesitzungen am 02.11.1998 abschließt. ${ }^{646}$ Die Materialien zu diesem virtuellen Patienten stammen nicht von einem Mitglied des festen Mitarbeiterstabes von The Company Therapist, sondern von einem freiwilligen Helfer namens Viktor Little. ${ }^{647}$ Im Gegensatz zu einigen anderen Kooperierenden des Projekts findet sich im Zusammenhang mit Little kein Verweis auf eine private Web-Seite, auf der genauere Informationen über seine Person vermerkt wären. Somit sind Rückschlüsse auf etwaige berufliche oder private Beziehungen zu Literatur, Software-Entwicklung oder Web-Design nicht möglich. Sofern die angegebene Adresse noch gültig ist, kann jedoch jeder Interessierte per E-Mail mit ihm persönlich in Kontakt treten.

Zum Zeitpunkt des ersten Gesprächs mit Dr. Balis ist Darius Booth, Jahrgang 1969, ursprünglich aus Australien stammend, seit ungefähr sechs Monaten Angestellter der Silicon Impressions Inc. Eigentlich fühlt sich Booth zum professionellen Komiker berufen, die geringe Zahl an Engagements erlaubt ihm jedoch nicht, seinen Lebensunterhalt ausschließlich mit dieser Tätigkeit zu bestreiten. Schuld hieran ist zum Teil Booth selbst: Es gelingt ihm nicht, sein Publikum zum Lachen zu bringen. Trotzdem ist er nicht bereit, sein Programm weniger intellektuell zu gestalten, mit der Folge, daß ihm bereits bei einigen Unterhaltungsshows gekündigt wurde. Aufgrund ihres beinahe belehrenden Charakters verschaffen ihm auch seine wenigen verbliebenen Auftritte in der Regel keine Anerkennung, sondern bedeuten im Gegenteil offene Ablehnung und Zurückweisung. Weil er mit diesen wiederholten Demütigungen nicht umzugehen vermag, sucht Booth den Psychiater Dr. Balis auf. Als Grund seines Erscheinens nennt er unspezifizierte Unzufriedenheit mit seiner Arbeit; über physische Beschwerden klagt er indes nicht.

Nach Dr. Balis' erstem Eindruck ist Booth ein ernsthafter, rastloser junger Mann, der offensichtlich dazu neigt, Lappalien maßlos überzubewerten. Seine vorläufige Diagnose lautet: "Delusional disorder with persecutory features"648 $-\mathrm{Be}$ -

\footnotetext{
${ }^{646}$ Pipsqueak Productions, Hg., The Company Therapist - Dr. Charles Balis' Files, $<$ http://www.thetherapist.com/File_Cabinet.html $>, 15.10 .2003$.

647 Dies., Hg., The Company Therapist - Authors, $<$ http://www.thetherapist.com/Authors_All.html $>, 15.10 .2003$.

648 Dies., Hg., The Company Therapist - Patient Form, $<$ http://www.thetherapist.com/Booth_File.html $>, 14.10 .2003$.
} 
wußtseinsstörung mit Zügen von Verfolgungswahn. Diese wie alle weiteren Erkenntnisse über Booth vermerkt Dr. Balis in seiner Patientenakte. Bei den Patientenakten handelt es sich um eine weitere Textsorte, die die Gruppe der Journals (sogenanntes Tagebuch), der Transcripts (Protokolle der Therapiesitzungen) und der Doctor's Notes (Auswertungen der Therapiesitzungen) erweitert. In den Akten werden nach einem festgelegten Schema zunächst die Personendaten wie Name, Geburtsdatum und berufliche Tätigkeit vermerkt, die physischen und psychischen Beschwerden zu Beginn der Therapie festgehalten, kurze Zusammenfassungen der Problemlage und des ersten Eindrucks notiert sowie die vorsichtige vorläufige Diagnose mit einem Hinweis auf die begleitende Medikation eingetragen.

Einen eigenen Eindruck von dem jeweiligen Patienten - also auch von Darius Booth - und seinen psychischen Problemen kann der Leser sodann mit Hilfe der wortgetreuen Protokolle der Sitzungen gewinnen. Hier geben die oben bereits genannten vier Mitschriften Aufschluß über den Dialogverlauf zwischen Psychiater und Patient.

Ganz im Sinne der Ankündigung von Werby und Werby auf deren Einstiegsseite sowie in ihrer Einführung - “There's a lot of content here to be explored." ${ }^{649}$ - bekommt der Leser mit den Sitzungsprotokollen sehr umfangreiches Material an die Hand. Dessen Aufarbeitung vermittelt ihm nicht nur Zugang zu dem Menschen Booth, sondern bringt ihm gleichzeitig die Belegschaft von Silicon Impressions Inc. nahe und verdeutlicht ihm das Verhältnis der Angestellten zueinander. Mit jedem Lesevorgang fügt sich ein weiteres Wissenselement zu den bereits erworbenen Erkenntnissen hinzu und formt das Gesamtbild aller Beteiligten. Darin eingeschlossen ist auch Dr. Balis selbst, den das Schicksal seiner Patienten teilweise stärker berührt, als der Neutralität seines Berufes zuträglich ist.

Ein Ausschnitt aus dem ersten Gespräch zwischen Booth und Dr. Balis mag einen Eindruck vom Sprachstil der beiden Dialogpartner und der Atmosphäre in Dr. Balis' Sprechzimmer vermitteln.

649 Dies., Hg., The Company Therapist, <http://www.thetherapist.com>, 14.10.2003. Dies., "The Company Therapist in General: The Premise", in: Dies., Hg., The Company Therapist,

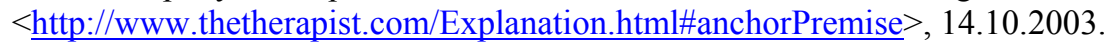


Transcript of $1^{\text {st }}$ Session between Charles Balis, M.D. and Mr. Darius Booth, Monday, October 5, 1998 at 3:00 pm.

Dr. Balis: [...] I assume it's your eventual goal to earn a living as a stand-up comedian?

Mr. Booth: Not necessarily.

Dr. Balis: The way you described it, I thought it was your primary goal.

Mr. Booth: It is. But the thing is I won't compromise. I have a vision, a path to follow, and I won't deviate even if I have to pay the ultimate price, even if it meant I had to go back to Big Jill, which I won't. Take the early explorers. Australia's history of European exploration is littered with desert corpses. Burke and Wills - statues of these brave and famous explorers can be found in every city - lugged a cast-iron bathtub a thousand miles into an uncharted desert and died of thirst five miles short of a pub. Before they set out, when they were packing, people said to Burke and Wills: "Whatever you do, don't take that huge tub with you." But they took it. And today, we salute what we still barely understand.

Dr. Balis: It does sound rather eccentric. So, from what you're telling me, stand-up comedy won't support you financially for the time being.

Mr. Booth: I haven't cracked it yet.

Dr. Balis: Meaning?

Mr. Booth: There's no laughter yet. At this stage, the crowds aren't responding to my routine. There's noise all right: the muttering and murmuring of dawning distress; the chain saw clarity of front-row invective; and always the unnecessary wailing from the back wall. But there's no laughter. And there's a growing list of venues from which I've been banned.

Dr. Balis: You've chosen a rather difficult path for yourself, Darius. [...] $]^{650}$

Die Wechselrede zwischen Arzt und Patient bildet den eigentlichen Hauptteil des webbasierten Dramas. Hier werden die menschlichen und sozialen Beziehungen bloßgelegt. Dabei dokumentieren die Dialoge, genauer die Zwiegespräche, die von den Autoren des Projekts zur Verfügung gestellt werden, gewissermaßen das Geschehen auf der Bühne des Company Therapist: Der Text versetzt den Leser in das "Theater [seiner] Vorstellungen". ${ }^{651}$

Da sich die gesamte Handlung im Sprechzimmer von Dr. Balis abspielt, sind Regieanweisungen zum Ort der Begebenheiten überflüssig. Hinweise zu Gestik, Mimik, Statur oder Physiognomie der Figuren wären für den Leser durchaus hilfreich gewesen, sind aber ebenfalls nicht vorhanden. Die Befindlichkeiten von Darius Booth als der Hauptfigur dieser Dramensequenz müssen sich allein aus

${ }^{650}$ Dies., "Transcript of 1st Session between Charles Balis, M.D. and Mr. Darius Booth", in: Dies., Hg., The Company Therapist - Darius Booth Transcripts ([ $\left.\left.{ }^{1} 05.10 .1998\right]\right)$, $<$ http://www.thetherapist.com/Booth_Session_100598.html > 14.10.2003.

651 Angelika Corbineau-Hoffmann, Die Analyse literarischer Texte: Einführung und Anleitung (Tübingen; Basel, 2002), 55. 
dem Dialogtext erschließen. Grundsätzlich wird der Leser nicht durch Monologe in die Gedanken der Figuren eingeweiht, das heißt es findet keine der beiden hier in Frage kommenden Formen des sogenannten Beiseite-Sprechens statt: Weder das monologische Beiseite-Sprechen (Aside), noch ein Beiseite-Sprechen ad spectatores (Addressing the audience).

Einzige neben den Dialogen existierende Informationsquelle sind die Doctor's Notes. Die Gedanken, die Dr. Balis nach seinen Gesprächen mit Booth aufzeichnet, die jenem gegenüber jedoch unerwähnt bleiben, werden dem Leser in diesem Abschnitt 'vertraulich' zur Verfügung gestellt. Auf diese Weise findet zugleich eine Kommentierung des 'Bühnen'-Geschehens statt, wobei diese allerdings nicht unmittelbar aus der Handlung heraus erfolgt. Da die Doctor's Notes jeweils direkt mit den Dialogen verknüpft sind, handelt es sich hier gewissermaßen um die hypertextuelle Variante des Aside.

Die Ausdrucksweise der beiden Dialogpartner ist - situationskonform umgangssprachlich, also durchaus realistisch. Als Psychiater versucht Dr. Balis, dem Problem seiner Patienten durch zurückhaltendes, aber nichtsdestoweniger konsequentes Fragen näherzukommen. Zu diesem Zweck gilt es zunächst herauszuarbeiten, welchen Vorstellungen und Zielen die Betreffenden verhaftet sind. Im Falle von Booth ist es notwendig, mehrmals nachzufassen, da dieser anfänglich statt therapieverwertbarer Auskünfte entweder nur äußerst kurze oder gar ausweichende Antworten gibt. An dieser Stelle meint man übrigens zu erkennen, daß der Dialog von einem Laien konzipiert worden ist; jeder Fachmann auf dem Gebiet der Psychiatrie hätte vermutlich vorzugsweise mit offenen Fragen operiert. Booth hingegen wird durch die geschlossenen Fragen eher dazu provoziert, sich hinter abwehrenden oder sibyllinischen Ausführungen zu verschanzen. Durchdrungen von dem Gedanken, den einmal eingeschlagenen Weg konsequent weiterzuverfolgen, versucht er, Dr. Balis die Bürde, aber auch die Notwendigkeit seines Vorhabens durch einen Vergleich begreiflich zu machen. Dieser scheint jedoch zu sehr auf die korrekte Beantwortung seiner Frage fixiert zu sein, um die Aussage von Booth angemessen zu bewerten. Wie sich im weiteren Verlauf der Therapie herausstellen wird, bleibt Booth noch lange Zeit ein Geheimnis für Dr. Balis.

Während die Dialoge das Geschehen unmittelbar wiedergeben, fassen alle darüber hinausgehenden weiteren Materialien die Gesprächsinhalte zusammen bezie- 
hungsweise geben sie aus Sicht von Dr. Balis wieder, so zum Beispiel im Falle der Doctor's Notes, in denen die Unterredungen analysiert und die Probleme diagnostiziert werden. Allerdings wurde bereits ausgeführt, daß die begleitenden Aufzeichnungen nicht von denselben Autoren stammen, die auch die Dialoge bereitstellten, sondern von Christopher Werby. ${ }^{652}$ Dies scheint zunächst nicht ganz verständlich, da wie man meinen möchte - niemand Inhalt und Intention der Sitzungen treffender und folgerichtiger wiedergeben kann als ihr Autor selbst, jedoch gewinnen die Analysen und Diagnosen dank dieser Arbeitsteilung tatsächlich an Glaubwürdigkeit. Ebenso wie der Psychiater Dr. Balis muß sich auch Christopher Werby mit einer fremden Person und einem ihm unbekannten Sachverhalt auseinandersetzen. Je weniger Werby mit diesen Hintergründen und eigentlichen Intentionen vertraut ist, umso überzeugender wirken seine allgemeine Vorsicht und Zurückhaltung sowie gelegentliche Urteilsschwächen oder gar Fehleinschätzungen.

Wie bereits angedeutet, wird Booth seinem Therapeuten im Laufe der Sitzungen zunehmend zum Rätsel, und es festigt sich bei Dr. Balis der Eindruck, sein Patient nehme seine Umwelt verzerrt war und sehe sie gewissermaßen durch ein Vergrößerungsglas, das Winzigkeiten riesig erscheinen läßt und gleichzeitig den Blick auf das Ganze verstellt. Bei dem Versuch, eine endgültigen Diagnose und damit auch eine Therapiemöglichkeit zu präsentieren, zieht der Psychiater auch physische Erkrankungen in Betracht und erwägt eine internistische Untersuchung. Zwischenzeitlich scheint sich eine Stabilisierung seines Patienten zu ergeben: Angespornt durch die positive Reaktion eines einzelnen Zuschauers seiner Show gelingt es Booth, - und auch hier zeigt sich sein Problem der maßlosen Übertreibung -, sich eine weitere Auftrittschance zu erbitten. Seine Hartnäckigkeit gegenüber den Verantwortlichen einer Unterhaltungsveranstaltung führt schließlich zum Erfolg. Booth erhält eine noch nicht definierte Anstellung in einer Kleinkunst- oder Schaubühne namens The Cha-Cha. Damit steht für ihn unwiderruflich fest, daß er Silicon Impressions Inc. verlassen wird, obgleich noch ungeklärt ist, welche berufliche Aufgabe auf ihn zukommt. Ob sich für Booth damit auch seine Besuche bei Dr. Balis gleichsam erledigt haben, geht aus dem Kontext nicht hervor, es ist aber davon auszugehen, daß die vermeintliche Schicksalswende zugleich den Abschied von der Therapie bedeutet.

${ }^{652}$ Pipsqueak Productions, Hg., The Company Therapist - File Cabinet, $<$ http://www.thetherapist.com/File_Cabinet.html $>, 15.10 .2003$. 
Mit Darius Booth hat sein Autor Victor Little eine Figur geschaffen, deren Gebrochenheit und Besessenheit von einer Vision des anderen, besseren Entertainments durch die Namenswahl - ob gewollt oder ungewollt, sei dahingestellt zusätzlich symbolisch überhöht wird.

So leistet die Figur des Darius Booth, dessen Vorname auf Altpersisch Darajawausch, "der das Gute bewahrt", zurückgeht, ${ }^{653}$ in gewisser Weise ihren Dienst an der Erhaltung des Guten, indem sie sich weigert, ihre künstlerische Integrität dem Massenkonsum zu opfern. Ähnlich Dareios I., auch Dareios der Große genannt, der den Herrscherthron erst nach seinem Sieg über den Magier Gaumata besteigen konnte, vermag auch Darius Booth sein Lebensziel erst dann zu erreichen, wenn es ihm gelingt, sich gegenüber seinen konkurrierenden Kollegen durchzusetzen, die das Publikum mit ihren Unterhaltungsprogrammen - gleich Magiern - in ihren Bann ziehen.

Der Familienname Booth könnte ebenfalls Hinweise auf die Mentalität seines Trägers geben. Welche Übersetzung des englischen Wortes booth man auch wählt, alle Bedeutungen erweisen sich als gleichermaßen charakteristisch für die Figur des verhinderten Alleinunterhalters, zum Beispiel ließe sich Booth in der Bedeutung von (Markt-)Bude/-Stand unschwer als Anspielung darauf verstehen, daß Booth auf dem Markt des Entertainment beharrlich seine handverlesene, hochwertige Ware anbietet, ohne $\mathrm{zu}$ registrieren, daß die maßgebliche Kundschaft durchschnittliche Erzeugnisse vorzieht. Unter Umständen hat Little bei der Wahl des Namens Booth auch des amerikanischen Schauspielers Junius Brutus Booth (1796-1852) gedacht, der zu den berühmtesten amerikanischen Bühnenschauspielern seiner Zeit gezählt haben soll, oder seines Sohnes John Wilkes Booth (1838-1865), der ebenso wie sein Vater und seine beiden Brüder Edwin Thomas und Junius Brutus jr. Bühnenschauspieler wurde und auf diesem Wege zu Popularität gelangte, heute aber vornehmlich als Attentäter Abraham Lincolns bekannt ist. $^{654}$

${ }^{653}$ Brockhaus - Die Enzyklopädie in vierundzwanzig Bänden, 20., überarb. und aktual. Aufl. (Mannheim, 1996-1999). Bd. 5, s.v. "Dareios".

${ }^{654}$ National Portrait Gallery, Smithsonian Institution, "Junius Brutus Booth, 1796-1852", in: Dies., Hg., Mathew Brady's Portraits: Images as History, Photography as Art, $<$ http://www.npg.si.edu/exh/brady/gallery/23gal.html >, 20.02.2004. The National Park Service, "A History of John Wilkes Booth", in: Dies., Hg., Ford's Theatre - National Historic Site ([108.12.1998)], <http://www.nps.gov/foth/booth.htm>, 20.02.2004. akademie.de asp GmbH, "John Wilkes Booth", in: Dies., Hg., Net-Lexikon, $<$ http://www.net-lexikon.de/John-Wilkes-Booth.html $>$, 20.02.2004. 
Ob Little Vor- und/oder Zunamen als 'sprechende' Namen zur Charakterisierung seiner Figur konzipiert hat, ist den Seiten des Company Therapist nicht zu entnehmen und bleibt damit letztlich der Phantasie des Rezipienten überlassen.

Mit seinen Dramensequenzen hat Little zweifellos zahlreiche gute Ideen und Anknüpfungspunkte in das Projekt eingebracht. Allerdings erweist sich diese Fülle letztlich als zu komplex, um in lediglich vier Sitzungen abgehandelt zu werden. Angesichts der Schwere der geschilderten Bewußtseinstörung wird der Therapieverlauf eindeutig zu schnell abgewickelt, was dem Tiefgang der Schilderungen notwendigerweise abträglich ist. Und auch wenn das Ende der Besuche bei Dr. Balis in der geschilderten Weise folgerichtig erscheint, bleibt es dennoch ein überstürzter Vorgang, der längerfristig, zum Beispiel durch unentschuldigtes oder mangelhaft entschuldigtes mehrfaches Fernbleiben von den Sitzungen, hätte vorbereitet werden müssen. ${ }^{655}$

\subsubsection{Auswertung}

Auch wenn der 'Fall Darius Booth' konsequenter hätte ausgearbeitet werden können, darf die Gesamtkonzeption von The Company Therapist als ausgesprochen stimmig und anregend beurteilt werden. Das Hypertext-System hält eine Fülle von Ressourcen bereit, die ebenso farbig wie realistisch erscheinen. Auch die Konzeption der thematischen wie chronologischen Nachvollziehbarkeit überzeugt: Der Leser kann entweder jede Figur einzeln erschließen, indem er die dazugehörigen Materialien assoziativ erschließt, oder aber er kann das Gesamtwerk sukzessive chronologisch durcharbeiten und dabei den Praxisablauf anhand von Dr. Balis' Terminkalender gewissermaßen nacherleben. In jedem Falle wird dem Leser eine Materialsammlung präsentiert, die in Umfang und Ausarbeitung beispielhaft ist.

Insofern konnte das Projekt im Hinblick auf die Zielsetzung der Herausgeber, ein neuartiges literarisches Produkt mit unterhaltsamem Charakter zu schaffen, zum Abschluß des Projekts im Jahre 1999 als ebenso erfolgreich beurteilt werden, wie es auch noch im Jahr 2003 als richtungweisend gelten konnte. Nur noch

655 Spätestens im Rahmen der vorletzten Sitzung wäre auch eine Andeutung, die Therapie abbrechen zu wollen, hilfreich gewesen. Die Behandlungspläne von Dr. Balis lassen zu diesem Zeitpunkt jedoch noch eindeutig auf einen längeren Therapiezeitraum schließen: "I think I'm going to have to embark on talk therapy where I neither condemn nor collude in Darius' delusions. After I gain his trust, I'll have to gently challenge his beliefs and show how they interfere with his life." Pipsqueak Productions, "Transcript of 3rd Session between Charles Balis, M.D. and Mr. Darius Booth", in: Dies., Hg., The Company Therapist - Darius Booth Transcripts ([ $\left.\left.{ }^{1} 19.10 .1998\right]\right),<$ http://www.thetherapist.com/Booth_Session_101998.html > 20.02.2004. 
bedingt berechtigt dürfte hingegen mittlerweile der didaktische Anspruch sein. Werby und Werby bekräftigen in ihren Ausführungen nachdrücklich den Wunsch, ein effektives edukatives Hilfsmittel zur Förderung literarischen Schaffens zu etablieren. Dies mag während der aktiven Phase des Projekts durchaus gegolten haben, als The Company Therapist Schreibinteressierten die Möglichkeit zur Steigerung ihrer schriftstellerischen Kompetenzen gab und ihnen Helfer und Korrektoren an die Seite stellte. Gegenwärtig erschöpft sich der didaktische Wert nurmehr in der Nachvollziehbarkeit der individuellen Fortschritte der Autoren hinsichtlich Stil, Folgerichtigkeit und Kreativität.

Während seiner Laufzeit vereinigte das Hypertext-System des Company Therapist in sich die entscheidenden Merkmale einer webbasierten Publikation, indem es unmittelbar, unausgesetzt und aktuell, interaktiv und kooperativ sowie mit externen Ressourcen aus dem World Wide Web verlinkt war. Aufgrund seiner Thematik gewährte es ungewöhnlich großen Spielraum für Kreativität und barg dadurch gleichzeitig ein hohes Identifikationspotential sowohl für Interagierende als auch für Kooperierende. Die Herausgeber haben mit ihrer Entscheidung für die Literaturgattung webbasiertes Drama und Festlegung auf ein psychotherapeutisches Szenario ein vorausschauendes Gespür für die Möglichkeiten und Chancen webbasierten Publizierens bewiesen.

Besonders deutlich zeigt das Beispiel des Company Therapist, wie andere Gattungen in das webbasierte Drama miteinfließen können. Werby und Werby haben Prätexte aus unterschiedlichsten Bereichen gewählt, so daß die Textsorten innerhalb des Werkes transzendieren. Durch die verschiedenartigen Texte, die sich neben den Dialogen finden, wie beispielsweise Patientenakten oder die Aufzeichnungen von Dr. Balis, kommt es zu einer Gattungsmischung.

Ungeachtet seines vorbildhaften Charakters in Intention, Konzeption und Realisation weist The Company Therapist als webbasiertes Drama in folgenden Punkten Schwächen auf:

1. Die internen Interaktionsmöglichkeiten des Forums waren gemessen an den multimedialen Gestaltungsmöglichkeiten des Web gering. Neben den visuellen Elementen wie Zeichnungen, Zeitungsausschnitten, Postkarten und Collagen hätten auch akustische Sequenzen zur Realismussteigerung des Dargestellten und zur Verstärkung der atmosphärischen Dichte eingesetzt werden können. Gerade die dialogische Präsentationsform hätte eine - zumindest auszugsweise - 
Umsetzung in das gesprochene Wort nahegelegt. Auf diese Weise wären die Figurenvielfalt des webbasierten Dramas sowie Charakter und Sprachduktus dieser Figuren wesentlich stärker zum Ausdruck gekommen.

Angesichts der speziellen Thematik wäre es zudem empfehlenswert gewesen, enzyklopädisches Material zur Verfügung zu stellen. So hätten zum einen ein Glossar speziell für alle im Forum des Company Therapist verwandten Fachtermini sowie zum anderen ein übersichtliches psychiatrisches Lexikon aufschlußreiche Referenzmedien darstellen können. Damit wären die Herausgeber nicht nur dem Leser entgegengekommen, indem sie ihm eine Hilfe für das Verständnis der Krankheitsbestimmungen des Psychiaters (Doctor's Notes) an die Hand gegeben hätten, sondern sie hätten gleichermaßen auch den Autoren beziehungsweise sich selbst in der Person des Dr. Balis mehr Entfaltungsmöglichkeiten bei der Gestaltung der Krankengeschichten beziehungsweise größeren Interpretationsspielraum bei der Konzeption der Diagnosen eröffnet.

2. Was den externen Bereich des Forums betrifft, machen sich die Herausgeber zwar die Hypertext-Systematik sowie die Unmittelbarkeit und allgemeine Verfügbarkeit des World Wide Web zunutze und erfüllen damit die entscheidenden Kriterien für eine webbasierte Publikation; der Aspekt der aktiven Vernetzung mit dem Veröffentlichungsmedium kommt dabei jedoch nur unzureichend zum Tragen: The Company Therapist beinhaltet bemerkenswert wenige Zugriffsmöglichkeiten auf Ressourcen im WWW. Der Link zur Homepage von Pipsqueak Productions, die im Grunde nur als ein Selbstdarstellungsmedium der Herausgeber gelten kann, sowie die von der Sponsorenseite ausgehenden Verweise auf die jeweiligen WebSeiten der fünf Förderer - ebenfalls ohne praktischen Nutzen für das Verständnis und die Fortentwicklung des webbasierten Dramas - bilden die einzigen externen Verknüpfungen des Forums.

Stichwort Hypertext: Für die effektive Nutzung und positive multimediale Fortentwicklung des Forums The Company Therapist war und ist die Kenntnis der Hypertext-Systematik von entscheidender Bedeutung. Aus diesem Grunde hätten die Herausgeber im Sinne sowohl der Interagierenden wie der Kooperierenden gehandelt, wenn sie eine übersichtliche Liste möglichst schrittweise aufeinander aufbauender Web-Ressourcen zur Definition, Konzeption und Rezeption von Hypertexten zur Verfügung gestellt hätten. Gerade vor dem Hintergrund des damaligen allgemeinen (eher noch geringen) Kenntnisstandes hin- 
sichtlich des - im Jahre 1996 noch vergleichsweise neuen - Publikationsmediums erhält die Bereitstellung umfangreichen Informationsmaterials besonderes Gewicht. Zum Zeitpunkt der Initiierung des Projekts war keineswegs ohne weiteres davon auszugehen, daß jeder Web-Besucher mit Struktur und Anwendung dieses Publikationsmediums vertraut war. Damit dürften Werby und Werby die Zielgruppe ihres Projektes nicht unerheblich eingeschränkt haben; möglicherweise ist dieser Effekt von ihnen aber auch ganz bewußt als einfaches Mittel der Selektion eingesetzt worden.

Stichwort webbasiertes Drama: Anknüpfend an die Liste der Web-Ressourcen zum Thema Hypertext hätte der Nutzer ganz allgemein über die Literaturgattung Drama und speziell über das webbasierte Drama in Kenntnis gesetzt werden können. Kenntnisse über Aufbau und Gestaltung hätte den potentiellen Autoren als Basis für ihr kooperatives Engagement dienen können. Es hätte ihnen Chancen und Grenzen dieser Literaturform aufgezeigt und geholfen, Fehler in der Konzeption ihrer Dramensequenzen zu vermeiden. Dies hätte den Autoren schon vorab mehr Selbstvertrauen und eventuell auch mehr Handlungsspielraum verliehen und den Mentoren gegebenenfalls einen Teil ihrer Arbeit erspart.

Stichwort San Francisco: Gerade zu Beginn des webbasierten Dramas, als sich Dr. Balis auf die Eröffnung seiner Praxis vorbereitete und zur besseren Orientierung in der neuen Stadt einen Spaziergang unternahm, hätte sich auch der Leser eine realistischere Vorstellung von San Francisco machen können, wenn beispielsweise Zugriff auf einen Stadtplan oder die Homepage der Stadt bestanden hätte. Auch Links zur örtlichen Presse oder anderen in den Dramenhandlungen auftauchenden Institutionen von San Francisco hätten das Geschehen illustrieren und noch realistischer gestalten können.

3. Die Inszenierung als Bühnengeschehen sowie die dialogische Gestaltung allein machen The Company Therapist noch nicht zu einem überzeugenden webbasierten Drama. Zwar dient es als Forum der Auseinandersetzung mit zeitspezifischen, allgemeinmenschlichen Problemen und entspricht damit durchaus dramaturgischem Konzept. Die Protagonisten der einzelnen Dramensequenzen verarbeiten in ihren Gesprächen alle ein persönliches Problem oder Schicksal, durch das sie sich selbst entfremdet fühlen oder an den Rand der Gesellschaft beziehungsweise teilweise sogar ins gesellschaftliche Abseits gestellt sind. Die einzelnen Dramensequenzen 
lassen allerdings zumeist ein definitives Ende, zumindest aber einen Höhepunkt oder sogenannten Aha-Effekt vermissen. Auf diese Weise geht ein entscheidendes Detail des traditionellen Dramas verloren. Während der Rezipient im Theater wie im Drucktext gezielt und erkennbar aus der Handlung entlassen wird, signalisieren ihm die jeweils letzten Therapiesitzungen zwischen Dr. Balis und seinen Patienten den Fortgang der Geschehnisse, der aber faktisch nicht erfolgt; mehrheitlich brechen die Dramensequenzen an irgendeinem Punkt unmotiviert $a b$. Ursächlich für derartige Konzeptionsschwächen ist vermutlich zum einen die Zusammensetzung der Autorenschaft, die größtenteils aus schriftstellerischen Amateuren ohne spezielle Erfahrungen auf dem Gebiet des (webbasierten) Dramas bestanden haben dürfte, zum anderen die mangelnde Nachbereitung seitens der Herausgeber und festen Mitarbeiter, die den Abschluß der kooperativen Beiträge als bindende Vorgabe für die Mitarbeit im Projekt hätten festsetzen sollen.

4. Der letzte Kritikpunkt betrifft die bereits diskutierte Urheberrechtsklausel, durch die den Kooperierenden alle Rechte an ihren Beiträgen entzogen wurden. Unbestritten ließe sich im nachhinein nur schwer bestimmen, mit wievielen Anteilen jeder einzelne Kooperierende am Gesamtwerk des Company Therapist beteiligt war. Dennoch paßt die Vorstellung, daß im Falle einer gewinnbringenden Verwertung des Werkes keiner der freiwilligen Mitarbeiter eine finanzielle Beteiligung erhielte, nicht in das Bild partnerschaftlichen Zusammenarbeitens. Auch dürfte die Zumutung, noch nicht einmal als (Mit-)Urheber erwähnt zu werden, der Kooperationsbereitschaft möglicherweise abträglich gewesen sein. Es ist erstaunlich, daß sich trotz der mehr oder weniger erzwungenen Einverständniserklärung doch noch so viele Freiwillige zu einer Teilnahme bereit erklärt haben. Um wirklich uneingeschränktes Engagement der Autoren gewährleisten zu können, sollte langfristig darüber nachgedacht werden, ob und wie das Urheberrecht bei den tatsächlichen Verfassern eines Hypertext-Elements verbleiben kann. Nur wenn ein Schriftsteller davon ausgehen darf, als geistiger Urheber seines Werkes identifiziert und anerkannt zu werden, wird er auch sein gesamtes Können einbringen. 


\subsubsection{M is for Nottingham?}

Das Projekt $M$ is for Nottingham? begann am 15. Mai 2002 und war auf eine Dauer von rund zwei Monaten angelegt. Seinen Höhepunkt fand es im Rahmen der vom 15. bis zum 17. Juli 2002 an der Universität von Nottingham stattfindenden internationalen Incubation 2-Konferenz zum Thema Writing and the Internet ${ }^{656}$. M is for Nottingham? war konzipiert als eine Mischung aus Übung zu kooperativem Schreiben, Kriminalspiel, Einführung in Geschichte und Sehenswürdigkeiten von Nottingham sowie real dargestelltem Drama. Es sollte Interessierten erlauben, sich im World Wide Web zu kreativem Austausch zusammenzufinden, um im virtuellen Bereich in und um Nottingham Anhaltspunkte oder Spuren zu einem mysteriösen Vorkommnis zu finden und adäquate Dramenfiguren zu schaffen. Aufgabe war es, ein rätselhaftes Verbrechen zu entschlüsseln. Der Verlauf der detektivischen Mitarbeit der Web-Gemeinschaft sollte zusammen mit der Auflösung des Mysteriums während des obigen Symposiums von Laienschauspielern dargestellt werden.

\subsubsection{Hintergründe}

Der Titel des Projekts $M$ is for Nottingham? entstand in Anlehnung an zwei literarische Vorbilder:

Zum einen erinnert er an die Titel einer Serie von Kriminalgeschichten der zeitgenössischen amerikanischen Schriftstellerin Sue Grafton. In einer alphabetisch fortschreitenden Reihe, beginnend mit $A$ is for Alibi und zum Zeitpunkt des Projektstarts im Mai 2002 angelangt bei $P$ is for Peril, schreibt Grafton seit dem Jahre 1982 Geschichten um und über die Privatdetektivin Kinsey Millhone. ${ }^{657}$

Eine andere Verbindung führt zu der Kurzgeschichte Death and the Compass, span. La muerte y la brújula, des argentinischen Autors Jorge Luis Borges. Schlüsselworte dieser Kurzgeschichte sind die Hinweise "The first letter [/second letter/last of the letters] of the Name has been spoken.", die jeden der Morde im Verlaufe der Geschichte nach Art einer Visitenkarte des Täters begleiten. Die Pro-

${ }^{656}$ trAce Online Writing Centre, Hg., Incubation 2 - The 2nd trAce International Conference on Writing and the Internet, $<$ http://trace.ntu.ac.uk/incubation/index2002.cfm $>, 22.10 .2003$.

${ }^{657}$ Im Oktober 2002 erschien Sue Graftons 17. Band dieser Reihe mit dem Titel $Q$ is for Quarry. Sue Grafton wurde 1940 in Louisville, Kentucky, geboren und studierte englische Literatur an der dortigen Universität. Sie lebt und arbeitet heute in Santa Barbara, Kalifornien und ist Präsidentin des Verbandes Mystery Writers of America. Henry Holt and Company, Inc., Sue Grafton Web Site ([ $\left.\left.{ }^{1} 1996\right]\right),<$ http://www.suegrafton.com/>, 24.10.2003. 
jektseiten von $M$ is for Nottingham? greifen jene Worte mit dem wiederholten Satz "The last letter of the Name has been written." auf. 658

Wie in den Geschichten von Grafton und Borges geht es auch bei $M$ is for Nottingham? um Mord, allerdings nicht um Mord an einem Menschen, sondern um Mord an dem traditionellen Medium Buch. Der Leser soll in diesem Fall nicht untätig und bloßer Zeuge der Überführung des Täters bleiben, vielmehr ist ihm eine aktive Rolle - sei es als Ermittler, sei es als Mitspieler oder als Koautor - zugedacht. Folgende Worte skizzieren die Ausgangssituation des webbasierten Dramas und ersuchen die Web-Besucher um Stellungnahme:

For some time now rumors have been surfacing - whispers here and there and even outright accusations - perhaps you have heard them. The word is that there has been a death concealed, or even a murder, within the literary corpus. Could this be true? Is the BOOK, indeed, dead? ${ }^{659}$

Der Leser erfährt des weiteren, der tödliche Anschlag auf das herkömmliche, papierne Buch sei möglicherweise in Nottingham erfolgt. Das Programmkomitee der Incubation 2-Konferenz habe in dieser Angelegenheit bereits einige vorbereitende Ermittlungen durchgeführt, die ergeben hätten, der Leichnam jenes erwähnten literarischen Korpus sei tatsächlich irgendwo im Bereich von Nottingham verborgen. Einige Stimmen mutmaßten, Hinweise zu diesem Fall fänden sich irgendwo in unterirdischen Gängen, Spukschlössern oder dunklen Kneipen, möglicherweise aber auch in den belebten Korridoren oder auf dem grünen Rasen der örtlichen Universität. Einer kühnen Behauptung zufolge hielten sich der oder die Täter vermutlich sogar ganz in der Nähe auf. Mit Hilfe der Web-Gemeinschaft hoffe man

${ }^{658}$ Marjorie Coverley Luesebrink u.a., "Borges Clue 1", in: Marjorie Coverley Luesebrink, Hg., $M$ is for Nottingham?, <http://califia.hispeed.com/Incubation2/borges.htm>, 23.10.2003. Luesebrink u.a., "Welcome", in: Luesebrink, Hg., $M$ is for Nottingham? - The Live Collaborative Mystery Drama for trAce Incubation 2, <http://califia.hispeed.com/IncubationDrama/nppresent2.htm>, 23.10.2003. - La muerte y la brújula, dt. Der Tod und der Kompaß, handelt von einem Mann, der von dem Gedanken besessen ist, den fremdverschuldeten Tod seines Bruders zu rächen, und hierfür einen perfiden Plan ersinnt. Die Visitenkartenmorde sind die drei Stationen auf dem blutigen Weg zu jenem vierten Mord, durch den der tote Bruder schließlich seine späte Genugtuung erhält. Jorge Luis Borges, "Der Tod und der Kompaß", in: Ders., Fiktionen, übers. von Karl August Horst, Wolfgang Luchting und Gisbert Haefs, (Frankfurt a.M., 1992 [Ficciones (Buenos Aires, 1974)]), $117-130$.

${ }^{659}$ Luesebrink u.a., "Your Invitation: How to participate", in: Luesebrink, Hg., $M$ is for Nottingham?, <http://califia.hispeed.com/Incubation2/welcome.htm>, 22.10.2003. 
nun, dem Geheimnis auf die Spur zu kommen und die endgültige Wahrheit über den angeblichen Tod des Buches herauszufinden. ${ }^{660}$

Die Idee zu diesem sogenannten whodunit ${ }^{661}$ war aus der seit langem schwelenden Debatte über das Ende der Druckliteratur erwachsen. Sue Thomas, künstlerische Direktorin des trAce Online Writing Centre ${ }^{662}$ der Universität von Nottingham und Mitinitiatorin und -gestalterin des Projektes, war in ihrer beruflichen Position selbst immer wieder Ziel von Vorwürfen geworden, Institutionen wie trAce seien für das Aussterben der gedruckten Medien verantwortlich. Thomas vermutete, die starke Zunahme schriftstellerischer Tätigkeiten im Web wecke diese Befürchtung bei vielen Autoren, eingeschlossen Vertreter der akademischen Welt, obgleich sie im Grunde genommen völlig unbegründet sei: “[O]nline writing will lead to more books that are better written". ${ }^{663}$

Um ihre eigene These zu untermauern und gleichzeitig einer Verhärtung der bestehenden Vorurteile vorzubeugen, beschlossen Sue Thomas und ihre Mitstreiter, darunter vor allem Marjorie Coverley Luesebrink ${ }^{664}$, die Beziehungen zwischen Buch, Wort, Text, Geschichte und Drama gründlich zu analysieren. Da es galt, eine möglichst breite Zielgruppe und damit vor allem auch die Skeptiker und Kritiker des Schreibens im elektronischen Medium zu erreichen, wollte man die Incubation 2-Konferenz, die erste internationale Veranstaltung für Schriftsteller im Web, als Gelegenheit zu öffentlicher Aufklärung nutzen. Es war vorgesehen, die Frage nach dem Ausmaß der Bedrohung traditioneller Bücher durch das Schreiben im Web zum Thema einer offiziellen Diskussion zu machen. Darüber hinaus sollte das

${ }^{660}$ Dies., "The Story", in: Luesebrink, Hg., $M$ is for Nottingham?, $<$ http://califia.hispeed.com/Incubation2/story.htm>, 23.10.2003.

661 Michael Arnone, "A British University Uses a Murder Mystery to Teach the Craft of Online Writing", The Chronicle of Higher Education, 29.05.2002, $<$ http://chronicle.com/free/2002/05/2002052901u.htm>, 27.09.2003.

662 Das trAce Inline Writing Centre ist eine Bildungseinrichtung mit alternativer Möglichkeit zum Fernstudium per WWW, die Interessierten Gelegenheit gibt, mit dem Medium World Wide Web zu experimentieren und neue literarische Werke zu schaffen. Das weltweit vorhandene schriftstellerische Potential soll dort kanalisiert und durch gezielte Förderung von Kreativität, kooperativem Arbeiten und Schreibtraining perfektioniert werden. Kurse und Workshops vor Ort sowie im WWW richten sich explizit auch an Kinder, denen ein eigener Bereich Kids on the Net vorbehalten ist. trAce Online Writing Centre, trAce Online Writing Centre - About, < http://trace.ntu.ac.uk/about/index.cfm>, 23.10.2003.

663 Arnone, "A British University Uses a Murder Mystery to Teach the Craft of Online Writing", $<$ http://chronicle.com/free/2002/05/2002052901u.htm>, 27.09.2003.

${ }_{64}$ Marjorie Coverley Luesebrink, Dozentin am amerikanischen Irvine Valley College, freiberufliche Journalistin und Verfasserin von computergestützter Literatur unter dem Künstlernamen M.D. Coverley. Marjorie Coverley Luesebrink, Marjorie Coverley Luesebrink: A Virtual, Vertical Bio (06.01.1997), <http://califia.hispeed.com/bio.htm>, 22.10.2003. 
WWW dazu genutzt werden, in Zusammenarbeit mit der Web-Gemeinschaft auf spielerisch-belehrende Weise die Hintergründe der Gerüchte um den Untergang der Druckkultur auszuleuchten und ihren Wahrheitsgehalt festzustellen. Die Vorliebe der Briten für Kriminalgeschichten und Detektivarbeit gab schließlich den Ausschlag, im Vorfeld der Konferenz ein Rätselspiel in Form eines kooperativen Schreibprojektes zu initiieren. ${ }^{665}$ Die geschichtsträchtige Stadt Nottingham mit ihren historischen Gemäuern und dem nahegelegenen Sherwood Forest bildeten in diesem Zusammenhang eine überzeugende Kulisse. Entwicklung und Ergebnisse des Projekts sollten im Rahmen der Konferenz aber nicht nur schauspielerisch nachgestellt werden; das Konferenzprogramm sah außerdem vor, daß ein Gremium die im Verlaufe des Projekts erzielten Meta-Erkenntnisse anhand des folgenden Fragenkatalogs diskutierte:

- "How can interactive, collaborative drama be structured?"

- "How can writers work together on the WWW?"

- "What elements of collaborative Web stories can be carried over into live drama?"

- "What interfaces arise as a result of the blending of the real with the virtual?"

- "What autonomies are possible for interactive agents?" 666

Das Projekt war als fünfstufiges Experiment konzipiert: ${ }^{667}$

1. Vorstellung des Projekts und Aufforderung zur Teilnahme

2. Auswahl aus bereits existierenden beziehungsweise Kreation neuer Figuren

3. Gegenseitiger Austausch der Projektteilnehmer und kooperatives Fortschreiben der Handlung

4. Ermittlungen vor Ort im Rahmen der Incubation 2-Konferenz in Nottingham

5. Auflösung des Geheimnisses in Form einer Theaterinszenierung

Der Ausgang des webbasierten Dramas war von Initiatoren und Gestaltern nach eigener Aussage keineswegs vorherbestimmt, sondern sollte allein von den Koope-

${ }^{665}$ Luesebrink räumt England auf dem Gebiet der Kriminalliteratur einen besonderen Platz ein: [T]he mystery genre - arguably a form most fully developed in England". Luesebrink u.a., "Welcome", <http://califia.hispeed.com/IncubationDrama/nppresent2.htm>, 23.10.2003

${ }^{666}$ Dies., "Critical Review”, in: Luesebrink, $\mathrm{Hg}$., $M$ is for Nottingham?, $<\underline{\text { http://califia.hispeed.com/IncubationDrama/criticalreview.htm }>, 22.10 .2003 .}$

667 Dies., "Description of the 'M is for Nottingham?' Project", in: Luesebrink, Hg., M is for

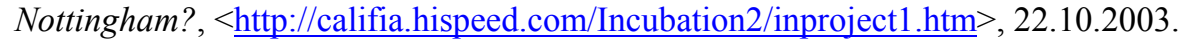


rierenden entwickelt werden. ${ }^{668} \mathrm{Da}$ sich die Organisatoren allerdings vorbehielten, gelegentlich neue Hinweise einzustreuen, ${ }^{669}$ ist die Vermutung nicht von der Hand zu weisen, daß letztlich doch Einfluß auf den Handlungsverlauf genommen wurde.

Zusammen mit ihrer Organisatorengruppe gingen Thomas und Luesebrink völlig neue Wege schriftstellerischen Schaffens. Es war vorgesehen, daß alle kooperativen Beiträge als Eintragungen in das Nachrichtenforum der Seiten eingehen sollten, die praktische Umsetzung zeigte aber bald, daß das Projekt nicht nur von seinem Konzept her experimentellen Charakter hatte, sondern daß bereits die Gestaltung zu einem Versuchsstück geriet; mehrmals mußten die Web-Seiten im Verlaufe der Dialogentwicklung modifiziert und den jeweiligen Erfordernissen angepaßt werden. ${ }^{670}$ Getestet wurden verschiedene Formen der DialogbeitragWiedergabe. Dies läßt sich auch heute noch bei Durchsicht der Aufzeichnungen und kritischem Vergleich ihrer einzelnen Abschnitte nachvollziehen.

\subsubsection{Verlauf}

Ebenso wie bei The Ultimate Writer wird der Web-Besucher auch bei $M$ is for Nottingham? mittels eines animierten Vorspanns in die Thematik des webbasierten Dramas eingeführt. Ein Lichtkegel wandert über eine abgedunkelte Seite und gibt den Blick zunächst jeweils nur auf einen Ausschnitt des Projekttitels " $M$ is for Nottingham? - a collaborative Mystery for Incubation 2" frei, bis er sich schließlich weitet und den gesamten Schriftzug erfaßt. Dem Titel ist das Bild einer mittelalterlichen Tischrunde unterlegt - vermutlich dem gesellschaftlichen Leben am Hofe von König John Lackland nachempfunden -, das im weiteren Verlauf als wesentliches Element der Handlung dient und leitmotivisch in den Kopfzeilen der Projektseiten erscheint. Dazu erklingt die Fantaisie-Impromptu, op. 66 von Frédéric Chopin, die den Web-Besucher während seines gesamten Aufenthalts auf den Seiten von $M$ is for Nottingham? begleitet. Es finden sich allerdings an keiner Stelle des Projekts Angaben zu dem Klavierwerk, obwohl es vermutlich ganz bewußt aufgrund seines aussagekräftigen Titels, der improvisierte schöpferische Kraft und spontane Phantasie beschwört, als Audioelement ausgewählt wurde.

\footnotetext{
668 Arnone, "A British University Uses a Murder Mystery to Teach the Craft of Online Writing", $<$ http://chronicle.com/free/2002/05/2002052901u.htm>, 27.09.2003.

669 Luesebrink u.a., "Your Invitation: How to participate", $<$ http://califia.hispeed.com/Incubation2/welcome.htm $>, 22.10 .2003$.

${ }^{670}$ Luesebrink, Hg., $M$ is for Nottingham? - Written Text of the Discussion List, $<\underline{\text { http://califia.hispeed.com/IncubationDrama/discussiontext.htm> }>22.10 .2003 . ~}$
} 
Die Gestalter haben die Seiten in teilweise bis zu fünf Teilflächen, sogenannte Frames aufgeteilt. Auf diese Weise war es ihnen zwar möglich, verschiedene Inhalte parallel darzustellen und dem Nutzer die Navigation innerhalb des Projektes zu erleichtern, gleichzeitig aber erscheinen die Seiten nun überladen und unübersichtlich. Darüber hinaus wirkt das Layout aufgrund Verwendung zahlreicher unterschiedlicher Schriftarten, -größen und -farben im Vergleich zur übrigen Präsentation des Forums verhältnismäßig unprofessionell.

In der untergliederten Bildschirmansicht befindet sich linker Hand eine Bedienungsleiste mit einem Verzeichnis des Seiteninhalts. Von dort aus erfolgt der Zugang zu allen Meta-Informationen des Projekts: Hintergründe, Zielsetzung und Konzeption, Sponsoren, eine Liste der meistgestellten Fragen, Teilnahmemodalitäten sowie eine Einführung in die Geschichte, die Figuren und die Handlungsorte. Aus dem Geschehen heraus kann der Nutzer über die Menüleiste rechter Hand navigieren und zwischen den Handlungsorten oder den Figuren wechseln beziehungsweise dem jeweiligen virtuellen Aufenthaltsort entsprechend Informationen im WWW abrufen. Zu Beginn des webbasierten Dramas wird dem Nutzer an dieser Stelle beispielsweise der Zugriff auf die Homepage des Nottinghamshire County Council ${ }^{671}$ oder wahlweise auch auf den Touristenführer zu Nottinghamshire $^{672}$ nahelegt.

Eine Fülle visueller Gestaltungsmittel wie beispielsweise Photographien, Gemälde, Zeichnungen oder Kartenmaterial verleihen dem Dargestellten hohe Anschaulichkeit und Wirklichkeitsnähe. Allen Figuren und Handlungsorten sind Illustrationen beigefügt, die nicht nur den besonderen Charakter dieser Dramenelemente unterstreichen, sondern vor allem dem Szenario eine hohe atmosphärische Dichte verleihen. Zudem sind die graphischen Darstellungen von großem Nutzen für die Verständlichkeit, insofern sie eine sofortige Zuordnung aller Figurenbewegungen innerhalb des Hyper-Raums sowie aller Kommentare und Ergänzungen zum Kriminalstück erlauben.

Mit der Gestaltung der Seiten in Schwarz und Ocker und dem Gebrauch von Licht und Schatten, nicht zuletzt aber auch mit dem erwähnten Bild der mittelalterlichen Tischgesellschaft haben die Projektgestalter versucht, dem web-

671 Nottinghamshire County Council, Nottinghamshire County Council Homepage (23.10.2003), $<$ http://www.nottscc.gov.uk/>, 24.10.2003.

${ }^{672}$ Nottinghamshire County Council, Official Visitors' Guide to Nottinghamshire, < $\underline{\text { http://www.robinhood.co.uk/index.htm }>, 24.10 .2003 . ~}$ 
basierten Drama die Aura des Altertümlichen und Mysteriösen zu verleihen. Durch Figuren wie "The White Lady", die als Geist von Newstead Abbey keine Ruhe findet, Eleanor of Aquitaine, die in Nottingham Castle gefangen gehalten wird, oder Mighty, den Hund von Jack the Ripper, wird dieser Eindruck ebenso unterstrichen wie beispielsweise durch die Handlungsorte Sherwood Forest, die The Ancient Caves under the City oder eben Nottingham Castle. Die Beschreibung der Situation zu Beginn der gemeinschaftlichen Untersuchungen soll Schaudern beim Leser erzeugen:

We collected reports of a White Lady, Byron-loving ghost of Newstead Abbey, who claimed to appear at will to writers. Others spoke of the Newstead Rooks, 'black monks' that haunted the ground and spoke aloud. Some witnesses alleged that they heard primeval screams at night from the ancient caves beneath the city. The boldest claim is that the ghostly murderer or murderers are nearby. When the moon shines clear over Nottingham Castle, even virtual visitors shiver at the implications. ${ }^{673}$

Insgesamt stellen die Herausgeber von $M$ is for Nottingham? in der Rubrik The Sites ${ }^{674} 11$ Handlungsorte zur Verfügung, nämlich The Great Hall ${ }^{675}$ als einen zentralen Sammelpunkt zum Erfahrungsaustausch mit den übrigen Beteiligten und zur Einstellung neuer Textbeiträge sowie zehn Schauplätze in und um Nottingham:

- The University

- The Gardens

- The Galleries of Justice

- Ye Olde Trip to Jerusalem Pub

- Newstead Abbey

- The Ancient Caves under the City

- Nottingham Castle

- Sherwood Forest

- The Council House and Square

- Green's Mill

Mittlerweile besteht nur noch die Möglichkeit der rekonstruierenden Aufklärung des angeblichen mysteriösen Mordfalles. Während der aktiven Phase des Projekts galt dagegen für die Erkundung der Schauplätze folgende Empfehlung:

${ }^{673}$ Luesebrink u.a., "The Story", $<$ http://califia.hispeed.com/Incubation2/story.htm>, 24.10.2003.

${ }^{674}$ Luesebrink, Hg., M is for Nottingham? - The Sites, <http://califia.hispeed.com/Incubation2/insite.htm>, 24.10.2003.

${ }^{675}$ Die Halle wird als ein riesiger hoher Raum von mittelalterlicher Schlichtheit und bar jeglichen Pomps beschrieben. Heinrich II veranlaßte den Bau, um dort seine Staatsangelegenheiten zu besprechen und Parlamentssitzungen abzuhalten. Aufgrund ihrer einstigen Tradition als Versammlungsort für die gesamte Gemeinschaft sollte die Great Hall auch im Rahmen des M is for Nottingham?-Projekts als Ort der Zusammenkunft dienen. Luesebrink, Hg., M is for Nottingham? - The Great Hall, $<$ http://califia.hispeed.com/Incubation2/sGreatHall.htm>, 28.10.2003. 
You can take a seat at the Head Table [...]

[ - eine Anspielung auf die besagte Tischgesellschaft, deren Bild leitmotivisch in den Kopfzeilen der Projektseiten wiederkehrt - ]

[...] and survey the doings, find out what your fellow sleuths have written, see the sites and look for clues, or you may choose or create a character and begin to search for evidence of the notorious corpse.... ${ }^{676}$

Die Besucher der Web-Seiten konnten zwischen drei Vorgehensweisen wählen: ${ }^{67}$

1. Zogen sie es vor, lediglich unauffällige Ermittlungen anzustellen, konnten sie während ihrer Navigation durch das Hypertext-System Hinweise zur Lösung des Kriminalfalles sammeln und ihre Informationen auf projektexternen Seiten im World Wide Web weiter vertiefen, beispielsweise auf der Homepage des Nottinghamshire County Council $6^{678}$, dem Informationsportal für die Grafschaft Nottinghamshire, oder der Regia Anglorum ${ }^{679}$, der Gesellschaft für historische Nachempfindung des frühen Mittelalters. Anschließend konnten sie ihre Erkenntnisse im Rahmen von Online-Konferenzen mit anderen Projektteilnehmern austauschen. ${ }^{680}$

2. Besucher, die mehr Einbindung in das Projekt wünschten, konnten sich die Zusammenhänge der Geschehnisse im Wege der Identifikation mit konkreten Personen beziehungsweise Figuren der englischen Geschichte oder Literatur erschließen, so zum Beispiel mit Richard the Lion Heart, Sherlock Holmes oder P.D. James, oder auch mit einem bestimmten Typus, wie beispielsweise dem des Butlers. Wem keine solche Figur zusagte, der hatte darüber hinaus die Möglichkeit, eine eigene zu entwickeln. In diesem Falle war er gehalten, Luesebrink per

676 Ebd., < $\underline{\text { http://califia.hispeed.com/Incubation2/sGreatHall.htm }>, ~ 25.10 .2003 . ~}$

677 Arnone, "A British University Uses a Murder Mystery to Teach the Craft of Online Writing", $<\underline{\text { http://chronicle.com/free/2002/05/2002052901u.htm }>, ~ 27.09 .2003 . ~}$

${ }^{678}$ Nottinghamshire County Council, Nottinghamshire County Council Homepage, $<$ http://www.nottscc.gov.uk/>, 24.10.2003.

679 Regia Anglorum Publications, Regia Anglorum - Anglo-Saxon, Viking, Norman and British Living History, $<$ http://www.regia.org/>, 28.10.2003. Die Interessengemeinschaft der Early Medieval Living History and Re-enactment Society, kurz Regia Anglorum, besteht aus einer Gruppe Enthusiasten, die sich zum Ziel gesetzt hat, Leben und Kampf der Angelsachsen, Wikinger, Normannen und weiteren Bewohner der britischen Inseln möglichst wirklichkeitsgetreu nachzustellen.

680 "As the readers progress through the story, they can click on clues in the text and visit Web sites to get more information. They can then go to online conference areas to share what they have learned with other participants." Arnone, "A British University Uses a Murder Mystery to Teach the Craft of Online Writing”, <http://chronicle.com/free/2002/05/2002052901u.htm>, 27.09.2003. 
E-Mail den Namen der neuen Figur zusammen mit den Antworten auf folgende Fragen zuzusenden:

- "Who are you?" (Name, geschichtliche Hintergründe und kurze Beschreibung der äußeren Erscheinung)

- "What brought you to Nottingham to search for a murder victim?"

- "What are your 'goals' for the investigation?"

- "What are your deepest 'fears' about what might happen to you?" (Trägt die Figur ein Geheimnis mit sich?)

- "What do you think happened to The Book?"681

3. Die engagierteste Form der Kooperation erschöpfte sich nicht in der Schaffung einer neuen Figur, sondern forderte die volle schriftstellerische Kreativität der Teilnehmer. Auf der Grundlage ihrer gewonnenen Erkenntnisse als agierende Figuren konnten die aktiv Mitwirkenden mit eigenen Textbeiträgen Hinweise zur Aufklärung des Falles geben sowie neue Handlungsstränge zum Gesamtwerk von $M$ is for Nottingham? hinzufügen.

Je nachdem für welchen Grad der Aktivität sich der Web-Besucher entschieden hatte, blieb er entweder Außenstehender ohne Einfluß auf das Geschehen oder er brachte sich - seiner Schreibbereitschaft entsprechend - mehr oder weniger nachhaltig in die Nachforschungen mit ein. Aus ihrer Erwartung machten die Organisatoren aber keinen Hehl: Zwar gestattete die Konzeption des webbasierten Dramas durchaus eine lediglich agierende beziehungsweise interagierende Nutzung; die Beschäftigung mit den diversen Handlungsorten und Figuren sei aber in erster Linie als eine inhaltliche Vorbereitung für eine intensivere, möglichst kooperative Auseinandersetzung mit $M$ is for Nottingham? gedacht:

You can read and browse through the sites and characters of Nottingham, but we hope you will decide to create or choose a character, participate in the discussion, and become a part of the writing team. ${ }^{682}$

Sofern er sich voll und ganz in das Geschehen einbrachte, bedeutete dies für den Kooperierenden allerdings ein unkalkulierbares Experiment. Es konnte durchaus zur Folge haben, daß seine neue Figur als tatverdächtig in den Fokus der übri-

${ }^{681}$ Luesebrink u.a., "Join In", in: Luesebrink, Hg., $M$ is for Nottingham?, $<$ http://califia.hispeed.com/Incubation2/injoin.htm>, 24.10.2003.

682 Dies., "Your Invitation: How to participate", $<$ http://califia.hispeed.com/Incubation2/welcome.htm $>$, 22.10 .2003 . 
gen Beteiligten geriet. Auf diese Möglichkeit wurde in der Einladung zur Mitarbeit ausdrücklich hingewiesen: "Become a Sleuth, Player, Writer, or Suspect!"683 Außerdem hieß es, in einem Kriminalstück seien die Dinge oftmals nicht so, wie sie schienen. Aus diesem Grunde wurde allen Beteiligten nahegelegt, den Exkurs in die virtuelle Welt inkognito durchzuführen ${ }^{684}$ : "It is safer if you assume a different identity for your foray into the world of mystery and intrigue." 685

Die Handlung beginnt mit dem Auftritt einer Gruppe von Ermittlern, die sich in dem alten Pub Ye Olde Trip to Jerusalem am Fuße von Nottingham Castle eingefunden hat, um die Begebenheiten der vorausgegangenen zwei Wochen zu besprechen:

Through the windows of the Ye Olde Trip to Jerusalem Pub, we see the characters discussing the activities of the last fortnight. Many of the sleuths have found the 'game' clues at the various sites around Nottingham, but they are keeping MuM about them, so far! The last letter of the Name has not yet been written! ${ }^{686}$

Das Geheimnis um das Schicksal des Buches läßt sich nur dadurch lüften, daß der Ermittelnde beziehungsweise Mitspieler oder Kooperierende alle Hinweise aufspürt, die an den zehn Handlungsorten in und um Nottingham versteckt sind. Unentbehrliches Requisit bei der Suche nach Anhaltspunkten ist - zumindest bei der überwiegenden Zahl der Schauplätze - die Computermaus, mit deren Hilfe die Darstellungen auf den jeweiligen Seiten gewissermaßen wie mit einer Lupe abgesucht werden können: "[S]ort through the clues with your own magnifying glass. " ${ }^{\circ 87}$ Jeder Ort liefert exakt ein einziges Indiz zur Lösung des Falles, und zwar in Form eines Buchstabens, der erst dann sichtbar wird, wenn der Cursor darüber gleitet. In die richtige Reihenfolge gebracht, ergeben alle zehn Buchstaben zusammengenommen schließlich das Lösungswort des Ermittlungsabschnitts The Sites.

${ }^{683}$ Dies., "The Story So Far", in: Luesebrink, Hg., $M$ is for Nottingham?, $<$ http://califia.hispeed.com/Incubation2/frame4.htm>, 24.10.2003.

684 Wie an späterer Stelle noch genauer erläutert werden wird, sind in $M$ is for Nottingham? drei verschiedene Sphären zusammengeführt: Die reale Gegenwart, die Kulisse des webbasierten Dramas mit insgesamt elf Handlungsorten in der Grafschaft Nottinghamshire und die unterschiedlichen Historien und Lebenskreise der fiktiven Charaktere und Persönlichkeiten der Geschichte Englands.

685 Dies., "The Story", < http://califia.hispeed.com/Incubation2/story.htm>, 24.10.2003.

${ }^{686}$ Ebd., 24.10.2003.

${ }^{687}$ Dies., “The Denouement”, in: Luesebrink, Hg., $M$ is for Nottingham?, $<$ http://califia.hispeed.com/IncubationDrama/npdenouement.htm $>, 23.10 .2003$. 
Die Handlungsorte sind jedoch nicht nur Fundstätten für Schriftzeichen, sondern bieten vor allem einen reichen Bestand an Tatsachen und Legenden, die dem Allgemeinwissen des Ermittelnden zugute kommen sollen. Gleichzeitig geben sie mehr oder weniger ostentative Denkanstöße zur Bedeutung schriftlicher Überlieferungen sowie zu Historie und Zukunft des Buches.

Alle zehn Schauplätze sind ähnlich strukturiert. Sie liefern in der Regel umfangreiche, teilweise sogar zweifach gestaffelte Ausführungen zu jenen Personen, Erscheinungen oder Einrichtungen, die eng mit der Geschichte des jeweiligen Handlungsortes verwoben sind. Mitunter sind sie geprägt von einer Atmosphäre der Mystik oder des Geheimnisvollen und kommen damit der britischen Leidenschaft für Spuk und Geister sowie Detektivarbeit entgegen.

Die Organisatoren haben für die zehn Abschnitte dieser Rubrik eine Fülle von Materialien aufbereitet, die den Ermittelnden mit anscheinend allen wissensund erzählenswerten Informationen zum Thema Nottinghamshire vertraut machen. Die Spannbreite reicht von touristischen Sehenswürdigkeiten (Nottingham ${ }^{688}$ und Major $O a k^{689}$ ) über einen sprachgeschichtlichen Abriß (Bedeutungsursprung des Wortes trip $^{690}$ ), historische Abhandlungen (Aufbruch zu den Kreuzzügen ${ }^{691}$, Entstehung der Magna Charta ${ }^{692}$ ), biographische Erläuterungen (Robin $\operatorname{Hood}^{693}$ und

${ }^{688}$ Luesebrink, Hg., M is for Nottingham? - The Council House and Square, $<$ http://califia.hispeed.com/Incubation2/sCouncil.htm $>, 28.10 .2003$. Wie sich der Stadtkern von Nottingham gegenwärtig präsentiert, erfährt der Ermittelnde über den externen Link dieses Abschnitts, der zu den Seiten von Britannia.com Touring Online führt. In dem virtuellen Reiseführer sind Vergangenheit und Gegenwart auf ansprechende Weise aufbreitet worden. Britannia.com, LLC, Britannia. com Touring Online - Tours: Robin Hood's Nottingham, $<$ http://www.britannia.com/tours/rhood/nottingham.html $>, 29.10 .2003$.

689 Auf einer Lichtung des Sherwood Forest befindet sich Englands größter und ältester Baum, die 800-jährige sogenannte Major Oak.

${ }^{690}$ Entgegen seiner heutigen Bedeutung geht das Wort Trip auf das Altenglische trip oder trypp, dt. Halt oder Stopp zurück. Luesebrink, Hg., M is for Nottingham? - Ye Olde Trip to Jerusalem $P u b,<$ http://califia.hispeed.com/Incubation2/sTrip.htm>, 28.10.2003.

691 Das Ye Olde Trip to Jerusalem Pub, ältestes Gasthaus Englands, wurde im Jahre 1189, dem Krönungsjahr von Richard I. Löwenherz, eröffnet und diente dessen Rittern als Sammelpunkt vor ihrem Aufbruch nach Jerusalem. Luesebrink, Hg., M is for Nottingham? - Ye Olde Trip to Jerusalem Pub, <http://califia.hispeed.com/Incubation2/sTrip.htm>, 28.10.2003.

692 Die königliche Allmacht von John Lackland wurde im Jahre 1215 durch ein geschichtsträchtiges Vertragswerk entscheidend eingeschränkt; Adel und Klerus nötigten den König zur Unterzeichnung der sogenannten Magna Charta, die entscheidenden Einfluß auf die weitere Gesetzgebung hatte. Luesebrink u.a., "Further Sherwood Forest Adventures 2", in: Luesebrink, Hg.,

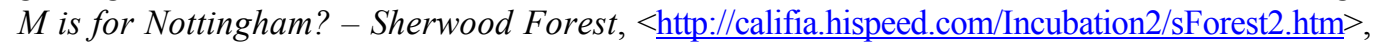
29.10.2003.

693 Der Marktplatz von Nottingham war Austragungsort des alljährlichen Wettbewerbs im Bogenschießen, bei dem ein goldener und ein silberner Pfeil zu gewinnen war. Auf einer dieser Veranstaltungen versuchte man, Robin Hood eine Falle zu stellen, doch er gewann die Ausscheidung und konnte entkommen. Luesebrink, Hg., M is for Nottingham? - The Council House and

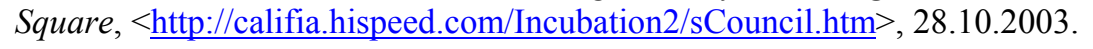


George Green ${ }^{694}$ ) und städtebauliche Auskünfte (Gewölbe unter der Stadt Nottingham $^{695}$ ) bis hin zu technischen Details aus den Bereichen Festungsbau und Kriegsführung (Nottingham Castle ${ }^{696}$ ) sowie Industrie (Mühlen ${ }^{697}$ ).

Anknüpfend an die historisch-legendenhaften Darstellungen ergehen sich die Organisatoren im allgemeinen in verschlüsselten Andeutungen über das Medium Buch. In einem letzten Absatz finden sich schließlich unter der Überschrift “About..." beziehungsweise "More about..." komprimierte Fakten zu den entsprechenden Schauplätzen, die über die Verknüpfungen der externen Links in der rechten Menüleiste nach Wunsch vertieft werden können. Je nach Themenverwandtschaft sind die Handlungsorte auch untereinander verbunden und gestatten somit auch eine assoziative Exploration der Rubrik The Sites.

Stellvertretend für alle zehn Abschnitte seien zwei Beispiele herausgegriffen und vorgestellt:

${ }^{694}$ In einer Art Empfehlungsschreiben aus dem Jahre 1828 wird George Green als ein ca. 26 Jahre alter, zurückhaltender Mann beschrieben, der sich schon von Kindheit an in außergewöhnlich hohem Maße für Mathematik interessiert habe; trotz seiner einfachen Schulbildung habe er es als Autodidakt zu erstaunlichen mathematischen Fertigkeiten gebracht. Green gilt heute als einer der bedeutendsten Mathematiker Großbritanniens. Luesebrink, Hg., M is for Nottingham? - Green's Mill, $<$ http://califia.hispeed.com/Incubation2/sGMill.htm>, 28.10.2003.

${ }^{695}$ Bezeichnenderweise wird Nottingham in seiner ersten historischen Erwähnung mit der Bezeichnung Tigguocobauc ("the house of caves") geführt. Andrew Nicholson, "Meaning and Origin of the Words: Shire and County", in: Ders., Hg., Nottinghamshire History and Archaeology - Nottingham and Nottinghamshire (09.05.2003), <http://www.nottshistory.org.uk/swinnerton1910/chapter1.htm>, 31.10.2003. Die Stadt ist über rund 200 Gewölben errichtet, die in den Buntsandstein getrieben sind und für die unterschiedlichsten Bereiche des Lebens genutzt wurden, zum Beispiel als Wohnraum oder Gefängnis oder auch als Arbeitsort für handwerkliche Tätigkeiten wie Fischverarbeitung oder Mälzen. Aufgrund ihrer konstanten Temperaturverhältnisse eigneten sie sich besonders für die Herstellung von Bier. Luesebrink, Hg., $M$ is for Nottingham? The Ancient Caves Beneath the City, $<$ http://califia.hispeed.com/Incubation2/sCaves.htm>, 28.10.2003.

${ }^{696}$ Der Abschnitt Nottingham Castle enthält detaillierte Beschreibungen der Angriffsmöglichkeiten auf die Festung zu Zeiten König Richards im Jahre 1194. Luesebrink, Hg., M is for Nottingham? Nottingham Castle, <http://califia.hispeed.com/Incubation2/sCastle.htm>, 28.10.2003. Weiterführende Informationen zu diesem Thema stellt das Archiv der Sherwood Times, zugänglich über den externen Link des Bereichs, in Form fingierter Berichterstattungen aus der Zeit ab 1190 bereit. "Nottingham Castle", Sherwood Times, $<$ http://www.times1190.freeserve.co.uk/castle.htm>, 29.10.2003.

${ }^{697}$ Green's Mill wurde zu Beginn des 19. Jahrhunderts errichtet und diente über 60 Jahre lang zur Produktion von Mehl und Pferdefutter. Aufgrund des wachsenden Konkurrenzdrucks durch größere und effizientere dampfgetriebene Walzwerke wurde sie schließlich aufgegeben und verfiel allmählich. Luesebrink, Hg., M is for Nottingham? - Green's Mill, $<$ http://califia.hispeed.com/Incubation2/sGMill.htm $>$, 28.10.2003. 
The Gardens ${ }^{698}$ : Die Gartenanlage, um die es sich hier handelt, ist der Park von Clifton Grove, Schauplatz der bekannten Romanze zwischen Paul Morel und der verheirateten Clara Dawes, den heimlich Liebenden des Roman Sons and Lovers von David Herbert Lawrence. ${ }^{699}$ Daran anknüpfend wird die Frage aufgeworfen, ob die Unterdrückung eines literarischen Werkes möglicherweise einem Mord gleichkomme; immerhin könne es durchaus sein, daß es sich bei dem hingeschiedenen Buch um das notorische Buch Lady Chatterley's Lover handele.

Nachdem D.H. Lawrences Roman auf diese Weise ins Visier des Ermittelnden geraten ist, führt die Suche nach dem Buchstaben des Lösungswortes zu einer der Hauptfiguren des Romans, dem zurückgezogen im Wald von Wragby lebenden Wildhüter Oliver Mellors und dessen Hütte: "The Gamekeeper's Cottage - the Clue is N."700 Die Entscheidung der Organisatoren für den Buchstaben "N" als Zwischenlösung dieses Ermittlungsabschnitts läßt sich möglicherweise damit erklären, daß die Natur (" $n$ "ature) eine Schlüsselrolle spielt als Zufluchtsort für Mellors und als elementare Kraft, die sich aufgrund der Liebe zu dem Wildhüter in Constance Chatterley Bahn bricht. ${ }^{701}$

${ }^{698}$ Luesebrink, Hg., $M$ is for Nottingham? - The Garden, $<$ http://califia.hispeed.com/Incubation2/sGarden.htm $>$, 28.10.2003.

${ }^{699}$ Eine Formulierung in diesem Abschnitt deutet darauf hin, daß an dieser Stelle ursprünglich eine Verknüpfung mit dem entscheidenden Textauszug aus Sons and Lovers vorlag: "And if you find the clue on this page, you can read the love scene that happened right below your residence halls at Nottingham Trent, on the River Trent - at least in a book. You can click on the clue in this Paragraph to be plunged into the love scene between Paul and Clara." Luesebrink, Hg., M is for Nottingham? - The Garden, < http://califia.hispeed.com/Incubation2/sGarden.htm>, 28.10.2003. Seit Projektende scheint dieser Link außer Kraft gesetzt zu sein. Damit ist ein wesentliches Element dieses Projektbereichs verloren gegangen, das eine Zusammenführung von fiktiver und realer Landschaft ermöglicht hätte.

${ }^{700}$ Luesebrink, Hg., M is for Nottingham? - The Garden, $<$ http://califia.hispeed.com/Incubation2/sGarden.htm $>, 28.10 .2003$.

701 Durch die Vereinigung mit Mellors findet Connie zu ihrem wahren Ich: "She felt, now, she had come to the real bedrock of her nature, [...]." D.H. Lawrence, Lady Chatterley's Lover, vollst. u. unzens. Ausg. (London u.a., 1960), 228. 


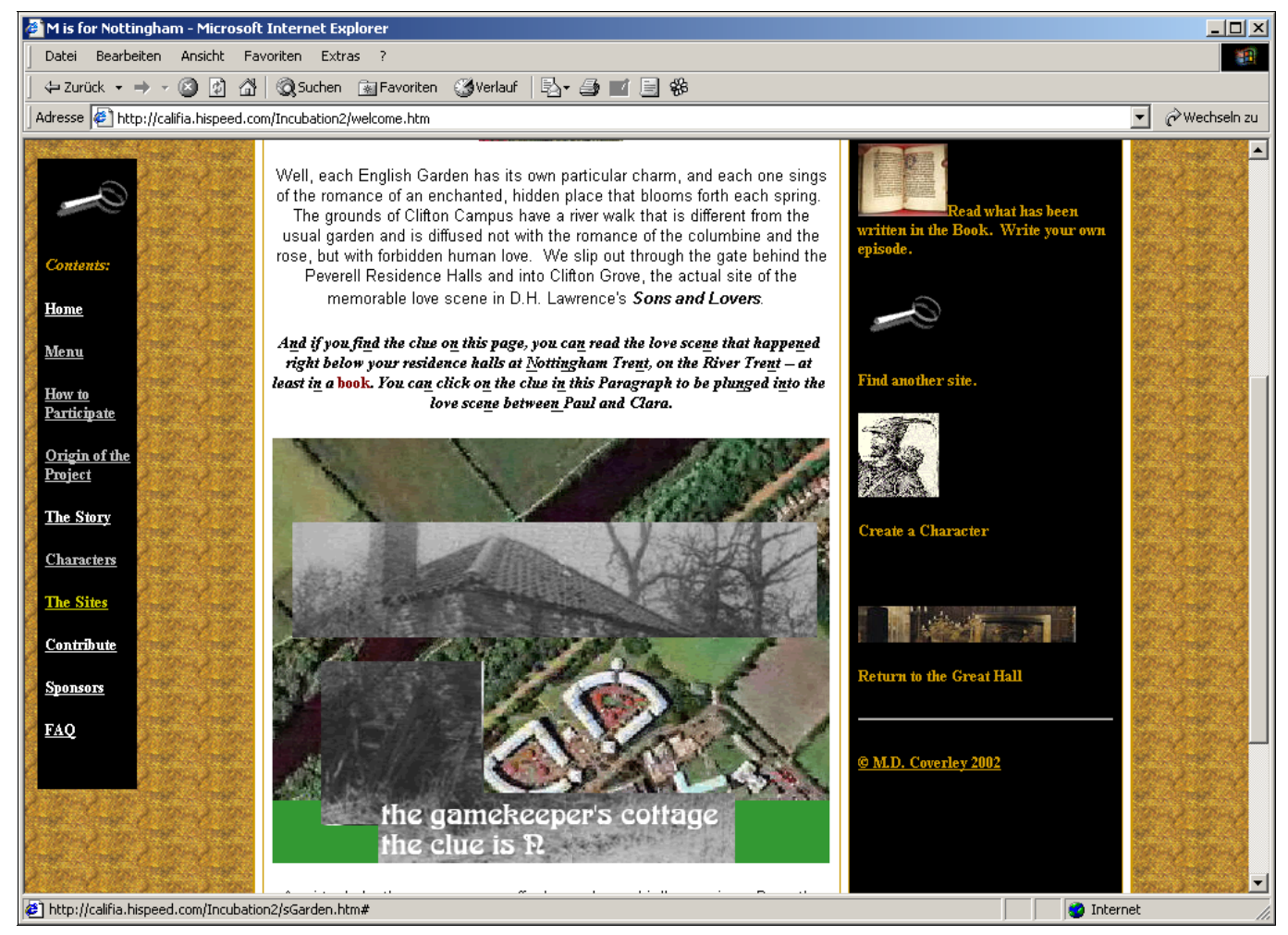

Abb. 7: 'M is for Nottingham? - The Gardens'

Der Entschluß der Organisatoren zum Rückgriff auf D.H. Lawrence und seine beiden Romane kommt nicht von ungefähr. In Eastwood in der Nähe von Nottingham geboren und aufgewachsen, ist Lawrence gewissermaßen der berühmte Sohn Nottinghamshires, zu dessen Ehren und Gedenken an der Nottingham Trent University seit rund 50 Jahren zwei Sammlungen aufgebaut und gepflegt werden, die D H Lawrence Manuscripts Collection und die D H Lawrence Printed Collection. ${ }^{702}$ Diese Sammlungen stellen mittlerweile eine der bedeutendsten internationalen Informationsquellen für die D.H. Lawrence-Forschung dar. Die Materialien liegen allerdings nicht in digitalisierter Form vor, vielmehr enthält der Informationsführer lediglich Beschreibungen der Sammelbestände, die vor Ort eingesehen werden müssen. ${ }^{703}$ Es gibt jedoch Hinweise auf andere Web-Seiten, die einen direkten Zugang zu Werken des Autors im WWW bereitstellen. ${ }^{704}$ Der Zugriff auf den elektronischen Informationsführer der Universität

702 The Nottingham Trent University, Hg., The University of Nottingham Information Services Manuscripts and Special Collections: D H Lawrence Resources, $<\underline{\text { http://mss.library.nottingham.ac.uk/dhl_home.html }>, 29.10 .2003 . ~}$

703 Dies., Hg., The University of Nottingham Information Services - D H Lawrence Resources: D H Lawrence Collections at the University of Nottingham, $<$ http://mss.library.nottingham.ac.uk/dhl_collections.html $>, 29.10 .2003$.

704 Dies., The University of Nottingham Information Services - D H Lawrence Resources: D H Lawrence Links, < http://mss.library.nottingham.ac.uk/dhl links.html\#literary>, 29.10.2003. 
von Nottingham ist aus den Ausführungen dieses Handlungsortes heraus über die rechte Menüleiste herstellbar.

Der Leser erfährt im Abschnitt The Gardens nicht nur etwas über eine Persönlichkeit der englischen Literatur und zweier ihrer Werke, was ihm möglicherweise Anlaß zu weitergehender Lektüre geben wird. Sue Thomas und die Universität von Nottingham nutzen auch die Gelegenheit, für die beiden oben genannten Sammlungen zu werben und die Erinnerung an Lawrence zu pflegen.

- The Galleries of Justice ${ }^{705}$ : Der Ermittelnde erhält in diesem Abschnitt ausführliche Hintergrundinformationen über Straftaten, ihre Verfolgung durch die Justiz und das Schicksal der Verurteilten. Einrichtung und Ausstellungsgegenstände des im Jahre 1995 eröffneten preisgekrönten Justizmuseums von Nottingham geben Zeugnis von englischer Jurisdiktion in den letzten drei Jahrhunderten. In dem Bemühen um eine möglichst realistische und interaktiv erlebbare Darstellung versucht die Museumsleitung, die Vergangenheit durch originalgetreue Ausstattung und nachgestellte Gerichtsszenen wieder lebendig werden zu lassen.

Angesichts dieser Kulisse wird der Ermittelnde vermutlich annehmen, er erhalte hier einen konkreten Anhaltspunkt, der helfe, das vermeintlich tödliche Attentat auf das Buch aufzuklären. Die Projektorganisatoren bestärken ihn in dieser Annahme, indem sie darauf hinweisen, daß bis zu diesem Punkt zwar weder ein Schuldiger gefaßt worden sei, noch Klarheit bestehe, ob überhaupt ein Verbrechen vorliege; der Rückblick in die Vergangenheit sei aber dennoch eine wesentliche Voraussetzung für eine erfolgreiche Ermittlungsarbeit.

Einen konkreten Hinweis auf das Buch enthält dieser Abschnitt letztlich nicht. Wohl aber wird unter anderem die im Abschnitt The University aufgeworfene Frage - "Why Snottingham?"706 - beantwortet: An jener Stelle, an der sich das Justizmuseum auf einem Felsen an einer der ältesten Durchgangsstraßen Nottinghams befindet, kam es zur ersten Ansiedlung von Angelsachsen, auf deren Oberhaupt Snot die Bezeichnung Snottingham vermutlich zurückgeht.

705 Luesebrink, Hg., $M$ is for Nottingham? - The Galleries of Justice, $<$ http://califia.hispeed.com/Incubation2/sGalleries.htm> , 28.10.2003.

706 Dies., Hg., M is for Nottingham? - The University, $<$ http://califia.hispeed.com/Incubation2/sUniver.htm>, 24.10.2003. 
Außerdem äußern die Projektorganisatoren - und dies führt den Ermittelnden wieder zurück zur Strafgerichtsbarkeit - deutliche Sympathien für die Angeklagten wegen der ihnen zuerkannten unverhältnismäßig harten Strafen; so ist beispielsweise von "harsh justice"707 die Rede. Die Abhandlung über die wechselvolle Geschichte der Galleries of Justice ${ }^{708}$, zu der der Ermittelnde über die externe Verknüpfung dieses Projektabschnittes geleitet wird, bestätigt dies durch ausgewählte Beispiele von ungewöhnlich harter oder fehlerhafter Jurisdiktion. Es entsteht der Eindruck, als sei die Geschichte der Rechtsausübung eine Geschichte der Willkür und des Unrechts.

Der versteckte Satz mit dem zusätzlichen Lösungsbuchstaben liefert schließlich den entscheidenden Brückenschlag zum Thema Buch: "The Clue is $\mathrm{T}$ - Tragedy, Truth, the Word Triumphs, Because We Have Truth in Books."709

Im Laufe der Bearbeitung aller zehn Schauplätze erkennt der Ermittelnde nach und nach, daß es nicht darum geht, einen der Handlungsorte als Tatort zu identifizieren oder an einem von ihnen das totgesagte Buch zu finden. Die Ausführungen weisen sogar darauf hin, daß es sich gar nicht um den Untergang eines ganz bestimmten Buches oder einer speziellen Gruppe von Büchern handelt, zum Beispiel - so ein Hinweis im Bereich The Gardens - um zensierte Literatur, sondern daß es um das Buch schlechthin, um das Druckwerk als Speicher universalen Wissens geht. Im Vordergrund stehen somit grundsätzliche Überlegungen zu Ursprung und Gebrauchswert von Büchern.

${ }^{707}$ Luesebrink, Hg., $M$ is for Nottingham? - The Galleries of Justice, $<$ http://califia.hispeed.com/Incubation2/sGalleries.htm>, 28.10.2003.

${ }^{708}$ Louise Connell, "A Just Measure of Pain? - The Galleries of Justice Nottingham", in: The Incorporated Council Of Law Reporting For England \& Wales, Hg., The ICLR Home Page, $<$ http://www.lawreports.co.uk/GalleriesofJustice.htm $>, 29.10 .2003$.

${ }^{709}$ Luesebrink, Hg., $M$ is for Nottingham? - The Galleries of Justice, $<$ http://califia.hispeed.com/Incubation2/sGalleries.htm $>, 28.10 .2003$. Wer wollte auch bestreiten, daß Bücher unterschiedliche Formen der Wahrheit enthalten können: So sind im Gesetzbuch jene Richtlinien niedergelegt, die dem Staat zur Wahrheitsfindung dienen. Die Bibel wiederum - das sogenannte Buch der Bücher - enthält die Offenbarung Gottes, die alle christlichen Kirchen und Gemeinschaften als ihre Wahrheit begreifen. Schließlich gibt jeder Autor eines Buches grundsätzlich - unter Umständen auch ungewollt - einen Teil von sich selbst preis; insofern ist sein Werk immer auch ein Dokument seines wahren Ichs. 
Dementsprechend unergiebig ist letztlich auch der Ausgang des Such- und Puzzlespiels. Das Lösungswort des Ermittlungsabschnitts The Sites lautet ${ }^{710}$, ein Ergebnis, das sich angesichts der bedeutungsvollen Thematik und in Anbetracht des erheblichen Zeitaufwandes, der mit den Recherchen verbunden ist, vergleichsweise banal ausnimmt. Das Resultat hat keinerlei Aussagekraft und verweist lediglich auf den Ort, an dem sich der Nutzer virtuell schon seit Beginn seiner Beschäftigung mit $M$ is for Nottingham? befindet. Im Gegensatz zu den Denkanstößen, die einigen Handlungsorten $\mathrm{zu}$ entnehmen sind, ergibt das Lösungswort keine neuen Aufschlüsse. Das Mysterium bleibt auch nach Bearbeitung dieses Abschnitts unentschlüsselt.

Statt dessen ist es der (ursprünglich) dynamische Teil des webbasierten Dramas, der Aufklärung verspricht. Der Gedankenaustausch, den die Kooperierenden im Verlaufe einer knapp zweimonatigen Kreativphase in der Rolle ihrer Figuren entwickeln, bildet gewissermaßen das Hauptelement von $M$ is for Nottingham?. Die Handlung entfaltet sich hier aus dem Gedankenaustausch von insgesamt 22 Figuren $^{711}$, deren Daten in der Rubrik Characters abgelegt sind. ${ }^{712} 16$ von ihnen wurden während des Dialogverlaufs neu in das Geschehen eingebracht, die übrigen waren bereits zu Beginn des Projekts Teil des Szenarios, wie beispielsweise “The White Lady”, Robin Hood oder George Green. ${ }^{713}$

710 " $[. .$.$] visit all of the 'virtual' clue locations and solve the initial puzzle - the letters of the$ word NOTTINGHAM." Luesebrink u.a., "Critical Review - Audience (Conference Attendees)", in: Luesebrink, Hg., $M$ is for Nottingham?,

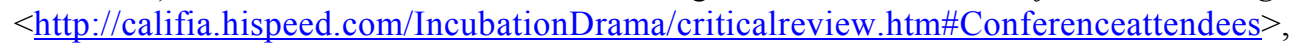
22.10 .2003 .

711 Es sei darauf hingewiesen, daß die Organisatoren von 22 Charakteren sprechen, die Liste in der Rubrik Characters hingegen 23 beteiligte Figuren enthält. Luesebrink u.a., "The Denouement", $<$ http://califia.hispeed.com/IncubationDrama/npdenouement.htm $>, 28.10 .2003$.

712 Luesebrink, Hg., $M$ is for Nottingham? - Characters, $<$ http://califia.hispeed.com/Incubation2/incharacters.htm $>, 22.10 .2003$.

713 “The White Lady" spielt als ruheloser Geist eine entscheidende Rolle im Abschnitt Newstead Abbey (Luesebrink, Hg., $M$ is for Nottingham? - Newstead Abbey, $<$ http://califia.hispeed.com/Incubation2/sAbbey.htm $>, 28.10 .2003)$, Robin Hood als legendärer Held des Schauplatzes Sherwood Forest (Luesebrink, Hg., M is for Nottingham? - Sherwood Forest, $<$ http://califia.hispeed.com/Incubation2/sForest.htm $>, 28.10 .2003)$ und George Green als Handlungsträger von Green's Mill (Luesebrink, Hg., M is for Nottingham? - Green's Mill, $<\underline{\text { http://califia.hispeed.com/Incubation2/sGMill.htm }>, 28.10 .2003) . ~}$ 


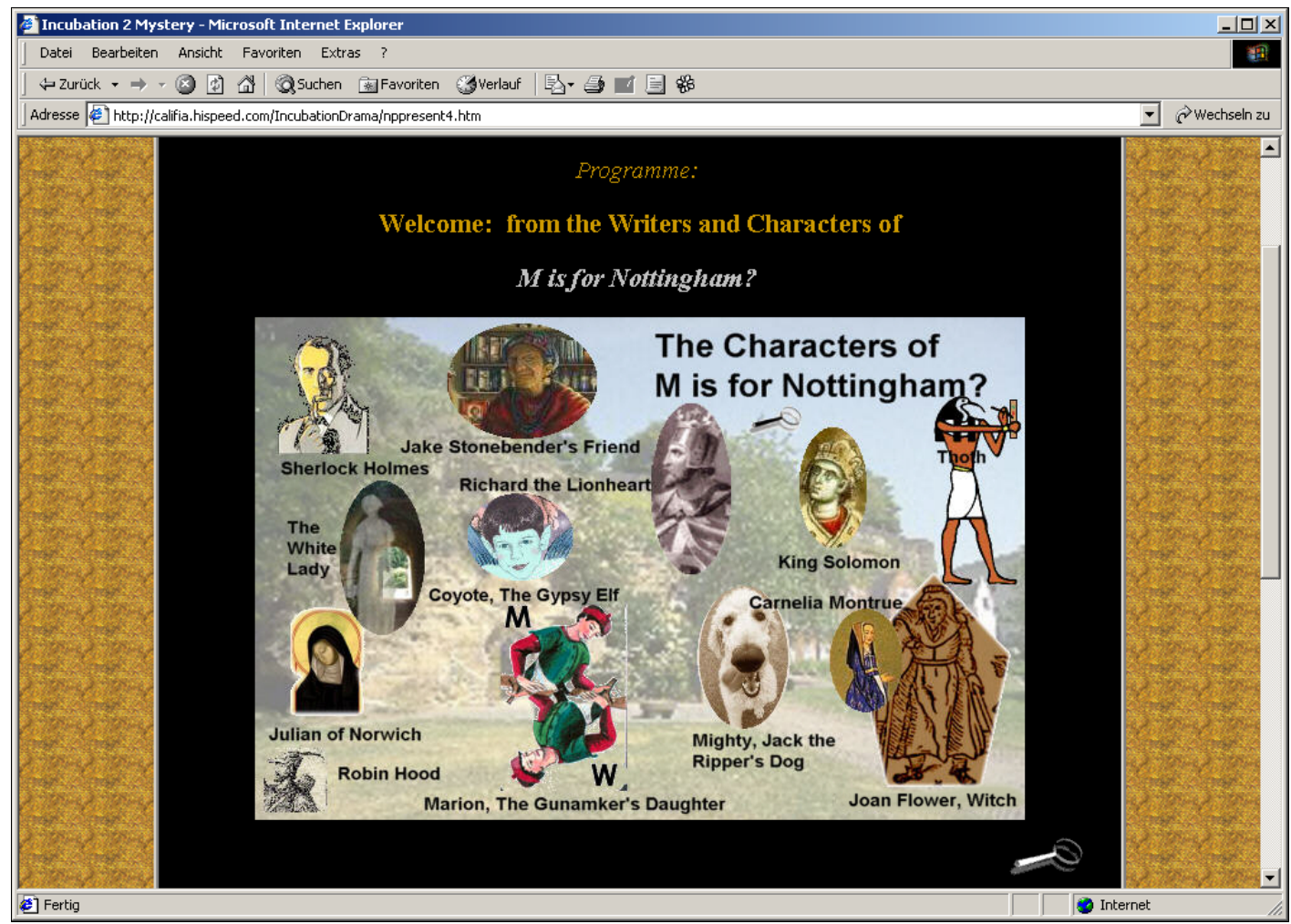

Abb. 8: 'M is for Nottingham? - Characters'

Bei fast allen Charakteren handelt es sich um bekannte Personen aus Literatur oder Geschichte. Zum Teil weisen ihre Biographien direkten Bezug zur Schriftkultur auf, wie im Falle des ägyptischen Mondgottes Thot, der als Schöpfer der verschiedenen Sprachen und Erfinder der Schreib- und Rechenkunst verehrt wurde. Andere Figuren stehen in Zusammenhang mit der Geschichte Nottinghamshires (King Henry II.) oder sind dem Genre Kriminalliteratur (Sherlock Holmes) zuzurechnen. Teilweise entwickeln diese Charaktere eine Eigendynamik, die Historie und Thema des webbasierten Dramas auf subtile Weise zusammenknüpft. Ein Beispiel hierfür ist Nedd Ludd, Namensgeber der ersten größeren Arbeiterunruhen, der Ludditenaufstände, der in $M$ is for Nottingham? bei Anbruch des 21. Jahrhunderts erneut als Kämpfer gegen den bedrohlichen Fortschritt auftritt, dieses Mal gegen die elektronischen Medien: "I think then I shall find the murderers of the book. Who knows, maybe I will revert to my old ways, maybe I will begin to smash the screens." ${ }^{, 714}$

${ }^{714}$ Die Ludditen wurden einst zu Maschinenstürmern, da sie die neuen Maschinen für die Auslöser der damaligen Beschäftigungskrise hielten. Die Parallele zu M is for Nottingham? liegt in der Wahrnehmung technischer Innovationen als Bedrohung für Traditionelles. Luesebrink u.a., "Nedd Ludd" in: Luesebrink, Hg., M is for Nottingham? - The New Characters, $<\underline{\text { http://califia.hispeed.com/Incubation2/newcharacters.htm\#NedLudd }>, ~ 22.10 .2003 . ~}$ 
Wie dieser Ausschnitt zeigt, bewegen sich die Figuren nicht in einer abgeschlossenen Welt und außerhalb der realen Gegenwart, es sind vielmehr drei verschiedene Sphären, die in $M$ is for Nottingham? mit dem Ziel der Verschmelzung zu einem größeren Ganzen zusammengeführt werden:

1. Die reale Gegenwart mit dem technischen Entwicklungsstand des beginnenden 21. Jahrhunderts und den daraus resultierenden Problemen wie beispielsweise der Sorge um die Zukunft der gedruckten Medien

2. Die Kulisse des webbasierten Dramas mit insgesamt elf Handlungsorten in der Grafschaft Nottinghamshire

3. Die ganz unterschiedlichen Historien und Lebenskreise der die Great Hall bevölkernden fiktiven Charaktere und Persönlichkeiten der Geschichte Englands.

Die Figuren treten miteinander in Dialog, tauschen sich aus, greifen gegenseitige Anregungen auf und besuchen im Rahmen ihrer Nachforschungen zum Schicksal des Buches alle zehn von den Organisatoren bereitgestellten Schauplätze.

Über das Zusammen- und Ineinanderübergehen der drei Sphären wird Robin Hood im Verlaufe der Geschehens sagen: "We had become supernatural, digital, ancient, natural, magic and modern. What a group of sleuths!"715

Der Auftakt des webbasierten Dramas mit den nachstehend widergegebenen ersten fünf kooperativen Elementen mag einen Eindruck von Art und Entstehung des Dialoges in $M$ is for Nottingham? vermitteln. Zwischen den Beiträgen bestehen je nach Bezugnahme aufeinander Verknüpfungen, die eine unmittelbare Rekonstruktion des Dialoges ermöglichen. Angesichts der hohen Zahl von Beiträgen bedeutet dies eine komfortable Navigationshilfe. Die optische Hervorhebung der Verfassernamen (hier durch Unterstreichung markiert) signalisiert, daß die Autoren per E-Mail erreichbar waren. ${ }^{716}$

715 Luesebrink u.a., “Green's Mill und Surround”, in: Luesebrink, Hg., M is for Nottingham? The Denouement at trAce Incubation 2 at Nottingham Trent University, $<$ http://califia.hispeed.com/IncubationDrama/npsofar7.htm $>, 27.10 .2003$.

716 Luesebrink, Hg., M is for Nottingham? - Written Text of the Discussion list, $<$ http://califia.hispeed.com/IncubationDrama/discussiontext.htm $>, 28.10 .2003$. 


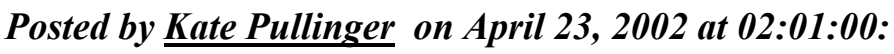

I saw a book lying on the bank of the river Trent. It had been ravaged by slavering readers and then cast aside. Its pages were coming out, it had lost its slipcover, in places the words were blurred, illegible.

\section{Posted by The White Lady on April 30, 2002 at 13:32:16:}

In Reply to: Looking for clues posted by Kate Pullinger on April 23, 2002 at 02:01:00:

It was my book... . I knew I had left it somewhere. I carried it with me as I followed David Herbert - he was abstracted, remembering a lost love - but even a ghost can slip down a bank and the steep cliffs by Clifton Grove were too steep for me. I saved myself but lost my book forever. It is all disaster, no other books can I bring to my non-corporeal world. It was by Byron...

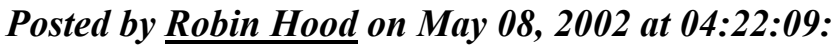

In Reply to: Re: Looking for clus posted by The White Lady on April 30, 2002 at 13:32:16:

Ah, The White Lady has appeared! I have scarcely ever seen her in the real world, a retiring ghost, she is.

But now, here, she mentions a book by Byron. I wonder if this book is from the Newstead Abbey library?

And is it DEAD, if it is wet and torn? And the Lady? Does she live?

\section{Posted by Richard the Lionheart on May 13, 2002 at 11:42:58:}

In Reply to: Re: Looking for clues posted by Robin Hood on May 08, 2002 at 04:22:09:

Returning from the Crusades I heard a mystic tell of the death of the book, but as the book was locked in my trunk I knew at least this book was not dead. For me it lived each night, told by a songbird in silk.

\section{Posted by The White Lady on May 14, 2002 at 08:28:33:}

In Reply to: Re: Looking for clues posted by Robin Hood on May 08, 2002 at 04:22:09:

The book comes not from Newstead but from a different library, one that did not exist when I did live in your world.

Yet perhaps one can say that I still live, but in a different world, a place of shadows and multiples which the "living" cannot see. And so I see the Book and its Death more clearly than the common multitude of flesh beings.

Wie der obigen Passage und dem weiteren Dialog zu entnehmen ist, hat Kate Pullinger auf einer Bank am Trent-Fluß ein abgegriffenes Buch von Byron gefunden und zur Universitätsbibliothek gebracht, um es wieder seinem Besitzer zuzuführen. Als Verliererin meldet sich daraufhin “The White Lady”, die überzeugt 
ist, daß es sich trotz seines schlechten Zustandes nicht um das totgeglaubte Buch handelt; allerdings gibt sie an, deutlicher als alle Sterblichen bereits jetzt sein baldiges Ende zu spüren. ${ }^{717}$

Nach diesem Auftakt sammeln sich die Kooperierenden beziehungsweise ihre Figuren, um gemeinsam das Mysterium um das mutmaßliche gewaltsame Ende des Buches aufzudecken. Zunächst besteht die Gruppe nur aus wenigen Teilnehmern, doch auf ihrer Spurensuche an den zehn Schauplätzen in und um Nottingham erhält sie rasch und regelmäßig neue Unterstützung durch wertvolle Hinweise und aktive Mithilfe.

In einem ersten Resümee erarbeiten die Ermittelnden die drei wesentlichen Schritte ihrer Spurensuche: ${ }^{718}$

- Zunächst gilt es, alle Hinweise an den Schauplätzen des webbasierten Dramas aufzuspüren.

- Zu klären ist ferner, ob die Veröffentlichung gedruckter Literatur zum Stillstand gekommen und wahre Kreativität somit nur noch in anderen Medien zu finden ist.

- Schließlich stellt sich die Frage: Gesetzt den Fall, das gedruckte Buch werde gänzlich von den elektronischen Medien verdrängt, so daß keine Druckseiten mehr zur Veröffentlichung gelangen - ist das Buch in diesem Falle auch dann tot, wenn noch alte Exemplare davon vorhanden sind beziehungsweise wenn zwar keine realen Bände mehr existieren, es aber noch eine Erinnerung an Bücher aus einer vergangenen Zeit gibt?

Im Verlaufe ihrer Recherchen in und um Nottingham melden sich die verschiedenen Figuren mit Vorschlägen zur Spurensuche und mit ihren Thesen zum Schicksal des Buches zu Wort.

Der überwiegende Teil sieht dessen Zukunft positiv. Bücher seien solange lebendig, wie sie eine besondere Bedeutung für ihre Leser besäßen. Aus diesem Grunde seien jedenfalls die religiösen beziehungsweise heiligen Bücher durchaus

717 Luesebrink u.a., “The Ancient Valley of Snottingham”, in: Luesebrink, Hg., M is for Nottingham? - The Denouement at trAce Incubation 2 at Nottingham Trent University, $<\underline{\text { http://califia.hispeed.com/IncubationDrama/npsofar2.htm }>, 27.10 .2003 . ~}$

718 Dies., "Ye Olde Trip to Jerusalem", in: Luesebrink, Hg., M is for Nottingham? - The Denouement at trAce Incubation 2 at Nottingham Trent University, $<\underline{\text { http://califia.hispeed.com/IncubationDrama/npsofar6.htm }>, ~ 27.10 .2003 . ~}$ 
nicht tot. ${ }^{719}$ Auch als Sammelbecken allen Wissens sei das Buch unverzichtbar, um zukünftigen Generationen Kenntnisse, vor allem solche der Vergangenheit zu vermitteln. Zudem sei die Sinnenfreude des Umblätterns einer Buchseite durch nichts zu ersetzen. Das Buch habe daher mit Sicherheit ewigen Bestand. ${ }^{720}$

Hierfür spreche außerdem seine Haltbarkeit; irgendwann seien die derzeitigen Computer nämlich veraltet und unbrauchbar und ihr Datenbestand nicht mehr zu rekonstruieren. Daher sei das Buch besser noch als der Computer geeignet, als Gedächtnis der Menschheit zu dienen. ${ }^{721}$ Im übrigen seien sogar die Handbücher für die Nutzung der technischen Hilfsmittel auf Papier gedruckt. Sobald das letzte Flackern der Monitore erloschen sei, werde man zweifellos das Buch zur Begründung einer neuen Zivilisation und Kultur wiederentdecken. ${ }^{722}$

Eine skeptische Diskussionsteilnehmerin sieht demgegenüber in der zunehmenden Mißachtung von Urheberrechtsgesetzen eine existentielle Bedrohung. Das Ende des Copyright, wenn es denn nicht abzuwenden sein sollte, bedeute gleichzeitig auch das Ende des Buches, da niemand mehr für die Entlohnung von Schriftstellern aufkommen werde: "[...] writers need to be kept in wine. How can they drink, eat and be merry without reward for their work? Those who disregard Copyright Laws are the ones who are killing the book."723

Eine andere Stimme sieht im Fernsehen die eigentliche, die tödliche Bedrohung des Buches. Unterhaltung sei nur noch auf Kommerz ausgerichtet und präge damit auf fatale Weise Sitten und Geschmack: "The great art patrons are gone and we are left with great patrons of crap.” In einer Atmosphäre der Verdummung und Unergiebigkeit sei das Buch zum Untergang verurteilt. "We don't need to burn books, we can put them on Television." 724

\footnotetext{
719 Ebd., 27.10.2003.

720 Dies., "The Resolution 1", in: Luesebrink, Hg., M is for Nottingham? - The Denouement at trAce Incubation 2 at Nottingham Trent University, $<$ http://califia.hispeed.com/IncubationDrama/npresoll.htm $>, 27.10 .2003$.

721 Dies., “The Resolution 2", in: Luesebrink, Hg., M is for Nottingham? - The Denouement at trAce Incubation 2 at Nottingham Trent University, $<\underline{\text { http://califia.hispeed.com/IncubationDrama/npresol2.htm }>, 27.10 .2003 . ~}$

722 Dies., "The Resolution 3", in: Luesebrink, Hg., M is for Nottingham? - The Denouement at trAce Incubation 2 at Nottingham Trent University, $<\underline{\text { http://califia.hispeed.com/IncubationDrama/npresol3.htm }>, 27.10 .2003 . ~}$

723 Ebd., 27.10.2003.

724 Ebd., 27.10.2003.
} 
Im Laufe der Diskussion über den gegenwärtigen Zustand des Buches und seine denkbaren Perspektiven gelangen die Figuren des webbasierten Dramas allmählich zu der Erkenntnis, die Fixierung auf ‘das Buch' als ein auf Papier gedrucktes, gebundenes literarisches Werk sei eventuell zu einseitig und vernachlässige das eigentliche Problem. Möge es auch tatsächlich zutreffen, daß die Druckliteratur irgendwann nicht mehr primärer Speicherort von Informationen sei oder sogar gänzlich durch alternative elektronische Medien verdrängt werde, so sei doch entscheidend, daß es immer eine Form der Wahrung und Übermittlung von Wissen geben werde, die nicht notwendigerweise an Bücher gebunden sei. Zahlreiche Niederschriften seien niemals zur Veröffentlichung in Buchform gelangt und gäben dennoch - beispielsweise als Manuskripte - Zeugnis früheren geistigen Schaffens. Es gehe allein darum, daß überhaupt Informationen weitergegeben würden, nicht auf welchem Wege dies erfolge; der Transport der Informationen sei entscheidender, weniger ihr Träger. Demzufolge lautet der wohl aufschlußreichste Satz der Diskussion: "Perhaps it is not the Book we seek at all, but the magic of making our stories heard." ${ }^{, 725}$ Die Figuren einigen sich darauf, der Schlüssel zur allgemeinen Verbreitung wichtigen Gedankenguts sei dessen Zugänglichkeit. Da das World Wide Web die Bedingung des globalen unausgesetzten Zugriffs erfülle - "Accessible to all who seek!" 726 - liege die Schlußfolgerung nahe, das Buch sei nicht tot, sondern vielmehr nach Art einer Metamorphose in einen neuen Zustand mit hinzugewonnenen Qualitäten übergegangen.

Höhepunkt und Abschluß der Spurensuche bildet die Deutung des Titels $M$ is for Nottingham?. Auf der Suche nach einem Interpretationsansatz erinnern sich die Teilnehmer schließlich der Erzählung The Garden of Forking Paths ${ }^{727}$. In Anlehnung an die Form des Ratespiels, die Borges in jener Erzählung entwickelt, nimmt man an, der versteckte Hinweis zur Auflösung des Mysteriums in $M$ is for Nottingham? liege möglicherweise in genau dem Wort, das während aller vorangegangenen Klärungsversuche unausgesprochen geblieben sei. Die Schlußfolge-

725 Ebd., 27.10.2003.

726 Ebd., 27.10.2003.

727 Borges spielt eine bedeutende Rolle im Auflösungsteil des Projekts. Der linke Rahmen der Seiten enthält zahlreiche Zitate, die sämtlich zu dem Thema Literatur in Beziehung stehen und dem Leser Gedankenanstöße liefern. Die Herausgeber von $M$ is for Nottingham? geben hierfür folgende Quelle an: Allen B. Ruch, Hg., The Modern Word-Jorge Luis Borges: The Garden of Forking Paths: Quotations $\left(\left[{ }^{1} 27.01 .2003\right]\right),<$ http://www.themodernword.com/borges/borges_quotes.html $>, 03.11 .2003$. 
rung lautet, es sei der Autor oder Schriftsteller als heimlicher Protagonist des Kriminalstücks, den bis zum Schluß niemand direkt genannt habe.

In der Tat ist nicht (nur) die allgemeine Zugänglichkeit Voraussetzung für Übermittlung und Wahrung von Wissen, sondern (auch) dessen geistiger Schöpfer. Die Existenz des Buches beziehungsweise jede Form der schriftlichen Überlieferung ist an Verfasser gebunden: "The secret of the Book is the creation of each new Writer - the preservation of the story that is otherwise lost." Kooperierenden des $M$ is for Nottingham?-Projektes über ihre Textelemente letztlich mit dazu beigetragen, die Schriftkultur am Leben zu erhalten.

Das webbasierte Drama endet mit der Feststellung:

The Last Letter of the Name is $\mathrm{M}$ - but we must be prepared to see things from many perspectives. If we look at the world a slightly different way, the $\mathrm{M}$ becomes a $\mathrm{W}-$ and the magic word is Writer. ${ }^{729}$

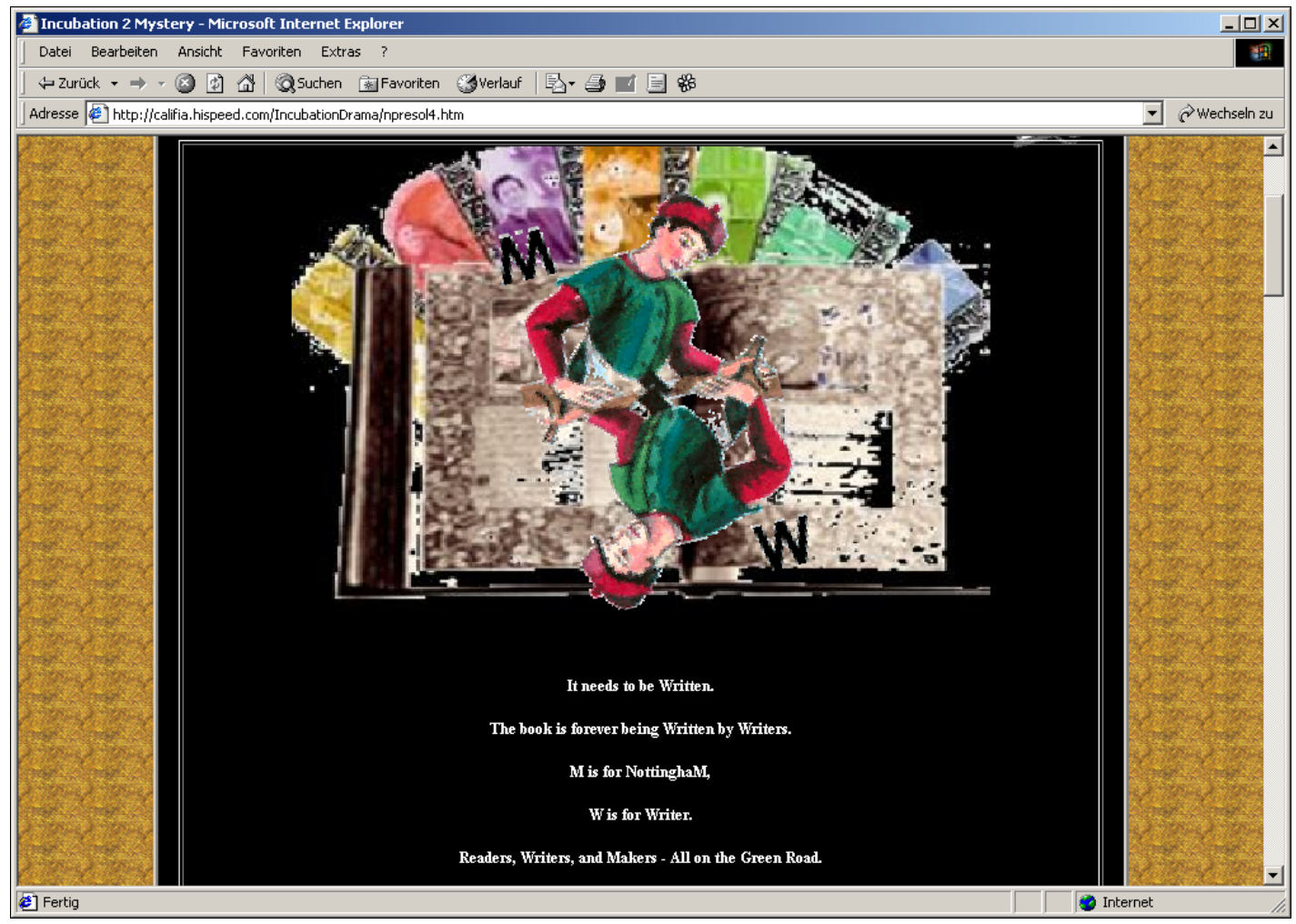

Abb. 9: 'M is for Nottingham? - The Denouement'

728 Luesebrink u.a., "The Resolution 4", in: Luesebrink, Hg., M is for Nottingham? - The Denouement at trAce Incubation 2 at Nottingham Trent University, $<\underline{\text { http://califia.hispeed.com/IncubationDrama/npresol4.htm }>, 27.10 .2003 . ~}$

729 Ebd., 27.10.2003. 
Die geplante Aufführung im Rahmen der Incubation 2-Konferenz hatte es mit sich gebracht, daß die lebhafte Kommunikation zwischen den Kooperierenden an einem bestimmten Punkt abrupt beendet werden mußte, um noch ausreichend Zeit für die Vorbereitung der Live-Darbietung zur Verfügung zu haben. Da die gemeinschaftliche Spurensuche zu jenem Augenblick gerade begonnen hatte, Kontur zu gewinnen, hätte eine längere Kreativphase vermutlich zu interessanten Entwicklungen führen können. Auch so war das Ergebnis des knapp zweimonatigen Kooperationszeitraumes mit 350 Textseiten derart umfangreich, daß es einer rigorosen Begrenzung unterzogen werden mußte. Es wurde von Marjorie Coverly Luesebrink auf ungefähr 40 Seiten Originaltext gekürzt und anschließend durch den festen Mitarbeiterstab des Projekts in weniger als einer Woche zu einem Drehbuch umgeschrieben. Den Darstellern blieben daraufhin lediglich ein Paar Tage zur Vorbereitung der Aufführung. Dennoch gelang es ihnen offenbar, das Produkt der Online-Zusammenarbeit adäquat in eine Bühnenhandlung umzusetzen.

Nach Aussage der Organisatoren vermochten die Darsteller den Figuren durch überzeugendes, flüssiges Schauspiel sowie stimmige Kostüme und Requisiten vollkommene Wirklichkeitsnähe zu verleihen. Es soll ihnen sogar gelungen sein, der Handlung unvorhergesehene Wendungen zu geben und improvisierend neue symbolische Elemente ${ }^{730}$ einzuführen. Die Leichtigkeit, mit der die Autoren die Rolle der Figuren adaptierten und die Handlung vom WWW auf die Bühne transportierten, wurde darauf zurückgeführt, daß schon die Spurensuche Aufführungscharakter gehabt habe. Die Verlagerung von der virtuellen auf die reale Bühne brachte daher offensichtlich keine grundlegenden Veränderungen mit sich. $^{731}$

${ }^{730}$ Der interessierte Nutzer, der sich intensiv mit Verlauf und Ergebnissen des $M$ is for Nottingham?-Projekts auseinandergesetzt hat, vermißt in den resümierenden Ausführungen der Organisatoren einige Beispiele für diese neueingeführten symbolischen Elemente.

${ }^{731}$ Dies., "Critical Review: Time Frames (Performance Event)", in: Luesebrink, Hg., $M$ is for Nottingham?, $<$ http://califia.hispeed.com/IncubationDrama/criticalreview.htm\#Performanceevent $>$, 27.10.2003. 
Nach Abschluß der Konferenz versuchten die Projektorganisatoren, das bis dahin dynamische, webbasierte Drama in ein statisches Gebilde, das heißt einen linearen Text umzugestalten. $M$ is for Nottingham? ging damit in seine dritte Zustandsform über: Nach seiner Entstehung im und über das Web hatte es sich aufgrund seiner Dialogstruktur und seiner Multilinearität verhältnismäßig unproblematisch in eine Theateraufführung umwandeln lassen; nunmehr mußte es eine feste Präsentationsgestalt annehmen, um künftig allen Web-Nutzern als Archiv zur Verfügung zu stehen. Die Schwierigkeit bestand dabei in der Simulation des projektphasen-typischen hypertextuellen Leseerlebnisses: "[...] since this story was entirely written through the threading of posts on the Message Board, it had all the qualities of a hypertext - linked lexias, multilinear plots, simultaneity, and multiple voices in time and space. ${ }^{, 732}$ Dementsprechend schwierig erschien es den Organisatoren, diese Dynamik in einem Fließtext wiederzugeben. ${ }^{733}$

Inzwischen präsentiert sich das Ergebnis der neuen Konzeption als ein Zusammenschluß der einzelnen Projektphasen sowie aller verfügbaren Hintergrundinformationen. Das Forum unter dem Titel A Mystery Solved ist in sechs Rubriken gegliedert, die dem Web-Nutzer jeweils unterschiedliche Herangehensweisen an das Dramenmaterial gestatten: Zum einen besteht die Möglichkeit des Zugriffs auf die gesamte ursprüngliche $M$ is for Nottingham?-Online Drama Site, der Nutzer kann jedoch auch der Einladung "Follow the Trail of Clues!" folgen und sich direkt der Spurensuche an den zehn Schauplätzen zuwenden. Im Abschnitt The Written Texts of the Story vermag der Leser einen Eindruck von der Entwicklung der kooperativen Dialogproduktion und dem konzeptionellen Wandel des Diskussionsforums zu gewinnen. Die eingangs erwähnte mehrmalige Überarbeitung der Darstellungsformate läßt sich dort - soweit sie im Verlaufe der aktiven Projektphase erfolgte - in ihren diversen Ausprägungsformen rekonstruieren. Die Auflösung des Mysteriums, wie sie sich im Rahmen der Incubation2-Konferenz vor Ort in Nottingham abspielte, ist in der Rubrik The Denouement at trAce Incubation 2 at Nottingham Trent University nachzuerleben. Allerdings steht dem Leser kein Mitschnitt, sondern lediglich eine hypertextuelle Nachschrift der Veranstaltung zur Verfügung. Eine kritische Auseinandersetzung mit Intention, Konzeption und Rea-

732 Luesebrink, Hg., M is for Nottingham? - The Denouement at trAce Incubation 2 at Nottingham Trent University, $<$ http://califia.hispeed.com/IncubationDrama/npdenouement.htm>, 27.10.2003.

733 Dies., Hg., $M$ is for Nottingham? - Written Text of the Discussion List,

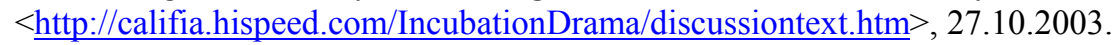


lisation des Projekts findet sich schließlich in den beiden letzten Kategorien The Critical Overview und Comments from Readers and Players. ${ }^{734}$

\subsubsection{Abschluß}

Die Projektorganisatoren hatten sich zum Ziel gesetzt, ein webbasiertes HypertextSystem mit einem möglichst hohen Identifikationspotential $\mathrm{zu}$ schaffen, das die Aufmerksamkeit eines zufällig auf die Web-Seiten stoßenden sogenannten Surfers ebenso binden sollte wie die eines Schriftstellers. Aufgrund der vergleichsweise großen Resonanz seitens der Web-Gemeinschaft und der hohen Aufmerksamkeit, die ihnen die Medien entgegenbrachten, durften sich die Verantwortlichen in ihrer Intention bestätigt fühlen. Unterstützt durch lokale Einrichtungen wie die Nottingham Trent University, den Nottingham City Council, die Initiative SOLON ${ }^{735}$ und das Justizmuseum (die sogenannten Galleries of Justice), durch die regionale Vertretung der englischen Kunstförderungsagentur Arts Council England (den East Midlands Council for the Arts ${ }^{736}$ ) sowie die amerikanische Electronic Literature Organization, konnte das Projekt an Profil gewinnen und nationales wie internationales Interesse auf sich ziehen: Die BBC, der Manchester Guardian, The Chronicle of Higher Education und das Wired Magazine berichteten über das Literaturexperiment, das neben den Teilnehmern der Incubation 2-Konferenz Kooperierende aus ganz Großbritannien und den USA vereinigte. ${ }^{737}$

$M$ is for Nottingham? scheint jeder Beachtung und Hilfe wert, denn die Erkenntnisse, die sich aus den Projektergebnissen ziehen lassen, sind für die weitere Entwicklung webbasierter Literatur von entscheidender Bedeutung. Aufschlußreich ist zunächst einmal das Verhalten der Web-Besucher, das in dreierlei Hinsicht eindeutige Tendenzen aufweist: In der Herangehensweise an die gestellte

734 Dies., Hg., $M$ is for Nottingham? - A Mystery Solved, $<\underline{\text { http://califia.hispeed.com/IncubationDrama/museumtoc.htm }>, 27.10 .2003 . ~}$

735 SOLON, ein Akronym aus Society, Order, Law, Offences und Notoriety, bezeichnet das Partnerschaftsprogramm Interdisciplinary Studies in Bad Behaviour and Socially Visible Crime zwischen der Trent-Universität und dem Justizmuseum von Nottingham. Nottingham Trent University/Museum of Law, SOLON - Interdisciplinary Studies in Bad Behaviour and Socially Visible Crime, $<$ http://solon.ntu.ac.uk/home.htm>, 28.10.2003.

736 Der Arts Council of England mit seinen zehn Regionalvertretungen ist verantwortlich für die Entwicklung, Aufrechterhaltung und Förderung der schönen Künste in England. Durch finanzielle und tatkräftige Unterstützung soll dem (multi-)kulturellen Leben mehr Raum und größere Aufmerksamkeit verschafft werden. Arts Council England, Arts Council England Homepage, $<$ http://www.artscouncil.org.uk/>, 28.10.2003.

737 Luesebrink, Hg., $M$ is for Nottingham? - Our Sponsors, $<$ http://califia.hispeed.com/Incubation2sponsors.htm $>, 24.10 .2003$. Luesebrink u.a., "Critical Review: Audience", in: Luesebrink, $\mathrm{Hg}$., $M$ is for Nottingham?, $<\underline{\text { http://califia.hispeed.com/IncubationDrama/criticalreview.htm\#Audience }>, ~ 22.10 .2003 . ~}$ 
Aufgabe, in den Erwartungen an das Ergebnis der Zusammenarbeit und in der Stetigkeit der Mitarbeit.

\section{Herangehensweise}

Gleich zu Beginn des Experiments war eine eindeutige Verhaltensübereinstimmung zu beobachten: Nachdem sich die Gruppe der Kooperationswilligen konstituiert hatte, ließ sich während der ersten Wochen zunächst noch keine Produktivität verzeichnen; vielmehr schienen die Teilnehmer in einer Art Inspirationsphase zu verharren. Die Organisatoren schlossen eine Fehlplanung des Projektkonzepts jedoch aus und verwiesen auf eine Parallelität zu Einzelschriftstellern. Stagnation zu Beginn eines Schaffensprozesses sei ein oft zu beobachtendes Phänomen und unabhängig davon, ob ein Autor in einer Gruppe oder allein tätig sei. ${ }^{738}$

Auch im weiteren Verlauf des Projekts bestand ungebrochener Konsens. Die Kooperierenden begriffen die Heterogenität der Teilnehmergruppe offensichtlich mehrheitlich als entscheidenden Vorteil sowohl für das Schaffen jedes einzelnen Autors als auch für das schriftstellerische Gesamtergebnis. Unbestritten verlieh sie dem webbasierten Drama einen Facettenreichtum, der nur der Phantasie Vieler, niemals nur der einer einzelnen Person entspringen konnte. ${ }^{739}$ Zudem gestattete die Größe und Verschiedenartigkeit der Gruppe eine unbefangenere Vorgehensweise, denn sie enthob die Kooperierenden der Sorge, sich mit ihren Dialogelementen möglicherweise in eine inhaltliche Sackgasse zu manövrieren. Alle Beiträge, die in eine falsche Richtung zu führen schienen, wurden wieder auf den richtigen Weg gebracht, sei es durch die Gruppe ${ }^{740}$ oder die Moderatorin des Diskussionsforums. ${ }^{741}$

738 Luesebrink u.a., "Critical Review: Time Frames (Writing Period)", in: Luesebrink, Hg., M is for Nottingham?, <http://califia.hispeed.com/IncubationDrama/criticalreview.htm\#Writingperiod $>, 27.10 .2003$.

739 "I have always felt collaborative projects add a richness to the whole that individuals on their own cannot achieve." Jane Dorner, "Commentary", in: Luesebrink, Hg., M is for Nottingham? Comments by Writers and Readers, <http://califia.hispeed.com/IncubationDrama/commentary.htm>, 27.10.2003.

740 "If something happened to produce a blind alley there was always someone else who could come up with a solution to the problem." Jeremy Duffield, "Commentary", in: Luesebrink, Hg., $M$ is for Nottingham? - Comments by Writers and Readers, $<\underline{\text { http://califia.hispeed.com/IncubationDrama/commentary.htm }>, 27.10 .2003 . ~}$

741 "I felt I was adding my bits on screen, but they were inconsequential to the whole thing - which was indeed taking off in all directions and was only pulled together because Margie [= Marjorie Coverley Luesebrink] acted as a director and drew out something meaningful from it." Dorner, "Commentary", <http://califia.hispeed.com/IncubationDrama/commentary.htm>, 27.10.2003. 


\section{Erwartungen an das Ergebnis der Zusammenarbeit}

Nachdem die Diskussion der Figuren in Gang gekommen war, wurde rasch deutlich, daß die Beteiligten unterschiedliche Erwartungen hegten, was den Ausgang des Projekts betraf. In dieser Hinsicht bot die Thematik besonders viel Zündstoff und sorgte für zahlreiche divergierende Mutmaßungen über das Schicksal des Buches. Einigkeit bestand allein darin, daß Aktion und Reaktion die Voraussetzung für die Entstehung eines inszenierungsfähigen Werkes waren. $^{742}$

Die Organisatoren waren letztlich überzeugt, daß auch dieser Prozeß seine Parallele in der Arbeit von Einzelautoren habe. Auch ein allein und unabhängig Schaffender müsse sich zunächst darüber klar werden, wie er sein Thema ausführen und zu welchem Schluß er gelangen wolle.

Es ist anzunehmen, daß tatsächlich in beiden Fällen zunächst grundlegende Konflikte zur Vorgehensweise zu bewältigen sind, mit dem Unterschied, daß sie in einer Gruppe offen zwischen allen beteiligten Personen ausgetragen werden müssen und dies die Konfliktlösung im allgemeinen nicht unwesentlich erschwert. Ein zentrales Problem, das immer wieder neu angegangen werden muß, ist beispielsweise die Frage, ob und wie sich ein anspruchsvolles Thema mit einer elementaren Aussage publikumswirksam umsetzen läßt. Vor dem Hintergrund eben dieser Frage hat eine Teilnehmerin des Projekts ihre Erfahrungen mit dem kooperativen Schreiben in einem Resümee dargelegt. Ein Ausschnitt gibt Zeugnis von ihren Überlegungen und den ernüchternden Konsequenzen:

[...] I was initially confused on the theme. I wanted to concentrate on the future of literature and the meaning of the word 'book' and to discuss this thread as it might appear from different cultures through history. However other writers, especially the committee, had different agenda, so I eased myself into a Falstaff-type role while watching what was going on. ${ }^{743}$

${ }^{742}$ Luesebrink u.a., "Critical Review: Time Frames (Writing Period)", $<$ http://califia.hispeed.com/IncubationDrama/criticalreview.htm\#Writingperiod $>, 27.10 .2003$.

743 Margaret Penfold, "Commentary", in: Luesebrink, Hg., M is for Nottingham? - Comments by Writers and Readers, <http://califia.hispeed.com/IncubationDrama/commentary.htm\#Writers: >, 27.10.2003. 
Es ist fraglich, ob den Organisatoren bewußt war, daß die Diskrepanz zwischen Thema und Inszenierung größer kaum hätte sein können. Hier wurde eine Grundsatzfrage, deren Beantwortung derzeit noch nicht möglich sein dürfte, zum Gegenstand von Unterhaltung gemacht. Es ging um eine Entwicklung, die seit der zunehmenden Verbreitung elektronischer Medien äußerst emotional und kontrovers diskutiert wird und deren weiterer Verlauf von ganz entscheidender Bedeutung für die kulturelle Perspektive der Menschheit ist. Eine andere Projektteilnehmerin hat den Widerspruch mit einem einzigen Satz auf den Punkt gebracht: "It is intriguing to couch a philosophical conversation as a mystery - what has happened to the book and where are we going now?"

\section{Stetigkeit der Mitarbeit}

$M$ is for Nottingham? bot zu jedem Zeitpunkt seiner Präsenz im World Wide Web Stoff für ausgiebige Lektüre. Schon zu Beginn des Projekts war eine fruchtbare Beschäftigung mit dem Hypertext-System mit hohem Zeitaufwand verbunden. Dies gilt mehr noch, seit mit seinem Abschluß der Umfang der Informationen durch die Berichterstattung über die Auflösung des Geheimnisses, ausführliche Projektauswertungen und Rezensionen beträchtlich angewachsen ist. Zwar waren die einführenden Hinweise so gestaltet, daß auch ein Kurzbesucher einen Eindruck von der Zielsetzung des Projekts und dem Inhalt des webbasierten Dramas gewinnen konnte, für das Verständnis aller Zusammenhänge war aber eine eingehendere Lektüre der Hintergrundinformationen sowie der historischen Zusammenhänge unerläßlich. Viele Konferenzteilnehmer verbrachten eigenen Aussagen zufolge eine beträchtliche Zeit mit der Rezeption von $M$ is for Nottingham?, obwohl sie nicht die Absicht hatten, sich kooperativ zu beteiligen. ${ }^{745}$

Dem enormen zeitlichen wie kreativen Aufwand, den die aktive Mitarbeit den Freiwilligen abverlangte, schienen offensichtlich - bis auf wenige Ausnahmen nur passionierte Freizeitliteraten und Profis gewachsen. In der Tat erforderte die Kooperation eine konsequente, aufmerksame Beteiligung. Die intensive Resonanz, die das Projekt erzielte, schlug sich in einem raschen Anstieg der schriftlichen Beiträge nieder, wodurch die einzelnen Handlungsstränge zunehmend komplexer

744 Deena Larsen, “Commentary", in: Luesebrink, Hg., M is for Nottingham? - Comments by Writers and Readers, <http://califia.hispeed.com/IncubationDrama/commentary.htm\#Writers: $>$, 27.10.2003.

${ }^{745}$ Luesebrink u.a., "Critical Review - Audience (Conference Attendees)", $<\underline{\text { http://califia.hispeed.com/IncubationDrama/criticalreview.htm\#Conferenceattendees }>, ~ 22.10 .2003 . ~}$ 
wurden. Um stets dem aktuellen Entwicklungsstand entsprechen zu können, waren die Autoren zusätzlich zu ihrem schriftstellerischen Engagement zur regelmäßigen Durchsicht aller Neueinstellungen genötigt. Bei einem der Teilnehmer führte dies sogar dazu, daß er sich schließlich als regelrecht abhängig betrachtete:

It actually became addictive. Logging on each day reading all the new entries and contributing. There were even stages (when we were arranging meetings) when it became difficult to distinguish between the real and the virtual. ${ }^{746}$

Die hohe Belastung, die das Projekt den Kooperierenden abverlangte, scheint schriftstellerische Laien und Anfänger bereits nach kurzer Zeit zur Aufgabe bewogen zu haben. Den Organisatoren wurde die Außerordentlichkeit des kooperativen Engagements aber offenbar erst im nachhinein bewußt: "As an analytical point, then, the preponderance of practiced writers suggests that this project required a high degree of focus and investment from the writing participants."

\subsubsection{Auswertung}

Das Ergebnis des $M$ is for Nottingham?-Projektes wurde von den Organisatoren selbst als "a kind of game, fantasy, history-mystery" "748 bezeichnet. Diese Einschätzung trifft ziemlich genau den endgültigen Charakter der Publikation.

Konzeption und Intention des Werkes hatten allen Anforderungen an eine niveauvolle webbasierte Publikation entsprochen. $M$ is for Nottingham? schien alle Eigenschaften zu vereinbaren, die in vorliegender Arbeit als unabdingbar für eine derartige Veröffentlichung herausgearbeitet worden sind: Konzipiert im und für das Web sowie in Zusammenarbeit mit der Web-Gemeinschaft, bot das Projekt Interaktivität, Kooperativität und direkten Zugriff auf weiterführende Ressourcen im World Wide Web. Der Einsatz audiovisueller Elemente versprach eine anschauliche und wirklichkeitsnahe Präsentation des Materials und damit eine Ausschöpfung aller Darstellungsmittel von Hypertext und WWW. Zudem verhieß die anschließende Umsetzung in ein Theaterstück eine neuartige Erfahrung auf dem Gebiet webbasierter Dramen. Die Fülle der bereitgestellten Informationen bot dem Web-Besucher darüber hinaus zahlreiche Ansatzpunkte für eine tiefergehende

${ }^{746}$ Duffield, "Commentary", <http://califia.hispeed.com/IncubationDrama/commentary.htm>, 27.10.2003.

${ }^{747}$ Luesebrink u.a., "Critical Review: Audience (Participating Writers)", in: Luesebrink, Hg., M is for Nottingham?, <http://califia.hispeed.com/IncubationDrama/criticalreview.htm\#ParticipatingWriters>, 22.10.2003.

${ }^{748}$ Ebd., 22.10.2003. 
Beschäftigung mit der jeweiligen Thematik und präsentierte ihm die Grafschaft Nottinghamshire und ihre Geschichte von einer gänzlich neuen Seite. Eingekleidet in ein Kriminalstück mit einer bewußt ausgelegten Fährte wurde das Interesse des Lesers auf unterhaltsame Weise gebunden. Die Möglichkeit zur Mitarbeit, die während der aktiven Phase des Projekts bestand, verlieh dem webbasierten Drama wietere Attraktivität.

Im Verlaufe der Bearbeitung erwiesen sich jedoch mehrere Punkte als nachteilig:

\section{Das Ergebnis der Spurensuche im Abschnitt 'The Sites'}

Es war von den Organisatoren zweifellos geschickt angedacht, die Spurensuche der allgemeinen Frage nach dem Schicksal des Buches, einer höchst subjektiven, nur schwer faßbaren Thematik, unterzuordnen, sie zugleich aber auf ein bestimmbares gewissermaßen handfestes - Ziel auszurichten. Damit wurden Neugier und ein gewisser Ehrgeiz des Ermittelnden wachgehalten. Der Nutzer würde sich allerdings wünschen, daß die Spurensuche zu einem wirklich entscheidenden Hinweis führte; infolge der Banalität ihres Resultats fühlt er sich in gewisser Weise um ein Erfolgserlebnis gebracht. Irgendeine konkrete Verbindung zum Thema Literatur beziehungsweise Buch wäre wünschenswert gewesen.

\section{Die Auflösung des Mysteriums}

Der Dialog der diversen Charaktere des webbasierten Dramas offenbarte die unterschiedlichsten Lösungsansätze zur Klärung des Buchschicksals. Die Kooperierenden brachten teilweise sehr ausgefallene Figuren und Gedanken in den Handlungsablauf ein, die in der Fülle der Ideen aber zunächst keine Beeinträchtigung der Glaubhaftigkeit der Handlung bewirken konnten. Wenn in der Sinndebatte um den letzten Buchstabens allerdings die unterschiedliche Sicht der Dinge beschworen und das "M" kurzerhand in ein "W" für Writer umgewandelt wird, mag der eine oder andere Rezipient diese Wendung als absurd empfinden und überzeugt sein, das gesamte Projekt habe dadurch mit einem Schlage eine nicht wieder gutzumachende Einbuße an Überzeugungskraft erlitten. Sicher wären auch andere Handlungsvarianten denkbar gewesen, die dem Werk einen eleganten, nichtsdestoweniger folgerichtigen Abschluß verliehen hätten. Der Rezipient gewinnt daher den Eindruck, daß sich die Organisatoren auf diese Weise um die Chance gebracht haben, die von ihnen erwünschte deutliche Bresche für die elektronischen Publi- 
kationsmittel zu schlagen; mit einem nur geringfügig anderen Handlungsverlauf hätte das Ergebnis ihres Projekts für internationale wissenschaftliche Aufmerksamkeit sorgen und möglicherweise auch zu einer neuen Wahrnehmung der Computermedien führen können.

\section{Der enge Zeitrahmen}

Es ist anzunehmen, daß der enge Zeitrahmen, der den Kooperierenden für die Entwicklung ihres Dialogs und den Organisatoren für die anschließende Aufbereitung dieses Textmaterials zur Verfügung stand, vor anderen Faktoren die Qualität des Ergebnisses in entscheidendem Maße beeinträchtigt hat. Wie die kritische Auswertung des Projekts zeigt, begann der Dialog der Kooperierenden gerade an Kontur zu gewinnen, als die Schreibphase beendet werden mußte. Vermutlich hätte die Kreativgemeinschaft bei kontinuierlicher Weiterarbeit einen wesentlich überzeugenderen Handlungsausgang entwickelt, als er von den Organisatoren unter Zeitdruck hervorgebracht worden ist.

\section{Mangel an akustischen und filmischen Elementen}

Mit Ausnahme von Chopins Fantaisie Impromptu wurde im gesamten Projektforum kein einziges Audioelement verwendet. Es wären aber durchaus einige Ansatzpunkte denkbar gewesen, wie beispielsweise im Abschnitt The Gardens, in dem eine Rezitation der Liebesszene zwischen Paul und Clara nahegelegen hätte. Ein ganz entscheidendes Manko ist überdies das Fehlen eines Mitschnitts der Theateraufführung im Rahmen der Incubation 2-Konferenz. Zum einen hätte ein solches Videoelement die Triade des Projekts als a) dynamisches Schreibexperiment, b) Umsetzung in eine Live-Inszenierung und c) statische Wiedergabe des Dramendialoges sinnvoll ergänzt, zum anderen hätte es aber auch hohen Anschauungswert gehabt und die Vorstellung des Web-Besuchers durch konkrete Bilder unterstützt und angeregt.

\section{Fehlende Orientierungshilfen}

Die Organisatoren haben ein breites Spektrum an Handlungsorten zusammengefügt, die allesamt für sich genommen aufschlußreiche Informationen liefern, aber trotz einiger hypertextueller Verknüpfungen keinen nachvollziehbaren Bezug zueinander aufweisen. Es wäre daher zumindest angebracht gewesen, eine Übersichtskarte bereitzustellen, die dem Leser beziehungsweise Ermittelnden von Fall 
zu Fall eine Standortbestimmung unter besonderer Berücksichtigung der übrigen Schauplätze ermöglicht hätte. ${ }^{749}$

Das Projekt $M$ is for Nottingham? hat aufgrund seiner Intention und beispielgebenden Konzeption gezeigt, daß künftige Vorhaben durchaus mit Publikumserfolg, aber auch mit wissenschaftlicher Anerkennung rechnen dürfen, wenn die Verantwortlichen einerseits Freiraum für die Kreativität der Kooperierenden zulassen, aber andererseits den spielerischen Charakter ihrer Publikation zugunsten von Seriosität, Plausibilität und Wissenschaftlichkeit begrenzen. Würden in Zukunft ähnlich engagierte Projekte folgen, wäre dies sicherlich geeignet, die Entwicklung auf dem Gebiet webbasierten Publizierens nachhaltig voranzutreiben.

\subsection{Webbasiertes Drama - Resümee}

Im Vergleich der Literaturgattungen webbasierte Prosa, webbasierte Lyrik und webbasiertes Drama bietet letzteres den wesentlich größeren Gestaltungs- und Handlungsspielraum, da es der Hypertext-Systematik durch seine szenische und dialogische Gestaltung strukturell bedeutend ähnlicher ist. Hypertext und WWW machen Literatur zu einem Ereignis mit stets neuen Perspektiven. Wenn darüber hinaus die Dramenhandlung so angelegt ist, daß sie mehr oder weniger unbegrenzte Entfaltungsmöglichkeiten hinsichtlich Figuren und Diskurs erlaubt, kommen die eingangs dieser Arbeit angesprochenen Synergieeffekte voll zum Tragen.

Aus diesem Grunde ist anzunehmen, daß das webbasierte Drama sich im World Wide Web wird etablieren können, da es tatsächlich neue Aspekte in der Literaturrezeption eröffnet. Hypertext und WWW haben dem traditionellen Drama eine Nische bereitet, die zwischen Buch und Theater liegt. Print-Medien können simultane Vorgänge in der Regel nur unzureichend wiedergeben. Das Theater ist für eine derartige Präsentationsform zwar durchaus geeignet, gewährt dem Zuschauer im allgemeinen aber nur Einblick in einen einzigen Handlungsablauf. Inhalt und Aussage des dort Dargestellten erschließen sich dem Zuschauer in einem einzigen, durchgängigen Erlebnisvorgang: "A traditional play is a single viewing from a single perspective of a story that is far richer and denser in

749 "It would have been great to have had [...] a map showing how to get round." Penfold, "Commentary", < $\underline{\text { http://califia.hispeed.com/IncubationDrama/commentary.htm\#Writers: }>, ~ 27.10 .2003 . ~}$ 
experience than Single Vision can ever communicate. ${ }^{, 750}$ Im webbasierten Drama hingegen sind mehrere Rezeptionsvorgänge notwendig, um alle theoretisch möglichen Handlungsabläufe miterleben zu können. Hier wird dem Leser Gelegenheit gegeben, den komplexen Inhalt in diversen Besuchen in selbstbestimmter Weise ohne zeitliche und örtliche Zwänge zu rezipieren. Dabei bildet jeder einzelne Lektürevorgang einen virtuellen Theaterbesuch, bei dem sich ein weiterer Teilaspekt der komplexen Gesamthandlung eröffnet.

Das traditionelle Drama scheint aber nicht nur in ganz besonderer Weise für die Umsetzung in eine webbasierte Publikation geeignet. Die Untersuchungen im Rahmen der vorliegenden Arbeit haben ergeben, daß diese Literaturgattung derzeit tatsächlich auch am überzeugendsten im WWW präsentiert wird. Die Möglichkeiten von Hypertext und World Wide Web scheinen hier am intensivsten genutzt zu werden, was als Beweis dafür gewertet werden kann, daß sich die erarbeiteten Maßstäbe für webbasierte Publikationen nicht fernab des Machbaren bewegen, sondern durchaus in die Praxis umzusetzen sind. Es obliegt allein dem Engagement und den programmiertechnischen Kenntnissen der Verantwortlichen, ob und inwieweit Medium und Sujet in wechselseitiger Befruchtung zu einem literarischen Kunstwerk mit völlig neuen Qualitäten gelangen.

Vor allem das letzte Literaturbeispiel hat gezeigt, daß im WWW durchaus überzeugende webbasierte Großwerke existieren, deren Rezeption sich für die Web-Besucher zu einem mehrstündigen multimedialen Erlebnis gestaltet. Sicherlich ist diesen Werken nicht jener durchschlagende Erfolg beschieden, den Rotermund sich von einem revolutionären Kunstwerk im Web verspricht ${ }^{751}$, jedoch besitzen sie durchaus das Potential, ihre Nutzer durch multimediale Inhalte in Erstaunen $\mathrm{zu}$ versetzen, durch spielerische Elemente $\mathrm{zu}$ fesseln und aufgrund ihres edukativen Ansatzes gleichzeitig einen didaktischen Auftrag zu erfüllen.

Es ist zu hoffen, daß die momentan noch recht geringe Auswahl an webbasierten Dramen einen Zuwachs an Werken im Stile von $M$ is for Nottingham erfährt. Was einer solchen Entwicklung entgegensteht, sind auch hier wieder zum einen der hohe Arbeits- und Zeitaufwand, zum anderen erhebliche Defizite an notwendigem technischen Hintergrundwissen. Was Umfang und Dauer des persön-

750 Charles Deemer, "The New Hyperdrama", <http://www.ibiblio.org/cdeemer/newhype.htm> 21.10.2003.

751 Rotermund, "Warten auf den Online-Ulysses", 78. 
lichen Einsatzes für ein webbasiertes Drama betrifft, ist allerdings zu bedenken, daß die Kooperation der Web-Gemeinschaft - ein wesentlicher Aspekt webbasierter Publikationen - gleichzeitig auch eine gewisse Entlastung des Herausgebers bedeutet. Das technische Problem wird sich möglicherweise dadurch langfristig lösen, daß nachfolgende Generationen von Autoren entweder das notwendige Wissen selbst mitbringen oder aber zur Veröffentlichung ihrer Werke ebenso wie im Print-Bereich auf die Unterstützung von Online-Herausgebern zurückgreifen.

Ein ganz großes Entwicklungshindernis besteht aber auch darin, daß Herausgeber webbasierter Werke gegenwärtig noch nicht mit nennenswerter öffentlicher Beachtung rechnen dürfen und dementsprechend geringe Aussicht auf nachhaltigen künstlerischen Erfolg haben. Gerade dies wäre aber ein Grund, sich dieser Literaturgattung als Autor anzunehmen. Mit Sicherheit könnte eine Zunahme der Produktion webbasierter Dramen langfristig einen allgemeinen Wahrnehmungswandel bewirken; auch ließe sich die Entwicklung unter Umständen durch gezielte Öffentlichkeitsarbeit deutlich fördern. In dieser Hinsicht ist auf Herausgeber- wie auf Publikumsseite zweifellos ein Umdenken notwendig. 


\section{Die Lage der englischsprachigen Literatur im World Wide Web}

\section{Vom allgemeinen Nutzen der neuen Technik für die englisch- sprachige Literatur}

In seinem Redebeitrag zur Konferenz Anglistik im Internet stellte Neumann, seines Zeichens Professor für Anglistische Literaturwissenschaft, bereits im Jahre 1996 fest:

Wesensmerkmale des elektronischen Mediums sind [...] nicht die Ewigkeit, seine Wiedergeburt in späteren Epochen oder die Kontinuität in einem Jahrhunderte übergreifenden Kanon, sondern die des schnellen und weltweiten Austauschs. ${ }^{752}$

Auch Sparschuh betont, der unbestrittene Vorzug der elektronischen Medien liege in ihrer Schnelligkeit. ${ }^{753}$

In dieser Hinsicht ist die elektronische Post derzeit sicherlich einer der nützlichsten Dienste des World Wide Web. Daten aller Art, das heißt sowohl Text als auch Graphik, Sound und Video lassen sich zeitsparend und kostengünstig an einen oder auch mehrere Teilnehmer des Netzes gleichzeitig versenden. Gerade im Hinblick auf schriftstellerische Gemeinschaftsproduktionen ist dieser Dienst von Nutzen und erweist sich als der Spontaneität der Kooperierenden sowie der Vielschichtigkeit der gemeinschaftlichen Werke förderlich. Elektronische Post kann unmittelbar und direkt vom Computer-Arbeitsplatz aus verschickt werden, wodurch die zeitraubende und möglicherweise kostspielige Zusendung per Post wegfällt. ${ }^{754}$ Kommunikation im Web gestaltet sich im allgemeinen unkompliziert und verzichtet auf die Formalitäten des herkömmlichen Briefverkehrs; ${ }^{755}$ die Nachrichten erreichen in kürzester Zeit

752 Neumann, "Geisteswissenschaften im Internet”, 60.

753 Jens Sparschuh, "Ein Geschenk für den Leser? - Tatjana Blobel im Interview mit Jens Sparschuh", Spiegel Spezial 10: Die Zukunft des Lesens - Vom Buch zum Internet (1999), 24.

754 Da es dem Empfänger überlassen bleibt, ob er die übersendeten Daten nach Kenntnisnahme umgehend löscht oder auf Papier ausdruckt, kann dieses Nachrichtenübermittlungsverfahren auch ökonomische und ökologische Vorteile gegenüber dem herkömmlichen Postversandsystem haben, für das es prinzipiell des Papiers als Informationsträgers bedarf.

755 Im Vergleich zu brieflichen Mitteilungen haben elektronische Nachrichten weniger formellen Charakter. Hemmschwellen, die die Abfassung eines Briefes begleiten - zum Beispiel offizielle Anrede, optische Gestaltung des Textes, Postversand - entfallen. Dies erleichtert und intensiviert den Nachrichtenaustausch. Andererseits besteht der Nachteil sinkender Briefästhetik. Der Sprachstil wird vernachlässigt und Groß- und Kleinschreibung mißachtet. Im selben Zuge entwickeln sich neuartige Kommunikationsformen wie beispielsweise die Verwendung von Symbolen, die als Emoticons (einer Verbindung der Worte Emotion und Icon) bezeichnet werden und mittels derer Gefühlsregungen dargestellt werden können. Per Konvention ergeben sich aus der Kombination bestimmter Satzzeichen unterschiedliche Bedeutungen. 
ihren Empfänger, so daß die Korrespondenz immer aktuell bleibt. Ein zusätzlicher Vorteil liegt in der Tatsache, daß eine Nachricht automatisch an mehrere Empfänger gleichzeitig gesendet werden kann. Auf diese Weise können mittels Rundspruchnachrichten Informationen oder Anfragen zwischen Kooperierenden konkurrenzlos zeit- und kostensparend ausgetauscht werden.

Aufgrund der genannten Vorteile wie Globalität, Schnelligkeit und Aktualität bietet sich das World Wide Web für die kooperative Gestaltung neuer Formen von Gegenwartsliteratur geradezu an, denn das elektronische Medium eröffnet und vereint Kommunikations- und Präsentationsformen, die vor seiner Verbreitung nicht denkbar gewesen wären und sich auch heute nicht auf andere Weise bereitstellen lassen.

Doch nicht nur den Literaturschaffenden kommt im WWW eine neue Rolle $\mathrm{zu}$, auch die Rezipienten stehen vor neuartigen Herausforderungen.

Zum einen versetzt die Möglichkeit zur Interaktion den Rezipienten in einen völlig neuartigen Lektüreprozeß, wie er bei linear-konzipierter beziehungsweise gedruckter Literatur nicht zu realisieren wäre. Dank der Hypertext-Systematik kann der Rezipient seinen individuellen Leseverlauf bestimmen und bei jedem neuen Rezeptionsvorgang einen unterschiedlichen Entwicklungshergang herbeiführen. Dabei unterstreichen multimediale Elemente den Erlebnischarakters der Lektüre. Das Bedürfnis nach weiterführenden Informationen wird durch hypertext-interne Verknüpfungen mit Hinweisen des Verfassers oder Links zu Ressourcen im World Wide Web befriedigt. Bei Fragen und Anregungen besteht die Gelegenheit, per E-Mail unmittelbaren Kontakt mit dem Autor aufzunehmen. In Kommunikationsforen kann der Rezipient ferner mit Gleichgesinnten in geistigen Austausch treten.

Besteht neben der Möglichkeit zur Interaktion auch Gelegenheit zur Kooperation, so wird der Leser gleichzeitig zum Autor und kann das betreffende Werk nach seinen eigenen Vorstellungen ergänzen. Auf diese Weise vermag er sich entweder unverbindlich kreativ zu betätigen oder auch ernsthaft seine persönliche Befähigung zum Schriftsteller zu erproben. Durch die zukunftsoffene, positiv eingestellte Annahme und Umsetzung der breiten Palette neuer technischer Angebote gewönne die englischsprachige Gegenwartsliteratur ein gänzlich verändertes aufregendes Erscheinungsbild. Sie würde heterogen, multimedial und multikulturell und präsentierte sich dynamisch, wandelbar und offen. 


\section{Die gegenwärtige englischsprachige Literatur im WWW kritisch gesehen}

Allerdings hat sich die neue Dimension des Schreibens im World Wide Web tatsächlich nicht in dem Maße durchgesetzt, wie es die große Zahl der theoretischen Texte zum Thema Hypertext-Systematik hätte vermuten lassen. Es scheint, als liege die Botschaft bei elektronischen Publikationen nach wie vor zu stark in der Präsentationsform, das heißt im Medium selbst. Offenbar erschöpft sich der revolutionäre Akt für die Herausgeber bereits im Wechsel vom Print-Bereich zu digitalen Datenträgern. Momentan herrscht genau jener Eindruck vor, den Douglas mit den Worten zum Ausdruck bringt, Hypertexte seien inhaltlich noch längst nicht ausgereift und im Vergleich zu Büchern - jenen ausgefeilten Ergebnissen einer simplen Technologie nur kümmerliche Exempel einer hochentwickelten Technik: "If the book is a highly refined example of a primitive technology, hypertext is a primitive example of a highly refined technology, [...]."

Wie die Bestandsaufnahme zeigte, findet sich im WWW überwiegend computergestützte, webunabhängige und mehr noch konventionelle, linear-konzipierte Literatur. Beide Darstellungsformen besitzen nicht das Potential, etwas grundsätzlich Neues und Revolutionäres hervorzubringen.

Lineare Textformen, die für eine kontinuierliche Leseweise konzipiert sind und mittels Digitalisierung - ungeachtet aller Möglichkeiten, die das World Wide Web bietet - in die ursprünglich nicht vorgesehene Form des fortlaufenden Fließtextes eingepaßt werden, büßen laut Glaser ihre Qualität ein. Aus diesem Grunde spricht er sich gegen die Online-Publikation von Drucktexten aus: "Literarische Texte, wie wir sie in Büchern lieben, verlieren im Netz ihre Würde und verkommen zur reinen Datei." 757

Computergestützte, webunabhängige Literatur vermittelt zwar einen deutlich innovativeren Charakter, da sie im Gegensatz zu konventioneller, für ein lineares Medium verfaßter Literatur speziell auf die Charakteristika des Computers abgestimmt ist. Gleichwohl bildet sie in ihrer Form als programmgesteuerte Literatur, sei es ohne oder mit Interaktionsmöglichkeiten, oft nicht mehr als eine spielerische Abwechselung; in ihrer anderen Form als Literatur im geschlossenen Hypertext birgt

\footnotetext{
756 Douglas, The End of Books, 15.

757 Peter Glaser, "Die große Odyssee - Wie haben das Internet und der Computer das Schreiben und Lesen verändert?”, Die Zeit - Magazin, 20.09.1996, 6-10, hier: 9.
} 
sie immerhin, wie in Kapitel IV.2. dargelegt, das Potential einer "Revolution des Lesens" ${ }^{\text {"758, }}$, vermag aber auch keinen grundlegenden Umbruch der literarischen Kunst auszulösen.

Der Befund läßt sich nicht damit erklären, das Web befinde sich derzeit gewissermaßen noch in statu nascendi, auch wenn dieser Einwand oftmals ins Feld geführt und angesichts der langen Anlaufzeit anderer Innovationen, die mittlerweile zum festen Bestandteil des Lebens zählen, vermutlich auch auf absehbare Zeit noch als Argument dienen wird. ${ }^{759}$ Auf dem Gebiet der Literatur hat das elektronische Medium mittlerweile schon mehrere Phasen durchlaufen und erfährt gegenwärtig erneut einen Umbruch. Nach einer Publikationswelle, in der es schon innovativ erschien, überhaupt im Web zu veröffentlichen, und in der vorwiegend die erwähnte konventionelle, linear-konzipierte Literatur ins WWW gestellt wurde, ließ sich eine Art Experimentierphase beobachten, die offenbar von dem Wunsch nach Befreiung von den Zwängen der herkömmlichen Form der Literaturpräsentation getragen war. Der zwischenzeitige Versuch, linear-konzipierte Gegenwartsliteratur kostenpflichtig $\mathrm{zu}$ veröffentlichen, fand nicht genug Anklang, um sich allgemein durchzusetzen. ${ }^{760}$ Vielmehr schien sich die Verbindung zwischen Literatur und World Wide Web zu Beginn dieses Jahrtausends allmählich aufzulösen. Momentan drängt sich der Eindruck auf, als stagniere die Literaturproduktion im Netz. In vereinzelten Foren verfassen Hobbyautoren - zumeist jeder für sich allein - vorwiegend Kurzgeschichten

758 Nestvold, "Das Ende des Buches", 23.

759 Vgl. hierzu auch den in Kapitel III.1. erwähnten Hinweis Bolters auf die ersten Jahrzehnte des Buchdrucks. Bolter, "Das Internet in der Geschichte der Technologien des Schreibens", 47.

760 Im März 2000 begann der US-Autor Stephen King mit der ausschließlich digitalen Veröffentlichung seines Romans Riding the Bullet. Die Bereitstellung erfolgte kapitelweise zum Preis von jeweils 1,- \$ pro Kapitel, allerdings waren nach 14 Tagen bereits Raubkopien des Werkes kostenfrei im Web erhältlich. King bewertete sein Buchexperiment dennoch als Erfolg; nach 41/2 Monaten sei immerhin für 93.000 von ca. 152.000 Downloads des ersten Kapitels gezahlt worden, weitere 20.000 Leser hätten die Bezahlung fest zugesagt. Golem.de, "Stephen Kings Buchexperiment erfolgreich", in: Dies., Hg., Golem.de - IT-News für Profis ([102.08.2000]), $<$ http://www.golem.de/0008/9049.html $>$, 08.03.2004. In einem Kommentar hierzu heißt es freilich, King profitiere von seinem bekannten Namen; es sei davon auszugehen, daß nicht ganz so bekannte Autoren mit ähnlichen Konzepten weniger Erfolg haben würden. Daher sei es falsch, das Ergebnis als Durchbruch neuer Vermarktungsstrategien zu werten. Ebd., 08.03.2004. Neben dem bekannten Namen dürfte nach Ansicht der Autorin auch die Neuartigkeit der Distribution zur hohen Zahl der Downloads beigetragen haben. Ein Artikel aus Oktober 2003 bestätigt dies: “ 'Die uneingeschränkte Euphorie der Anfangszeit gehört der Vergangenheit an', so Arnoud de Kemp, Sprecher des Arbeitskreises Elektronisches Publizieren. 'Die Möglichkeiten und Chancen des Elektronischen Publizierens werden durchaus erkannt, aber in einem realistischen Licht betrachtet.' " Über 70 Prozent der Verlage gingen davon aus, der Anteil elektronischer Produkte am Gesamtumsatz werde in zehn Jahren maximal 30 Prozent betragen.” Golem.de, “ Elektronisches Publizieren: Ernüchterung macht sich breit", in: Dies., Hg., Golem.de - IT-News für Profis ([ $\left.\left.{ }^{1} 09.10 .2003\right]\right),<$ http://www.golem.de/0310/27827.html $>, 08.03 .2004$. 
und Gedichte, denen jedoch kein Erfolg beschieden ist; das webbasierte Drama findet paradoxerweise trotz seiner nachweislich hervorragenden Eignung für eine Darstellung im WWW kaum Verbreitung. Neue innovative Projekte zur Kreation kooperativer Werke bleiben derzeit offensichtlich aus.

Die Qualität der augenblicklich zugängigen Dokumente ist zudem kritisch zu beurteilen. Ein erheblicher Teil von ihnen ist offensichtlich schon kurze Zeit nach der Veröffentlichung keiner weiteren Überarbeitung mehr unterzogen worden. Bei geschlossenen Hypertext-Systemen wäre dies nicht notwendigerweise als Nachteil anzusehen. Auch gedruckte Veröffentlichungen werden oftmals nicht mehr verändert, sondern lediglich neu aufgelegt; zudem besteht bei einem rein literarischen Hypertext nicht die Notwendigkeit der Überarbeitung. Beinhaltet der Text jedoch auch Sekundärmaterial, so kann je nach Thematik eine Anpassung an den neuesten Informationsstand nötig sein. Bei einem offenen Hypertext schließlich ist eine kontinuierliche Pflege unumgänglich, um die Aktualität der Zugriffsmöglichkeiten auf systemexterne Verknüpfungen zu gewährleisten.

Es erscheint nachvollziehbar, wenn sich der Nutzer angesichts des momentanen Erscheinungsbildes der Veröffentlichungen im WWW des Eindrucks nicht erwehren kann, alles, was das Web zu bieten habe, verströme “den Geruch einer Naivität und Provinzialität, die mit den Verheißungen einer weltweiten Vernetzung, eines globalen Aufbruchs zu neuen Ufern von vornherein nicht mithalten kann." ${ }^{\text {761 }}$ Diese Diskrepanz zwischen überschwenglichen Vorankündigungen und ernüchternder Realität mag generell Zweifel aufwerfen, wie sie Neumann speziell im Hinblick auf den wissenschaftlichen Bereich äußert, nämlich “ob sich hinter dem allgemeinen laxen Sprachgebrauch und dem ständigen präverbalen Mausklicken auf den Icons des globalen Surfens überhaupt ein komplexes Denken entwickeln und auf diesem Wege eine echte Konkurrenz zu gedruckten wissenschaftlichen Veröffentlichung entstehen kann." 762

Viele Abhandlungen zum World Wide Web sind nur sehr oberflächlich und emotionsgelenkt und beschränken sich mehr oder weniger auf eine allgemeine Erörterung von Sinn und Unterhaltungswert der Online-Literatur. Während gewisse The-

761 Bolter, "Das Internet in der Geschichte der Technologien des Schreibens", 110.

762 Neumann, "Geisteswissenschaften im Internet", 53. 
menbereiche keinerlei oder nur flüchtige Erwähnung finden, werden andere Fragen umso häufiger und ausgiebiger behandelt. ${ }^{763}$

Die Forderung nach einer eigenen Terminologie für die neue Art der Literatur wurde zwar wiederholt erhoben, tatsächlich wurden aber bisher nur wenige ernsthafte, konstruktive Lösungsansätze vorgetragen. ${ }^{764}$ Zumeist erweckten die vorgebrachten Alternativvorschläge den Eindruck, als hätten sich ihre Namensgeber nur sehr oberflächlich mit dem Literaturangebot im World Wide Web beschäftigt.

Wenn die elektronischen Medien im Laufe der vergangenen Jahre auch in vielen Bereichen einen selbstverständlichen Platz einnehmen und zu Bereicherung und Fortschritt der jeweiligen Disziplinen eingesetzt werden konnten, scheint es doch, als befänden wir uns jedenfalls im englischsprachigen Literaturbetrieb zum gegenwärtigen Zeitpunkt tatsächlich noch immer in einer Art Übergangsphase zwischen herkömmlichem und zukünftigem Selbstverständnis. Um in dieser Hinsicht Abhilfe zu schaffen, ist ein grundsätzliches Umdenken notwendig. Es empfiehlt sich, zum Abbau innerer Hemmungen zunächst technische Kompetenzen zu vermitteln, da nur das Gefühl für einen sicheren Umgang mit der Materie auch die Bereitwilligkeit schafft, sich auf Neues einzulassen.

Dementsprechend ist die Voraussetzung für eine signifikante Hebung von Quantität und Qualität speziell für das World Wide Web erstellter literarischer Werke erst dann gegeben, wenn das Defizit an technischer Kompetenz im Kreise der Autoren behoben ist. Angesichts des veränderten Erscheinungsbildes der Literatur, das aus der zunehmenden Integration elektronischer Medien in den Literaturbetrieb resultiert, muß die Vorgabe dort lauten, die digitale Computertechnik entsprechend den Bedürfnissen des modernen Lesers in höchstmöglichem Maße auszuschöpfen. Es

763 "Da jeder verständlicherweise nur das ins Netz stellt, was er persönlich für gut und wichtig erachtet, werden manche Fragestellungen quasi ad nauseam traktiert, während andere schlicht und einfach nicht vorkommen." Gersmann, "Schöne Welt der bunten Bilder", 110.

764 Auch Hautzinger stellt angesichts der Heterogenität elektronischer "Textgeflechte" die Frage nach den Interpretationskriterien. Hautzinger, Vom Buch zum Internet?, 117. Als Ausgangspunkt für eine wissenschaftliche Herangehensweise führt sie die Positionierung der Links innerhalb der Texte an. Ihrer Ansicht nach bildet die Analyse ihrer Anordnung die entscheidende Basis für eine vom jeweils leserspezifischen Vorgehen unbeeinflußte Beschäftigung mit dem Text. Hautzinger schreibt hierzu: "Es läßt sich vermuten, daß der Anordnung der Links besondere Bedeutung im Text zukommt, die es ermöglicht, eine objektivere Auseinandersetzung unabhängig vom individuellen Leseverlauf zu führen.” Ebd. Daß der Autor eines Hypertextes die Position der Verknüpfungen nach Folgerichtigkeit und Sinnzusammenhang bestimmt, sollte vorauszusetzen sein. Es darf jedoch bezweifelt werden, daß die Analyse der Link-Struktur zu einer "objektiveren Auseinandersetzung" mit dem Text verhilft. Ebd. Hautzingers These scheint auf der Annahme zu basieren, Links enthielten nur z u s ätzli c he Angaben, tatsächlich aber bilden sie das Hypertext-System. 
muß das Ziel aller Autoren sein, so souverän über die Darstellungsmöglichkeiten von Hypertext und WWW zu verfügen und gleichsam spielerisch mit ihnen zu verfahren, daß es gelingt, "neuartige Konflikte zwischen visueller Gestalt und semantischem Gehalt von Texten anzuzetteln oder eine neue Einheit von visueller und akustischer Bearbeitung von Sprachmaterial zu schaffen" ${ }^{765}$ Die Verfasser literarischer Hypertexte müssen technisch stets auf der Höhe des Machbaren sein, um eine ausgewogene und übersichtliche Umsetzungen des Hypertext-Prinzips zu gewährleisten. Unter dem Stichwort "Integration" "766 müssen die charakteristischen hypertextuellen und webspezifischen Merkmale, nämlich Interaktivität, Kooperativität, Multimedialität und Verknüpfung mit Ressourcen aus dem World Wide Web auf überzeugende Weise in das literarische Werk eingebracht werden.

Neben den technischen Kenntnissen, ohne die der Versuch einer Veröffentlichung im WWW bereits an der äußeren Konzeption scheitert, ist die Vertrautheit mit der inhaltlichen Gestaltung von Hypertexten oberste Voraussetzung für die Kreation digitaler Literatur. Trotz zahlreicher inhaltlicher Varianten muß ein Hypertext-Werk die Bedingungen der Plausibilität, Attraktivität und Abgeschlossenheit erfüllen, damit die Lektüre den Leser überzeugt, ihn gleichzeitig aber auch fasziniert und ihm nach Beendigung des Leseprozesses ein Gefühl der Befriedigung verschafft.

Nach wie vor steht die Herausforderung im Raum, etwas revolutionär Andersartiges zu schaffen, das vollkommen auf die Gegebenheiten des Web abgestimmt und nicht ohne erhebliche Qualitätseinbußen in einem anderen Medium zu reproduzieren ist. Phantasie und Kreativität zählen daher zu den Attributen, die für die Erstellung von Hypertext-Literatur absolut unabdingbar sind. Aus diesem Grunde sei die Empfehlung ausgesprochen, dem WWW Aufgeschlossenheit und Neugier entgegenzubringen, da nur aus einer gewissen Experimentierfreudigkeit innovative Ideen für eine grundsätzlich neuartige Form der Literaturpräsentation erwachsen können.

Da theoretisch jeder, der die technischen Voraussetzungen mitbringt, seinen Beitrag zur (englischsprachigen) Literatur im World Wide Web leisten kann, obliegt die Beurteilung der dort präsentierten Dokumente letztlich dem Nutzer. Es stellt sich allerdings die Frage, ob dieser bei der Auswahl elektronischer Dokumente wirklich eine höhere Verantwortung trägt beziehungsweise größere Sorgfalt anwenden muß,

765 Rotermund, "Warten auf den Online-Ulysses", 78.

766 Neumann, "Geisteswissenschaften im Internet", 58. 
als dies bei der Selektion gedruckter Literatur der Fall ist. Kaiser zieht hier einen Vergleich zwischen herkömmlichen Bibliotheken und der digitalen Bibliothek des WWW und weist darauf hin, daß der Nutzer konventioneller Bibliotheken im allgemeinen aus der Masse des verfügbaren Materials wie selbstverständlich die ihm wichtige Literatur auswählt. Viele Bücherregale - so Kaiser - läßt der Nutzer bei seiner Literaturrecherche intuitiv unbeachtet, um aus einem einzigen, ganz bestimmten Regal gezielt das gesuchte Buch auszuwählen. Diese Zielstrebigkeit basiere jedoch auf Erfahrung und auf der Nutzung von Katalogen und Lageplänen, und so kommt Kaiser zu dem Schluß, daß die Informationsflut im Internet keineswegs derart bedrohliche Ausmaße aufweist, daß sie nicht ebenso wie in herkömmlichen Bibliotheken zu bewältigen wäre. ${ }^{767}$

Die Hoffnung, daß literarische Texte aus dem Web und vor allem webbasierte Literatur ein aufgeschlossenes Publikum und Beachtung durch die Literaturwissenschaft finden möchten, muß mit der Erkenntnis einhergehen, daß Computerkompetenz künftig eine Basisqualifikation aller derer, die sich kreativ im WWW betätigen wollen, aber auch des Literaturwissenschaftlers sein wird. ${ }^{768}$

Die Teilhabe des Literaturwissenschaftlers an den Fortschritten der elektronischen Kommunikation dient dabei keineswegs allein der Literaturwissenschaft. Sobald dieser nämlich der Schritt in das Zeitalter neuartiger Kommunikationstechnologien und völlig neuer Literaturformen gelingt, ist anzunehmen, daß von ihr auch deutliche positive Impulse in Richtung auf Herausgeber, Produzenten und Autoren ausgehen.

Das Signal zum Aufbruch in eine neue Ära der Literatur ist gegeben. Ob dieser Aufbruch wirklich stattfindet, hängt ab von der Bereitschaft derer, an die es gerichtet ist, es aufzunehmen und sich in Bewegung zu setzen.

767 Kaiser, Literarische Spaziergänge im Internet, 11.

768 Rutenfranz sah diese Eignung bereits im Jahre 1997 in seinen Ausführungen zur Bedeutung des Mediums Computer für das Kommunikationssystem Wissenschaft als unabdingbar an. Uwe Rutenfranz, Wissenschaft im Informationszeitalter: Zur Bedeutung des Mediums Computer für das Kommunikationssystem Wissenschaft (Opladen, 1997), 152. Der Initiativkreis Bildung der BertelsmannStiftung sieht die modernen Kommunikationstechnologien gar als einen "Garant[en] für eine Effizienzsteigerung im Bildungsbetrieb" und spricht in diesem Zusammenhang von einem "Quantensprung der Wissensvermittlung". Neumann, "Geisteswissenschaften im Internet”, 55. Noch einen Schritt weiter geht Kuhlen, der in dem modernen Wissenschaftler nicht nur einen sachkundigen Computernutzer sieht, sondern ihm bereits eine weitere Kompetenz zuschreibt: "Entfällt [...] die Lektoratsleistung seitens des Verlags und stehen der Wissenschaft die elektronischen Distributionskanäle ohnehin zur Verfügung, so ist der Schritt nicht weit vom schreibenden zum elektronisch verlegenden Wissenschaftler." Rainer Kuhlen, "Zur Virtualisierung von Bibliotheken und Büchern”, in: Matejovski und Kittler, Hg., Literatur im Informationszeitalter, 112-142, hier: 119. 


\section{Die besondere Situation der webbasierten englischsprachigen Literatur, deren Ursache und Überwindung}

Wie die vorliegende Arbeit gezeigt hat, ist es innerhalb der digitalen Literatur vor allem der Bereich der webbasierten Literatur, der tatsächlich epochemachend wirken könnte. Umso desillusionierender ist das Ergebnis der Bestandsaufnahme: Trotz einiger aufwendiger Veröffentlichungen mit vielversprechendem, hohem konzeptionellen Anspruch bleibt das Angebot an webbasierter Literatur im großen und ganzen sowohl nach Umfang ${ }^{769}$ wie Qualität weit hinter allen euphorischen Prognosen zurück und präsentiert sich in seiner gegenwärtigen Form als leider eher dürftig. Ausweislich der Fallbeispiele in Kapitel VI. konnte keine der Veröffentlichungen uneingeschränkt wirklich alle Kriterien für eine webbasierte Publikation in befriedigender Weise erfüllen. In der Regel lag der Schwerpunkt auf nur einer der geforderten Eigenschaften für webbasierte Publikationen, während andere Merkmale lediglich sehr oberflächlich oder mitunter gar nicht vertreten waren. Erlaubt "Poem Number 334" beispielweise keine Interaktion, so gestattet The Ultimate Writer wiederum keine Kooperation; Mahoney the Cat hingegen sah keine Zugriffe auf das Web vor, und "PERL Poetry" ließ multimediale Elemente gänzlich vermissen. $M$ is for Nottingham? kam der Zielsetzung als einzige der Veröffentlichungen sehr nahe, gab aber gleichwohl noch Anlaß zu Kritik.

So gesehen wird der Rezipient vorwiegend mit Mittelmaß konfrontiert. Die Veröffentlichungen präsentieren sich als durchaus innovativ, aber keine der Publikationen brachte etwas so grundlegend Neues hervor, daß sie das Prädikat "revolutionär" verdient hätte: Nirgendwo ist mit anderen Worten etwas Außergewöhnliches auszumachen, das sich durch Attribute auszeichnet, die in ihrer Einzigartigkeit und Dynamik nur im Web entstehen und existieren können. Damit sind selbstverständlich nicht jene Merkmale gemeint, die bereits aus gedruckten Medien bekannt sind und im Web lediglich reproduziert und durch werkinterne und werkübergreifende Interaktivität sowie Kooperativität und Multimedialität ergänzt werden. Im

\footnotetext{
769 Angesichts der Unübersichtlichkeit des Web sollte allerdings gerechterweise darüber nachgedacht werden, ob der Eindruck, webbasierte Literatur sei selten, wirklich der Realität entspricht. Möglicherweise entzieht sich ein wesentlicher Teil der Online-Veröffentlichungen der Wahrnehmung und Recherche, weil sich die Produktivität der Autoren nur in speziellen, für die Allgemeinheit unzugänglichen Foren offenbart. Gegebenenfalls gehen in diese Richtung auch die Gedanken Suters, der meint, es habe den Anschein, als wachse abseits des herkömmlichen Literaturbetriebes eine neue kulturelle Sphäre heran: "Autoren und Künstler haben sich im Netz einen neuen sozialen Raum geschaffen." Suter, "Ein neues Literaturmilieu", <http://www.netzliteratur.net/suter/kassel.htm>, 07.07.2003.
} 
Grunde geht es um Elemente, die sich derzeit noch außerhalb der Vorstellung bewegen.

Mit der allgemeinen Verbreitung des Web schienen Gesamtkunstwerke unter Verwendung solcher Idealelemente plötzlich möglich zu werden. Die Frage lautet allerdings, warum kein Autor das Wunschbild einer webbasierten Publikation in die Realität umzusetzen vermochte.

Eine mögliche Erklärung wäre die Komplexität von Hypertexten und speziell jenen im Web, die neben einer internen Verknüpfungsstruktur auch noch Verbindungen nach außen aufweisen sollten. Aus dieser Komplexität wurde bereits in den Kapiteln III.3.1. und V.3.1. gefolgert, hypertextuelle Werke verlangten eine tiefergehende und gewissenhaftere Analyse als gedruckte Werke. Die zunächst nur auf die bloße Rezeption bezogene Annahme könnte auch - und möglicherweise mit noch größerer Berechtigung - für die K o n z e p ti o n (offener) Hypertext-Werke gelten.

Um diese Hypothese eingehender zu prüfen, sei das Verhältnis zwischen Leser und Text unter Berücksichtigung der praktischen Erfahrungen im Umgang mit webbasierter Literatur der beschriebenen Art nachfolgend genauer analysiert.

\subsection{Von der unterschiedlichen Grundeinstellung zu Hypertext und Buch}

Wenn die Nutzung von Hypertext-Systemen ${ }^{770}$ wirklich eine höhere geistige und motorische Flexibilität erfordert, liegt dies offenbar daran, daß Hypertexte aufgrund ihres Aufbaus und der damit verbundenen Anforderungen an den Leser dessen grundsätzlicher Denkweise und seiner Psyche prinzipiell widersprechen. Umgekehrt formuliert: Linear-konzipierte Print-Literatur kommt unserem Wesen und Bewußtsein anscheinend mehr entgegen. Die Gründe hierfür sind vielfältig. ${ }^{771}$

Erstens: Aufgrund seiner (relativen) Unveränderbarkeit bedeutet das gedruckte Dokument für den Leser gewissermaßen einen materiellen, greifbaren Besitz. Es vermittelt ihm die Gewißheit, das Dokument jederzeit in gleichbleibendem Zustand wieder vorzufinden, und verschafft ihm damit ein Gefühl der Solidität. Gleichzeitig bildet das Print-Dokument auch ein Medium der Bezugnahme, das heißt eine gemeinsame Gesprächsgrundlage.

\footnotetext{
770 An dieser Stelle geht es lediglich um elektronische Hypertext-Systeme im allgemeinen, unabhängig davon, ob sie computergestützt und webunabhängig oder aber webbasiert sind; der anschließende, letzte Abschnitt hingegen nimmt konkreten Bezug auf offene Hypertext-Systeme.

771 Bezüglich der nachfolgenden Argumente vgl. auch Birkerts, Die Gutenberg-Elegien, 166.
} 
Ein Hypertext hingegen zeichnet sich gerade dadurch aus, daß er möglichst vielfältig und mehrschichtig strukturiert ist, was einschließt, daß er in der Regel niemals in identischer Konstellation wieder aufgerufen werden kann. Auch wenn die einzelnen Textelemente unverändert bleiben, wird der Leser doch bei jedem Neueinstieg mit andersgelagerten Verhältnissen konfrontiert. Somit ist es nicht ohne weiteres möglich, eine in Bearbeitung befindliche Geschichte eine gewisse Zeit lang ruhen zu lassen. Entweder ist der Rezipient in der Lage, das jeweilige Lesepensum während eines einzigen Lesevorgangs zu bewältigen, oder aber er bricht das Hypertext-System - und somit auch die entsprechende Geschichte vorzeitig ab. Dies ist beispielsweise bei The Ultimate Writer der Fall, wo der Agierende gezwungen ist, alle Gedichtübungen in einem Durchgang zu bewältigen, andernfalls er bei seinem nächsten Besuch wieder neu beginnen muß, da keine Möglichkeit der Ergebnisspeicherung vorgesehen ist.

Zweitens: Ein Print-Text - als feste Größe - läßt dem Leser die Freiheit desjenigen Lesetempos, das seinen jeweiligen geistigen und körperlichen Fähigkeiten entspricht und frei von äußeren Zwängen ausschließlich durch den aktuellen Grad seiner Auffassungsgabe und Konzentration bestimmt wird.

Ein Hypertext hingegen nötigt dem Leser gelegentlich eine bestimmte Bearbeitungs- oder Verlaufsgeschwindigkeit auf. Beispielsweise sind manche Systeme so programmiert, daß sich die Bildschirmanzeigen regelmäßig nach Ablauf bestimmter Zeitspannen automatisch verändern, was einen nicht zu unterschätzenden Druck auf den Rezipienten ausüben kann.

Drittens: Analysen des Nutzerverhaltens und der persönlichen Vorlieben hinsichtlich gedruckter und elektronischer Texte haben ergeben, daß letztere - was die Leseatmosphäre betrifft - als "unsinnlich", in ihrer Handhabung als "unpraktisch" sowie "unübersichtlich" und hinsichtlich der körperlichen Auswirkungen als "anstrengend [und] ermüdend" empfunden werden. ${ }^{772}$ Als Vorteile des Online-Mediums gelten demgegenüber seine Aktualität, sein hoher Informationsgehalt und die schnelle Zugriffsmöglichkeit auf das angebotene Datenmaterial. ${ }^{773}$ Das WWW soll danach hauptsächlich zum Nachschlagen von Informationen und Abfragen von Auskünften dienen; von Lektüretexten erwartet die überwiegende Nutzermehrheit hingegen, daß

\footnotetext{
772 Sabine Gross, “Das Buch in der Hand: Zum situativ-affektiven Umgang mit Texten”, in: Leseverhalten in Deutschland im neuen Jahrtausend, 175-197, hier: 189.

773 Ebd., 188.
} 
sie möglichst in greifbarer Form vorliegen, das heißt nötigenfalls als Papierausdruck des Online-Materials. ${ }^{774}$ Bestätigt wird dies durch das Ergebnis einer von Spiegel Spezial in Auftrag gegebenen Untersuchung: auf die Frage, ob sie sich ihre Zeitung beziehungsweise Zeitschrift lieber am Bildschirm oder auf Papier wünschten, gaben 90 Prozent aller Deutschen an, die Papierversion zu bevorzugen. ${ }^{775}$

Viertens: Die zumeist lineare Konzeption eines gedruckten Dokuments empfiehlt in der Regel eine sequentielle, schrittweise aufeinander aufbauende Bearbeitung, wie sie üblicherweise dem Umgang des Menschen mit unbekannten Situationen und Sachverhalten entspricht. Das Verständnis von Zusammenhängen entwickelt sich im allgemeinen ebenso wie eine Problemlösungsstrategie als Schlußfolgerung aus einem Gesamteindruck, der sich wiederum sukzessive aus allen vorhandenen Informationselementen zusammensetzt. Laut Heim helfen lineare Gedankenprozesse, alle aufgenommenen Informationen zu strukturieren. Diesen Vorgängen liege die Tendenz zugrunde, einen Anfang aufzubauen, eine gedankliche Mitte zu konstruieren und mit einem Ausblick abzurunden. Geordnete Logik werde zur Norm gedanklicher Abläufe. ${ }^{776}$

An dem Wechselspiel kontinuierlichen Sich-Fortbewegens im Text und proportional dazu anwachsenden Wissens und Verständnisses lassen sich also bekannte menschliche Verhaltensmuster aufzeigen. Dazu zählt neben den rationalen Beweggründen auch die emotionale Komponente. Die Beschäftigung mit einer linear konzipierten Lektüre kann dem Leser eine Befriedigung verschaffen, die ein Hypertext in der Regel nicht gewähren kann: Der Leser hat alle vom Autor zur Verfügung gestellten Informationen übersichtlich und faßbar vorliegen und kann nach kompletter Lektüre des Werkes sicher sein, es vollständig erschlossen zu haben.

\footnotetext{
774 Ebd.

775 “Lieber Papier”, Spiegel Spezial 10: Vom Buch zum Internet: Die Zukunft des Lesens, 11. - Interessanterweise liegt die Lesegeschwindigkeit bei Bildschirmtexten im Vergleich zu Gedrucktem um 2030 Prozent niedriger. Welche Umstände die Geschwindigkeit verlangsamen, das heißt ob Nutzererfahrungen, Bildschirmdarstellungen oder andere Faktoren dafür verantwortlich sind, konnte allerdings bislang noch nicht ermittelt werden. Jürgen Redelius, Der 'digitale' Gutenberg: Untersuchungen zur Lesbarkeit digitaler Bildschirmschriften (Ludwigsburg, 1998), 81, 135. Die Dissertation von Redelius weist einen entscheidenden (Tipp-)Fehler auf: 170 Sekunden für 500 Worte entsprechen ca. 176 Wörtern pro Minute (wpm), womit die Lesegeschwindigkeit für das Lesen von Bildschirmen um ca. 20 Prozent niedriger liegt. Nur so bestätigen sich auch die Forschungsergebnisse von Gould und Grischkowsky. - Die Erkenntnis, daß ein Leser deutlich mehr Zeit benötigt, um einen Text am Bildschirm zu rezipieren, könnte eine weitere mögliche Erklärung dafür sein, warum er die Lektüre am Bildschirm meidet und statt dessen gedruckte Texte vorzieht.

776 Vgl. Heim, The Metaphysics of Virtual Reality, 42
} 
Insofern kommt ein gedruckter Text dem Leser hinsichtlich seiner Erfahrungen und üblichen Vorgehensweisen eher entgegen als ein Hypertext, der sowohl gezieltes Handeln als auch zielloses Sich-Bewegen gestattet. Letzteres entspricht zwar grundsätzlich dem assoziativen Denken; Textelemente erscheinen auf dem Bildschirm in ebenso rascher und unvermittelter Weise, wie Gedanken gelegentlich in den Sinn kommen. ${ }^{777}$ Aufgrund seiner vielschichtigen, verzweigten Struktur kann ein Hypertext aber durchaus auch als Labyrinth empfunden werden. Wingert sieht bezeichnenderweise den "Sprung" als das wesentliche Merkmal eines Hypertextes. ${ }^{778}$ Durch die diskontinuierliche Leseweise werde die Aufmerksamkeit verstärkt auf diejenigen Verknüpfungen innerhalb des Textes gelenkt, die dem Leser weitere Informationen versprächen und ihn somit von dem gerade Gegenwärtigen fortleiteten. Wingert spricht hier von sogenannten "zentrifugalen Kräften”. 779

Mag dies auch eine etwas überspitzte Aussage sein, so ist doch festzuhalten, daß es dem Leser schwerfallen dürfte, bei seiner ruhelosen Bewegung in einem undurchsichtigen Netz von Verknüpfungen ein klares Ziel auszumachen. Getrieben von Neugierde und der Befürchtung, eine wichtige oder interessante Information zu verpassen, wird er vermutlich von einem Hypertext-Element zum nächsten fortgelenkt, ohne unbedingt ein befriedigendes Ergebnis zu erzielen.

Im Grunde genommen befindet sich der Rezipient nicht selten in einer undankbaren Rolle: Entweder bleibt er ungefordert, da innerhalb der Dokumente zu wenige Interaktions- oder Kooperationsmöglichkeiten bestehen oder abgeschlossene Kunstwerke präsentiert werden, die wie ein Film in einer endlosen Multimedia-Darstellung vor dem Auge des Betrachters ablaufen, oder aber er fühlt sich angesichts kompliziert strukturierter und schwer durchschaubarer Hypertexte mit der Bedienung dieser Systeme überfordert. Hypertexte verlangen - ob sie nun online oder offline vorliegen - eine kritische Leseweise sowie eine kontrollierte und abwägende Nutzung der informationellen Einheiten. Es ist unbedingt erforderlich, sich eine selektive Wahrnehmung anzutrainieren, um nicht die Übersicht $\mathrm{zu}$ verlieren oder sich online in den Weiten des WWW zu verirren. So bedarf der Nutzer bei der HypertextLektüre eines hohen Maßes an Orientierungsvermögen und Disziplin, um sich nicht zu sehr von der eigentlichen Erzählung zu entfernen. Der Grat zwischen diskontinu-

\footnotetext{
777 Ebd., 43.

778 Wingert, “Kann man Hypertexte lesen?”, 202.

779 Ebd.
} 
ierlichem, aber dennoch homogenem Lesevergnügen und zur Resignation führender Leseverwirrung ist schmal. Wirth sieht in der Orientierungslosigkeit im Umgang mit Hypertext-Systemen eine Parallele zum topographischen Lesen und zieht einen Vergleich zu "dem Problem, eine Karte zu lesen, ohne den eigenen Standpunkt zu kennen" ${ }^{\text {780 }}$. Das Problem der Angebotsvielfalt beschäftigt auch Schmundt, für den die "Polylinearität" eines Hypertextes in kritischem Maße labyrinthischen Charakter aufweist und der darin die Gefahr eines "cognitive overload"781 sieht.

Es sind also insgesamt vier Aspekte, die allesamt die Feststellung tragen, die Leseweise gedruckter Texte decke sich mit menschlichen Verhaltensmustern und komme dem Leser folglich eher entgegen als die Rezeption von Hypertexten. Demgegenüber läßt sich vermutlich nur ein einziges Feld erkennen, auf dem umgekehrt Hypertexte dem Leser eher gerecht werden als Print-Medien: Hypertext-Systeme befriedigen das Bedürfnis nach spielerischer Zerstreuung und Abenteuer.

In der Tat kommt dieser Komponente eine wichtige Rolle im Verhältnis von Leser und Text zu. Nicht ohne Grund sind es Bezeichnungen wie player, gambler $^{782}$ und play $^{783}$, die zur Charakterisierung von Hypertext-Nutzern und Aktivitäten innerhalb eines Hypertext-Systems verwendet werden. ${ }^{784}$ Die Freude am spielerischen Umgang ist ein entscheidender Impuls für die Beschäftigung mit Hypertexten, verspricht sie doch die Erfüllung von Wünschen, denen die meisten Menschen aus vielerlei Gründen üblicherweise nicht nachgeben. Im Hypertext ist es möglich, selber Regie zu führen, sich auf unbekannte Situationen einzulassen und in fremde Rollen zu schlüpfen. Dort zählen Aufgeschlossenheit, Neugier und Ausdauer zu den treibenden Kräften. Die Ausführungen zu The Ultimate Writer, The Company Therapist und vor allem $M$ is for Nottingham? haben dies anschaulich dargelegt. Insbesondere letzteres Projekt stellte hohe Anforderungen an die Kooperierenden.

780 Ebd., 323.

781 Schmundt, "Strom, Spannung, Widerstand", 47.

782 Aarseth, Cybertext, 4. Nach Aarseth sind Hypertexte ebensowenig als Texte im herkömmlichen Sinne zu verstehen, wie die Nutzer dieser Texte als Leser bezeichnet werden dürfen. Aufgrund von Faktoren wie Einflußnahme, Improvisation und Risikobereitschaft sei der Leser nunmehr eindeutig als Spieler (player) zu sehen. (Vgl. auch den Hinweis in Kapitel III.3.1.).

783 Ebd., 49.

784 Suter und Böhler beispielsweise sprechen von der "Lust am Spiel mit dem Text”. Sie sehen die NutzerAktivitäten in Hypertexten nicht nur in literarischer Produktivität sondern auch in spielerischer Aktivität: "Der Herausgeber (und eigentliche Autor) einer Hyperfiction [...] schlüpft mehr und mehr in die Rolle des Bereitstellers von Strukturen und Materialien. Man erwartet von ihm keine abgeschlossenen Fiktionen mehr, sondern eine grosse Fülle von gegliederten Materialien und Beziehungen, eventuell eine ganze simulierte Welt, mit eigenen Strukturen zur Kommunikation, zur spielerischen Interaktion und zur Produktion von narrativen Strängen.” Suter und Böhler, "Hyperfiction”, 19. 
Wenngleich das Spielerische in der Literatur durchaus nicht neu und keineswegs eine Erscheinung des Web-Zeitalters ist, so wird sich doch das Bild vom Spieler und vom Spielen für manchen Rezipienten nur schwer mit den traditionellen Vorstellungen von Leser und Literatur in Einklang bringen lassen. In Bezug auf The Ultimate Writer wurde diese Vermutung bereits aufgeworfen. Die Bewertung hatte ergeben, daß der spielerische Charakter des Forums zu stark im Vordergrund stehe und damit seinen literarischen und didaktischen Wert mindere. Eventuell ist deshalb die Bezeichnung Spieler im Zusammenhang mit Hypertexten unvorteilhaft. Worum es geht, ist aufzuzeigen, daß es einen gewissen kreativen, erwartungsfrohen, offenen, mithin im positiven Sinne spielerischen Umgang mit Hypertexten gibt, der zweifellos bei vielen Hypertext-Nutzern ein erhebliches Gegengewicht darstellt zu der Neigung, sich eher auf linear-konzipierte Print-Literatur einzulassen.

\subsection{Ein leidenschaftlicher Spieler und mehr - Oder: Über die Minimalqualifikationen eines erfolgreichen Autors}

Unbestritten setzt die Erschaffung offener Hypertexte ein hohes Maß an konzeptuellem Gestaltungsvermögen und imaginärer Vorstellungskraft voraus. Bildschirmliteratur entsteht gleichsam skulptural. Der Schöpfungsvorgang läßt sich mit der Arbeit eines Töpfers oder Bildhauers vergleichen, der stets aufs neue "um das Grobgeformte herumgeht, um es zu verbessern" ${ }^{\text {,755 }}$. Textbruchstücke dergestalt miteinander zu verbinden, daß jede mögliche Leseweise die Rezipienten befriedigt und überzeugt, erfordert demnach ein ebenso arbeits- wie zeitaufwendiges Verfahren, das zudem entsprechende technische Kenntnisse verlangt. Diese Bedingungen kommen nur in seltenen Fällen in produktiver Weise zusammen, so daß nur wenige der speziell für elektronische Medien konzipierte Werke als webbasierte Literatur bezeichnet werden dürfen.

Ein weiterer Grund mag darin liegen, daß den Autoren die Bereitschaft dazu fehlt, ihre Texte in das unendliche Netzwerk von Online-Texten einzugliedern beziehungsweise ihre Texte dem Netzwerk unterzuordnen. In der Tat würde das Publizieren im Web eine weitgehende Aufgabe der persönlichen Individualität erfordern, ginge man von einer konsequenten Einordnung aller Einzelprojekte in das Gesamtsystem aus. Jeder Herausgeber von Web-Veröffentlichungen - gleichgültig, ob es sich um literarische Texte, Spiele oder Computerprogramme handelt - muß in der Lage sein, nicht nur

${ }^{785}$ Glaser, "Die große Odyssee”, 8. 
mit seinem schöpferischen Werk hinter dem Gesamtwerk World Wide Web zurückzustehen, sondern sich auch in seiner Bedeutung als Autor zurückzunehmen. Bolter hat diese Tatsache erkannt und gelangt zu folgender Feststellung: "Die Produkte selbst sind wichtiger als ihre Autoren, und diese Produkte stellen Resultate einer hochgradig kooperativen Organisation dar." ${ }^{\text {786 }}$ Für eine konsequente Umsetzung der Prinzipien webbasierter Literatur werden die Autoren daher mehr Bereitschaft zur Kooperation und wird sich diese Literaturform somit zukünftig zu einer Art Gemeinschaftsprojekt entwickeln müssen.

Wie die vorstehenden Betrachtungen gezeigt haben, ist die Komplexität von Hypertexten und speziell der webbasierten Literatur also ganz ohne Frage ein wichtiger, wenn nicht gar entscheidender Grund dafür, weshalb die Entwicklung in diesem Bereich hinter den Erwartungen zurückgeblieben ist. Indes ist diese Komplexität beherrschbar und überwindbar.

Luserke hatte die programmatische Frage formuliert:

"Wo ist der Ort der Literatur in den Medien und wo ist der Ort der Medien in unserer Kultur?"787

Wie die Dinge derzeit stehen, lautet die Antwort im Rahmen der vorliegenden Arbeit:

Die elektronischen Medien verfügen über einen festen Platz in unserer Kultur. Hingegen hat die Literatur den ihren in den elektronischen Medien noch nicht gefunden.

${ }^{786}$ Bolter, "Das Internet in der Geschichte der Technologien des Schreibens", 50. - Bolters Bezeichnung des World Wide Web als notwendig (hochgradig) kooperativ bestätigt die Ausführungen zur Kooperativität (siehe Kapitel V.3.2.2.).

${ }^{787}$ Luserke, "Kultur, Literatur, Medien ", 185. Siehe auch Kapitel I.4. der vorliegenden Arbeit. 


\section{Bibliographie}

\section{Hilfsmittel}

akademie.de asp GmbH, "John Wilkes Booth", in: Dies., Hg., Net-Lexikon,

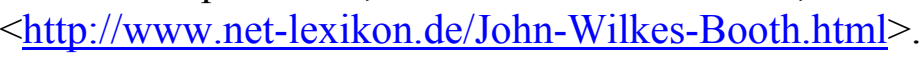

Britannia.com, LLC, Hg., Britannia - British History, Life \& Travel, $<$ http://www.britannia.com/history/londonhistory/oldsomhse.JPG $>$.

Brockhaus - Die Enzyklopädie in vierundzwanzig Bänden, 20., überarb. und aktual. Aufl. (Mannheim, 1996-1999).

BTex Ltd., Streetmap.co.uk $\left(\left[{ }^{1} 1997\right]\right),<\underline{\mathrm{http}}$ ://www.streetmap.co.uk/>.

cd-rom-produktion, Hg., DicData Wörterbuch Deutsch-Englisch, $<$ http://www.dicdata.de/>.

Diderot, Denis, Encyclopédie ou Dictionnaire raisonné des sciences, des arts et des métiers, par une Société de Gens de lettres III. Tome VII, (Paris, 1976).

Drosdowski, Günther, Hg., Duden Etymologie: Herkunftswörterbuch der deutschen Sprache, 2., völl. neu bearb. u. erw. Aufl. (Mannheim; Wien; Zürich, 1989 [1963]).

Engesser, Hermann, Hg., Duden Informatik: Ein Sachlexikon für Studium und Praxis, 2., vollst. bearb. u. erw. Aufl. (Mannheim; Wien; Zürich, 1993 [ $\left.{ }^{1} 1988\right]$ ).

Ernst Klett Sprachen GmbH, Hg., PONSline - Das Online-Wörterbuch, $<$ http://www.pons.de/>.

Gibaldi, Joseph, MLA Handbook for Writers of Research Papers (New York, 1999 [ $\left.\left.{ }^{1} 1977\right]\right)$.

Hehn, Paul und R.F. Holznagel, Hg., Who2?, < $\underline{\text { http://www.who2.com/>. }}$

Jens, Walter, Hg., Kindlers Neues Literaturlexikon, Bd. 13 (München, 1991).

Klußmann, Niels, Lexikon der Kommunikations- und Informationstechnik: Telekommunikation, Internet, Mobilfunk, Multimedia, Computer, E-Business, 3., neu bearb. und stark erw. Aufl. (Heidelberg, 2001 [ $\left.^{1} 1997\right]$ ).

London Ancestor, Hg., Genealogy, Local \& Family History in London, England, $<$ http://www.londonancestor.com/maps/baynards.htm $>$.

Meid, Volker, Sachwörterbuch zur deutschen Literatur (Stuttgart, 1999).

Microsoft Corporation, Hg., Microsoft Encarta Online Encyclopedia (2003 [ $\left.\left.{ }^{1} 1997\right]\right)$, $<$ http://encarta.msn.com/>.

MOTCO Enterprises Limited, MOTCO UK Directory and Image Database - Antique Maps, Prints \& Books, <http://www.motco.com/>.

Schneider, Hans-Jochen, Hg., Lexikon Informatik und Datenverarbeitung, 4., aktual. u. erw. Aufl. (München; Wien, 1997 [ $\left.{ }^{1} 1983\right]$ ).

The New Encyclopoedia Britannica in 32 volumes - Vol. 9: Micropcedia: Ready Reference, 15. Aufl. (Chicago, 2002 [ $\left.^{1} 1768\right]$ ).

Wilpert, Gero von, Sachwörterbuch der Literatur, 7., verb. u. erw. Aufl. (Stuttgart, $\left.1989\left[{ }^{1} 1955\right]\right)$. 


\section{Primärliteratur}

Borges, Jorge Luis, "Der Tod und der Kompaß”, in: Ders., Fiktionen, übers. von Karl August Horst, Wolfgang Luchting und Gisbert Haefs, (Frankfurt a.M., 1992 [Ficciones (Buenos Aires, 1974)]), 117-130.

Cline, Alison, "Joshua", in: Chris Holstrom, Hg., The Mahoney Project ([ $\left.\left.{ }^{1} 18.04 .2002\right]\right)$, $<$ http://www.mahoneythecat.org/node.asp?node $=66>$.

Lawrence, D.H., Lady Chatterley's Lover, vollst. u. unzens. Ausg. (London, 1960).

Locke, John, Some thoughts concerning education, 4. erw. Ausg. (London, 1699 $\left.\left[{ }^{1} 1693\right]\right)$.

Maximus, Toddius, "PERL Poetry" [Strophe 1], in: Prosebush.com, Hg., Prosebush $\left(\left[{ }^{1} 28.08 .2000\right]\right),<$ http://www.prosebush.com/viewnode.cgi?NID=32>.

Scott, "PERL Poetry" [Strophe 2a], in: Prosebush.com, Hg., Prosebush ([ 28.08 .2000$])$, $<$ http://www.prosebush.com/viewnode.cgi?NID $=36>$.

- "PERL Poetry" [Strophe 2b], in: Prosebush.com, Hg., Prosebush ([101.09.2000]), $<$ http://www.prosebush.com/viewnode.cgi?NID=79>.

Smart, Billy und Hannah E. Rudman, The Benefactor - a Short Hyperdrama (30.04.1998), <http://www.rhul.ac.uk/drama/research/benefactor/>.

White, Lee, "Double Crossings", in: Gutenberg Litegraphic Society, Hg., Pictures and Stories - The Online Community of Creative Minds, $<$ http://www.picturesandstories.org/doublecrossings.html $>$.

- "Double Crossings", Pictures and Stories - Ezine of Literature \& Art 2 (2003), $<$ http://www.picturesandstories.org/vol2issue2.html $>$.

\section{Sekundärliteratur}

Aarseth, Espen J., Cybertext: Perspectives on Ergodic Literature (Baltimore; London, 1997).

About, Inc., Hg., About Poetry, <http://poetry.about.com/>.

Affected Publishing Ltd., Affected Publishing Ltd. (27.03.2003), $<\underline{\text { http://www.affectedpublishing.co.uk/>. }}$.

Albrecht, Wolfgang, Arno Schmidt (Stuttgart; Weimar, 1998).

American Psychological Association, Hg., Reference Examples for Electronic Source Materials, $<$ http://www.apastyle.org/elecsource.html $>$.

Apitz, Rico, Andreas Guther und Gero Hoffmann, Wissenschaftliches Arbeiten im World Wide Web: HTML - Style-Guide - Sicherheit (Bonn, 1996).

Arbor, "Annotation on 'Navy Office'", in: Gyford, Hg., The Diary of Samuel Pepys ([01.07.2003]), <http://www.pepysdiary.com/p/483.php $>$.

Arnone, Michael, "A British University Uses a Murder Mystery to Teach the Craft of Online Writing", The Chronicle of Higher Education, 29.05.2002, $<$ http://chronicle.com/free/2002/05/2002052901u.htm>.

Arts Council England, Arts Council England Homepage,


Asher, Levi, “About Us”, in: Ders., Hg., Literary Kicks, $<$ http://www.litkicks.com/AboutUs.html $>$.

Assfalg, Rolf, Udo Goebels und Heinrich Welter, Internet-Datenbanken: Konzepte, Modelle, Werkzeuge (Bonn, 1998).

Auer, Johannes, "Der Leser als DJ oder was Internetliteratur mit HipHop verbindet", in: Beat Suter und Michael Böhler, Hg., Hyperfiction. Hyperliterarisches Lesebuch: Internet und Literatur (Basel; Frankfurt a.M., 1999), 173-181.

Barth, John, "The State of the Art", WQ - The Wilson Quarterly 20 (1996), 36-45.

Barth, Robert, "Die Bibliothek der Zukunft", in: Peter Rusterholz und Rupert Moser, Hg., Die Bedeutung des Buches - gestern-heute-morgen, Referate einer Vorlesungsreihe des Collegium generale der Universität Bern (Bern; Stuttgart, 1996), 53-75.

Bédoyère, Guy de la, Who was John Evelyn?, $<$ http://www.bedoyere.freeserve.co.uk/johnevelyn.htm $>$.

Berlich, Peter, "Das Internet als innerer Ort - 'Das digitale Kloster'”, in: Beat Suter und Michael Böhler, Hg., Hyperfiction. Hyperliterarisches Lesebuch: Internet und Literatur (Basel; Frankfurt a.M., 1999), 137-148.

Bertelsmann Stiftung, Hg., Lesen fördern in der Welt von morgen: Modelle für die Partnerschaft von Bibliothek und Schule (Gütersloh, 2000).

Bertelsmann Stiftung und Heinz Nixdorf Stiftung, Hg., Studium Online: Hochschulentwicklung durch neue Medien (Gütersloh, 2000).

Birkerts, Sven, Die Gutenberg-Elegien: Lesen im elektronischen Zeitalter, übers. von Kurt Neff, (Frankfurt a.M., 1997 [The Gutenberg Elegies. The Fate of Reading in an Electronic Age (New York, 1994)]).

Bischof, Ulrike und Horst Heidtmann, "Warum sind Film- und Fernsehbücher so erfolgreich? Das IfaK forscht zur Kinder- und Jugendliteratur im Medienverbund", Buch und Bibliothek: Medien, Kommunikation, Kultur 52 (2000), 414417.

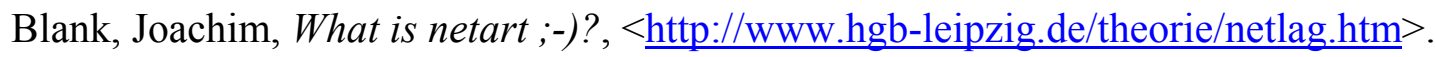

Bleuel, Jens, Online Publizieren im Internet: Elektronische Zeitschriften und Bücher. Mit einem Vorw. von Norbert Mundorf (Pfungstadt; Bensheim, 1995).

Block, Friedrich W., "Acht poetologische Thesen zur digitalen Poesie”, in: Johannes Auer, Christiane Heibach und Beat Suter, Hg., netzliteratur.net: Netzliteratur Internetliteratur - Netzkunst ( $\left[{ }^{1}\right.$ März 2001] ), $<\underline{\text { http://www.netzliteratur.net/block/acht thesen.html }}>$.

Böhle, Knud, Ulrich Riehm und Bernd Wingert, Vom allmählichen Verfertigen elektronischer Bücher: ein Erfahrungsbericht (Frankfurt a.M.; New York, 1997).

Boesken, Gesine, 'Lesen am Bildschirm: Wer ist 'drin', und sind Bücher jetzt 'out'?", in: Stiftung Lesen, Hg., Leseverhalten in Deutschland im neuen Jahrtausend: eine Studie der Stiftung Lesen (Hamburg, 2001), 127-149.

Bollmann, Stefan, und Christiane Heibach, "Sucht keine Wurzeln, folgt dem Kanal: Die frohen Botschaften der französischen Zahnärzte", in: Dies., Hg., Kursbuch Internet: Anschlüsse an Wirtschaft und Politik, Wissenschaft und Kultur (Reinbek bei Hamburg, 1998), 466-474. 
Bolter, Jay David, "Das Internet in der Geschichte der Technologien des Schreibens", in: Stefan Münker und Alexander Roesler, Hg., Mythos Internet (Frankfurt a.M., 1997), 37-55.

Botz, Gerhard und Gerald Sprengnagel, "Zeitgeschichte als Multimedia-Geschichte und Hi-Tech-History - Allgemeine Überlegungen zur Implementierung an österreichischen Universitäten”, Zeitschrift für Hochschuldidaktik 1-2 (1996), $<$ http://www.oeghd.or.at/zeitschrift/1996h1-2/16 art.html>.

Britannia.com, LLC, Britannia.com Touring Online - Tours: Robin Hood's Nottingham, <http://www.britannia.com/tours/rhood/nottingham.html $>$.

Bürger, Thomas, "Modelle zum Umgang mit originalen und digitalen Drucken. Zur Bereitstellung alter Bücher", in: Sabine Wefers, Hg., Von Gutenberg zum Internet / 7. Deutscher Bibliothekskongreß, 87. Deutscher Bibliothekartag in Dortmund 1997, Zeitschrift für Bibliothekswesen und Bibliographie: Sonderheft 68 (Frankfurt a.M., 1997), 51-60.

Burdorf, Dieter, Einführung in die Gedichtanalyse (Stuttgart, 1995).

Cantelow, Ann, Hg., Interactive Poetry Pages - Completed Poems, $<$ http://atlas.csd.net/ cantelow/cgi-bin/poem_history.pl/crit4/1/>.

- Hg., The Interactive Poetry Pages - Salon of Poetry for Critique - Four, $<\underline{\text { http://atlas.csd.net/ cantelow/cgi-bin/poem_view.pl/crit4/current poems/1/0/> }}$.

- “A Very Brief Definition of Haiku”, in: Dies., Hg., Ann Cantelow's Homepage,

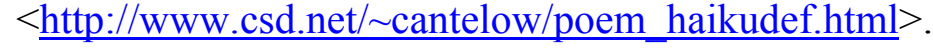

- "Welcome to the Interactive Poetry Pages", in: Dies., Hg., Interactive Poetry Pages, <http://www.csd.net/ cantelow/poem_welcome.html $>$.

Charlier, Michael, "Von der Armenbibel zu Myst - Zurück zu einer Literatur ohne Worte?", in: Beat Suter und Michael Böhler, Hg., Hyperfiction. Hyperliterarisches Lesebuch: Internet und Literatur (Basel; Frankfurt a.M., 1999), 125-136.

Chip, "Annotation on 'Seething Lane'”, in: Gyford, Hg., The Diary of Samuel Pepys ([112.07.2003]), <http://www.pepysdiary.com/p/1023.php $>$.

Clearingstelle Multimedia für Verwertungsgesellschaften von Urheber- und Leistungsschutzrechten GmbH, Hg., CMMV-Info, <http://www.cmmv.de/cmmvinfo.htm>.

Cölfen, Elisabeth, Hermann Cölfen und Ulrich Schmitz, Linguistik im Internet: Das Buch zum Netz - mit CD-ROM (Opladen, 1997).

Connell, Louise, "A Just Measure of Pain? - The Galleries of Justice Nottingham", in: The Incorporated Council Of Law Reporting For England \& Wales, Hg., The ICLR Home Page, <http://www.lawreports.co.uk/GalleriesofJustice.htm>.

Coover, Robert, "The End of Books", New York Times Book Review, 21.06.1992, 1 und 23-25.

Corbineau-Hoffmann, Angelika, Die Analyse literarischer Texte: Einführung und Anleitung (Tübingen; Basel, 2002).

Costanzo, William, "Reading, Writing, and Thinking in an Age of Electronic Literacy", in: Cynthia L. Selfe und Susan Hilligoss, Hg., Literacy and Computers: The Complications of Teaching and Learning with Technology (New York, 1994), 11-21. 
Cramer, Florian, "Literatur im Internet", in: Johannes Auer, Christiane Heibach und Beat Suter, Hg., netzliteratur.net $\left(\left[{ }^{1} 01.12 .1999\right]\right),<\underline{\text { http://www.netzliteratur.net/cramer/alg- }}$ literatur im internet.html>.

- "Warum es zuwenig interessante Netzdichtung gibt. Neun Thesen", in: Johannes Auer, Christiane Heibach und Beat Suter, Hg., netzliteratur.net ([ 27.04 .2000$])$, $<$ http://www.netzliteratur.net/cramer/karlsruher thesen.html $>$.

Creative Commons Corporation, Hg., Creative Commons - Commons Deed: Attribution - Noncommercial - Share Alike - Key License Terms, $<$ http://creativecommons.org/licenses/by-nc-sa/1.0/>.

Crump, Eric und Nick Carbone, English Online: A Student's Guide to the Internet and World Wide Web (Boston, 1997).

Daiber, Jürgen, "Literatur und Nicht-Linearität: Ein Widerspruch in sich?”, Jahrbuch für Computerphilologie - online, 1 (1999), < $\underline{\mathrm{http}}$ ://computerphilologie.unimuenchen.de/jahrbuch/jb1/daiber.html>.

- "Literatur und Nicht-Linearität: Ein Widerspruch in sich?", Jahrbuch für Computerphilologie 1 (1999), 21-38.

Deemer, Charles, "How do I number these pages", in: Center for the Public Domain/University of North Carolina - Chapel Hill, Hg., ibiblio - the public's library and digital archive, <http://www.ibiblio.org/cdeemer/howdoi.htm>.

- "Hyperdrama and Virtual Development: Notes on Creating New Hyperdrama in Cyberspace", in: Center for the Public Domain/University of North Carolina - Chapel Hill, Hg., ibiblio - the public's library ans digital archive, $<\underline{\text { http://www.ibiblio.org/cdeemer/virtdev.htm }>\text {. }}$

- "The New Hyperdrama: How hypertext scripts are changing the parameters of dramatic storytelling", in: Center for the Public Domain/University of North Carolina - Chapel Hill, Hg., ibiblio - the public's library ans digital archive,

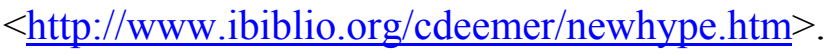

- "What is Hypertext?" in: Center for the Public Domain/University of North Carolina - Chapel Hill, Hg., ibiblio - the public's library ans digital archive

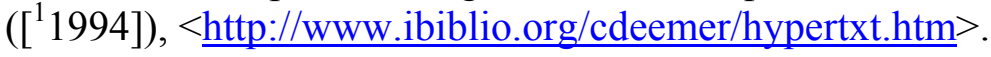

Dörr, Marianne, "Bibliotheken im Internet - Auf dem Weg zur virtuellen Bibliothek?", in: Gudrun Gross, Uwe Langer und Rudolf Seising, Hg., Studieren und Forschen im Internet: Perspektiven für Wissenschaft, Wirtschaft, Kultur und Gesellschaft (Frankfurt a.M., 1997), 163-172.

Dorner, Jane, "Comment", in: Luesebrink, Hg., M is for Nottingham? - Comments by Writers and Readers, $<\underline{\text { http://califia.hispeed.com/IncubationDrama/commentary.htm }>\text {. }}$

Douglas, Jane Yellowlees, The End of Books - Or Books without End?: Reading Interactive Narratives (Ann Arbor, 2000).

Drake, Luigi-Bob, Wr-eye-tings Scratchpad (21.05.1999 [ ${ }^{1}$ Februar 1997]), $<$ http://www.burningpress.org/wreyeting/>.

Dublin Core Metadata Intiative, Hg., Dublin Core Metadata Initiative Elements and Element Refinements - a current list (06.10.2002), $<$ http://dublincore.org/documents/2002/10/06/current-elements/>. 
Duffield, Jeremy, "Comment", in: Luesebrink, Hg., M is for Nottingham? Comments by Writers and Readers, $<\underline{\text { http://califia.hispeed.com/IncubationDrama/commentary.htm }>\text {. }}$

Duncan, Bonnie, "Citing Hypermedia: Solving the Indexing Dilemma", (Re)Soundings 2.1 (1998), <http://www.millersv.edu/ resound/*vol2iss1/topframe.html>.

Eckermann, Johann Peter, Gespräche mit Goethe in den letzten Jahren seines Lebens, Hg. Heinz Schlaffer (München, 1986).

Enitharmon Press, Hg., Enitharmon Press Online - Contemporary Poetry Publisher (21.07.2003), <http://www.enitharmon.co.uk/>.

Faßler, Manfred und Wulf Halbach, "Vorwort", in: Dies., Hg., Geschichte der Medien (München, 1998), 7-15.

Feldmann, Doris, "Multimedia Shakespeares", in: Dies., Neumann und Rommel, Hg., Anglistik im Internet, 129-143.

Feldmann, Doris, Fritz-Wilhelm Neumann und Thomas Rommel, "Introduction: Hanging in/on to the Net", in: Dies., Hg., Anglistik im Internet. Proceedings of the 1996 Erfurt Conference on Computing in the Humanities (Heidelberg, 1997), i-iii.

- "Anglistik im Internet - Proceedings of the Erfurt Conference on Computing in the Humanities, April 1996. Web Sites - Uniform Resource Locators", Erfurt Electronic Studies in English, <http://webdoc.gwdg.de/edoc/ia/eese/urls.html>.

Flusser, Vilém, Die Schrift: Hat Schreiben Zukunft? (Göttingen, 1987).

Gaiser, Wilfried, Hg., "Urheberrechte im Internet", in: Ders., Verantw., Cyberlaw $\left(\left[{ }^{1} 2002\right]\right),<$ http://www.cyberlaw.de/Recht/Urheberrecht/ReUr025.pdf $>$.

- Hg., "Warum Info zum Internet-Recht?”, in: Ders., Verantw., Cyberlaw, ([ $\left.\left.{ }^{1} 2002\right]\right)$, $<$ http://www.cyberlaw.de/Recht/Onlinerecht/ReOn049.pdf $>$, 04.11.2002.

Gates, Bill (mit Nathan Myhrvold und Peter Rinearson), The Road Ahead (Rockland, MA, 1995).

Gatterburg, Angela und Karen Andresen, “'Man muß den Charakter bilden.' Die 'heute'-Moderatorin Petra Gerster und ihr Mann, der Journalist Christian Nürnberger, über ihr Buch zum 'Erziehungsnotstand' in Deutschland, die Unsicherheit von Eltern und Lehrern und die Folgen der Globalisierung für die Familie", Der Spiegel 35 (2001), 58-61.

Gauron, André, "Das digitale Zeitalter", in: Stefan Bollmann, Hg., Kursbuch neue Medien. Trends in Wirtschaft und Politik, Wissenschaft und Kultur, 2. Aufl. (Mannheim, 1996 [ $\left.\left.{ }^{1} 1995\right]\right), 24-40$.

Gemmeke, Claudia, Hartmut John und Harald Krämer, "Vorwort", in: Dies., Hg., Euphorie digital? Aspekte der Wissensvermittlung in Kunst, Kultur und Technologie (Bielefeld, 2001), 9-10.

Gendolla, Peter und Jörgen Schäfer, "Auf Spurensuche. Literatur im Netz, Netzliteratur und ihre Vorgeschichte(n)", in: Digitale Literatur [Text + Kritik: Zeitschrift für Literatur 152 (2001)], 75-86.

Gersmann, Gudrun, "Schöne Welt der bunten Bilder: Kritische Anmerkungen zur Geschichtsdarstellung in den Neuen Medien", in: Claudia Gemmeke, Hartmut John und Harald Krämer, Hg., Euphorie digital? Aspekte der Wissensvermittlung in Kunst, Kultur und Technologie (Bielefeld, 2001), 105-119.

Gilster, Paul, Digital Literacy (New York, 1997). 
Glaser, Peter, "Was wohl wird (WWW). Wohin führt uns die digitale Technologie in den nächsten zehn Jahren?", Süddeutsche Zeitung, 27.08.1996, 31.

- "Die große Odyssee - Wie haben das Internet und der Computer das Schreiben und Lesen verändert?", Die Zeit - Magazin, 20.09.1996, 6-10.

Glazier, Loss Pequeño u.a., Hg., Electronic Poetry Center, <http://epc.buffalo.edu/>.

- Hg., Electronic Poetry Center - E-Poetry, <http://epc.buffalo.edu/e-poetry/>.

- "Intro", in: Dies., Hg., Electronic Poetry Center, <http://epc.buffalo.edu/intro.html $>$.

Glotz, Peter und Uwe Thomas, Das dritte Wirtschaftswunder. Aufbruch in eine neue Gründerzeit (Düsseldorf; New York, 1994).

Grandel, Andreas, "Internet - Grundlagen und Anwendungen", in: Gudrun Gross, Uwe Langer und Rudolf Seising, Hg., Studieren und Forschen im Internet: Perspektiven für Wissenschaft, Wirtschaft, Kultur und Gesellschaft (Frankfurt a.M., 1997), 51-67.

Greter, Reinhold, "Versuch über Welttexte", in: Beat Suter und Michael Böhler, Hg., Hyperfiction. Hyperliterarisches Lesebuch: Internet und Literatur (Basel; Frankfurt a.M., 1999), 85-100.

Grey, Duncan, Samuel Pepys Diary (27.05.2003), <http://www.pepys.info/>

- "Samuel Pepys at Hinchingbrooke", in: Ders., Hg., The History of Hinchingbrooke House, $<$ http://www.hinchbk.cambs.sch.uk/historical/hinchhistory/spepys/spepys.html $>$.

Gross, Sabine, "Das Buch in der Hand: Zum situativ-affektiven Umgang mit Texten", in: Stiftung Lesen, Hg., Leseverhalten in Deutschland im neuen Jahrtausend: eine Studie der Stiftung Lesen (Hamburg, 2001), 175-197.

Guggenberger, Bernd, Das digitale Nirwana (Hamburg, 1997).

Gyford, Phil, The Diary of Samuel Pepys (29.07.2003 [ [ 01.01 .2003$])$, $<\underline{\text { htp://www.pepysdiary.com/>. }}$

- "About the text", in: Ders., Hg., The Diary of Samuel Pepys (03.08.2003 $\left.\left[{ }^{1} 01.01 .2003\right]\right),<$ http://www.pepysdiary.com/about/text/>.

- "About this site", in: Ders., Hg., The Diary of Samuel Pepys (03.08.2003 [01.01.2003]), $<\underline{\mathrm{http}}$ //www.pepysdiary.com/about/>.

- “Admiralty”, in: Ders., Hg., The Diary of Samuel Pepys (06.08.2003 [0101.01.2003]),

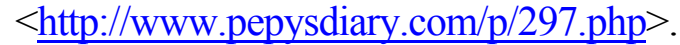

- “Annotation guidelines", in: Ders., Hg., The Diary of Samuel Pepys (03.08.2003 $\left.\left[{ }^{1} 01.01 .2003\right]\right),<\underline{\mathrm{http}}: / / \mathrm{www}$. pepysdiary.com/about/annotation/>.

- “Archive for July 1660”, in: Ders., Hg., The Diary of Samuel Pepys (05.08.2003 $\left.\left[{ }^{1} 01.01 .2003\right]\right),<$ http://www.pepysdiary.com/archive/1660/07/index.php $>$.

- "Background information", in: Ders., Hg., The Diary of Samuel Pepys (03.08.2003 $\left.\left[{ }^{1} 01.01 .2003\right]\right),<$ http://www.pepysdiary.com/background/>.

- "Banned user", in: Ders., Hg., The Diary of Samuel Pepys (02.09.2003 [101.01.2003]), $<$ http://www.pepysdiary.com/about/archive/2003/05/06/748.php $>$.

- "Cover text", in: Ders., Hg., The Diary of Samuel Pepys (04.08.2003 [01.01.2003]), $<$ http://www.pepysdiary.com/intro/>.

- "Frequently Asked Questions", in: Ders., Hg., The Diary of Samuel Pepys (29.07.2003 $\left.\left[{ }^{1} 01.01 .2003\right]\right),<\underline{\mathrm{http}} / / / \mathrm{www}$. pepysdiary.com/about/faq/>. 
- "Further reading", in: Ders., Hg., The Diary of Samuel Pepys (05.08.2003 $\left.\left[{ }^{1} 01.01 .2003\right]\right),<$ http://www.pepysdiary.com/about/further/>.

- “Glossary”, in: Ders., Hg., The Diary of Samuel Pepys (04.08.2003 [ $\left.\left.{ }^{1} 01.01 .2003\right]\right)$,

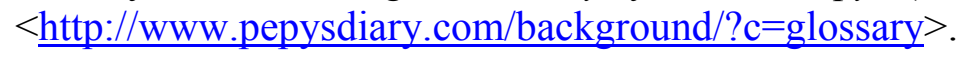

- "Hinchingbroke", in: Ders., Hg., The Diary of Samuel Pepys (31.08.2003 [101.01.2003]), <http://www.pepysdiary.com/p/104.php $>$.

- “Join the discussion forum!", in: Ders., Hg., The Diary of Samuel Pepys (03.08.2003 [ $\left.\left.{ }^{1} 01.01 .2003\right]\right),<$ http://www.pepysdiary.com/about/archive/2003/07/27/1137.php $>$.

- "King's Great Wardrobe", in: Ders., Hg., The Diary of Samuel Pepys (31.08.2003 $\left.\left[{ }^{1} 01.01 .2003\right]\right),<$ http://www.pepysdiary.com/p/946.php $>$.

- "Lemons", in: Ders., Hg., The Diary of Samuel Pepys (31.08.2003 [ $\left.\left.{ }^{1} 01.01 .2003\right]\right)$, $<$ http://www.pepysdiary.com/p/1084.php $>$.

- "Navy Board", in: Ders., Hg., The Diary of Samuel Pepys (06.08.2003 [101.01.2003]),

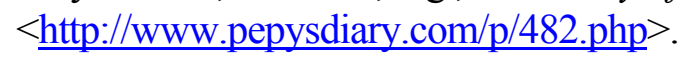

- "Navy Office", in: Ders., Hg., The Diary of Samuel Pepys (06.08.2003 [101.01.2003]), $<$ http://www.pepysdiary.com/p/483.php $>$.

- "New Exchange", in: Ders., Hg., The Diary of Samuel Pepys (31.08.2003 [101.01.2003]), <http://www.pepysdiary.com/p/1085.php >.

- "New glossary in Background Info", in: Ders., Hg., The Diary of Samuel Pepys (04.08.2003 [ $\left.\left.{ }^{1} 01.01 .2003\right]\right)$, $<$ http://www.pepysdiary.com/about/archive/2003/08/04/1168.php>.

- "Other formats", in: Ders., Hg., The Diary of Samuel Pepys (05.08.2003 [101.01.2003]), $<$ http://www.pepysdiary.com/about/formats/>.

- "Particulars of the life of Samuel Pepys", in: Ders., Hg., The Diary of Samuel Pepys (29.07.2003 [ $\left.\left.{ }^{1} 01.01 .2003\right]\right),<$ http://www.pepysdiary.com/intro/pepys/>.

- "Pepys, Elizabeth (wife)", in: Ders., Hg., The Diary of Samuel Pepys (06.08.2003 [101.01.2003]), <http://www.pepysdiary.com/p/150.php $>$.

- "Pepys, John (father)", in: Ders., Hg., The Diary of Samuel Pepys (06.08.2003 [ $\left.\left.{ }^{1} 01.01 .2003\right]\right),<$ http://www.pepysdiary.com/p/154.php $>$.

- "Pepys, Margaret (mother)", in: Ders., Hg., The Diary of Samuel Pepys (06.08.2003 [01.01.2003]), <http://www.pepysdiary.com/p/282.php $>$.

- "Places", in: Ders., Hg., The Diary of Samuel Pepys (06.08.2003 [ $\left.\left.{ }^{1} 01.01 .2003\right]\right)$, $<$ http://www.pepysdiary.com/background/?c=places $>$.

- "Previous editions of the diary", in: Ders., Hg., The Diary of Samuel Pepys (05.08.2003 [01.01.2003]), <http://www.pepysdiary.com/about/text/>.

- "Should entries display the weekday?", in: Ders., Hg., The Diary of Samuel Pepys (04.08.2003 [ $\left.\left.{ }^{1} 01.01 .2003\right]\right)$, <http://www.pepysdiary.com/about/archive/2003/01/13/200.php>.

- "Somerset House", in: Ders., Hg., The Diary of Samuel Pepys (06.08.2003 $\left.\left[{ }^{1} 01.01 .2003\right]\right),<$ http://www.pepysdiary.com/p/243.php $>$.

- "Support this site", in: Ders., Hg., The Diary of Samuel Pepys (05.08.2003 $\left.\left[{ }^{1} 01.01 .2003\right]\right),<$ http://www.pepysdiary.com/about/support/>.

- "The story so far", in: Ders., Hg., The Diary of Samuel Pepys (06.08.2003 $\left.\left[{ }^{1} 01.01 .2003\right]\right),<$ http://www.pepysdiary.com/about/history/>. 
- "Venison", in: Ders., Hg., The Diary of Samuel Pepys (31.08.2003 [ $\left.\left.{ }^{1} 01.01 .2003\right]\right)$, $<$ http://www.pepysdiary.com/p/383.php $>$.

- "Wednesday 18 July 1660 - Annotations", in: Ders., Hg., The Diary of Samuel Pepys (06.08.2003 [ $\left.\left.{ }^{1} 01.01 .2003\right]\right)$, $<$ http://www.pepysdiary.com/archive/1660/07/18/index.php\#annotations $>$.

- "What is RSS?", in: Ders., Hg., Writing, $<$ http://www.gyford.com/phil/writing/2003/01/05/an_introduction_php $>$.

Gyford, Phil (Verantw.), Pepys Diary Discussion, in: Freeserve Plc, Hg., SmartGroups. com, $<$ http://www.smartgroups.com/groups/pepysdiary $>$.

Hart, Michael, Hg., Project Gutenberg (12.02.2003 [ $\left.\left.{ }^{1} 1971\right]\right),<$ http://gutenberg.net/>.

- "About Project Gutenberg? - The Selection of Project Gutenberg Etexts", in: Ders., Hg., Project Gutenberg (28.10.2003 [ $\left.\left.{ }^{1} 1971\right]\right)$, $<$ http://www.gutenberg.net/about.shtml $>$.

- "Welcome to Project Gutenberg", in: Ders., Hg., Project Gutenberg (12.02.2003 [ $\left.\left.{ }^{1} 1971\right]\right),<$ http://gutenberg.net/index.html $>$.

- "What books will I find in Project Gutenberg?", in: Ders., Hg., Project Gutenberg (20.06.2002 [ $\left.\left.{ }^{1} 1971\right]\right),<$ http://promo.net/pg/helpex.html\#What-books $>$.

- "What is PG? - The Beginning of the Gutenberg Philosophy", in: Ders., Hg., Project Gutenberg (13.05.2002[ [ $\left.\left.{ }^{1} 1971\right]\right),<$ http://gutenberg.net/history.html\#beginningphil>.

Hautzinger, Nina, Vom Buch zum Internet? Eine Analyse der Auswirkungen hypertextueller Strukturen auf Text und Literatur (St. Ingbert, 1999).

Heibach, Christiane, “'Creamus, ergo sumus' - Ansätze zu einer Netz-Ästhetik”, in: Beat Suter und Michael Böhler, Hg., Hyperfiction. Hyperliterarisches Lesebuch: Internet und Literatur (Basel; Frankfurt a.M., 1999), 101-112.

- "Ins Universum der digitalen Literatur. Versuch einer Typologie", in: Digitale Literatur [Text + Kritik: Zeitschrift für Literatur 152 (2001)], 31-42.

Heidtmann, Horst, “'Im Buch geht alles ohne dich, aber im Computer nichts...'. Multimedia in Kinder- und Jugendbibliotheken: Ergebnisse einer Umfrage", Buch und Bibliothek: Medien, Kommunikation, Kultur 53 (2001), 406-410.

Heim, Michael, The Metaphysics of Virtual Reality (New York, 1993).

Henry Holt and Company, Inc., Hg., Sue Grafton Web Site ([ $\left.\left.{ }^{1} 1996\right]\right)$, $<$ http://www.suegrafton.com/>.

Hesse, Friedrich W. und Heinz Mandl, unter Mitarbeit von Gabi Reinmann-Rothmeier und Steffen-Peter Ballstaedt, "Neue Technik verlangt neue pädagogische Konzepte: Empfehlungen zur Gestaltung und Nutzung von multimedialen Lehr- und Lernumgebungen", in: Bertelsmann Stiftung und Heinz Nixdorf Stiftung, Hg., Studium Online: Hochschulentwicklung durch neue Medien (Gütersloh, 2000), 31-49.

Hhomeboy, "Annotation on "Annotation length and errors", in: Gyford, Hg., The Diary of Samuel Pepys ([102.05.2003]), $<$ www.pepysdiary.com/about/archive/2003/04/30/742.php>.

Hippler, Hans-Jürgen, “Tummelplatz Internet oder: Ist Lesen eine veraltete 'Technologie'?", in: Stiftung Lesen, Hg., Leseverhalten in Deutschland im neuen Jahrtausend: eine Studie der Stiftung Lesen (Hamburg, 2001), 165-174. 
Hofmann, Martin und Lothar Simon, Problemlösung Hypertext: Grundlagen, Entwicklung, Anwendung (München, 1995).

Holstrom, Chris, The Mahoney Project $\left(\left[^{1} 2002\right]\right),<\underline{\mathrm{http}} / / /$ www.cholstro.net/mahoney/>.

- Mahoney the Cat $\left(\left[{ }^{1} 08.02 .2002\right]\right),<$ http://www.mahoneythecat.org/>.

- "Collaborative Fiction Writing as an Example of Online Community", in: Ders., Hg., The Mahoney Project ([12002]), <http://www.cholstro.net/mahoney/thesis3.shtml $>$.

- "Conclusions", in: Ders., Hg., The Mahoney Project ([12002]), $<$ http://www.cholstro.net/mahoney/thesis5.shtml $>$.

- "Hypertext", in: Ders., Hg., The Mahoney Project ([ $[2002])$, $<$ http://www.cholstro.net/mahoney/thesis4.shtml $>$.

- "Introduction", in: Ders., Hg., The Mahoney Project ([ [2002]), $<$ http://www.cholstro.net/mahoney/thesis1.shtml $>$.

- "About the story", in: Ders., Hg., Mahoney the Cat ([ $\left.\left.{ }^{1} 2002\right]\right)$,

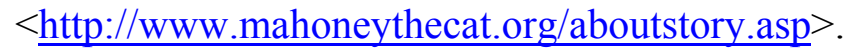

- "How Mahoney started", in: Ders., Hg., Mahoney the Cat ([ $\left.\left.{ }^{1} 2002\right]\right)$,

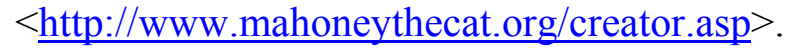

- "Mahoney considers the world outside the cottage", in: Ders., Hg., The Mahoney Project $\left(\left[{ }^{1} 18.04 .2002\right]\right),<$ http://www.mahoneythecat.org/node.asp?Node=23 $>$.

- "Mahoney Outline View", in: Ders., Hg., Mahoney the Cat $\left.\left({ }^{1}{ }^{1} 2002\right]\right)$, $<$ http://www.mahoneythecat.org/outline.asp $>$.

- "Message in 'Mahoney Discussion Topics"', in: Ders., Hg., The Mahoney Project $\left(\left[{ }^{1} 12.06 .2003\right]\right)$, $<\underline{\mathrm{http}}: / /$ www.mahoneythecat.org/Discussion/forum/start.asp?forumid $=3 \&$ select=491\& $>$.

- "Sign Up", in: Ders., Hg., Mahoney the Cat ([ $\left.\left.{ }^{1} 2002\right]\right)$, $<$ http://www.mahoneythecat.org/signup.asp $>$.

Huhn, Uwe, "Break On Through", in: Ders., Hg., The Doors, <http://www.doorsonline.de/1.htm>.

- "Die Band", in: Ders., Hg., The Doors, $<$ http://www.doors-online.de/Bandframe.htm $>$.

Humboldt-Forum Recht, Hg., Humboldt-Forum Recht, < $\underline{\text { http://www.humboldt-forum- }}$

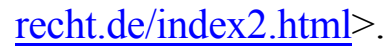

Idensen, Heiko, "Schreiben/Lesen als Netzwerk-Aktivität. Die Rache des (Hyper-) Textes an den Bildmedien”, in: Martin Klepper, Ruth Mayer und Ernst-Peter Schneck, Hg., Hyperkultur: Zur Fiktion des Computerzeitalters (Berlin; New York, 1996), 81-107.

- "Hyper-Scientifiction”, in: Beat Suter und Michael Böhler, Hg., Hyperfiction. Hyperliterarisches Lesebuch: Internet und Literatur (Basel; Frankfurt a. M., 1999), 61-83.

Illich, Ivan, Im Weinberg des Textes: Als das Schriftbild der Moderne entstand. Ein Kommentar zu Hugos 'Didascalicon', übers. von Ylva Eriksson-Kuchenbuch, (Frankfurt a.M., 1991 [In the Vineyard of the Text: A Commentary to Hugh's 'Didascalicon' (Chicago, 1993)]).

ISBN-Agentur für die Bundesrepublik Deutschland, Hg., Erste Informationen für Erzeuger, Verlage und Informationsanbieter zur Anwendung des Digital Object Identifier, < $\underline{\text { http://www.german-doi.org/LEITFADE.HTM>. }}$. 
Jenny, Zoë, "Ein Geschenk für den Leser? - Tatjana Blobel im Interview mit acht Autoren", Spiegel Spezial 10: Die Zukunft des Lesens - Vom Buch zum Internet (1999), 20-24.

Jochum, Uwe und Gerhard Wagner, Hg., Am Ende - das Buch: semiotische und soziale Aspekte des Internet (Konstanz, 1998).

Jones, Steven, "Kommunikation, das Internet und Elektromagnetismus", in: Stefan Münker und Alexander Roesler, Hg., Mythos Internet (Frankfurt a.M., 1997), 131-146.

Joyce, Michael, Othermindedness: the Emergence of Network Culture (Ann Arbor, 2000).

Jung, Alexander, "Rilke, elektronisch", Spiegel Spezial 10: Die Zukunft des Lesens Vom Buch zum Internet. (1999).

Kaiser, Reinhard, Literarische Spaziergänge im Internet: Bücher und Bibliotheken online, 2. Aufl. (Frankfurt a.M., 1997 [ $\left.\left.{ }^{1} 1996\right]\right)$.

Karpenstein-Eßbach, Christa, "Medien als Gegenstand der Literaturwissenschaft. Affären jenseits des Schönen", in: Julika Griem, Hg., Bildschirmfiktionen: Interferenzen zwischen Literatur und neuen Medien (Tübingen, 1998), 13-32.

Keil-Slawik, Reinhard, Werner Beuschel, Birgit Gaiser, Michael Klemme, Cornelia Pieper und Harald Selke, "Multimedia in der universitären Lehre: Eine Bestandsaufnahme an deutschen Hochschulen", in: Ingrid Hamm und Detlef Müller-Böling, Hg., Hochschulentwicklung durch neue Medien: Erfahrungen Projekte - Perspektiven. Mit einer Bestandaufnahme über Multimedia-Projekte an deutschen Hochschulen (Gütersloh, 1997), 73-122.

Kieninger, Martina, "Vom Schreiben auf glatten Oberflächen - Zur Geschichte des zweisprachigen Mehrautorenprojekts Tango", in: Beat Suter und Michael Böhler, Hg., Hyperfiction. Hyperliterarisches Lesebuch: Internet und Literatur (Basel; Frankfurt a.M., 1999), 183-199.

Kist, Joost, Elektronisches Publizieren: Übersicht, Grundlagen, Konzepte, bearb. u. hrsg. Manfred Krüger (Stuttgart, 1988).

Klepper, Martin, Ruth Mayer und Ernst-Peter Schneck, "Glossar", in: Dies., Hg., Hyperkultur: Zur Fiktion des Computerzeitalters (Berlin; New York, 1996), 267-285.

Klostermann, Vittorio E., Verlegen im Netz: Zur Diskussion um die Zukunft des wissenschaftlichen Buches (Frankfurt a.M., 1997).

- “'Von Gutenberg zum Internet' - Wie bitte?!”, BuB-Journal 49 (1997), Sonderheft Bibliothekskongreß, 25.

Knorr, Dagmar und Eva-Maria Jakobs, "Textproduktion in elektronischen Umgebungen: Einleitung und Überblick”, in: Dagmar Knorr und Eva-Maria Jakobs, Hg., Textproduktion in elektronischen Umgebungen (Frankfurt a.M., 1997), 1-7.

Kock, Markus, "Biographie Raymond Daniel Manzarek", in: Ders., Hg., The Doors Die inoffizielle Fanpage, <http://private.addcom.de/schafi/raybio.html $>$.

Komninos University of Queensland Press, "Articles on Komninos", in: Komninos Zervos, Hg., cyberpoetry ([ $\left.\left.{ }^{1} 1991\right]\right)$, $<$ http://www.gu.edu.au/ppages/k zervos/hsc/fascist.html $>$. 
Koring, Bernhard, Lernen und Wissenschaft im Internet: Anleitungen und Reflexionen zu neuen Lern-, Forschungs- und Beratungsstrukturen (Bad Heilbrunn, 1997).

Krahberger, Franz, "Hypertext, Hyperraum, Kulturentwicklung", in: Franz Krahberger, Hg., Das Babylonprojekt: Ein Textcompendium zur Computerkultur, Electronic Journal Literatur Primär, $<$ http://ezines.onb.ac.at:8080/ejournal/pub/ejour97-II/buecher/babyl/hypertext.html $>$.

Krajewski, Markus, "Spür-Sinn. Was heißt einen Hypertext lesen?”, in: Lorenz Gräf und Markus Krajewski, Hg., Soziologie des Internet: Handeln im elektronischen Web-Werk (Frankfurt a.M.; New York, 1997), 60-78.

Kress, Gunther, "Visual and verbal modes of representation in electronically mediated communication: the potentials of new forms of text", in: Ilana Snyder, Hg., Page to Screen: Taking literacy into the electronic era, 53-79.

Krieger, H. Jochen, Verantw., Das deutsche Urheberrechtsgesetz - Teil 1 Urheberrecht, \& 8 Miturheber (14.08.2002), <http://transpatent.com/gesetze/urhg1.html\#8>.

Kröher, Michael O. R., "Studium im Cyberspace - Teil 4: Netzwerke von Gleichgesinnten", in: SPIEGELnetAG, manager-magazin.de, $\left(\left[{ }^{1} 18.04 .2001\right]\right)$, $<$ http://www.manager-magazin.de/koepfe/uniguide/0,2828,127848,00.html $>$.

Kuhlen, Rainer, Hypertext: Ein nicht-lineares Medium zwischen Buch und Wissensbank (Berlin; Heidelberg, 1991).

- "Zur Virtualisierung von Bibliotheken und Büchern", in: Dirk Matejovski und Friedrich Kittler, Hg., Literatur im Informationszeitalter (Frankfurt a.M., 1996), 112142.

Laciefae, "Break on through", in: Dies., Hg., Waiting for the Sun - The Spirit of Jim Morrison, $<$ http://archives.waiting-forthesun.net/Pages/Legacy/Albums/TheDoors/Songs/BreakOnThrough.html>.

Landon, Brooks, "Hypertext and Science Fiction", Science Fiction Studies 20 (1993), 449-456.

Landtag von Baden-Württemberg, Bericht und Empfehlungen der Enquête-Kommission "Entwicklung, Chancen und Auswirkungen neuer Informations- und Kommunikationstechnologien in Baden-Württemberg” (Multimedia-Enquête), Drucksache 11/6400 (1995).

Langen, Claudia, "Das Lesebarometer 1999 - Tendenz steigend?", in: Claudia Langen und Ulrike Bentlage, Hg., Das Lesebarometer - Lesen und Mediennutzung in Deutschland, 11-17.

- "Der Griff zum Buch - warum wird gelesen?", in: Claudia Langen und Ulrike Bentlage, Hg., Das Lesebarometer - Lesen und Mediennutzung in Deutschland, 19-24.

- "Lesen in einer veränderten Medienlandschaft", in: Claudia Langen und Ulrike Bentlage, Hg., Das Lesebarometer - Lesen und Mediennutzung in Deutschland (Gütersloh, 2000), 25-40.

language hat, "Annotation on "Navy Office",, in: Phil Gyford, Hg., The Diary of Samuel Pepys ([109.03.2003]), <http://www.pepysdiary.com/p/483.php $>$.

- "Annotation on 'Seething Lane"”, in: Gyford, Hg., The Diary of Samuel Pepys ([15.07.2003]), <http://www.pepysdiary.com/p/1023.php $>$. 
Larsen, Deena, "Comment", in: Luesebrink, Hg., M is for Nottingham? - Comments by Writers and Readers, $<$ http://califia.hispeed.com/IncubationDrama/commentary.htm\#Writers: $>$.

Leimberg, Inge und Matthias Bauer, Hg., Connotations - A Journal for Critical Debate, <http://www.connotations.de/>, 22.05.2003. Parallelpublikationen: Connotations - A Journal for Critical Debate 1 (1991)-11 (2002).

Lévy, Pierre, "Cyberkultur: Universalität ohne Totalität", in: Stefan Bollmann und Christiane Heibach, Hg., Kursbuch Internet: Anschlüsse an Wirtschaft und Politik, Wissenschaft und Kultur (Reinbek bei Hamburg, 1998), 60-87.

“Lieber Papier", Spiegel Spezial 10: Die Zukunft des Lesens - Vom Buch zum Internet, 11.

Lipinski, Klaus, Hg., Lexikon der Datenkommunikation: 2.000 Begriffe von ATM bis Z-Modem - 3.500 Abkürzungen, 4., aktual. und erw. Aufl. (Bonn, 1996 [ $\left.\left.{ }^{1} 1994\right]\right)$.

Liu, Alan, Hg., Voice of the Shuttle - Restoration \& $18^{\text {th }}$ Century, $<$ http://vos.ucsb.edu/browse.asp?id=2738>.

lizzard, "Message in "Mahoney Discussion Topics", in: Chris Holstrom, Hg., The Mahoney Project ([ $\left.\left.{ }^{1} 24.04 .2003\right]\right)$, $<$ http://www.mahoneythecat.org/Discussion/forum/start.asp?forumid $=3 \&$ select $=490 \&>$.

Luesebrink, Marjorie Coverley, Marjorie Coverley Luesebrink: A Virtual, Vertical Bio (06.01.1997), <http://califia.hispeed.com/bio.htm>.

Luesebrink, Marjorie Coverley, Hg., $M$ is for Nottingham? - A Mystery Solved, $<$ http://califia.hispeed.com/IncubationDrama/museumtoc.htm $>$.

- Hg., M is for Nottingham? - Characters, $<$ http://califia.hispeed.com/Incubation2/incharacters.htm $>$.

- Hg., M is for Nottingham? - Green's Mill, $<$ http://califia.hispeed.com/Incubation2/sGMill.htm>.

- Hg., M is for Nottingham? - Newstead Abbey,

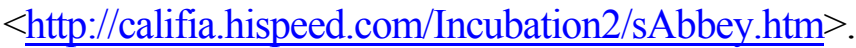

- Hg., M is for Nottingham? - Nottingham Castle,

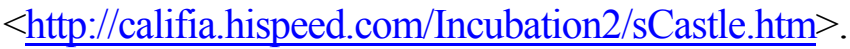

- Hg., M is for Nottingham? - Our Sponsors, $<$ http://califia.hispeed.com/Incubation2/sponsors.htm $>$.

- Hg., M is for Nottingham? - Sherwood Forest,

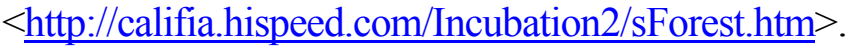

- Hg., M is for Nottingham? - The Ancient Caves Beneath the City, $<$ http://califia.hispeed.com/Incubation2/sCaves.htm $>$.

- Hg., M is for Nottingham? - The Council House and Square, $<\underline{\mathrm{http}}$ //califia.hispeed.com/Incubation2/sCouncil.htm>.

- Hg., M is for Nottingham? - The Denouement at trAce Incubation 2 at Nottingham Trent University, $<$ http://califia.hispeed.com/IncubationDrama/npdenouement.htm $>$.

- Hg., M is for Nottingham? - The Galleries of Justice, $<$ http://califia.hispeed.com/Incubation2/sGalleries.htm $>$.

- Hg., M is for Nottingham? - The Garden, $<$ http://califia.hispeed.com/Incubation2/sGarden.htm $>$. 
- Hg., M is for Nottingham? - The Great Hall, $<\underline{\mathrm{http}}$ //califia.hispeed.com/Incubation2/sGreatHall.htm $>$.

- Hg., M is for Nottingham? - The Sites, $<$ http://califia.hispeed.com/Incubation2/insite.htm $>$.

- Hg., Mis for Nottingham? - The University, $<$ http://califia.hispeed.com/Incubation2/sUniver.htm $>$.

- Hg., M is for Nottingham? - Written Text of the Discussion List, $<$ http://califia.hispeed.com/IncubationDrama/discussiontext.htm $>$.

- Hg., M is for Nottingham? - Ye Olde Trip to Jerusalem Pub, $<$ http://califia.hispeed.com/Incubation2/sTrip.htm>.

Luesebrink, Marjorie Coverley u.a., "Borges Clue 1", in: Marjorie Coverley Luesebrink, Hg., M is for Nottingham?, $<$ http://califia.hispeed.com/Incubation2/borges.htm $>$.

- "Critical Review", in: Marjorie Coverley Luesebrink, Hg., $M$ is for Nottingham?, $<$ http://califia.hispeed.com/IncubationDrama/criticalreview.htm $>$.

- "Critical Review: Audience", in: Luesebrink, Hg., M is for Nottingham?, $<$ http://califia.hispeed.com/IncubationDrama/criticalreview.htm\#Audience>.

- "Critical Review - Audience (Conference Attendees)", in: Luesebrink, Hg., M is for Nottingham?, $<\underline{\text { ttp }}$ //califia.hispeed.com/IncubationDrama/criticalreview.htm\#Conferenceattendees $>$.

- "Critical Review: Audience (Participating Writers)", in: Luesebrink, Hg., M is for Nottingham?,

$<\underline{\text { http://califia.hispeed.com/IncubationDrama/criticalreview.htm\#Participatingwriters }>}$.

- "Critical Review: Time Frames (Performance Event)", in: Luesebrink, Hg., $M$ is for Nottingham?, $<\underline{\text { http://califia.hispeed.com/IncubationDrama/criticalreview.htm\#Performanceevent }>}$.

- "Critical Review: Time Frames (Writing Period)", in: Luesebrink, Hg., $M$ is for Nottingham?, $<$ http://califia.hispeed.com/IncubationDrama/criticalreview.htm\#Writingperiod $>$.

- "Description of the ' $\mathrm{M}$ is for Nottingham?' Project", in: Marjorie Coverley Luesebrink, Hg., M is for Nottingham?, $<$ http://califia.hispeed.com/Incubation2/inproject1.htm $>$.

- "Further Sherwood Forest Adventures 2", in: Luesebrink, Hg., M is for Nottingham? - Sherwood Forest, <http://califia.hispeed.com/Incubation2/sForest2.htm>.

- "Green's Mill und Surround", in: Luesebrink, Hg., M is for Nottingham? The Denouement at trAce Incubation 2 at Nottingham Trent University, $<$ http://califia.hispeed.com/IncubationDrama/npsofar7.htm $>$.

_ “Join In", in: Luesebrink, Hg., M is for Nottingham?, $<$ http://califia.hispeed.com/Incubation2/injoin.htm $>$.

- "Nedd Ludd" in: Luesebrink, Hg., M is for Nottingham? - The New Characters,

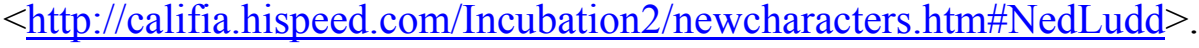

- "The Ancient Valley of Snottingham", in: Luesebrink, Hg., M is for Nottingham? - The Denouement at trAce Incubation 2 at Nottingham Trent University, $<\underline{\text { http://califia.hispeed.com/IncubationDrama/npsofar2.htm }>\text {. }}$ 
- "The Denouement", in: Luesebrink, Hg., $M$ is for Nottingham?, $<$ http://califia.hispeed.com/IncubationDrama/npdenouement.htm $>$.

- "The Resolution 1", in: Luesebrink, Hg., M is for Nottingham? - The Denouement at trAce Incubation 2 at Nottingham Trent University, $<$ http://califia.hispeed.com/IncubationDrama/npresoll.htm $>$.

- "The Resolution 2", in: Luesebrink, Hg., M is for Nottingham? - The Denouement at trAce Incubation 2 at Nottingham Trent University, $<$ http://califia.hispeed.com/IncubationDrama/npresol2.htm $>$.

- "The Resolution 3", in: Luesebrink, Hg., M is for Nottingham? - The Denouement at trAce Incubation 2 at Nottingham Trent University, $<$ http://califia.hispeed.com/IncubationDrama/npresol3.htm $>$.

- "The Resolution 4", in: Luesebrink, Hg., M is for Nottingham? - The Denouement at trAce Incubation 2 at Nottingham Trent University,

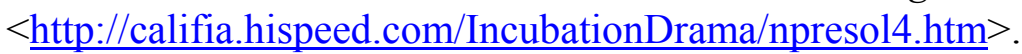

- "The Story", in: Marjorie Coverley Luesebrink, Hg., M is for Nottingham?, $<$ http://califia.hispeed.com/Incubation2/story.htm $>$.

- "The Story So Far", in: Luesebrink, Hg., $M$ is for Nottingham?, $<\underline{\text { http://califia.hispeed.com/Incubation2/frame4.htm }>\text {. }}$

- "Welcome", in: Marjorie Coverley Luesebrink, Hg., M is for Nottingham? The Live Collaborative Mystery Drama for trAce Incubation 2,

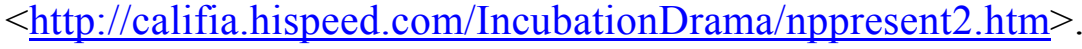

- "Ye Olde Trip to Jerusalem", in: Luesebrink, Hg., M is for Nottingham? - The Denouement at trAce Incubation 2 at Nottingham Trent University, $<$ http://califia.hispeed.com/IncubationDrama/npsofar6.htm $>$.

- "Your Invitation: How to participate", in: Marjorie Coverley Luesebrink, Hg., $M$ is for Nottingham?, < $\underline{\text { http://califia.hispeed.com/Incubation2/welcome.htm>. }}$.

Luserke, Matthias, "Kultur, Literatur, Medien. Aspekte einer verwickelten Beziehung", in: Renate Glaser und Matthias Luserke, Hg., Literaturwissenschaft - Kulturwissenschaft: Positionen, Themen, Perspektiven (Opladen, 1996), 169-191.

Maczewski, Jan-Mirko, Studium digitale. Geisteswissenschaften und WWW (Hannover, 1996).

Magdalene College, Hg., Pepys Library, <http://www.magd.cam.ac.uk/pepys/>.

Make-A-Wish Foundation, Hg., Make A Wish, <http://www.wish.org/>.

Martin, Sigurd, "Nachwort", in: Sven Birkerts, Die Gutenberg-Elegien: Lesen im elektronischen Zeitalter, übers. von Kurt Neff, (Frankfurt a.M., 1997 [The Gutenberg Elegies. The Fate of Reading in an Electronic Age (New York, 1994)]), 310-320.

Marx, Thomas Christoph, "Zur Problematik des Zitierens aus dem Internet: Anforderungen, Möglichkeiten und Grenzen - Tabelle 2: Zitier-Muster und Beispiele", in: Albert-Ludwigs-Universität Freiburg, Historisches Seminar: Forum, $<$ http://www.geschichte.uni-freiburg.de/histsem/forum/zitieren.html\#tabelle2>, S.9f. Als Vorabversion erschienen: Thomas Christoph Marx, "Zur Problematik des Zitierens aus dem Internet: Anforderungen, Möglichkeiten und Grenzen", Geschichte in Wissenschaft und Unterricht (GWU), 52 (2001), 238-245. 
- "Zur Problematik des Zitierens aus dem Internet: Anforderungen, Möglichkeiten und Grenzen - Übersicht 2: Problemfelder bei Nachweisen aus dem Internet", in: Albert-Ludwigs-Universität Freiburg, Historisches Seminar: Forum, $<$ http://www.geschichte.uni-freiburg.de/histsem/forum/zitieren.html $>$, S.12. In Kurzfassung als Vorabversion erschienen: Thomas Christoph Marx, "Zur Problematik des Zitierens aus dem Internet: Anforderungen, Möglichkeiten und Grenzen", Geschichte in Wissenschaft und Unterricht (GWU), 52 (2001), 238245.

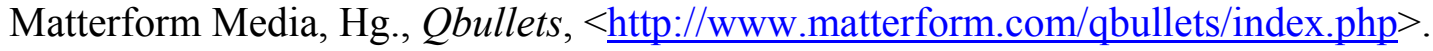

Maximus, Toddius, "Biography”, in: Prosebush.com, Hg., Prosebush,

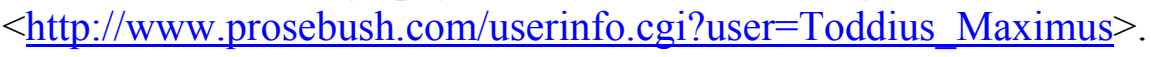

Mayer, Ruth und Ernst-Peter Schneck, "Hyperkultur - die ganze Welt ist ein Text. Eine Einleitung", in: Martin Klepper, Ruth Mayer und Ernst-Peter Schneck, Hg., Hyperkultur: Zur Fiktion des Computerzeitalters (Berlin; New York, 1996), 1-13.

McClelland, John, “Grace Neill's”, in: Irish Country Sports and Country Life, Hg., Countrysports and Countrylife.com,

$<$ http://www.countrysportsandcountrylife.com/sections/restaurants/grace-neills/ $>$.

McCready, Michael P., "What is a work for hire?", in: Ders., Hg., Music Law Offices, $<$ http://www.music-law.com/workforhire.html $>$.

Meckel, Miriam, "Schleusenwärter in Digitalien”, Zeit-Punkte 5: Der Mensch im Netz: Kultur, Kommerz und Chaos in der digitalen Welt (1996), 64.

Mengel, Ewald, "Drama- und Theaterressourcen im Internet", in: Doris Feldmann, Fritz-Wilhelm Neumann und Thomas Rommel, Hg., Anglistik im Internet. Proceedings of the 1996 Erfurt Conference on Computing in the Humanities (Heidelberg, 1997), 157-182.

Modick, Klaus, "Textnetzwerke. Prolegomena zu einer Literatur des Bildschirmtextes", in: Ders., Hg., Das Stellen der Schrift. Essays (Siegen, 1988), 91-104.

Möbius, Michael Uwe, "Elektronische Zeitschriften über Internet”, in: Helmut Jüngling, Hg., Internet und Bibliotheken: Entwicklung - Praxis - Herausforderung (Köln, 1995), 136-169.

Modern Language Association, Hg., MLA Style: Citing Sources from the World Wide Web (14.05.1998 [ $\left.\left.{ }^{1} 07.04 .1998\right]\right),<$ http://www.mla.org/main stl.htm>.

- MLA Style: How do I document sources from the World Wide Web in my workscited list?,

$<$ http://www.mla.org/www mla org/style/style main.asp?level=2\&mode=page \&page $=1 \&$ link $=$ sty $72800121438 \&$ section $=$ sty $51800124510>$.

Möbius, Ralf, "Urheberrechte im Internet", in: Ralf Möbius, Hg., Internet-Rechtonline, $<\underline{\text { http://mitglied.lycos.de/InternetRechtOnline/urhr.htm }>\text {. }}$

Möckel-Rieke, Hannah, "Der virtuelle Text", in: Martin Klepper, Ruth Mayer und Ernst-Peter Schneck, Hg., Hyperkultur: Zur Fiktion des Computerzeitalters (Berlin; New York, 1996), 68-80.

Morgan, Eric Lease, Hg., Alex Catalogue of Electronic Texts,

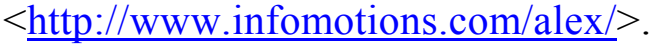


MOTCO Enterprises Limited, "John Rocque London, Westminster and Southwark, First Edition 1746 (26 inches to a mile); Segment 81", in: Dies., Hg., MOTCO UK Directory and Image Database - Antique Maps, Prints \& Books ([ $\left.\left.{ }^{1} 2001\right]\right)$, $<$ http://www.motco.com/Map/81002/SeriesSearchPlatesFulla.asp?mode=query \&artist $=384 \&$ other $=315 \& \mathrm{x}=11 \& \mathrm{y}=11>$.

- "John Rocque London, Westminster and Southwark, First Edition 1746 (26 inches to a mile); Segment 87", in: Dies., Hg., MOTCO UK Directory and Image Database - Antique Maps, Prints \& Books ([ $\left.\left.{ }^{1} 2001\right]\right)$,

$<$ http://www.motco.com/map/81002/SeriesSearchPlatesFulla.asp?mode=query\& title $=$ Seething + Lane \&artist $=384 \&$ other $=321 \& \mathrm{x}=11 \& \mathrm{y}=11>$.

- "John Rocque London, Westminster and Southwark", in: Dies., Hg., MOTCO UK Directory and Image Database - Antique Maps, Prints \& Books ([ $\left.\left.{ }^{1} 2001\right]\right)$, $<$ http://www.motco.com/MapImages/81002/81002113.jpg $>$.

Moulthrop, Stuart und Nancy Kaplan, "They became What They Beheld: The Futility of Resistance in the Space of Electronic Writing", in: Cynthia L. Selfe und Susan Hilligoss, Hg., Literacy and Computers: The Complications of Teaching and Learning with Technology (New York, 1994), 220-237.

Moynihan, David, "Distributed Proofing site goes through the roof: One Page Per

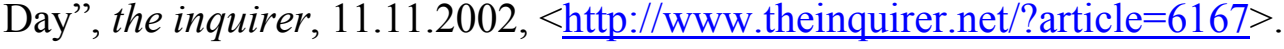

Müller, Walter, "Literatur im technisch-elektronischen Zeitalter", Merkur 402 (1981), $1270-1277$.

Murphy, Helena, "Annotation on "Admiralty", in: Gyford, Hg., The Diary of Samuel Pepys ([ $\left.\left.{ }^{1} 15.03 .2003\right]\right),<$ http://www.pepysdiary.com/p/297.php $>$.

National Library of Canada, Hg., ISO 690-2: Information and documentation Bibliographic references - Part 2: Electronic documents or parts thereof - 5: Outline of Bibliographic References (22.08.2002), $<\underline{\text { http://www.nlc- }}$ bnc.ca/iso/tc46sc9/standard/690-2e.htm\#5>.

National Portrait Gallery, Smithsonian Institution, "Junius Brutus Booth, 1796-1852", in: Dies., Hg., Mathew Brady's Portraits: Images as History, Photography as Art, $<$ http://www.npg.si.edu/ exh/brady/gallery/23gal.html $>$.

Nestvold, Ruth, "Das Ende des Buches: Hypertext und seine Auswirkungen auf die Literatur", in: Martin Klepper, Ruth Mayer und Ernst-Peter Schneck, Hg., Hyperkultur: Zur Fiktion des Computerzeitalters (Berlin; New York, 1996), 14-30.

- "Der neue Autor. Die elektronische Gemeinschaft und das Ende des einsamen Schriftstellers", in: Julika Griem, Hg., Bildschirmfiktionen: Interferenzen zwischen Literatur und neuen Medien (Tübingen, 1998), 205-221.

Neumann, Fritz-Wilhelm, "Geisteswissenschaften im Internet - Möglichkeiten und Grenzen einer neuen Technologie", in: Doris Feldmann, Fritz-Wilhelm Neumann und Thomas Rommel, Hg., Anglistik im Internet. Proceedings of the 1996 Erfurt Conference on Computing in the Humanities (Heidelberg, 1997), 47-72.

Nicholson, Andrew, "Meaning and Origin of the Words: Shire and County", in: Ders., Hg., Nottinghamshire History and Archaeology - Nottingham and Nottinghamshire (09.05.2003), <http://www.nottshistory.org.uk/swinnerton1910/chapter1.htm>.

Nielsen, Jakob, Multimedia and Hypertext: The Internet and beyond (Boston, 1995).

"Nottingham Castle", Sherwood Times, $<$ http://www.times1190.freeserve.co.uk/castle.htm>. 
Nottinghamshire County Council, Nottinghamshire County Council Homepage (23.10.2003), < http://www.nottscc.gov.uk/>.

- Official Visitors' Guide to Nottinghamshire, <http://www.robinhood.co.uk/index.htm>.

Nottingham Trent University/Museum of Law, SOLON - Interdisciplinary Studies in Bad Behaviour and Socially Visible Crime, $<$ http://solon.ntu.ac.uk/home.htm>.

Ockerbloom, John Mark, Hg., The Online Books Page - Banned Books Online ([ $\left.\left.{ }^{1} 1993\right]\right),<$ http://digital.library.upenn.edu/books/banned-books.html>.

- Hg., The Online Books Page - Prize-Winning Books On-Line ([ $\left.\left.{ }^{1} 1993\right]\right)$, $<$ http://digital.library.upenn.edu/books/prize.html $>$.

Ortmann, Sabrina, netz literatur projekt: Entwicklung einer neuen Literaturform von 1960 bis heute (Berlin, 2001)

Pauline, "Annotation on 'Seething Lane", in: Gyford, Hg., The Diary of Samuel Pepys ([11.07.2003]), <http://www.pepysdiary.com/p/1023.php $>$.

Payer, Margarete, Grundlagen der Formalerschließung: Skript-Kap. 1.3.: Welche Informationen sollen erfaßt werden? (18.10.1999),

$<$ http://www.payer.de/grundlagenfe/fegscr01.htm>.

Penfold, Margaret, "Comment", in: Luesebrink, Hg., M is for Nottingham? Comments by Writers and Readers, $<$ http://califia.hispeed.com/IncubationDrama/commentary.htm\#Writers: $>$.

Pipsqueak Productions, Pipsqueak Productions - New Media Creators, $<\underline{\text { http://www.pipsqueak.com/>. }}$.

- "Educational Philosophy", in: Dies., Hg., Pipsqueak Productions, $<$ http://www.pipsqueak.com/Educational_Philosophy-fs.html $>$.

- Hg., The Company Therapist $\left(\left[{ }^{1} 1996\right]\right),<$ http://www.thetherapist.com>.

- Hg., The Company Therapist-Authors, $<\underline{\mathrm{http}}$ ://www.thetherapist.com/Authors_All.html $>$.

- Hg., The Company Therapist - Backstage, $<\underline{\mathrm{http}} / / / \mathrm{www} \cdot$ thetherapist.com/Backstage Frontscreen.html $>$.

- Hg., The Company Therapist - Diaries \& Logs, $<$ http://www.thetherapist.com/Balis Log.html $>$.

- Hg., The Company Therapist - Doctor's Schedule, $<$ http://www.thetherapist.com/Appointments.html $>$.

- Hg., The Company Therapist - Dr. Charles Balis' Files, $<\underline{\mathrm{http}}$ ://www.thetherapist.com/File_Cabinet.html $>$.

- Hg., The Company Therapist - File Cabinet, $<$ http://www.thetherapist.com/File Cabinet.html $>$.

- Hg., The Company Therapist - Original Front Page,

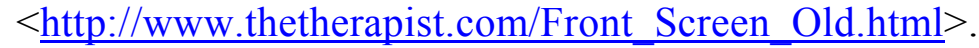

- Hg., The Company Therapist - Patient Form, $<$ http://www.thetherapist.com/Booth File.html $>$.

- Hg., The Company Therapist - Professional Correspondence, $<\underline{\mathrm{http}}$ ://www.thetherapist.com/TCTSeason1/Letter From HMO 071796.html $>$.

- The Company Therapist - Sponsors Page, $<\underline{\mathrm{http}}$ :/Www.thetherapist.com/Sponsors_Page_2.html $>$. 
- "Agent John Kline of the FBI regarding Joseph Mazurka", in: Dies., Hg., The Company Therapist - Transcripts ([103.12.1996]),

$<$ http://www.thetherapist.com/TCTSeason1/Mazurka_Rele_120396.html>.

- "Charles Balis' Journal for the Week ending 7/19/96", in: Dies., Hg., The Company Therapist - Diaries \& Logs,

$<$ http://www.thetherapist.com/TCTSeason1/DrNotes_071996.html $>$.

- "Credits Page", in: Dies., Hg., The Company Therapist,

$<$ http://www.thetherapist.com/Credits_Page.html $>$.

- "Educational Principles and Considerations: Collaborative Hyperdrama", in: Dies., Hg., The Company Therapist,

$<$ http://www.thetherapist.com/Explanation.html\#anchorCollaborativeHyperdrama $>$.

- "Educational Principles and Considerations: Conceptual Changes", in: Dies., Hg., The Company Therapist,

$<$ http://www.thetherapist.com/Explanation.html\#anchorConceptualChanges $>$.

- "Newspaper Article on Joseph Mazurka's Death", in: Dies., Hg., The Company Therapist-Article ([118.12.1996]),

$<$ http://www.thetherapist.com/TCTSeason1/Mazurka_Rele_121896.html $>$.

- "Postcard from Helen Gregory", in: Dies., Hg., The Company Therapist Correspondence ([127.09.1996]),

$<$ http://www.thetherapist.com/TCTSeason1/Greg_Letter_092796.html $>$.

- "Submission Guidelines", in: Dies., Hg., The Company Therapist, $<$ http://www.thetherapist.com/Submission_Guidelines.html $>$.

- "The Company Therapist in General: A New Form", in: Dies., Hg., The Company Therapist, $<$ http://www.thetherapist.com/Explanation.html\#anchorNewForm>.

- "The Company Therapist in General: The Premise", in: Dies., Hg., The Company

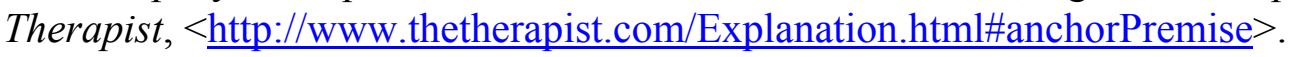

- "The Company Therapist in General: Two Goals", in: Dies., Hg., The Company Therapist, $<$ http://www.thetherapist.com/Explanation.html\#anchorTwoGoals $>$.

- “The Company Therapist in General: Write for Free?", in: Dies., Hg., The Company

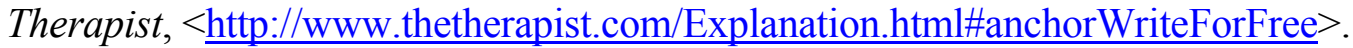

- "Transcript of 1st Session between Charles Balis, M.D. and Mr. Darius Booth", in: Dies., Hg., The Company Therapist - Darius Booth Transcripts $\left(\left[{ }^{1} 05.10 .1998\right]\right)$, $<$ http://www.thetherapist.com/Booth_Session_100598.html $>$.

- "Transcript of 3rd Session between Charles Balis, M.D. and Mr. Darius Booth", in: Dies., Hg., The Company Therapist - Darius Booth Transcripts ([ $\left.\left.{ }^{1} 19.10 .1998\right]\right)$, $<$ http://www.thetherapist.com/Booth Session 101998.html $>$.

- "Work for Hire Agreement", in: Dies., Hg., The Company Therapist, $<$ http://www.thetherapist.com/Contract.html $>$.

PoetryPoem.com, Hg., PoetryPoem.com, <http://poetrypoem.com/>.

- PoetryPoem.com - More Info, $<$ http://poetrypoem.com/info.htm>.

Politycki, Matthias, "Auch ein Schock. Autoren online: der Zukunftsroman? - HannaSophia Bucher im Interview mit dem Autor Matthias Politycki”, Spiegel special 3: Info-Sucht-Der Mensch im Netz der Medien (1999), 118.

Popham, Michael, Hg., The Oxford Text Archive (12.02.2003), $<$ http://ota.ahds.ac.uk/ $>$. 
- The Oxford Text Archive - About the Oxford Text Archive (12.02.2003), $<$ http://ota.ahds.ac.uk/>.

Prosebush.com, Hg., PERL Poetry - Story Map ([101.09.2000]), $<\underline{\mathrm{http}}$ //www.prosebush.com/storymap.cgi?NID=79>.

- Hg., Prosebush - Collaborative Fiction Community, <http://www.prosebush.com/>.

Pross, Harry, Der Mensch im Mediennetz: Orientierung in der Vielfalt (Düsseldorf; Zürich, 1996).

- Publizistik: Thesen zu einem Grundcolloquium (Neuwied; Berlin, 1970).

Redelius, Jürgen, Der 'digitale' Gutenberg: Untersuchungen zur Lesbarkeit digitaler Bildschirmschriften (Ludwigsburg, 1998).

Regia Anglorum Publications, Regia Anglorum - Anglo-Saxon, Viking, Norman and British Living History, <http://www.regia.org/>.

Reif, Holger, "Datensicherheit in vernetzten Systemen", in: Doris Feldmann, FritzWilhelm Neumann und Thomas Rommel, Hg., Anglistik im Internet. Proceedings of the 1996 Erfurt Conference on Computing in the Humanities (Heidelberg, 1997), 85-98.

Rendell, Jon, "Exquisite Corpse - Definition”, in: Ders., Hg., Exquisite Corpse Exquisite Links, <http://www.exquisitecorpse.com/definition.html $>$.

Rhizome.org, “cyberpoetry 1995-1997: Komninos Zervos”, in: Dies., Hg., Rhizome. org - The New Media Art Resource ([101.01.1995]),

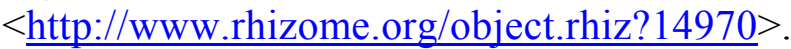

Rojan, Norbert und Joachim Schroth, "Leser als Buchkäufer: Wie kommt der Mensch zum Buch?", in: Stiftung Lesen, Hg., Leseverhalten in Deutschland im neuen Jahrtausend: eine Studie der Stiftung Lesen (Hamburg, 2001), 85109.

Rost, Martin und Michael Schack, Hg., Der Internet-Praktiker: Referenz und Programme (Hannover, 1995).

Rotermund, Hermann, "Warten auf den Online-Ulysses: Über das Problem des Internet, eine eigene Kunstform zu finden”, Die Zeit 41 (1997), 78.

Ruch, Allen B., Hg., The Modern Word - Jorge Luis Borges: The Garden of Forking Paths: Quotations ([ $\left.\left.{ }^{1} 27.01 .2003\right]\right)$,

$<$ http://www.themodernword.com/borges/borges quotes.html $>$.

Rumsey, Kenneth, Hg., The Beat Page, <http://www.rooknet.com/beatpage/>.

Runkehl, Jens und Torsten Siever, Das Zitat im Internet: Ein Electronic Style Guide zum Publizieren, Bibliografieren und Zitieren (Hannover, $\left.2001\left[^{1} 2000\right]\right)$.

Rutenfranz, Uwe, Wissenschaft im Informationszeitalter: Zur Bedeutung des Mediums Computer für das Kommunikationssystem Wissenschaft (Opladen, 1997).

Samuel Pepys Club, Samuel Pepys Club, <http://www.pepys-club.org.uk/>.

- Samuel Pepys Club - Major tercentenary events and exhibitions, $<$ http://www. pepys-club.org.uk/events.html>.

Sand, Thomas und Kay Wahlen, Mediennutzungskonzepte im Hochschulbereich: Planung, Organisation, Strategien (Hannover, 2000).

Schäfer, Gabriele und Martin Stengel, Hg., Vollgas auf der Datenautobahn? Perspektiven digitaler Telekommunikation (München, 1996). 
Schäffer, Vera, Hg., Deutsche Klassiker von Andersen bis Wieland im Internet und auf CD-ROM,

$<\underline{\text { http://www.leu.bw.schule.de/beruf/projektg/online/news4/tips/cdtip.htm>. }}$.

Schanze, Helmut, "Was kann ein Geisteswissenschaftler mit einem Informationsnetz anfangen? Vorüberlegungen zu einer Nutzungstheorie eines 'Neuen Mediums'", in: Helmut Jüngling, Hg., Internet und Bibliotheken: Entwicklung - Praxis Herausforderungen (Köln, 1995), 102-111.

Schellong, Marcel, “'how to ...': DOI - Digital Object Identifier”, in: Martin Huber u.a., Hg., Zentrum Elektronisches Publizieren in den Literaturwissenschaften ([127.08.2003]), < http://www.zepl.uni-muenchen.de/reports/how to doi.htm>.

Schmidt, Mareya und Peter Schmidt, "Komninos Constantine Zervos", in: Dies., Hg., The Ozlit Site ([ $\left.\left.{ }^{1} 26.01 .1996\right]\right)$, $<\underline{\text { http://blackdog.vicnet.net.au/ozlit/writers.cfm?id=914> }}$.

Schmundt, Hilmar, "Strom, Spannung, Widerstand: Hyperfictions - die Romantik des elektronischen Zeitalters", in Martin Klepper, Ruth Mayer und Ernst-Peter Schneck, Hg., Hyperkultur: Zur Fiktion des Computerzeitalters (Berlin; New York, 1996), 44-67.

Schneider, Irmela, “'Please Pay Attention Please'. Überlegungen zur Wahrnehmung von Schrift und Bild in der Medienkunst", in: Julika Griem, Hg., Bildschirmfiktionen: Interferenzen zwischen Literatur und neuen Medien (Tübingen, 1998), $223-243$.

Schön, Erich, "Kein Ende von Buch und Lesen: Entwicklungstendenzen des Leseverhaltens in Deutschland - Eine Langzeitbetrachtung", in: Stiftung Lesen, Hg., Lesen im Umbruch - Forschungsperpektiven im Zeitalter von Multimedia (Baden-Baden, 1998), 39-77.

Schreiber, Gerhard Andreas, Neue Wege des Publizierens: Das Handbuch zu Einsatz, Strategie und Realisierung aller elektronischen Medien (Braunschweig, 1997).

Schröder, Dirk, "Der Link als Herme und Seitensprung - Überlegungen zur Komposition von Webfiction", in: Suter und Böhler, Hg., Hyperfiction. Hyperliterarisches Lesebuch: Internet und Literatur (Basel; Frankfurt a.M., 1999), 4360 .

- “Was kann die Literatur für das Internet tun?", Universitas: Zeitschrift für interdisziplinäre Wissenschaft 54 (1999), 282-292.

Scotland On Line, "Venison Haunch", in: Dies., Hg., scotland online.com, $<$ http://www.scotlandonline.com/heritage/cookery_august_recipe two.cfm $>$.

Seidel, Chris, Heretical Rhyme Generator, <http://www.pangloss.com/seidel/Poem/>.

Seising, Rudolf, "Internet - neue Kulturtechnik zwischen den Kulturen”, in: Gudrun Gross, Uwe Langer und Rudolf Seising, Hg., Studieren und Forschen im Internet: Perspektiven für Wissenschaft, Wirtschaft, Kultur und Gesellschaft (Frankfurt/Main, 1997), 11-50.

Selfe, Cynthia L. und Susan Hilligoss, "Introduction", in: Selfe und Hilligoss, Hg., Literacy and Computers, 1-7.

Simanowski, Roberto, "Autorschaft in digitalen Medien. Eine Einleitung”, in: Ders., Hg., Digitale Literatur (München, 2001) [Text + Kritik: Zeitschrift für Literatur 152 (2001)], 3-21. 
- “Interfictions. Vom Schreiben im Netz", in: Ders., Hg., dichtung-digital, $<$ http://www.dichtung-digital.de/buch/einleitung.htm\#2 $>$.

Smart, Billy und Hannah E. Rudman, The Benefactor - Table of Scenes (30.04.1998), $<$ http://www.rhul.ac.uk/drama/research/benefactor/tabler.html\#table $>$.

Snyder, Ilana, "Page to Screen", in: Dies., Hg., Page to Screen: Taking literacy into the electronic era (London; New York, 1998), xx-xxxvi.

Sommer, Winfried, "E-learning: Schöne neue Welt des Lernens", MEORUM: Menschen, Erfolge, Organisationen, Resultate \& Märkte 3 (2001), 12-13.

Jens Sparschuh, "Ein Geschenk für den Leser? - Tatjana Blobel im Interview mit Jens Sparschuh”, Spiegel Spezial 10: Die Zukunft des Lesens - Vom Buch zum Internet (1999), 24.

Spiegel special 3: Info-Sucht-Der Mensch im Netz der Medien (1999).

Spiegel Spezial 10: Die Zukunft des Lesens - Vom Buch zum Internet (1999).

Staats- und Universitätsbibliothek Göttingen, "Dublin-Core-Metadata-Element-Set", in: Dies., Hg., Metadata Server - Einführung in Metadaten (27.03.2001 [ [1997]), $<$ http://www2.sub.uni-goettingen.de/intrometa.html $>$.

- "Was sind Metadaten?", in: Dies., Hg., Metadata Server - Einführung in Metadaten (27.03.2001 [ [ 1997$]),<$ http://www2.sub.uni-goettingen.de/intrometa.html >.

- "SSG-FI Metadatenkonzept (Template)", in: Dies., Hg., SSG-Fachinformation (SSG-FI) (28.04.1999 [ [1997]), $<\underline{\text { http://www.sub.uni-goettingen.de/ssgfi/projekt/doku/templ-de.html }>\text {. }}$

Sterz, Max, "marietta - novell [sic!] in progress", in: Ders., Hg., publizieren im internet (08.03.2001 [ $\left.\left.{ }^{1} 23.11 .2000\right]\right)$, $<$ http://www.lrz-muenchen.de/ piiseminar/2311nlmarietta.htm $>$.

Stoll, Clifford, Die Wüste Internet: Geisterfahrten auf der Datenautobahn, 3. Aufl., übers. von Hans Jörg Friedrich, (Frankfurt a.M., 1996 [ $\left.{ }^{1} 1996\right]$ [Silicon Snake Oil: Second Thoughts on the Information Highway, (New York, 1995)]).

Storrer, Angelika, "Vom Text zum Hypertext: Die Produktion von Hypertexten auf der Basis traditioneller wissenschaftlicher Texte", in: Dagmar Knorr und Eva-Maria Jakobs, Hg., Textproduktion in elektronischen Umgebungen (Frankfurt a.M., 1997), 121-139.

Suter, Beat, "Ein neues Literaturmilieu (zwischen Transfugalität und 'Eventualität')", in: Johannes Auer, Christiane Heibach und Beat Suter, Hg., netzliteratur.net: Netzliteratur - Internetliteratur - Netzkunst ([ $\left.\left.{ }^{1} 30.11 .2000\right]\right)$, $<$ http://www.netzliteratur.net/suter/kassel.htm>.

Suter, Beat und Michael Böhler, "Hyperfiction - ein neues Genre? Einleitung", in: Dies., Hg., Hyperfiction. Hyperliterarisches Lesebuch: Internet und Literatur (Basel; Frankfurt a.M., 1999). 7-25.

Tangens, Rena und padeluun, "Auf dem Weg zu einer Informationsgesellschaft", in: Enquête-Kommission 'Zukunft der Medien in Wirtschaft und Gesellschaft; Deutschlands Weg in die Informationsgesellschaft' Deutscher Bundestag, Hg., Medienkompetenz im Informationszeitalter (Bonn, 1997), 147-161.

The Gutenberg Litegraphic Society, Hg., Pictures and Stories - The Online Community of Creative Minds, <http://www.picturesandstories.org/>.

- Hg., Pictures and Stories: Awards, <http://www.picturesandstories.org/awards.html $>$. 
The National Park Service, “A History of John Wilkes Booth", in: Dies., Hg., Ford's Theatre - National Historic Site ([108.12.1998)], <http://www.nps.gov/foth/booth.htm>.

The Nottingham Trent University, Hg., The University of Nottingham Information Services - Manuscripts and Special Collections: D H Lawrence Resources, $<$ http://mss.library.nottingham.ac.uk/dhl home.html $>$.

- Hg., The University of Nottingham Information Services - D H Lawrence Resources: D H Lawrence Collections at the University of Nottingham, $<\underline{\mathrm{http}}$ ://mss.library.nottingham.ac.uk/dhl_collections.html $>$.

The University of Missouri-Kansas City Libraries, Hg., Guide to Evaluating Resources on the World Wide Web (18.09.2002),

$<$ http://www.umkc.edu/lib/Resources/webeval.htm $>$.

Tischer, Wolfgang, “'Anfangserscheinungen und Kinderkrankheiten' - Interview mit Dr. Hermann Rotermund über den Zeit-Literaturpreis '97 und die Lage der Netzliteratur", in: Ders., Verantw., Das Literatur-Café - Der literarische Treffpunkt im Internet, $<$ http://www.literaturcafe.de/berichte/roterm.shtml $>$.

Todesco, Rolf, "Hyperkommunikation - Schrift-Um-Steller statt Schriftsteller", in: Beat Suter und Michael Böhler, Hg., Hyperfiction. Hyperliterarisches Lesebuch: Internet und Literatur (Basel; Frankfurt a.M., 1999), 113-124.

Townsend, John Rowe, Written for Children: An Outline of English-language Children's Literature, 2., überarb. Aufl. (Harmondsworth, 1983 [ $\left.\left.{ }^{1} 1965\right]\right)$.

trAce Online Writing Centre, Hg., Incubation 2 - The 2nd trAce International Conference on Writing and the Internet, <http://trace.ntu.ac.uk/incubation/index2002.cfm>.

- Hg., trAce Online Writing Centre - About, <http://trace.ntu.ac.uk/about/index.cfm $>$.

Travis, Graham, "Annotation on 'Seething Lane'”, in: Gyford, Hg., The Diary of Samuel Pepys $\left(\left[{ }^{1} 11.07 .2003\right]\right),<\underline{\mathrm{http}}: / / \mathrm{www}$.pepysdiary.com/p/1023.php $>$.

Tremmel Verlag, Hg., Urheberrechte im Netz, $<$ http://www.tremmel.de/html/urheberrecht.htm $>$.

Trigg, Randell Hagner, A Network-Based Approach to Text Handling for the Online Scientific Community [Ph.D. diss. Maryland] (Maryland, 1983).

- A Network-Based Approach to Text Handling for the Online Scientific Community - Chapter 4: A Taxonomy of Link Types [Ph.D. diss. Maryland] (05.11.2001 $\left.\left[{ }^{1} 19.05 .1999\right]\right),<$ http://www.workpractice.com/trigg/thesis-chap4.html $>, 16.04 .2003$.

Tullius, Christiane, "Typologien der Leser und Mediennutzer", in: Stiftung Lesen, Hg., Leseverhalten in Deutschland im neuen Jahrtausend: eine Studie der Stiftung Lesen (Hamburg, 2001), 61-83.

“Tumbe Computerkids”, Der Spiegel 21 (2002), 187.

Uçkan, Alp, Was ist RSS?, in: Marianne Bunyan und Alp Uçkan, Hg., windhunde.de Das Windhunde-Portal (12.07.2003 [ $\left.\left.{ }^{1} 09.03 .2003\right]\right)$, $<$ http://www.windhunde.de/wasistrss.php $>$.

Urbach, Tilman, "Matthias Politycki: Ein Mann von vierzig Jahren”, in: BR-online, Hg., \{literatur + \} Lesezeichen, $<\underline{\mathrm{http}} / / / \mathrm{www}$. br-online.de/kultur/literatur/lesezeichen/20000220/20000220_1.html $>$.

Urbanek, Walter, lyrische signaturen: zeichen und zeiten im deutschen gedicht-eine anthologie mit einer einführung in die poetik des gedichts (Bamberg, 1995). 
Van Leeuwen, Steven H., Hg., Bartleby.com: Great Books Online ([ ${ }^{1}$ Dez. 1995]), $<$ http://www.bartleby.com/ $>$.

- Hg., Welcome to Bartleby.com: Great Books Online ([ ${ }^{1}$ Dez. 1995]), $<$ http://www.bartleby.com/sv/welcome.html $>$.

- Hg., Bartleby.com: Great Books Online: Mission Statement ([ ${ }^{1}$ Dez. 1995]), $<$ http://www.bartleby.com/press/profile.html $>$.

Vincent, "Annotation on 'Admiralty", in: Gyford, Hg., The Diary of Samuel Pepys

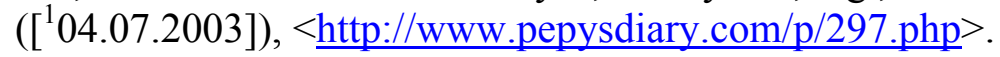

- "Annotation on 'Navy Office"”, in: Gyford, Hg., The Diary of Samuel Pepys ([101.07.2003]), <http://www.pepysdiary.com/p/483.php $>$.

- "Annotation on 'Seething Lane"”, in: Gyford, Hg., The Diary of Samuel Pepys ([105.07.2003]), <http://www.pepysdiary.com/p/1023.php $>$.

Walker, Janice R. und Todd Taylor, The Columbia Guide to Online Style (New York, NY; Chichester, West Sussex, 1998).

Wall, Larry, Tom Christiansen und Randal L. Schwartz, Programmieren mit Perl, übers. von Peter Klicman, (Köln, 1997 [Programming Perl (Cambridge, 1996)]).

Wallmannsberger, Josef, Virtuelle Textwelten: Theoretische Grundlagen und Implementationsperspektiven der anglistischen Computerphilologie (Heidelberg, 1994).

Warnke, Martin, "Informationstechnologie - das digitale Monopol", in: Claudia Gemmeke, Hartmut John und Harald Krämer, Hg., Euphorie digital? Aspekte der Wissensvermittlung in Kunst, Kultur und Technologie (Bielefeld, 2001), 21-33.

Weber, Hartmut und Gerald Maier, Hg., Digitale Archive und Bibliotheken: Neue Zugangsmöglichkeiten und Nutzungsqualitäten (Stuttgart; Berlin, 2000).

Wefers, Sabine, Hg., Von Gutenberg zum Internet/7. Deutscher Bibliothekskongreß, 87. Deutscher Bibliothekartag in Dortmund 1997, Zeitschrift für Bibliothekswesen und Bibliographie: Sonderheft 68 (Frankfurt a.M., 1997).

Wegener, Basil, "Chaos im Netz macht der Internet-Ökonomie zu schaffen", heise online, 26.07.2000, <http://www.heise.de/newsticker/data/jk-26.07.00-000>.

Wegmann, August, "Elektronische Bücher im Internet: Modell und standardisiertes Werkzeug zur Präsentation sequentiell abfolgender Seiten", in: Hartmut Weber und Gerald Maier, Hg., Digitale Archive und Bibliotheken: Neue Zugangsmöglichkeiten und Nutzungsqualitäten (Stuttgart; Berlin; Köln, 2000), 287-296.

Wenz, Karin, "Die unerträgliche Leichtigkeit des Textes", in: Julika Griem, Hg., Bildschirmfiktionen: Interferenzen zwischen Literatur und neuen Medien (Tübingen, 1998), 245-254.

Werby, Christoper, "Message Board is Back Up", in: Pipsqueak Productions, Hg., The Company Therapist $\left(\left[^{1} 26.09 .2003\right]\right)$,

$<$ http://www.thetherapist.com/cgi/BBS2/config.pl?read=894>.

Widger, David, "Entire Gutenberg Edition of the Diary of Samuel Pepys", in: Center for the Public Domain/University of North Carolina - Chapel Hill, Hg., ibiblio - the public's library ans digital archive (Juni 2003 [ $\left.{ }^{1} 12.12 .2001\right]$ ), $<\underline{\text { http://www.ibiblio.org/gutenberg/etext03/sp85g10.txt }>}$.

- "Samuel Pepys - Unabridged Diary", in: Hart, Hg., Project Gutenberg, $<$ http://www. gutenberg.net.au/widger/home.html\#pepys $>$. 
Willamowski, Marcus, "Zitierfähigkeit von Internetseiten", JurPC Web-Dok. 78 (2000), Abs. 3, 22.05.2000, <http://www.jurpc.de/aufsatz/20000078.htm>.

Willett, Perry, Hg., Victorian Women Writers Project (19.01.2001 [ [1995]), $<$ http://www.indiana.edu/ letrs/vwwp $>$.

Wingert, Bernd, “Kann man Hypertexte lesen?”, in: Dirk Matejovski und Friedrich Kittler, Hg., Literatur im Informationszeitalter (Frankfurt a.M., 1996), 185-218.

- "Der Leser im Hypertext: Im Weinberg oder im Steinbruch?", in: Beat Suter und Michael Böhler, Hg., Hyperfiction. Hyperliterarisches Lesebuch: Internet und Literatur (Basel; Frankfurt a.M., 1999), 159-172.

Winter, Helmut, Literaturtheorie und Literaturkritik (Düsseldorf, 1975).

Wirth, Uwe, “Literatur im Internet. Oder: Wen kümmert's, wer liest?”, in: Stefan Münker und Alexander Roesler, Hg., Mythos Internet (Frankfurt a.M., 1997), 319-337.

Wishart, Martha, "Annotation on 'Navy Office'”, in: Gyford, Hg., The Diary of Samuel Pepys ([131.03.2003]), < http://www.pepysdiary.com/p/483.php>.

Wolf, Stefan, Mensch - Maschine - Metapher: Zur Exemplifikation des menschlichen Geistes durch den Computer. Eine wissenschaftsphilosophische Rekonstruktion der Kognitionswissenschaft als Technologie [Diss. Bamberg] (Bamberg, 1994).

Wooley, Benjamin, Die Wirklichkeit der virtuellen Welten, übers. von Gabriele Herbst, (Basel; Boston; Berlin, 1994 [Virtual Worlds (Oxford, 1992)]).

World Wide Web Consortium, Hg., Naming and Addressing: URIs, URLs,... (09.07.2002), <http://www.w3.org/Addressing/>.

Zervos, Komninos, cyberpoetry, $<$ http://www.gu.edu.au/ppages/k_zervos/>.

- The Ultimate Writer, <http://live-wirez.gu.edu.au/cyber/ultimate/index.html>.

- The Ultimate Writer - Body: Sound Poetry, $<$ http://live-wirez.gu.edu.au/cyber/ultimate/index.html $>$.

- The Ultimate Writer - Build: Haiku,

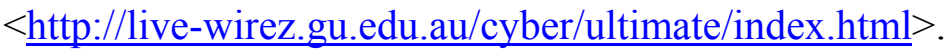

- The Ultimate Writer - Feet: Beat Poetry, $<$ http://live-wirez.gu.edu.au/cyber/ultimate/index.html $>$.

- "Avoid", in: Ders., Hg. The Ultimate Writer - Final Section,

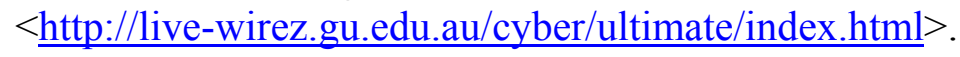

- "Be organised", in: Ders., Hg., The Ultimate Writer - Final Section, <http://livewirez.gu.edu.au/cyber/ultimate/index.html>.

- "Biography - Komninos Zervos", infLect - a journal of multimedia writing 1(2003),

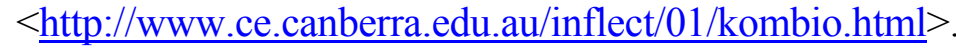

- "Control your breathing", in: Ders., Hg., The Ultimate Writer - Final Section, $<$ http://live-wirez.gu.edu.au/cyber/ultimate/index.html $>$.

- "Don't be frightened by the microphone", in: Ders., Hg., The Ultimate Writer Final Section, < $<$ ttp://live-wirez.gu.edu.au/cyber/ultimate/index.html $>$.

- “Don't go overtime", in: Ders., Hg., The Ultimate Writer - Final Section, $<$ http://live-wirez.gu.edu.au/cyber/ultimate/index.html $>$. 
- “easter offering”, in: Luigi-Bob Drake, Hg., Wr-eye-tings Scratchpad ([ ${ }^{1}$ Oktober 1997]), <http://www.burningpress.org/wreyeting/zervos/easter.html $>$.

- "Establish eye contact", in: Ders., Hg., The Ultimate Writer - Final Section, $<$ http://live-wirez.gu.edu.au/cyber/ultimate/index.html $>$.

- "How to read", in: Ders., Hg., The Ultimate Writer - Final Section, $<\underline{\text { http://live- }}$ wirez.gu.edu.au/cyber/ultimate/index.html>.

- "Intro", in: Ders., Hg., The Ultimate Writer,

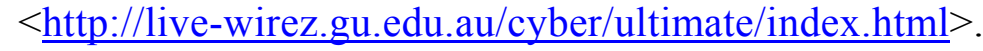

- "Performance", in: Ders., Hg., The Ultimate Writer - Final Section, < $\underline{\text { http://live- }}$ wirez.gu.edu.au/cyber/ultimate/index.html>.

- "Poetry - Cyber Beginnings", in: The Thylazine Foundation, Hg., Thylazine: Australian Arts and Literature on Landscape and Animals, $<$ http://www.thylazine.org/archive1/thyla6/thyla6f.html $>$.

Ziegfeld, Richard, "Interactive Fiction: A New Literary Genre?", New Literary History: a Journal of Theory and Interpretation 20 (1989), 341-372.

Ziegler, Joachim, Programmieren lernen mit Perl (Berlin, 2002).

Zimmer, Dieter E., Die Bibliothek der Zukunft: Text und Schrift in den Zeiten des Internet, 3. Aufl. (Hamburg, 2000 [ $\left.{ }^{1} 2000\right]$ ).

- "Die digitale Bibliothek: Eine fünfteilige Artikelserie für Nutzer und Verächter der Computernetze", in: Georg Ruppelt, Hg., Bibliothekspolitik in Ost und West: Geschichte und Gegenwart des Deutschen Bibliotheksverbandes. [Zeitschrift für Bibliothekswesen und Bibliographie: Sonderheft 72] (Frankfurt a.M., 1998), 265-317.

\section{Abbildungsnachweis}

Abb. 1: "PERL Poetry" - Strophe 1, in: Prosebush.com, Hg., Prosebush $\left(\left[{ }^{1} 28.08 .2000\right]\right),<\underline{\mathrm{http}}: / /$ www.prosebush.com/viewnode.cgi? $\mathrm{NID}=32>, 22.07 .2003$.

Abb. 2: "PERL Poetry" - Strophe 2a, in: Prosebush.com, Hg., Prosebush ([128.08.2000]), <http://www.prosebush.com/viewnode.cgi?NID=36>, 22.07.2003.

Abb. 3: "PERL Poetry" - Strophe 2b, in: Prosebush.com, Hg., Prosebush ([128.08.2000]), $<$ http://www.prosebush.com/viewnode.cgi?NID=79>, 22.07.2003.

Abb. 4: 'The Ultimate Writer - Surreal Poetry', in: Komninos Zervos, The Ultimate Writer, $<$ http://live-wirez.gu.edu.au/cyber/ultimate/index.html $>$, 28.07.2003.

Abb. 5: 'The Ultimate Writer - Performance', in: Komninos Zervos, The Ultimate Writer, <http://live-wirez.gu.edu.au/cyber/ultimate/index.html $>$, 10.09.2003.

Abb. 6: 'The Company Therapist' - Sprechzimmer, in: Pipsqueak Pipsqueak Productions, Hg., The Company Therapist-Backstage,

$<$ http://wwwthetherapist.com/Backstage Frontscreen.html $>, 02.10 .2003$.

Abb. 7: 'M is for Nottingham? - The Gardens', in: Marjorie Coverley Luesebrink, Hg., $M$ is for Nottingham? - The Gardens,

$<$ http://califia.hispeed.com/Incubation2/welcome.htm>, 28.10.2003. 
Abb. 8: 'M is for Nottingham? - Characters', in: Marjorie Coverley Luesebrink, Hg., $M$ is for Nottingham? - Characters, $<\underline{\text { http://califia.hispeed.com/IncubationDrama/nppresent4.htm }>, 28.10 .2003 . ~}$

Abb. 9: 'M is for Nottingham? - The Denouement', in: Luesebrink u.a., "The Resolution 4", in: Marjorie Coverley Luesebrink, Hg., M is for Nottingham? - The Denouement at trAce Incubation 2 at Nottingham Trent University,

$<\underline{\text { http://califia.hispeed.com/IncubationDrama/npresol4.htm }>, 27.10 .2003 . ~}$ 